This item was submitted to Loughborough's Research Repository by the author.

Items in Figshare are protected by copyright, with all rights reserved, unless otherwise indicated.

\title{
Experimental and computational study of hybrid diffusers for gas turbine combustors
}

PLEASE CITE THE PUBLISHED VERSION

PUBLISHER

(C) A.D. Walker

LICENCE

CC BY-NC-ND 4.0

REPOSITORY RECORD

Walker, Alastair Duncan. 2019. "Experimental and Computational Study of Hybrid Diffusers for Gas Turbine Combustors". figshare. https://hdl.handle.net/2134/5854. 
This item was submitted to Loughborough's Institutional Repository (https://dspace.lboro.ac.uk/) by the author and is made available under the following Creative Commons Licence conditions.

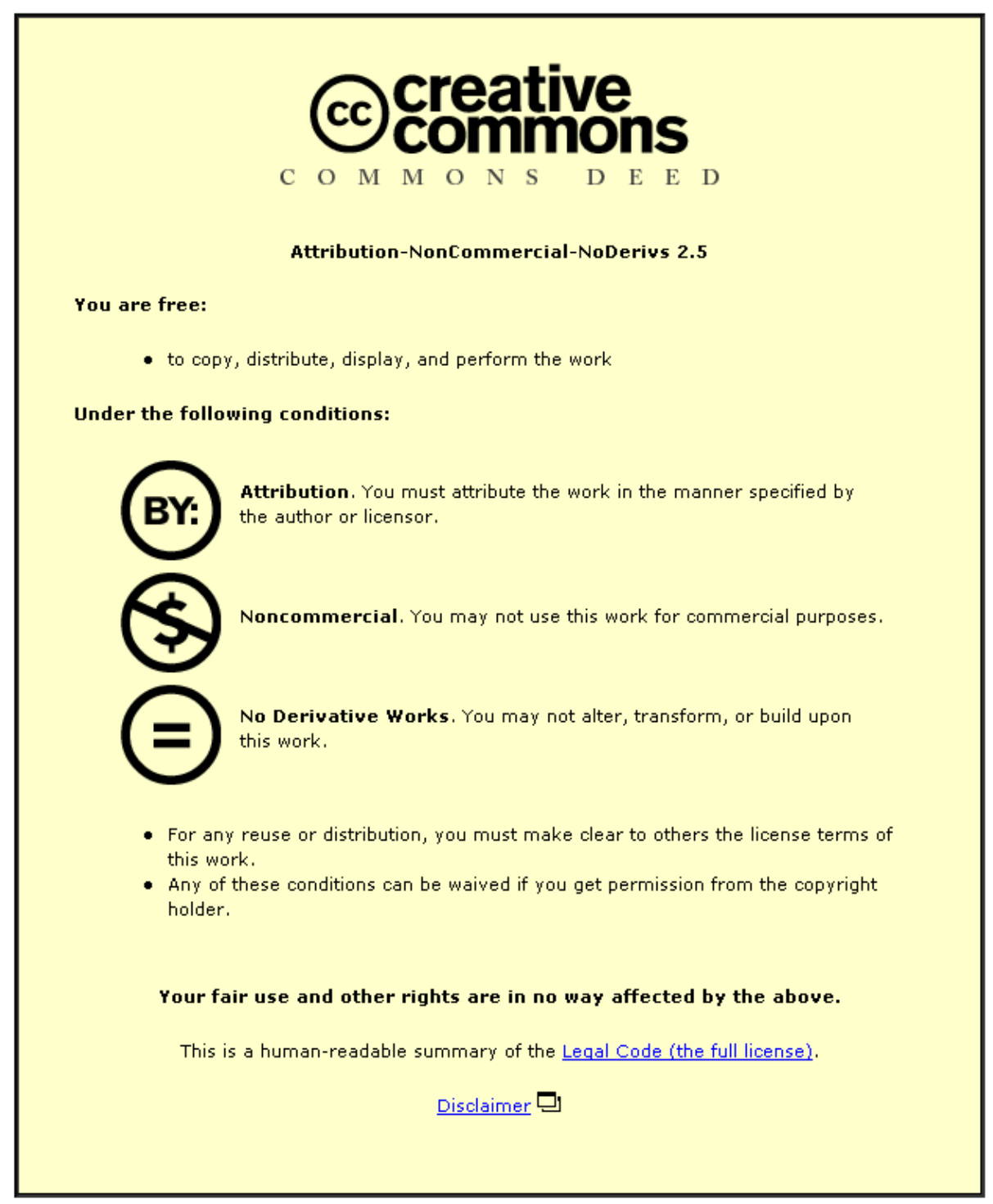

For the full text of this licence, please go to: http://creativecommons.org/licenses/by-nc-nd/2.5/ 


\title{
Experimental and Computational Study of Hybrid Diffusers for Gas Turbine Combustors
}

\author{
Alastair Duncan Walker
}

Submitted in partial fulfilment of the requirements

for the award of Doctor of Philosophy of Loughborough University

Department of Aeronautical and Automotive Engineering

2002

(C) A D Walker 2002 


\section{Experimental and Computational Study of Hybrid Diffusers For Gas Turbine Combustors}

Diffusers are essential in gas turbine combustors, decelerating the compressor efflux prior to the combustion chamber to reduce total pressure losses. Modern, low emission, radially staged combustors require even more diffusion due to the increased flame tube depth of this type of combustor. Furthermore, these high rates of deceleration are accompanied by large adverse pressure gradients and an associated risk of flow separation. Previous studies have shown that hybrid diffusers can achieve high rates of efficient diffusion in far shorter lengths than conventional faired diffusers or dump diffuser systems, representing a potential performance gain and weight saving. Hybrid diffusers consist of a wide angle diffuser immediately downstream of a sudden expansion, with flow separation prevented by bleeding off a small amount of the mainstream flow. However, previous studies have not provided a conclusive understanding of the associated flow mechanisms leading to hybrid diffusers currently being considered high risk. Additionally definitive data does not exist on the influence of bleed gap geometry and therefore hybrid diffusers cannot, currently, be optimised for use in a modern gas turbine. Further issues also not addressed by earlier studies, but concerning the use of hybrid diffuser in gas turbine combustors, are the effect of representative inlet conditions incorporating vane wakes at diffuser inlet, the quality of the bleed air and its potential for use for component cooling, the effect of radial struts within a hybrid diffuser and the quality of the flow delivered to the combustor feed annuli (total pressure losses). Therefore, a predominately experimental study, coupled with CFD predictions, was undertaken to investigate the controlling flow mechanisms of hybrid diffusers and address the questions necessary to evaluate the suitability of hybrid diffusers for use in modern, low emission, radially staged combustion systems.

An existing isothermal test facility was used comprising a fully annular, staged combustor downstream of a single stage axial compressor incorporating engine representative outlet guide vanes. Initial experimental work led to rig modifications which allowed a range of hybrid diffusers to be studied. To act as a benchmark the performance of a conventional single-passage, dump diffuser system was first studied. A hybrid diffuser demonstrated a $53 \%$ increase in area ratio within the same axial length as the conventional 
diffuser. Results showed that this hybrid diffuser achieved a $13 \%$ increase in static pressure recovery which, in turn, improved the feed to the combustor feed annuli and decreased total pressure loses by $25 \%$. Notably this brought the annulus losses within accepted target values; something the conventional diffuser system was unable to do. Additionally, it was clearly shown, in contradiction to previous studies, that bleeding air via a vortex chamber was not necessary. Bleeding air via a simple duct arrangement achieved the same results without altering the governing flow mechanisms.

To provide a better understanding of these flow mechanisms, a computational investigation was also undertaken. A commercial CFD code, Fluent, was used to solve the Reynolds averaged Navier-Stokes equations for an incompressible flow regime, employing a blended second order upwind/central differencing scheme and the SIMPLE pressure correction algorithm. The turbulence was modelled using the $\mathrm{k}-\varepsilon$ model in conjunction with a standard wall function. Several generic two-dimensional hybrid diffusers were studied in order to reveal the controlling flow mechanisms and enable optimisation of the bleed gap geometry. Importantly, this revealed that many features previously thought to contribute to the flow mechanisms were, in fact, unnecessary. A detailed examination of the flow field, including an analysis of the terms within the momentum equation, demonstrated that the controlling flow mechanisms were not simply a boundary layer bleed but involve a much more complex interaction between the accelerating bleed flow and the diffusing mainstream flow. Firstly, momentum is transferred from the accelerating bleed flow to the diffusing mainstream flow, enabling a fresh boundary layer to be formed on the diffuser wall which is sufficiently energetic to overcome the high rates of diffusion and high adverse pressure gradient. Secondly, the radial pressure gradient created by the bleed causes deflection of the mainstream flow which also transports higher momentum fluid into the boundary layer. Understanding this resulted in a greatly simplified design for the hybrid diffuser not only potentially reducing weight but also reducing bleed flow total pressure losses.

Predictions for a three-dimensional representation of the experimental facility displayed many similarities in the flow field and similar performance trends to the experimental data. However, predicted values of total pressure loss and static pressure recovery differed from experimental data and it was thought that this was due to an incomplete 
description of the turbulence ( $\mathrm{k}$ and $\varepsilon$ ) at inlet and/or known problems the k- $\varepsilon$ turbulence model has with predicting some unconfined flows. Nonetheless, three-dimensional predictions revealed an interaction between the OGV wake fluid and bleed flow causing localised, but small, modification of the flow mechanisms. Furthermore, it was shown that without the levels of turbulence produced downstream of an axial compressor the hybrid diffuser under study would, in fact, stall.

Overall, experimental and computational results obtained in the current research suggest that the performance of hybrid diffusers is more than satisfactory for use within lowemission, staged, gas turbine combustion systems. An understanding of the governing flow mechanisms and the effect of features such as OGV wakes or radial struts has lead to a more practical design of hybrid diffuser, simplifying the geometry and reducing bleed flow total pressure losses (increasing the possibility of this air being used for component cooling). 


\section{ACKNOWLEDGEMENTS}

A debt of gratitude is owed to my supervisors, Dr. Paul Denman and Dr. Adrian Spencer and to my Director of Research, Professor Jim McGuirk. Their knowledge, understanding, guidance and encouragement has been invaluable throughout my research. A good balance of professionalism and a sense of humour made the work both rewarding and enjoyable.

I would also like to express my appreciation to the other members of the Rolls-Royce/Loughborough University Technology Centre. Particularly to Dr. Jon Carrotte, Ashley Barker, John Luff, Nicola Hughes, Bill Rasmussen, Les Monk, Bill Niven and Dave Glover. Not only were they a source of help and advice but also friendship for which I am truly grateful.

Finally, to both family and friends who provide that necessary escape from work. 


\section{EXPERIMENTAL AND COMPUTATIONAL STUDY OF HYBRID DIFFUSERS FOR GAS TURBINE COMBUSTORS}

\section{TABLE OF CONTENTS}

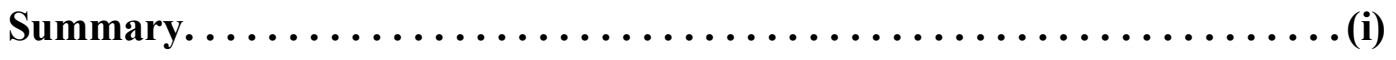

Acknowledgements $\ldots \ldots \ldots \ldots \ldots \ldots \ldots \ldots \ldots \ldots \ldots \ldots$ (iv)

Table Of Contents $\ldots \ldots \ldots \ldots \ldots \ldots \ldots \ldots \ldots \ldots \ldots \ldots \ldots \ldots \ldots \ldots \ldots \ldots \ldots$ (v)

List Of Figures $\ldots \ldots \ldots \ldots \ldots \ldots \ldots \ldots \ldots \ldots \ldots \ldots \ldots \ldots \ldots \ldots \ldots \ldots \ldots \ldots$ (x)

List Of Tables. . . . . . . . . . . . . . . . . . . . . . . (xx)

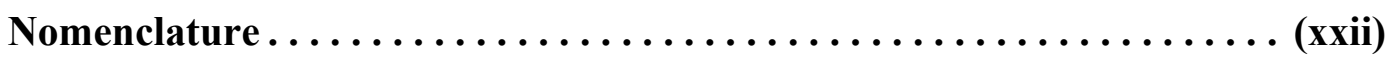

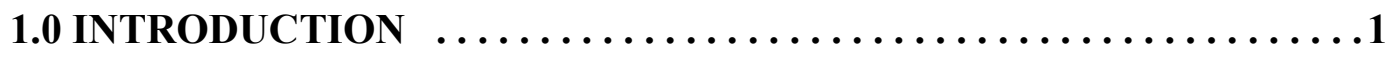

1.1 Emission Concerns and Regulations $\ldots \ldots \ldots \ldots \ldots \ldots \ldots$

1.2 Reducing $\mathrm{NO}_{\mathrm{x}}$ and Low-NO $\mathrm{N}_{\mathrm{x}}$ Combustion Systems .........5

1.2.1 Variable Geometry Combustors $\ldots \ldots \ldots \ldots \ldots \ldots$

1.2.2 Lean Premix Prevaporise Combustion ............6

1.2.3 Rich-Burn, Quick-Quench, Lean-Burn Combustor . . . . . . .6

1.2.4 Staged Combustion $\ldots \ldots \ldots \ldots \ldots \ldots \ldots \ldots \ldots$

1.3 Combustor Total Pressure Loss .................

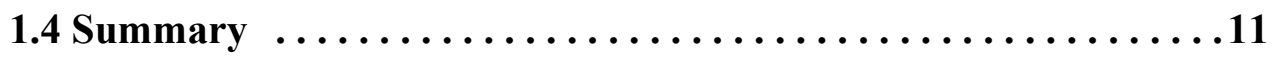

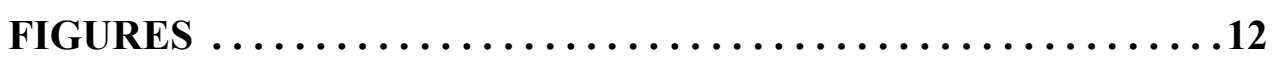

2.0 A DISCUSSION OF DIFFUSER TECHNOLOGY $\ldots \ldots \ldots \ldots \ldots \ldots$

2.1 Basic Diffuser Considerations $\ldots \ldots \ldots \ldots \ldots \ldots \ldots \ldots$

2.2 Expressions for Diffuser Performance $\ldots \ldots \ldots \ldots \ldots \ldots$. . $\ldots \ldots$

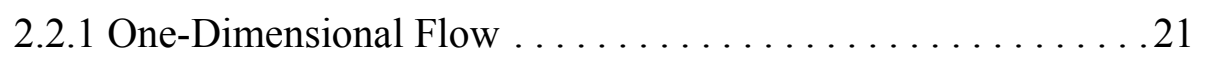

2.2.2 Non-Uniform Flows and Suitable Mean Values . . . . . . . . 22

2.3 Annular Diffuser Performance $\ldots \ldots \ldots \ldots \ldots \ldots \ldots \ldots \ldots$

2.3.1 Influence of Geometrical Parameters . . . . . . . . . . 25

(i) Length and Divergence Angle (Area Ratio) .......... 25

(ii) Curvature . . . . . . . . . . . . . . . . . 27

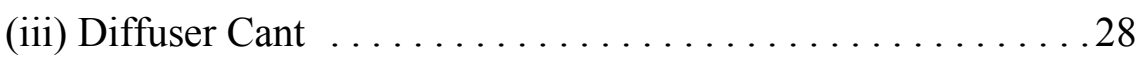


2.3.2 Effect of Inlet Conditions on Diffuser Performance . . . . . . . 29

(i) Reynolds Number . . . . . . . . . . . . . . . . . . . . 29

(ii) Mach Number . . . . . . . . . . . . . . . . . . . . 29

(iii) Inlet Boundary Layer $\ldots \ldots \ldots \ldots \ldots \ldots \ldots \ldots \ldots$

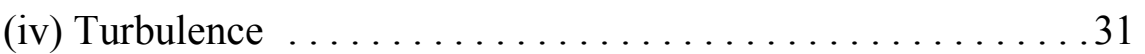

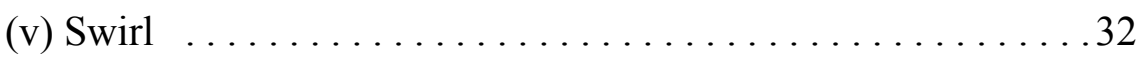

(vi) Outlet Guide Vane Wakes ................... 33

2.4 Influence of Combustor Flow Characteristics on Dump Diffusers 34

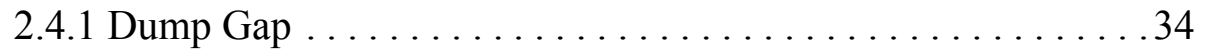

2.4.2 Flame Tube Depth . . . . . . . . . . . . . . . . . . 35

2.5 High Area Ratio, Wide Angle, Multi-Passage Diffusers . . . . . 37

2.6 Bled, Vortex Controlled and Hybrid Diffusers ..............39

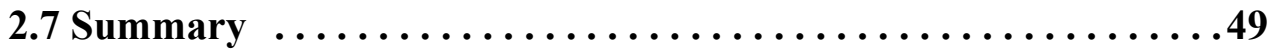

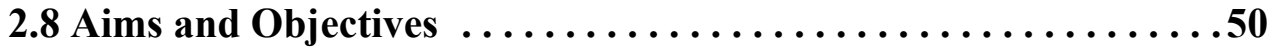

2.9 Structure of Thesis $\ldots \ldots \ldots \ldots \ldots \ldots \ldots \ldots \ldots \ldots \ldots \ldots \ldots$

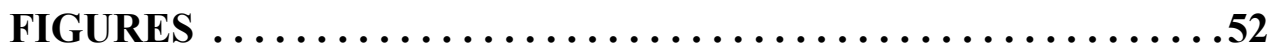

3.0 EXPERIMENTAL ARRANGEMENT AND PROCEDURE . . . . . . 80

3.1 Description of the Test Facility $\ldots \ldots \ldots \ldots \ldots \ldots \ldots \ldots$. $\ldots \ldots 1$

3.1.1 The Test Rig $\ldots \ldots \ldots \ldots \ldots \ldots \ldots \ldots \ldots \ldots \ldots \ldots \ldots \ldots \ldots \ldots$

3.1 .2 Test Rig Traversing $\ldots \ldots \ldots \ldots \ldots \ldots \ldots \ldots \ldots \ldots \ldots \ldots \ldots \ldots \ldots$

3.1.3 Compressor Stage Design $\ldots \ldots \ldots \ldots \ldots \ldots \ldots \ldots$

3.2 Instrumentation and Data Analysis $\ldots \ldots \ldots \ldots \ldots \ldots \ldots$

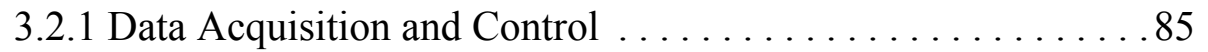

3.2.2 Operating Conditions $\ldots \ldots \ldots \ldots \ldots \ldots \ldots \ldots \ldots \ldots$

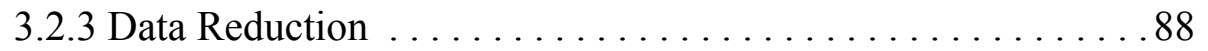

3.2.4 Graphical Techniques for Data Presentation . . . . . . . . .89

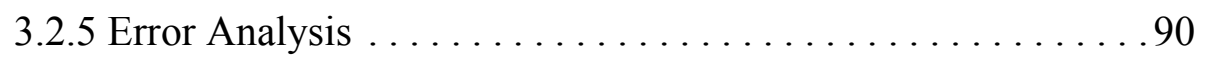

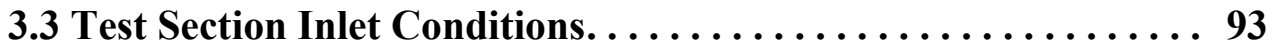

3.4 Test Section Geometry $\ldots \ldots \ldots \ldots \ldots \ldots \ldots \ldots \ldots \ldots$

3.4.1 Flame Tube Geometry $\ldots \ldots \ldots \ldots \ldots \ldots \ldots \ldots$

3.5 A Discussion of the Pre-Diffuser Geometries ............97

3.5.1 Conventional Datum Pre-Diffuser . . . . . . . . . . . 997 
3.5.2 Optimised Conventional Pre-Diffuser . . . . . . . . . .997

3.5.3 Hybrid Diffusers - Build 1 Flame Tube . . . . . . . . . . .98

3.5.4 Hybrid Diffuser - Build 2 Flame Tube . . . . . . . . . 100

3.6 Two-Dimensional, Axi-Symmetric Flame Tube Arrangement . . 101

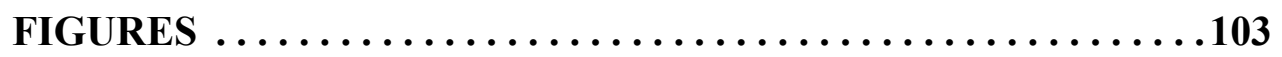

4.0 EXPERIMENTAL RESULTS AND DISCUSSION ...........116

4.1 Build 1 Flame Tube $\ldots \ldots \ldots \ldots \ldots \ldots \ldots \ldots \ldots \ldots \ldots \ldots$

4.1.1 Diffuser Performance $\ldots \ldots \ldots \ldots \ldots \ldots \ldots \ldots \ldots \ldots$

4.1.2 Hybrid 4 Stage 3 Wall Static Pressure Tapping Data . . . . . 122

4.1.3 Outer Feed Annulus Performance . . . . . . . . . . . . 124

4.1.4 Splitter Feed Annulus Performance . . . . . . . . . 126

4.1.5 Inner Feed Annulus Performance . . . . . . . . . . . . . 127

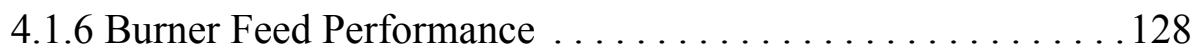

4.1.7 Dump Losses . . . . . . . . . . . . . . . . . . . . . . . . 129

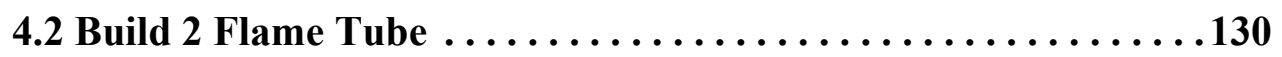

4.2.1 Diffuser Performance $\ldots \ldots \ldots \ldots \ldots \ldots \ldots \ldots \ldots \ldots$

4.2.2 Feed Annulus Performance . . . . . . . . . . . . . 132

4.3 Bleed Flow Assessment $\ldots \ldots \ldots \ldots \ldots \ldots \ldots \ldots \ldots \ldots \ldots \ldots$

4.3.1 Effect of Bleed Rate on Diffuser Performance .......... . 133

4.3.2 Vortex Chamber versus Duct Bleed .............. 134

4.3.3 Bleed Flow Total Pressure Loss . . . . . . . . . . . . . . 135

4.4 Two-Dimensional, Axi-Symmetric Flame Tube Arrangement . .137

4.4.1 Two-Dimensional Inlet (Clean) $\ldots \ldots \ldots \ldots \ldots \ldots \ldots$

4.4.2 Three-Dimensional Inlet Conditions (Rotor) . . . . . . . 138

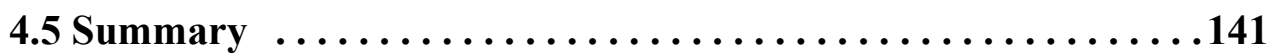

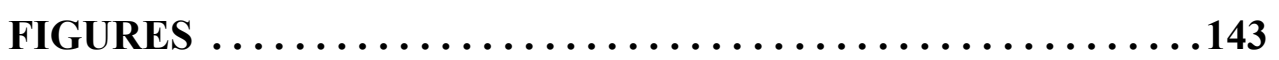

5.0 COMPUTATIONAL METHODOLOGY $\ldots \ldots \ldots \ldots \ldots \ldots \ldots$

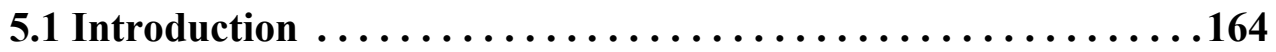

5.2 Governing Equations . . . . . . . . . . . . . . . 165

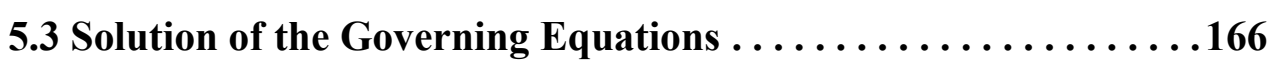

5.4 Closure - Turbulence Modelling $\ldots \ldots \ldots \ldots \ldots \ldots \ldots \ldots$ 
5.4.1 The k-e model . . . . . . . . . . . . . . . . . . 167

5.4.2 The Reynolds Stress Equation Model . . . . . . . . . . . . . 172

5.5 Modelling of Near-Wall Flows for Wall-Bounded Flows . . . . 175

5.5.1 Wall Functions ......................... 176

5.6 Numerical Implementation $\ldots \ldots \ldots \ldots \ldots \ldots \ldots \ldots \ldots \ldots$

5.7 Computational Investigation of Hybrid Diffusers $\ldots \ldots \ldots 183$

5.7.1 Geometry Definition and Boundary Conditions . . . . . . . 184

5.7.1.1 Plane Two-Dimensional Hybrid Diffusers . . . . . . . 184

5.7.1.2 Two-Dimensional Axi-Symmetric Hybrid Diffuser . 188

5.7.1.3 Three-Dimensional, Fully Annular Hybrid Diffuser . 189

5.7.2 Post-Processing and Presentation of Results .......... 190

5.8 Grid Generation and Solution Development $\ldots \ldots \ldots \ldots \ldots 1$

5.8.1 Initial Grid Definition . . . . . . . . . . . . . . 192

5.8.2 Grid Refinement and Near Wall Treatment ........... 193

5.8.3 Turbulence Model Assessment . . . . . . . . . . . . . . . . 194

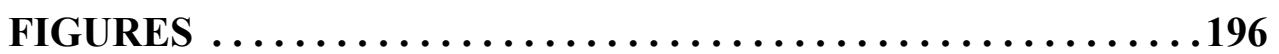

6.0 COMPUTATIONAL RESULTS AND DISCUSSION ..........209

6.1 Two-Dimensional, Plane Hybrid Diffusers $\ldots \ldots \ldots \ldots \ldots \ldots 210$

6.1.1 Overall Diffuser Performance . . . . . . . . . . . . 210

6.1.1.1 Vortex Chamber and Duct Bled Hybrid Diffusers . . .212

6.1.1.2 Alternative Bleed Geometries . . . . . . . . . 216

6.1.2 Bleed Flow Assessment . . . . . . . . . . . . . 220

6.1.3 Flow Mechanisms - Momentum Transfer Analysis . . . . . . 222

6.1.3.1 The Momentum Equation . . . . . . . . . . . . . 224

6.1.3.2 Flow Field Discussion (2.5\% Bleed) . . . . . . . . 229

6.1.3.3 Flow Field Discussion $(0.0 \%$ Bleed $) \ldots \ldots . . .232$

6.2 Two-Dimensional, Axi-Symmetric Hybrid Diffuser with Flame Tube

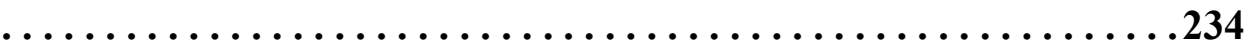

6.3 Hybrid Diffuser with OGV Wakes $\ldots \ldots \ldots \ldots \ldots \ldots \ldots . \ldots 238$

6.3.1 Diffuser Performance and OGV Wake Effects . . . . . . . . 238

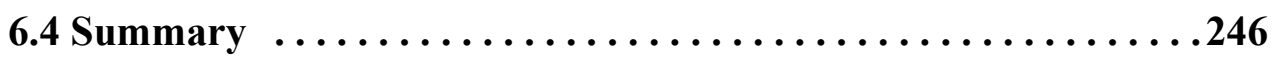

FIGURES $\ldots \ldots \ldots \ldots \ldots \ldots \ldots \ldots \ldots \ldots \ldots \ldots \ldots \ldots . \ldots \ldots$ 
7.0 CONCLUSIONS AND RECOMMENDATIONS ............291

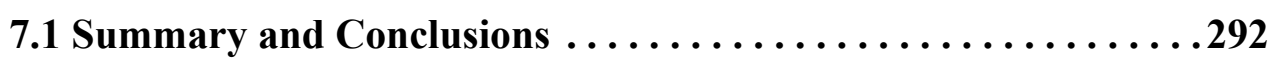

7.2 Recommendations for Further Work .............297

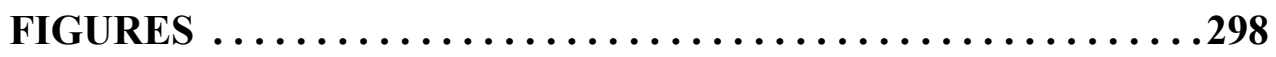

REFERENCES................................

APPENDIX A1 PRLIMINARY EXPERIMENTAL INVESTIGATION

APPENDIX A2 BUTTON HOOK PROBE THEORY

APPENDIX A3 FIVE HOLE PROBE THEORY

APPENDIX A4 BLEED FLOW: CALIBRATION AND CONTROL

APPENDIX A5 PROBE LINEAR TRAVERSE MECHANISM 


\section{LIST OF FIGURES}

\subsection{INTRODUCTION}

1.1 Emissions Characteristic of a Gas Turbine, Lefebvre ${ }^{[1995]}$................................. 12

1.2 Variation of NOx Characteristics with Engine Pressure Ratio, Birch ${ }^{[2000]}$........ 12

1.3 Main Components of a Gas Turbine Combustor, Lefebvre ${ }^{[1995]}$........................ 13

1.4 Influence of Primary Zone Temperature on CO and NOx Emissions,

Lefebvre $^{[1995]}$ 13

1.5 Relationship between Primary Zone and Overall Stoichiometries for Fixed

Combustor Geometries, Wray ${ }^{[1995]}$ 14

1.6 Relationship between Primary Zone and Overall Stoichiometries for a

Staged Combustor, Wray ${ }^{[1995]}$ 14

1.7 Pratt and Whitney Axially Staged Combustor, Koff ${ }^{[1993]}$ 15

1.8 General Electric Radially Staged Combustor, Lefebvre ${ }^{[1995]}$ 15

1.9 Variation of Total Pressure Loss to the Feed Annuli with Mean Deflection

Ratio, Carrotte and Bailey ${ }^{[1994]}$ 16

1.10 A Comparison of Diffuser Performance with the Nominal Design Curves,

Sovran and Klomp ${ }^{[1967]}$ 16

1.11 Typical Flow Regime Chart for Curved Diffusers, Sagi and Johnston ${ }^{[1967]}$.... 17

\subsection{A DISCUSSION OF DIFFUSER TECHNOLOGY}

2.1 Basic Diffuser Geometry 52

2.2 Faired and Dump Diffusers 52

2.3 Influence of Divergence Angle on Pressure Loss, Lefebvre ${ }^{[1983]}$.......................5 52

2.4 Annular Diffuser Performance Data, Sovran and Klomp ${ }^{[1967]}$..........................53

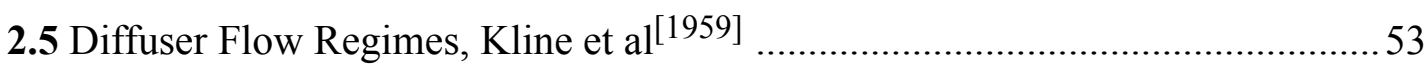

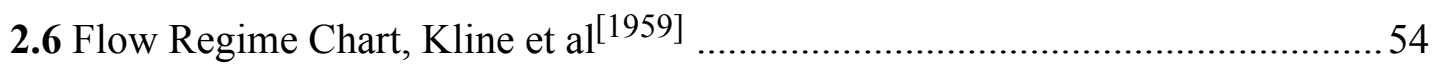

2.7 Motion of a Displaveed Element in a Curved Flow ............................................54

2.8 Effect of Inlet Boundary Layer Blockage on Pressure Recovery of Two AR

2.0 Diffusers, Stevens and Williams ${ }^{[1980]}$ 55 
2.9 Effect of Inlet Swirl on Axial Velocity Profile, Elkersh et al ${ }^{[1985]}$.....................56

2.10 Effect of Inlet Swirl on Diffuser Performance, Elkersh ${ }^{[1985]}$............................57

2.11 Effect of Profile Distortion on Diffuser Performance, Stevens et al ${ }^{[1978]}$........58

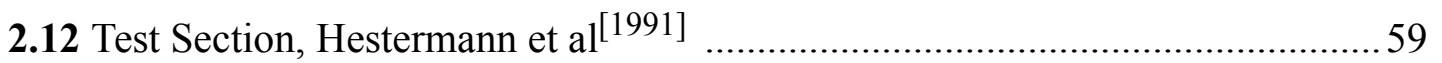

2.13 Influence of Dump Gap Ratio on Diffuser Exit Profile, Hestermann et $\mathrm{al}^{[1991]}$

2.14 Test Section, Fishenden and Stevens ${ }^{[1977]}$

2.15 Influence of Dump Gap Ratio on Overall Pressure Loss Coefficient,

Fishenden and Stevens ${ }^{[1977]}$

2.16 Flow Pattern in the Dump Region, Fishenden and Stevens ${ }^{[1977]}$.....................61

2.17 Test Section Geometry, Carrotte and Bailey ${ }^{[1994]}$............................................61

2.18 Variation of Pressure Loss to the Feed Annuli with Flame Tube Depth,

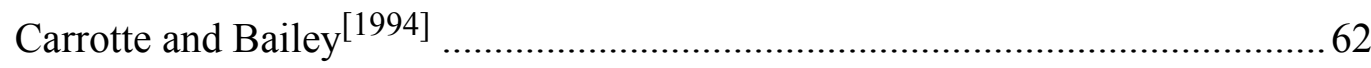

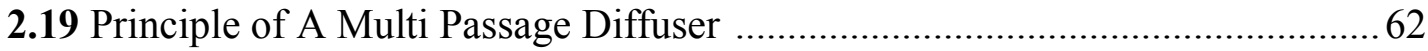

2.20 Exit Profile Distortion Caused by Splitter, Moore ${ }^{[1976]}$....................................6 63

2.21 General Electric Twin Passage Diffuser, Salba et al ${ }^{[1982]}$................................63

2.22 Cross Section of Double Annular Sector Rig, Shedden ${ }^{[1993]}$............................64 64

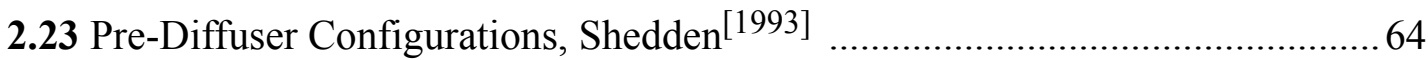

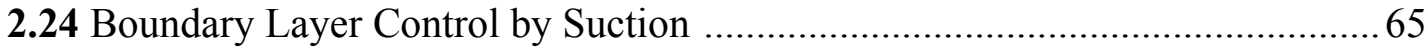

2.25 Effect of Suction Control on Diffuser Exit Velocity Profile for a $30^{\circ}$ Conical

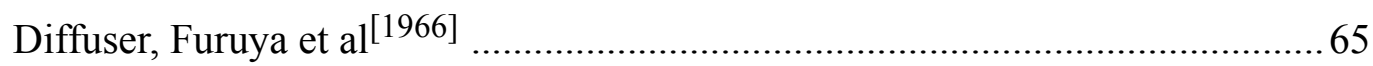

2.26 Heskestad ${ }^{[1968]}$ Bleed Gap Geometry ...........................................................66

2.27 Effect of Suction on Pressure Recovery, Heskestad ${ }^{[1968]}$.................................66

2.28 Suggested Flow in Vortex Controlled Diffuser with No Suction and Suction

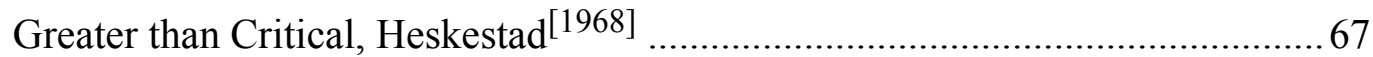

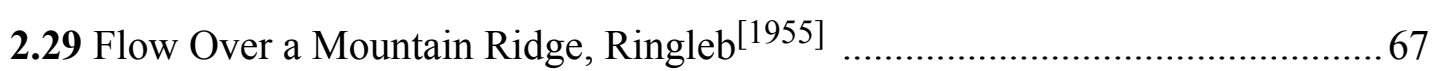

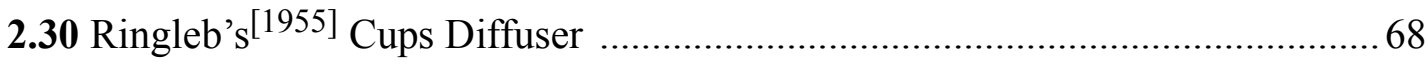

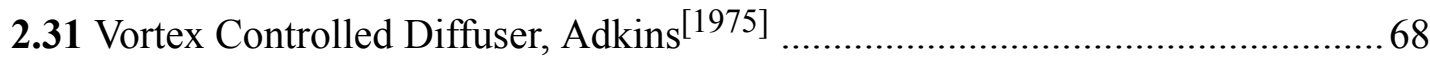

2.32 Suggested Flow Mechanism of Vortex Controlled Diffuser, Adkins ${ }^{[1975]}$........68

2.33 Performance Characteristics of VCD, Adkins ${ }^{[1975]}$.......................................69 
2.34 The Hybrid Vortex Controlled Diffuser, Adkins et al ${ }^{[1980]}$................................69

2.35 Hybrid Vortex Controlled Diffuser Nomenclature, Adkins et al ${ }^{[1980]}$............... 70

2.36 Combined Diffuser Arrangement, Adkins and Yost ${ }^{[1979]}$................................ 70

2.37 Effect of Bleed Gap on Pressure Recovery, Adkins and Yost ${ }^{[1979]}$....................71

2.38 HVCD Pressure Recovery, Adkins et $\mathrm{al}^{[1980]}$................................................. 71

2.39 Vortex Controlled Diffusers at Low and Zero Bleed, Adkins et al ${ }^{[1980]}$........... 72

2.40 A Compact Diffuser System for Annular Combustors, Adkins and

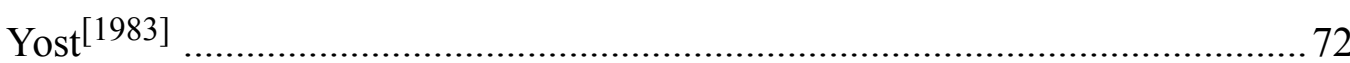

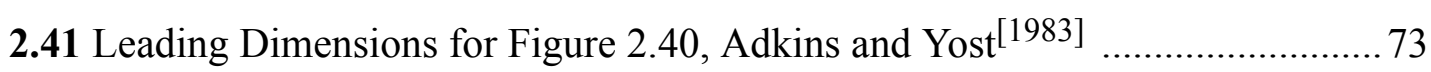

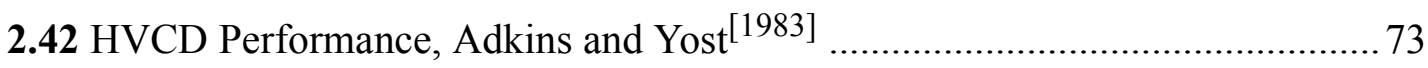

2.43 Test Arrangement, Adkins and Montazerin ${ }^{[1985]}$............................................ 74

2.44 Inlet Velocity Profiles, Adkins and Montazerin ${ }^{[1985]}$...................................... 74

2.45 Effect of Inlet Distortion, Adkins and Montazerin ${ }^{[1985]}$.................................. 75

2.46 Canted Diffuser Arrangement, Adkins and Kuile ${ }^{[1985]}$.................................. 75

2.47 Diffuser Performance with Unbalanced Bleed, Adkins and Kuile ${ }^{[1985]}$...........76

2.48 Effect of Bleed Imbalance of Annuli Mass Flow, Adkins and Kuile ${ }^{[1985]}$....... 77

2.49 Myres et $\mathrm{al}^{[1993]}$ Test Facility ................................................................... 78

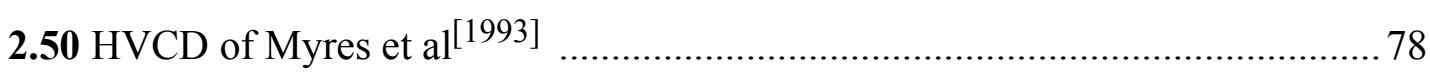

2.51 Leading Dimensions, Myres et $\mathrm{al}^{[1993]}$.......................................................... 79

2.52 Ratio of Bleed Static to Inlet Total Pressure, Myres et al ${ }^{[1993]}$........................ 79

\subsection{EXPERIMENTAL ARRANGEMENT AND PROCEDURE}

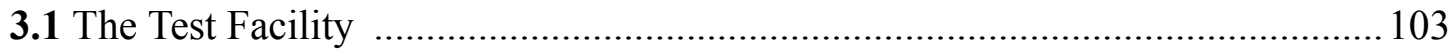

3.2 The Test Rig (showing Hybrid Diffuser and Build 1 Flame Tube) .................... 104

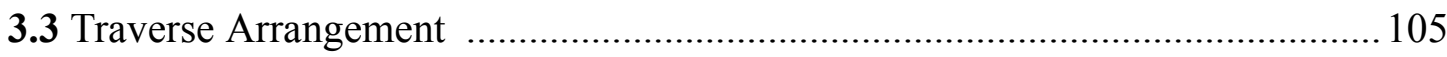

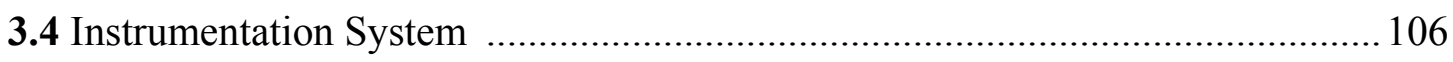

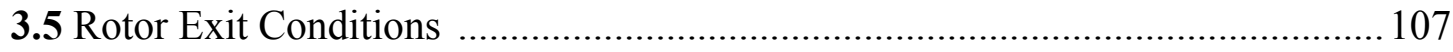

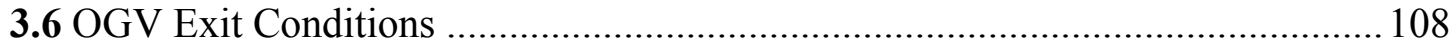

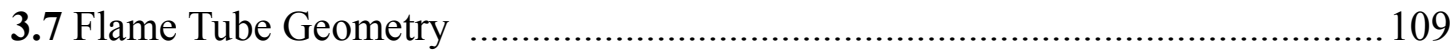

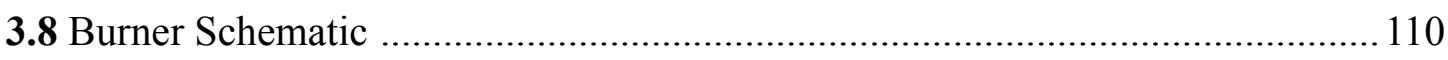


3.9 Conventional Datum Diffuser

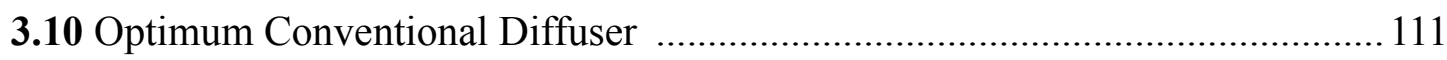

3.11 A Comparison with Nominal Design Curves ................................................... 111

3.12 Hybrid Pre-Diffuser Geometry Definition .................................................... 112

3.13 Hybrid Diffuser Area Ratio Distribution ..................................................... 112

3.14 Hybrid Diffuser Options (Refer to Table 3.6)................................................ 113

3.15 Static Pressure Tapping Arrangement on Stage 3 Outer Wall .......................... 114

3.16 Modified (Two-Dimensional, Axi-Symmetric) Test Section ........................... 115

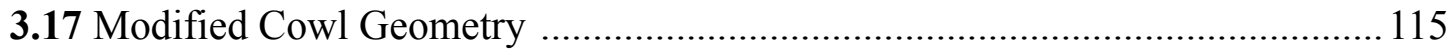

\subsection{EXPERIMENTAL RESULTS AND DISCUSSION}

4.1 Variation of Diffuser Exit Profile with Bleed ................................................... 143

4.2 Circumferentially Averaged Axial Velocity Profiles at Diffuser Exit ................ 143

4.3 Axial Velocity Contours at Diffuser Exit ........................................................ 144

4.4 Projected Total Velocity Vectors at Diffuser Exit ............................................. 145

4.5 Circumferentially Averaged Pitch Angle at Diffuser Exit ................................. 146

4.6 Circumferentially Averaged Swirl Angle at Diffuser Exit ............................... 146

4.7 Flow Mechanism of a Hybrid Diffuser ........................................................... 147

4.8 Circumferentially Averaged Wall Static Pressure Recovery (Hybrid 4) ............ 147

4.9 Variation of Maximum Static Pressure Recovery ............................................ 148

4.10 Contours of Wall Static Pressure Recovery (Hybrid 4, $\mathrm{B}_{\text {inner }}=2.75 \%$,

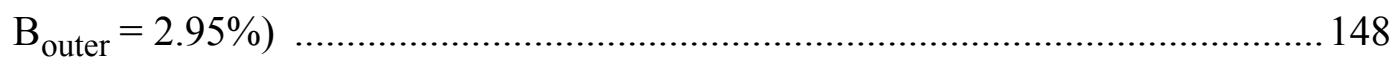

4.11 Circumferentially Averaged Axial Velocity Profiles in Outer Annulus ........... 149

4.12 Axial Velocity Contours in the Outer Annulus .............................................. 150

4.13 Projected Total Velocity Vectors in the Outer Annulus ...................................151

4.14 Circumferentially Averaged Swirl Angle in Outer Annulus ........................... 152

4.15 Circumferentially Averaged Velocity Profiles in Inner Annulus ...................... 152

4.16 Axial Velocity Contours in Inner Annulus ..................................................... 153

4.17 Projected Total Velocity Vectors in Inner Annulus ......................................... 154

4.18 Circumferentially Averaged Swirl Angle in Inner Annulus ........................... 155

4.19 Circumferentially Averaged Axial Velocity Profiles at Diffuser Exit .............. 155

4.20 Axial Velocity Contours at Diffuser Exit (Build 2) ...................................... 156 
4.21 Projected Total Velocity Vectors at Diffuser Exit (Build 2) ............................ 157

4.22 Differences between Vortex and Duct Bleed .................................................. 158

4.23 Optimum Conventional Dump Bleed ........................................................... 158

4.24 Variation of Outer Bleed Flow Loss with Bleed Rate (Hybrid 4) .................... 159

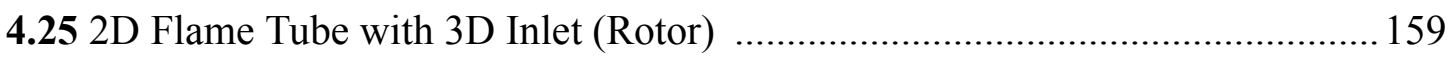

4.26 Circumferentially Averaged Axial Velocity Profile at Diffuser Exit ................ 160

4.27 Circumferentially Averaged Axial Velocity Profile at Pre-Diffuser Inlet ........ 160

4.28 Axial Velocity Contours at Diffuser Exit ....................................................... 161

4.29 Circumferentially Averaged Axial Velocity Profiles in Inner Annulus ............ 161

4.30 Circumferentially Averaged Axial Velocity Profiles in Outer Annulus ............ 162

\subsection{COMPUTATIONAL METHODOLOGY}

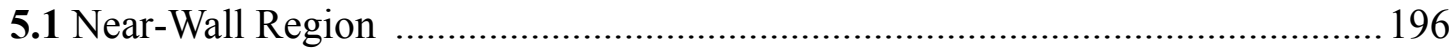

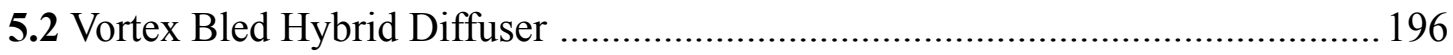

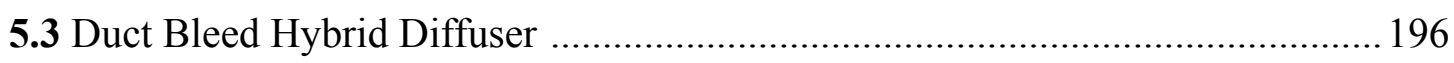

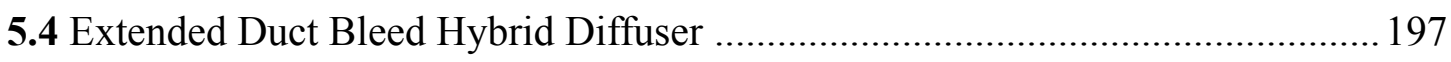

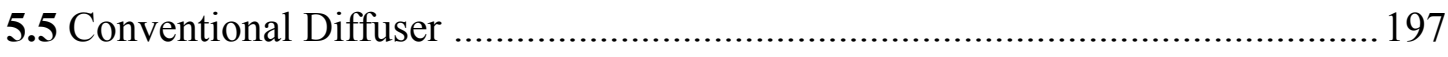

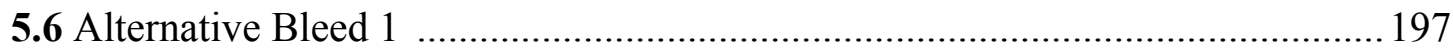

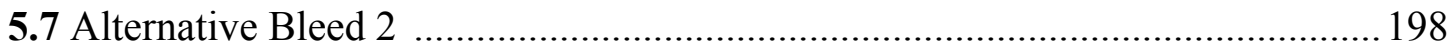

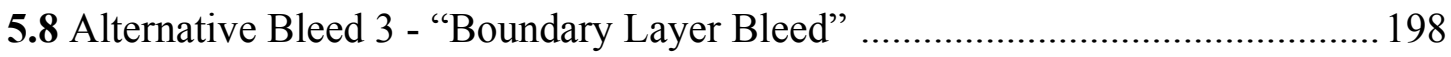

5.9 Alternative Bleed 4 - "Profiled Bleed Gap" ...................................................... 198

5.10 Alternative Bleed 5 - “Angled Bleed Duct” .................................................... 199

5.11 Inlet Velocity Profile for Plane Two-Dimensional CFD .................................. 199

5.12 Effect of Inlet Turbulence Intensity on Solution ..........................................200

5.13 Two-Dimensional, Axi-Symmetric Hybrid Diffuser with Flame Tube ............ 200

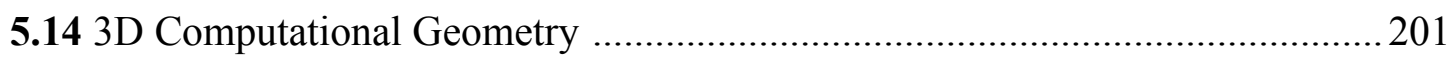

5.15 3D CFD Inlet Conditions - Normalised Axial Velocity Contours ....................201

5.16 3D CFD Inlet Conditions - V-W Velocity Vectors ..........................................202

5.17 Initial 2D Grid in Stage 2 Diffuser (shown with Duct Bleed) ........................202

5.18 Non-Physical Near-Wall Flow (Initial 2D Grid, k- $\varepsilon$, Standard Wall

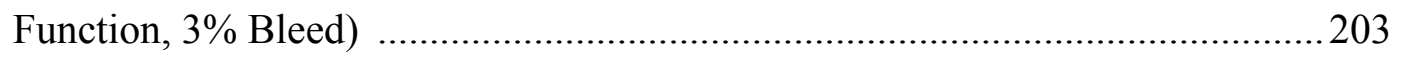

5.19 Non-Physical Near-Wall Flow (Initial 2D Grid, RNS, Standard Wall

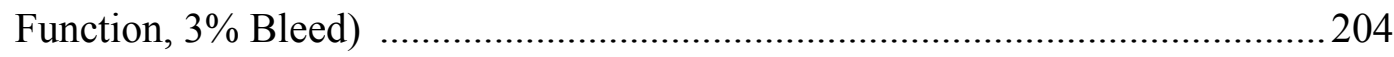


5.20 Near Wall Modified 2D Grids (Near Wall Cell 16.5\%, 25\% and 50\% of

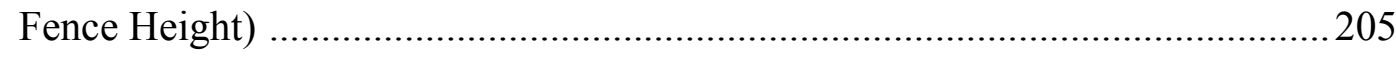

5.21 Axial Velocity in Stage 3 Near Wall Cell (k-e, 3\% Bleed) ..............................206

5.22 Effect of Grid Density on Solution (k- $\varepsilon$, Standard Wall Function, 3\% Bleed) 206

5.23 Effect of Turbulence Closure Method on Solution ..........................................207

5.24 Streamlines at Reattachment (k- $\varepsilon$, Standard Wall Function, 3\% Bleed) .........207

5.25 Streamlines at Reattachment (RNS, Standard Wall Function, 3\% Bleed) ........208

5.26 Anomaly in RNS Boundary Layer Profile on Satge 3 Wall ...........................208

\subsection{COMPUTATIONAL RESULTS AND DISCUSSION}

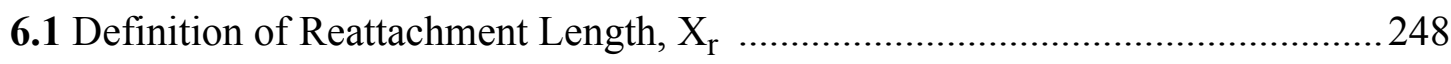

6.2 Axial Velocity Profiles at Diffuser Exit (Vortex Chamber) ..............................2248

6.3 Axial Velocity Profiles at Diffuser Exit (Duct Bleed) .......................................2249

6.4 Variation of Diffuser Total Pressure Loss with Bleed ......................................249

6.5 Variation of Diffuser Static Pressure Recovery with Bleed ..............................250

6.6 Variation of Reattachment Length with Bleed ..............................................250

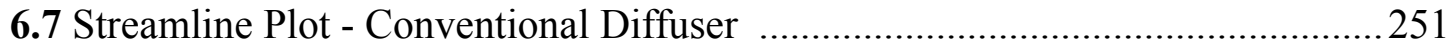

6.8 Streamline Plot - Duct-bled Diffuser, 2.5\% Bleed ..............................................2. 251

6.9 Streamline Plot - Duct-bled Diffuser, 1.5\% Bleed ............................................. 252

6.10 Streamline Plot - Duct-bled Diffuser, 0.0\% Bleed ......................................... 252

6.11 Axial Velocity Profile at Stage 1 Exit (Duct-bled Diffuser) ............................253

6.12 Radial Velocity Profile at Stage 1 Exit (Duct-bled Diffuser) ...........................253

6.13 Axial Velocity Profile at Stage 2 Exit (Duct-bled Diffuser) ............................254

6.14 Radial Velocity Profile at Stage 2 Exit (Duct-bled Diffuser) ...........................254

6.15 Radial Velocity Across Bleed Gap (Duct-bled Diffuser) .................................255

6.16 Static Pressure Gradient Across Bleed Gap (Duct-bled Diffuser) .....................255

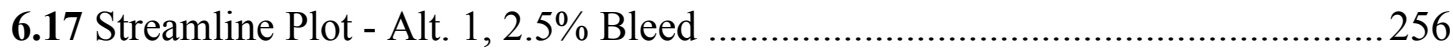

6.18 Cell Centred Axial Velocity in Wall Adjacent Cell, 2.5\% Bleed ......................256

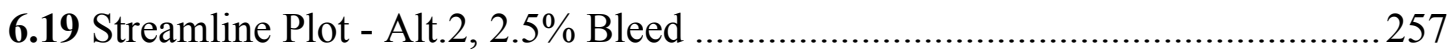

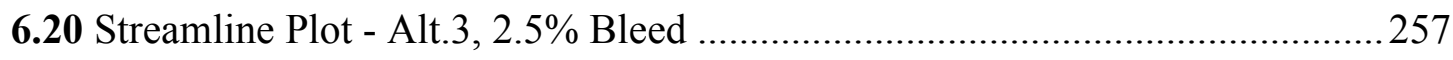

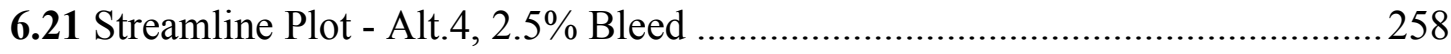

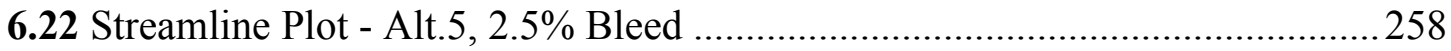


6.23 Static Pressure Contours - Duct, 2.5\% Bleed .................................................259

6.24 Static Pressure Contours - Alt.1, 2.5\% Bleed .................................................259

6.25 Static Pressure Contours - Alt.2, 2.5\% Bleed .................................................260

6.26 Static Pressure Contours - Alt.3, 2.5\% Bleed ...................................................260

6.27 Static Pressure Contours - Alt.4, 2.5\% Bleed .................................................. 261

6.28 Static Pressure Contours - Alt.5, 2.5\% Bleed ................................................. 261

6.29 Development of Mass Weighted Static Pressure Recovery ............................. 262

6.30 Rate of Mass Weighted Static Pressure Recovery ............................................262

6.31 Streamline Plot - Alt.4, 0\% Bleed, Stalled ...................................................263

6.32 Streamline Plot - Alt.4, 1.0\% Bleed, Stalled ...................................................263

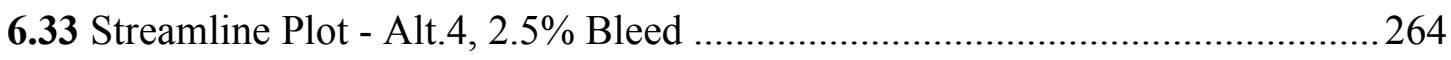

6.34 Axial Velocity Profiles at Diffuser Exit, Alt.4 ...............................................264

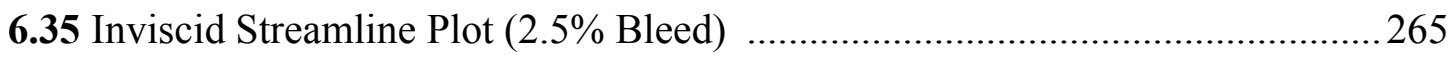

6.36 Diffuser Nomenclature (Momentum Analysis) .............................................265

6.37 Typical Variation of $\beta$ from Inviscid Solution ..............................................266

6.38 Gain/Loss Processes of Streamwise Momentum ..........................................266

6.39 Flow Regions within Alt.4 Hybrid Diffuser .................................................267

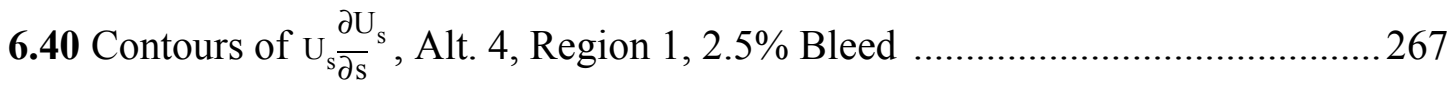

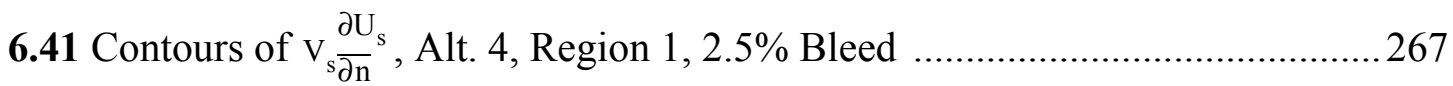

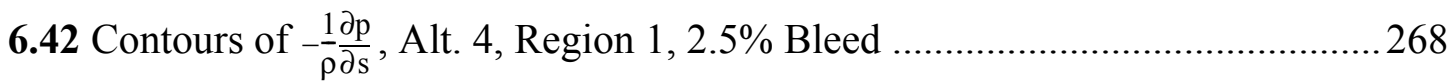

6.43 Contours of $-\left(\frac{\partial}{\partial \mathrm{s}} \overline{\mathrm{u}}+\frac{\partial}{\partial \mathrm{u}} \overline{\mathrm{uv}}\right)$, Alt. 4, Region 1, 2.5\% Bleed ..............................268

6.44 Turbulent Flow Streamlines, Alt. 4, Region 1, 2.5\% Bleed .............................269

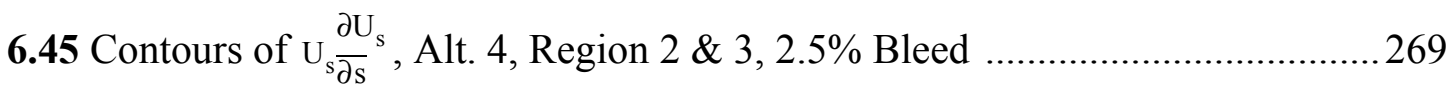

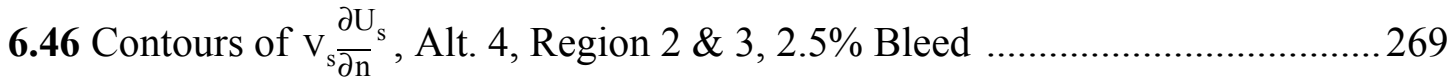

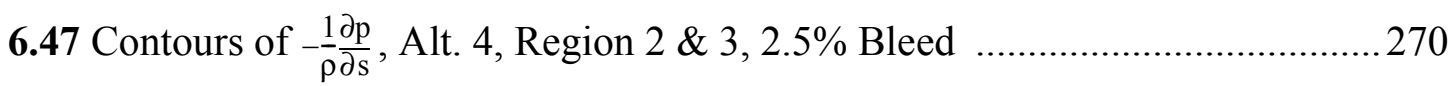

6.48 Contours of $-\left(\frac{\partial}{\partial \mathrm{s}} \overline{\mathrm{uu}}+\frac{\partial}{\partial \mathrm{n}} \overline{\mathrm{uv}}\right)$, Alt. 4, Region $2 \& 3,2.5 \%$ Bleed ......................... 271

6.49 Streamlines, Alt. 4, Region $2 \&$ 3, 2.5\% Bleed ............................................ 271

6.50 Streamlines, Alt. 4, Region 4, 2.5\% Bleed ................................................. 272 
6.51 Contours of $U_{s} \frac{\partial U_{s}}{\partial s}$, Alt. 4, Region 4, 2.5\% Bleed 272

6.52 Contours of $v_{s} \frac{\partial U_{s}}{\partial n}$, Alt. 4, Region 4, 2.5\% Bleed 273

6.53 Contours of $-\frac{1}{\rho} \frac{\partial \mathrm{p}}{\partial \mathrm{s}}$, Alt. 4 , Region $4,2.5 \%$ Bleed 273

6.54 Contours of $-\left(\frac{\partial}{\partial \mathrm{s}} \overline{\mathrm{u}}+\frac{\partial}{\partial \mathrm{n}} \overline{\mathrm{uv}}\right)$, Alt. 4, Region 4, 2.5\% Bleed 274

6.55 Streamlines, Alt. 4, Region 5, 2.5\% Bleed .274

6.56 Contours of $U_{s} \frac{\partial U_{s}}{\partial s}$, Alt. 4, Region 5, 2.5\% Bleed 275

6.57 Contours of $v_{s} \frac{\partial U_{s}}{\partial n}$, Alt. 4, Region 5, 2.5\% Bleed 275

6.58 Contours of $-\frac{1}{\rho} \frac{\partial \mathrm{p}}{\partial \mathrm{s}}$, Alt. 4, Region $5,2.5 \%$ Bleed 276

6.59 Contours of $-\left(\frac{\partial}{\partial \mathrm{s}} \overline{\mathrm{uu}}+\frac{\partial}{\partial \mathrm{n}} \overline{\mathrm{uv}}\right)$, Alt. 4, Region 5, 2.5\% Bleed 276

6.60 Contours of $U_{s} \frac{\partial U_{s}}{\partial s}$, Alt. 4, 0.0\% Bleed

6.61 Contours of $\mathrm{v}_{\mathrm{s}} \frac{\partial \mathrm{U}_{\mathrm{s}}}{\partial \mathrm{n}}$, Alt. $4,0.0 \%$ Bleed 277

6.62 Contours of $-\frac{1}{\rho} \frac{\partial \mathrm{p}}{\partial \mathrm{s}}$, Alt. $4,0.0 \%$ Bleed 278

6.63 Contours of $-\left(\frac{\partial}{\partial \mathrm{s}} \overline{\mathrm{u}}+\frac{\partial}{\partial \mathrm{n}} \overline{\mathrm{uv}}\right)$, Alt. $4,0.0 \%$ Bleed 278

6.64 Streamline Plot, 2D, Axi-Symmetric, $\mathrm{B}_{\mathrm{i}}=\mathrm{B}_{\mathrm{o}}=2.5 \%, \mathrm{~T}_{\mathrm{i}}=0.5 \%$ 279

6.65 Streamline Plot, 2D, Axi-Symmetric, $\mathrm{B}_{\mathrm{i}}=\mathrm{B}_{\mathrm{o}}=2.5 \%, \mathrm{~T}_{\mathrm{i}}=5.0 \%$ 279

6.66 Effect of Inlet Turbulence on Axial Velocity Profile at Diffuser Exit, 2D, Axi-Symmetric, (Experimental Data, $\mathrm{T}_{\mathrm{i}}<1.0 \%$ ) 280

6.67 Static Pressure Recovery, $2 \mathrm{D}$, Axi-Symmetric, $\mathrm{T}_{\mathrm{i}}=5.0 \%, \mathrm{~B}_{\mathrm{i}}=\mathrm{B}_{\mathrm{o}}=2.5 \% \quad \ldots \ldots .280$

6.68 Static Pressure Recovery, $2 \mathrm{D}$, Axi-Symmetric, $\mathrm{T}_{\mathrm{i}}=0.5 \%, \mathrm{~B}_{\mathrm{i}}=\mathrm{B}_{\mathrm{o}}=2.5 \% \quad \ldots \ldots .281$

6.69 Streamline Plot, 2D, Axi-Symmetric, $\mathrm{T}_{\mathrm{i}}=5.0 \%, \mathrm{~B}_{\mathrm{i}}=\mathrm{B}_{\mathrm{o}}=1.0 \%$ 281

6.70 Effect of Bleed Rate on Axial Velocity Profile at Diffuser Exit, 2D,

Axi-Symmetric, $\mathrm{T}_{\mathrm{i}}=5.0 \%$ 282

6.71 Normalised Axial Velocity Contours at Diffuser Exit (Experimental Data,

$\left.\mathrm{B}_{\mathrm{i}}=2.5 \%, \mathrm{~B}_{\mathrm{o}}=3.0 \%\right)$ 282 
6.72 Normalised Axial Velocity Contours at Diffuser Exit (CFD Data, $\mathrm{T}_{\mathrm{i}}=5.0 \%$, $\left.\mathrm{B}_{\mathrm{i}}=2.5 \%, \mathrm{~B}_{\mathrm{o}}=3.0 \%\right)$ 283

6.73 Velocity Vectors at Diffuser Exit (Experimental Data, $B_{i}=2.5 \%$, $\left.\mathrm{B}_{\mathrm{o}}=3.0 \%\right)$ 283

6.74 Velocity Vectors at Diffuser Exit (CFD Data, $\mathrm{T}_{\mathrm{i}}=5.0 \%, \mathrm{~B}_{\mathrm{i}}=2.5 \%$, $\left.\mathrm{B}_{\mathrm{o}}=3.0 \%\right)$ 284

6.75 Normalised Axial Velocity Contours at Diffuser Exit (CFD Data $T_{i}=1.0 \%$, $\left.\mathrm{B}_{\mathrm{i}}=2.5 \%, \mathrm{~B}_{\mathrm{o}}=3.0 \%\right)$ .284

6.76 Normalised Axial Velocity Contours at Diffuser Exit (CFD Data $T_{i}=2.0 \%$, $\left.\mathrm{B}_{\mathrm{i}}=2.5 \%, \mathrm{~B}_{\mathrm{o}}=3.0 \%\right)$ 285

6.77 Circumferential Variation of Reattachment .................................................225

6.78 Outer Wall Static Pressure Contours $\left(\mathrm{T}_{\mathrm{i}}=5.0 \%, \mathrm{~B}_{\mathrm{i}}=2.5 \%, \mathrm{~B}_{\mathrm{o}}=3.0 \%\right) \ldots \ldots .286$

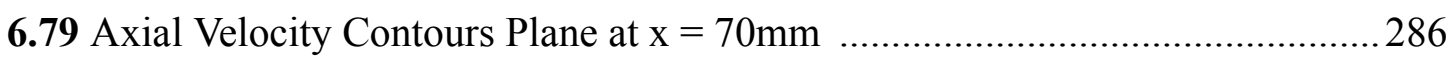

6.80 Inner Bleed Gap Radial Velocity Contours ......................................................28

6.81 Outer Bleed Gap Radial Velocity Contours ...................................................28

6.82 Stage 2 Axial Velocity Contours within OGV Wake Fluid ..............................228

6.83 Stage 2 Radial Velocity Contours within OGV Wake Fluid ...........................228

6.84 Stage 2 Axial Velocity Contours Between OGV Wake Fluid ...........................289

6.85 Stage 2 Radial Velocity Contours Between OGV Wake Fluid .........................28

6.86 Circumferential Variation of Reattachment ....................................................220

\subsection{CONCLUSIONS AND RECOMMENDATIONS}

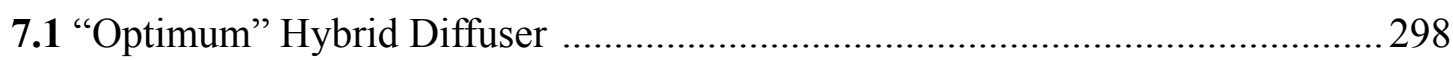

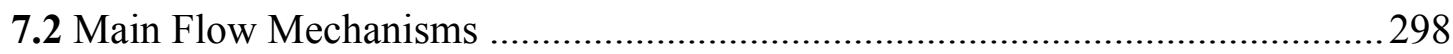

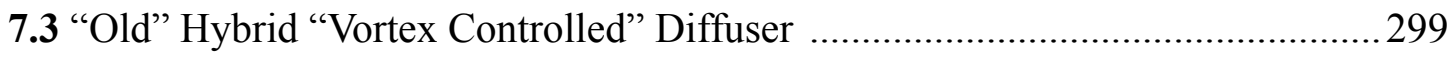

7.4 Further Hybrid Diffuser Development …….....................................................299

\section{APPENDIX A1 PRLIMINARY EXPERIMENTAL INVESTIGATION}

A1.1 Rectangular/Planar Test Facility ............................................................ A1-8

A1.2 Hybrid Diffuser Nomenclature .................................................................. A 1-8

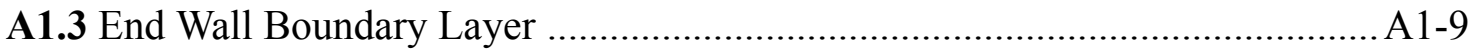

A1.4 Vortex 'Roll-Up' at End Wall ..................................................................... A1-9 
A1.5 Regions of Vorticity A1-9

\section{APPENDIX A2 BUTTON HOOK PROBE THEORY}

A2.1 Button Hook Probe Geometry A2-3

\section{APPENDIX A3 FIVE HOLE PROBE THEORY}

A3.1 Five Hole Probe A3-7

A3.2 Typical Five Hole Probe Dimensions A3-7

A3.3 Contours of Constant Pseudo Pitch Angle, PPS A3-8

A3.4 Contours of Constant Yaw Angle, YTR A3-8

A3.5 Contours of Constant Dynamic Pressure Parameter, $D_{p}$ A3-9

A3.6 Contours of Constant Stagnation Pressure Parameter, $S_{p}$ A3-9

A3.7 FIve Hole Probe Calibration Rig A3-10

\section{APPENDIX A4 BLEED FLOW: CALIBRATION AND CONTROL}

A4.1 Inner Bleed Throttle and Orifice A4-3

A4.2 Outer Bleed Bell Mouth Trumpet A4-4

A4.3 Inner Bleed Calibration A4-4

A4.4 Vortex Bleed Calibration Curves A4-5

\section{APPENDIX A5 PROBE LINEAR TRAVERSE MECHANISM}

A5.1 Probe Linear Traverse Mechanism A5-2

A5.2 Section A-A A5-2

A5.3 Section B-B A5-3

A5.4 Section C-C A5-3 


\section{LIST OF TABLES}

\subsection{EXPERIMENTAL ARRANGEMENT AND PROCEDURE}

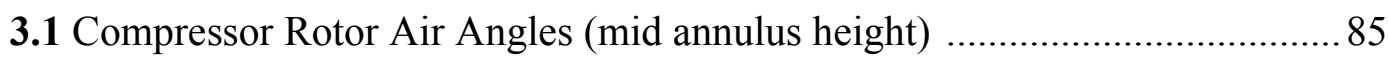

3.2 Compressor Stage Performance(mid annulus height) .................................85

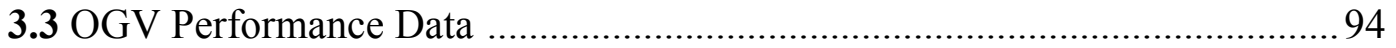

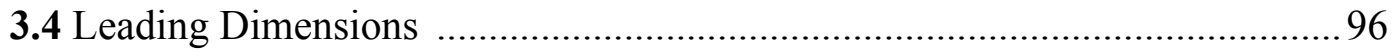

3.5 Flametube Air Mass Flow Distribution - Design Values ...............................96

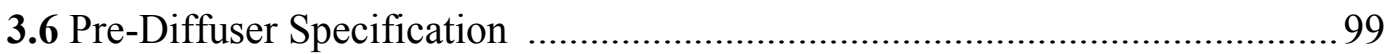

\subsection{EXPERIMENTAL RESULTS AND DISCUSSION}

4.1 Pre-Diffuser Performance Data (* - indicates strutted diffusers) ................. 119

4.2 Outer Feed Annulus Performance Data ........................................................... 125

4.3 Splitter Feed Annulus Performance Data ................................................... 127

4.4 Inner Feed Annulus Performance Data ...................................................... 128

4.5 Burner Flow Total Pressure Loss Coefficient (w.r.t. Rotor Exit) ................. 129

4.6 Total Pressure Loss Coefficients in the Dump ........................................... 129

4.7 Pre-Diffuser Performance Data (Build 2 Flame Tube) ................................ 131

4.8 Outer Annulus Performance Figures - Build 2 .......................................... 132

4.9 Inner Annulus Performance Figures - Build 2 ......................................... 132

4.10 Variation of Diffuser Performance with Bleed Rate (Hybrid 4) ............... 133

4.11 Bleed Flow Total Pressure Loss Coefficients ............................................ 136

4.12 Diffuser Performance (2D Flame Tube, 3D Inlet Conditions, $\mathrm{B}_{\mathrm{i}}=2.5 \%$ ) ... 139

4.13 Annuli Performance (2D Flame Tube, 3D Inlet Conditions, $\mathrm{B}_{\mathrm{i}}=2.5 \%$ ) ..... 140

\subsection{COMPUTATIONAL METHODOLOGY}

5.1 Effect on Solution of Inlet Characteristic Length $\left(\mathrm{T}_{\mathrm{i}}=5.0 \%, \mathrm{~B}=3.0 \%\right) \ldots . .187$

5.2 2D Axi-Symmetric Geometry - Leading Dimensions 188 


\subsection{COMPUTATIONAL RESULTS AND DISCUSSION}

6.1 Diffuser Performance - Hybrid Diffuser with Vortex Chamber ...................2212

6.2 Diffuser Performance - Hybrid Diffuser with Duct Bleed ...........................2 213

6.3 Diffuser Performance - Hybrid Diffuser with Extended Duct Bleed ...........213

6.4 Diffuser Performance - Alternative Bleed Hybrid Diffusers (2.5\% Bleed) . 217

6.5 Bleed Flow Mass Weighted Total Pressure Loss ........................................221

6.6 Bleed Flow Mass Weighted Total Pressure Loss (2.5\% Bleed) ................... 222

6.7 Mass Weighted Diffuser Performance Figures - Alt. Step 4 ........................224

6.8 Variation of Performance with Inlet Turbulence $\left(\mathrm{B}_{\mathrm{i}}=\mathrm{Bo}=2.5 \%\right) \quad \ldots \ldots \ldots \ldots . .235$

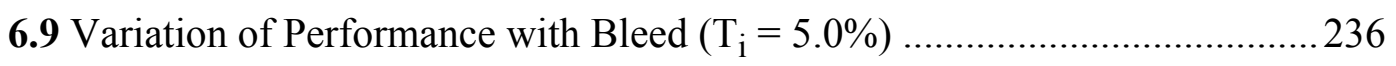

6.10 Performance Data, Three-Dimensional Predictions, $\mathrm{B}_{\mathrm{i}}=2.5 \%, \mathrm{~B}_{\mathrm{o}}=3.0 \% \quad .242$ 


\section{NOMENCLATURE}
A area
$A_{R}, A R \quad$ ratio, aspect ratio
B percentage of mainstream flow which is bled out through the vortex
$\mathrm{C}_{\mathrm{d}} \quad$ discharge coefficient
$\mathrm{C}_{\mathrm{p}} \quad$ coefficient of static pressure recovery (e.g. $\mathrm{C}_{\mathrm{p} 2-3}=\frac{\tilde{\mathrm{p}}_{3}-\tilde{\mathrm{p}}_{2}}{\tilde{\mathrm{q}}_{2}}$ )
$\mathrm{C}_{\mu} \quad$ dimensionless constant $(\approx 0.09)$
$\mathrm{D}_{\mathrm{ij}} \quad$ transport of Reynolds stress by diffusion
$\mathrm{D}_{\mathrm{p}} \quad$ dynamic pressure parameter
h height
$\mathrm{i}, \mathrm{j}, \mathrm{k} \quad$ unit vectors in general orthoganol co-ordinate directions
$\mathrm{k} \quad$ turbulent kinetic energy
L length, characteristic length
$\dot{\mathrm{m}} \quad$ mass flow rate
P local total pressure
$\mathrm{P}_{\mathrm{ij}} \quad$ production of Reynolds stress
p local static pressure
q dynamic pressure
Re Reynolds number
$\mathrm{r} \quad$ radius
r, $\theta \quad$ polar co-ordinates
$\mathrm{r}_{\mathrm{i}} \quad$ inner radius
$\mathrm{r}_{\mathrm{o}} \quad$ outer radius
S $\quad$ source term
$\mathrm{S}_{\mathrm{p}} \quad$ stagnation pressure parameter
$\mathrm{U}, \mathrm{V}, \mathrm{W} \quad$ velocity components in $\mathrm{x}, \mathrm{y}, \mathrm{z}$ directions
$\mathrm{U} \quad$ rotor blade speed
$\mathrm{U}_{\mathrm{i}} \quad$ component of mean velocity in $\mathrm{x}$ direction 
$\mathrm{u}_{\mathrm{i}} \quad$ fluctuating velocity component

$U_{\mathrm{i}} \quad$ instantaneous velocity component $\left(=\mathrm{U}_{\mathrm{i}}+\mathrm{u}_{\mathrm{i}}\right)$

$\mathrm{U}_{\tau} \quad$ friction velocity $\left(\left(\frac{\tau_{\mathrm{w}}}{\rho}\right)^{\frac{1}{2}}\right)$

$\mathrm{V}_{\mathrm{a}} \quad$ flow axial velocity through rotor (mid annulus height)

X pitch pressure parameter

$\mathrm{Xr} \quad$ reattachment length

$\mathrm{x}, \mathrm{y}, \mathrm{z} \quad$ Cartesian co-ordinates

axial gap between inlet duct and vortex retaining fence

Y yaw pressure parameter

y radial gap between inlet duct and vortex retaining fence

$\alpha \quad$ kinetic energy coefficient

$\beta \quad$ diffuser mean turning angle

$\delta_{\mathrm{ij}} \quad$ Kronecker delta $(=1$ when $\mathrm{i}=\mathrm{j}$, and $=0$ when $\mathrm{i} \neq \mathrm{j})$

$\varepsilon \quad$ dissipation rate of turbulent kinetic energy

$\eta \quad$ diffuser effectiveness

$\theta \quad$ diffuser wall angle, circumferential position

$\vartheta \quad$ velocity scale $\left(\mathrm{k}^{1 / 2}\right)$

$\ell \quad$ length scale $\left(\frac{\mathrm{k}^{2 / 3}}{\varepsilon}\right)$

$\kappa \quad$ von-Karman's constant (0.41)

$\lambda \quad$ total pressure loss coefficient (e.g. $\lambda_{2-3}=\frac{\tilde{\mathrm{P}}_{2}-\tilde{\mathrm{P}}_{3}}{\tilde{\mathrm{q}}_{2}}$ )

$\mu \quad$ molecular viscosity

$\mu_{\mathrm{t}} \quad$ dynamic turbulent viscosity $\left(\rho \mathrm{C}_{\mu} \frac{\mathrm{k}^{2}}{\varepsilon}\right)$

$v \quad$ kinematic viscosity $(\mu / \rho)$

$\xi \quad$ absolute swirl angle

$\Pi_{\mathrm{ij}} \quad$ transport of Reynolds stress by pressure-strain interaction

$\rho \quad$ density

$\overline{\rho u_{\mathrm{i}} \mathrm{u}_{\mathrm{j}}} \quad$ Reynolds stress 


$\begin{array}{ll}\tau & \text { shear stress } \\ \tau_{\mathrm{w}} & \text { wall shear stress } \\ \phi & \text { general scalar quantity } \\ \phi & \text { fence subtended angle, } \tan ^{-1}(\mathrm{y} / \mathrm{x}), \text { rotor flow coefficient }(\mathrm{Va} / \mathrm{U}) \\ \Omega_{\mathrm{ij}} & \text { transport of Reynolds stress by rotation }\end{array}$

\section{Subscripts}

$\begin{array}{ll}1 & \text { inlet } \\ 2 & \text { rotor exit } \\ 3 & \text { OGV exit } \\ 4 & \text { diffuser exit } \\ 5 & \text { inner feed annulus } \\ 6 & \text { outer feed annulus } \\ \text { amb } & \text { ambient conditions } \\ \text { i } & \text { inner (bleed) } \\ \text { o } & \text { outer (bleed) }\end{array}$

\section{Superscripts}

$-\quad$ spacial mean value
$\sim \quad$ mass weighted spatial mean value




\subsection{INTRODUCTION}

1.1 Emission Concerns and Regulations $\ldots \ldots \ldots \ldots \ldots \ldots \ldots$

1.2 Reducing $\mathrm{NO}_{\mathrm{x}}$ and Low-NO $\mathrm{N}_{\mathrm{x}}$ Combustion Systems .........5

1.2.1 Variable Geometry Combustors $\ldots \ldots \ldots \ldots \ldots \ldots \ldots$

1.2.2 Lean Premix Prevaporised Combustion ............6

1.2.3 Rich-Burn, Quick-Quench, Lean-Burn Combustor . . . . . . .6

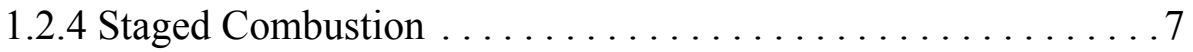

1.3 Combustor System Total Pressure Loss $\ldots \ldots \ldots \ldots \ldots$

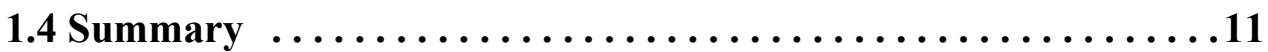

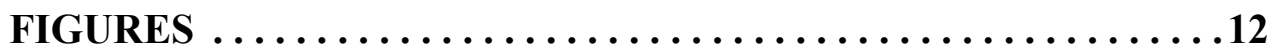




\subsection{INTRODUCTION}

\section{$\underline{\text { 1.1 Emission Concerns and Regulations }}$}

In order to discuss the major focus of this thesis - hybrid diffusers for gas turbine combustion - it is important to understand the reasons why, and the direction in which, this technology is being driven. Traditionally, for aeronautical gas turbines it has been the desire for better fuel efficiency and reduced weight which has forced development of the combustion system. More recently, however, strict legislation has meant that the emission of pollutants is now a major factor behind the design of combustion chambers. The reduction of emissions is not only important from an environmental view point but it has also become a significant issue for engine manufacturers in an increasingly competitive market.

The exhaust from an aircraft gas turbine is composed of carbon monoxide (CO), carbon dioxide $\left(\mathrm{CO}_{2}\right)$, oxides of nitrogen $\left(\mathrm{NO}_{\mathrm{x}}\right)$, unburned hydrocarbons (UHC), water vapour $\left(\mathrm{H}_{2} \mathrm{O}\right)$ and particulate matter or smoke. The two main areas of concern are, according to Lefebvre $^{[1983]}$, urban pollution in the vicinity of airports and pollution of the stratosphere at aircraft cruise altitudes. Lefebvre ${ }^{[1995]}$ comments that carbon dioxide and water vapour have not, traditionally, been considered as pollutants as they are natural products of the complete combustion of a hydrocarbon fuel. However, they both contribute to global warming and can only be reduced by burning less fuel. Conversely, carbon monoxide is a toxin and reduces the blood's capacity to absorb oxygen and, in high concentrations, can even be fatal. Unburned hydrocarbons are also toxic but in addition to this they can combine with oxides of nitrogen to form photochemical smog. Smoke emissions are undesirable as they contain carcinogens and very fine particulates have been associated (Seaton et $\mathrm{al}^{[1995]}$ ) with asthma and other respiratory diseases. At ground level, oxides of nitrogen not only contribute to the formation of smog but also add to the problem of acid rain. Furthermore, at altitudes of less than $3 \mathrm{~km}$ a major concern of $\mathrm{NO}_{\mathrm{x}}$ emissions is the resulting formation of low level ozone. Bahr ${ }^{[1991,2]}$ reports that in Western Europe low level ozone concentrations are approaching 50 parts per billion (ppb). At concentrations around 100ppb prolonged exposure to ozone can be detri- 
mental to health causing impaired vision and headaches. In addition to this, at higher altitudes, typical of aircraft cruise, $\mathrm{NO}_{\mathrm{x}}$ reacts with and causes depletion of the stratospheric ozone layer. Reduction of the ozone layer increases ground level ultraviolet radiation which, in turn, leads to an increase in the incidence of skin cancer. Thus, the effect of $\mathrm{NO}_{\mathrm{x}}$ emissions is serious and far-reaching and has become a major concern for aircraft engine designers.

Aircraft gas turbine emissions are, relative to other sources, only a minor contributor to global emissions. A 1999 report by the Intergovernmental Panel on Climate Change (IPCC) entitled "Aviation and the Global Atmosphere" summarised that:

- in 1992 aviation contributed $3-5 \%$ of the total man-made pollutants that add to the greenhouse effect.

- in 1999 aircraft will produce approximately $2 \%$ of all $\mathrm{CO}_{2}$ emissions (or $13 \%$ from all transport sources).

- emissions of $\mathrm{NO}_{\mathrm{x}}$ from subsonic aircraft are estimated to have increased ozone concentrations at cruise altitude by $6 \%$ since 1992 and this is projected to increase to about $13 \%$ by 2050 .

For many years aircraft gas turbines have operated within the limits prescribed by the International Civil Aviation Organisation (ICAO). These regulations define acceptable limits for the emission of unburned hydrocarbons, smoke, carbon monoxide and oxides of nitrogen. However, due to an increased awareness of the environmental impact of aircraft emissions the ICAO's Committee on Aviation Environmental Protection (CAEP) was asked to recommend a more stringent set of regulations. In 1993 the ICAO accepted the CAEP/2 proposals (ICAO Annex 16 Vol.2) which, in essence, represent a two tier system whereby the emissions legislation is linked to the date an engine type is certified or an engine unit is manufactured. For example ICAO Annex 16, Vol. 2 gives the $\mathrm{NO}_{\mathrm{x}}$ limits as:

- new engine types certified before the end of 1995 or engine units manufactured before the end of 1999: $\quad \frac{D_{p}}{F_{o o}}=40+2 \pi_{o o}$ 
- and for new engine types certified after January 1996 or engine units manufactured after January 2000: $\quad \frac{\mathrm{D}_{\mathrm{p}}}{\mathrm{F}_{\mathrm{oo}}}=32+1.6 \pi_{\mathrm{oo}}$

where $\mathrm{D}_{\mathrm{p}}$ is the mass in grams of pollutant, $\mathrm{F}_{\mathrm{oo}}$ is the rated thrust $(\mathrm{kN})$ of the engine and $\pi_{\mathrm{oO}}$ is the engine pressure ratio at takeoff.

Mortimer ${ }^{[1998]}$ reports that in 1995 CAEP revised their legislation and issued the CAEP/ 3 guidelines which recommended further reductions in $\mathrm{NO}_{\mathrm{x}}$. However, these new limits were not imposed as engine manufacturers argued they were too stringent. In April 1998 CAEP reworked their proposals and slightly relaxed the $\mathrm{NO}_{\mathrm{x}}$ limit for high engine pressure ratios. These $\mathrm{CAEP} / 4$ proposals have since been approved and are due to come into force in 2004 and therefore represent the standard to which engine manufacturers are currently designing.

With reference to Figure 1.1 the mechanisms for the production of pollutants are such that levels of $\mathrm{CO}$ and $\mathrm{UHC}$ are at a maximum at low power levels whereas the levels of $\mathrm{NO}_{\mathrm{x}}$ and smoke are highest at full power. Lefebvre ${ }^{[1995]}$ states that, for modern gas turbine engines, levels of $\mathrm{CO}$ and $\mathrm{UHC}$ have been greatly reduced at all low power conditions and therefore it is the level of $\mathrm{NO}_{\mathrm{x}}$ emissions which is of paramount importance. Thus to remain competitive within the market place engine manufacturers must produce combustion systems which minimise the formation of $\mathrm{NO}_{\mathrm{x}}$. Figure 1.2 graphically illustrates the current $\mathrm{CAEP} / 2$ and future $\mathrm{CAEP} / 4 \mathrm{NO}_{\mathrm{x}}$ limits with respect to engine pressure ratio. Of interest are the shaded areas which represent the performance of old, current and future combustor technologies. Figure 1.2 indicates that in order to meet the CAEP/2 requirements older single annular combustion technology had to be refined and developed. At the upper limit of development this technology may satisfy CAEP/4 but it is clear that to meet future and more stringent emissions regulations engine manufacturers must look towards new technology such as staged double annular combustors or even more radical designs. However, the development and implementation of new technology is an expensive process and far from risk free. 


\section{$\underline{1.2 \text { Reducing NO}}{ }_{\underline{x}}$ and Low-NO $\underline{\underline{x}}$ Combustion Systems}

The primary mechanism, according to Lefebvre ${ }^{[1995]}$, for the formation of $\mathrm{NO}_{\mathrm{x}}$ is by the oxidation of atmospheric nitrogen in the high temperature regions of the flame. Above temperatures of $1850 \mathrm{~K}$ this reaction is significantly rapid and therefore the prime objective in reducing $\mathrm{NO}_{\mathrm{x}}$ is to reduce the reaction temperature or decrease residence time at high temperature. Included in this aim is the elimination of "hot-spots" as these will result in localised $\mathrm{NO}_{\mathrm{x}}$ production. Adding more air to the primary zone (refer to Figure 1.3) will lower the reaction temperature or an increase in liner pressure drop will enhance mixing and eliminate hot-spots. One further method of lowering the flame temperature is to inject water into the flame tube and Hung ${ }^{[1974,85]}$ claimed to achieve $\mathrm{NO}_{\mathrm{x}}$ reductions of $80 \%$. However, White et al ${ }^{[1982]}$ report this method has many drawbacks such as increased capital cost, increased fuel consumption and the impracticality, for aero applications, of physically carrying the required water. Furthermore, another drawback of reducing $\mathrm{NO}_{\mathrm{x}}$ emissions by lowering the flame temperature is that it will inevitably lead to increased levels of UHC and CO. Figure 1.4 shows that for a conventional combustor an optimum temperature range of $1670-1900 \mathrm{~K}$ exists for which $\mathrm{NO}_{\mathrm{x}}$ and $\mathrm{CO}$ emissions can be kept below 15 and 25ppm respectively. Thus the underlying principle in the design of low emission, specifically low $\mathrm{NO}_{\mathrm{x}}$, combustion systems is to ensure that the reaction temperature remains within this range from engine idle to full power.

\subsubsection{Variable Geometry Combustors}

Variable geometry combustion, as a concept, is not new but designers have been reluctant to employ this type of combustor due to the mechanical complications involved. However, it is a measure of the magnitude of the emissions issue that variable geometry is again being considered, especially for non-aeronautical applications. The concept is simple; at full-power the geometry is varied such that large quantities of air are admitted into the primary zone thus reducing the temperature. And, as engine power is reduced air is diverted into the dilution zone, hence maintaining the primary zone temperature within the low-emissions band illustrated in Figure 1.4. To achieve this variation in airflow Bayle-Labouré ${ }^{[1991]}$ used variable-area swirlers and Roberts et al ${ }^{[1982]}$ and Sasaki et 
$\mathrm{al}^{[1991]}$ used variable air openings into the dilution zone. The main drawback with variable geometry is the complex mechanical control and feedback systems which add to weight, reduce reliability and make this type of low-emission combustion chamber impractical for aeronautical applications.

\subsubsection{Lean Premix Prevaporised Combustion}

In a conventional combustion chamber fuel is injected into the primary zone with little or no premixing with air. The result of this is that combustion occurs in localised stoichiometric pockets producing high temperatures and thus high amounts of $\mathrm{NO}_{\mathrm{x}}$. The underlying principle behind lean, premix, prevaporisation (LPP) is elimination of these pockets by mixing the fuel and air prior to injection and then operating the primary zone at a low fuel/air ratio or ideally an equivalence ratio very close to the lean extinction limit. Essentially LPP systems require a fuel injector which can achieve the complete evaporation and mixing of the fuel and air. Combustion of this mixture can then take place at a fairly low and uniform temperature thus minimising $\mathrm{NO}_{\mathrm{x}}$ production. Poeschl et $\mathrm{al}^{[1994]}$ reported $\mathrm{NO}_{\mathrm{x}}$ concentrations as low as 10ppm. Furthermore, Leonard and Stegmaier ${ }^{[1994]}$ note that the flame temperature never exceeds $1900 \mathrm{~K}$ and as such the amount of $\mathrm{NO}_{\mathrm{x}}$ formed does not increase with residence time. Therefore, LPP systems can utilise a relatively long residence time in order to reduce production of $\mathrm{CO}$ and $\mathrm{UHC}$ whilst still maintaining low $\mathrm{NO}_{\mathrm{x}}$. Nonetheless, LPP is not without its problems. For example there is a danger of autoignition or flashback at high power settings due to premixing of the fuel with air at a high inlet temperature. Also the high quantity of air required at maximum power to produce lean conditions may result in a flame blow out at low power conditions.

\subsubsection{Rich-Burn, Quick-Quench, Lean-Burn Combustor}

Rich-burn, quick-quench, lean-burn (RQL) as proposed by Mosier and Pierce ${ }^{[1980]}$ and

Pierce el al ${ }^{[1980]}$ utilises a staged burning concept. Initially a fuel-rich primary zone (equivalence ratio 1.2-1.6) is used to reduce $\mathrm{NO}_{\mathrm{x}}$ by lowering the flame temperature and lowering the available oxygen. Immediately after this the fuel-rich combustion products 
are exposed to jets of air which rapidly cool them to a level where $\mathrm{NO}_{\mathrm{x}}$ production is negligible. This is the quick-quench stage and must be almost immediate to prevent formation of local regions of near-stoichiometric fuel/air ratios as this will result in high $\mathrm{NO}_{\mathrm{x}}$ production. It is this area where most of the problems associated with RQL lie but the concept has enough potential for the Pratt and Whitney Company (Lefebvre ${ }^{[1995]}$ ) to be actively pursuing it.

\subsubsection{Staged Combustion}

The aim of staged combustion is to switch the fuel flow between two or more primary zones in order to maintain an overall combustion temperature within the low emissions band. A commonly used technique to achieve this is "selective fuel injection" $\left(\right.$ Bahr $\left.^{[1987]}\right)$ in which at low power settings only a limited number of the available fuel injectors are employed and the full complement of injectors is only used at maximum power. A disadvantage of this method is that it results in "chilled" regions downstream of the injectors not in use. This reduces combustion efficiency, increases $\mathrm{CO}$ and UHC production and is detrimental to turbine performance and life due to a non-uniform combustor exit temperature distribution. The search to overcome these problems has led to the development of the true "staged" combustor in which one annular combustion zone is replaced by two or more annular zones. This principle is illustrated in Figure 1.5 and Figure 1.6. For a conventional single annular combustor, as more power is required the fuel/ air ratio is increased and thus the flame temperature and $\mathrm{NO}_{\mathrm{x}}$ production also increase. However in a staged system, before $\mathrm{NO}_{\mathrm{x}}$ emissions become excessive fuel is diverted to a separate primary zone. Now both primary zones (usually termed pilot and main) operate but at fuel/air ratios low enough to prevent high flame temperatures and high $\mathrm{NO}_{\mathrm{x}}$. These primary zones can be arranged axially as in the Pratt and Whitney combustor shown in Figure 1.7 or radially as in the General Electric dual-annular combustor (Figure 1.8). In the latter case the outer primary zone is designated the pilot zone and provides the combustion at low engine power levels such as start up and idle. At higher power levels fuel is added to the main zone which is optimised for higher fuel flow rates with a short residence time and low equivalence ratio thus limiting the formation of $\mathrm{NO}_{\mathrm{x}}$ and smoke. The General Electric combustor is currently an option for the CFM56-5B engine 
used on the Airbus Industrie A320 and A321 aircraft. Lefebvre ${ }^{[1995]}$ reports that it achieves around 35\% reduction in $\mathrm{CO}$ and $\mathrm{UHC}$, and $45 \%$ reduction in $\mathrm{NO}_{\mathrm{x}}$ over a corresponding single annular combustor fitted in the same engine type. Disadvantages of the staged combustion systems are added weight and mechanical complexity plus the need for more cooling air as the combustion chamber liners comprise more surface area. Axial staging does have certain advantages over radial staging due to the main stage being downstream of the pilot which ensures ignition of the main stage is rapid and reliable. Furthermore, the hot gases from the pilot zone ensure that the main zone has a high combustion efficiency. However, in addition to having many of the same disadvantages as radial staging, axially staged combustion also suffers from an extra length and weight penalty.

A further development of staged combustors, suggested by Lefebvre ${ }^{[1995]}$, is to include an LPP unit in place of the main zone fuel injector, thus taking advantage of the low emission properties of both staged and LPP combustion.

However, a major drawback of staged combustion is that, relative to conventional single annular technology, staged systems have an extremely deep flame tube. This presents a problem as compressor delivery air must be supplied as efficiently as possible (i.e. with minimum total pressure loss) to the various features of the combustor. This implies that very high rates of diffusion and turning must be achieved in a short length. Conventional diffuser technology can only achieve a limited amount of diffusion and turning in any given length and only then with the penalty of increasing total pressure loss (Sections 1.3 and 2.0). Thus it is clear that if staged combustion is to be used to meet future emissions legislation then diffuser technology must also be developed. However, the fact that General Electric and Pratt and Whitney have already invested in staged combustion technology suggests that they believe this could provide a solution to the emissions problem. Furthermore, General Electric have recognised the fact that diffuser technology must also be advanced and employ a split, twin passage pre-diffuser in their dual-annular combustor (see Figure 1.8). 


\section{$\underline{1.3 \text { Combustor System Total Pressure Loss }}$}

Any loss in total pressure between inlet and outlet of a combustor system leads (Cohen at $\left.\mathrm{al}^{[1996]}\right)$ to both an increase in specific fuel consumption and a reduction in specific power. These losses arise from two causes;

(i) skin friction and turbulence, and

(ii) a rise in temperature due to combustion.

The latter of these is termed the 'fundamental' loss; the combustion process increases the temperature of the air, its density drops, its velocity increases and this results in a total pressure loss. However, Cohen et al ${ }^{[1996]}$ comment that the fundamental loss is typically $1-2 \%$ of the inlet dynamic pressure which is small compared to the loss due to skin friction and turbulence. It is therefore imperative that this aerodynamic loss is minimised in order to maximise the fuel efficiency of an engine.

Overall combustor total pressure loss is non-dimensionalised by the mean total pressure at compressor exit to give a pressure loss parameter. In any given configuration this increases proportionally to the square of the compressor exit velocity (Klein ${ }^{[1959]}$ ). A typical value for this parameter is, according to Kaiser and McDonald ${ }^{[1980]}$, 6\% (not including the fundamental loss) and Oates ${ }^{[1985]}$ reports that an increase of $1 \%$ will result in a drop in thrust of $1 \%$ and a rise in specific fuel consumption of $0.5-0.75 \%$.

Lefebvre $^{[1983]}$ states that the aerodynamic pressure loss can be divided into two main components; total pressure drop in the diffuser and total pressure drop across the flame tube liner. It is essential that all the elements of a gas turbine combustion system are adequately fed, via the diffuser, with air from the compressor exit. If the fuel injectors, swirlers (especially LPP units) or feed annuli are not correctly fed then even the best design of low emission combustion chamber will ultimately fail in its goal. For example, the condition of the flow within the combustor feed annulus will effect the delivery of air into the flame tube via the primary and dilution ports. A high velocity will be beneficial for convective cooling of the liner walls but generally lower velocities are preferred because 
this leads to:

(i) lower losses (skin friction and 'sudden expansion' losses downstream of liner holes),

(ii) steeper angles of jet penetration, and

(iii) lower circumferential variations in annulus velocity and static pressure which ensures all liner holes in the same row pass the same airflow.

It is the task of the pre-diffuser to slow the compressor efflux, turning the airflow and directing it towards the downstream components of the combustion system. Diffusers must achieve this in a short axial length to minimise weight and with the minimum of total pressure loss to maximise specific fuel consumption. Carrotte and Bailey ${ }^{[1994]}$ and Carrotte and Barker ${ }^{[1994]}$ produced data (Figure 1.9) which demonstrated that as the ratio of flame tube depth to combustor length increases, inevitable for staged combustion, the aerodynamic losses to the combustor feed annuli rapidly rise. To overcome this the diffuser must further reduce the velocity of the airflow (i.e. increase area ratio) and produce a higher degree of turning. However, conventional diffusers have performance limits and simply increasing area ratio will eventually result in flow separation (Sovran and Klomp ${ }^{[1967]}$, Figure 1.10). Additionally, the introduction of curvature will further reduce the area ratio at which this occurs (Sagi and Johnston ${ }^{[1967]}$, Figure 1.11). In simple terms these performance limits mean that conventional diffusers will struggle to meet the necessary performance required for staged combustion. Thus there is a need to study and develop an understanding of more advanced and unconventional diffuser technology. 


\subsection{Summary}

In the space of the last two decades the need to reduce the emissions of pollutants, in particular $\mathrm{NO}_{\mathrm{x}}$, from aero gas turbines has become perhaps the most important driving force behind the design of combustion chambers. Furthermore, in order to meet the emissions legislation designers are having to consider new and unconventional designs. According to Lefebvre ${ }^{[1995]}$ one of the most attractive options is the combination of staged combustion with lean, premixed, prevaporised fuel injection. However, for this combination to reach its full potential diffuser technology needs to be developed. Thus the specifics of conventional diffuser performance and a review of the relevant literature is presented in Section 2.0. Furthermore, Section 2.0 also reviews the methods, both conventional and unconventional, by which diffusers have been developed to achieve higher area ratios and controlled turning without incurring excessively high total pressure losses. Additionally Section 2.0 identifies the "Hybrid diffuser" as a attractive alternative to conventional pre-diffuser designs due to its ability to achieve high rates of diffusion in a short length. The detailed aims and objectives of this study are presented in Section 2.8 but can be summarised as:

- identification of an advanced diffuser concept (hybrid diffuser) which has the potential to acheive the necessary performance for use with low emission, staged comustion systems,

- investigation of the effect on hybrid diffuser and system performance of, for example, various geometric partameters, diffuser radial struts and OGV wakes,

- identification of the pertenent aerodynamic mechanisms in order to design an optimised hybrid diffuser,

- finally, to determine if hybrid diffusers offer a solution to the requirements of modern, low emission, staged combustion systems. 


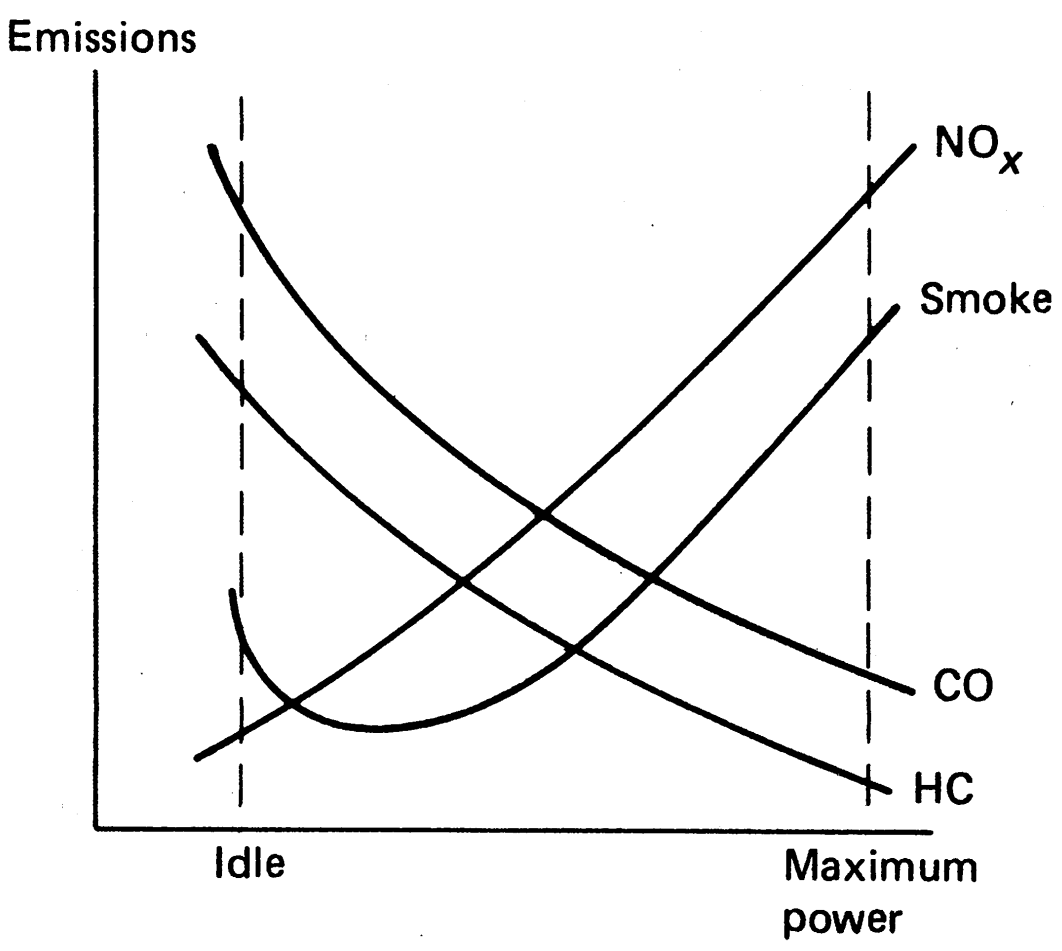

Figure 1.1 Emissions Characteristics of a Gas Turbine, Lefebvre ${ }^{[1995]}$

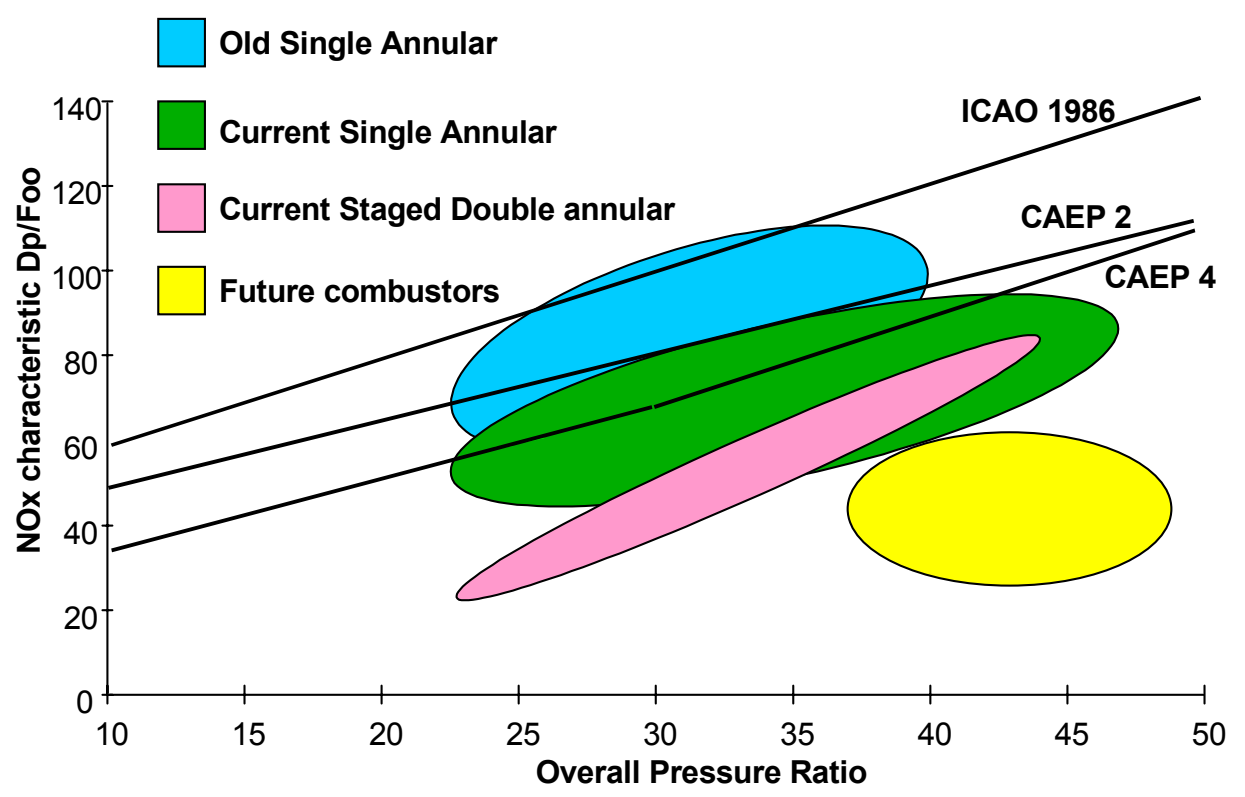

\section{圈 Rolls-Royce}

Figure 1.2 Variation of $\mathrm{NO}_{\mathrm{x}}$ Characteristics with Engine Pressure Ratio, Birch ${ }^{[2000]}$ 


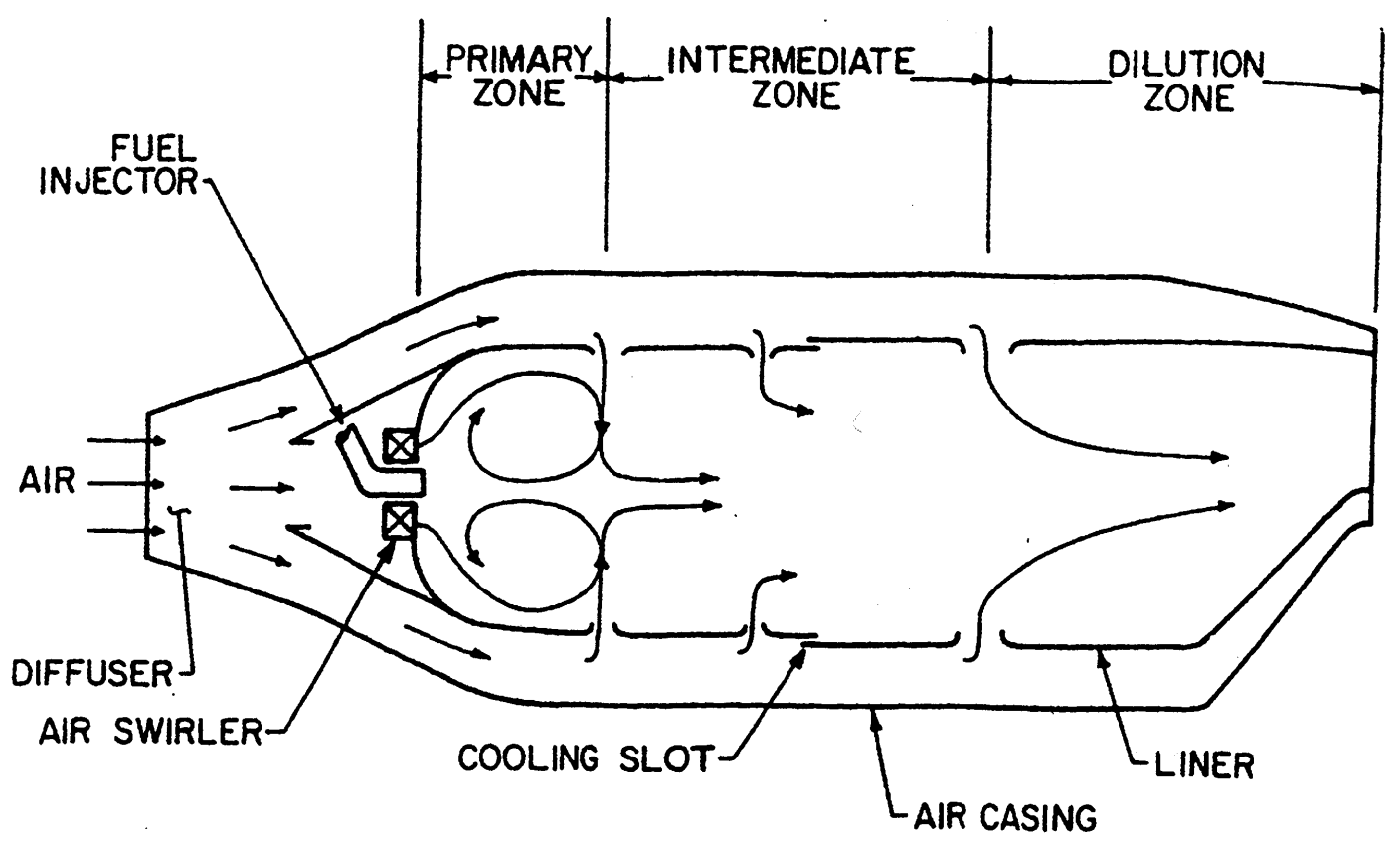

Figure 1.3 Main Components of a Gas Turbine Combustor, Lefebvre ${ }^{[1995]}$

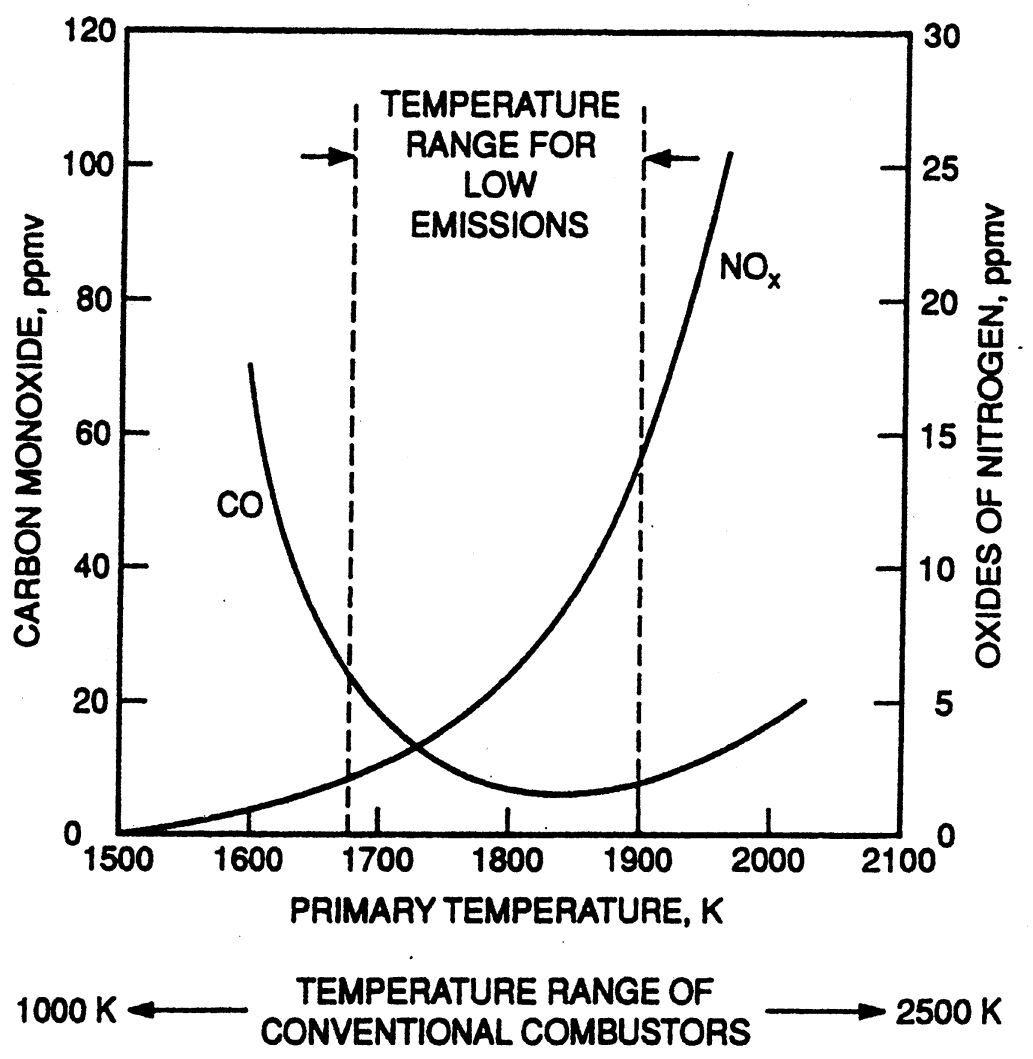

Figure 1.4 Influence of Primary Zone Temperature on $\mathrm{CO}$ and $\mathrm{NO}_{\mathrm{x}}$ Emissions, Lefebvre $^{[1995]}$ 


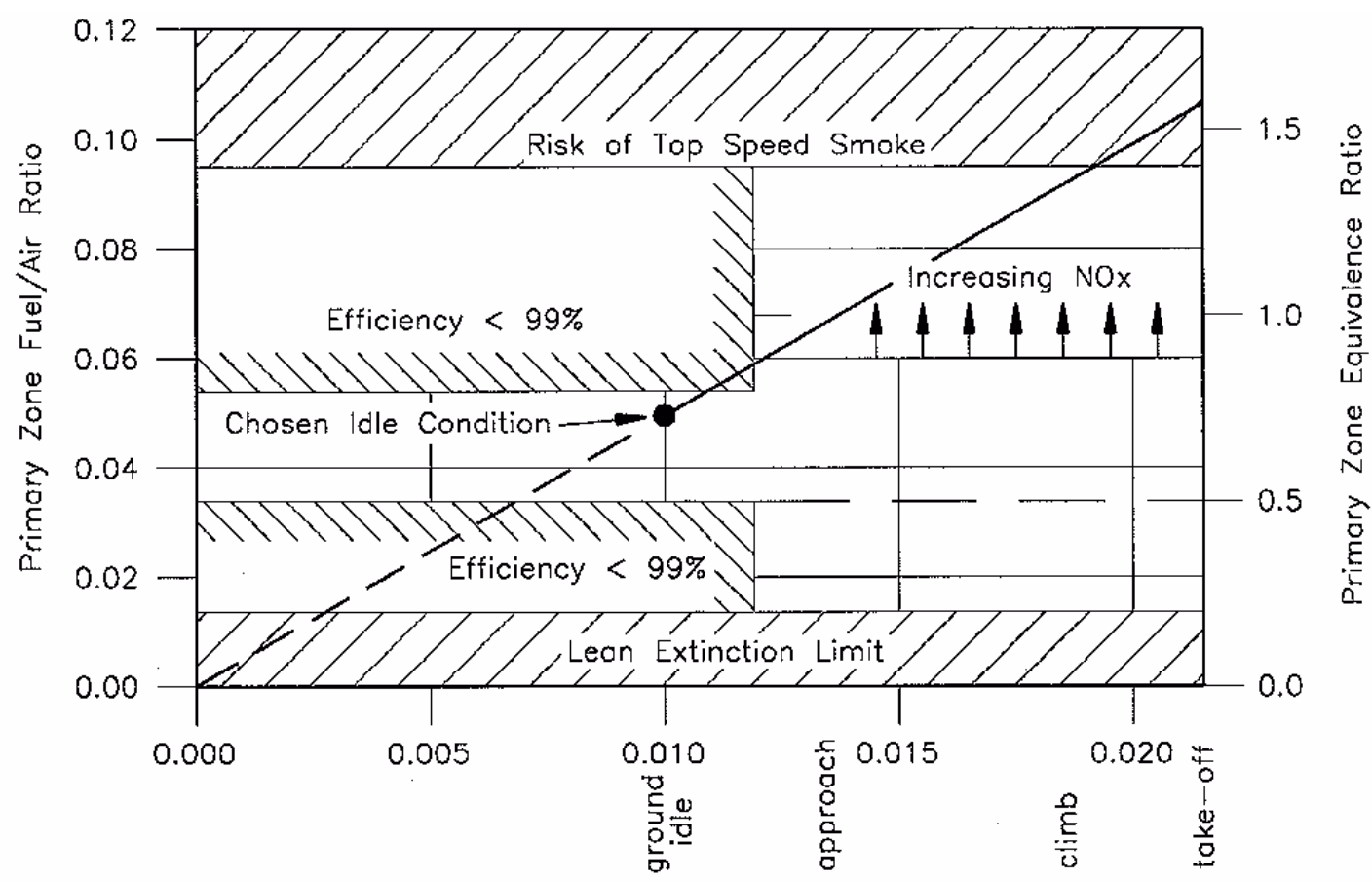

Overall Fuel/Air Ratio

Figure 1.5 Relationship between Primary Zone and Overall Stoichiometries for Fixed Combustor Geometries, Wray ${ }^{[1995]}$

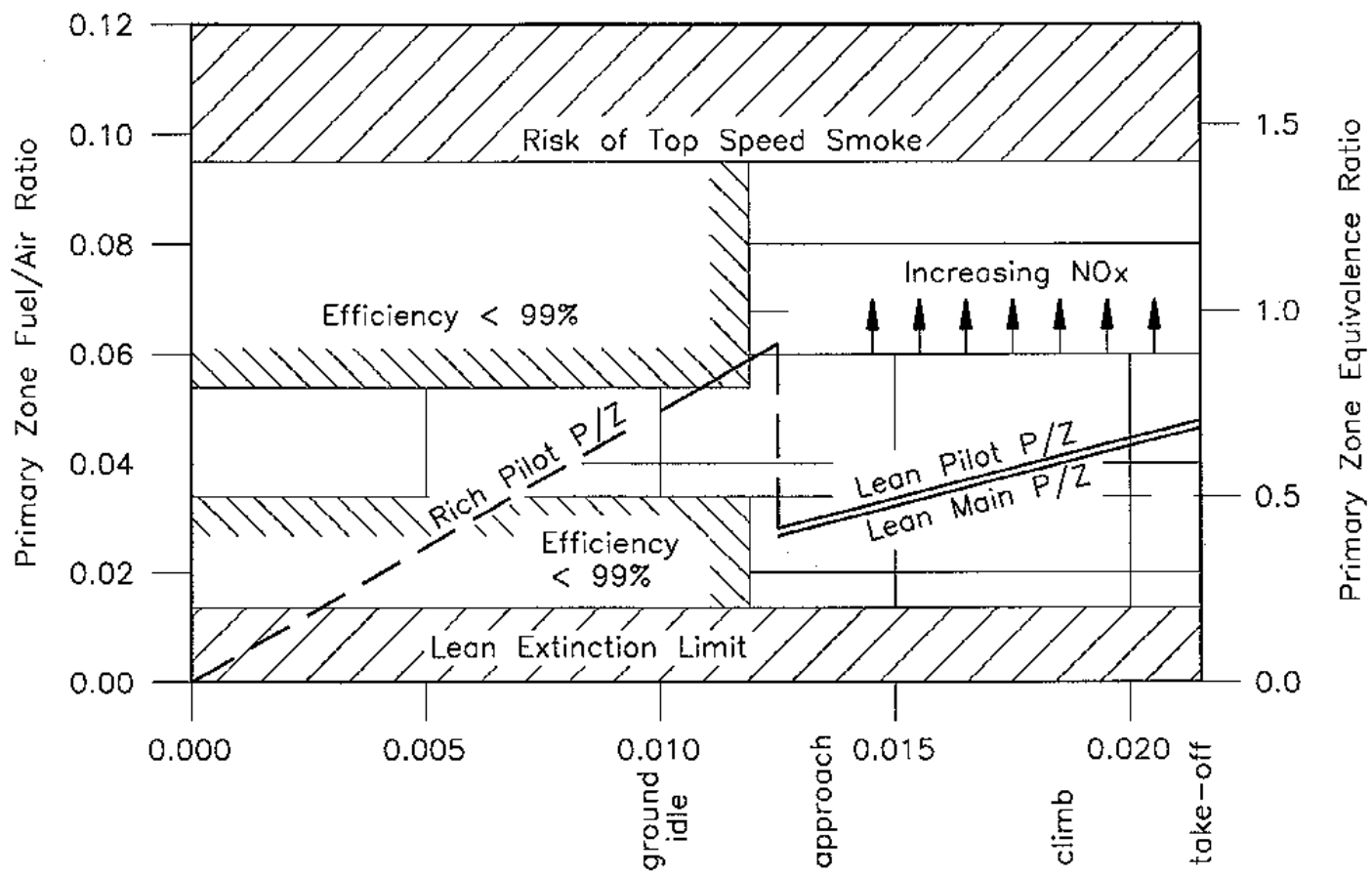

Overall Zone Fuel/Air Ratio

Figure 1.6 Relationship between Primary Zone and Overall Stoichiometries for a Staged Combustor, Wray ${ }^{[1995]}$ 


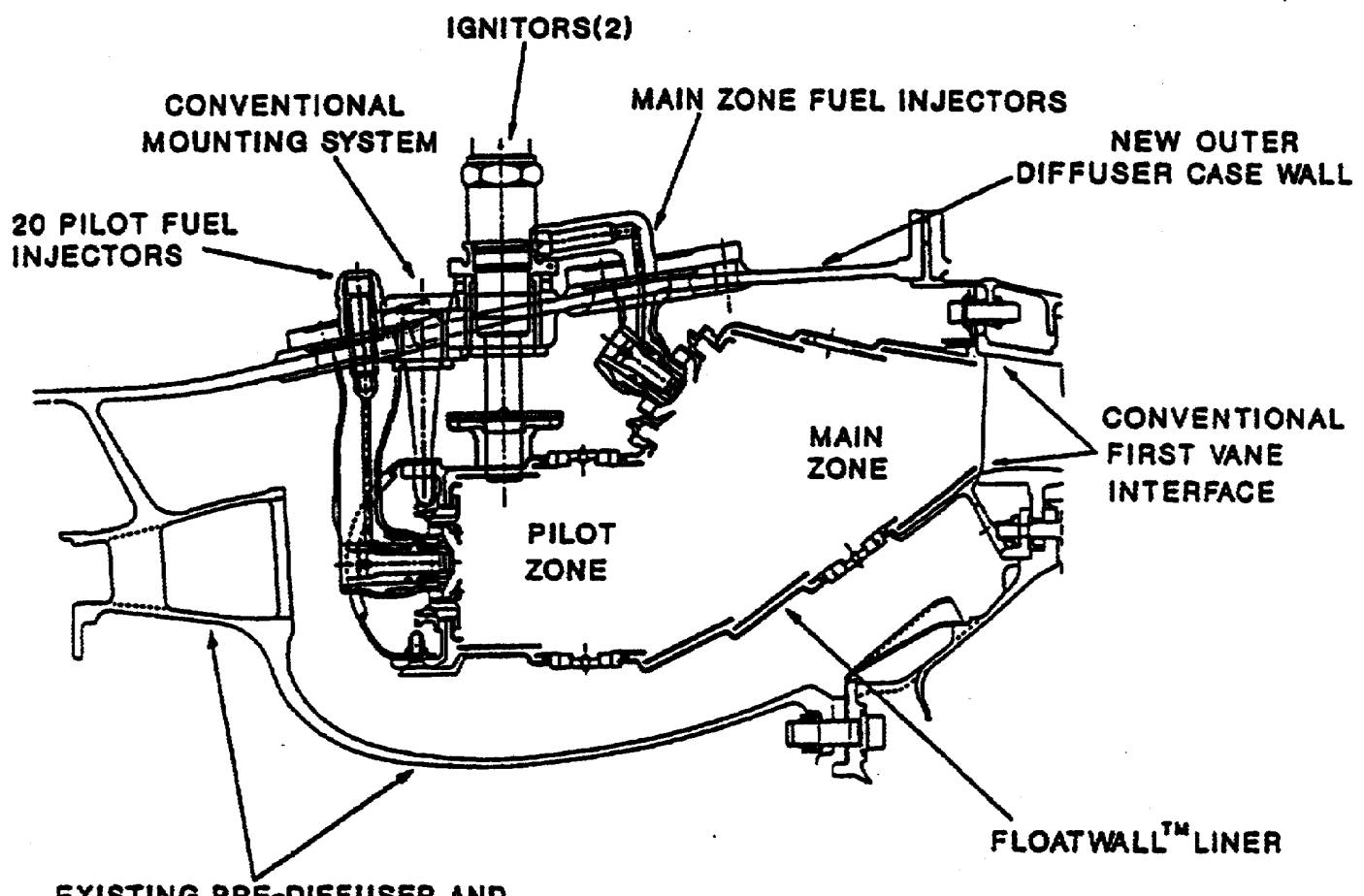

EXISTING PRE-DIFFUSER AND

INNER DIFFUSER CASE WALL

Figure 1.7 Pratt and Whitney Axially Staged Combustor, Koff ${ }^{[1993]}$

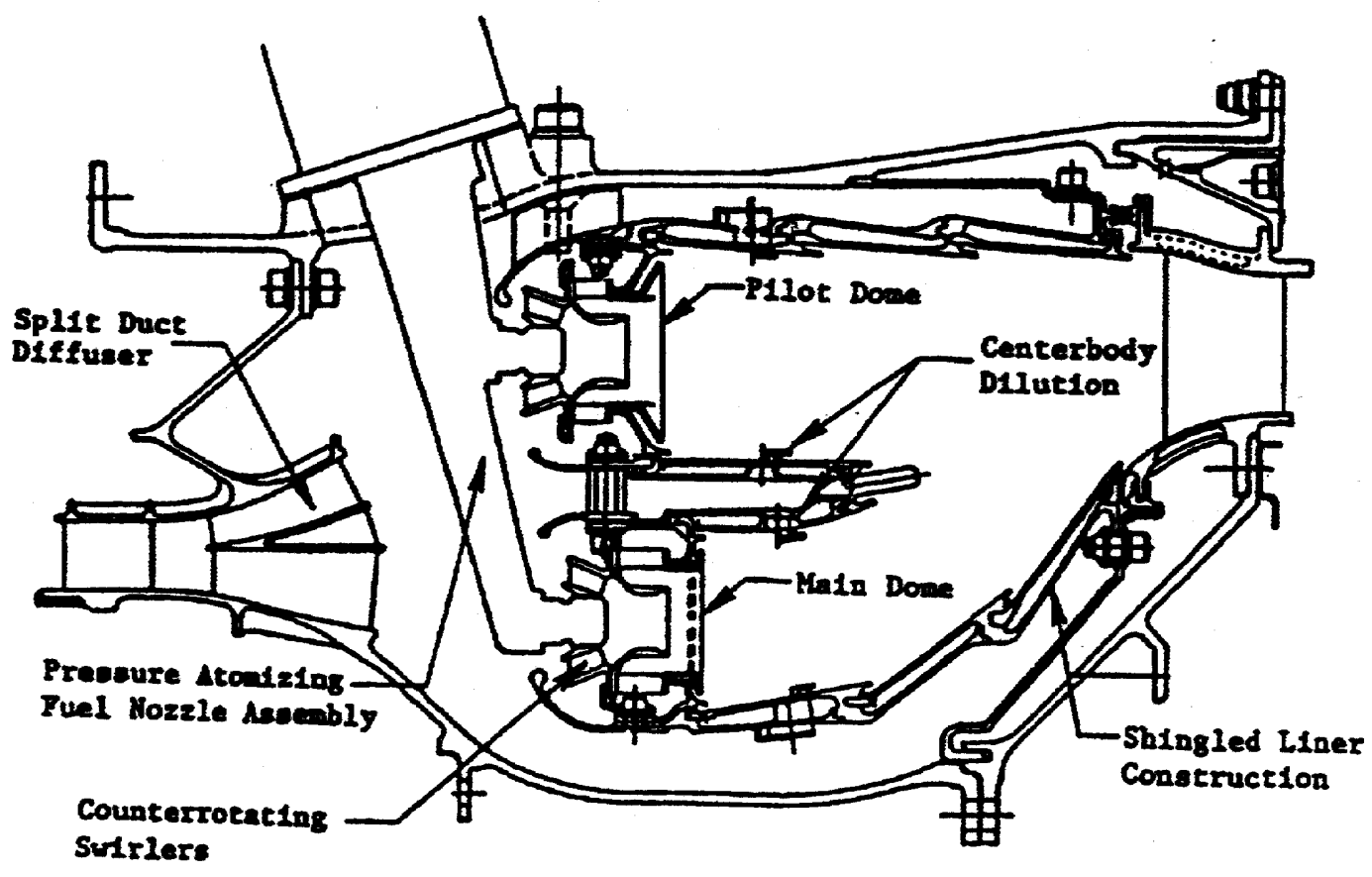

Figure 1.8 General Electric Radially Staged Combustor, Lefebvre ${ }^{[1995]}$ 


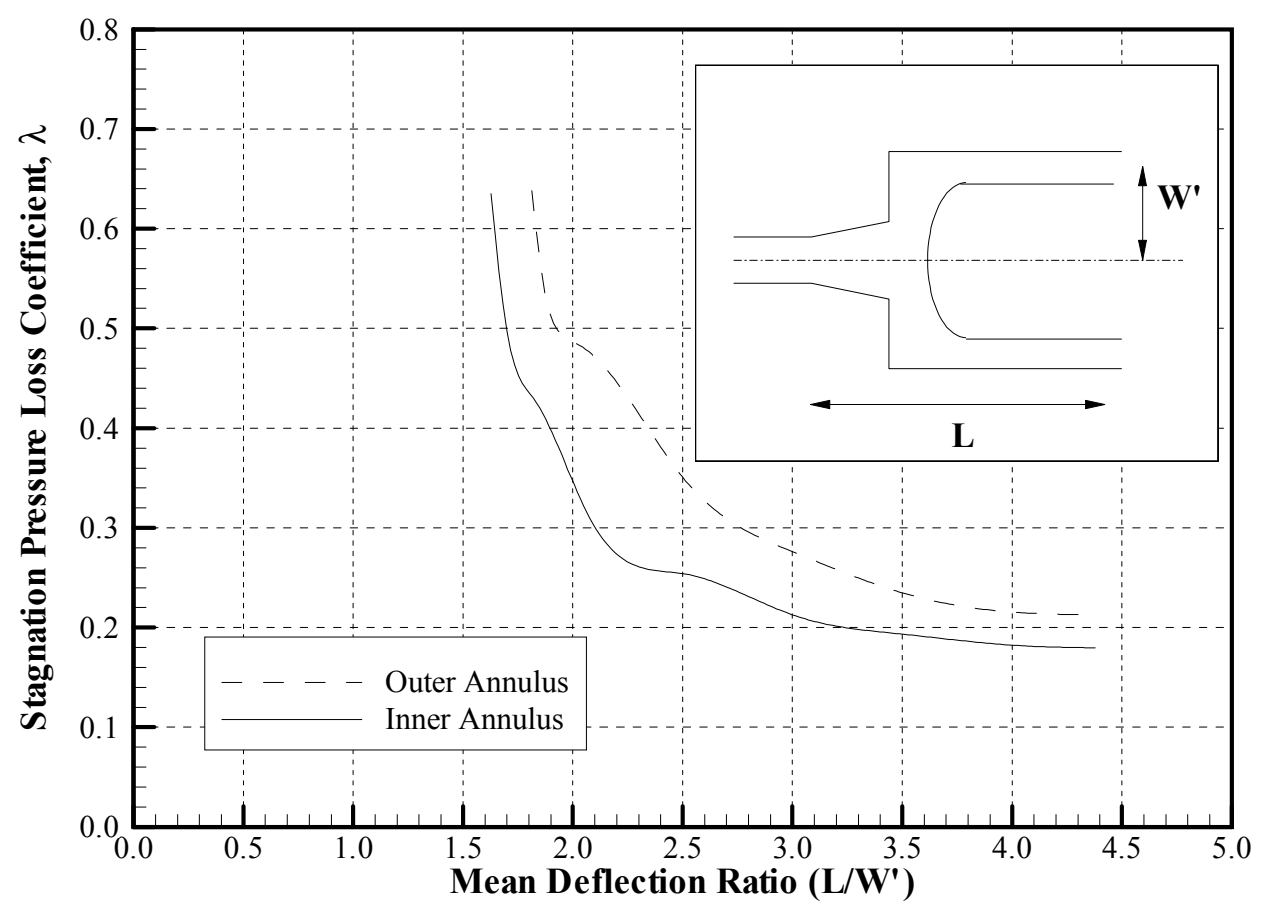

Figure 1.9 Variation of Total Pressure Loss to the Feed Annuli with Mean Deflection Ratio, Carrotte and Bailey ${ }^{[1994]}$

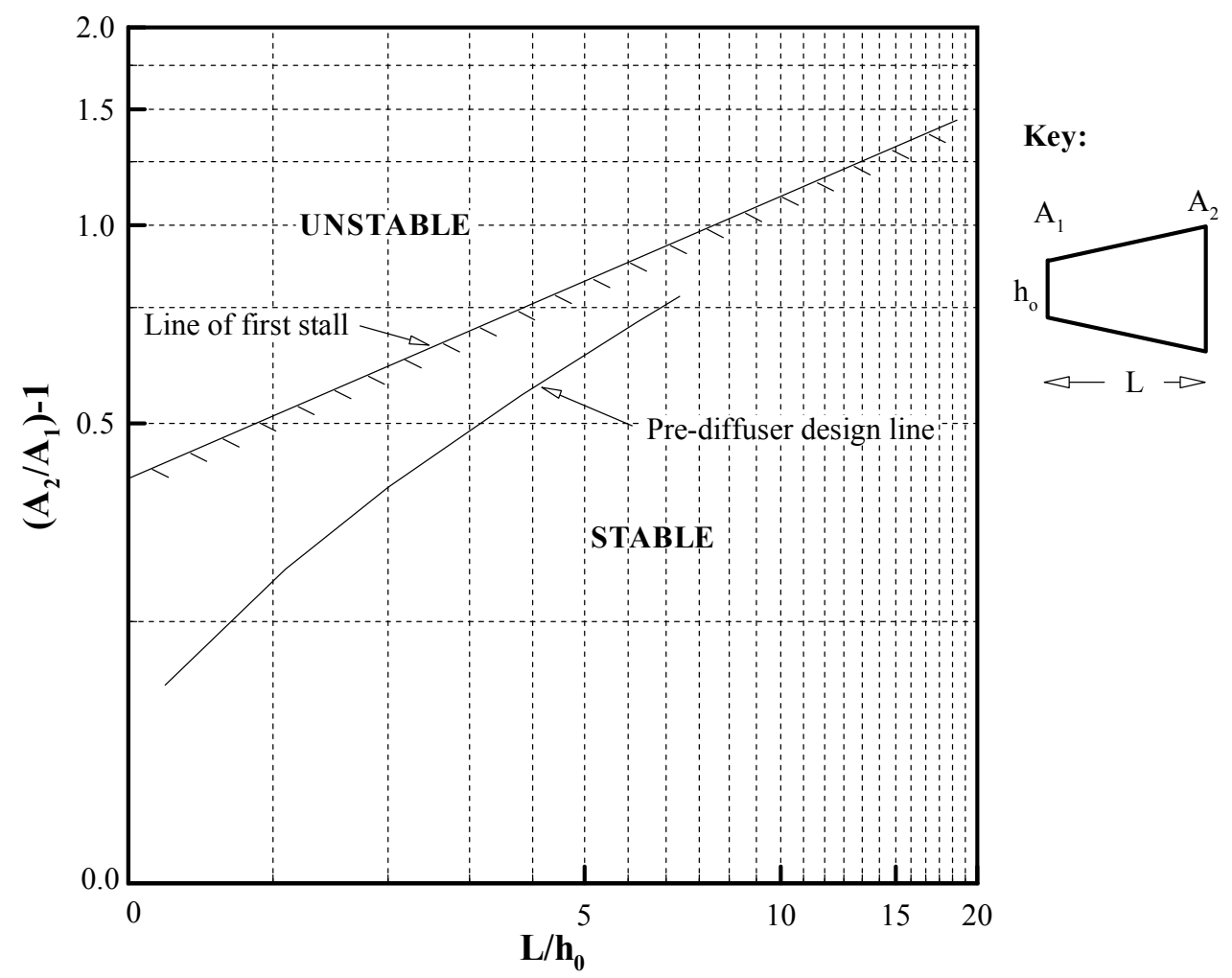

Figure 1.10 A Comparison of Diffuser Performance with the Nominal Design Curve, Sovran and Klomp ${ }^{[1967]}$ 


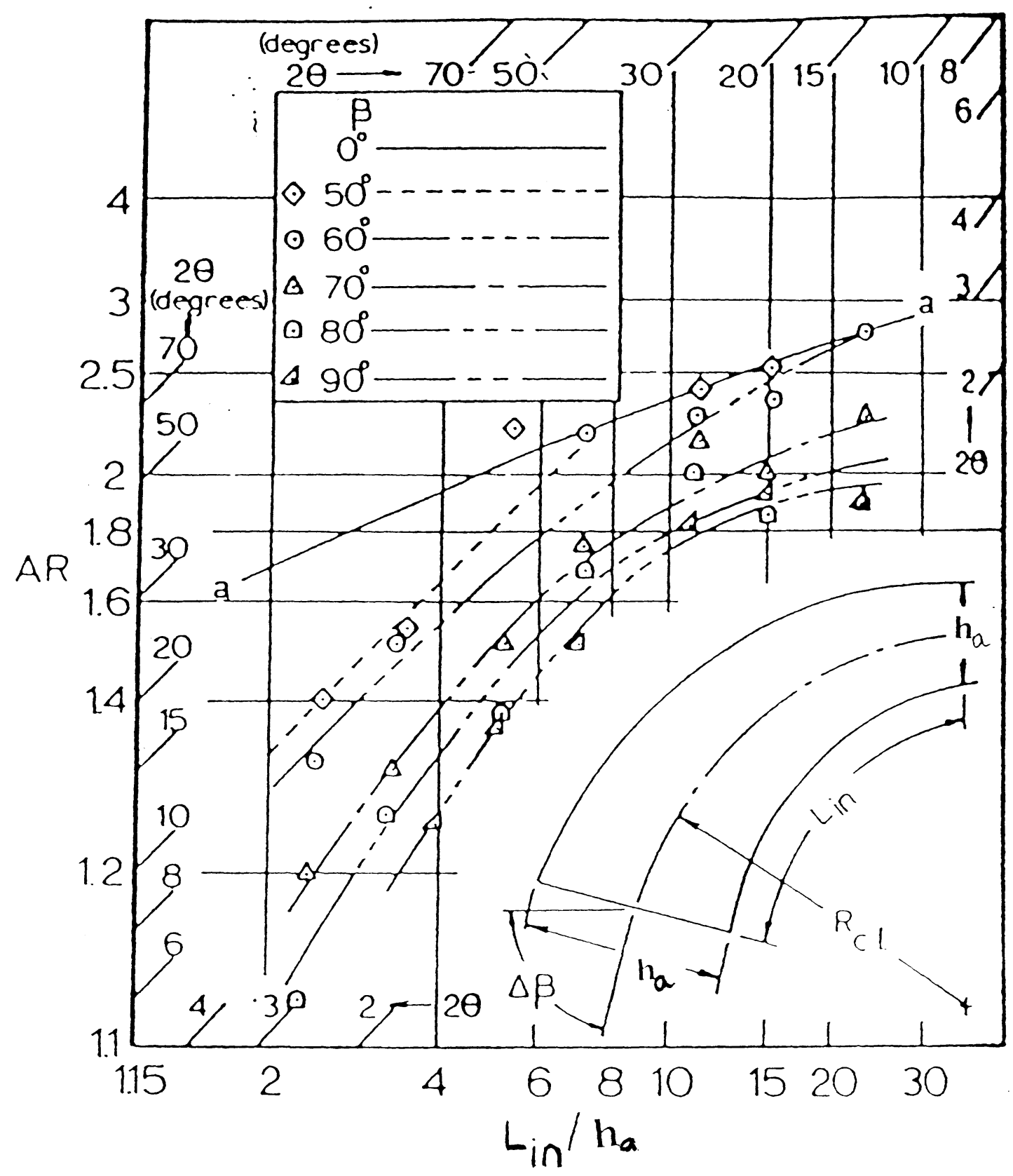

Figure 1.11 Typical Flow Regime Chart for Curved Diffusers, Sagi and Johnston $^{[1967]}$ 


\subsection{A DISCUSSION OF DIFFUSER TECHNOLOGY}

2.1 Basic Diffuser Considerations $\ldots \ldots \ldots \ldots \ldots \ldots \ldots \ldots$

2.2 Expressions for Diffuser Performance $\ldots \ldots \ldots \ldots \ldots \ldots 21$

2.2.1 One-Dimensional Flow Analysis $\ldots \ldots \ldots \ldots \ldots \ldots \ldots$

2.2.2 Non-Uniform Flows and Suitable Mean Values . . . . . . . . 22

2.3 Annular Diffuser Performance $\ldots \ldots \ldots \ldots \ldots \ldots \ldots \ldots \ldots$

2.3.1 Influence of Geometrical Parameters . . . . . . . . . . . 25

(i) Length and Divergence Angle (Area Ratio) . . . . . . . . 25

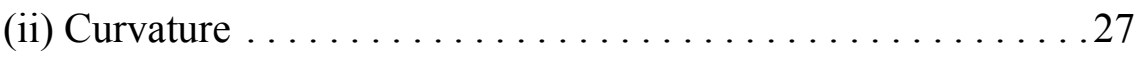

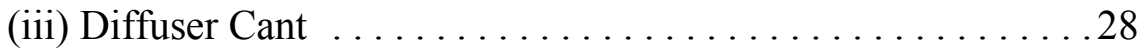

2.3.2 Effect of Inlet Conditions on Diffuser Performance . . . . . . 28

(i) Reynolds Number $\ldots \ldots \ldots \ldots \ldots \ldots \ldots \ldots \ldots \ldots \ldots \ldots \ldots \ldots$

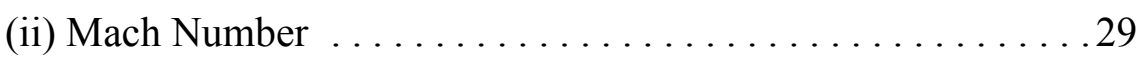

(iii) Inlet Boundary Layer . . . . . . . . . . . . . . . . 29

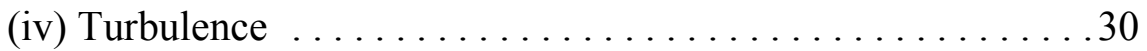

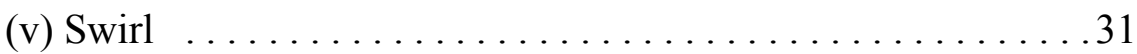

(vi) Outlet Guide Vane Wakes .................... 32

2.4 Influence of Combustor Characteristics on Dump Diffusers . . . 33

2.4.1 Dump Gap . ..............................

2.4.2 Flame Tube Depth . . . . . . . . . . . . . . . . . 35

2.5 High Area Ratio, Wide Angle, Multi-Passage Diffusers .......36

2.6 Bled, Vortex Controlled and Hybrid Diffusers .............38

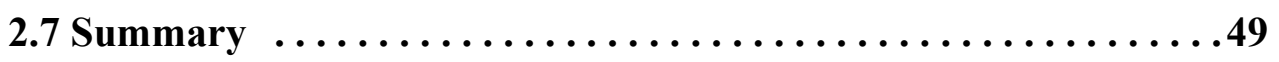

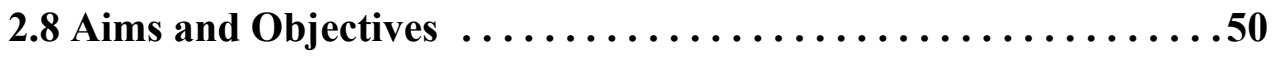

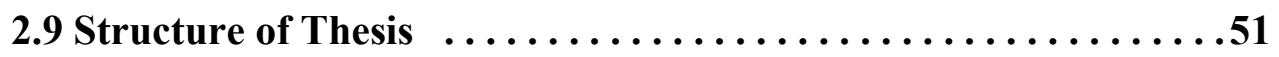

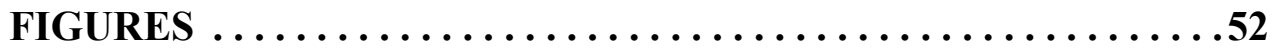




\subsection{A DISCUSSION OF DIFFUSER TECHNOLOGY}

Chapter 1.0 has highlighted the fact that staged combustion systems represent an attractive option to meet projected $\mathrm{NO}_{\mathrm{x}}$ emission regulations. However, evidence has also been presented which suggests that conventional diffusers will have difficulty in meeting the performance requirements imposed by the deep flame tubes associated with this type of combustor. Current conventional diffusers cannot achieve the required area ratios and degree of flow turning within the axial length permitted without incurring unacceptable levels of loss. To understand why this is the case and how diffusers might be developed to meet these requirements a discussion of diffuser technology is presented next. The data discussed comes solely from experimental sources. There are several reasons why computational data are not presented; the main one being that much of the work discussed was conducted before computational methods were available. Additionally the experimental techniques have been well established whereas much of the available computational data is concerned with using experimental data to validate computational methodology. Although computational techniques are now well established much of the later work discussed comprises complex systems incorporating, for example, representative combustion systems and inlet conditions including vane wakes. Computational studies have not, as yet, reached this level of complexity.

\section{$\underline{\text { 2.1 Basic Diffuser Considerations }}$}

At the exit from a high pressure compressor in a modern gas turbine the axial velocity of the flow is typically $150 \mathrm{~ms}^{-1}$ (Mach $0.2-0.3$ ) or higher. The pressure rise in an axial compressor is directly related to the velocity and as such high exit velocities are unavoidable. Unfortunately it is not practical to burn aviation fuel in air flowing at such high velocities and therefore, before steady-state combustion can be achieved, the axial velocity of the airflow must be significantly reduced. To accomplish this a diffuser system is employed to slow the compressor efflux to about $20 \%$ of its original value.

In essence a diffuser is a duct along which the kinetic energy of the flow is reduced and the static pressure increased. For subsonic flow a diffuser is a diverging passage (Figure 2.1) in which the cross-sectional area increases with axial length. Conservation of mass 
dictates that the higher the area ratio, the greater the reduction in velocity and hence increase in dynamic pressure. For high efficiency, the diffusion process must be accomplished with the maximum recovery of static pressure and a minimum total pressure loss. High losses have a detrimental effect on the engine cycle efficiency and thus increase specific fuel consumption (Oates ${ }^{[1985]}$ ). In addition to recovering static pressure a combustor diffuser system must also efficiently turn the air flow such that it is distributed correctly around the various components of the combustion system. This latter function must also be accomplished with the minimum total pressure loss, because both the total and static pressures upstream of fuel injectors, combustor liners and air admission holes are important for the efficient operation of these components (e.g. distribution of cooling air and control of internal combustor flow patterns).

Traditionally there are two main methods of diffusing the flow in a gas turbine combustion system. The first is to employ an aerodynamic or 'faired' diffuser (Figure 2.2a) which will achieve the maximum static pressure recovery but will incur a high skin friction loss due to its length. The second method is to use a short 'pre'-diffuser and then 'dump' the flow into a region of sudden expansion (Figure 2.2b). Carrotte et al ${ }^{[1994]}$ conducted a detailed performance comparison of dump and faired diffuser systems within an isothermal representation of a gas turbine combustion system. The dump pre-diffuser reduced the overall system length by $14 \%$, which would represent a significant weight reduction in any final engine design. However, total pressure losses to the inner and outer feed annuli were found to increase by $40 \%$ and $30 \%$ respectively. Carrotte et al ${ }^{[1994]}$ commented that, although dump systems incur higher losses, they are favoured in most modern gas turbines due to their short length, inherent flow stability and insensitivity to manufacturing tolerances and thermal expansions. 


\subsection{Expressions for Diffuser Performance}

\subsubsection{One-Dimensional Flow Analysis}

The task of a diffuser is to reduce the inlet kinetic energy of the flow with maximum efficiency. Or, in terms of pressure; to increase the static pressure as much as possible but with minimum loss in total pressure. To measure the performance of a simple diffuser (Figure 2.1) the static pressure recovery and total pressure losses are non-dimensionalised by the inlet dynamic pressure. This gives rise to the two most important parameters describing diffuser performance. For an incompressible, one-dimensional uniform flow, the static pressure recovery coefficient, $\mathbf{C}_{\mathbf{p}}$, between inlet (1) and exit (2) is defined as:

$$
\mathrm{C}_{\mathrm{p} 1-2}=\frac{\mathrm{p}_{2}-\mathrm{p}_{1}}{\mathrm{q}_{1}}
$$

and the total pressure loss coefficient, $\lambda$, as:

$$
\lambda_{1-2}=\frac{\mathrm{P}_{1}-\mathrm{P}_{2}}{\mathrm{q}_{1}}=\frac{\Delta \mathrm{P}_{1-2}}{\mathrm{q}_{1}}
$$

Where $\mathrm{q}_{1}$ is the dynamic pressure at inlet $\left(\frac{1}{2} \rho \mathrm{U}_{1}^{2}\right)$

Further, if the flow is assumed incompressible $\left(\mathrm{M}_{1}<0.3\right)$ a simple relationship between these two parameters follows from Bernouilli's equation:

$$
\mathrm{p}_{1}+\frac{1}{2} \rho \mathrm{U}_{1}^{2}=\mathrm{p}_{2}+\frac{1}{2} \rho \mathrm{U}_{2}^{2}+\Delta \mathrm{P}_{1-2}
$$

where $\Delta \mathrm{P}_{1-2}$ is the total pressure loss. Therefore:

$$
\mathrm{p}_{2}-\mathrm{p}_{1}=\frac{1}{2} \rho\left(\mathrm{U}_{1}^{2}-\mathrm{U}_{2}^{2}\right)-\Delta \mathrm{P}_{1-2}
$$

and if the mass flow is given by:

$$
\dot{\mathrm{m}}=(\rho \mathrm{AU})_{1}=(\rho \mathrm{AU})_{2}=\mathrm{constant}
$$

then assuming constant density:

$$
\mathrm{U}_{2}=\mathrm{U}_{1} \frac{\mathrm{A}_{1}}{\mathrm{~A}_{2}}=\frac{\mathrm{U}_{1}}{\mathrm{AR}}
$$

where AR is the area ratio of the diffuser, giving: 


$$
\mathrm{C}_{\mathrm{p}_{1-2}}=\left[1-\mathrm{AR}^{-2}\right]-\lambda_{1-2}
$$

or an ideal (zero loss) pressure recovery can be defined as:

$$
\mathrm{C}_{\mathrm{p}_{1-2}}{ }^{\prime}=1-\mathrm{AR}^{-2}
$$

Furthermore, an overall effectiveness, $\eta$, can now be defined as the ratio of actual to ideal pressure recovery, i.e.

$$
\eta=\frac{\mathrm{C}_{\mathrm{p}_{1-2}}}{\mathrm{C}_{\mathrm{p}_{1-2}}}
$$

or,

$$
\eta=\frac{p_{2}-p_{1}}{q_{1}\left(1-A R^{-2}\right)}
$$

\subsubsection{Non-Uniform Flows and Suitable Mean Values}

Equations 2.1 to 2.10 are valid for one-dimensional flow, but it is clear that diffuser flows will posses non-uniform profiles of velocity and pressure over the duct cross-section, particularly downstream of a compressor and its OGVs where spatial variations will occur. However, provided valid and suitable average values are used the above definitions may remain valid. Livesey and Hugh ${ }^{[1966]}$ stress the importance of adopting a consistent system such that the average values for a flow field still satisfy the relevant aerothermal relationships in the same manner as the non-uniform flow field. In a duct flow, the total pressure will fall without the presence of external work or heat exchange due to work done against internal friction stresses and as such the total pressure loss is used almost universally in assessing duct performance. Thus, Livesey and Hugh ${ }^{[1966]}$ consider the suitable definition of an average total pressure to be of fundamental importance. For example an area-weighted average (given the symbol "-") can be defined as:

$$
\overline{\mathrm{P}}=\frac{1}{\mathrm{~A}} \int_{\mathrm{A}} \mathrm{PdA}
$$

However, in real flows a boundary layer exists at the wall and the mass flow in this region is small compared to the central portion of the duct. Equation 2.11 does not account for this, giving equal weighting to the low mass flow and high mass flow regions. This is inconsistent and dictates that the area-weighted mean total pressure is 
biased towards the low total pressure close to the wall.

\section{(i) Mass-Weighted Mean Values}

Ferguson ${ }^{[1962]}$ states that, for incompressible flow, "the true average total pressure for loss assessment in a flow passage is a mass flow averaged pressure".

The mass-weighted mean total pressure (given the symbol " $\sim$ ") properly accounts for the low mass flow regions in the boundary layer and is defined as:

$$
\tilde{\mathrm{P}}=\frac{1}{\dot{\mathrm{m}}} \int \mathrm{Pd \dot {m }}=\frac{1}{\rho \overline{\mathrm{u} A}} \int \mathrm{P} \rho \mathrm{pud}
$$

In addition a kinetic energy flux coefficient $(\alpha)$ can be defined which compares the kinetic energy flux of the real profile with a uniform profile of identical mass flow (i.e. $\alpha=1$ represents a flat profile). For a uniform flat profile the kinetic energy flux is given by:

$$
\int_{\mathrm{A}} \frac{1}{2} \rho \mathrm{u}^{3} \mathrm{dA}=\frac{1}{2}(\rho \overline{\mathrm{u}} \mathrm{A}) \overline{\mathrm{u}}^{2}=\frac{1}{2} \dot{\mathrm{m}} \overline{\mathrm{u}}^{2}
$$

and for a general non-uniform profile:

$$
\int \frac{1}{2} \rho u^{3} \mathrm{dA}
$$

therefore:

$$
\alpha=\frac{\int \frac{1}{2} \rho u^{3} d A}{\frac{1}{2} \rho \bar{u} A \bar{u}^{2}}
$$

such that:

$$
\tilde{\mathrm{P}}=\tilde{\mathrm{p}}+\alpha \frac{1}{2} \rho \overline{\mathrm{u}}^{2}=\tilde{\mathrm{p}}+\tilde{\mathrm{q}}
$$

The definition of a kinetic energy flux coefficient provides a method by which the distortion of the profile can be assessed; increasing from a value of unity as the profile distorts from uniformity. However, a mass-weighted mean total pressure does not include losses which are 'locked' into this distorted profile. These are losses which would be incurred by mixing out the actual flow distribution to a uniform profile in a duct of constant area 
in the absence of heat transfer.

\section{(ii) Momentum Mix Mean Values}

Mean values of total pressure calculated by this method include the loss described above which is 'locked' into the profile non-uniformities. Hence, a momentum mix total pressure (here given the symbol “^”) will be less than a mass-weighted value since it includes the mixing loss.

Momentum mean values by definition, pertain to the totally uniform flow (i.e. all fluid properties constant over the flow area) which has the same energy, mass and momentum flow rates as the real flow (Livesey and Hugh ${ }^{[1966]}$ ) and therefore, must satisfy the following conservation equations:

Energy Flux:

$$
\int C_{p}\left(t+\frac{u^{2}}{2 C_{p}}\right) \frac{p}{R t} u d A=\dot{m} C_{p} \hat{t}\left(1+\frac{\gamma-1}{2} \hat{M}^{2}\right)
$$

Mass:

$$
\dot{\mathrm{m}}=\int \frac{\mathrm{p}}{\mathrm{Rt}} \mathrm{udA}=\hat{\mathrm{p}} \hat{\mathrm{M}} \mathrm{A} \sqrt{\frac{\gamma}{\mathrm{Rt}}}
$$

Momentum:

$$
\int_{A} \rho u(u) d A=\int_{A} \frac{p u^{2}}{R t} d A=\hat{p} A\left(\gamma \hat{M}^{2}\right)
$$

The unknowns $(\hat{\mathrm{p}}, \hat{\mathrm{M}}, \hat{\mathrm{t}})$ are found using the above equations and the momentum mix total pressure is then given by:

$$
\hat{\mathrm{P}}=\hat{\mathrm{p}}\left(1+\frac{\gamma-1}{2} \hat{\mathrm{M}}^{2}\right)^{\frac{\gamma}{\gamma-1}}
$$

Care must be taken when using momentum mix values to calculate diffuser total pressure loss downstream of an axial compressor. Inlet and exit values of total pressure will include the losses associated with mixing to a uniform profile. Thus at diffuser exit the momentum mix value of total pressure will include the mixing losses associated with 
non-uniform velocity profile at diffuser exit. However, at entry the extra mixing losses are caused by non-uniformities generated by the compressor (i.e. not a function of the diffuser).

Other methods of obtaining consistent mean values have been developed (Livesey and Hugh $^{[1966]}$ and Livesey ${ }^{[1972]}$ ) but Klein ${ }^{[1995]}$ comments that they have not been applied to experimental studies of diffusers and combustion chamber aerodynamics. Both massweighted and momentum mix methods are valid but on review of the general literature it is clear the mass-weighting is by far the most popular method. This is because when using mass-weighted average pressures to calculate a total pressure difference across a component then only the losses associated with that component are included.

\subsection{Annular Diffuser Performance}

\subsubsection{Influence of Geometrical Parameters}

The main geometrical parameters (Figure 2.1) which are of prime importance when considering the efficiency of the diffusion process are the divergence angle, $\boldsymbol{\theta}$, the nondimensional length, $L / h_{1}$, the area ratio, $A R$, the angle of curvature or cant, $\beta$, (i.e. the inclination of the diffuser to/from the axial direction).

The static pressure recovery of a diffuser is a function of the area ratio and the effectiveness of the diffusion process. However, for practical diffusers, reduction in effectiveness or total pressure losses are caused by:
a) skin friction losses at the wall,
b) turbulence, and
c) boundary layer separation losses (stall).

\section{(i) Length and Divergence Angle (Area Ratio)}

Figure 2.3 shows the relationship between total pressure loss and the diffuser divergence angle for a fixed area ratio. For a given area ratio a diffuser with a small divergence angle 
will be long and suffer from high skin friction losses. Conversely a diffuser with a larger divergence angle will be shorter and suffer less from frictional losses but will have high stall losses due to breakdown of the flow at the walls. The wall angle for minimum combined loss is found to be about 7 degrees for simple geometries with thin inlet boundary layers and low turbulence conditions. However, in a gas turbine the inlet conditions, downstream of an axial compressor, are very different and incorporate OGV wakes and high levels of turbulence. Section 2.3.2 explains how these features delay the onset of separation with the result that in practice a wall angle of 11 degrees is more typical. This gives a significant saving in weight and length with only a small penalty in increased total pressure loss.

Many authors have investigated the effect of divergence angle and length on diffuser performance. For example, McDonald and Fox ${ }^{[1966]}$ and Sovran and Klomp ${ }^{[1967]}$ have produced extensive performance maps cataloguing the static pressure recovery, total pressure loss and effectiveness for a wide range of conical diffusers with varying lengths and area ratios. Similar maps for annular diffusers, as used in gas turbines, have also been produced by Sovran and Klomp ${ }^{[1967]}$ and Howard et al ${ }^{[1967]}$. Figure 2.4 is an example performance map from Sovran and Klomp ${ }^{[1967]}$ and shows lines of constant diffuser effectiveness on a plot of area ratio versus non-dimensional length. Essentially Figure 2.4 shows that the data from Sovran and Klomp ${ }^{[1967]}$ confirm the relationship shown in Figure 2.3. A short length coupled with a high area ratio incurs a high loss (low effectiveness) due to flow breakdown near the high angle walls. Alternatively, longer diffusers also have poor effectiveness due to increased skin friction losses.

The rise in static pressure within a diffuser dictates that, on the diffuser walls, the boundary layers develop rapidly under the action of a strong adverse pressure gradient. As the area ratio of a diffuser increases the adverse pressure gradient also increases. At a certain level this gradient will become too high, the boundary layer will thicken rapidly and separate. Kline et al ${ }^{[1959]}$ studied two-dimensional diffusers and found that if the divergence angle is continually increased, while the inlet conditions and wall length remain constant, four distinct flow regimes can be observed. These regimes are illustrated schematically in Figure 2.5 and as a function of area ratio or divergence angle and non- 
dimensional length in Figure 2.6. Kline et al ${ }^{[1959]}$ described them as follows:

1. No "appreciable" stall, with the main flow well behaved and apparently unseparated.

2. Large transitory stall, in which the separation varies in size, position and intensity with time.

3. Fully developed stall, where the major portion of the diffuser is filled with a large recirculation region, extending along the majority of the diffuser. The main flow follows along one wall continuously and relatively smoothly.

4. Jet flow, in which the main flow is separated from both walls. The separation begins almost immediately and the flow does not reattach until well downstream of the diffuser. This flow regime is only observed at high angles of divergence.

\section{(ii) Curvature}

In a review of diffuser characteristics Klein ${ }^{[1995]}$ comments that wall curvature is also an important parameter when considering the performance of diffusers. Curvature of the wall affects the production of turbulence, which in turn will affect boundary layer development. Bradshaw ${ }^{[1973]}$ reported that boundary layers grow more quickly on a convex surface, compared to a flat surface while, conversely, boundary layers grow more slowly on a concave surface. This can be explained with reference to Figure 2.7. The trajectory of a fluid element (with velocity $U_{1}$ at radius $r_{1}$ ) is maintained through a balance of the centripetal force and the pressure gradient.

$$
\frac{\partial \mathrm{p}}{\partial \mathrm{n}}=\frac{\rho \mathrm{U}_{1}^{2}}{\mathrm{r}_{1}}
$$

If the fluid element is then displaced, by some external force, to a new radius $\left(r_{2}\right)$ its new velocity (U') can be calculated by assuming constant angular momentum (i.e. U.r $=$ constant and $\left.U^{\prime}=U_{1} r_{1} / r_{2}\right)$. However, there may now exist a imbalance between the centripetal force and the pressure gradient which is required to maintain the trajectory of the mean flow with velocity $\mathrm{U}_{2}$ at radius $\mathrm{r}_{2}$ as:

$$
\frac{\rho U_{1}^{2} r_{1}^{2}}{r_{2}^{3}} \neq \frac{\rho U_{2}^{2}}{r_{2}}
$$


Thus, if the angular momentum of the mean flow increases with radius (as in a boundary layer on a convex surface) the element will be forced back to its original position. Conversely, if the angular momentum of the mean flow decreases with radius (as in a boundary layer on a concave surface) the fluid element will be forced further from its original course. Hence on a convex wall turbulence production is suppressed causing the boundary layer to develop more quickly. Whereas on a concave wall turbulence is enhanced reducing boundary layer growth.

In general, curvature has a negative effect on the performance of annular diffusers due to the presence of a convex surface and the earlier onset of separation. Sagi and Johnston $^{[1967]}$ investigated a range of diffusers with varying degrees of curvature $(\beta)$. From their data, lines of first stall are plotted on a flow regime chart (Figure 1.11) and these clearly show that as the angle of curvature increases the onset of stall will occur, for a given non-dimensional length, at a lower area ratio.

\section{(iii) Diffuser Cant}

Cant is defined as the inclination of the diffuser towards or from the axial direction. In gas turbines the mean radius at compressor exit is often different from that at turbine entry. Thus it is often necessary to cant the combustion chamber and/or diffuser. Cant generates an implied flow curvature at diffuser inlet. For example, most common is outward cant which results in a biased mass flow distribution with a hub-peaked velocity profile. Furthermore for an outwardly canted diffuser the outer wall has, in general, a relatively high divergence angle and in conjunction with the velocity deficit just described will incur a higher total pressure loss and be prone to early separation. Thus, although often necessary, diffuser cant can introduce penalties to diffuser performance. 


\subsubsection{Effect of Inlet Conditions on Diffuser Performance}

The performance of a given diffuser geometry is, to a large extent, dependant on the condition of the inlet flow. Changes in many of the flow parameters can, some more than others, alter the performance of a diffuser in a positive or negative manner. Therefore, in any investigation into the performance of diffusers in a specific role it is important to generate the correct inlet conditions. For example, in a gas turbine the diffuser inlet conditions are generated by an axial flow compressor and will be characterised by high turbulence, radial and circumferential variations in pressure/velocity, swirl and OGV wakes (large scale turbulence). This represents substantially different conditions to a fully developed flow and thus great care and attention must be paid to the condition of the flow at diffuser inlet otherwise is it possible that the results could be misleading.

\section{(i) Reynolds Number}

Klein ${ }^{[1981]}$ reported that the Reynolds number has no effect on the performance of a conical diffuser if the flow at inlet is fully turbulent. Japikse ${ }^{[1984]}$ also indicates that the Reynolds number is a relatively weak parameter provided that flow is fully turbulent. Furthermore, at Reynolds numbers greater than $8 \times 10^{4}$ at diffuser inlet McDonald and Fox ${ }^{[1966]}$ found that performance and flow regime become independent of the Reynolds number. The operational Reynolds number of aero gas turbine combustion systems at diffuser inlet it is typically in excess of $10^{5}$ and therefore does not play a major role in diffuser performance.

\section{(ii) Mach Number}

Japikse ${ }^{[1984]}$ states that the static pressure recovery is insensitive to Mach numbers below 0.25 and has almost negligible influence up to Mach numbers of 0.60. Above this the pressure gradient near the inlet of a straight walled diffuser becomes excessive and the performance begins to deteriorate. For Mach numbers above 0.70 large scale recirculation can occur resulting in a dramatic reduction in performance. However, a Mach number of around 0.2-0.3 is more typical at inlet to a modern combustion chamber. 


\section{(iii) Inlet Boundary Layer}

The action of a diffuser imposes an adverse pressure gradient on the flow. Thus a boundary layer will grow more rapidly than in a non-diverging passage. A reasonably highly loaded diffuser which initially shows no sign of separation will, as the inlet boundary layer thickness increases, eventually stall. An already thick and well developed boundary layer exposed to an adverse pressure gradient will separate in a shorter length than a thin, less developed boundary layer. Thus the boundary layer at inlet to a diffuser can, depending upon its condition, have a large effect on the performance of the diffuser.

Klein ${ }^{[1995]}$ reports that the main fluid dynamic boundary layer parameter which affects diffuser performance is the area fraction blocked by the boundary layer displacement thickness. Sovran and Klomp ${ }^{[1967]}$ define a blockage factor, B, for low Mach Numbers, as:

$$
\mathrm{B}=1-\frac{\mathrm{U}}{\mathrm{u}_{\max }}
$$

where $U$ is the mean velocity.

Stevens and Williams ${ }^{[1980]}$ conducted experiments on two diffusers (both AR 2.0 but L/ $\mathrm{h}_{1}=5.0$ and 7.5 ) altering the inlet boundary layer by using approach sections of varying length. Static pressure recovery data from these experiments was summarised by Klein ${ }^{[1995]}$ and is shown in Figure 2.8. The plot of static pressure recovery coefficient $\left(C_{p}\right)$ versus inlet blockage factor $\left(B_{1}\right.$, where ' 1 ' indicates that the measurement is taken at diffuser inlet) shows that, for approach flows with very thin boundary layers the pressure recovery falls rapidly as the inlet blockage increases from $0 \sim 0.05$. As the blockage increases the opposing boundary layers become thicker and begin to interact. The shear stress in the core flow is then re-distributed as the boundary layers merge creating a higher turbulence intensity and a slightly increased static pressure recovery. 


\section{(iv) Turbulence}

Stevens and Williams ${ }^{[1980]}$ found that the turbulence produced in a long approach duct has a beneficial effect on diffuser performance as it reduces the distortions in the flow. Additionally they found that introducing a grid to generate an increased turbulence intensity could increase diffuser static pressure recovery by up to $20 \%$ (Figure 2.8 ) with only a small penalty in total pressure loss. This was due to the increased turbulence enhancing mixing and producing a drastic decrease in the flow distortion. Combining Equations 2.6, 2.7 and 2.16 the following expression for pressure recovery can be written:

$$
\mathrm{C}_{\mathrm{p} 1-2}=\left[1-\frac{\alpha_{2}}{\alpha_{1}} \mathrm{AR}^{-2}\right]-\lambda_{1-2}
$$

From this expression it can be seen that any reduction in distortion of the profile at diffuser exit (i.e. reduction in the term $\alpha_{2} / \alpha_{1}$ ) will potentially increase the static pressure recovery, providing the loss does not also increase greatly.

Hoffman $^{[1981]}$ and Hoffman and Gonzalez ${ }^{[1983,84]}$ investigated the effect of varying the turbulence intensity and character on the performance of two-dimensional diffusers by placing various rods in the flow upstream of the diffuser. The turbulence intensity was increased from less than $1 \%$ to $3-4 \%$ and this had the effect of reducing profile distortions and delaying separation. For divergence angles $(2 \theta)$ of $9^{\circ}$ and $20^{\circ}$ the pressure recovery was seen to increase by $10 \%$ and $22 \%$ respectively. Furthermore, in the latter case the diffuser was in a state of transitory stall for the low turbulence inlet condition but remained fully attached at the high inlet turbulence levels.

In a gas turbine combustion system the diffuser generally sits downstream of an axial flow compressor. The turbulence structure of the compressor efflux is characterised by relatively high turbulence intensities in the region of 5\%. Furthermore, the presence of outlet guide vane wakes introduces relatively large scales of turbulence. Authors such as Stevens et al ${ }^{[1978]}$ and Stevens and Williams ${ }^{[1980]}$ have investigated the effect compressor exit conditions have on diffuser performance and in summary Klein ${ }^{[1995]}$ states that the turbulence associated with the flow field downstream of an axial compressor has a beneficial effect on diffuser performance. The onset of separation is delayed thus allow- 
ing larger area ratios than those suggested by the classical performance charts.

\section{(v) Swirl}

A compressor efflux usually contains some degree of swirl. In general, in an annular diffuser, this will have a similar effect on the boundary layer as wall curvature because, tangentially, the inner and outer wall represent a convex and concave surface. Thus the outer wall boundary layer flow will be enhanced due to transfer of high momentum fluid towards the wall. However, the opposite is true of the inner wall boundary layer making it prone to early separation. Liepe ${ }^{[1963]}$ reported that inlet swirl can, therefore, sometimes prevent flow separation on diffusers with high angle outer walls. However, Lohmann et $\mathrm{al}^{[1976]}$ showed that for swirling flows in annular diffusers the onset of flow separation at the hub occurs earlier than for axial flow.

Elkersh et al ${ }^{[1985]}$ studied the effect of inlet swirl of up to $45^{\circ}$ on the performance of an outwardly canted annular diffuser. Figure 2.9 shows normalised velocity profiles at inlet to, and several stations along, one of the diffusers studied by Elkersh et al ${ }^{[1985]}$. The effect of inlet swirl on the condition of the boundary layers is clearly demonstrated. As the flow develops along the diffuser the inner wall boundary layer grows much more rapidly at higher levels of inlet swirl. Conversely the condition of the outer wall boundary layer is seen to improve. Elkersh et al ${ }^{[1985]}$ report that the pressure recovery increased and the total pressure loss decreased as inlet swirl was increased up to 30 degrees. However, as illustrated in Figure 2.10 diffuser performance then begins to fall as swirl is further increased and the inner wall boundary layer becomes close to stall.

Compressor outlet guide vanes are generally designed to provide an efflux with zero swirl but this is only true when the compressor is operating on its design condition. At off-design conditions swirl angles as high as 30-40 degrees occur and as such the impact diffuser on performance will vary with the compressor operating conditions. 


\section{(vi) Outlet Guide Vanes (OGV) Wakes}

In a gas turbine combustion system wakes from the compressor outlet guide vanes will be present at diffuser inlet and therefore many authors have studied the effect of OGV wakes on diffuser performance. For example, Stevens et al ${ }^{[1978]}$ studied the flow in diffusers downstream of both a multi stage axial compressor and a tandem cascade. They found no significant penalty on overall performance when the outlet guide vanes were situated close to the pre-diffuser inlet. Using mass-weighted mean values they used a reworked version of Equation 2.24 and defined the pressure recovery as:

$$
\mathrm{C}_{\mathrm{p}_{1-2}}=\left[1-\frac{\alpha_{2}}{\alpha_{1}}\left(\frac{\mathrm{A}_{1}}{\mathrm{~A}_{2}}\right)^{2}\right]-\lambda_{1-2}
$$

where ' 1 ' and ' 2 ' represent diffuser inlet and exit.

Equation 2.25 shows that potentially the pressure recovery will be reduced by an increasing velocity profile distortion $\left(\alpha_{2} / \alpha_{1}>1\right)$ and by total pressure losses $\left(\lambda_{1-2}>0\right)$. Stevens et $\mathrm{al}^{[1978]}$ comment that with blade wakes present the extra turbulent mixing can enhance the pressure recovery through improvement of the diffuser exit velocity profile. Figure 2.11 summarises some of their results and clearly shows that as $\alpha_{2}$ decreases static pressure recovery $\left(\mathrm{C}_{\mathrm{p}}\right)$ increases.

Klein et al ${ }^{[1980]}$ also investigated the effect of flow wakes, generated by a tandem cascade, on the performance of a dump diffuser. However, their findings seemed to contradict those of Stevens et $\mathrm{al}^{[1978]}$. Klein et al ${ }^{[1980]}$ found that the losses increased substantially when the cascade was close to the diffuser inlet. The losses were found to be a minimum when the cascade was at a distance of about two chord lengths from the inlet. In a later study Stevens et $\mathrm{al}^{[1984]}$ tested several diffusers downstream of a single stage axial compressor. They commented that Klein et al ${ }^{[1980]}$ had reported blade wakes still present in the flame tube feed annuli. This suggests that the wakes have grown rather than decayed and this would clearly lead to an increased overall loss. Furthermore, Stevens et ${ }^{[1984]}$ expressed doubts concerning the use of an annular tandem cascade to simulate compressor exit flows, since they do not recreate the unsteadiness associated with the flow downstream of a rotor. Nor do they generate the same turbulence structure 
which will contribute to the decay of the wakes by promoting vigorous mixing.

\section{$\underline{2.4 \text { Influence of Combustor Characteristics on Dump Diffusers }}$}

The performance of a dump diffuser within a gas turbine combustion system is not only influenced by its geometry and inlet conditions but also by the presence, downstream, of a combustion chamber. Not only does this effect the diffuser exit characteristics but these characteristics must be tailored to suit the combustion chamber. The two requirements are interdependent as one is influenced by the other. The main downstream considerations which effect a combustor dump diffuser are discussed below.

\subsubsection{Dump Gap}

The distance (d) from the pre-diffuser exit plane to the flame tube, namely the dump gap, is an influential parameter in terms of diffuser and system performance. Expressed as a fraction of diffuser exit height $\left(\mathrm{d} / \mathrm{h}_{2}\right)$ the effect of the dump gap ratio was investigated by Hestermann et al ${ }^{[1991]}$ within the configuration shown in Figure 2.12. As the ratio is reduced the presence of the flame tube induces flow curvature at the diffuser exit plane resulting in a migration of mass flow towards the diffuser walls. Hestermann et al ${ }^{[1991]}$ showed that this results in a more uniform diffuser exit velocity profile and suppresses flow separation due to the introduction of higher momentum flow to the boundary layer. This is graphically illustrated in Figure 2.13 showing flatter, more uniform profiles; the results also show that a diffuser (Figure 2.13c) which would normally be in a state of transient stall becomes fully attached.

Fishenden and Stevens ${ }^{[1977]}$ also investigated the effect of altering the dump gap ratio in the annular configuration illustrated in Figure 2.14. The reduction in flow distortion caused by the proximity of the flame tube was seen to increase diffuser pressure recovery. However, it does not directly follow that a higher diffuser pressure recovery will result in lower losses to the feed annuli. Figure 2.15 shows that Fishenden and Stevens ${ }^{[1977]}$ observed that as the dump gap ratio reduces beyond a certain point the annulus losses begin to rapidly increase. This is because on exiting the diffuser the flow must turn in a much shorter space before entering the annuli and, in doing so, incurs high turning 
losses. Figure 2.16 shows a typical flow pattern downstream of a dump diffuser where it enters a region of free expansion. The flow close to the flame tube generally accelerates while a vortex is produced in the dump region. A dividing streamline exists in the time averaged flow field between these two areas across which there are high levels of turbulence and a steep velocity gradient. As the flow progresses towards the outer annuli the velocity profile is highly sheared but will eventually become more uniform as it passes down the annuli. Fishenden and Stevens ${ }^{[1977]}$ note that it is the amount of turning and diffusion attempted within the dump region that is the main cause of loss to the annuli. Thus it is important to achieve as much diffusion and turning as possible within the diffuser prior to the dump in order to limit system loss.

The dump gap must be large enough to allow the flow to diffuse around the flame tube head without incurring excessive losses in total pressure. However, large dump gaps are not desirable as this increases the system length which is contrary to the aim of using dump diffusers. Srinivasan et al ${ }^{[1990 a, b]}$ attempted to optimise the dump gap ratio and found that, for an uncanted system, the optimum value for this ratio was about 1.0. At this value the flame tube is sufficiently close to diffuser exit to provide a beneficial effect on the diffuser but not too close such that the turning loss in the dump region becomes excessive.

\subsubsection{Flame Tube Depth}

The investigation of Fishenden and Stevens ${ }^{[1977]}$ used a flame tube of depth, expressed as a multiple of pre-diffuser inlet height (i.e. $\mathrm{W} / \mathrm{h}_{1}$ ), of 3.5 . They concluded that total pressure loss to the feed annuli was a function of the amount of diffusion being attempted and the radius of curvature undertaken as the flow passes around the flame tube. The amount of flow curvature depends upon the relative depth of the flame tube as well as the dump gap and this, therefore, is another important parameter. The conclusions of Fishenden and Stevens were confirmed by the work of Srinivasan et al ${ }^{[1990]}$ who observed a $60 \%$ increase in total pressure loss as the flame tube depth ratio, $\mathrm{W} / \mathrm{h}_{1}$, was increased from 3.1 to 4.1 . 
The current trend in aero gas turbines is for deeper flame tubes especially with emissions legislation making staged combustion systems more likely. For example, the General Electric radially staged combustor shown in Figure 1.8 has a flame tube depth ratio in excess of 8 .

Carrotte and Bailey ${ }^{[1994]}$ conducted an extensive series of tests on an annular dump diffuser system downstream of a single stage axial compressor. They employed a conventional pre-diffuser of area ratio 1.35 and non-dimensional length 1.5 as shown in Figure 2.17. The flame tube was relatively deep with a depth ratio of 5.6. They investigated many of the parameters which affect system performance including the losses generated as the flow passes around the flame tube. They defined a mean flow deflection ratio as the ratio of a diffusing length (L) to a flow deflection (W'). The diffusing length is defined as the axial distance from pre-diffuser inlet to the combustor primary ports whereas flow deflection was defined as the radial distance between the combustor centre line and outer feed annulus mean radius. Carrotte and Bailey ${ }^{[1994]}$ altered the ratio L/W' primarily by changing the dump gap (i.e. changing L). However, the data should still be applicable to a ratio altered by changing the deflection (W') as it remains a measurement of the required flow turning in the dump. Figure 2.18 shows how the total pressure loss to the feed annuli varied with mean deflection ratio. Clearly as the ratio of length to deflection decreases the loss increases rapidly. With the current trend towards staged combustion the mean deflection ratio will be significantly smaller than investigated by Carrotte and Bailey ${ }^{[1994]}$, potentially as low as 1.5 . The data would suggest that a radially staged combustion system with a mean deflection ratio of 1.5 to 2 would suffer unacceptably high total pressure losses to the feed annuli. To reduce the losses in this type of system it would be necessary (Fishenden and Stevens ${ }^{[1977]}$ ) to reduce the amount of diffusion and turning performed within the dump gap. However, the overall requirement for diffusion and turning would not change and, therefore, the only option would be to perform more diffusion and turning of the flow within the pre-diffuser. Unfortunately, all the available data suggests that conventional pre-diffusers would be unable to achieve the large area ratios and pressure recoveries required within an acceptable length or without the onset of stall. Thus it is clear that it is necessary to develop pre-diffuser technology to increase achievable area ratios in a given length, increasing the pressure recovery but without incurring excessive extra loss penalties. This is an important area for future 
pre-diffuser development and potential methods for achieving this aim are discussed in the next section (including presentation of any relevant data).

\section{$\underline{2.5 \text { High Area Ratio, Wide Angle, Multi-Passage Diffusers }}$}

One method of increasing the amount of diffusion and turning within a conventional combustor pre-diffuser is to introduce a splitter or divider vane (or vanes). This separates the diffuser into a number of passages each with a lower aerodynamic loading. In Figure 2.19 diffuser (a) represents a conventional single passage diffuser of area ratio 1.45 and non-dimensional length of 3.5. Performance maps, such as Figure 2.6, indicate that this geometry sits at the upper limit of the stable flow regime. However, diffuser (b) has the same overall non-dimensional length but an area ratio of 1.8. Introducing a splitter vane produces two passages each having twice the effective non-dimensional length thus, with reference to Figure 2.6, enabling an area ratio increase to 1.8. Furthermore, Figure 2.19 illustrates that a splitter vane can also be used to increase the flow deflection within the diffuser; both passages now direct the flow outward to a greater degree. These benefits are, however, not without penalty. First of all the presence of a splitter vane has introduced more surface area and as such losses due to skin friction will increase. Secondly, as shown in Figure 2.20, a splitter vane can, according to Moore ${ }^{[1976]}$, induce distortions in the exit profile. Finally, there is a risk of flow separation from the leading edge of the splitter at off-design conditions. Not only will this increase diffuser loss and reduce static pressure recovery but will also impact the airflow distribution to the downstream components.

The General Electric radially staged combustor shown in Figure 1.8 employs a twin passage pre-diffuser in an attempt to overcome the problem of an excessively deep flame tube. The pre-diffuser geometry is shown in Figure 2.21, each passage having an area ratio of 1.8. This split diffuser (Salba et al ${ }^{[1982]}$ ) reduces the length relative to a single passage diffuser of the same area ratio by some $50 \%$ with the added benefit that each passage directs the flow towards the main and pilot burners.

Shedden ${ }^{[1993]}$ conducted an aerodynamic study of several diffusers within an isothermal representation of a radially staged combustion system. Using a $48^{\circ}$ sector test rig (Figure 
2.22) Shedden ${ }^{[1993]}$ assessed a datum system performance using a conventional single passage pre-diffuser of area ratio 1.65 and non-dimensional length 4.6. In addition, Shedden tested two twin-passage pre-diffusers (referred to here as 'Vane' and 'Wedge') both with an overall area ratio of 2.0. These two diffusers are shown in Figure 2.23 and include a thin splitter vane and a thick splitter wedge respectively. Diffuser static pressure recovery was seen to increase for the high area ratio Vane and Wedge diffusers. For example, the Wedge diffuser achieved a recovery coefficient of 0.49/0.48 (w.r.t. OGV exit) in the inner and outer branches respectively which represents a $20 \%$ increase over the conventional, single passage diffuser $\left(\mathrm{C}_{\mathrm{p} 1-2}=0.4\right)$. However, due to the increased skin friction and curvature the total pressure loss within the twin passage diffusers also increases significantly. Shedden ${ }^{[1993]}$ found the conventional diffuser incurred a loss coefficient of 0.08 and this was almost doubled for the Wedge diffuser which suffered a loss coefficient in the inner and outer passage of 0.13 and 0.16 respectively. Nevertheless, Shedden observed that the increased magnitude of controlled turning and diffusion within the Vane and Wedge diffusers resulted in lower losses in the dump region and hence lower losses to the feed annuli. The loss coefficient to the inner feed annuli reduced from 0.48 with the single passage diffuser to 0.46 and 0.37 for the Vane and Wedge diffusers. Similarly, outer annulus losses reduced from 0.52 to 0.48 and 0.49 respectively.

Although it is clear that a multi-passage diffuser can increase the area ratio and enhance system performance, the losses to the feed annuli observed by Shedden ${ }^{[1993]}$ are still high. Adenubi ${ }^{[1976]}$ comments that the total pressure loss to the combustor feed annuli in a gas turbine should be no more than $30 \%$ to $40 \%$ of the OGV exit dynamic pressure (to ensure the pressure drop across the combustor liners is sufficient to drive flow through air admission ports or cooling features). However, losses of almost $50 \%$ observed by Shedden ${ }^{[1993]}$ significantly exceed this and would have a detrimental effect on the engine cycle efficiency increasing specific fuel consumption (Oates ${ }^{[1985]}$ ). 


\subsection{Bled, Vortex Controlled and Hybrid Diffusers}

Under the action of the adverse pressure gradient within a diffuser the boundary layer will rapidly grow and eventually separate. One method of preventing this, and potentially allowing higher divergence angles to be achieved, is to remove the boundary layer by suction. This method was originally suggested by Prandtl and the concept is illustrated in Figure 2.24 and Figure 2.25 which shows how the exit velocity profile can be significantly enhanced (Furuya et al ${ }^{[1966]}$ ). It must be noted, however, that the boundary layer may still separate downstream of the suction if the adverse pressure gradient is strong enough.

Heskestad $^{[1966,1968]}$ studied the effect of suction on incompressible flow through a step expansion in a circular pipe, removing air though an annular gap in the convex corner of the step (Figure 2.26). He found that for a fixed geometry gradual increase in the suction rate from zero caused progressively more rapid expansion of the flow into the larger diameter pipe. This process continued rapidly until a 'critical' suction rate was reached and then continued only slowly after that. Heskestad observed that at above the critical suction rate the system produced a more favourable static pressure recovery than optimised conical diffusers of the same area ratio. Figure 2.27, taken from Heskestad ${ }^{[1968]}$, illustrates the effect of suction on pressure recovery for various geometries and it is clear that each set of data exhibits a rapid increase in $C_{p}$ above a particular suction rate. Heskestad $^{[1968]}$ further comments that this critical suction rate depends upon the expansion ratio $\left(D_{2} / D_{1}\right)$; the required suction increasing as the expansion ratio is increased. However, no mention is made of any effect the geometry of the bleed gap may have on the suction rate, reattachment length or static pressure recovery.

When the critical suction rate is reached Heskestad suggests (Figure 2.28) that "the dividing streamline, $d_{l}$, and the stagnation streamline, $S$, contain the suction flow which proceeds as a jet along the lower wall of the suction passage. The flow exterior to $S$ continues for a short distance along the downstream leg of the corner and then, in general, separates at point $C$. The dividing streamline, $d_{2}$, represents the boundary of the altered exterior (main) flow". 
Ringleb $^{[1955]}$ studied cross winds flowing over snow covered mountain ridges where the snow had formed into cusps. Using snow flakes to visualize the flow Ringleb noticed that the cross winds were rapidly turned by the ridge to a point of re-attachment on the leeward side of the ridge. He theorized that this was due to the formation of a vortex as illustrated in Figure 2.29. A streamline starting at $A$ enclosing the vortex ends up at stagnation point $B$ where snow is deposited. Snow is also deposited at $A$. The build up of snow at $A$ and $B$ form the cusp shape which acts to stabilize the vortex. It is this stabilized standing vortex which now causes the flow to re-attach at $B$ and remain attached as it flows down the leeward side of the ridge.

Those observations led Ringleb to design a diffuser which became known as Ringleb's Cusp Diffuser (Figure 2.30) which consisted of a sudden expansion with cusps in the walls. Ringleb implemented his diffuser in a closed loop wind tunnel immediately upstream of a $90^{\circ}$ bend. Others were unable to repeat this success as they found that the eddies were periodically shed resulting in high levels of instability. It was suggested that the success of Ringleb's diffuser was due to the bend causing secondary flows which stabilized the eddies.

Adkins ${ }^{[1975]}$ commented that Ringleb's diffuser relied solely on the aerodynamic design of the cusps in the diffuser wall to locate vortices as shown in Figure 2.30. Adkins ${ }^{[1975]}$ suggests that these vortices, rotating preferentially in the direction of the flow, reduce the boundary layer shear stress experienced by the flow. Furthermore, Adkins ${ }^{[1975]}$ remarks that the success of Ringleb's diffuser was limited due to the difficulty in maintaining a stable vortex system, since energy was lost within the cusp by skin friction. To solve this problem Adkins designed a vortex controlled diffuser (Figure 2.31) featuring a bleed directly from within the vortex/cusp region. The reasoning being that the vortex is then replenished by air of a higher energy level from the mainstream flow.

Beatty ${ }^{[1970]}$ showed that by introducing a fence downstream of the vortex the required amount of bleed could be reduced to a reasonable level. This configuration introduces a vortex "chamber" and leads to what Adkins ${ }^{[1975]}$ terms a "vortex controlled" diffuser (Figure 2.31). 
The flow mechanisms of this type of vortex controlled diffuser are not fully understood but Adkins ${ }^{[1975]}$ puts forward the following theory; the application of bleed reduces the static pressure of the vortex chamber and this causes stream tube $a$ (Figure 2.32), which is being drawn into the chamber to accelerate. On the other hand, stream tube $b$, which flows down the diffuser, is flowing in a region of greater static pressure and therefore decelerates. A shearing action is produced between the streams resulting in an energy transfer from $a$ to $b$. Some of the fluid in stream $b$ which would have been too energy deficient for diffusion is now able to flow through the vortex controlled diffuser without stalling. However, Adkins offers no experimental evidence to support his theory.

Adkins ${ }^{[1975]}$ also investigated the relationship between bleed level and diffuser effectiveness, $\eta$. Summarized in Figure 2.33 he found that there are three distinct regions of performance:-

(a) - (b) bleed is insufficient to draw the flow into chamber from upstream but fluid is drawn in from leeward of the fence resulting in a small increase in pressure recovery.

(b) - (c) static pressure in the vortex chamber is low enough to draw in the mainstream flow causing a significant increase in diffuser effectiveness.

(c) - (d) the vortex has been stabilized and any further increase in bleed does not greatly improve performance.

Figure 2.33 shows that there is a Minimum Bleed Requirement for this type of diffuser to work effectively. Adkins ${ }^{[1975]}$ correlated experimental data to give the following expression for minimum bleed requirement;

$$
\mathrm{B}_{\min }(\%)=0.393 \eta\left(1-\mathrm{AR}^{-2}\right)\left(\frac{\mathrm{AR}}{\mathrm{d}_{\mathrm{e}}}\right)^{0.5}
$$

where $\quad \eta=$ percentage effectiveness of the diffuser

$$
\begin{aligned}
& A R=\text { area ratio of the diffuser, } A_{2} / A_{1} \\
& d_{e}=\text { equivalent diameter at diffuser inlet, } \frac{4 \times \text { Cross Sectional Area }}{\text { Vortex Controlled Perimeter }}
\end{aligned}
$$


Note that no account of the bleed gap geometry is made here; thus it is questionable as to whether this is a valid design rule as it is applicable to one specific case only. Furthermore, in this study Adkins ${ }^{[1975]}$ has not considered, in any detail, the effect of bleed gap geometry. Although he varied the axial and radial bleed gaps (x and y in Figure 2.31) Adkins made no definite conclusions as to their influence. It would be surprising if changes to the bleed gap geometry did not significantly effect the required bleed rate, reattachment length and/or overall performance.

The minimum bleed requirement is also important when considering the potential use of hybrid diffusers in an aero gas turbine. Air is required not only for combustion but also for component cooling and auxiliary services. Thus if the required bleed rate is too high this type of diffuser may not be practical. Furthermore, the quality of the bleed air is also an issue if it is to be used in, for example, component cooling.

In his work Adkins ${ }^{[1975]}$ tested various vortex controlled diffusers, both tubular and annular, with area ratios of 1.9:1 to $3.2: 1$ and summarised that vortex controlled diffusers (with respect to conventional diffusers);

- have short length,

- have a low total pressure loss and high static pressure recovery, and

- require a bleed of about $3 \%$ of the main stream flow (Adkins ${ }^{[1975]}$ suggested this may be used for turbine blade cooling).

The logical extension of the vortex controlled diffuser is to place a conventional diffuser downstream of the vortex controlled step expansion. This type of diffuser was first proposed by Adkins and Yost ${ }^{[1979]}$ and termed a hybrid vortex controlled diffuser (HVCD). Figure 2.34 and Figure 2.35 illustrate the concept as described by Adkins et al ${ }^{[1980]}$. In this design the vortex controlled step expansion accounts for a smaller increase in area than previously and thus requires only a small amount of bleed. The re-energised turbulent layer generated by the step is then used to inhibit flow separation from a large angled conventional diffuser which accounts for a larger increase in flow area. 
Adkins and Yost ${ }^{[1979]}$ investigated the use of a three stage hybrid vortex controlled diffuser in a fan test installation as illustrated in Figure 2.36. This three stage diffuser comprised of a conventional pre-diffuser (Stage 1) followed by a vortex controlled diffuser (Stage 2) followed by another conventional diffuser (Stage 3). The Stage 1 diffuser had an equivalent cone angle of $10.2^{\circ}$ and an area ratio of 1.19 . The Stage 2 vortex controlled diffuser had an area ratio of 1.47:1 and the Stage 3 diffuser had an equivalent cone angle of $16.1^{\circ}$ with an area ratio of 1.53 . This resulted in the combination having an equivalent cone angle of $20.06^{\circ}$ and an overall area ratio of 2.69 . The area ratio of $1.47: 1$ for the vortex controlled step expansion was chosen because Adkins ${ }^{[1975]}$ had found that above 1.5:1 there was some risk of stall. Adkins and Yost ${ }^{[1979]}$ tested this configuration with several different combinations of axial (x) and radial (y) bleed gaps, measuring the static pressure recovery coefficient $\left(\mathrm{C}_{\mathrm{p}}\right)$ for various values of bleed. Furthermore, a measure of the pressure lost by the bleed air was made by comparing the static pressure inside the vortex with that at diffuser inlet. A vortex chamber depression coefficient, $V_{c}$, was defined:

$$
\mathrm{V}_{\mathrm{c}}=\frac{\mathrm{p}_{1}-\mathrm{p}_{\mathrm{c}}}{\mathrm{q}_{1}}
$$

Adkins and Yost ${ }^{[1979]}$ concluded that a hybrid vortex controlled diffuser can achieve a much higher area ratio within a given length than a conventional diffuser. For example, for the same length constraint a conventional diffuser will be limited to an area ratio of about 1.7 compared to 2.69 achieved by this HVCD. Consequently, Adkins and Yost $^{[1979]}$ measured a significantly higher pressure recovery than would be attainable by an optimised conventional diffuser $\left(C_{p}>0.80\right.$ compared to $\left.C_{p}=0.45\right)$. Furthermore, they report that the amount of bleed required for a hybrid vortex controlled diffuser is much less than for a simple vortex controlled diffuser; $3 \%$ of inlet mass flow compared to the $6 \%$ required by Adkins ${ }^{[1975]}$ for a similar overall area ratio. Figure 2.37 illustrates the effect on pressure recovery of varying the axial and radial bleed gaps. Adkins and Yost $^{[1979]}$ comment that the effect on diffuser performance is relatively small and changes in bleed gap geometry only really effect the level of suction required to extract the bleed air $\left(\mathrm{V}_{\mathrm{c}}\right)$. However, they did not offer any conclusion on whether this bleed air would be of sufficient quality for the suggested use for turbine blade cooling. Furthermore, Adkins and Yost ${ }^{[1979]}$ made only simple changes to the bleed gap geometry and 
were unable to make any conclusions regarding an optimum geometry. Adkins and Yost ${ }^{[1979]}$ were satisfied to use bleed gap geometries defined from earlier work and did not attempt to investigate the effect on the flow of more radical changes in the bleed geometry. This is unfortunate as any description of the flow mechanisms cannot be complete without understanding the interactions of the mainstream flow, the bleed air, the bleed geometry, the step/fence arrangement and the downstream boundary layer.

Adkins et al ${ }^{[1980]}$ conducted experiments on tubular/conical vortex controlled and hybrid diffusers with a fully developed inlet flow with the aim of investigating the bleed requirements, effects of axial and radial bleed gaps ( $\mathrm{x}$ and $\mathrm{y})$, fence subtended angle $(\phi=$ $\left.\tan ^{-1} y / x\right)$, step area ratio $\left(A_{2} / A_{1}\right)$, overall area ratio $\left(A_{3} / A_{1}\right)$ and the angle of divergence of the conventional diffuser. (Refer to Figure 2.35). They were unable to put forward any theories about the flow mechanism other than those already described. Figure 2.38 (a) and (b) demonstrate how the static pressure recovery varied with non-dimensional length at various bleed rates. Both (a) and (b) show plots for two HVCD's of overall area ratio 2.0 but having a step expansion of area ratios 1.2 and 1.34 respectively. Both HVCD's have similar overall performance characteristics but importantly both achieve significantly higher pressure recoveries than a conventional diffuser of area ratio 2.0 (data also plotted on Figure 2.38(a) and (b)). Interestingly this is also true for zero bleed and can be seen in Figure 2.38(a). At zero or very low bleed rates Adkins et al ${ }^{[1980]}$ note that vortex will break down and suggest that the bleed flow will now originate from downstream of the fence and will still produce a significant region of low pressure as it accelerates over the top of the fence (Figure 2.39). In addition to deflecting the main jet by the influence of this depression, the reverse flow will also generate a highly turbulent area. The resultant turbulent mixing is not aerodynamically efficient and high static pressure recoveries will not be produced unless the flow reattaches to the diffuser wall.

Although the study by Adkins et al ${ }^{[1980]}$ examined a wide range of bleed geometries they were still unable to make any conclusions regarding optimum geometry. Nor were they able to offer firm or conclusive evidence concerning the flow mechanisms of this type of diffuser. These are two very important points as it is likely they are mutually dependant. The definition of an optimum bleed configuration will require an understanding of the 
flow mechanisms involved. Importantly, this would need to include an understanding of how the flow is altered (or perhaps improved) by changes in the configuration of the bleed gap geometry.

Adkins and Yost ${ }^{[1983]}$ tested a fully annular hybrid vortex controlled diffuser of area ratio 2.5 and non-dimensional length of 2.33 upstream of a simple combustion chamber model (Figure 2.40 and Figure 2.41). The flame tube head was manufactured from wood with no attempt to model porosity or the presence of burners and burner arms. Realistic inlet conditions were not used; a fully developed inlet profile was used rather than a flow with blade wakes, high turbulence and swirl such as that generated by a compressor. Adkins and Yost ${ }^{[1983]}$ reported a high diffuser static pressure recovery coefficient of 0.80 and a very low total pressure loss coefficient of 0.04 at a bleed rate of $4.0 \%$. Loss and recoveries to the combustor feed annuli are summarised in Figure 2.42 and at $4.0 \%$ bleed are well below the $30 \%$ to $40 \%$ (of inlet dynamic pressure) target suggested by Adenubi ${ }^{[1976]}$.

A pre-diffuser was incorporated upstream of the vortex controlled expansion by Adkins and Yost ${ }^{[1983]}$ in order to raise the pressure of the bleed air. In a modern gas turbine cycle air is a valuable commodity. Not only is the correct amount required for the combustion process itself but air is also needed for dilution of the combustion products and cooling of components such as flame tube walls and turbine blades. Thus, if a portion of air is required for a bled diffuser it would be imperative that this air can then be used for another purpose. Adkins ${ }^{[1975]}$ suggests the most suitable purpose would be turbine blade cooling and additionally comments that an increase in bleed air pressure would be advantageous, hence the pre-diffusion in their HVCD. To assess the quality of the bleed air they measured a bleed air static pressure recovery coefficient, $\mathrm{C}_{\mathrm{B}}$, defined as:

$$
\mathrm{C}_{\mathrm{B}}=\frac{\text { bleed air static pressure }-\mathrm{p}_{1}}{\mathrm{P}_{1}-\mathrm{p}_{1}}
$$

This quantity is also plotted in Figure 2.42 and has a value of above 0.3 at $4.0 \%$ bleed; Adkins and Yost remark that this value indicates that the air would have sufficient pressure to be used as a turbine coolant. However, they offer no other evidence to support this statement and do not identify why they consider a value of $\mathrm{C}_{\mathrm{B}}=0.3$ to be significant. 
Montazerin and Adkins ${ }^{[1985]}$ used the same test rig of Adkins and Yost ${ }^{[1983]}$ (Figure 2.40) but removed the representation of a combustor flame tube. They varied the included angle of the conventional diffuser from $15^{\circ}$ to $45^{\circ}$ and found that to maintain attached flow at the higher angles the bleed rate had to be increased to $6 \%$ and above. However, it was highlighted in Section 2.4 that the presence of a flame tube has a significant effect on the condition of the diffuser exit profile, reducing profile distortion and potentially suppressing separation. Thus the levels of diffuser performance recorded by Montazerin and Adkins ${ }^{[1985]}$ are likely to be below that which would be observed with a flame tube present.

Adkins and Montazerin ${ }^{[1985]}$ conducted tests on a conical hybrid vortex diffuser in order to asses the effect of inlet flow distortions. Using the same test facility as Adkins et $\mathrm{al}^{[1980]}$ (Figure 2.43) non uniform inlet velocity profiles were generated by inserting perforated plates upstream of the diffuser. These plates all feature a large central hole in which a settling tube was located, producing peaked profiles as shown in Figure 2.44. Figure 2.45 summarises performance data for one configuration and clearly shows that as the inlet profile becomes more peaked ( $\alpha$ increases) the effectiveness of the diffuser reduces and higher bleed rates are required to achieve the same level of pressure recovery.

Adkins and Kuile ${ }^{[1985]}$ added a flame tube canted radially outward at $15^{\circ}$ to the test facility used by Montazerin and Adkins ${ }^{[1985]}$ as shown in Figure 2.46. They reported difficulties in setting the bleed rates; at an inner bleed above $1 \%$ of the inlet mass flow the outer wall stalled and the effect of this on annulus performance $\left(\mathrm{C}_{\mathrm{p}}\right.$ and $\left.\lambda\right)$ is illustrated in Figure 2.47. The outer bleed was kept at a constant $4 \%$ but the inner bleed varied from $0-5 \%$. For low levels of inner bleed, annuli performance is good; loss coefficients below 0.4 and recovery coefficients above 0.8 . However, above an inner bleed of $1 \%$ performance rapidly declines. The introduction of pre-diffuser cant has increased the loading on the outer wall but decreased loading on the inner wall to the extent that it would probably remain attached without the action of bleed.

Adkins and Kuile suggest the outer diffuser wall stalls and performance rapidly 
decreases. In conclusion, it would appear that bleed is not necessary on the inner wall and on examination of the geometry this is not surprising. The effect of outwardly canting the diffuser and flame tube results in a modestly loaded inner wall which would probably remain attached without bleed.

With reference to Figure 2.48, Adkins and Kuile ${ }^{[1985]}$ also report that an unbalanced bleed significantly alters the mass flow split between the two feed annuli. They do not plot diffuser exit profiles but this effect is more than likely due to the unbalanced bleed producing a biased diffuser exit velocity profile hence presenting one of the annuli with a higher mass flow.

A comprehensive piece of research was undertaken by Myres et al ${ }^{[1993]}$ who performed a series of tests to investigate the effect of geometric and flow parameters on the performance of an isothermal, fully annular, inwardly canted, hybrid vortex diffuser in a representation of a modern combustion chamber. The tests were designed to simulate, realistically, the aerodynamic features of an advanced engine core from the exit of a high pressure compressor to the entry of the turbine. The test facility used by Myres et al is illustrated in Figures 2.49 and 2.50 with the leading dimensions shown in Figure 2.51.

Measurements were taken at pre-diffuser inlet (i.e. immediately downstream of the deswirl vanes), pre-diffuser exit, inner and outer combustor feed annuli. The instrumentation consisted of wall static pressure tappings and total pressure rakes at inlet and in the feed annuli with a traversable five hole probe at pre-diffuser exit.

The hybrid vortex controlled diffuser was designed to be similar to that used by Adkins and Yost ${ }^{[1983]}$ with a step expansion of area ratio 1.3. The conventional diffuser downstream of the step expansion had an outer wall angle of $9.5^{\circ}$ and inner wall angle of $32^{\circ}$ or $37^{\circ}$. Combined with a cant angle of $20^{\circ}$ this produced an diffuser of overall area ratio 2.36 or 2.6 with a non-dimensional length of about 3.6.

The initial tests conducted by Myres et al ${ }^{[1993]}$ involved a clean inlet duct which produced a fully developed velocity profile. This configuration produced disappointing results; $C_{p 1-2}$ values of 0.34 to 0.42 which represents a diffuser effectiveness, $\eta$, of less 
than $50 \%$. Diffuser total pressure losses were also high with $\lambda_{1-2}$ ranging from 0.15 to 0.28 .

In an attempt to increase the performance of their diffuser Myres et al ${ }^{[1993]}$ installed a set of swirl/de-swirl vanes immediately upstream of the diffuser. They comment that this introduced a higher level of inlet turbulence intensity, added blade wakes, energised hub/ shroud boundary layers and promoted circumferential movement of the flow in the prediffuser. They do not, however, present any evidence of this other than an increase in performance of the diffuser to a level in line with previous studies.

The inward cant and modest angle of the outer diffuser wall should not present much of an aerodynamic problem for the flow. Thus it is of no surprise that Myres et al ${ }^{[1993]}$ were able to remove vortex bleed from this area. Once a working system had been established some promising results were observed. For a nominal bleed rate of about $1.2 \%$ of the total inlet flow the pre-diffuser static pressure recovery was high $\left(\mathrm{C}_{\mathrm{p} 1-2} \approx 0.75\right)$ and the total pressure loss was low $\left(\lambda_{1-2} \approx 0.1-0.17\right)$. The condition of the flow to the feed annuli was also relatively good $\left(\mathrm{C}_{\mathrm{p} 1-4} \approx 0.8\right.$ and $\left.\lambda_{1-4} \approx 0.2-0.25\right)$.

Myres et al ${ }^{[1993]}$ recognised the fact that high performance engines cannot afford to waste even $2 \%$ of the airflow, and that the utility of the bleed air is proportional to its pressure. Thus, they measured and plotted the ratio of bleed chamber static pressure to diffuser inlet total pressure for $2 \%$ bleed. Figure 2.52 shows the ratio reduces as inlet Mach number increases but no qualitative assessment of this data is offered

A further observation on the work of Adkins and Yost ${ }^{[1983]}$, Montazerin and Adkins ${ }^{[1985]}$ and Adkins and Kuile ${ }^{[1985]}$ is that the geometries studied (Figure 2.36, Figure 2.40 and Figure 2.46) do not incorporate a definite vortex chamber. Much of the work discussed above suggests a vortex chamber is an essential component of a hybrid vortex controlled diffuser having it's origins in Ringleb's ${ }^{[1955]}$ cusp diffuser. However, the lack of a definite vortex chamber here would cast doubts on this conclusion. Furthermore, on inspection, it appears that the presence of a Coanda bubble immediately downstream of the step expansion may more closely simulate Ringleb's cusp vortex. Thus it 
must be asked what is the purpose of a vortex chamber; does it represent an important part of the flow mechanisms or is it simply a means of removing the bleed air? In addition, what of the Coanda bubble itself? Such a phenomenon will always be formed downstream of a backward step but to what extent does it contribute to the flow mechanisms of a hybrid vortex controlled diffuser? Indeed what precisely are these flow mechanisms? The evidence is not conclusive in the support of Adkins et al ${ }^{[1980]}$.

\section{$\underline{\text { 2.7 Summary }}$}

To reiterate, it is has been highlighted that conventional diffuser technology is unlikely to offer a solution to the problems posed by future, low emission, staged combustion systems. However, as reported in Section 2.6, the work of Adkins et al ${ }^{[1980]}$, Myres et $\mathrm{al}^{[1993]}$ and others has demonstrated that hybrid diffusers can, indeed, achieve the required rates of diffusion and turning and offer an attractive option for use with this type of combustion system. Unfortunately, these authors have left some important questions which must be addressed:

- All the studies reported have based their bled diffusers on the basic geometry developed by Adkins et al ${ }^{[1980]}$. There have been no studies into the development of alternative bleed geometry and how this could be used to augment the performance of the diffuser or improve the quality of the bleed flow. Additionally, there is some doubt that a 'vortex chamber' is really a necessary feature.

- Although Adkins et al ${ }^{[1980]}$ have suggested flow mechanisms for hybrid diffusers, no detailed measurements or computational predictions have been made which can verify or disprove these theories. Furthermore, since no conclusive data exist on the effect of bleed gap configuration any suggested mechanisms cannot be complete.

- When considering hybrid diffusers for use in modern gas turbine combustion systems previous studies have not incorporated realistic or representative configurations. It was highlighted in Sections 2.3.2 and 2.4 that the upstream and downstream conditions have a large impact on the performance of a diffuser. The studies reported here have not included an inlet flow typical of axial compressor efflux (including OGV 
wakes) or a detailed reproduction of a modern flame tube design. The effect of these conditions must analysed in order to assess the potential use of hybrid diffusers.

- Furthermore, detailed and high quality data allowing back-to-back performance comparisons of hybrid and conventional diffuser technologies are not available. Again, without this type of data it is impossible to conclude as to the suitability of hybrid diffusers for use in combustion systems of the future.

- Comment has been made concerning utilising the bleed air for component cooling but no conclusive data exist with which to assess this possibility. Nor has any attempt been made to improve the quality of the bleed air. If the bleed air cannot be usefully employed elsewhere then it unlikely this type of diffuser will be used in a gas turbine combustion system. Modern engine cycles cannot afford to waste any air.

\subsection{Aims and Objectives}

Based on the survey presented in the previous section, the overall aim of this study is to analyse the performance and flow mechanisms of hybrid diffusers in modern, low emission, radially staged gas turbine combustion systems. This includes:

- determining the effect on pre-diffuser and system performance of:

(i) altering geometrical parameters, including investigating the purpose of the vortex chamber and a generic study into alternative bleed/step arrangements,

(ii) varying bleed rates,

(iii) inclusion of diffuser struts, and

(iv) representative inlet conditions incorporating OGV wakes.

- assessing the quality of the bleed air flow, possible methods of improving this and its potential use for component cooling.

- a detailed examination of the flow mechanisms of hybrid diffusers in order to determine the pertinent aerodynamic processes and thus allow the description of an optimum hybrid diffuser for use in a modern, low emission, radially staged gas turbine combustion 
system.

To achieve these aims both an experimental and analytical approach should be used. Firstly, a representative isothermal experimental test facility was available to provide the realistic environment necessary to assess the performance and suitability of hybrid diffusers for use in aero gas turbines. Secondly, the application of Computational Fluid Dynamic (CFD) would enable the prediction of the entire flow field within a hybrid diffuser thus, facilitating a more detailed assessment of the flow structure and aerodynamic mechanisms. Furthermore, CFD is an ideal vehicle for a generic study of bleed gap geometry and how this can be optimised.

\subsection{Structure of Thesis}

This thesis will, in the main, discuss the experimental and computational work separately until these are drawn together in the final conclusions section.

Following the general introduction (Chapter 1.0) and review of past work (Chapter 2.0) the experimental investigation will be discussed in Chapters 3.0 and 4.0, beginning with a presentation, in detail, of the experimental test facility, instrumentation, data acquisition/reduction and error analysis. This is followed, in Chapter 4.0, by a detailed presentation and discussion of data and observations from the experimental test programme.

The computational work is described in Chapters 5.0 and 6.0. Chapter 5.0 introduces the techniques and methods employed in the computational modelling. Following on from this, Chapter 5.0 also discusses the geometry and meshes used in the computation coupled with a subsequent assessment of the solution techniques. Results from the computational study are presented and discussed in Chapter 6.0 and, where relevant, compared to the experimental results. Finally, conclusions will be made and re-iterated in Chapter 7.0 with any suggestions for further work also being made. 

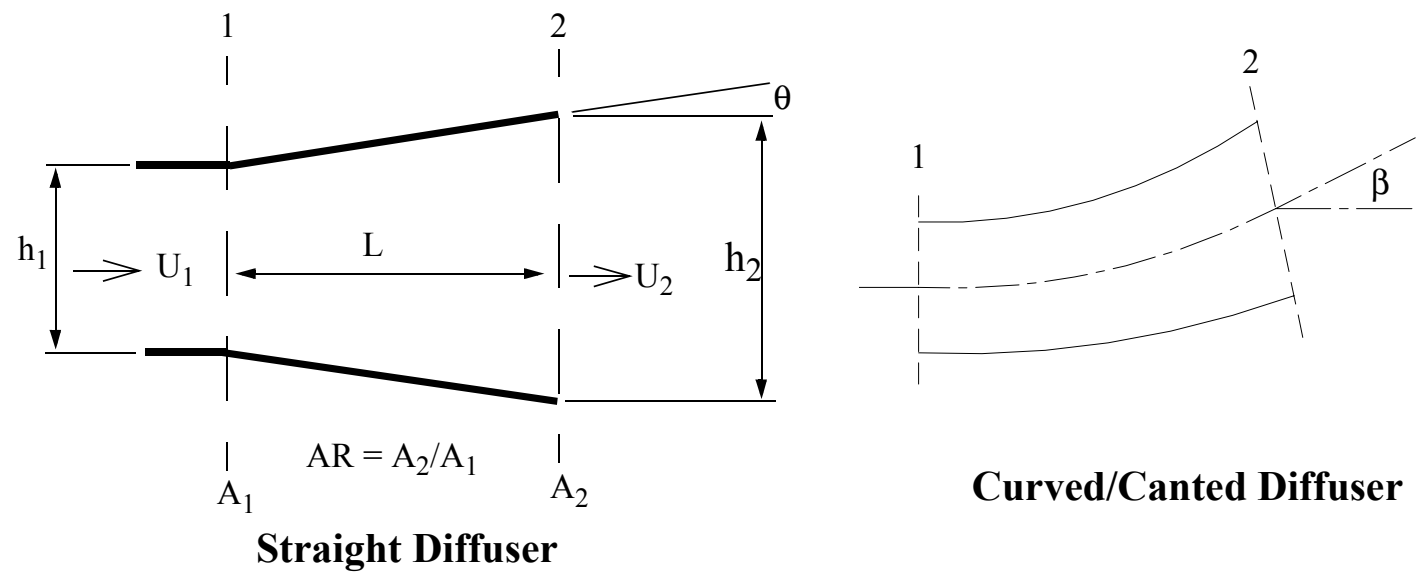

Curved/Canted Diffuser

Straight Diffuser

Figure 2.1 Basic Diffuser Geometry

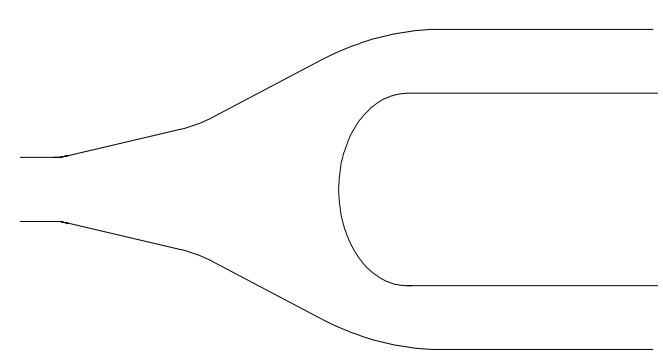

(a) Faired Diffuser

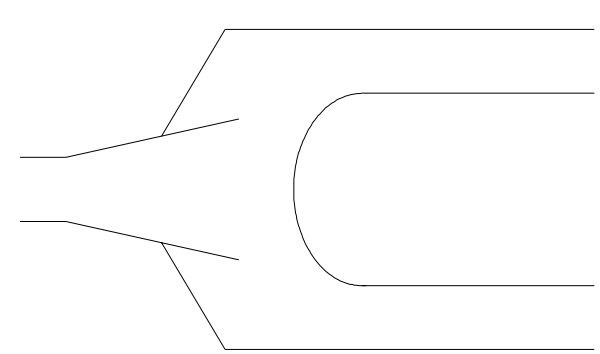

(b) Dump Diffuser

Figure 2.2 Faired and Dump Diffusers

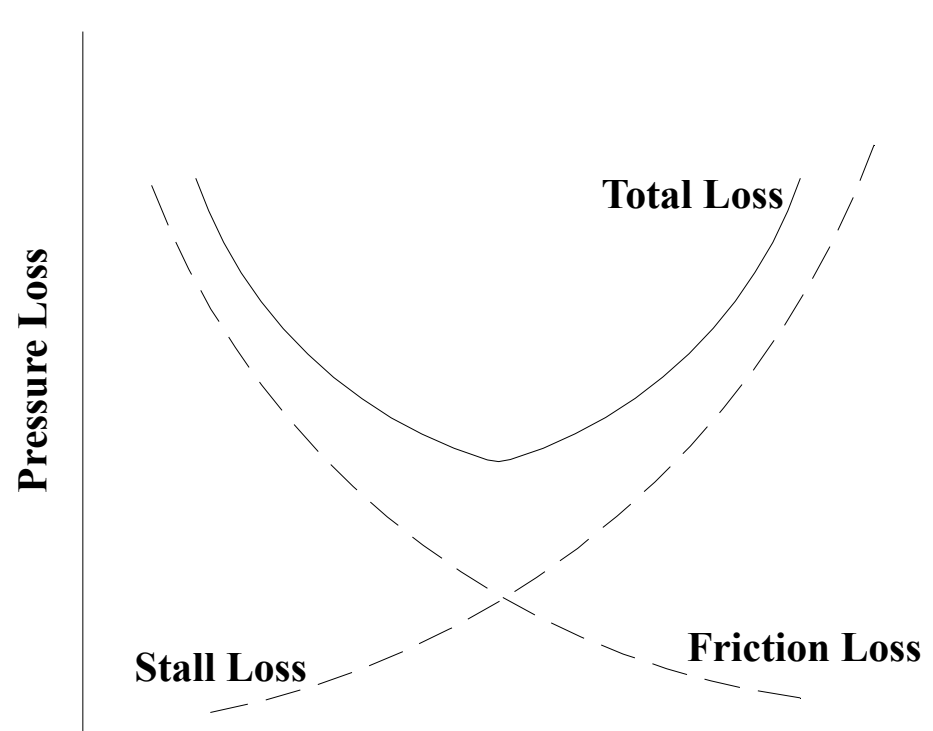

Divergence Angle

Figure 2.3 Influence of Divergence Angle on Pressure Loss, Lefebvre ${ }^{[1983]}$ 


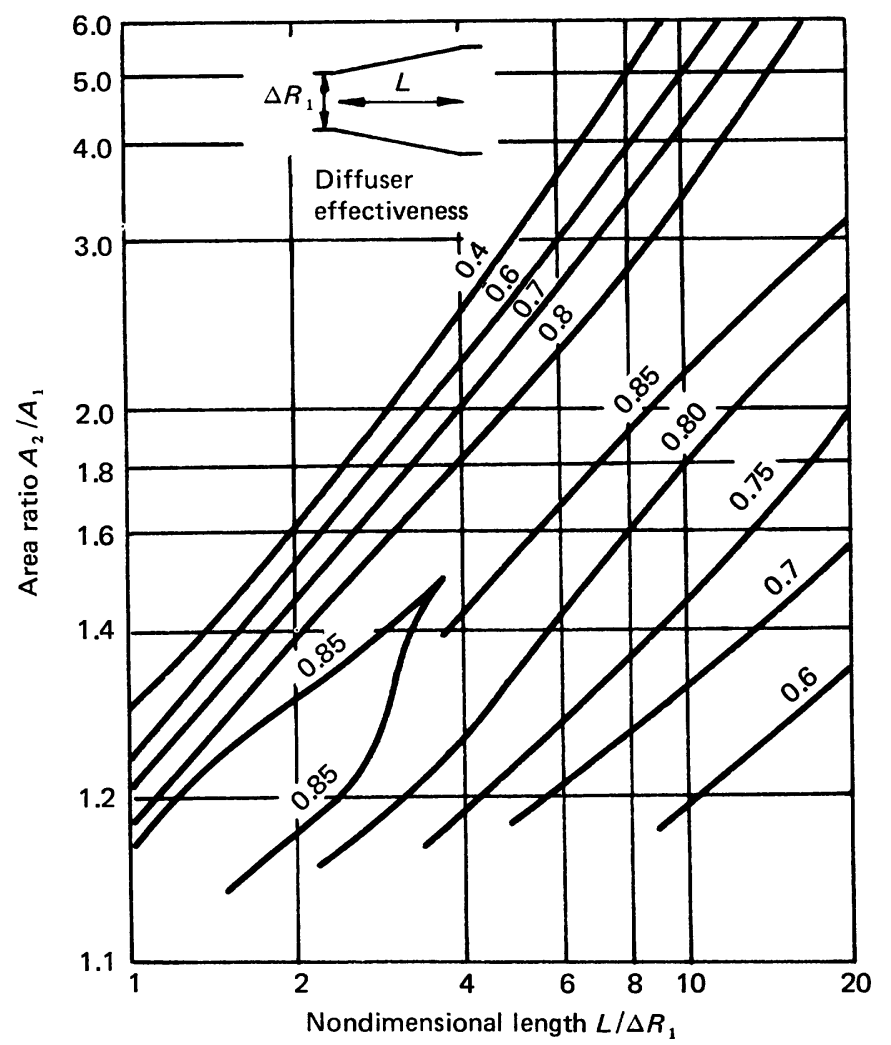

Figure 2.4 Annular Diffuser Performance Data, Sovran and Klomp ${ }^{[1967]}$
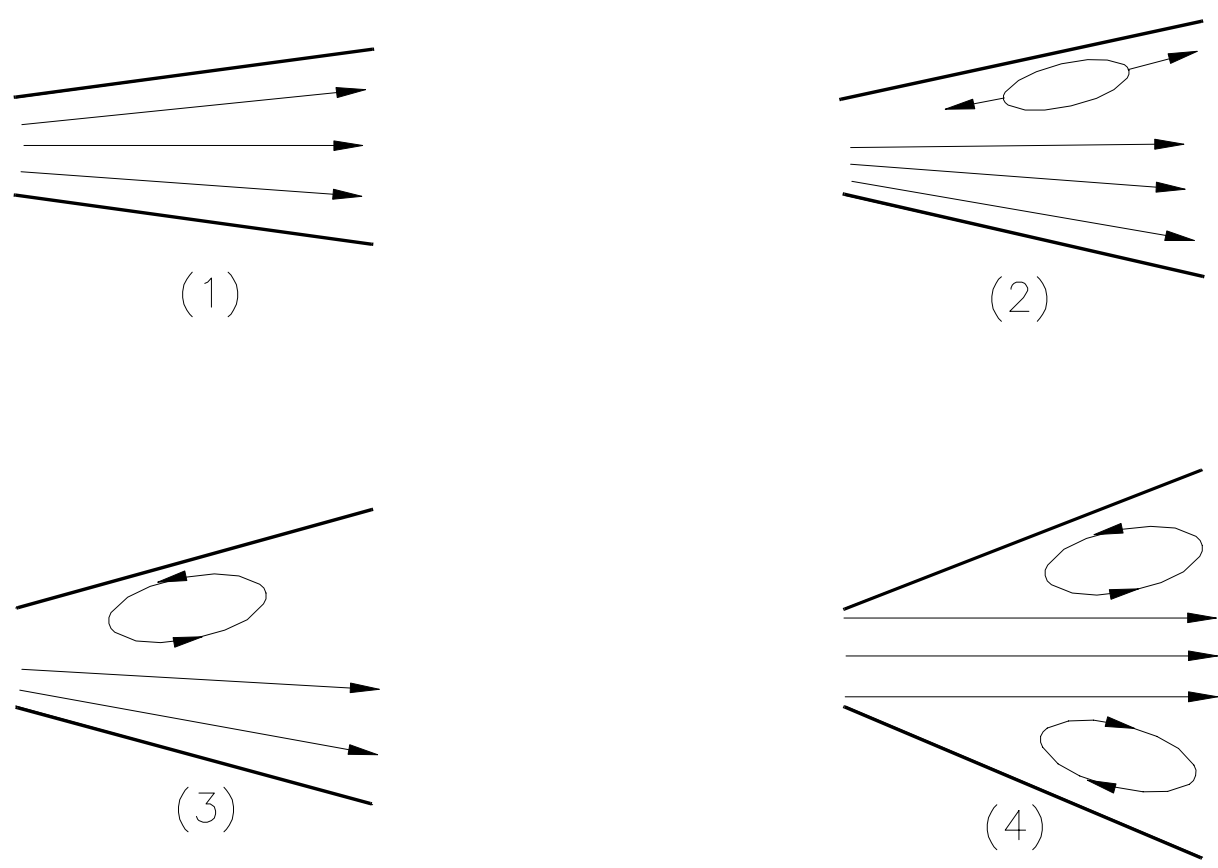

Figure 2.5 Diffuser Flow Regimes, Kline et al ${ }^{[1959]}$ 


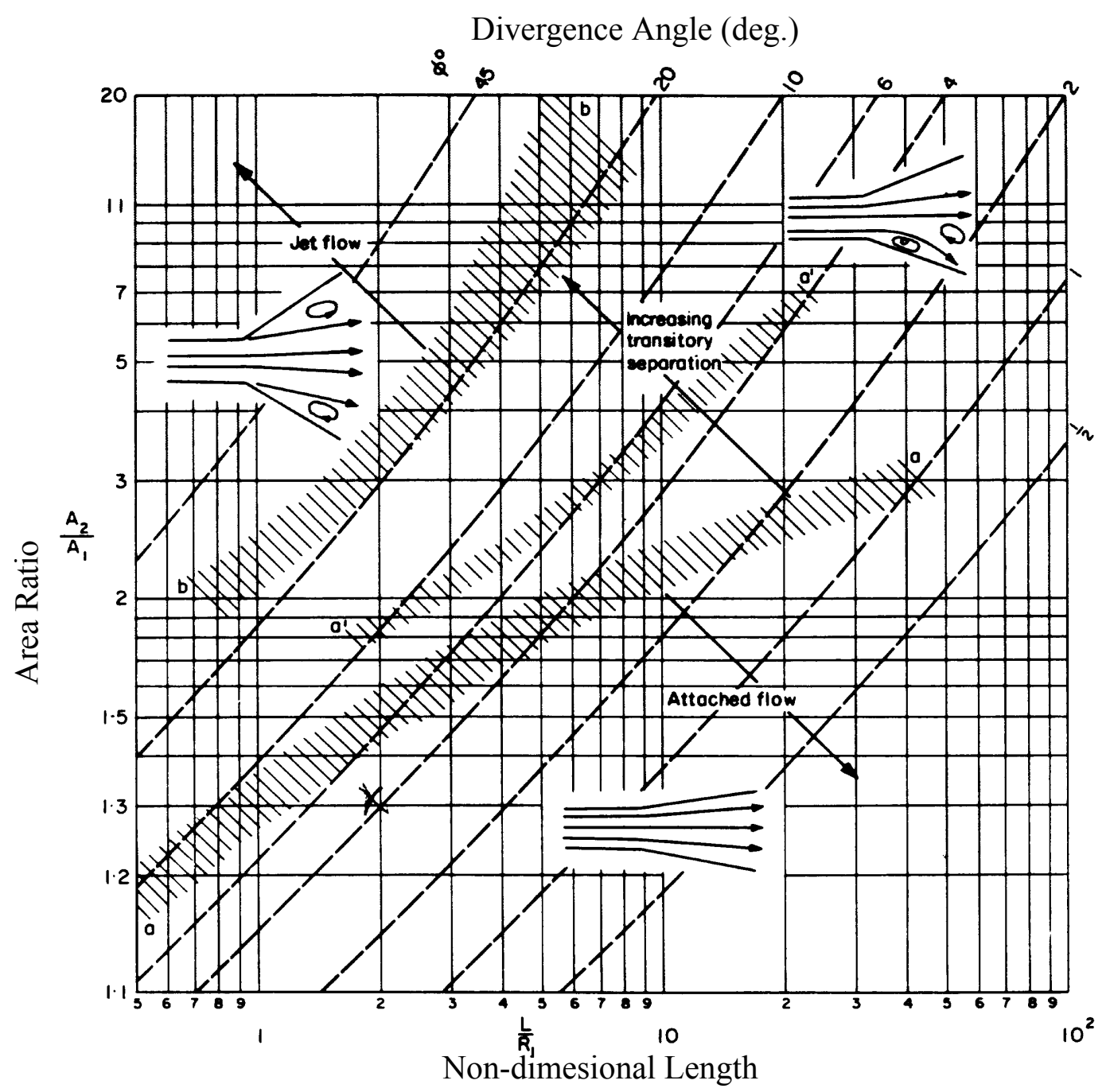

Figure 2.6 Flow Regime Chart, Kline et al ${ }^{[1959]}$

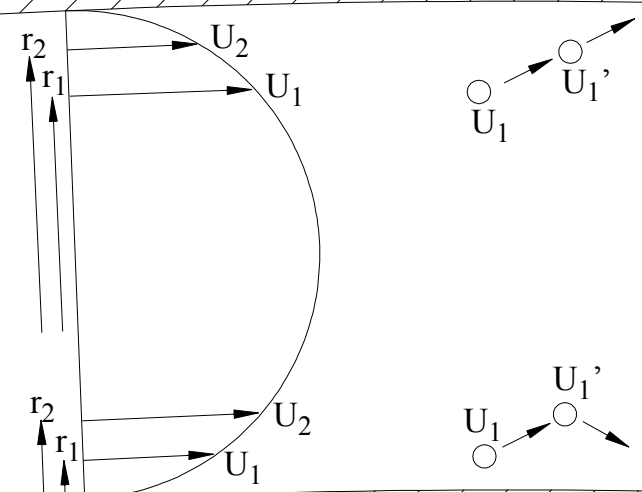

Figure 2.7 Motion of a Displaced Element in a Curved Flow 


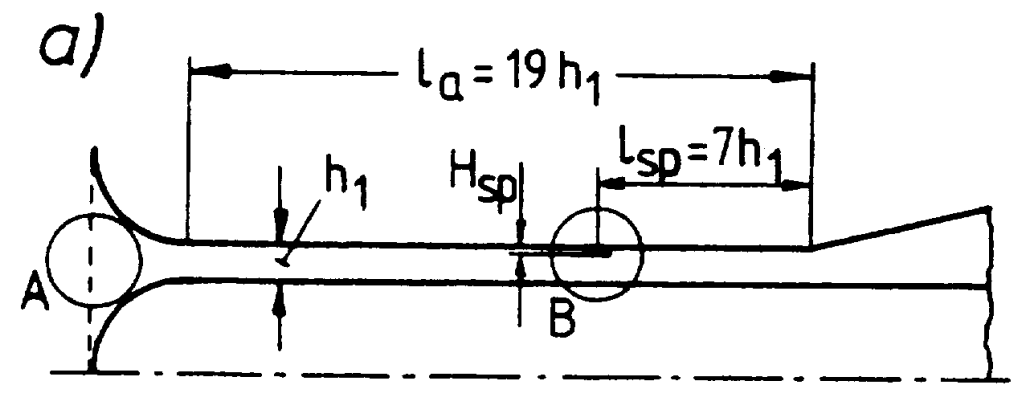
A: Coarse grid
$B$ : Spoiler $H_{s p} / h_{1}=0.10$
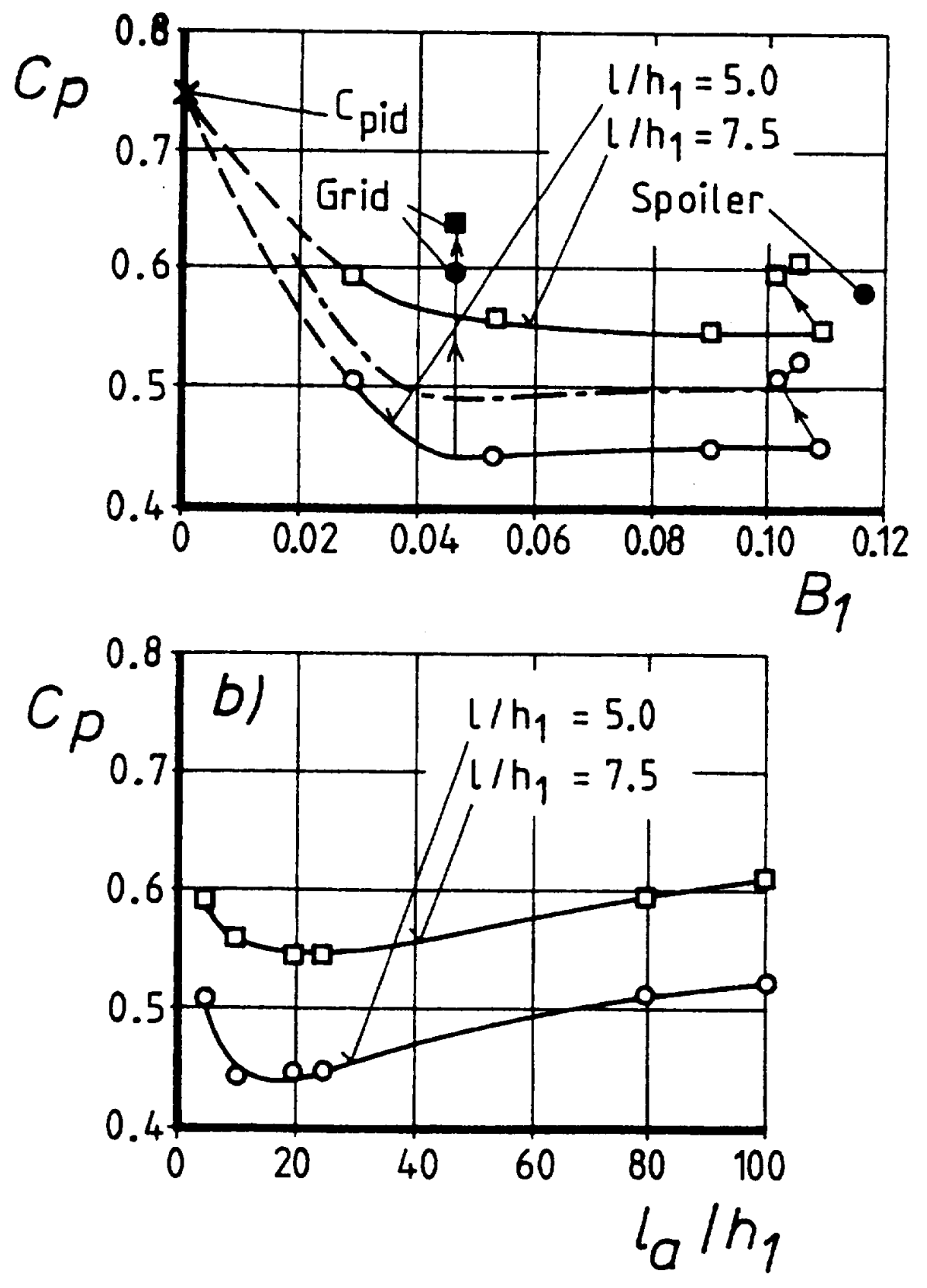

Figure 2.8 Effect of Inlet Boundary Layer Blockage on Pressure Recovery of Two AR 2.0 Diffusers, Stevens and Williams ${ }^{[1980]}$ 


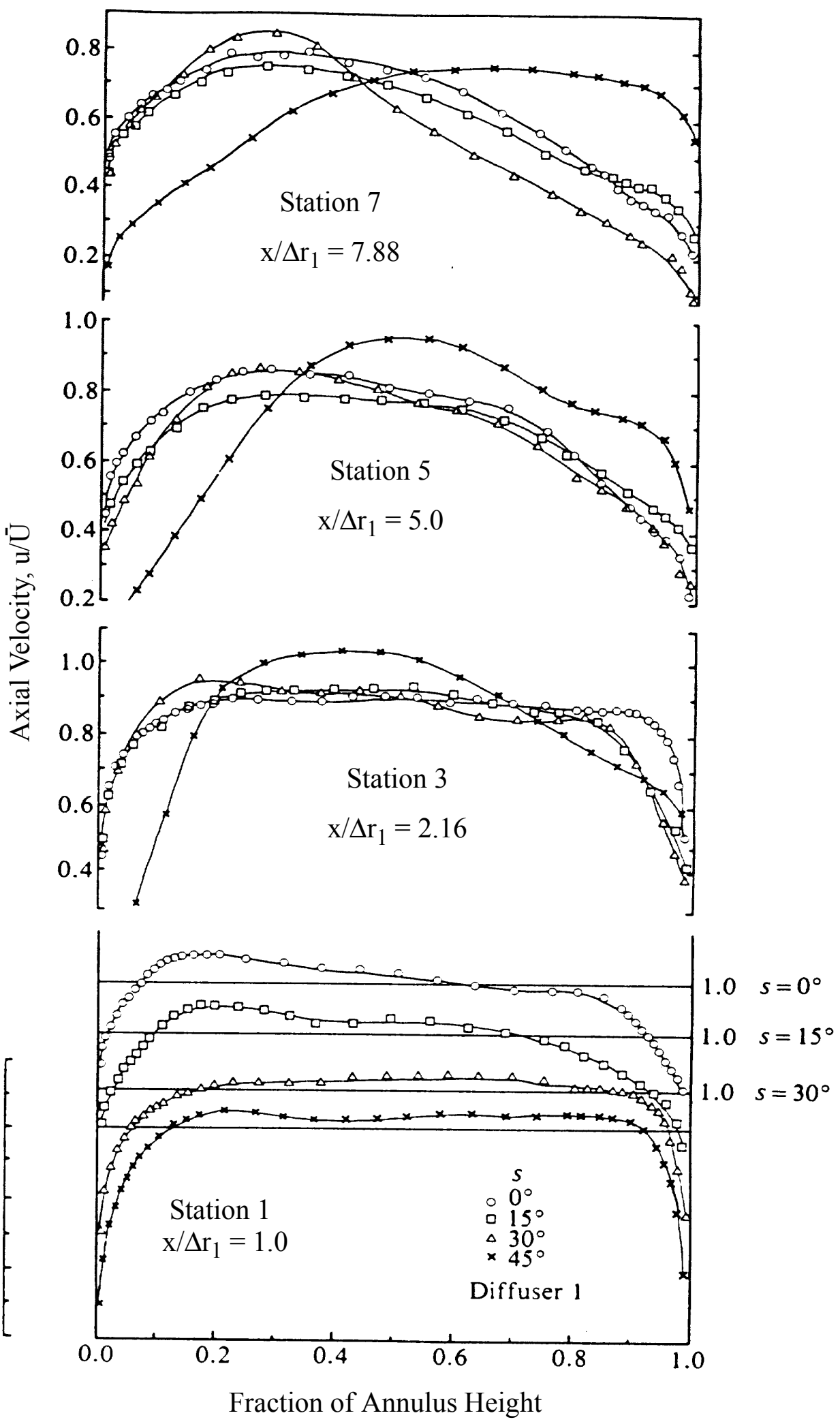

Figure 2.9 Effect of Inlet Swirl on Axial Velocity Profile, Elkersh et al ${ }^{[1985]}$ 


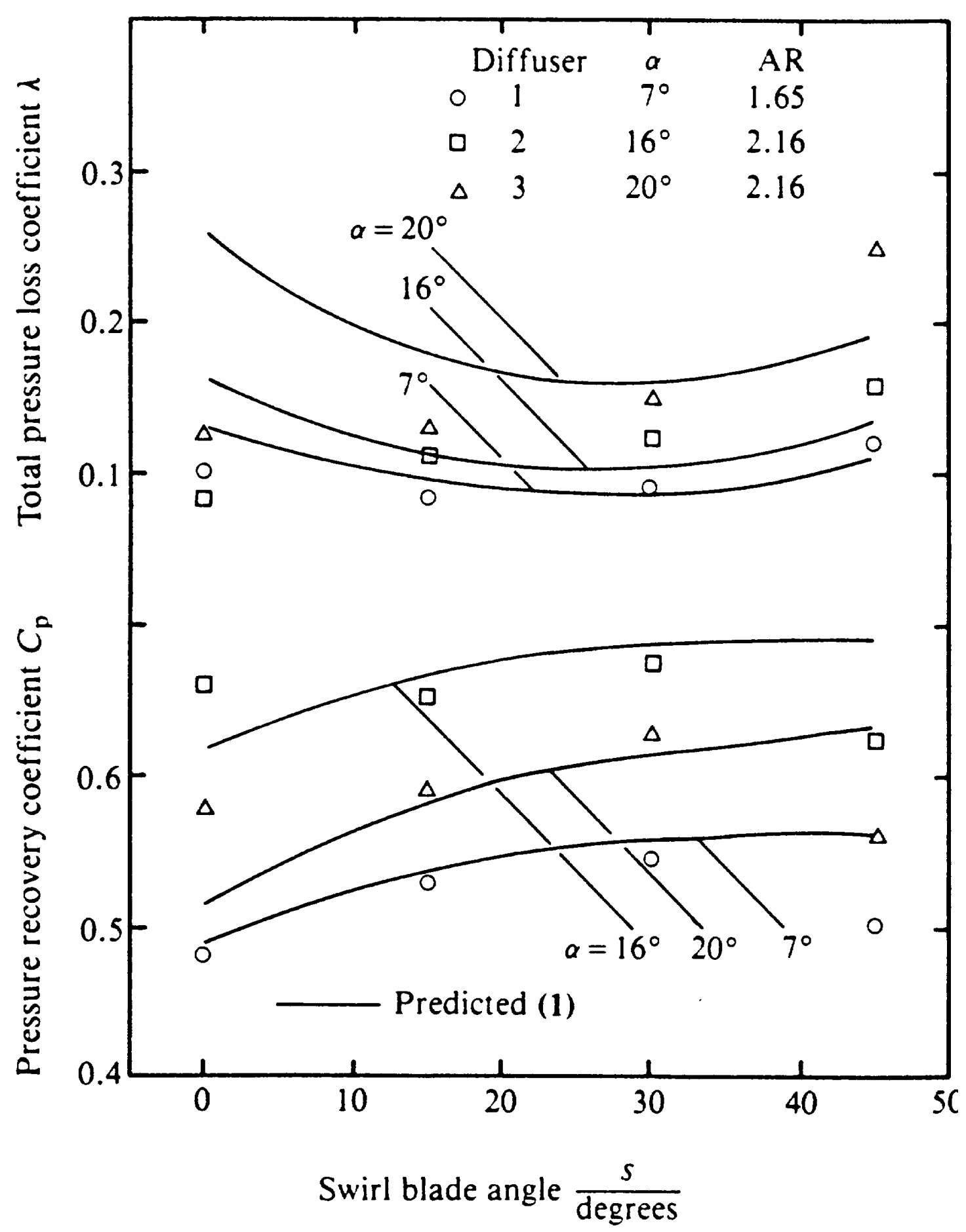

Figure 2.10 Effect of Inlet Swirl on Diffuser Performance, Elkersh ${ }^{[1985]}$ 


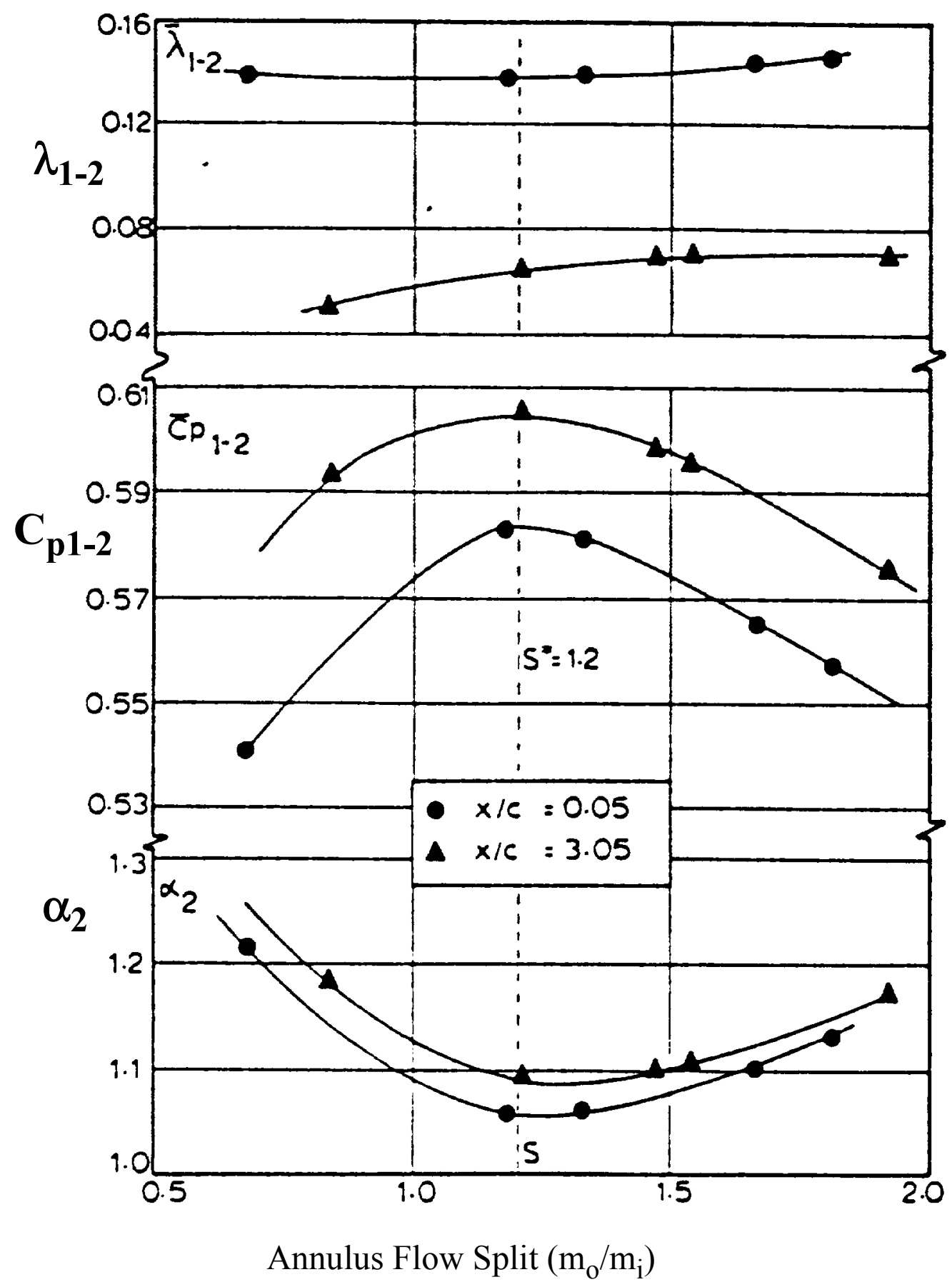

Figure 2.11 Effect of Profile Distortion on Diffuser Performance, Stevens et al ${ }^{[1978]}$ 


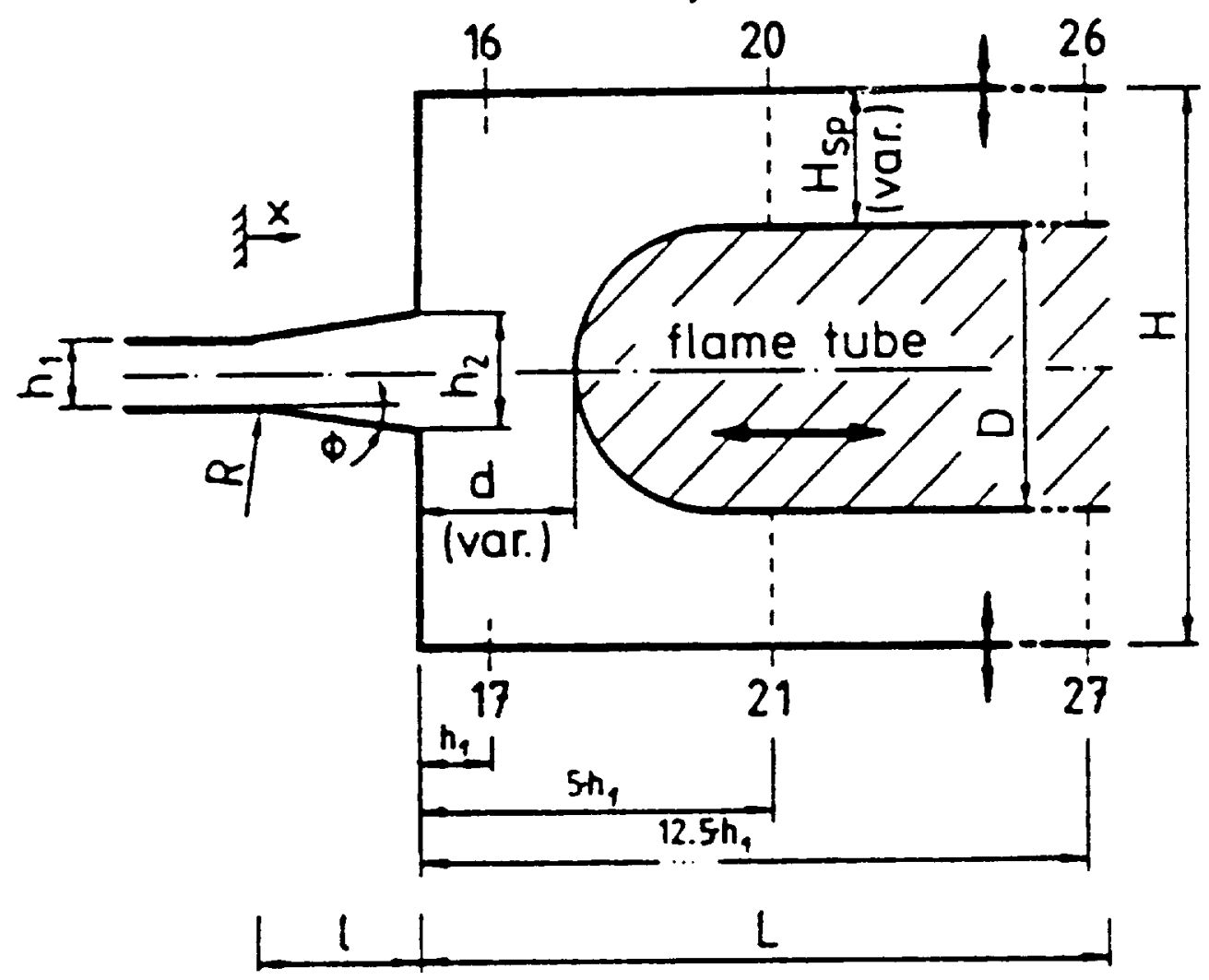

Figure 2.12 Test Section, Hestermann et al ${ }^{[1991]}$

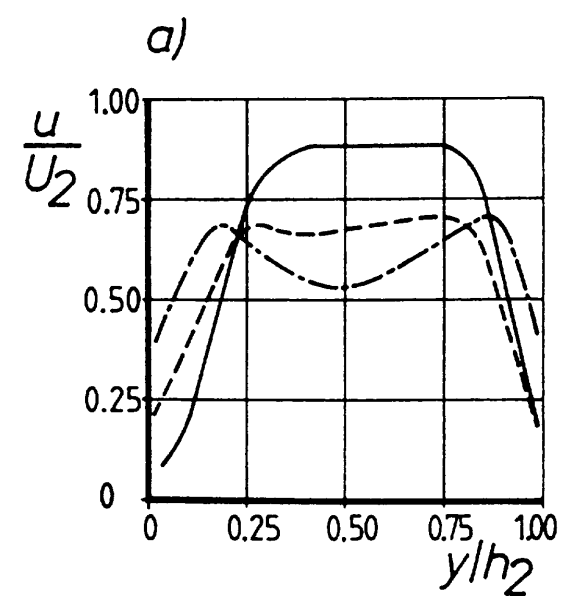

b)

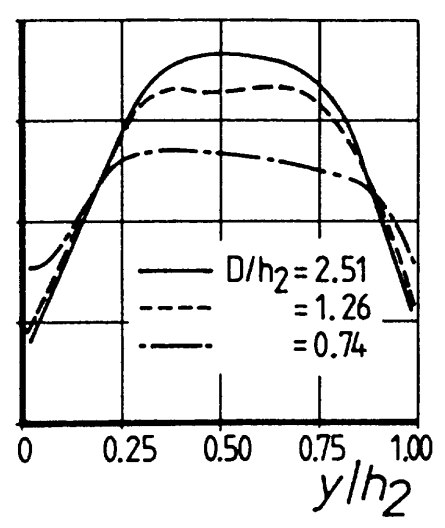

c)

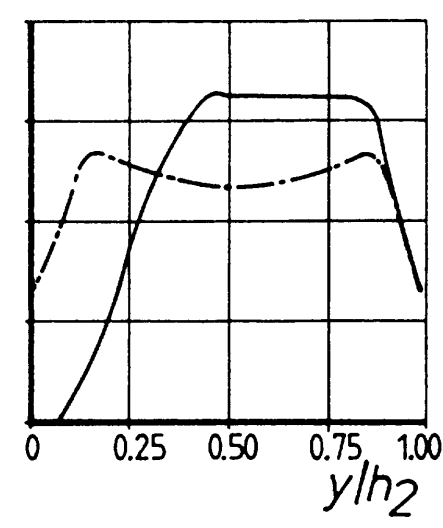

a) $\left.\mathrm{L} / \mathrm{h}_{1}=3.0, \mathrm{AR}=1.78, \mathrm{~B}_{1}=0.05, \mathrm{~b}\right) \mathrm{L} / \mathrm{h}_{1}=3.0, \mathrm{AR}=1.78, \mathrm{~B}_{1}=0.12$,

c) $\mathrm{L} / \mathrm{h}_{1}=1.84, \mathrm{AR}=1.78, \mathrm{~B}_{1}=0.05$

Figure 2.13 Influence of Dump Gap Ratio on Diffuser Exit Profile, Hestermann et al $^{[1991]}$ 


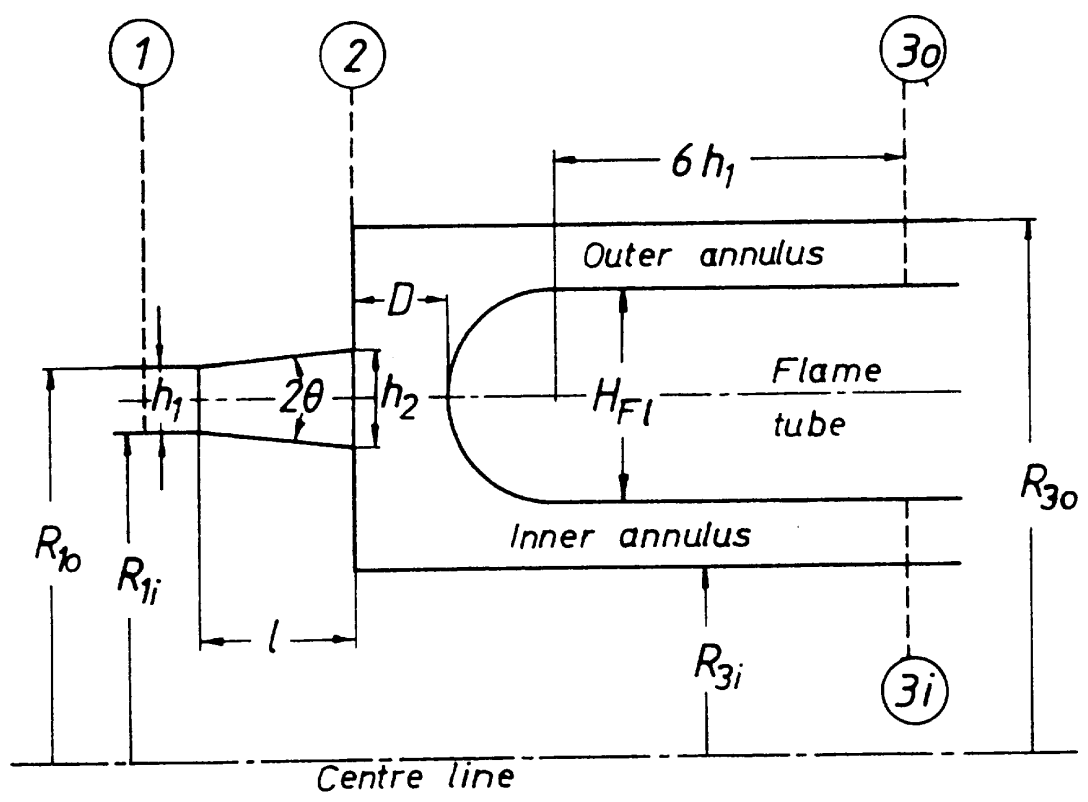

\begin{tabular}{|l|l|l|l|l|l|l|}
\hline$\frac{R_{1 i}}{R_{70}}$ & $\frac{R_{3 i}}{R_{7 i}}$ & $\frac{R_{30}}{R_{3 i}}$ & $\frac{H_{F 1}}{h_{1}}$ & $\frac{A_{3 i}}{A_{1}}$ & $\frac{A_{30}}{A_{1}}$ & $\frac{A_{30}}{A_{3 i}}$ \\
\hline 0.85 & 0.60 & 2.61 & 3.50 & 0.635 & 1.365 & 2.15 \\
\hline
\end{tabular}

Figure 2.14 Test Section, Fishenden and Stevens ${ }^{[1977]}$

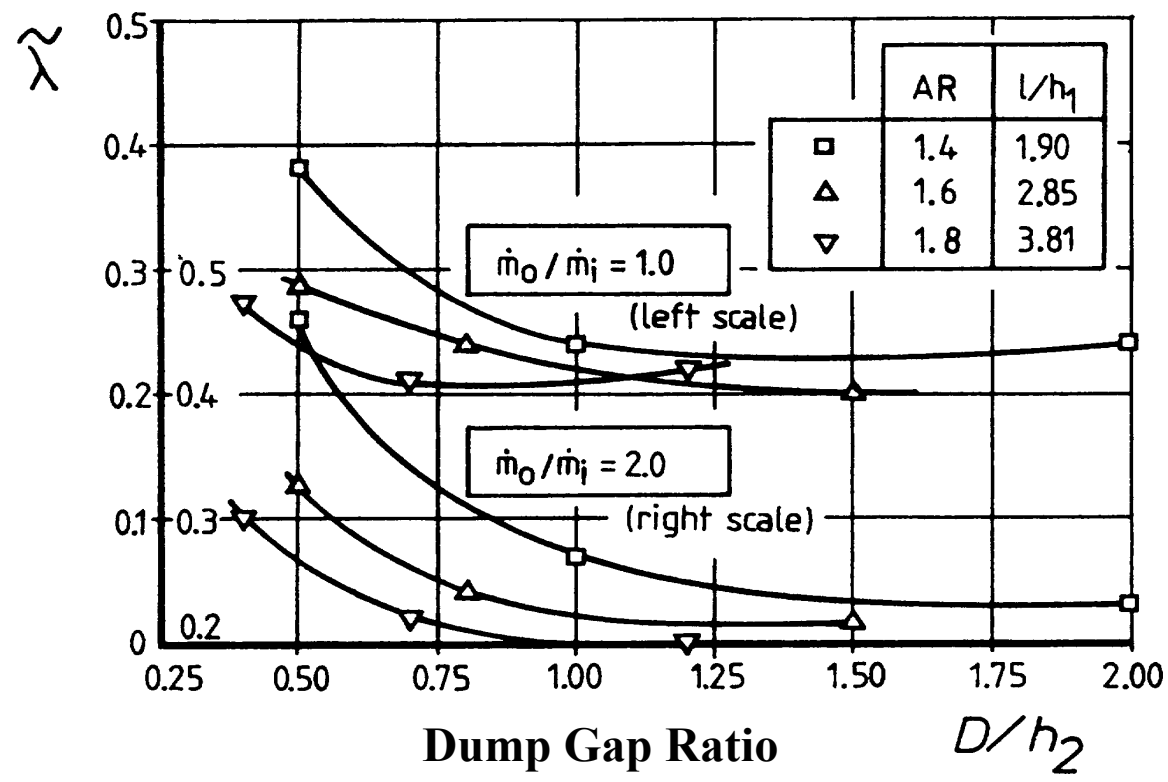

Figure 2.15 Influence of Dump Gap Ratio on Overall Pressure Loss Coefficient, Fishenden and Stevens ${ }^{[1977]}$ 


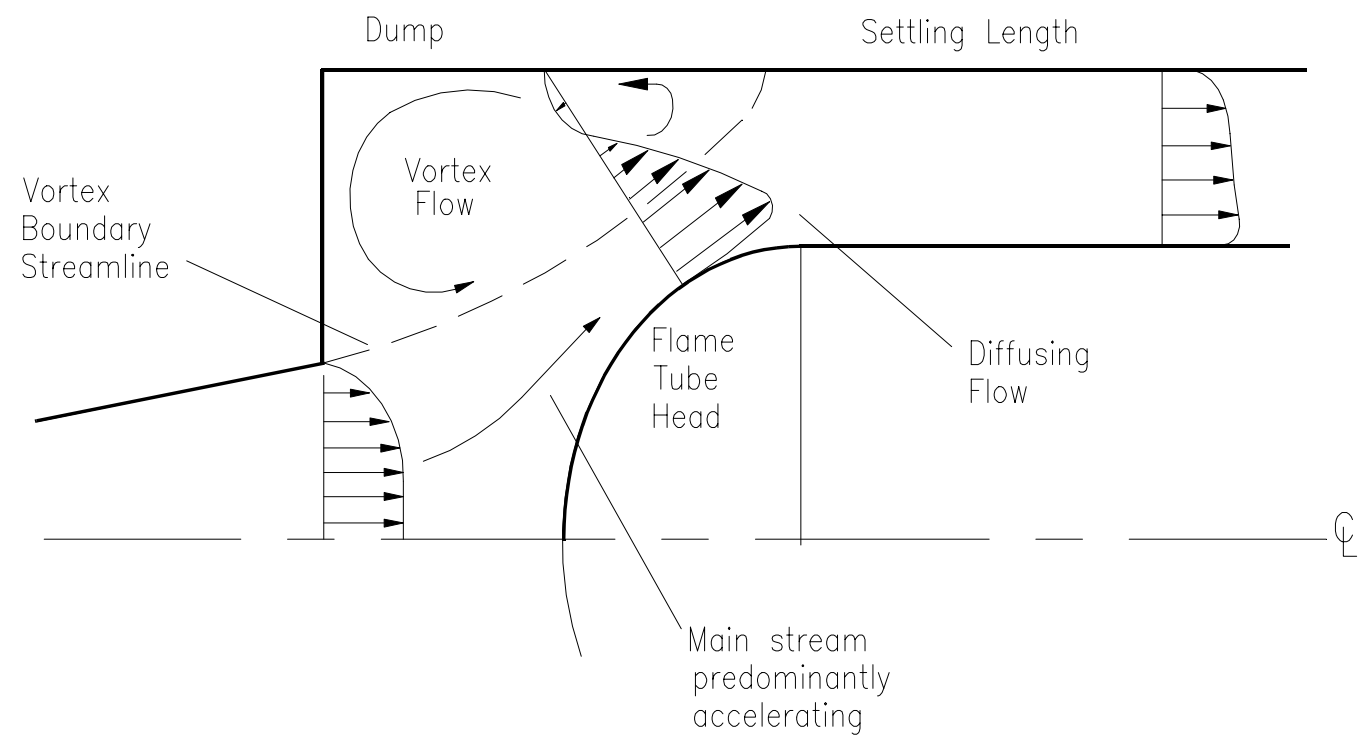

Figure 2.16 Flow Pattern in the Dump Region, Fishenden and Stevens ${ }^{[1977]}$

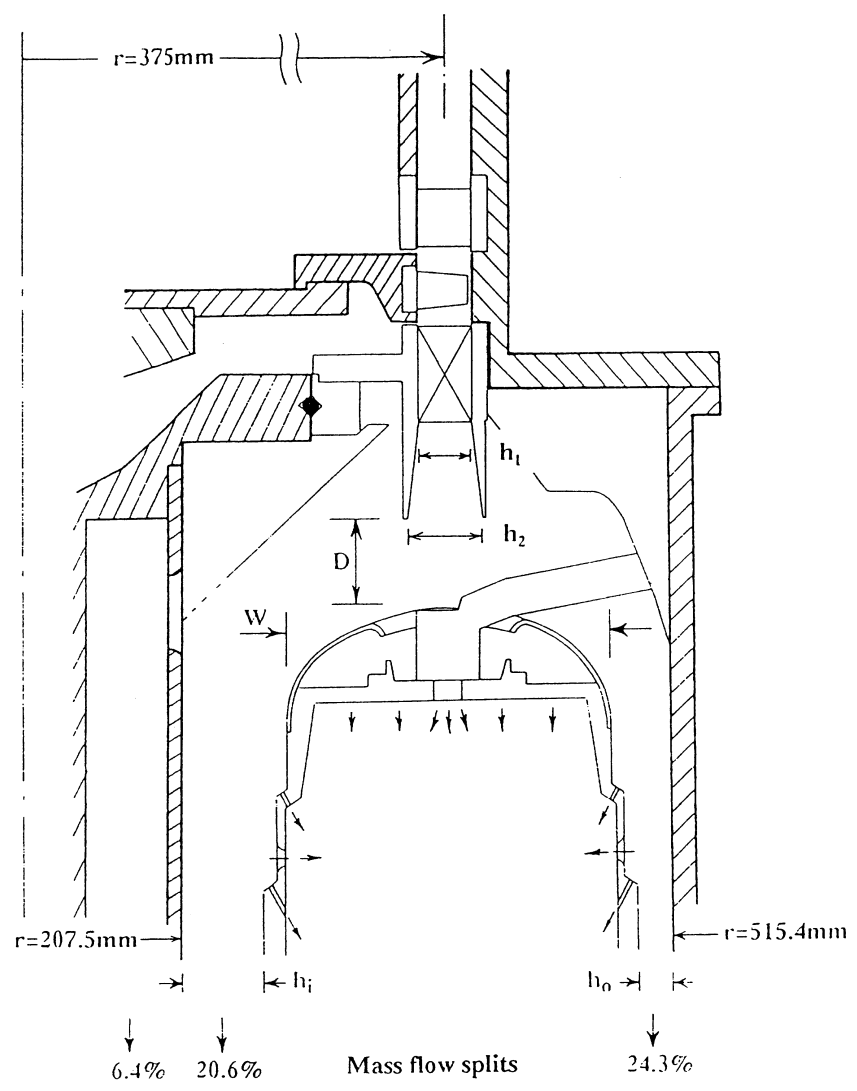

$$
\begin{array}{ll}
\text { Pre-diffuser inlet height }\left(h_{1}\right) & =36.6 \mathrm{~mm} \\
\text { Pre-diffuser exit height }\left(\mathrm{h}_{2} / h_{1}\right) & =1.35 \\
\text { Inner annulus height }\left(\mathrm{h}_{\mathrm{i}} / \mathrm{h}_{1}\right) & =1.41 \\
\text { Outer annulus height }\left(\mathrm{h}_{\mathrm{d}} / \mathrm{h}_{1}\right) & =0.67 \\
\text { Flame tube radial depth }\left(\mathrm{W} / \mathrm{h}_{1}\right) & =5.5
\end{array}
$$

Figure 2.17 Test Section Geometry, Carrotte and Bailey ${ }^{[1994]}$ 


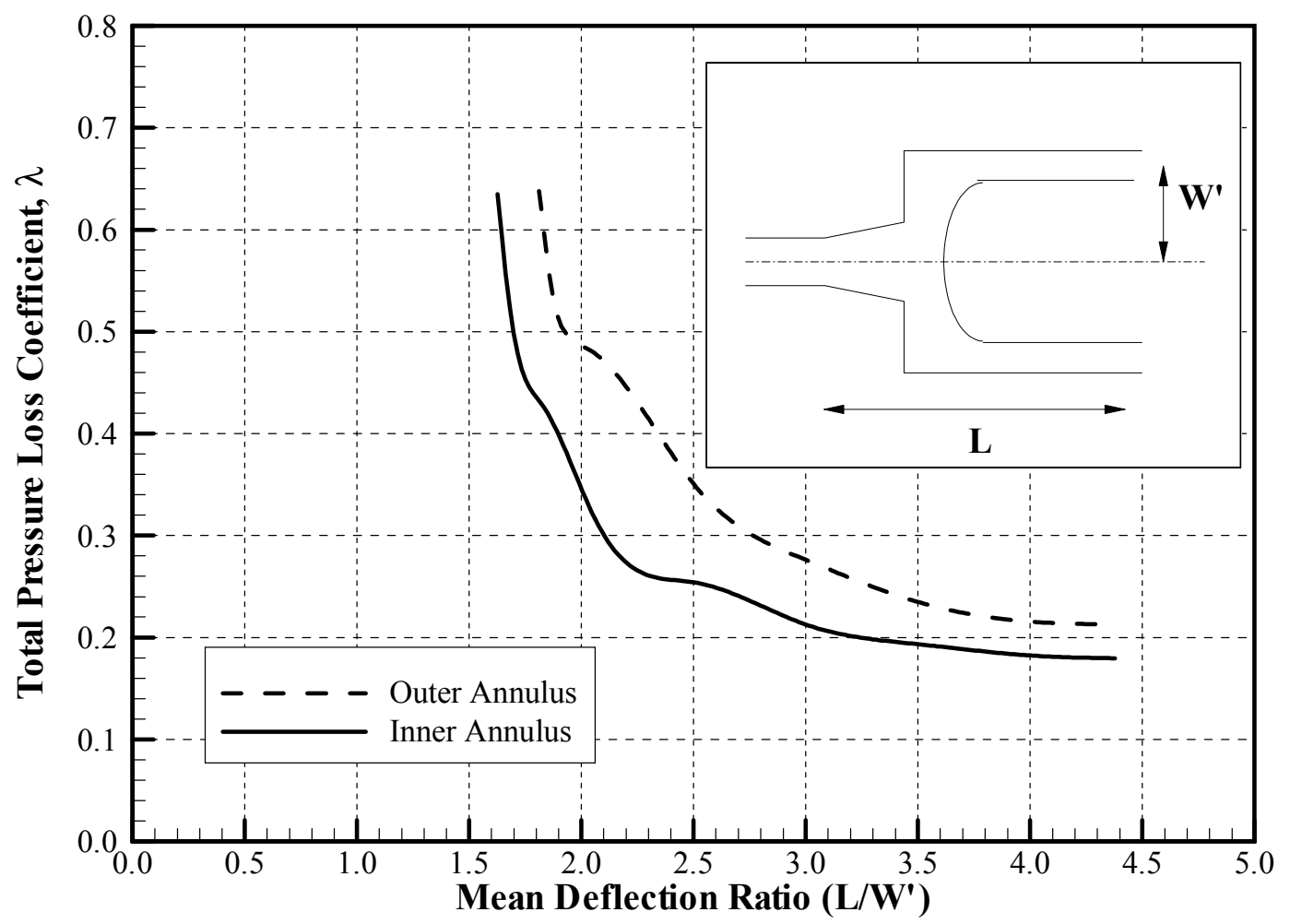

Figure 2.18 Variation of Pressure Loss to the Feed Annuli with Flame Tube Depth, Carrotte and Bailey ${ }^{[1994]}$

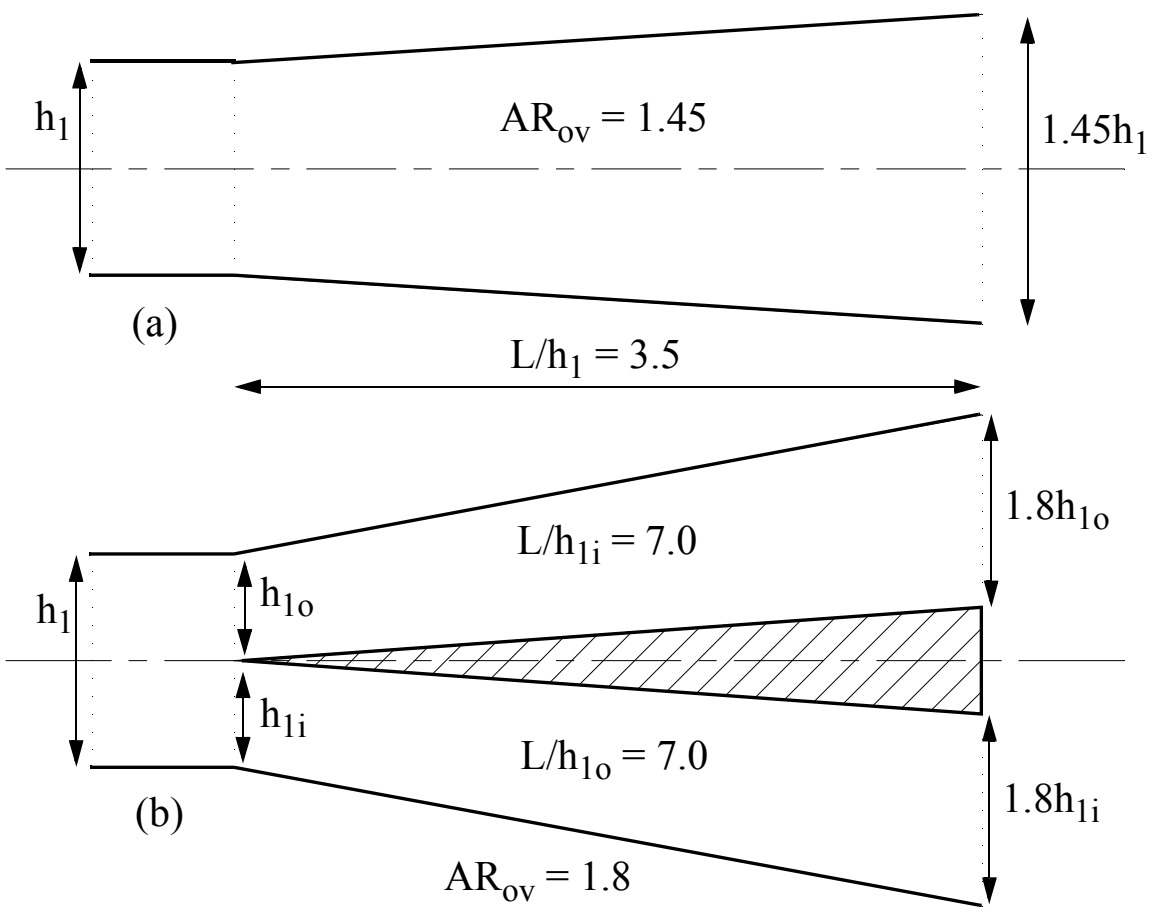

Figure 2.19 Principle of A Multi Passage Diffuser 


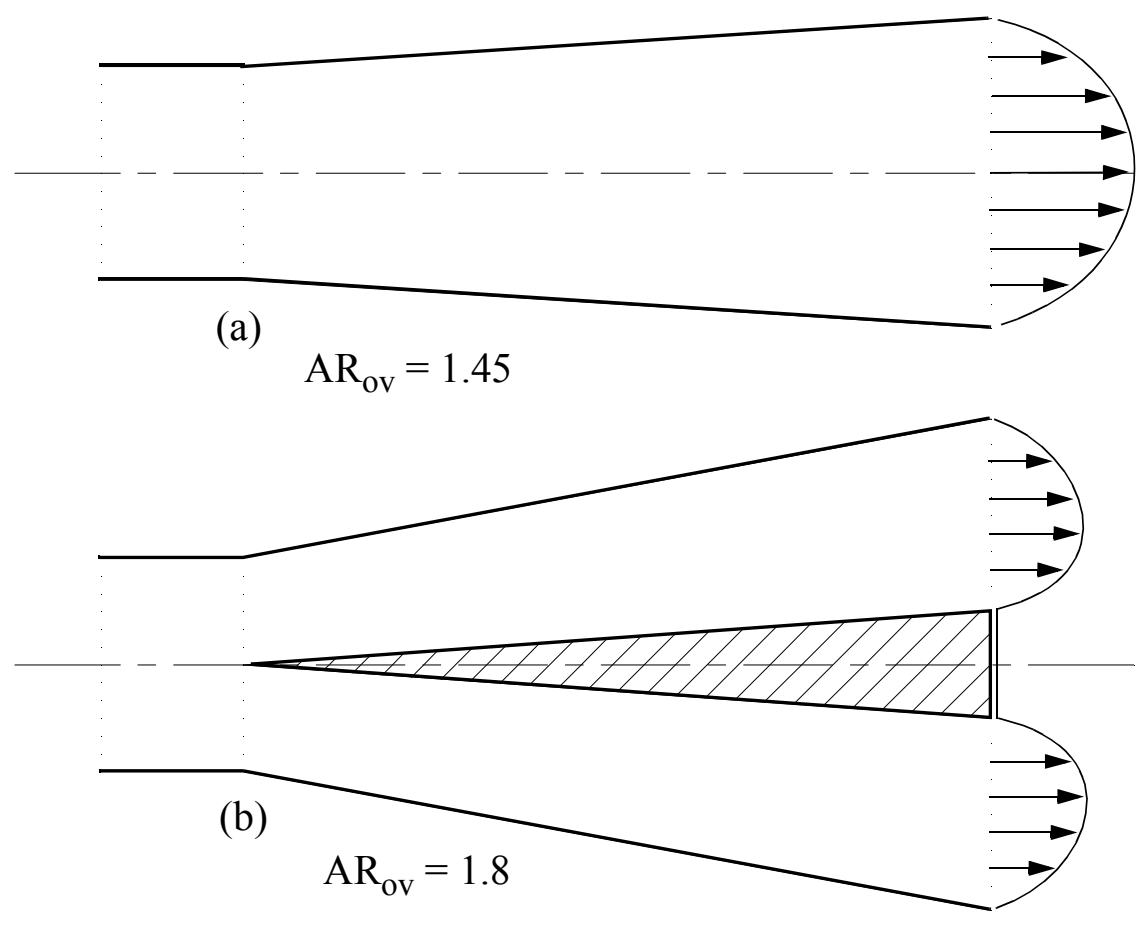

Figure 2.20 Exit Profile Distortion Caused by Splitter, Moore ${ }^{[1976]}$

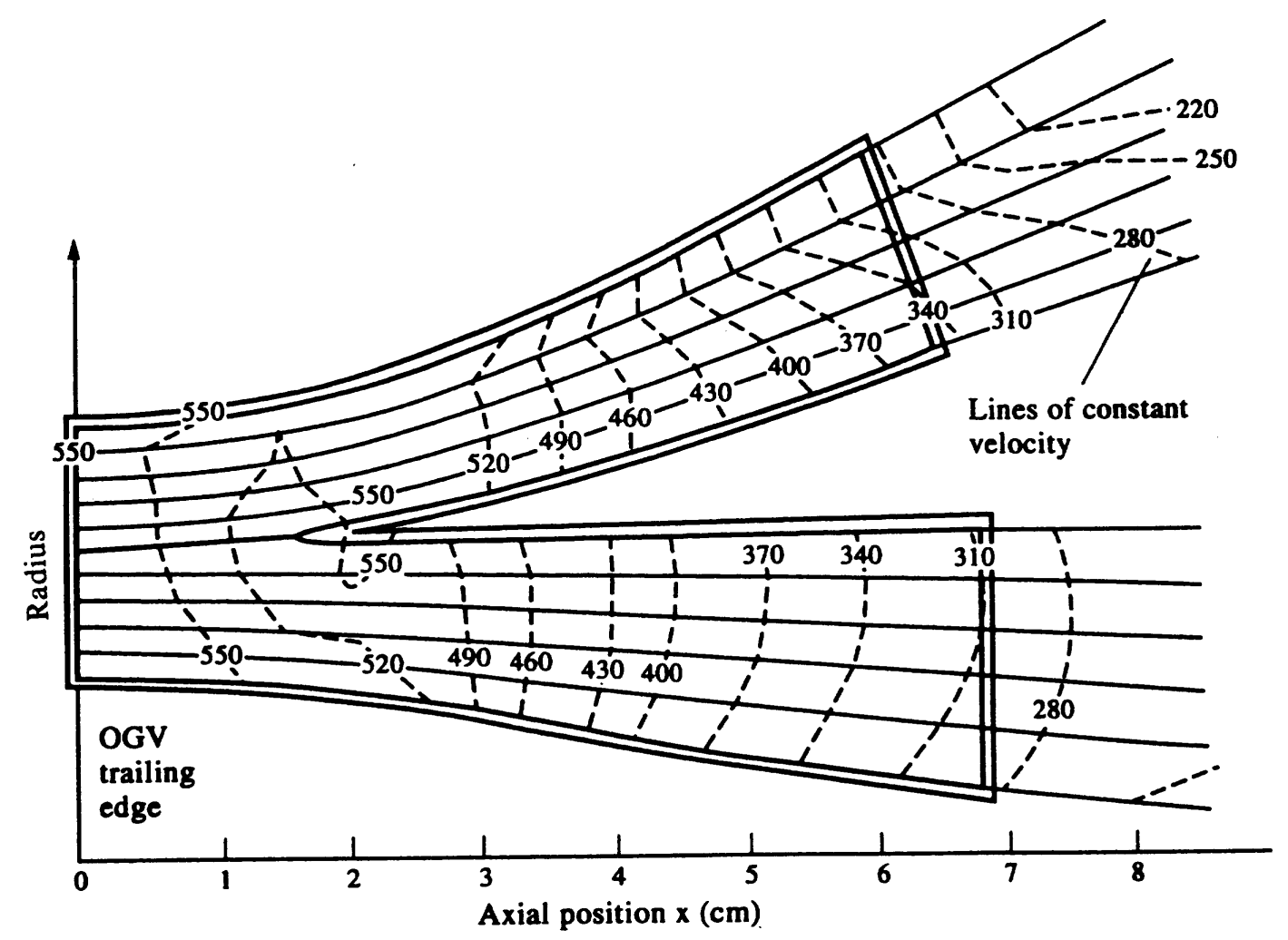

Figure 2.21 General Electric Twin Passage Diffuser, Salba et al ${ }^{[1982]}$ 


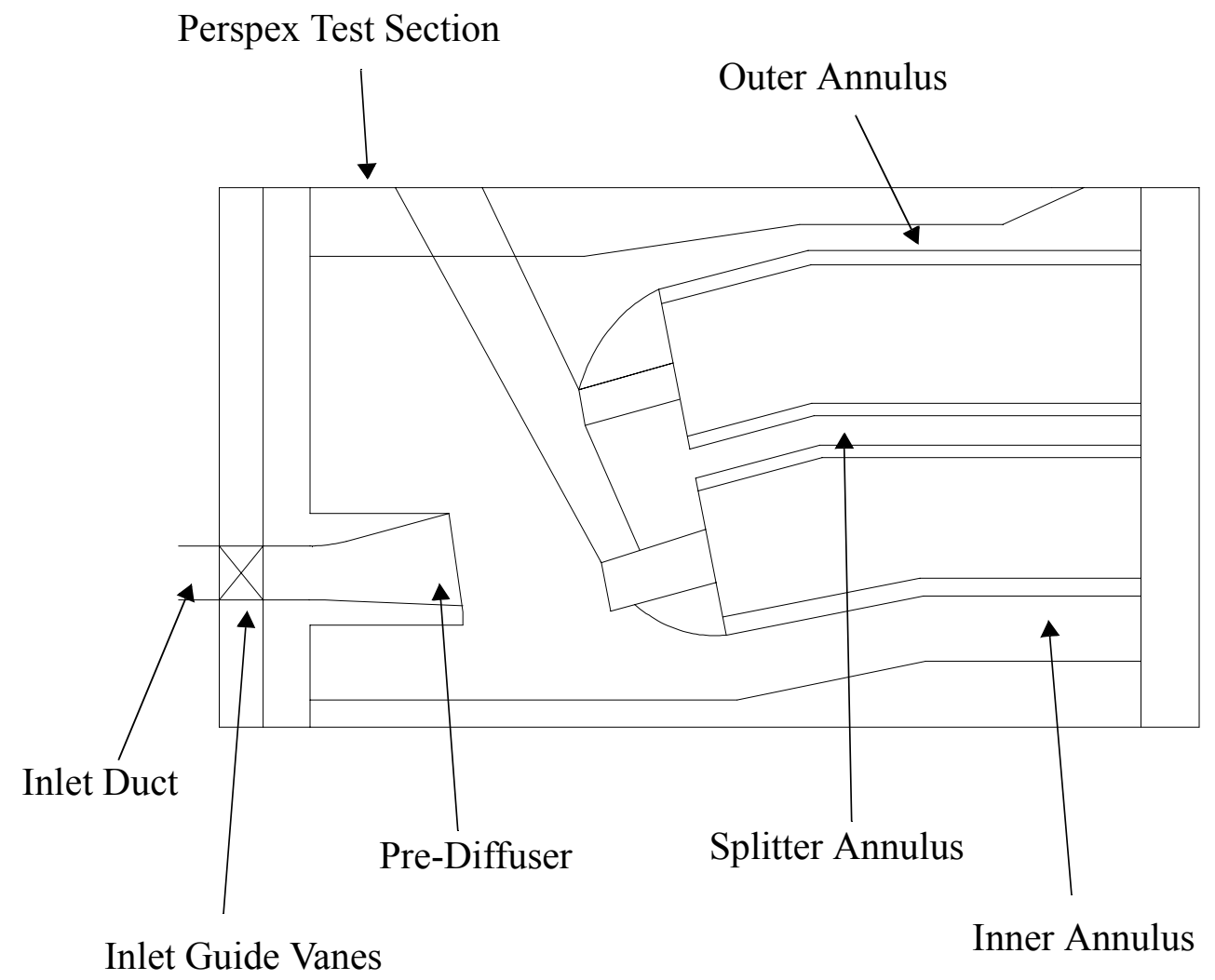

Figure 2.22 Cross Section of Double Annular Sector Rig, Shedden ${ }^{[1993]}$

$$
\mathrm{L} / \mathrm{h}_{1}=4.6, \beta=11.5^{\mathrm{o}}
$$

Conventional; AR 1.65

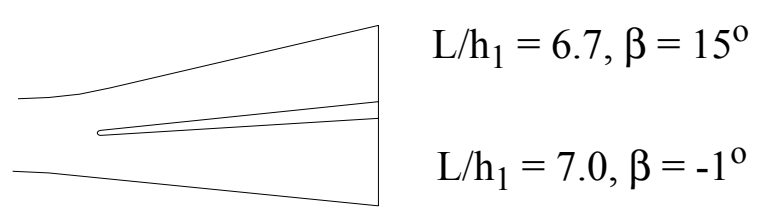

Twin-Passage Vane; AR 2.0

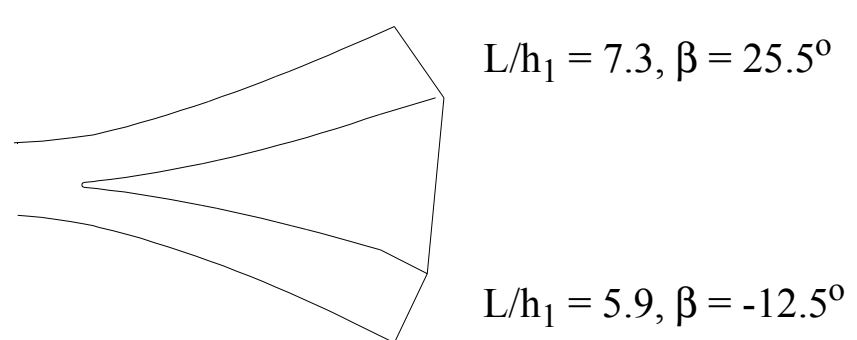

Twin-Passage Wedge; AR 2.0

Figure 2.23 Pre-Diffuser Configurations, Shedden ${ }^{[1993]}$ 


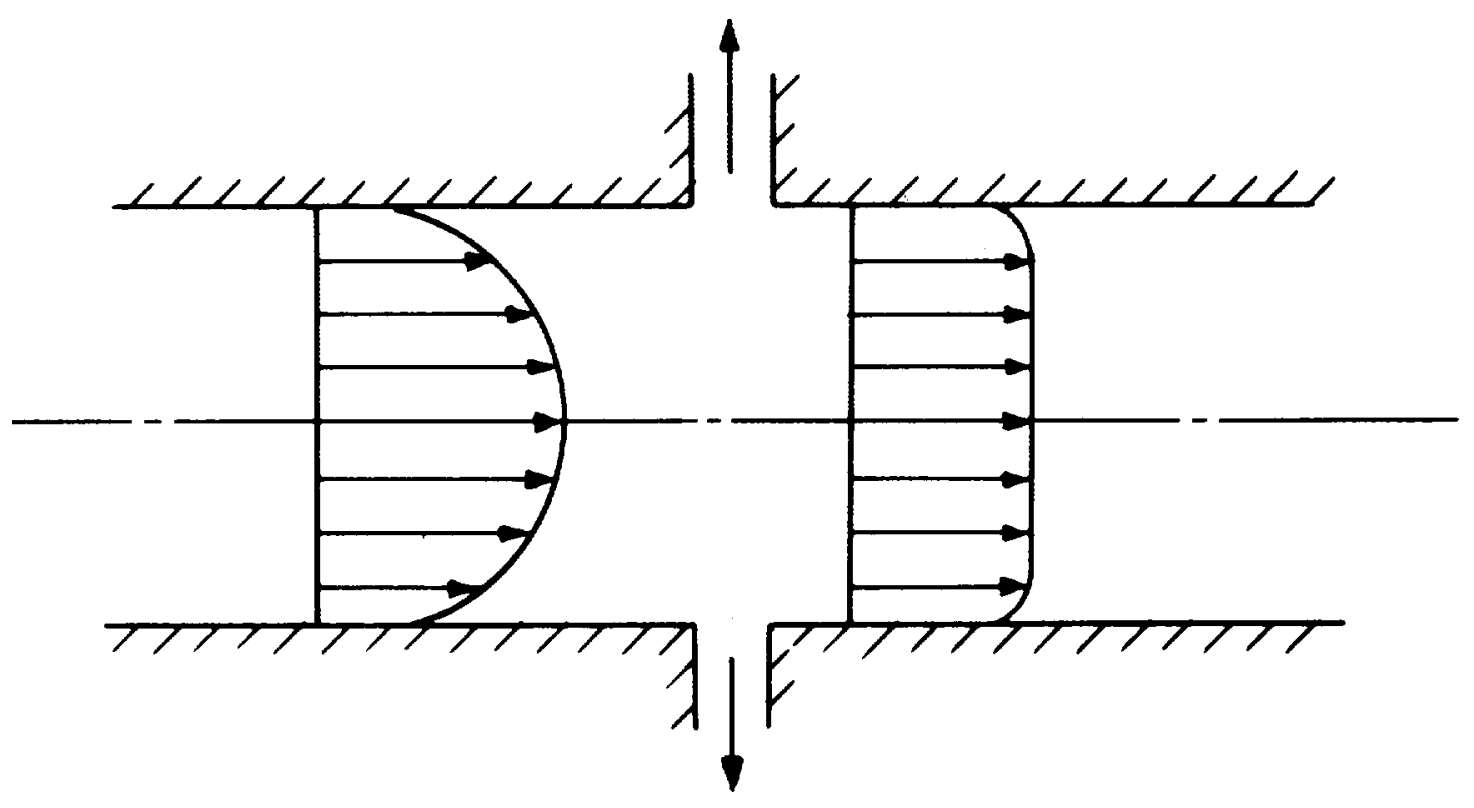

Figure 2.24 Boundary Layer Control by Suction

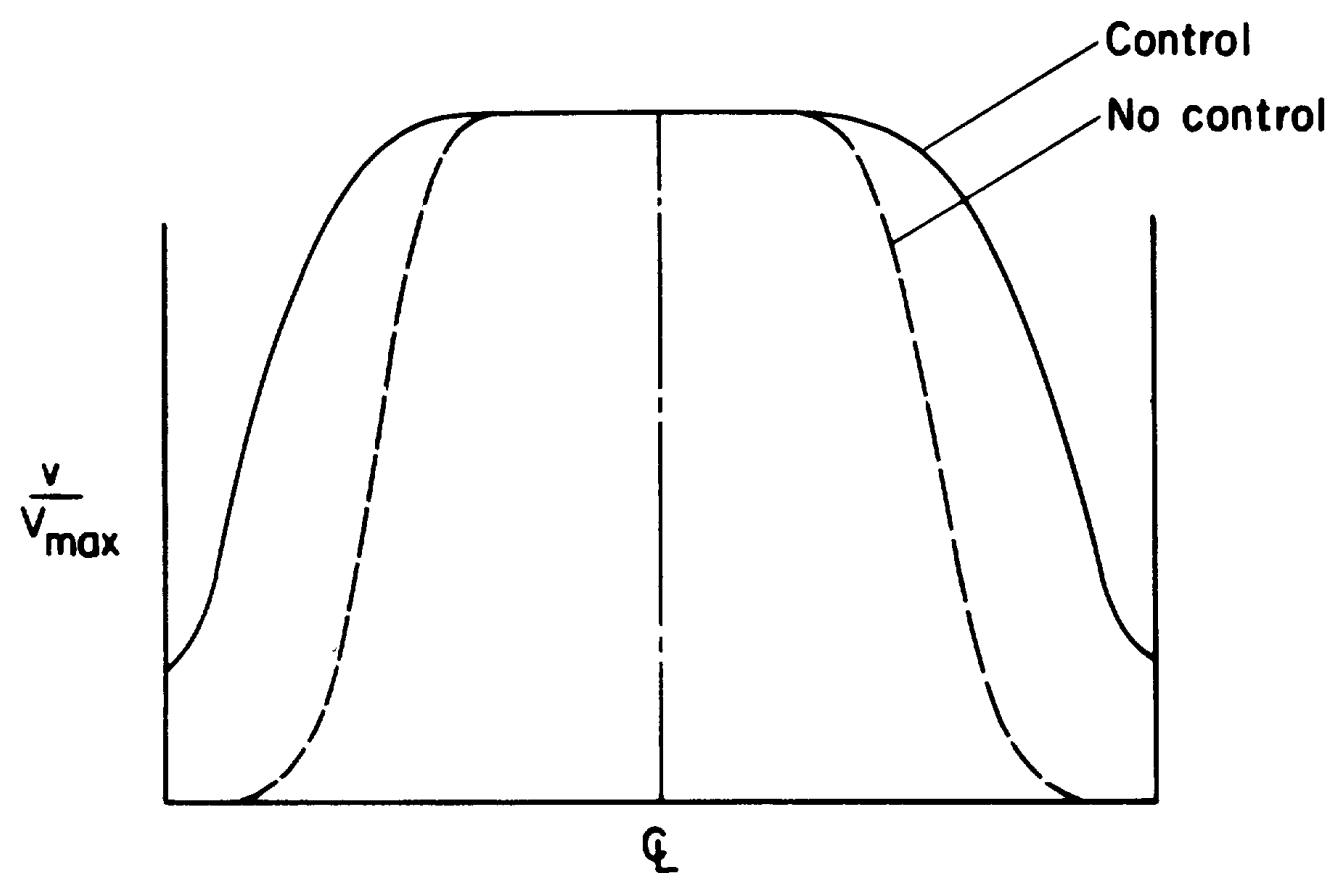

Figure 2.25 Effect of Suction Control on Diffuser Exit Velocity Profile for a 30 Conical Diffuser, Furuya et al ${ }^{[1966]}$ 

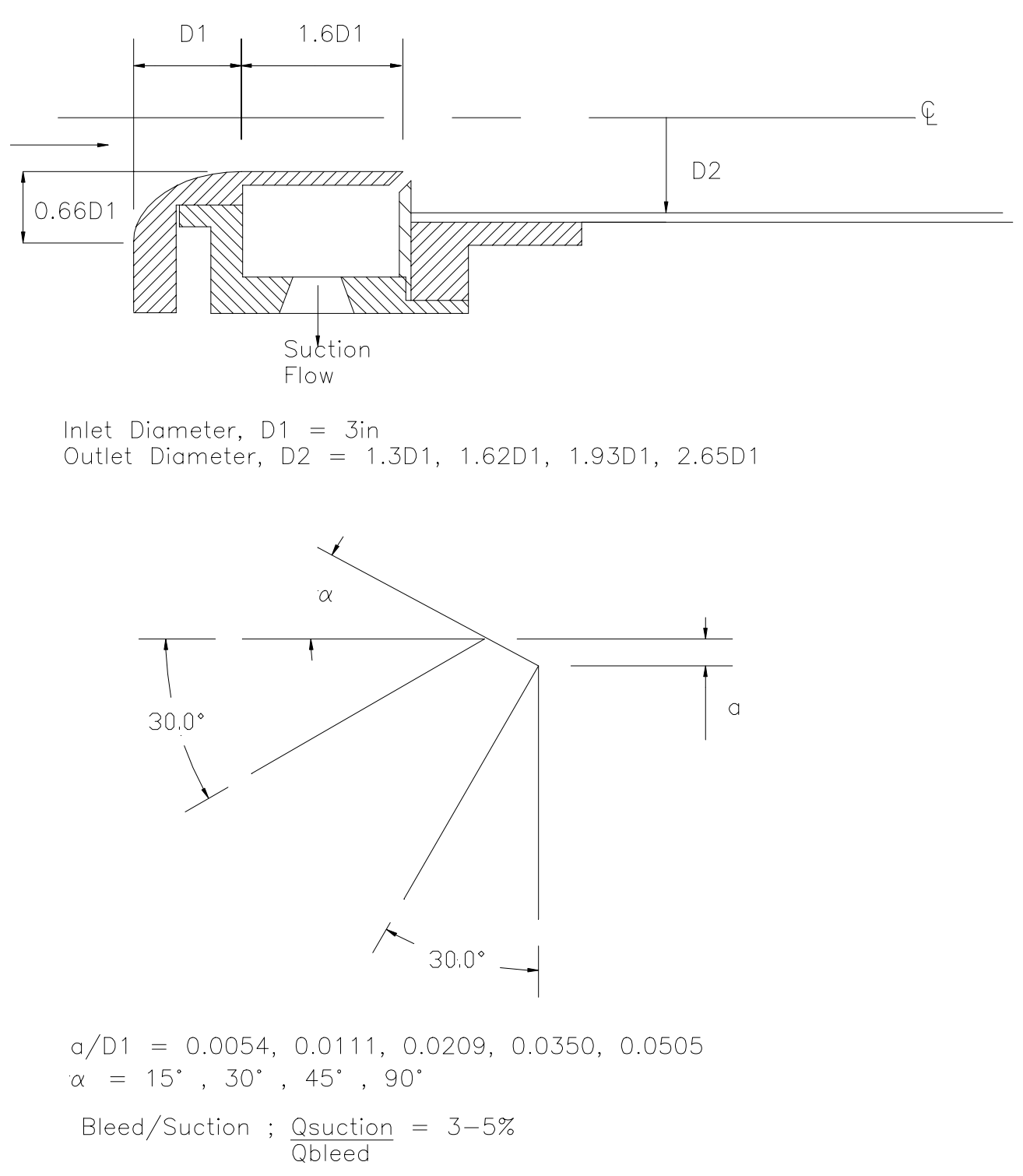

Figure 2.26 Heskestad ${ }^{[1968]}$ Bleed Gap Geometry

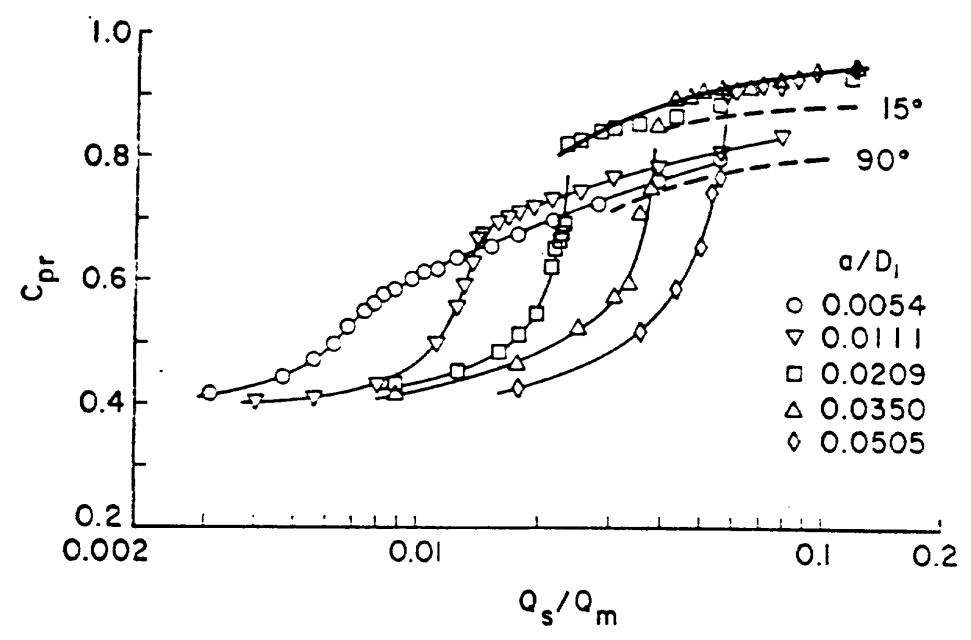

Figure 2.27 Effect of Suction on Pressure Recovery, Heskestad ${ }^{[1968]}$ 

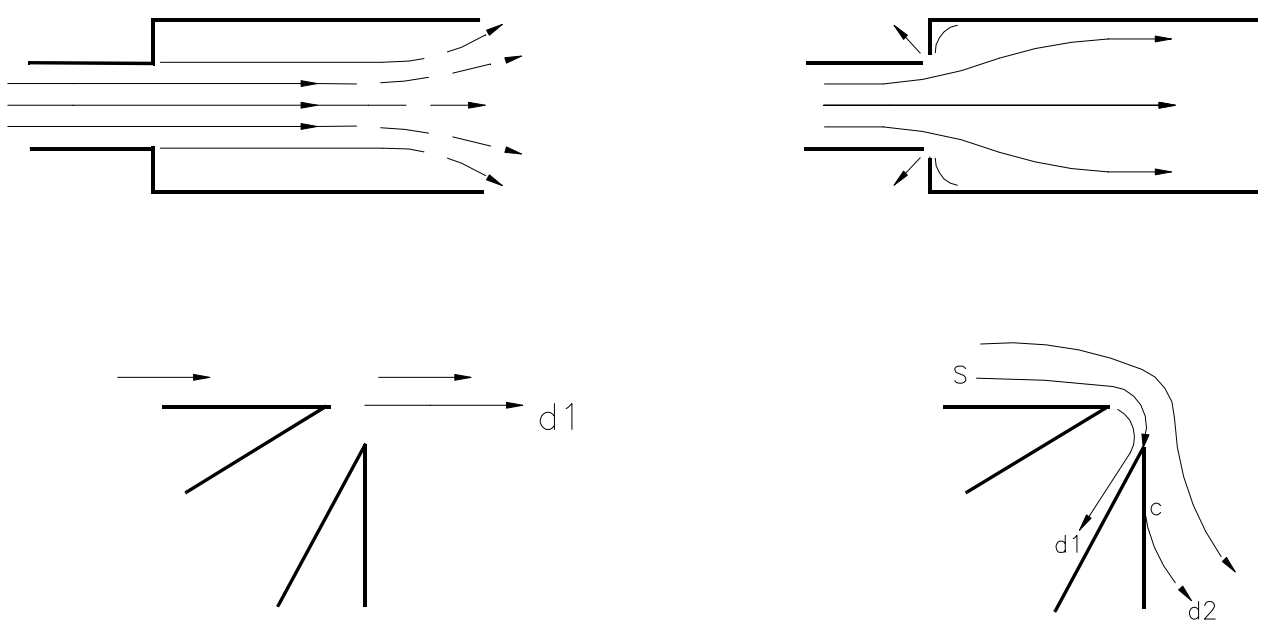

No Suction Flow

Figure 2.28 Suggested Flow in Suction Controlled Diffuser with No Suction and Suction Greater than Critical, Heskestad ${ }^{[1968]}$

Figure 2.29 Flow Over a Mountain Ridge, Ringleb ${ }^{[1955]}$ 


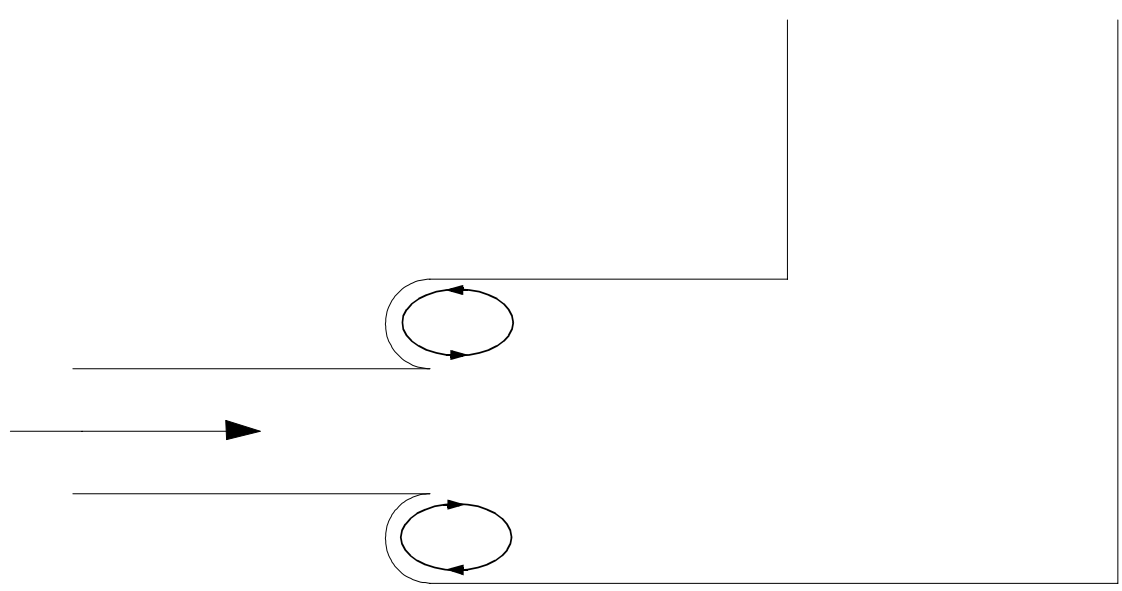

Figure 2.30 Ringleb's ${ }^{[1955]}$ Cusp Diffuser
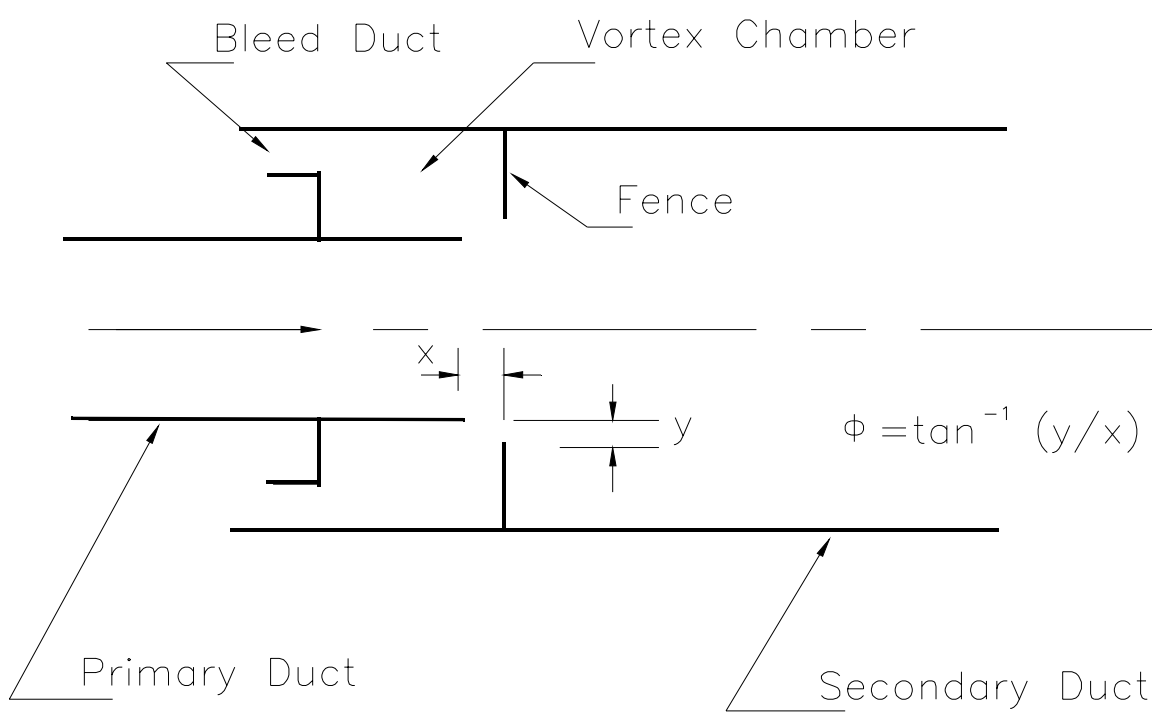

Figure 2.31 Vortex Controlled Diffuser, Adkins ${ }^{[1975]}$

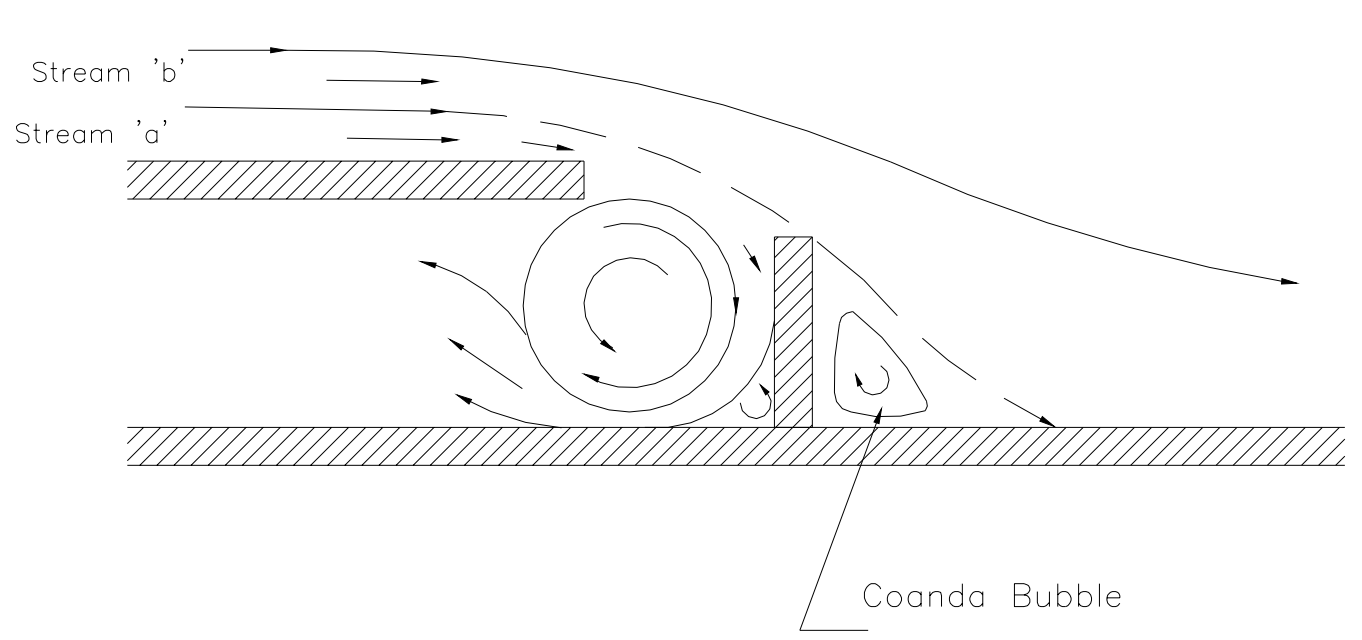

Figure 2.32 Suggested Flow Mechanism of Vortex Controlled Diffuser, Adkins ${ }^{[1975]}$ 


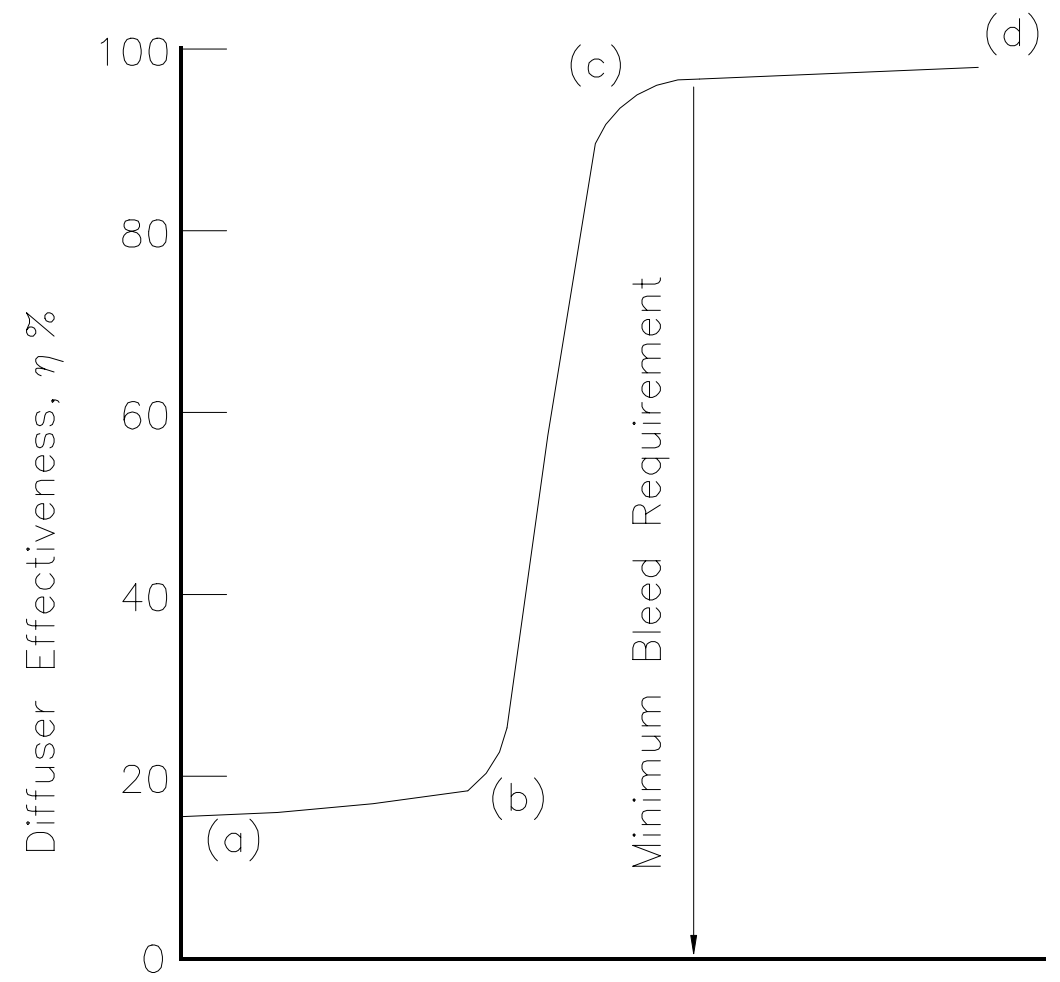

Percentage Bleed, B\%

Figure 2.33 Performance Characteristics of VCD, Adkins ${ }^{[1975]}$

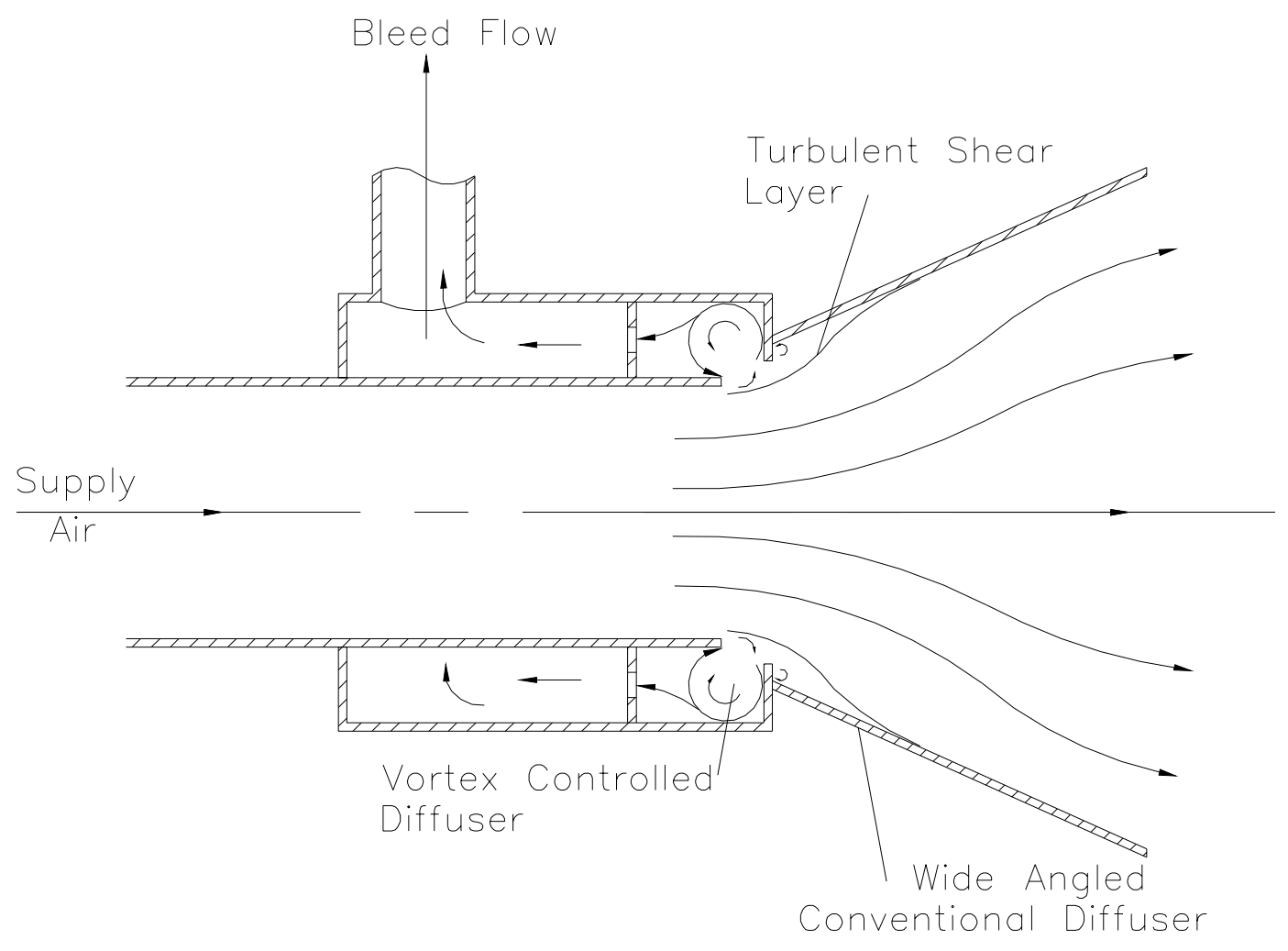

Figure 2.34 The Hybrid Vortex Controlled Diffuser, Adkins et al ${ }^{[1980]}$ 


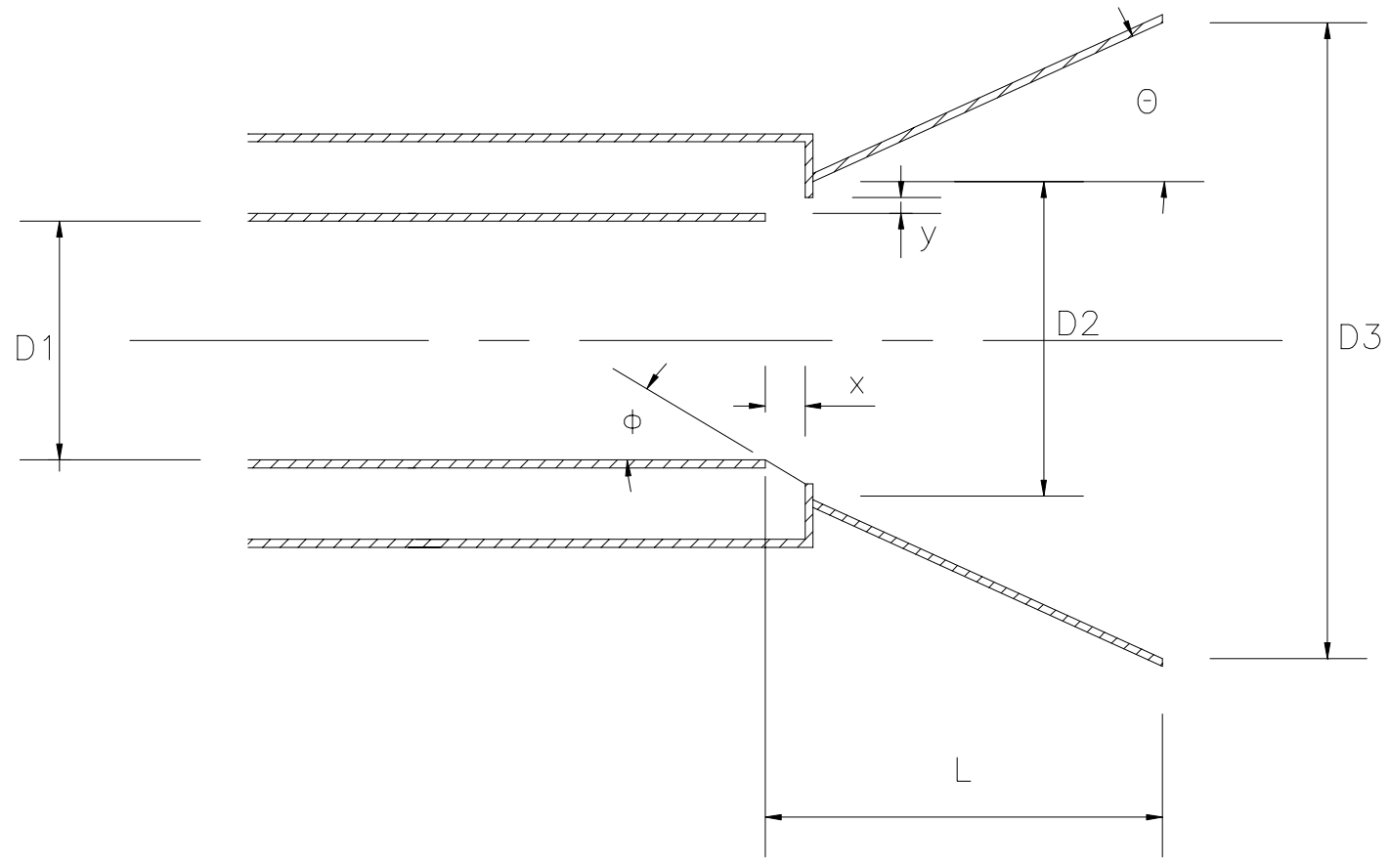

Figure 2.35 Hybrid Vortex Controlled Diffuser Nomenclature, Adkins et al ${ }^{[1980]}$

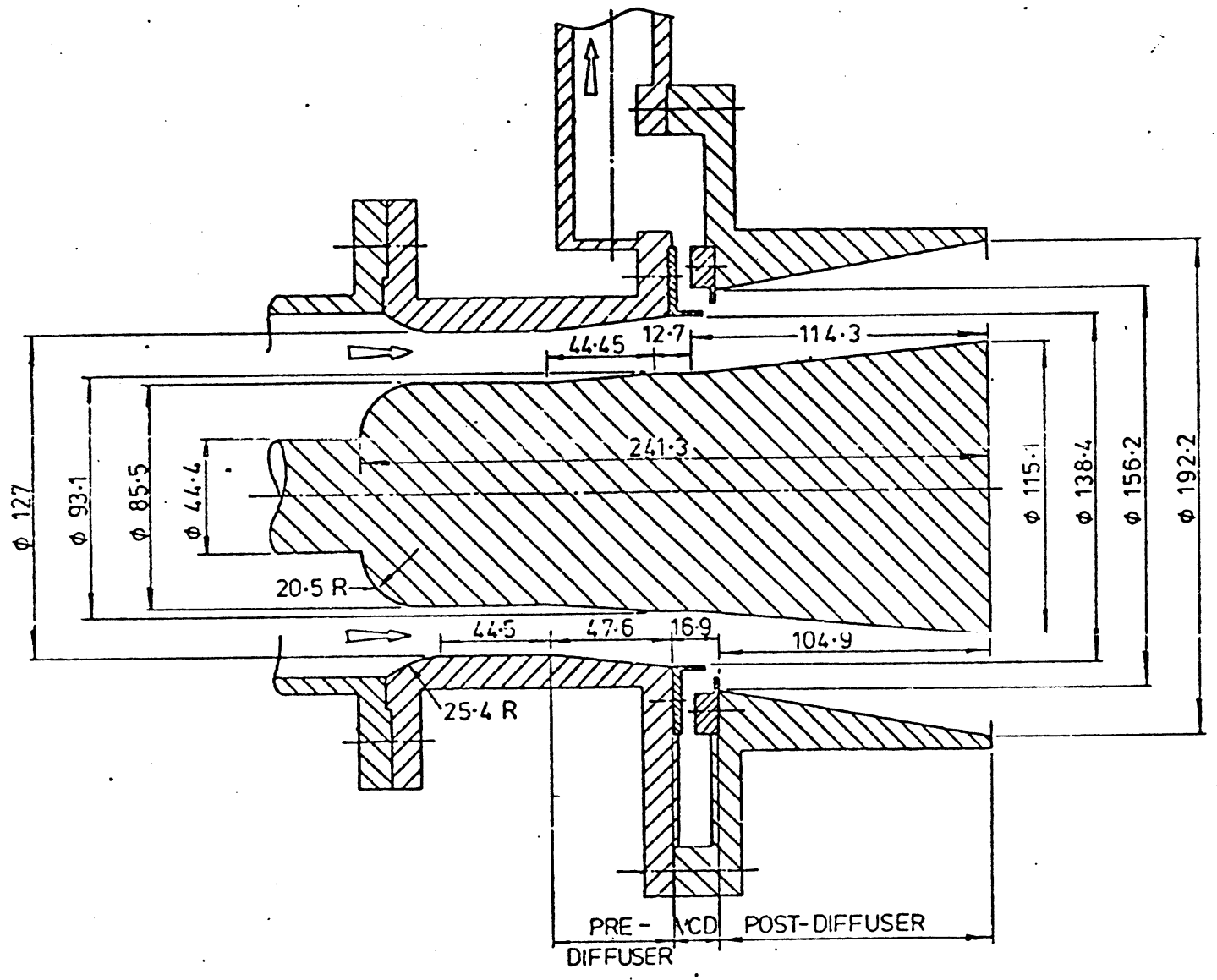

Figure 2.36 Combined Diffuser Arrangement, Adkins and Yost ${ }^{[1979]}$ 
$1 / 6$ SCALE MODEL TESTS

FENCE RADLAL GAP, $y=2.54 \mathrm{~mm}$
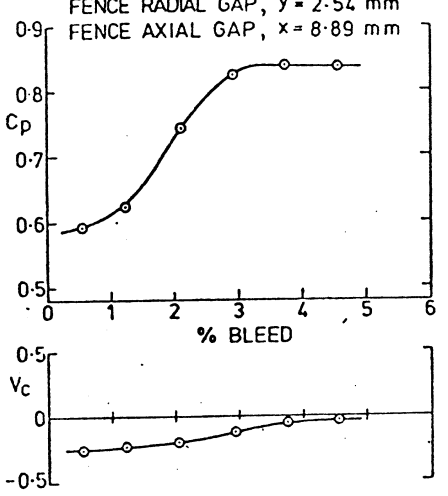

$1 / 6$ SCALE MODEL TESTS

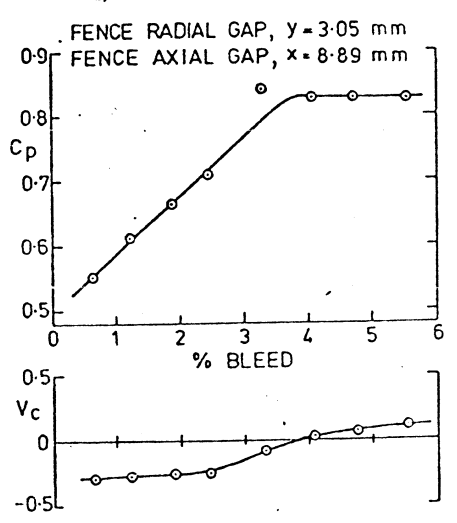

$1 / 6 \quad$ SC.ALE MGOEL TESTS

FENCE RADLLL GAP, $y=2.56 \mathrm{~mm}$
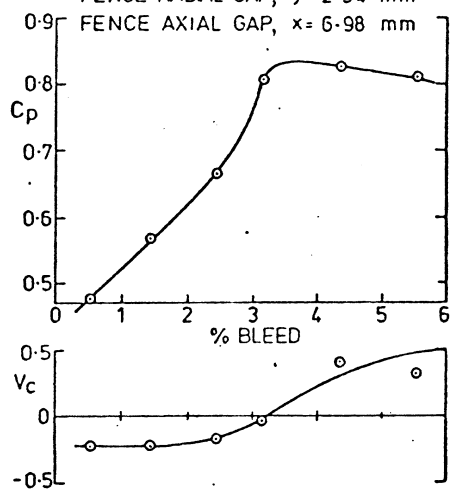

Figure 2.37 Effect of Bleed Gap on Pressure Recovery, Adkins and Yost ${ }^{[1979]}$

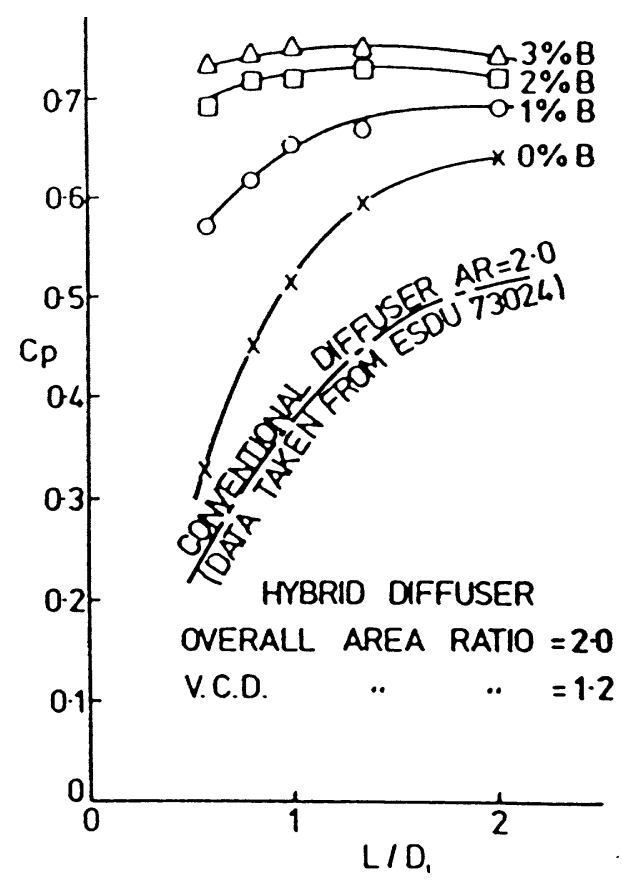

(a)

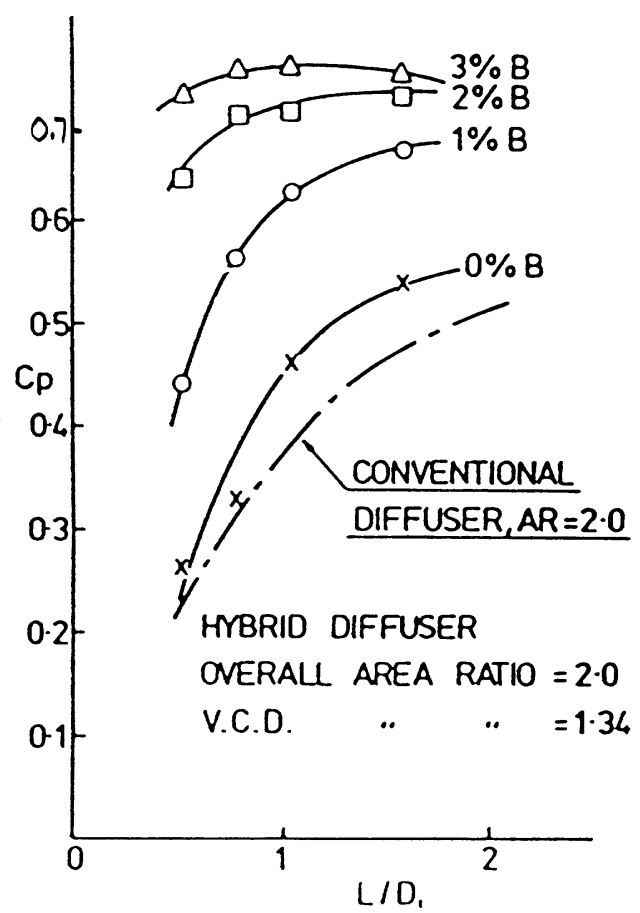

(b)

Figure 2.38 HVCD Pressure Recovery, Adkins et al ${ }^{[1980]}$ 


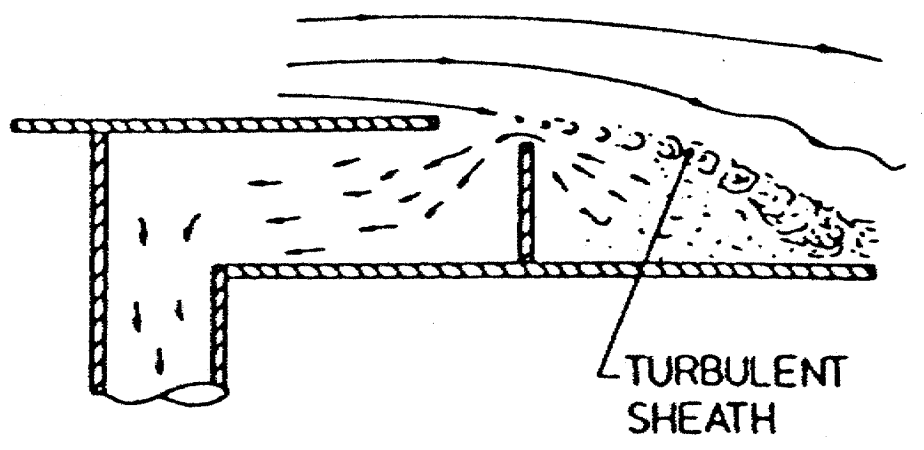

Low Bleed

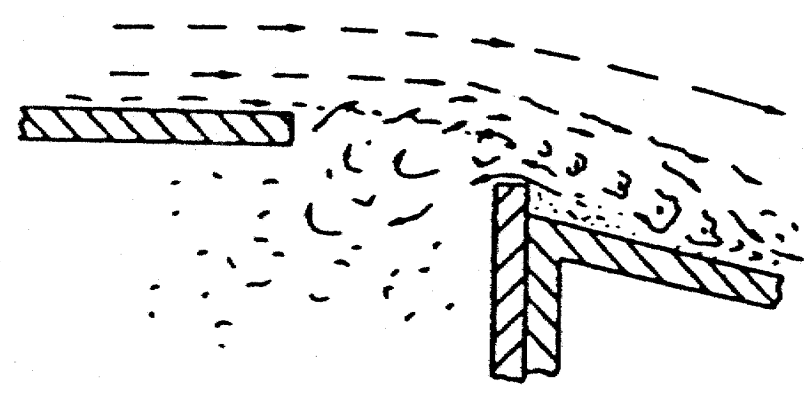

Zero Bleed

Figure 2.39 Vortex Controlled Diffusers at Low and Zero Bleed, Adkins et al ${ }^{[1980]}$

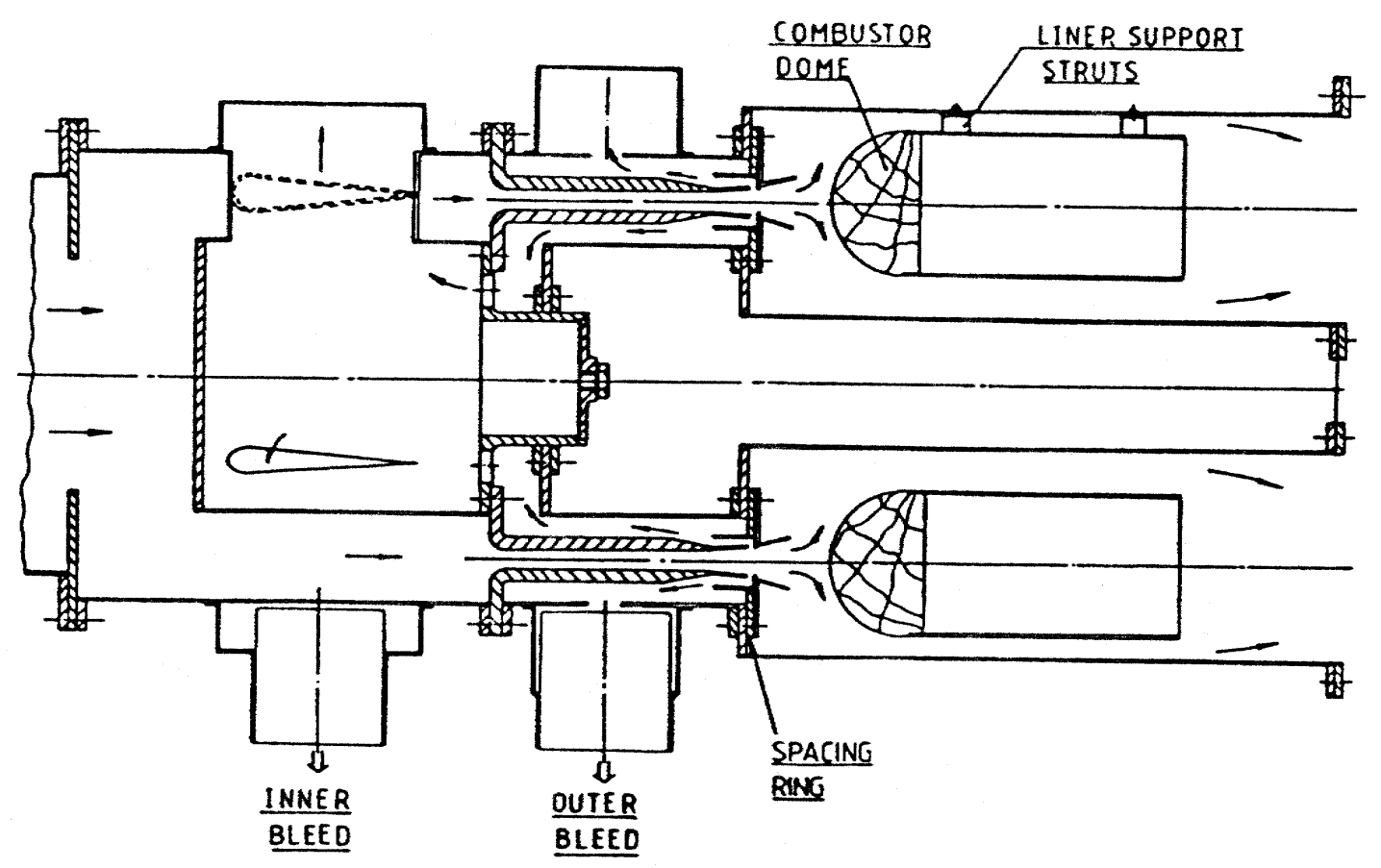

Figure 2.40 A Compact Diffuser System for Annular Combustors, Adkins and Yost $^{[1983]}$ 


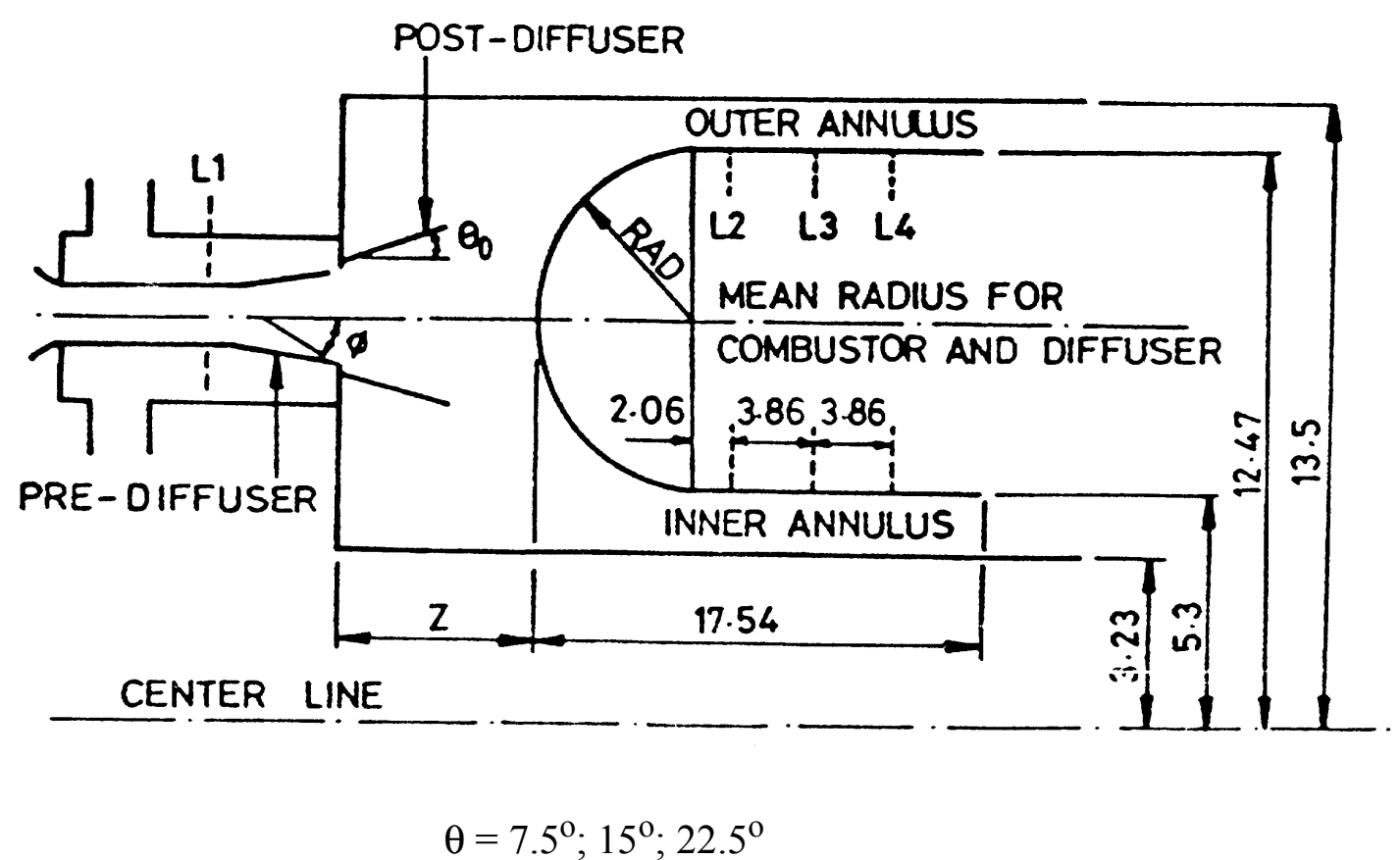

Note: all dimensions are divided by annulus height at inlet

Figure 2.41 Leading Dimensions for Figure 2.40, Adkins and Yost ${ }^{[1983]}$

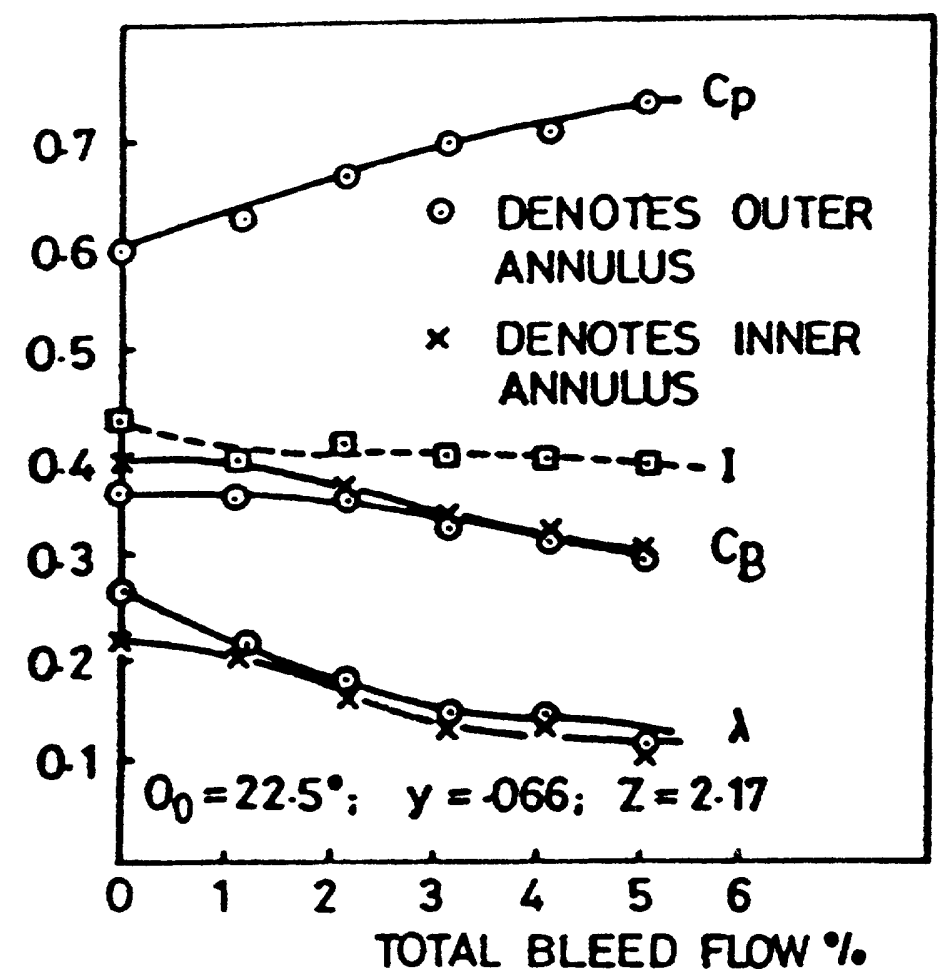

Figure 2.42 HVCD Performance, Adkins and Yost ${ }^{[1983]}$ 


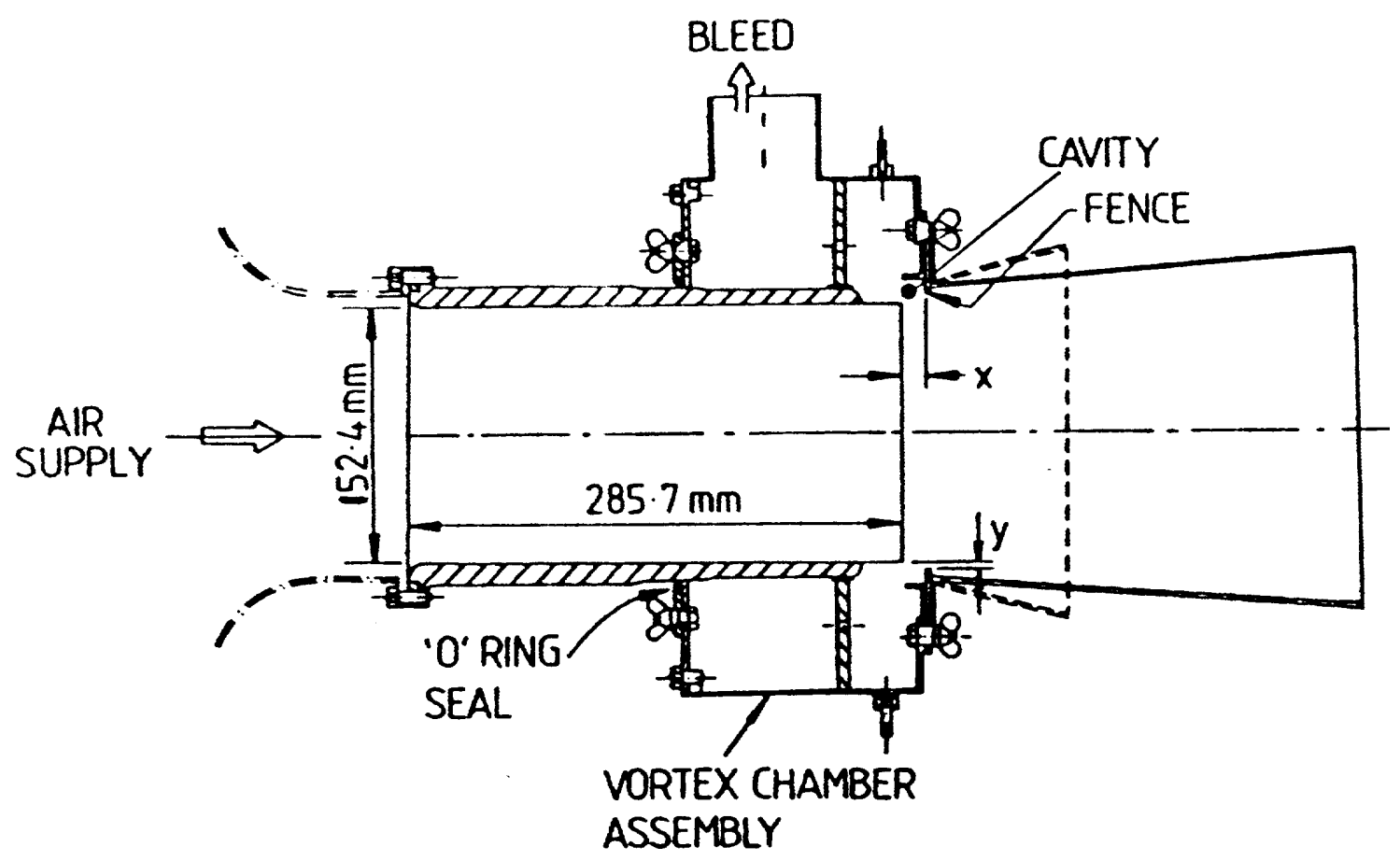

Figure 2.43 Test Arrangement, Adkins and Montazerin ${ }^{[1985]}$

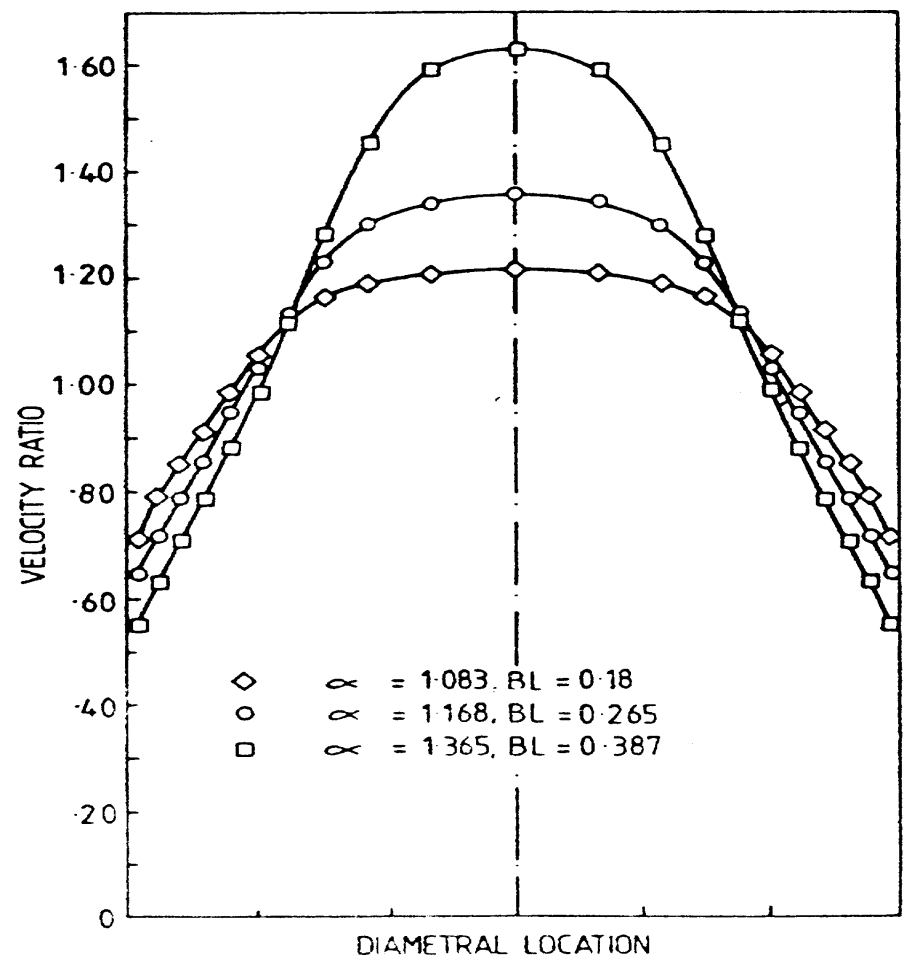

Figure 2.44 Inlet Velocity Profiles, Adkins and Montazerin ${ }^{[1985]}$ 

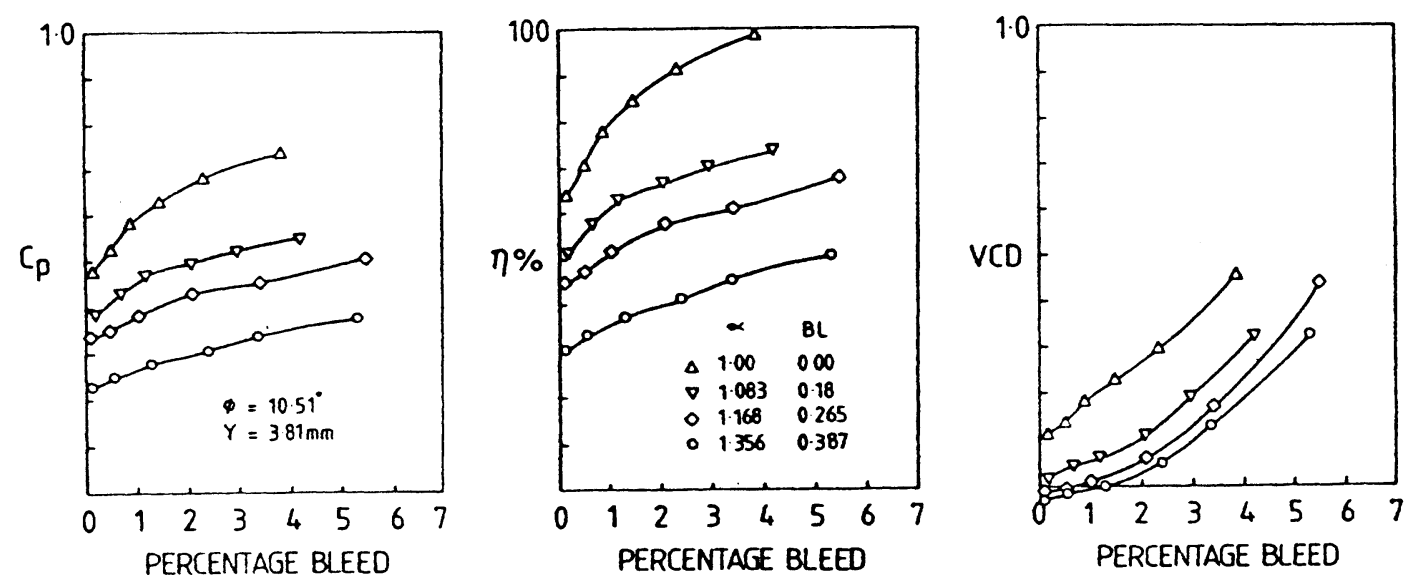

Figure 2.45 Effect of Inlet Distortion, Adkins and Montazerin ${ }^{\text {[1985] }}$

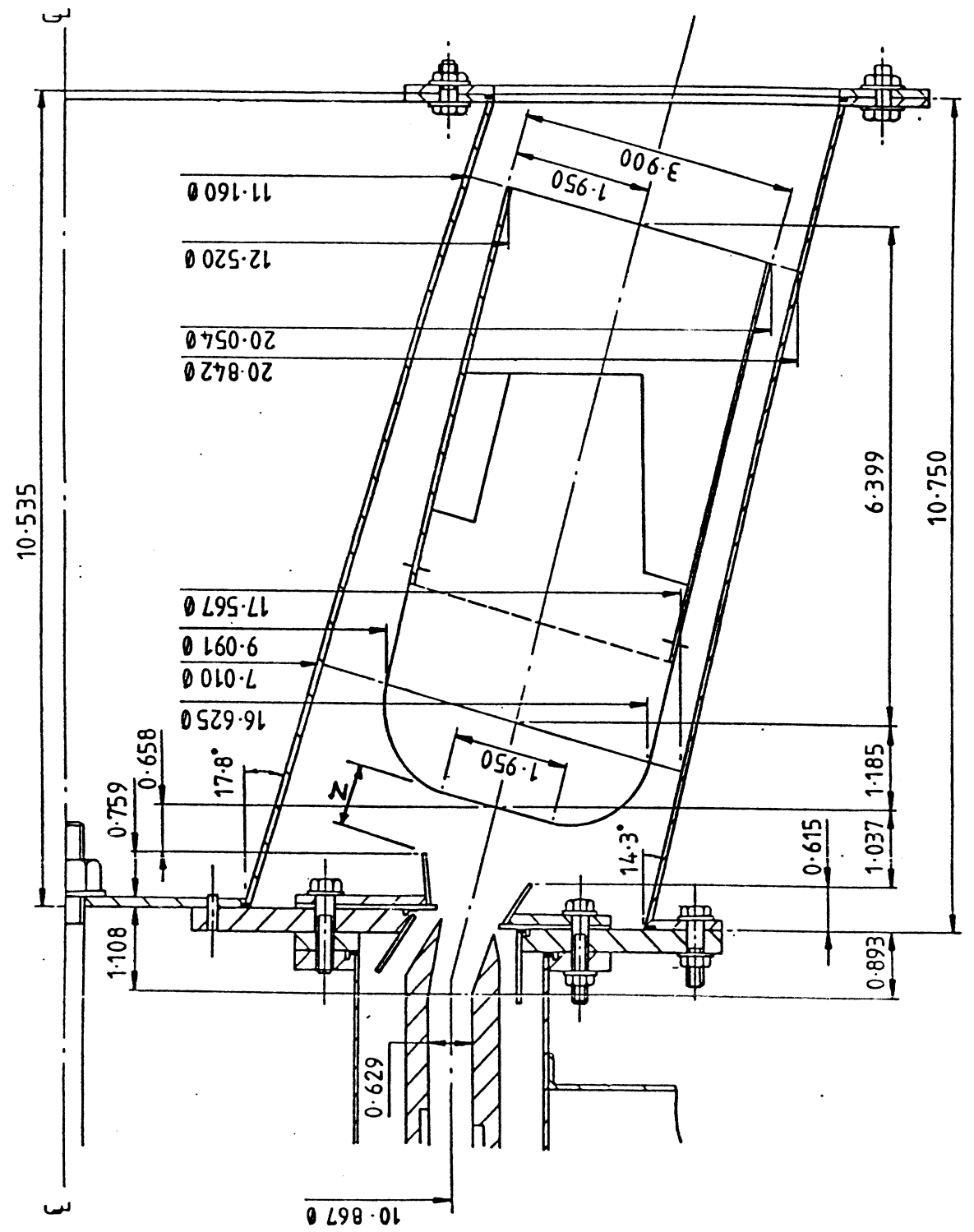

Figure 2.46 Canted Diffuser Arrangement, Adkins and Kuile ${ }^{[1985]}$ 
TESTS CONDUCTED WITH A CONSTANT OUTER BLEED EQUAL TO

$4 \%$ OF TOTAL MASS FLOW AT DAFFUSER INLET (BUILD 1)

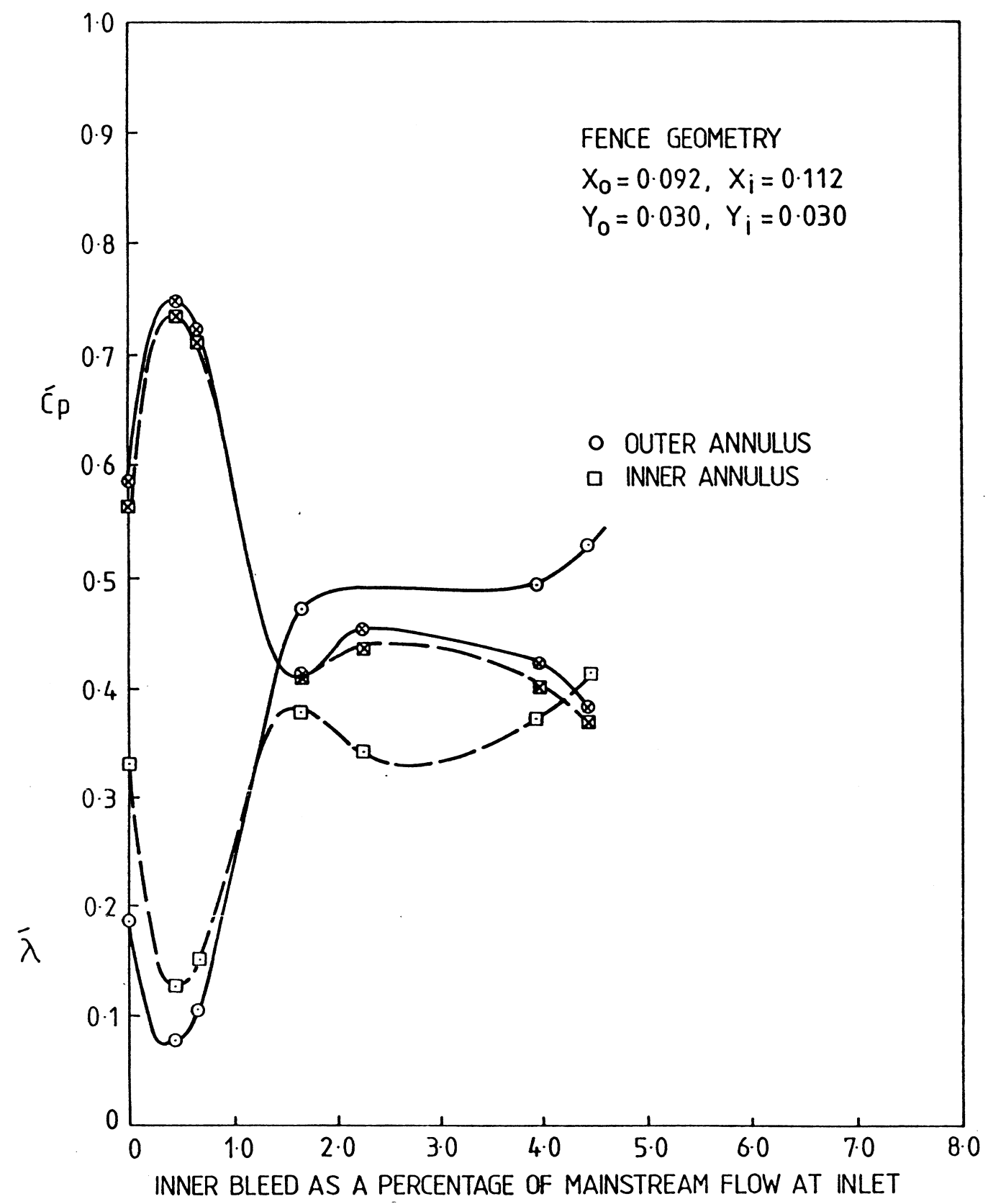

Figure 2.47 Diffuser Performance with Unbalanced Bleed, Adkins and Kuile ${ }^{[1985]}$ 
ANNULAR MASS FLOW RATIO VERSUS INNER BLEED FOR BUILD TWO TEST CONDITIONS : MACH. No. 0.35, OUTER BLEED $5 \%$

(ANNULAR AREA RATIO $A_{0} / A_{i}=1.237$ )

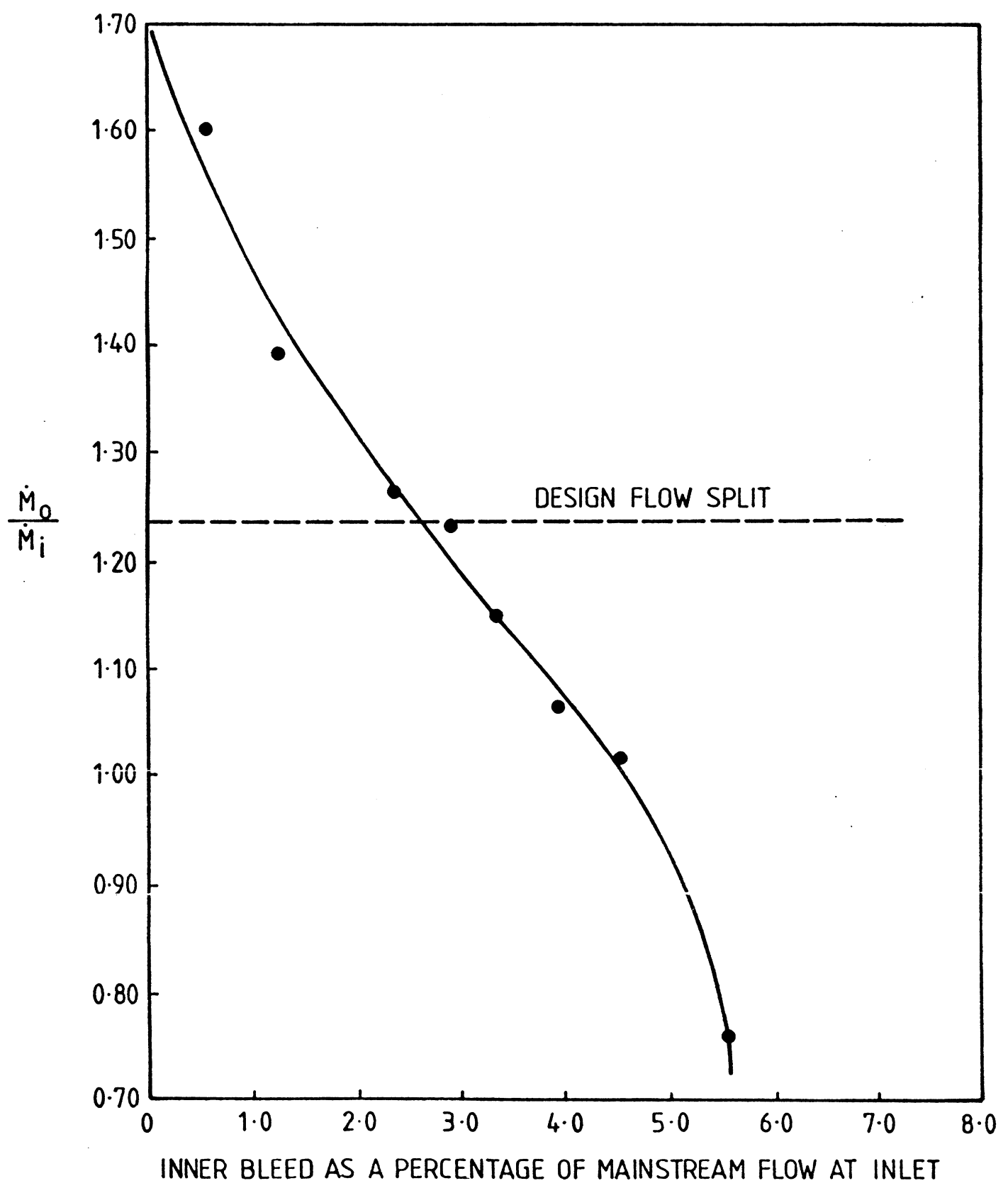

Figure 2.48 Effect of Bleed Imbalance of Annuli Mass Flow, Adkins and Kuile ${ }^{[1985]}$ 


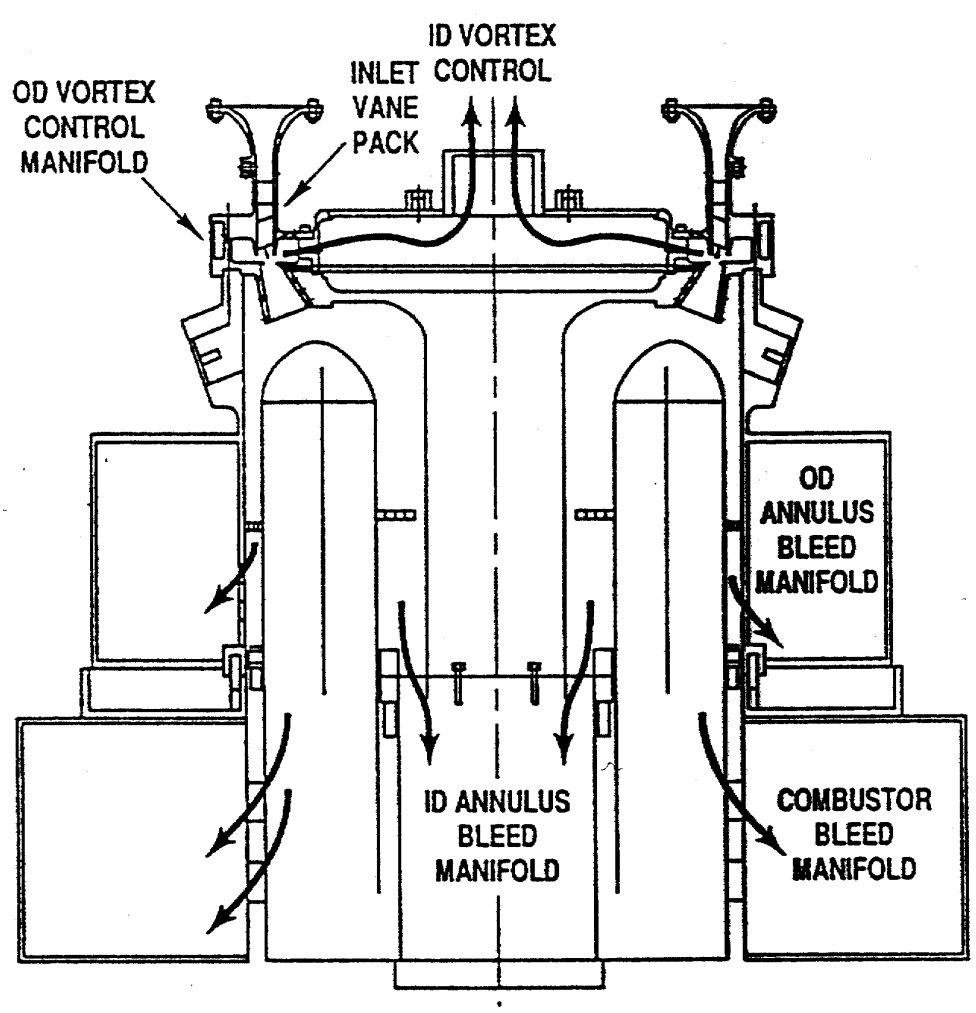

Figure 2.49 Myres et al ${ }^{[1993]}$ Test Facility

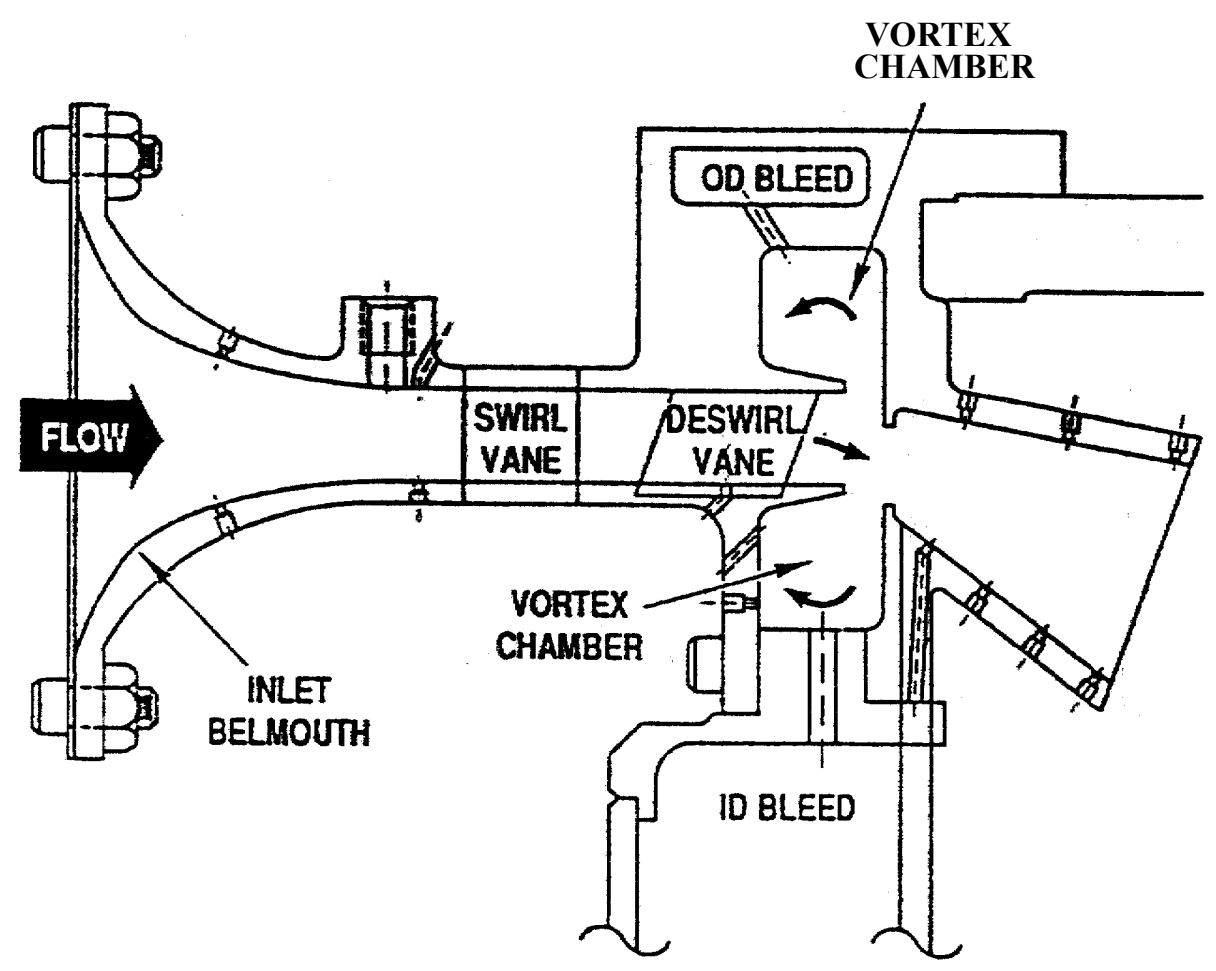

Figure 2.50 HVCD of Myres et al ${ }^{[1993]}$ 


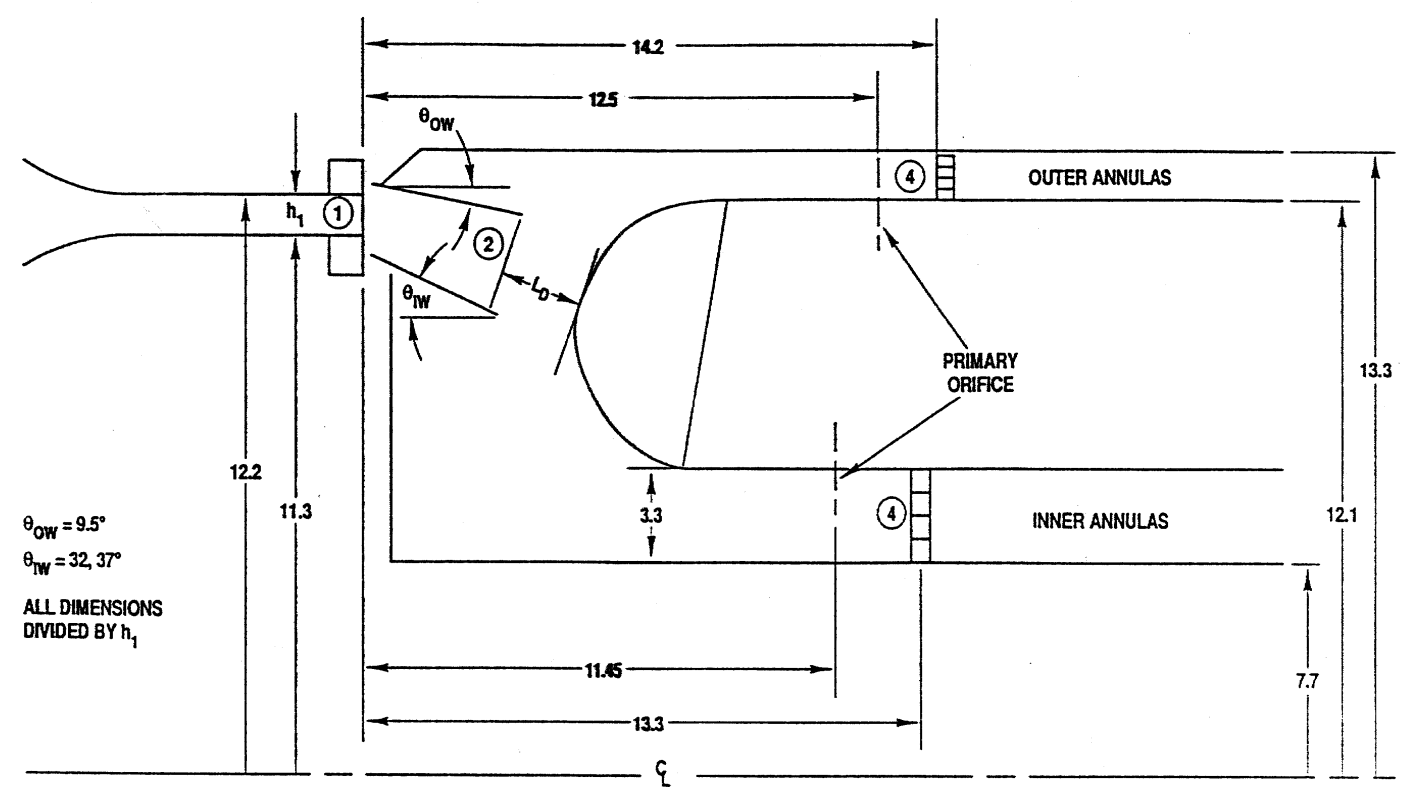

Figure 2.51 Leading Dimensions, Myres et al ${ }^{[1993]}$

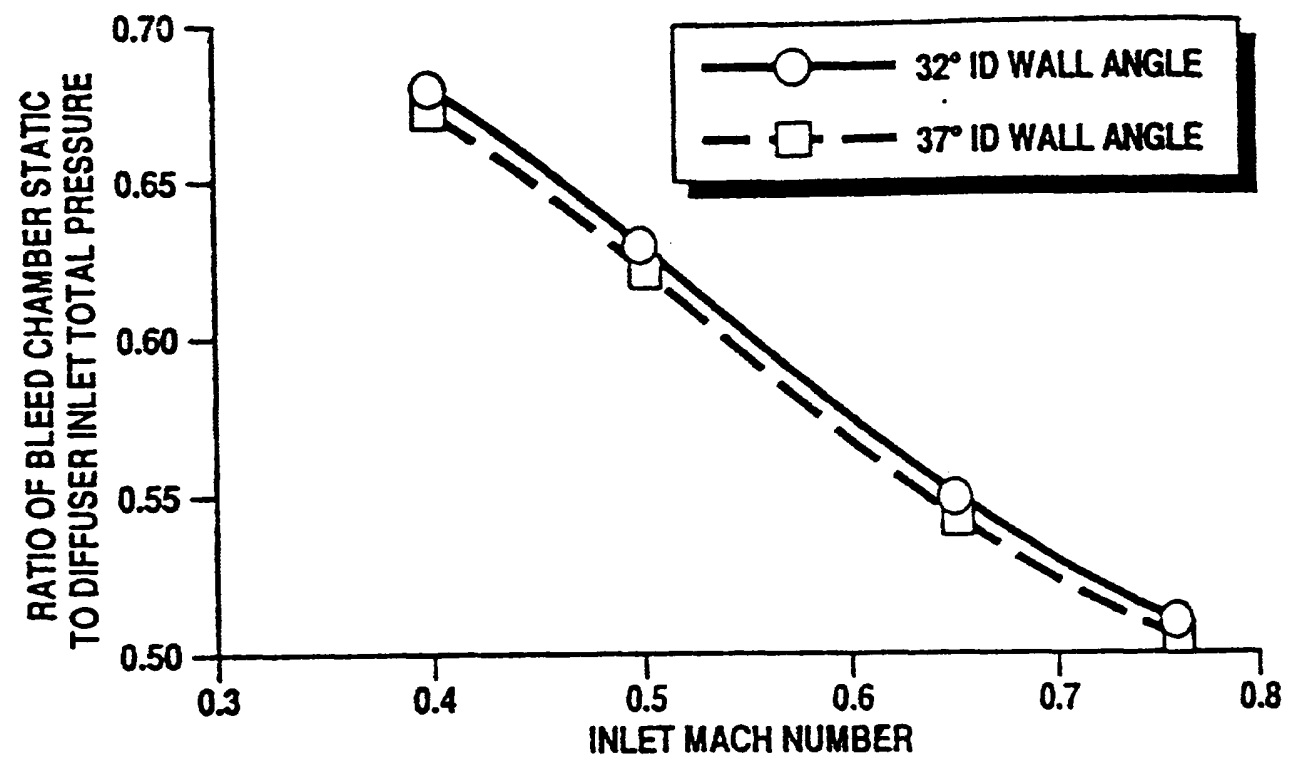

Figure 2.52 Ratio of Bleed Static to Inlet Total Pressure, Myres et al ${ }^{[1993]}$ 


\subsection{EXPERIMENTAL ARRANGEMENT AND PROCEDURES}

3.1 Description of the Test Facility $\ldots \ldots \ldots \ldots \ldots \ldots \ldots \ldots$. $\ldots \ldots$

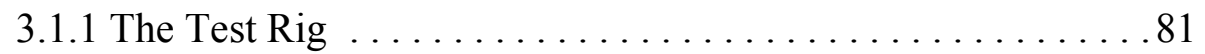

3.1.2 Test Rig Traversing $\ldots \ldots \ldots \ldots \ldots \ldots \ldots \ldots \ldots \ldots \ldots \ldots \ldots \ldots \ldots$

3.1.3 Compressor Stage Design and Operating Conditions . . . . . . 83

3.2 Instrumentation and Data Analysis $\ldots \ldots \ldots \ldots \ldots \ldots \ldots$

3.2.1 Data Acquisition and Control $\ldots \ldots \ldots \ldots \ldots \ldots \ldots$

3.2.2 Operating Condition Control $\ldots \ldots \ldots \ldots \ldots \ldots \ldots 7$

3.2.3 Data Reduction . . . . . . . . . . . . . . . . . 88

3.2.4 Graphical Techniques for Data Presentation ... . . . . . . .89

3.2 .5 Error Analysis $\ldots \ldots \ldots \ldots \ldots \ldots \ldots \ldots \ldots \ldots \ldots \ldots \ldots \ldots \ldots \ldots \ldots$

3.3 Test Section Inlet Conditions. ................ 93

3.4 Test Section Geometry $\ldots \ldots \ldots \ldots \ldots \ldots \ldots \ldots \ldots \ldots$

3.4.1 Flame Tube Geometry $\ldots \ldots \ldots \ldots \ldots \ldots \ldots \ldots$

3.5 A Discussion of the Pre-Diffuser Geometries $\ldots \ldots \ldots \ldots \ldots \ldots 7$

3.5.1 Conventional Datum Pre-Diffuser $\ldots \ldots \ldots \ldots \ldots . \ldots 97$

3.5.2 Optimised Conventional Pre-Diffuser $\ldots \ldots \ldots \ldots \ldots 97$

3.5.3 Hybrid Diffusers - Build 1 Flame Tube . . . . . . . . . . .98

3.5.4 Hybrid Diffuser - Build 2 Flame Tube . . . . . . . . . . . 100

3.6 Two-Dimensional, Axi-Symmetric Flame Tube Arrangement ..101

FIGURES .............................103 


\subsection{EXPERIMENTAL ARRANGEMENT AND PROCEDURE}

\subsection{Description of the Test Facility}

Findings from a preliminary experimental study using a simple two-dimensional, planar test rig are presented in Appendix A1. Initially it was hoped that this would aid the investigation by simplifying geometry modification and providing easier access for instrumentation. However, due to flow interactions with the test rig end walls the hybrid diffuser became unstable. Thus, the main experimental investigation was undertaken utilising an existing fully annular test facility which was then modified. Illustrated in Figure 3.1 , this facility comprises of a test cell containing the test rig, a plenum chamber and a fan room adjacent to the test cell. Contained within the fan room is a large centrifugal fan driven by a $75 \mathrm{~kW}$, variable speed d.c. motor. This draws approximately $4 \mathrm{kgs}^{-1}$ of ambient air through a system of filters (not shown in Figure 3.1) before delivering the clean air, via a diffusing passage, to a plenum chamber of volume $18 \mathrm{~m}^{3}$. The air then flows through a honeycomb flow straightener and a bell mouth intake into a single stage axial compressor. This compressor is driven by a $100 \mathrm{~kW}$, variable speed d.c. motor through a 4:1 step up spiral bevel gearbox and a vertical shaft allowing it to run at rotational speeds up to $5200 \mathrm{rpm}$.

\subsubsection{The Test Rig}

The test rig is mounted vertically in order to simplify the overall mechanical design and allows each section to be kept concentric using only lightly bolted, spigotted flanges and, only when absolutely necessary, thin aerodynamic struts. This configuration then allows the rig to be assembled using only an overhead hoist and simple lifting frames and thus removes the need for complicated tooling.

Figure 3.2 details the rig which is manufactured predominately from Plexiglass, other acrylics, aluminium for main load bearing components and in a few selected areas, such as the intake flare, hardwood.

The test rig is designed to simulate all of the likely airways present in a modern combus- 
tion system. Thus on exiting the pre-diffuser system the flow is split into a maximum of eight separate streams. Five of these are fundamental to radially staged combustion systems; namely the flame tube inner feed annulus, outer feed annulus, splitter annulus, inner cowl/burner (pilot) and outer cowl/burner (main) (refer to Figure 1.8). The remaining streams are optional and can be used to provide off-takes for hybrid diffuser bleed flow, or to simulate turbine blade cooling flow or cabin service off-takes. Each of these flows passes through an infinitely variable throttle and flow metering system (either calibrated bellmouths or orifice plates - refer to Appendix A4) before dumping into the test cell.

The flame tube is a representative model of a radially staged combustor front-end (external aerodynamics) only and does not include air admission ports or wall cooling features. This allows the five fundamental air flows to remain separate until they have passed through the flow metering section (Figure 3.2). This consists of five, fully annular, infinitely variable throttles followed by a settling length and a series of calibrated orifice holes designed using the data of Hay and Spencer ${ }^{[1992]}$.

The test rig is essentially of modular design which allows alternative configurations to be tested. Consequently, various designs of IGV, rotor blading, OGV, pre-diffuser, flame tube cowl, fuel injectors, dump cavity liners and bleed off-take features can all be fitted without disruption to other components.

\section{$\underline{\text { 3.1.2 Test Rig Traversing }}$}

In order to assess the aerodynamic performance of the system it is necessary to obtain complete area surveys of the flow at rotor exit, OGV exit, pre-diffuser exit, various positions around the flame tube head and at entry to the feed annuli. To achieve this a pressure probe must be moved both radially and circumferentially. Radial movement is achieved using a purpose-built linear traverse mechanism providing radial positional accuracy of $\pm 0.025 \mathrm{~mm}$. This consists of a precision linear guide fitted with a lead screw and a stepper motor as described in Appendix A5. However, circumferential movement is more complicated and is accomplished by rotating both the inner and outer pressure casings (see Figure 3.3), onto which the linear traverse is secured. It is essential that the 
timing between the OGVs, pre-diffuser, dump liner porosity and flame tube head remains fixed. Consequently, these are all held stationary, whilst the casings rotate on upper and lower pairs of bearings (Figure 3.3). Circumferential movement is achieved using a high powered DC servo motor fitted with a large reduction, low backlash gearhead. This rotates the outer casing using a heavy duty, high precision toothed timing belt and provides a circumferential positional accuracy of $\pm 0.01^{\circ}$. The inner casing is mechanically locked to the outer casing.

When it is necessary to simulate an air bleed from the outer dump cavity then an airtight seal is needed between the outer casing, which rotates, and the outer dump liner which is held stationary. During the collection of test data, the radial gap between these two items is sealed using an inflatable tube shown in Figure 3.3. This seal is deflated and the air vented to atmosphere leaving enough clearance to allow circumferential movement of the outer casing.

\section{$\underline{\text { 3.1.3 Compressor Stage Design and Operating Conditions }}$}

Section 2.0 highlighted that the inlet conditions are of great importance to the overall performance of a diffuser/combustor system. In an aero gas turbine the diffuser inlet conditions are generated by a multi-stage axial compressor and the flow is characterised by many complex features. The outlet guide vane wakes, the turbulence structure and the radial distribution of total pressure downstream are all very different to that produced by, for example, a tandem cascade. A truly representative flow structure can only be reproduced by using representative blading, operating at the correct angles of incidence. Unfortunately a multi-stage compressor has many technical problems associated with it. For example they are extremely complex and expensive, require large power requirements to drive and produce a high pressure rise which results in structural difficulties. However, a single stage axial compressor, whilst not perfect, reproduces representative inlet conditions with none of the problems associated with multi-stage compressors.

Compressor operating conditions are usually defined by a pair of parameters; a flow function $\dot{\mathrm{m}} \sqrt{\mathrm{T}} / \mathrm{AP}$ and a speed function $\mathrm{N} / \sqrt{\mathrm{T}}$. These are usually referred to as the 'non-dimesional' mass flow and 'non-dimensional' rotational speed although they are 
not truly dimensionless. Cohen at al ${ }^{[1977]}$ show that all operating conditions covered by a pair of 'non-dimensional' mass flow and speed should give rise to similar velocity triangles, so that the vane angles and air flow directions will match and the compressor should yield the same performance in terms of pressure ratio, temperature ratio and isentropic efficiency.

Ideally, the flow in the test rig would be such that the 'non-dimensional' mass flow and compressor blade Reynolds Numbers are engine representative. However, due to the low temperatures and pressures in the test rig it is not possibly to satisfy both these requirements. Thus, the design mass flow was set to give a sufficiently high OGV Reynolds Number of approximately $1.8 \times 10^{5}$, which is above the critical value, and a prediffuser inlet Mach Number of 0.15 , which is close to that of many engines.

The 154 OGVs are engine standard, whereas the 77 IGV/rotor blades were designed specifically to provide a representative swirl angle and total pressure distribution at the design flow coefficient. The ratio of OGVs to IGVs was deliberately set at an integer value (2:1) to prevent a vernier effect. A non-integer ratio would result in the alignment of the IGV/rotor and OGVs changing slightly around the annulus. This would present each OGV with a slightly different upstream condition and thus impact the performance of the OGV.

The rotor blades employ a NACA (A) camber line, a mid-height lift coefficient of 0.62 and a mid-height stagger angle of $59.4^{\circ}$. Superimposed on this was a NACA-65 profile with mid-height maximum thickness-chord ratio of $6 \%$ and a thickened trailing edge.

Details of the compressor rotor air angles and overall stage performance at mid annulus height are given below in Table 3.1 and Table 3.2. 


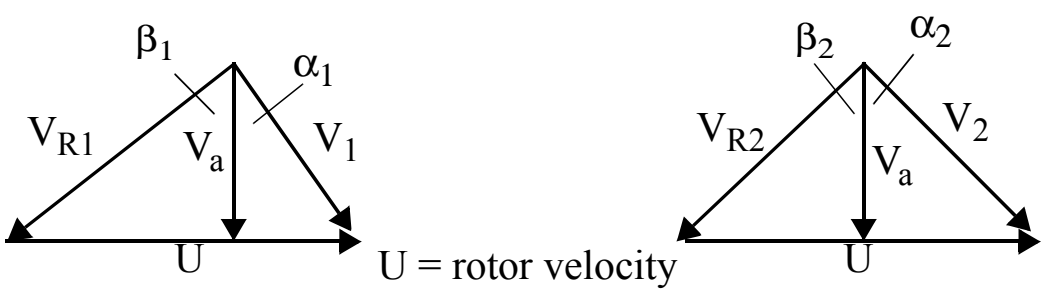

\begin{tabular}{|l|l||l|l|}
\hline \multicolumn{2}{|l||}{ Rotor Entry } & \multicolumn{2}{l|}{ Rotor Exit } \\
\hline \hline$\alpha_{1}$ & $\beta_{1}$ & $\alpha_{2}$ & $\beta_{2}$ \\
\hline $15.9^{\circ}$ & $65.5^{\circ}$ & $46.0^{\circ}$ & $53.3^{\circ}$ \\
\hline
\end{tabular}

Table 3.1 Compressor Rotor Air Angles (mid annulus height)

\begin{tabular}{|l|l|}
\hline Speed $(\mathrm{N})$ & 4000 r.p.m. \\
\hline $\mathrm{N} / \mathrm{J}_{\mathrm{o} 1}$ & 235.6 \\
\hline Mass Flow Rate & $3.79 \mathrm{kgs}^{-1}$ \\
\hline Temperature Rise & $6.5 \mathrm{~K}$ \\
\hline Total Pressure Rise & $6600 \mathrm{~Pa}$ \\
\hline Degree of Reaction & $73.4 \%$ \\
\hline Flow Coefficient, $\mathrm{V}_{\mathrm{a}} / \mathrm{U}$ & 0.385 \\
\hline Blade Loading, $\mathrm{C}_{\mathrm{p}} \Delta \mathrm{T}_{\mathrm{o}} / \mathrm{U}^{2}$ & 0.373 \\
\hline
\end{tabular}

Table 3.2 Compressor Stage Performance (mid annulus height)

\subsection{Instrumentation and Data Analysis}

\subsubsection{Data Acquisition and Control}

A schematic representation of the various devices used to monitor and control the test facility, perform probe radial/circumferential traversing and sample/record data is shown in Figure 3.4. The system is based around an IBM compatible Personal Computer (PC) linked to a 16 bit data acquisition system. This provides analogue to digital conversion of up to 16 channels of time averaged voltage information, together with the conditioning and recording of five K-type thermocouple readings. Three RS232 serial links enable the PC to communicate with a DC servo traverse system and the two DC motors driving the 
centrifugal fan and compressor rotor. Operating conditions and data provided by pneumatic pressure probes are converted to voltages using Furness FCO44 differential pressure transducers. These transducers are calibrated, including compensation of zero drift due to temperature, resulting in an accuracy of approximately $\pm 0.5 \mathrm{mmH}_{2} \mathrm{O}$.

Several safety systems are also built into the instrumentation due to the complex mechanical nature of the turbo machinery. This enables the performance of critical components to be closely monitored and the test facility automatically shut down or a warning given to the operator if certain parameters exceed operational limits. For example, the rotor blade tip clearance was set to $1.8 \%$ of the blade height and a fine wire is positioned in the rotor housing to monitor this. Should, for any reason, the tip running clearance decrease then this wire would be broken initialising automatic shut down. In addition, the temperature and pressure of the oil fed to the gearbox was also monitored as was the temperature of the main drive shaft bearings.

To assess the aerodynamic performance of the system under test, data were obtained using a miniature five hole probe used in the 'non-nulled' configuration (refer to Appendix A3). Area traverses were conducted at rotor exit, OGV exit, pre-diffuser exit and at entry to the inner and outer combustor feed annuli. These measurement stations are illustrated in Figure 3.2 and designated 2, 3, 4, 5 and 7 respectively. Pressure information from the splitter annulus (station 6) was recovered using a system of fixed pitot rakes and wall static tappings via a Furness FCO510 scanivalve. Burner feed was assessed via a series of pressure tappings arranged around the main and pilot injectors. Positioning of the five hole probe was achieved radially using a stepper motor powered linear guide (Appendix A5) in conjunction with electronic wall proximity detection. Driven by a PC this system enabled radial probe positioning with an accuracy of $\pm 0.025 \mathrm{~mm}$. This radial traverse system could be attached to either of the external casings which were free to rotate relative to the test section geometry. Circumferential probe movement was achieved using a DC servo drive unit which provided a positional accuracy of better than $\pm 0.01^{\circ}$. 


\subsubsection{Operating Condition Control}

A suite of software was developed by Denman ${ }^{[1996]}$ to utilise the instrumentation system described above. Control of the facility relies upon successful integration of the centrifugal fan and the rotor. Initially, on start-up, the centrifugal fan must assist the rotor in overcoming the system pressure loss. However, as the rotor accelerates towards the desired $\mathrm{N} / \sqrt{ } \mathrm{T}_{\mathrm{o}}$ it begins to work more effectively. Thus the speed of the centrifugal fan must be gradually reduced to allow the rotor to achieve the correct flow coefficient (Va/ U). Similarly, during shut down, a balance must be achieved to prevent a compressor surge.

Two Furness FCO44 pressure transducers were used to monitor the static and dynamic pressures in the inlet section upstream of the IGV row (Station 1, Figure 3.2). A K-type thermocouple was used to monitor the inlet stagnation temperature, $\mathrm{To}_{1}$, and the rotor speed feedback signal was provided by a shaft encoder. Using these signals the control system was able to maintain a rotor speed, $\mathrm{N} / \sqrt{ } \mathrm{To}_{1}$, and a flow coefficient, $\mathrm{Va} / \mathrm{U}$, to within $\pm 0.08 \%$ and $\pm 0.18 \%$ respectively. For this investigation the flow coefficient was fixed at the design value of 0.385 . However, the control algorithms have the ability to allow the operator to preset the value of $\mathrm{Va} / \mathrm{U}$ and thus investigate off-design performance. A third FCO44 pressure transducer was used to monitor a stable reference pressure situated below the outer dump liner (Figure 3.3). This pressure was used as reference for all measured pressures as a $\Delta \mathrm{p}$ measured with reference to ambient would be (a) too large for the pressure tansducers and (b) reduce measurement resolution because changes in $\Delta \mathrm{p}$ would small and difficult to resolve.

One module within the control software allows the operator to set the various mass flow splits using the metering section shown in Figure 3.2. Using this device the mass flow rate to each combustor feature can be set to within $\pm 0.1 \%$ of the total rig intake mass flow rate. The ability to perform this task accurately is of paramount importance as a development of this allows the hybrid diffuser bleed flow to be set to within $\pm 0.05 \%$ of the total rig mass flow rate. Figure 3.2 illustrates the hybrid diffuser bleed system. Outer bleed flows out of the vortex chamber, through a throttle and is vented to the test cell via twelve calibrated bellmouths. The inner bleed flows from the inner bleed chamber via a 
throttle and through a single calibrated orifice designed in line with Spencer and Hay ${ }^{[1992]}$.

\subsubsection{Data Reduction}

A polar $(\mathrm{x}, \mathrm{r}, \theta)$ co-ordinate system was used as this is naturally suited to an annular test facility. The x co-ordinate is aligned axially, in a streamwise direction, with the test rig centre line. The $\mathrm{r}$ co-ordinate extends radially outwards from the centre line and rotates circumferentially in an anti-clockwise direction (i.e. right-handed co-ordinate system) about the $\mathrm{x}$-axis subtending an angle of $\theta$ from some datum. Velocity components are $\mathrm{u}$, $\mathrm{v}$ and $\mathrm{w}$ such that $\mathrm{u}$ is the axial velocity in the $\mathrm{x}$ direction, $\mathrm{v}$ is the radial velocity in the $\mathrm{r}$ direction and $\mathrm{w}$ is the swirl velocity in the $\theta$ direction.

To achieve a meaningful assessment of the relative performance of the various diffuser configurations it is necessary to derive a system of performance parameters, which in turn can be related to each measurement station. Five hole probe area surveys consisting typically of 21 radial x 21 circumferential data points were used to aerodynamically assess the flow. Throughout the data acquisition procedure the operator was required to periodically input the ambient pressure into the control program. By maintaining constant operating conditions $\left(\dot{\mathrm{m}} \sqrt{\mathrm{T}} / \mathrm{AP}\right.$ and $\left.\mathrm{N} / \sqrt{\mathrm{T}_{\mathrm{o} 1}}\right)$ and hence a constant inlet Mach number, this allowed for the pressure readings from the five hole probe to be corrected to the ICAO Standard Day $\left(\mathrm{P}_{\mathrm{amb}}=760 \mathrm{mmHg}\right.$ and $\left.\mathrm{T}_{\mathrm{amb}}=288.15 \mathrm{~K}\right)$. Given that pressures were all measured with respect to a reference pressure (i.e. $\Delta p=p-p_{\text {ref }}$ ) the measured difference will be proportional to the mean dynamic pressure of the flow $\left(1 / 2 \rho \bar{U}^{2}\right)$

i.e.

$$
\mathrm{p}-\mathrm{p}_{\mathrm{ref}} \propto \frac{1}{2} \rho \overline{\mathrm{U}}^{2}
$$

but

$$
\mathrm{M}=\frac{\overline{\mathrm{U}}}{\sqrt{\gamma \mathrm{Rt}_{\mathrm{amb}}}}, \quad \underline{\mathrm{p}}=\mathrm{Rt} \text { and } \gamma \text { can be assumed constant }
$$


thus

$$
\mathrm{p}-\mathrm{p}_{\mathrm{ref}} \propto \mathrm{M}^{2} \mathrm{p}_{\mathrm{amb}}
$$

and since the Mach number is constant a measured pressure can be corrected to an ICAO Standard Day equivalent as follows:

$$
\left(p-p_{\text {ref }}\right)_{\text {standard day }}=\left(p-p_{\text {ref }}\right)_{\text {measured }} x \frac{\left(p_{a m b}\right)_{\text {standard day }}}{\left(p_{a m b}\right)_{\text {measured }}}
$$

Appendix A3 presents, in full, the relevant five hole probe theory, method of calibration and discusses how the five pressure readings are reduced to values of velocity, total pressure, static pressure and dynamic pressure for each data point. From this data, as discussed in Section 2.2, a set of quantitative performance parameters can be derived, such as mass weighted static pressure recovery (Equation 3.1) and total pressure loss (Equation 3.2) coefficients,

$$
\begin{gathered}
\mathrm{C}_{\mathrm{p} 1-2}=\frac{\mathrm{p}_{2}-\mathrm{p}_{1}}{\tilde{\mathrm{P}}_{1}-\tilde{\mathrm{p}}_{1}} \\
\lambda_{1-2}=\frac{\tilde{\mathrm{P}}_{1}-\tilde{\mathrm{P}}_{2}}{\tilde{\mathrm{P}}_{1}-\tilde{\mathrm{p}}_{1}}
\end{gathered}
$$

\subsubsection{Graphical Techniques for Data Presentation}

Graphical representation of the experimental data is afforded using a commercial plotting package; Tecplot (Amtec Engineering Inc.). This allows visualisation of technical data in a number of formats including simple ' $\mathrm{X}-\mathrm{Y}$ ' plots and two-dimensional surface plots (contours, vectors etc.). Presentation of data using these techniques enables quick and easy visualisation and significantly aids analysis.

' $\mathrm{X}-\mathrm{Y}$ ' plots are used to display various parameters including circumferentially averaged profiles of axial velocity, pitch angle, yaw angle, etc. These profiles are usually plotted against the non-dimensionalised radial position (i.e. plotted on a scale of 0 to 1.0 ):

$$
\frac{\left(\mathrm{r}-\mathrm{r}_{\mathrm{i}}\right)}{\left(\mathrm{r}_{\mathrm{o}}-\mathrm{r}_{\mathrm{i}}\right)}
$$


where $r$ is the radial position, $r_{i}$ and $r_{o}$ are the traverses station inner and outer radii respectively.

The independent variable is also generally non-dimensionalised, and the non-dimensionalising parameter indicated on the figure. For example, circumferentially averaged velocity profiles are non-dimensionalised by the area weighted mean axial velocity, $\overline{\mathrm{u}}$.

In most instances ' $\mathrm{X}-\mathrm{Y}$ ' plots display the data points and where possible Tecplot is used to fit a spline curve.

Two-dimensional contour plots are used to illustrate the radial and circumferential variation of parameters across a measurement sector, viewing upstream in a negative $\mathrm{x}$-direction. Similarly, vector plots are used to project the total velocity vectors onto the measurement sector. Although viewing in two-dimensions does not give a true indication of total velocity it allows the variation of radial and swirl velocity across an axial plane to be easily visualised.

\section{$\underline{\text { 3.2.5 Error Analysis }}$}

It is important to recognise that experimental errors can be introduced and equally important to quantify these errors and attempt, where possible, to reduce them to an acceptable level. Traditionally errors occur in several key areas; hardware, data collection and data reduction. However it is difficult to separate the latter two as the final errors are a combination of many factors.

In general the experimental test facility was manufactured to the specified dimensions with an accuracy of $\pm 0.1 \mathrm{~mm}$. This leads to a possible variation of the area aG OG entry of $\pm 0.3 \%$ which is deemed tolerable.

Section 3.2 and Appendix A3 describe how miniature five hole probes have been used to assess the relative performance of the various diffusers under investigation. The positional accuracy of the probe within the test rig is difficult to quantify. The traverse mechanisms used are accurate to $\pm 0.025 \mathrm{~mm}$ in the radial direction and $\pm 0.01^{\circ}$ in the 
circumferential direction. The probes are held in the test rig by accurately manufactured blocks and guides. These ensure that the probe stem is held rigid and the head is aligned correctly in pitch. To align the probe head in yaw the probe is first held in the traverse mechanism and guide/block and then using an engineer's flat table, set squares and a datum face on the block, the head is positioned at the required angle $\left(0^{\circ}\right.$ or $45^{\circ}$ to the rig axis). The probe head is always aligned using the same datum face to ensure consistancy. The probe, traverse and guide block can then be attached to the test rig ensuring, with the use of a spirit level, that the datum face on the block is square. The resultant pitch and yaw setting error is then about $\pm 1^{\circ}$. Furthermore, this same method of probe alignment is used when calibrating the probes (Appendix A3) ensuring that alignment is consistent between calibration and data collection.

A detailed and quantitative description of the accuracy of the five hole probe is, in itself, a substantial piece of work and thus beyond the scope of the current project. However, other authors have examined the accuracy of the five hole probe. Appendix A3 describes the calibration method used for the five hole probe and the accuracy of this was analysed by Wray ${ }^{[1997]}$. He found that, away from any solid surface, the errors in the velocities and flow angles were negligible for a calibration range of $\pm 36^{\circ}$ in yaw and pitch. The presence of a wall close to the probe head can introduce errors and Sitaram et al ${ }^{[1981]}$ found that the measured velocity could vary by $\pm 1 \%$. Similarly, close to a wall, Tamigniaux and Oats ${ }^{[1986]}$ revealed that a maximum error of $2^{\circ}$ in measured flow angle was seen. However, these errors are confined to a region less than one probe head diameter from a wall and therefore have a negligible overall effect.

Turbulence levels also effect the accuracy of a five hole probe; an error of $\pm 0.3 \%$ was seen by Sitaram et al for a turbulence intensity of about $10 \%$. At rotor exit and diffuser exit turbulence intensities are, in general, less than $10 \%$. However, at entry to the feed annuli this value can approach $30 \%$, thus at the annuli measurement stations a slightly higher error may be observed.

The physical size and presence of the probe can also introduce errors as it presents a blockage to the flow. It is also possible that, in confined regions, the presence of a pressure probe can modify the flow mechanisms under study. However, at the various meas- 
urement stations the five hole probe presents only a small blockage. For example, at rotor exit (station 2) the five hole probe represents about $6 \%$ of the passage height but only $0.004 \%$ of the area.

Ultimately the accuracy of any pressure measurements also depends upon the accuracy of the Furness FC044 differential pressure transducers, and the associated data acquisition system. The pressure transducers were calibrated against a Furness FC0510 Micromanometer which has a quoted accuracy of better than $\pm 0.05 \%$ at $\pm 500 \mathrm{mmH}_{2} \mathrm{O}$. Calibration was performed by logging the pressure in a vessel as it was varied through the range $\pm 500 \mathrm{mmH}_{2} \mathrm{O}$. The analogue voltages produced by the transducers were converted to a digital signal by an analogue-to-digital convertor (ADC) which itself was calibrated by comparison, over a range of voltages, with a very accurate DC voltage source. The accuracy of the ADC was found to be $\pm 0.1 \%$ which yields an accuracy for the transducer calibration of about $\pm 0.15 \%$. Thus, the accuracy of pressure measurements from the test rig is $\pm 0.25 \%$ and is a combination of the overall transducer accuracy coupled with accuracy of the voltage measurements. However, this is true only for the maximum pressure difference; the accuracy will decrease as the pressure difference drops. Typical measurements record a pressure difference in the region $100-200 \mathrm{mmH}_{2} \mathrm{O}$ which will result in an accuracy of about $\pm 0.5 \%$.

The individual five hole pressures are used to calculate the dynamic pressure of the flow and this is then used to calculate the flow total velocity. It is sensible to assume that the dynamic pressure is obtained with a similar accuracy to the individual pressures, and since dynamic pressure is proportional to velocity squared then the error in the measured velocity would be halved to $\pm 0.25 \%$.

Throughout this work diffuser and system performance is discussed in terms of massweighted static pressure recovery and total pressure loss coefficients as defined in Equations 3.1 and 3.2. An error analysis for typical mass-weighted pressures at rotor exit and diffuser exit indicated that these coefficients have an accuracy of better than $\pm 2.0 \%$. 


\subsection{Test Section Inlet Conditions}

All experimental data were recorded at a design rotor flow coefficient, $\mathrm{V}_{\mathrm{a}} / \mathrm{U}$, of 0.385 and an OGV Reynolds number of approximately 1.8 x $10^{5}$. Mean flow conditions measured at rotor exit (station 2) and OGV exit (station 3) are illustrated in Figure 3.5 and Figure 3.6. The data, recorded over a single IGV blade space, represent and define the inlet conditions for all the various test geometries described later.

Non-dimensionalised, circumferentially averaged axial velocity, total pressure and swirl angle distributions at rotor exit are presented in Figure 3.5(a), (b), and (c) respectively. Reasonably thick boundary layers can be seen in Figure 3.5(a) on the inner and outer wall each amounting to some $10-15 \%$ of the passage height.

The effect of rotor tip leakage losses are clearly visible in the total pressure profile shown in Figure 3.5(b). This local loss in total pressure is caused by secondary flows across the tip of the rotor from the pressure to the suction side of the blade.

The rotor was designed to have an exit swirl angle of -46 degrees and achieves this at mid annulus height (Figure 3.5(c)), however there is a sharp reduction in swirl angle close the passage walls due to the reduced axial velocity of the boundary layers.

Variations in the total pressure contours illustrated in Figure 3.5(d) are due to the influence of IGV wakes. Local flow angle variations caused by these wakes will effect the performance of the rotor and introduce local total pressure losses. However, the characteristics shown in Figure 3.5 are typical of the flow downstream of a rotor.

Due to an OGV/IGV ratio of 2 the OGV exit conditions are periodic and thus OGV exit conditions were recorded over a sector made up of two OGV spaces and then integrated over a full annulus. The rig instrumentation allows several of these sectors to be traversed as described in Section 3.2.1 and this enabled confirmation that this sector was indeed repeated.

Figure 3.6(a) details the non-dimensionalised axial velocity contours over a two OGV 
sector. The OGV wakes are easily identified as the two regions of low axial velocity (i.e. darker contours). The slight difference between the two wakes results from a propagation of the IGV wake through the rotor which influences local OGV performance.

A plot of total velocity vectors (Figure 3.6(b)) reveals the secondary flows generated by the static pressure gradients within the OGVs. These can be seen as a rotational tendency in the flow.

The circumferentially averaged axial velocity at OGV exit (Figure 3.6(c)) is slightly inboard biased but generally does not differ greatly from the average. The slight divergence towards the outer casing is a result of the slightly deeper OGV wake flow and secondary flows at this radius (i.e. there is a loss core generated on the suction surface of the OGV at $75 \%$ height). This also impacts the circumferentially averaged swirl angle distribution as shown in Figure 3.6 (d). Towards the outer casing the swirl angle drops then rapidly climbs as, firstly, the wake flow, and then secondly, the boundary layer causes the flow to deviate from the axial direction. The negative angle indicates that the flow is under turning out of the OGV passage; the design swirl angle being zero.

The aerodynamic performance of the OGV row is detailed in Table 3.3 in terms of the mass weighted total pressure loss, static pressure recovery and kinetic energy coefficients. Measurements of mass flow at rotor exit (station 2) and OGV exit (station 3) by means of five-hole probe area traverses produce a balance of better than $\pm 1.0 \%$

\begin{tabular}{|c||c||c|}
\hline$\lambda_{2-3}$ & $\mathrm{C}_{\mathrm{p} 2-3}$ & $\alpha_{3}$ \\
\hline \hline 0.10 & 0.40 & 1.17 \\
\hline
\end{tabular}

Table 3.3 OGV Performance Data 


\subsection{Test Section Geometry}

\subsubsection{Flame Tube Geometry}

A detailed view of the two flame tube geometries employed in this study is given in Figure 3.7(a) and (b), with Table 3.4 detailing some leading dimensions. These will be referred to as Build 1 and Build 2 respectively. The test facility was originally designed to accommodate the Build 1 flame tube with the Build 2 configuration arising from a development of radially staged combustor technology driven by the need to significantly reduce the length and weight of the combustion system.

Both build standards represent typical radially staged combustion systems with the main combustor positioned outboard of the pilot. Generically similar, both present a difficult aerodynamic challenge for the diffuser; namely very high required levels of diffusion and controlled turning in order to prevent excessively high annuli total pressure losses.

To allow for investigation of the flow between rotor exit and the feed annuli the cowl flows are modelled. However, combustor wall porosity is not modelled as this enables the design mass flow distribution (Table 3.5) to be forced using the metering section as described earlier. There are, however, several major geometrical differences in the two builds, and these are summarised below:

- The Build 2 pilot flame tube was moved axially upstream of its location in Build 1.

- In Build 1 a single burner feed arm was employed. However, to accommodate the axial movement of the pilot combustor separate burner feed arms were introduced. This significantly reduced the length available for the pre-diffuser.

- The full elliptical cowl in Build 1 was replaced in Build 2 by simple annular fairings.

- Although 22 burners were employed per bank in each build, the circumferential stagger was $1 / 2$ and $1 / 4$ of a burner sector for Builds 1 and 2 respectively.

- The splitter annulus in Build 1 was replaced by an effusion cooled wall in Build 2. 


\begin{tabular}{|l||l||c|c||c|c|}
\hline \multicolumn{1}{|c||}{ Station No. } & \multirow{2}{*}{ Description } & \multicolumn{2}{c||}{ Build 1 } & \multicolumn{2}{c|}{ Build 2 } \\
\cline { 3 - 6 } & & $\mathbf{r}_{\text {mean }}$ & $\mathbf{h ~ ( m m )}$ & $\begin{array}{l}\mathbf{r}_{\text {mean }} \\
\text { (mm) }\end{array}$ & h (mm) \\
\hline \hline 2 & Rotor Exit & 316.75 & 30.5 & 316.75 & 30.5 \\
\hline 3 & OGV Exit & 316.75 & 30.5 & 316.75 & 30.5 \\
\hline 4 & Diffuser Exit & 316.75 & varies & 316.75 & varies \\
\hline 5 & Inner Annulus & 195.60 & 27.2 & 214.60 & 21.5 \\
\hline 6 & Splitter Annulus & 334.00 & 19.0 & $\mathrm{n} / \mathrm{a}$ & $\mathrm{n} / \mathrm{a}$ \\
\hline 7 & Outer Annulus & 462.25 & 24.5 & 465.20 & 18.6 \\
\hline
\end{tabular}

Table 3.4 Leading Dimensions

The main and pilot (Figure 3.8) injectors are modelled, for both build standards, using dummy injectors of suitable porosity. By considering mass continuity, Equation 3.4 was used to calculate the appropriate number of plain holes $(\mathrm{N})$ to replace the flow area represented by the inner, outer and dome elements of each swirler.

$$
A_{\text {hole }}=\text { Scale } \times \frac{A_{\text {swirler }}}{N} \times \frac{C_{\text {dswirler }}}{C_{\text {dhole }}}
$$

\begin{tabular}{|c|c|c|}
\hline Feature & $\begin{array}{l}\text { Build } 1 \\
\frac{\dot{\mathrm{m}}}{\dot{\mathrm{m}}_{\text {total }}}(\%)\end{array}$ & $\begin{array}{l}\text { Build } 2 \\
\frac{\dot{\mathrm{m}}}{\dot{\mathrm{m}}_{\text {total }}}(\%)\end{array}$ \\
\hline Inner Annulus & 17.7 & 23.0 \\
\hline Pilot Combustor & 7.7 & 14.6 \\
\hline Splitter / Effusion Wall & 18.3 & 1.7 \\
\hline Main Combustor & 25.6 & 29.1 \\
\hline Outer Annulus & 30.7 & 31.6 \\
\hline
\end{tabular}

Table 3.5 Flametube Air Mass Flow Distribution - Design Values 


\subsection{A Discussion of the Pre-Diffuser Geometries}

\subsubsection{Conventional Datum Pre-Diffuser}

The conventional, single passage Datum pre-diffuser geometry, shown in Figure 3.9, represents current engine technology and is included to provide benchmark performance from which any improvements can be measured. The diffuser has a modest overall area ratio of 1.48 , with a non-dimensionalised length $\left(\mathrm{L} / \mathrm{h}_{1}\right)$ of 3.13 and at exit it is canted radially outwards at an angle of $\beta=+4.0^{\circ}$. Also included are 22 radial struts circumferentially aligned mid-way between the main and pilot injectors. The strut leading edge has a radius of $0.75 \mathrm{~mm}$ and is centred on a local OGV blade space. The strut trailing edge thickness is approximately $28 \%$ of diffuser exit height. Designed using conventional design rules the Datum diffuser is also area ruled to account for the strut blockage. (Area ruling simply increases the area of a strutted diffuser to account for the blockage created by the struts).

\subsubsection{Optimised Conventional Pre-Diffuser}

Illustrated in Figure 3.10 this diffuser represents an attempt to optimise conventional diffuser technology using the multi-passage concept described in Section 2.5 in conjunction with conventional design rules. By introducing a splitter vane the effective non-dimensional length of the two resulting passages is increased by a factor of approximately 2 . Thus, with reference to Figure 3.11, the area ratio of each passage can be increased. Furthermore, this configuration also allows a significant increased degree of turning within the diffuser which will improve the feed to the injectors and combustor feed annuli. The Optimum Conventional diffuser, therefore, will demonstrate the performance limits associated with conventional diffusers and act as a secondary benchmark with which to compare hybrid diffuser performance.

Both diffuser passages have an area ratio of 2.0 resulting in an overall diffuser area ratio of 2.0. The inner passage has a non-dimensionalised length of 6.79 and at exit is canted radially inwards at an angle of $-14^{\circ}$. The outer passage has a non-dimensional length of 4.95 and at exit is also radially canted at an angle of $+14^{\circ}$. Figure 3.10 clearly shows that 
the Optimised Conventional diffuser is considerably longer than the Datum diffuser and this significantly reduces the dump gap in the Build 1 configuration but, more notably, results in the fact that it physically will not fit into the Build 2 test section. Again this diffuser has 22 equi-spaced radial struts with the leading edges centred on an OGV blade space and the trailing edges aligned between the injectors. The leading edge is fairly blunt with a radius of approximately $4 \mathrm{~mm}$ and a trailing edge equal to $19 \%$ of diffuser exit height.

\subsubsection{Hybrid Diffusers - Build 1 Flame Tube}

A review of previous work with hybrid diffusers has been presented in Section 2.6 and revealed that there is a significant lack in the understanding of the flow mechanisms involved. However, a basic hybrid configuration was developed using a knowledge of the specific requirements of an aero gas turbine combustion system coupled with data from the work of Adkins et $\mathrm{al}^{[1980]}$ and Myres et $\mathrm{al}^{[1993]}$. The final design adopted for this study consisted of a three stage hybrid diffuser as illustrated in Figure 3.12 and Figure 3.14. The initial geometry (Hybrid1) represents a conservative design which was then extended into five distinct diffusers, for the Build 1 flame tube, whilst maintaining the same fundamental configuration. For the Build 1 flame tube configuration all the hybrid diffusers have a modest static pressure rise imposed immediately downstream of the OGVs using a conventional diffuser. This Stage 1 diffuser has an area ratio of 1.3, a non-dimensional length of 1.4 and a wall angle of $7^{\circ}$. The Stage 2 diffuser consists of a sudden step expansion as discussed in Section 2.6. Two values of area ratio were used for the Stage 2 diffuser; 1.3 and 1.42. The control of the Stage 2 diffuser is afforded by bleeding air into vortex chambers through circumferential slots located in the inner and outer walls. The bleed slot geometry was defined in terms of parameters " $\mathrm{x}$ " and " $\mathrm{y}$ " which were inferred non-dimensionally from published data (Section 1.4). These are detailed in Figure 3.12 and remained unaltered throughout the experimental work. For all the hybrid diffuser geometries tested the Stage 3 diffuser comprised of a moderately loaded conventional diffuser immediately downstream of Stage 2. The wall angle and hence area ratio progressively increases throughout, as detailed in Table 3.6 and illustrated in Figure 3.11 and Figure 3.13. The main function of the Stage 3 component was essentially to encourage radial flow movement and, although diffusive in nature, provide 
an environment for rapid flow reattachment. Describing the configuration of the hybrid diffuser in three stages is in no way meant to imply that the flow physics can be separated into these same three distinct regions. On the contrary, strong interactions between the step expansion and Stages 1 and 3 are likely to dominate the overall performance.

The decision to impose a moderate static pressure recovery on the flow immediately prior to the vortex controlled step expansion is logical when considered in the context of an aero gas turbine. It is essential that any air removed can be used to replace or augment air that is normally removed from elsewhere in the combustion system. Normally air removed from this region is used for turbine blade or disc cooling. Furthermore the condition of the bleed flow must be of sufficient pressure to be useful. Thus a conventionally diffusing section upstream of the off-take can be advantageous in providing a significant level of pressure recovery before the air is removed. Additionally, the presence of strong, fresh OGV wakes was also thought to be important. The presence of the step expansion imposes a significant adverse pressure gradient upon the flow passing over the bleed. If the OGV exit plane is too close to the bleed off-take then the low energy wake flow represents the flow least likely to overcome this pressure gradient. Consequently this flow would then form the majority of the bleed flow resulting in only poor quality air being removed which would be unsuitable for use elsewhere. The presence of the conventional Stage 1 diffuser will allow the blade wakes to begin to mix out

\begin{tabular}{|l||c|c|c|c|c|c|c|c|c|c|}
\hline Diffuser & $\mathrm{AR}_{1}$ & $\mathrm{~L}_{1} / \mathrm{h}_{1}$ & $\mathrm{AR}_{2}$ & $\mathrm{AR}_{3}$ & $\mathrm{~L}_{3} / \mathrm{h}_{3}$ & $\theta_{3}$ & $\mathrm{AR}$ & $\mathrm{L} / \mathrm{h}_{1}$ & $\beta$ & Strut \\
\hline \hline Hybrid 1 & 1.3 & 1.4 & 1.3 & 1.34 & 1.22 & $7^{\circ}$ & 2.27 & 3.5 & $0^{\circ}$ & 7 \\
\hline Hybrid 2 & 1.3 & 1.4 & 1.3 & 1.42 & 1.22 & $9^{\circ}$ & 2.40 & 3.5 & $0^{\circ}$ & 7 \\
\hline Hybrid 3 & 1.3 & 1.4 & 1.3 & 1.47 & 1.22 & $11^{\circ}$ & 2.49 & 3.5 & $0^{\circ}$ & 7 \\
\hline Hybrid 4 & 1.3 & 1.4 & 1.42 & 1.35 & 1.11 & $9^{\circ}$ & 2.49 & 3.5 & $0^{\circ}$ & 7 \\
\hline Hybrid 5 & 1.3 & 1.4 & 1.42 & 1.23 & 1.11 & $9^{\circ}$ & 2.27 & 3.5 & $0^{\circ}$ & 3 \\
\hline Hybrid 6 & 1.05 & 0.22 & 1.60 & 1.23 & 1.48 & $9^{\circ}$ & 2.06 & 3.0 & $0^{\circ}$ & 3 \\
\hline Datum & - & - & - & - & - & - & 1.48 & 3.13 & $+4^{\circ}$ & 3 \\
\hline $\begin{array}{l}\text { Opt. Conv. } \\
\text { (inner/outer) }\end{array}$ & - & - & - & - & - & - & 2.00 & 6.79 & $-12^{\circ}$ & 3 \\
+
\end{tabular}

Table 3.6 Pre-Diffuser Specification 
In order to investigate the reattachment process immediately downstream of the step, static pressure tappings were positioned along the outer wall of the Hybrid 4 Stage 3 diffuser as illustrated in Figure 3.15. The design of the instrumentation was strongly influenced by the desire to resolve the OGV wakes and their effect on flow reattachment. Thus 40 static pressure tappings were arranged in an array distributed over one OGV space. The instrumentation system described earlier and shown in Figure 3.4 included a remotely operated scanni-valve which allowed data to be automatically sampled from each of the static pressure tappings. Figure 3.15 also illustrates how P.T.F.E. bearings were used to allow the Stage 3 outer wall to be circumferentially rotated relative to the OGV row. Thus a full area survey of the wall static pressure could be easily achieved covering one or several OGV spaces.

The desire to assess the effect of radial struts in the Stage 3 diffuser led to the transition of the Hybrid 4 pre-diffuser into Hybrid 5. The two pre-diffusers are identical except for 22 radial struts located in the Stage 3 component of Hybrid 5. The leading edge of each strut was positioned $12 \mathrm{~mm}, 2.5$ step heights (refer to Figure 3.12) downstream of the step expansion. This was done to limit the effect the presence of the strut had on reattachment and to allow the integrity of the Coanda bubble shown in Figure 2.31 to be maintained. The leading edge of each strut was elliptical in shape to increase their tolerance to any non-axial flow. The struts had a simple, straight taper to a trailing edge thickness of 8 $\mathrm{mm}$ which is approximately $10.5 \%$ of the annulus height at pre-diffuser exit. This thickness was chosen such that the overall area ratio of Hybrid 5 was returned to that of Hybrid 1 (i.e. $A R=2.27$ ). Thus direct comparison of performance data would then be possible.

\subsubsection{Hybrid Diffuser - Build 2 Flame Tube}

To accommodate the reduction in length imposed by moving the pilot combustor forward for Build 2, the Stage 1 diffuser has been almost removed from the Hybrid 6 diffuser (Table 3.6 and Figure 3.14(b)). Tests, to be reported later, suggested that the area ratio of the Stage 2 diffuser was quite modest. Thus, to offset the loss of Stage 1 diffusion the Stage 2 area ratio was increased to 1.6. In order to maintain consistency and continuity with the hybrid diffusers tested in Build 1 the Stage 3 section of Hybrid 6 is designed 
to have the same area ratio as in Hybrid 5. It is, however, slightly longer and thus the aerodynamic loading is slightly less. The Hybrid 6 diffuser also includes 22 engine representative radial struts within the Stage 3 section with a trailing edge thickness equivalent to some $22 \%$ of the diffuser exit height and the leading edge positioned approximately 6.3 step heights downstream of the step expansion.

In addition to removing most of the Stage 1 diffuser the bleed chambers have also been significantly redesigned. The space afforded by the Build 2 flame tube was insufficient to accommodate the 'vortex chambers' as seen in Build 1. These were replaced by diffusing passages shown in Figure 3.14. If the vortex chambers are not necessary and only exist as a means to produce a stable bleed flow then a diffusing passage rather than a chamber could be used. This would reduce the extra complexity and length introduced by the vortex chambers with the additional benefit that simple diffusing passages will improve the quality of the bleed flow making its use for component cooling a more attractive option.

\subsection{Two-Dimensional, Axi-Symmetric Flame Tube Arrangement}

One aim of this project was to use Computational Fluid Dynamics (CFD) to supplement experimental data and reveal, in greater detail, the structure of the controlling flow mechanisms of a hybrid diffuser. However, as described in Section 5.0, the generation of a computational representation of the experimental test facility and calculation of the associated flow field is a highly complex task. It is not practical to generate an exact computational model of the experiment and simplifications to the geometry must be introduced. This then enables computational predictions to be undertaken but raises the question of their validity and accuracy. Without a direct comparison to an identical experimental test it is not possible to conclude that the CFD results are free from numerical error. Therefore, to enable validation of the computational methodology the experimental test facility was modified to produce a two-dimensional, axi-symmetric flame tube configuration which can be more closely modelled using CFD. A comparison of experimental data from the modified facility with computational data would then satisfactorily indicate the accuracy of the computational methods and allow a more confident discussion of further CFD predictions. 
In order to produce an entirely two-dimensional, axi-symmetric configuration of the test facility the compressor stage must be removed entirely. The structure of the flow downstream of an axial compressor is highly three dimensional and varies considerably in the circumferential direction. Although changing the inlet conditions will alter diffuser performance it is reasonable to assume it will not greatly affect the controlling flow mechanisms of the hybrid diffuser. Thus, in order to study these mechanisms, simplifying the inlet conditions is reasonable. Therefore the IGV row, rotor and OGV row were replaced by simple perspex rings as shown in Figure 3.16. Data acquisition and control was afforded using the same system as the un-modified rig (described in Section 3.2). To achieve the same inlet Reynolds Number of about $1.8 \times 10^{5}$ (based now on inlet duct height) as for the un-modified rig the centrifugal fan was run at a higher speed. Further to removing the compressor the downstream geometry was also modified by removal of the burners and simplification of the cowl. The replacement cowl is shown in Figure 3.16 and no longer includes the 'double-bubble' shape of the original. Initially the new cowl was designed to have no porosity but for reasons described in Section 4.4 limited porosity was introduced as illustrated in Figure 3.17.

There are several ramifications caused by removing the compressor and modifying the cowl geometry. The inlet conditions to the pre-diffuser will change significantly and the modified cowl will impose a different set of constraints on the diffuser exit flow. Both of these will effect diffuser performance (refer to Section 2.0). Previously $51.6 \%$ of the total mass flow passed through the cowl into the burners or splitter annulus but now the mass flow splits will be significantly different. The mass flow splits used are outlined in Section 4.4 and were set in the same way as before using the metering section (Section 3.2). However, if inlet conditions for the computational prediction are taken from experimental measurements on the modified facility then the results will serve to provide the direct comparison necessary for CFD validation. Furthermore, data to match the threedimensional computational prediction can be easily obtained by simply replacing the rotor. 


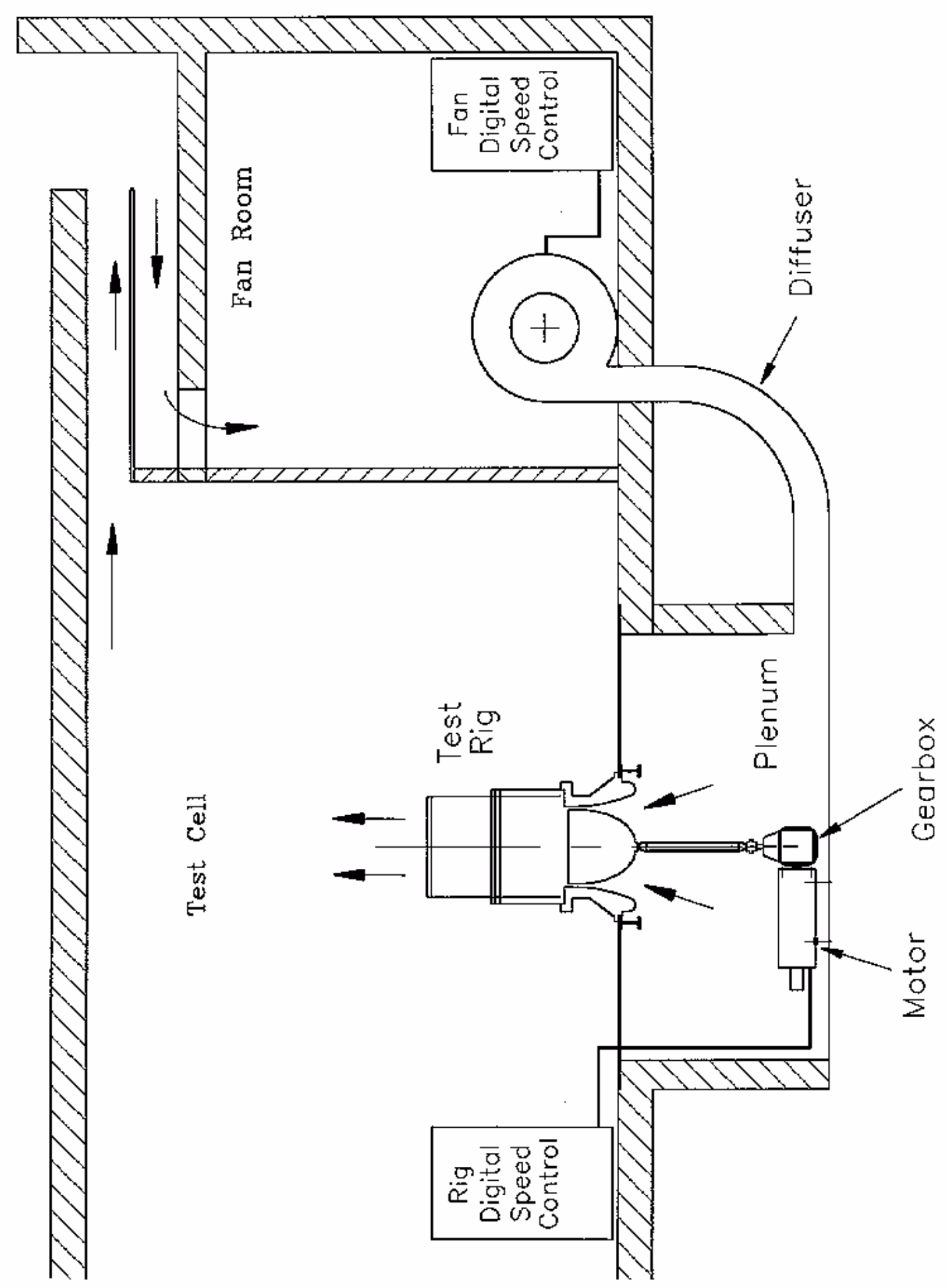

Figure 3.1 The Test Facility 


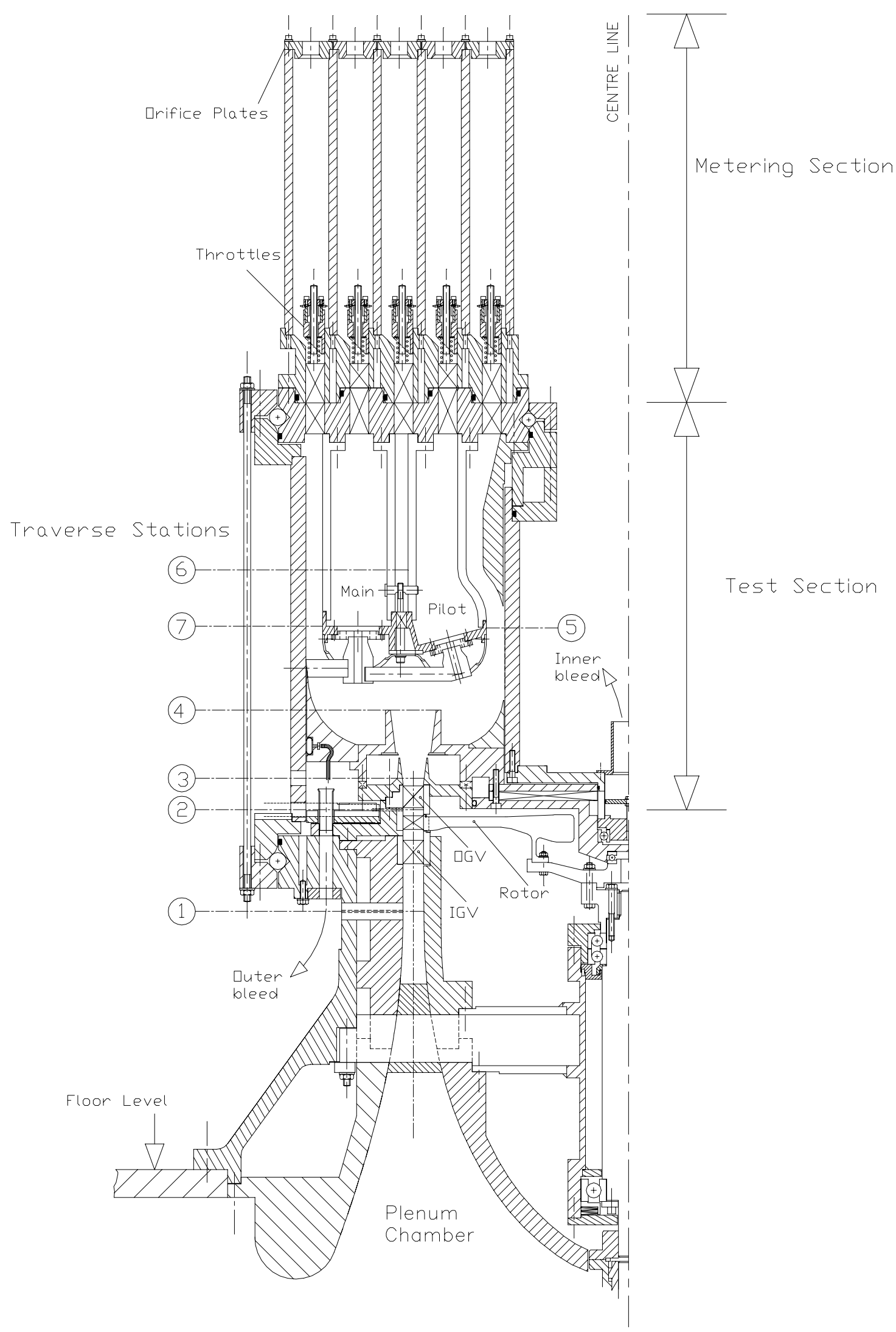

Figure 3.2 The Test Rig (showing Hybrid Diffuser and Build 1 Flame Tube) 


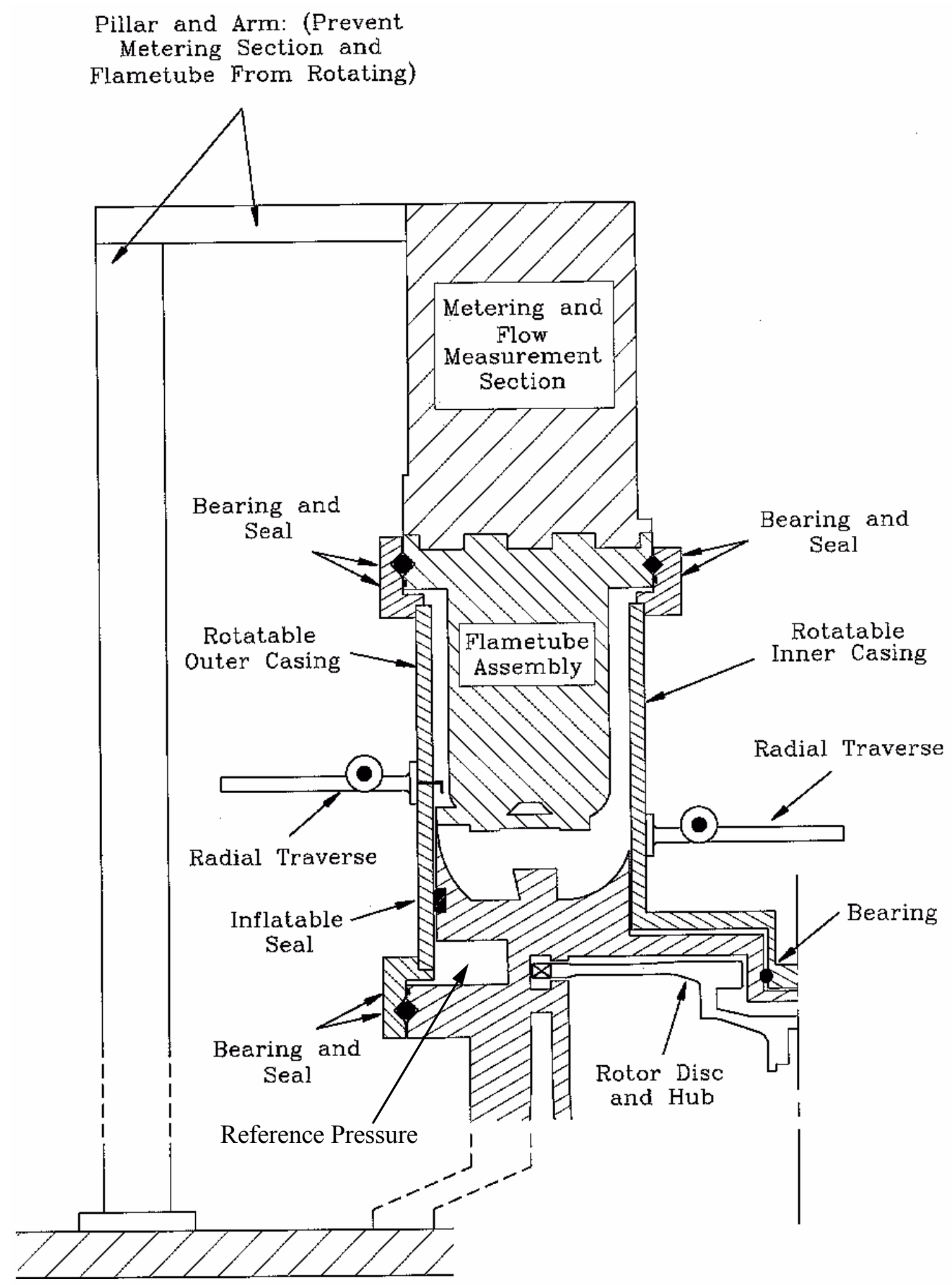

Figure 3.3 Traverse Arrangement 


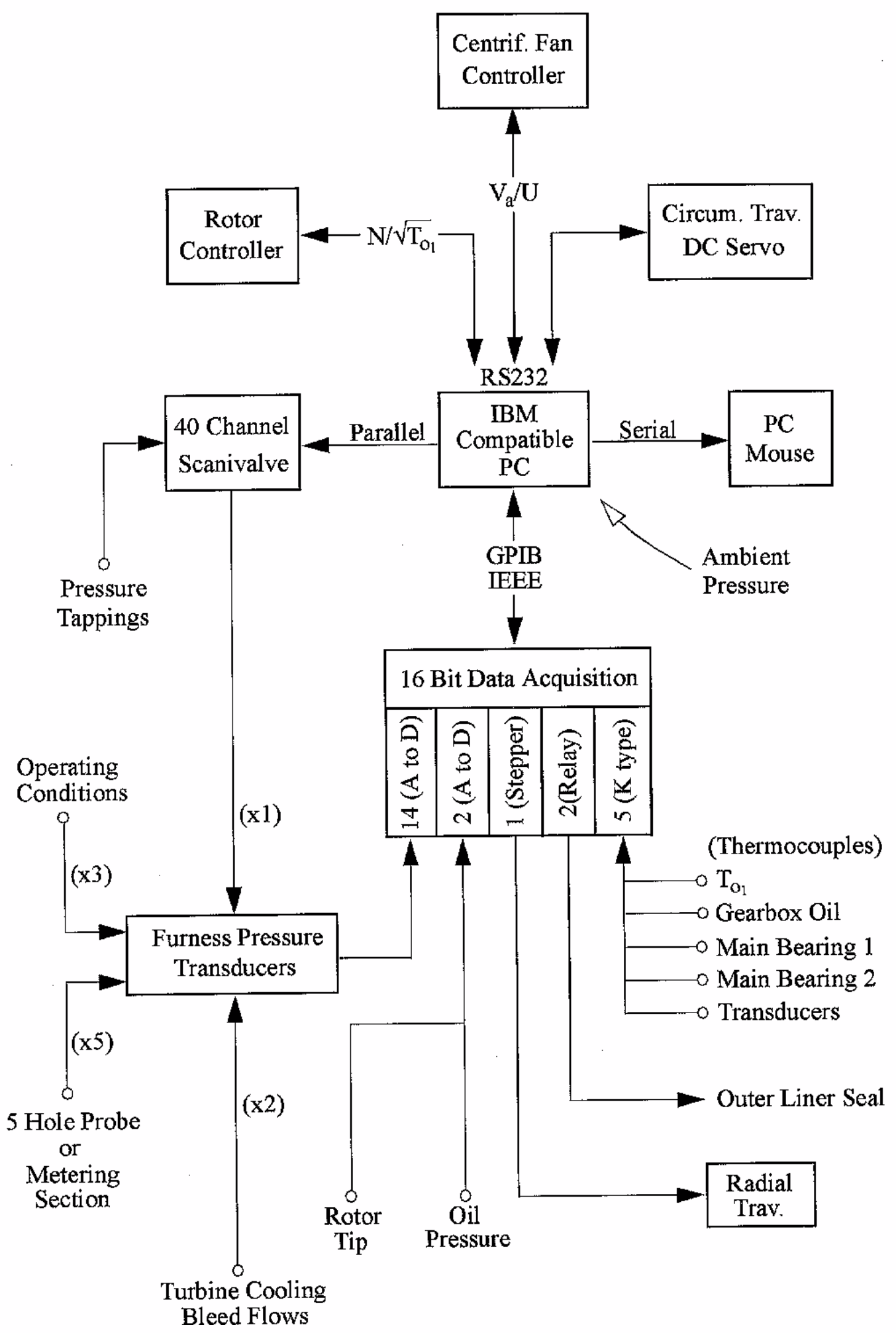

Figure 3.4 Instrumentation System 


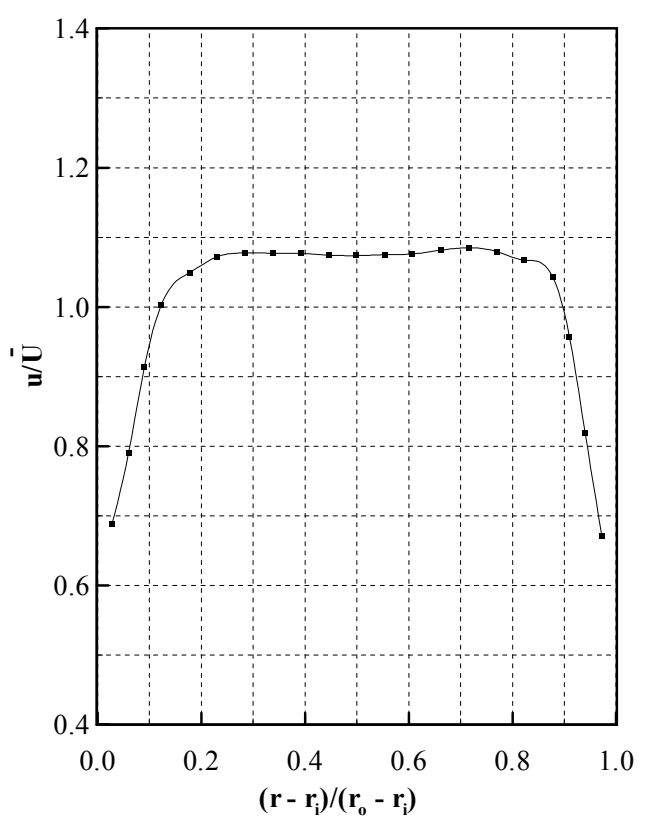

(a) Circumferentially Averaged Axial Velocity Profile

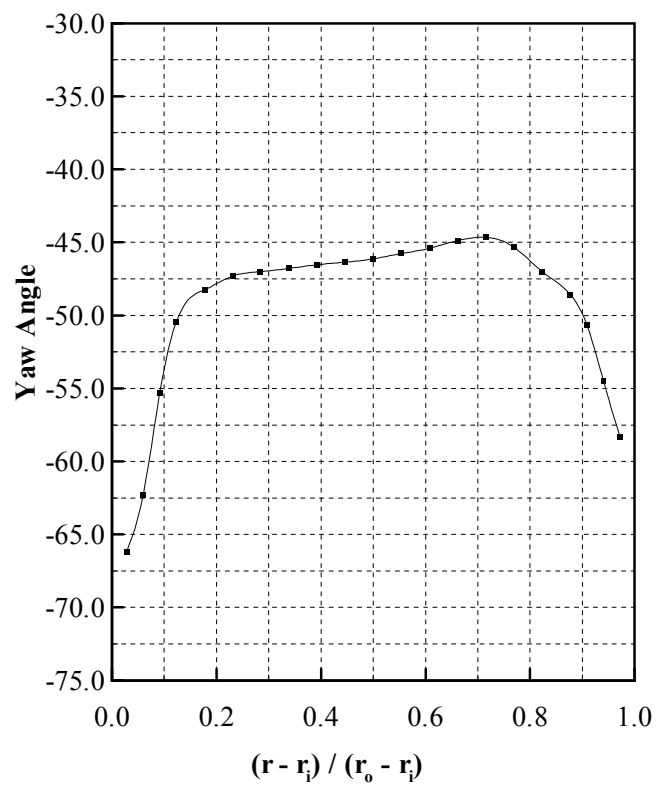

(c) Circumferentially Averaged Swirl Angle

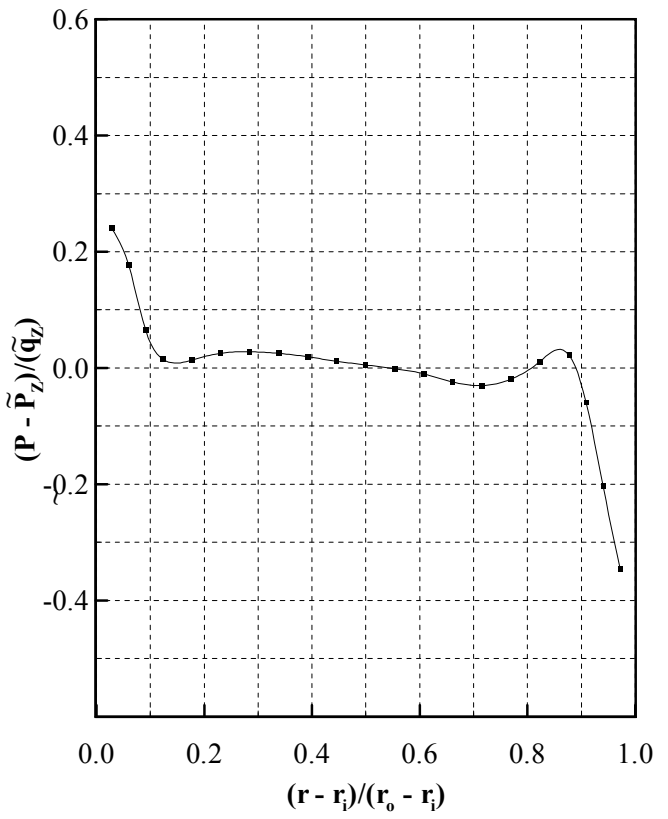

(b) Circumferentially Averaged Total Pressure Profile

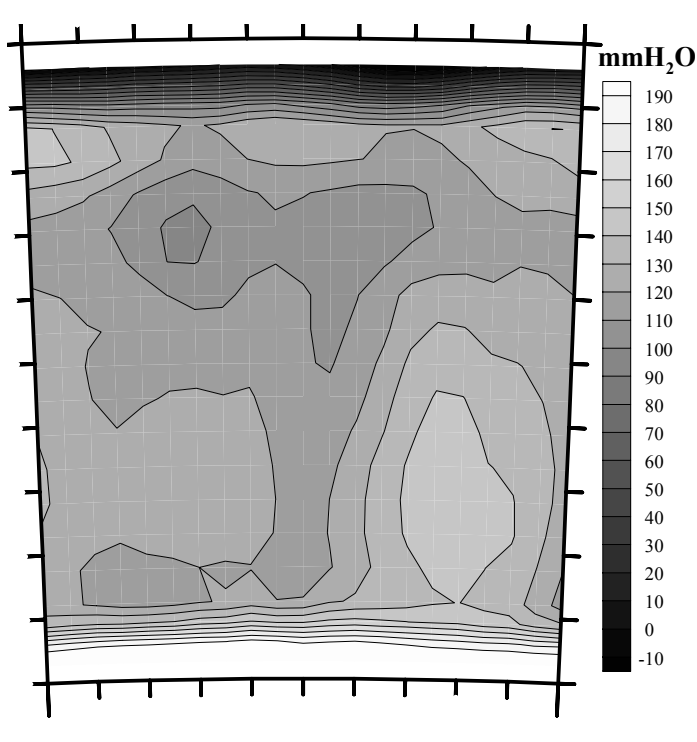

(d) Total Pressure Contours

Figure 3.5 Rotor Exit Conditions 


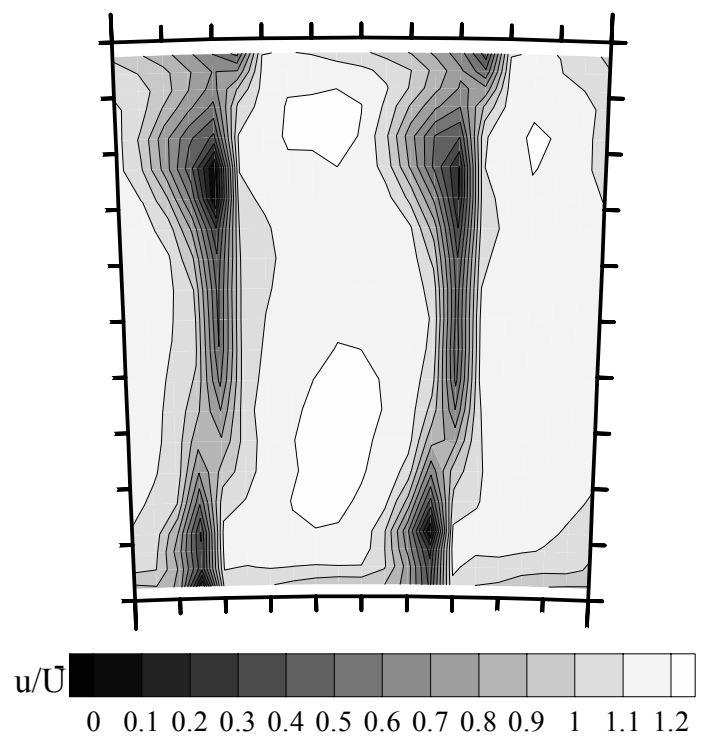

(a) Normalised Axial Velocity Contours

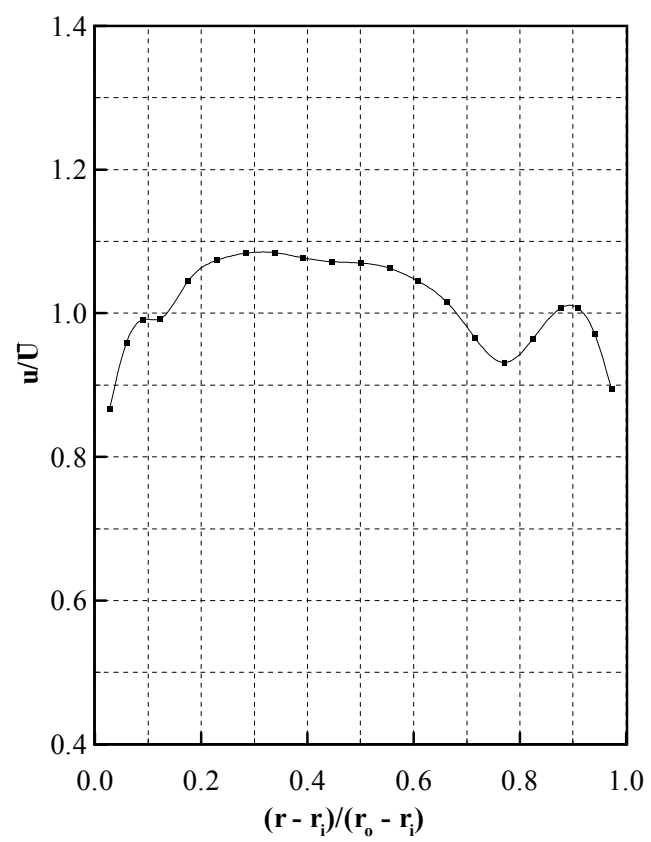

(c) Circumferentially Averaged Axial Velocity Profile

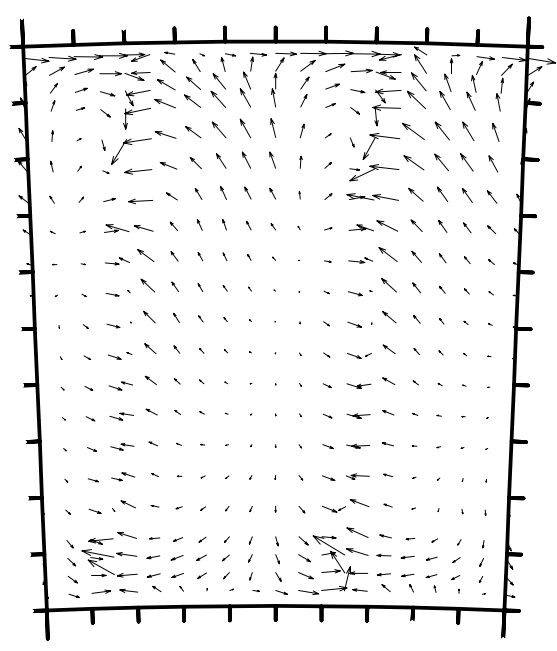

(b) Projected Total Velocity Vectors

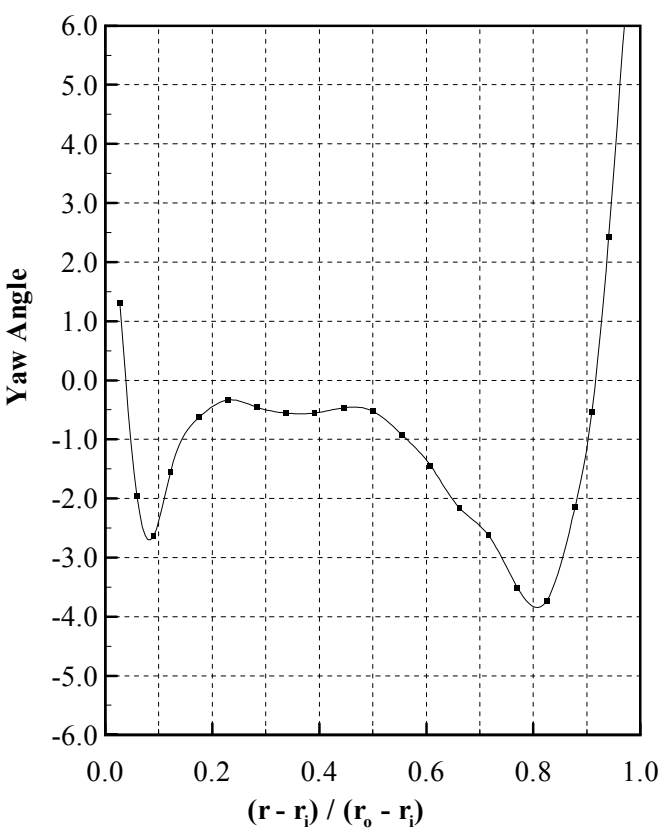

(d) Circumferentially Averaged Swirl Angle

Figure 3.6 OGV Exit Conditions 


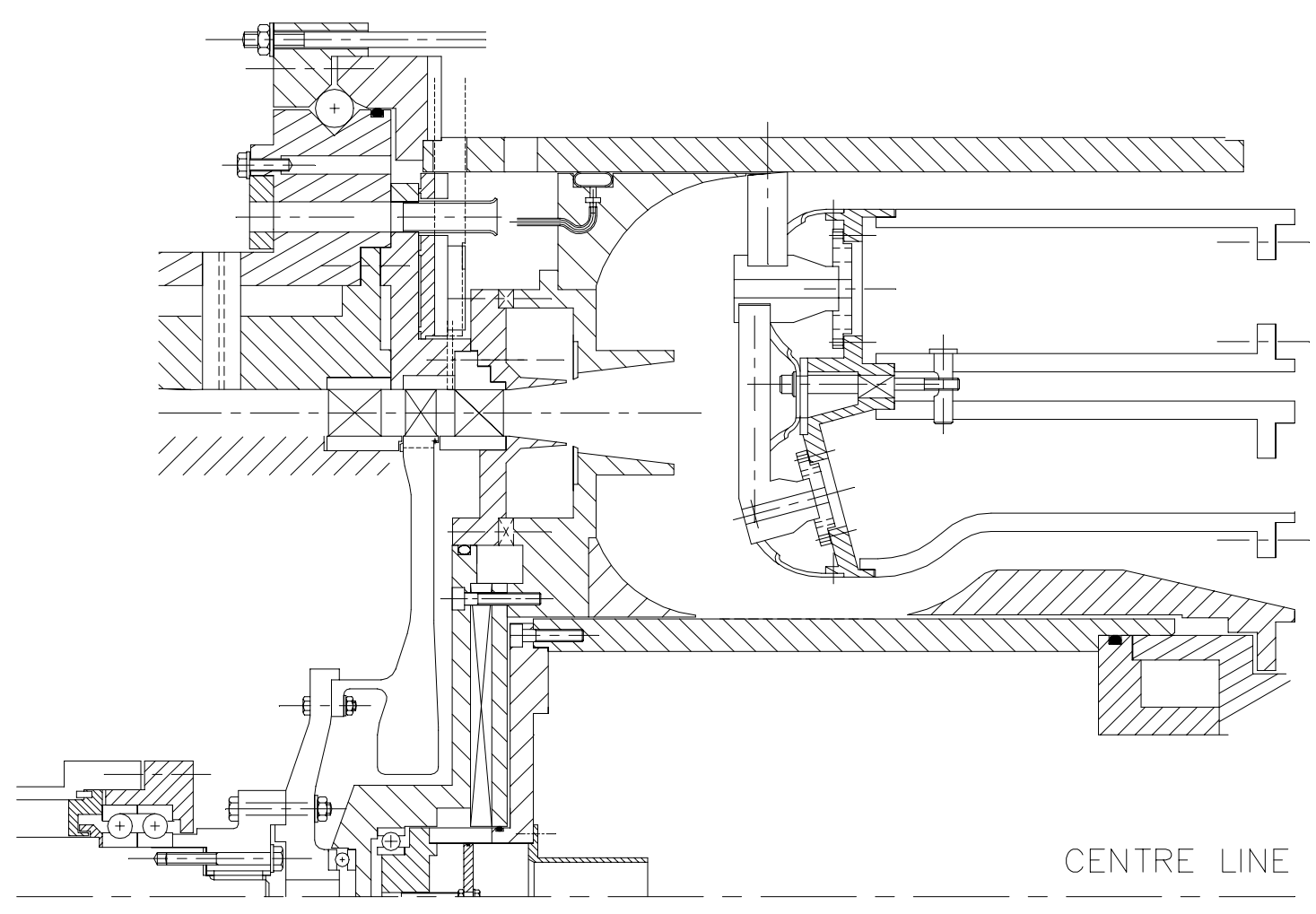

(a) Build 1

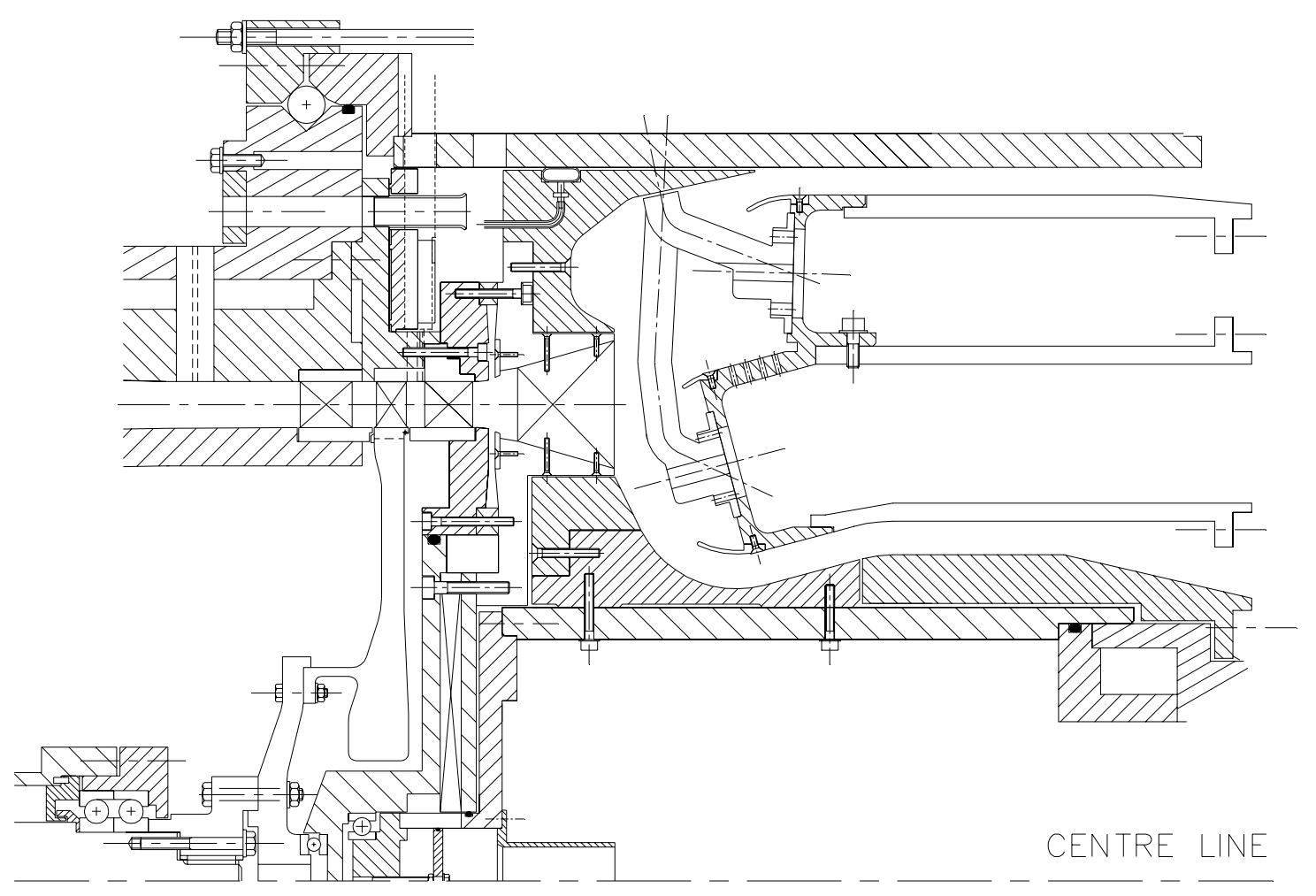

(b) Build 2

Figure 3.7 Flame Tube Geometry 


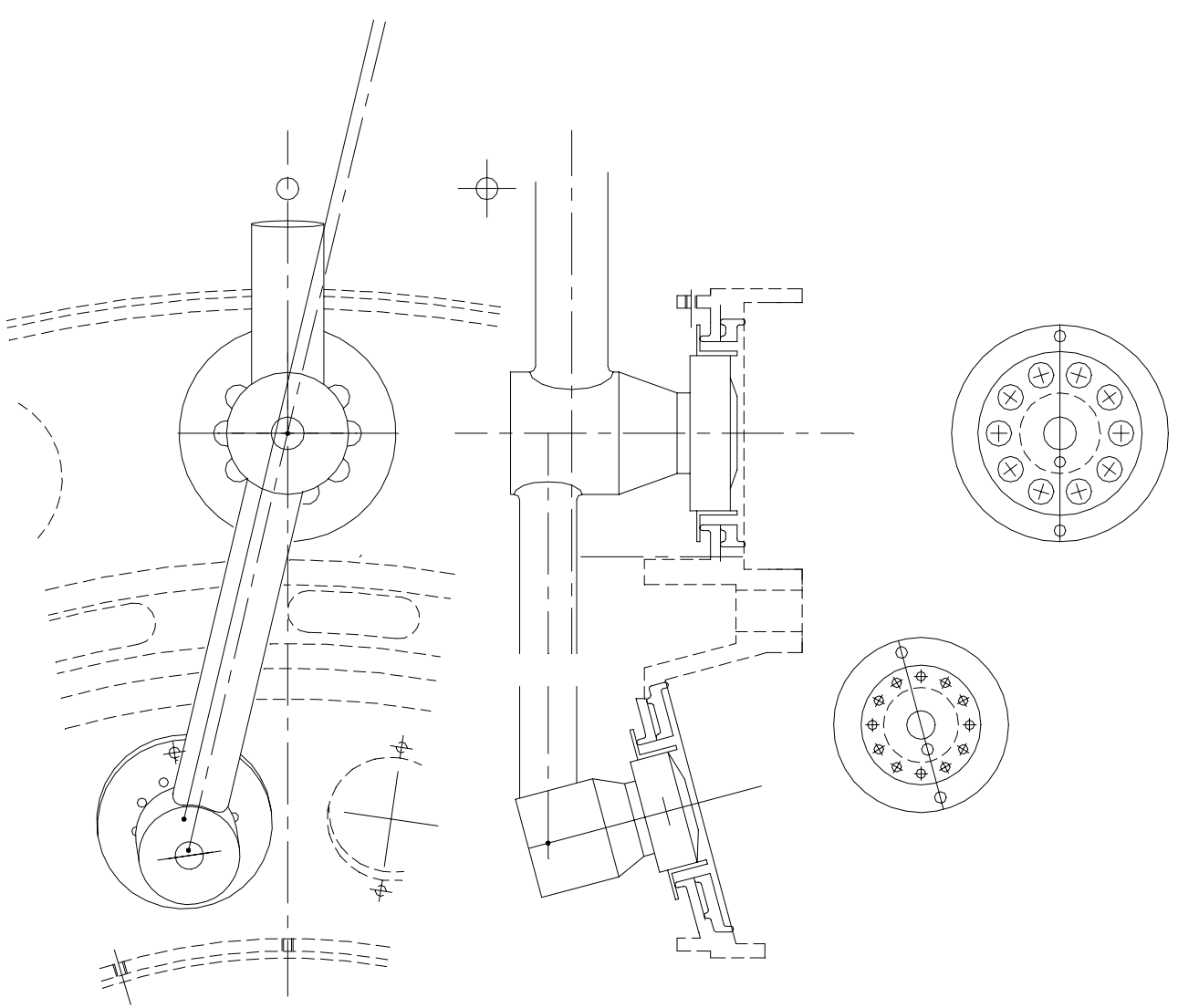

Figure 3.8 Burner Schematic

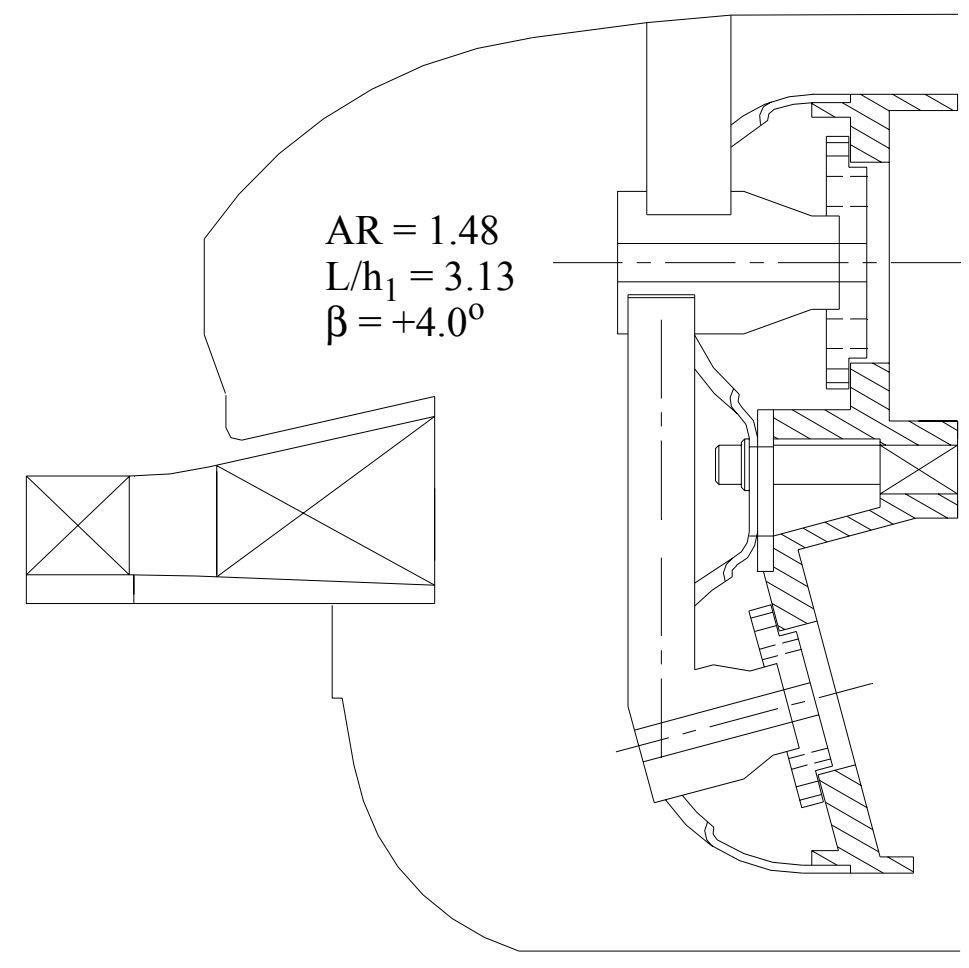

Figure 3.9 Conventional Datum Diffuser 


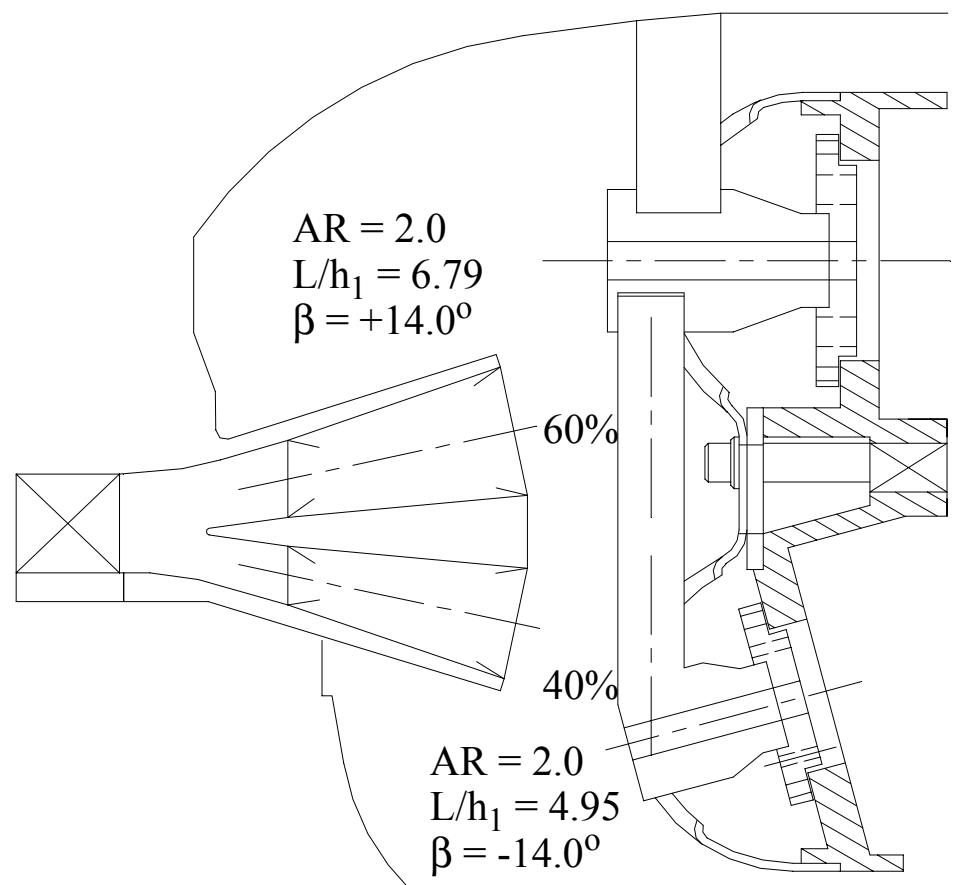

Figure 3.10 Optimum Conventional Diffuser

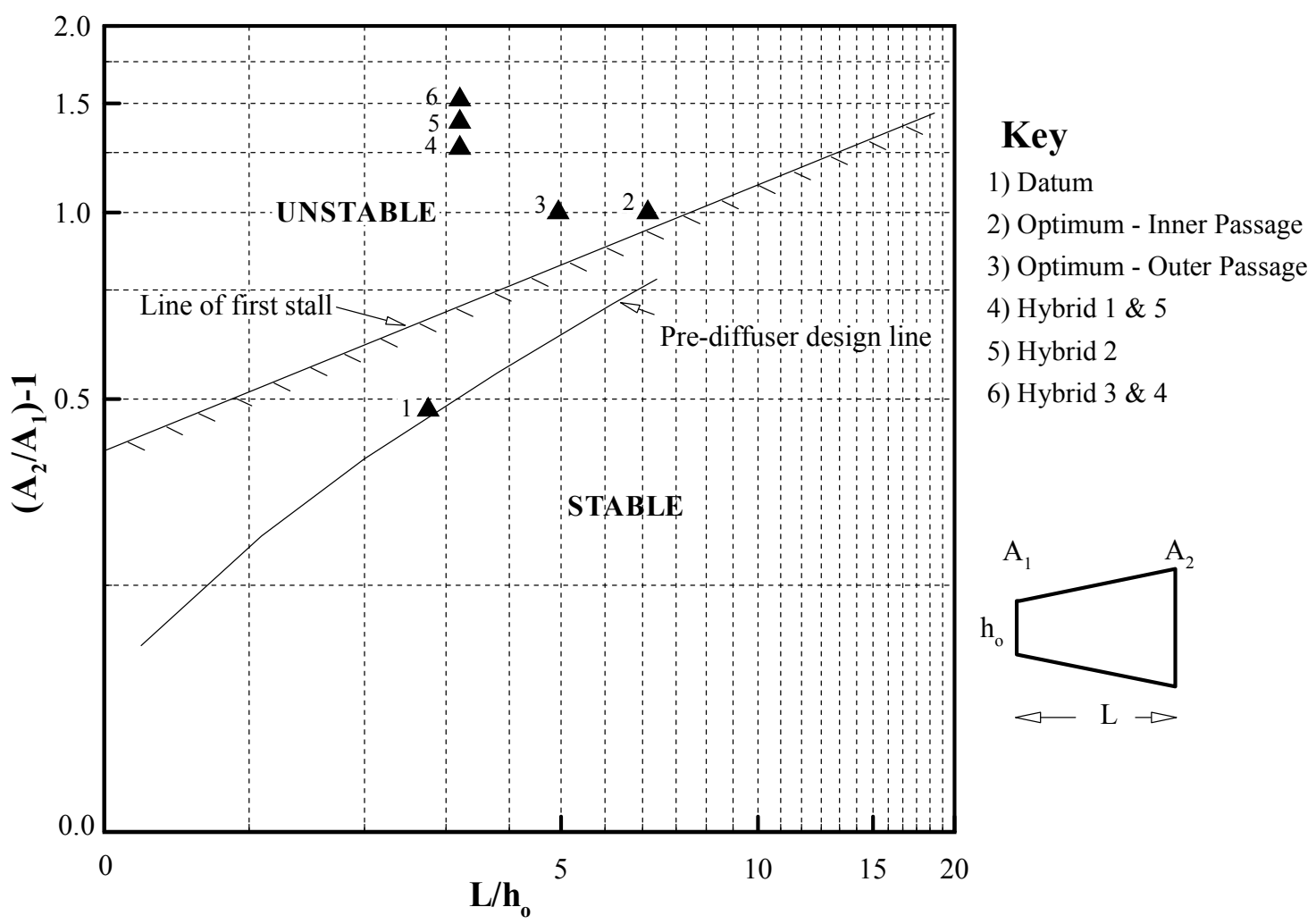

Figure 3.11 A Comparison with Nominal Design Curves 


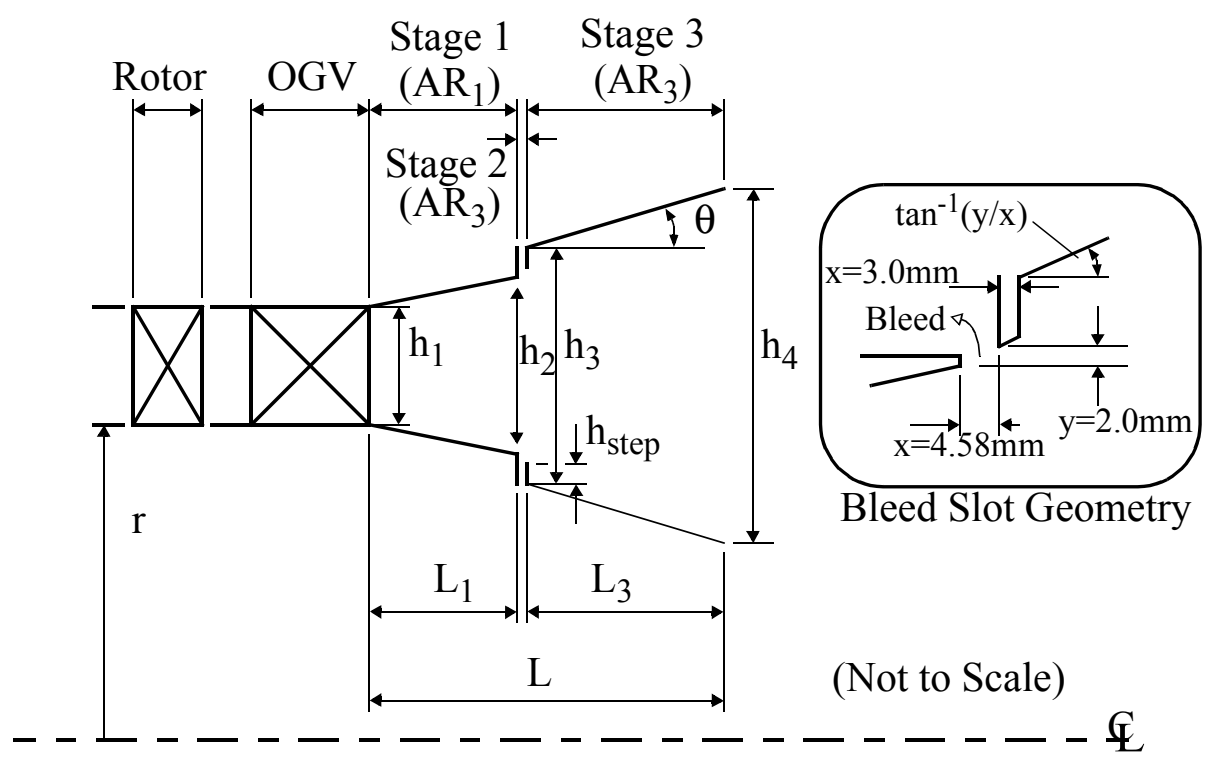

Figure 3.12 Hybrid Pre-Diffuser Geometry Definition

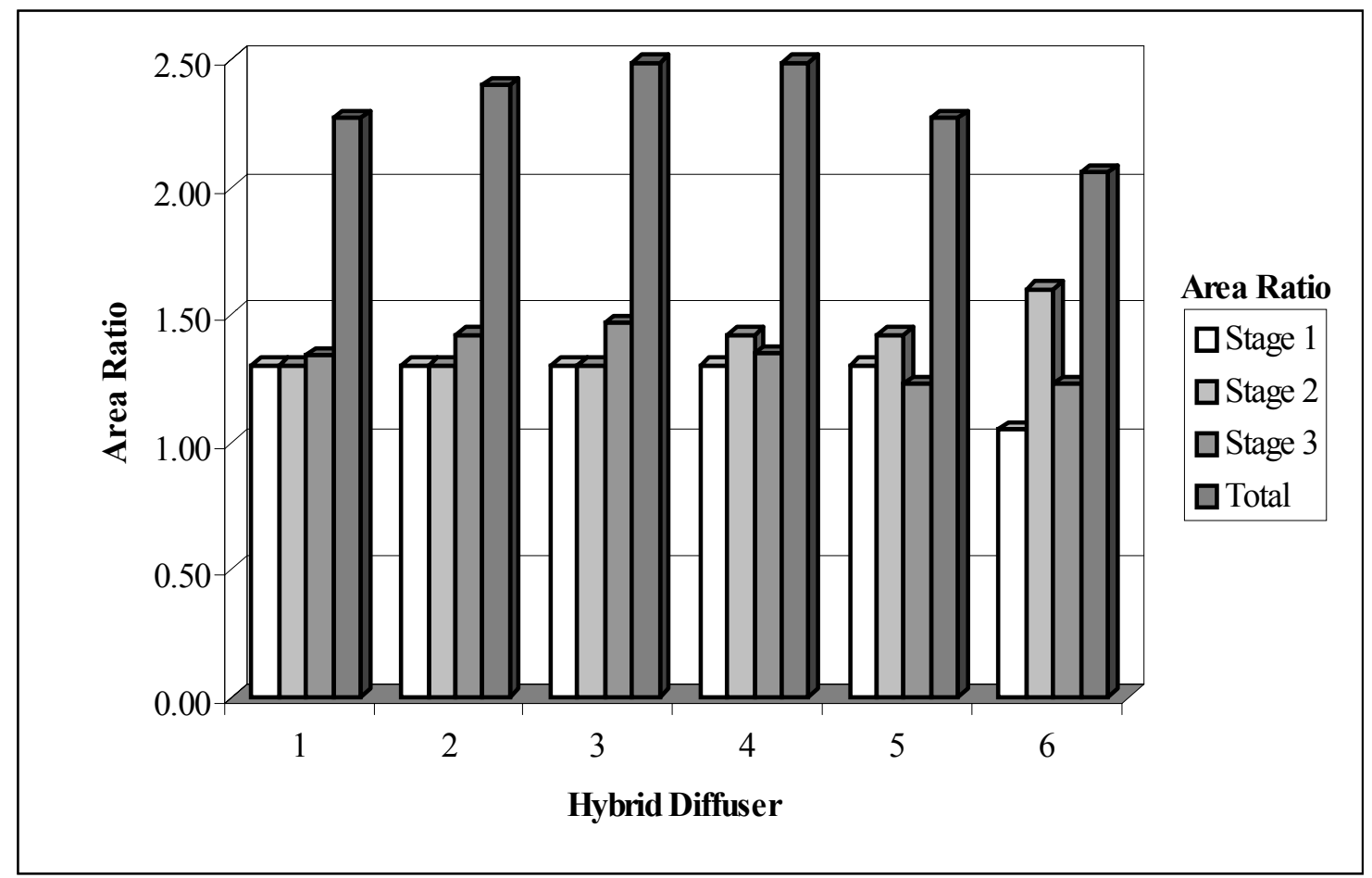

Figure 3.13 Hybrid Diffuser Area Ratio Distribution 


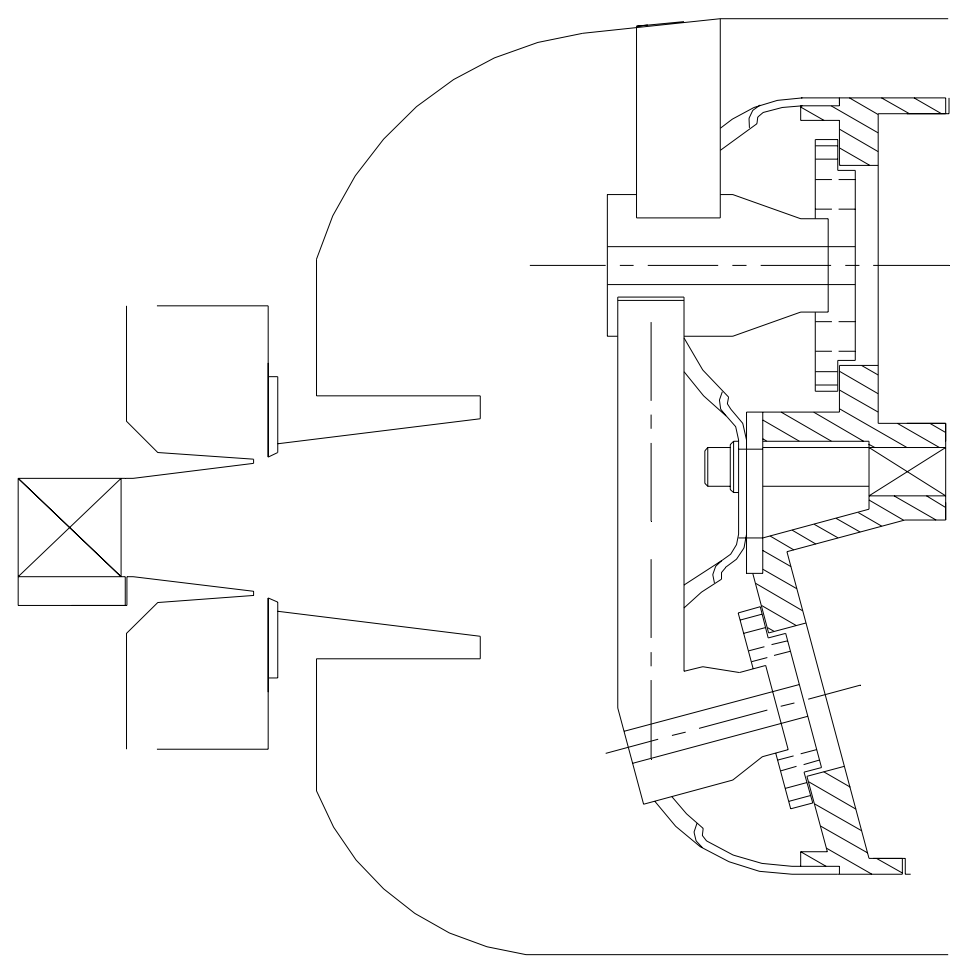

(a) Hybrid Vortex Controlled Diffuser

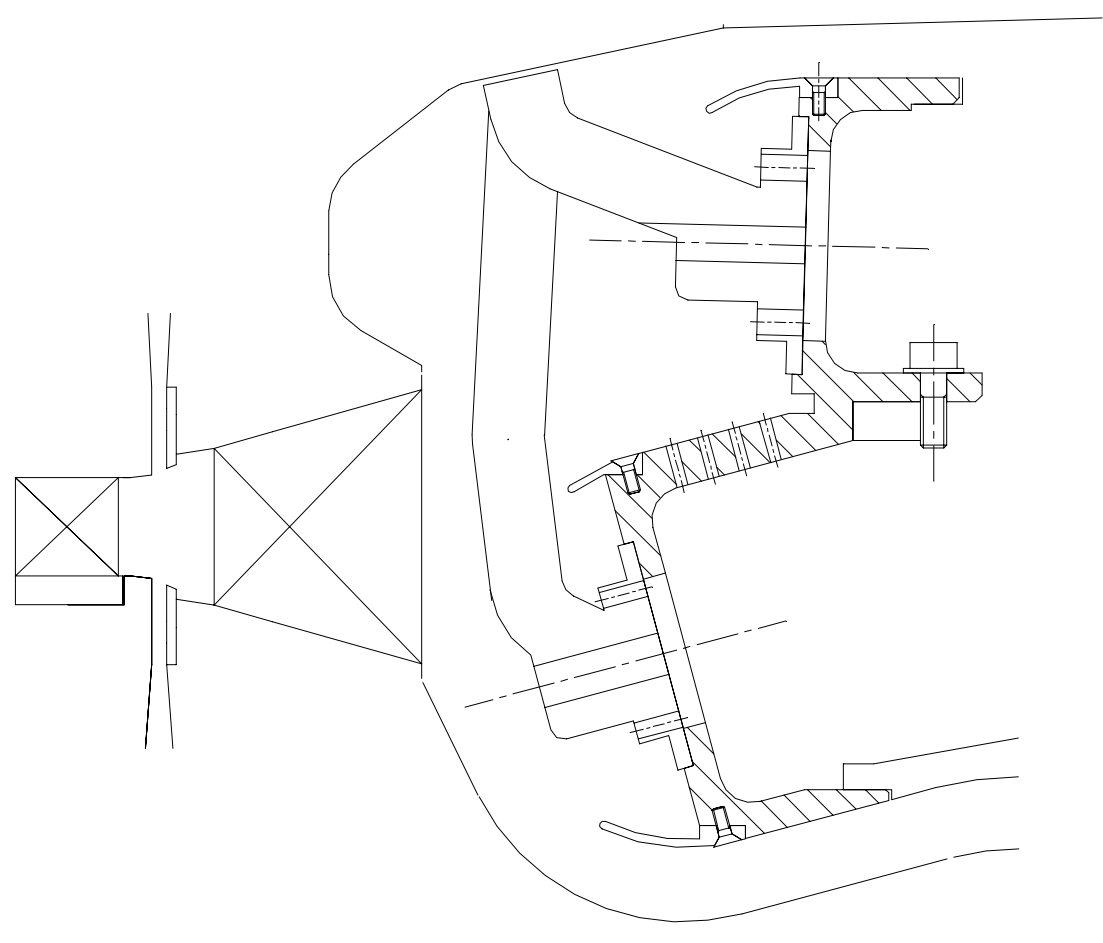

(b) Hybrid Diffuser

Figure 3.14 Hybrid Diffuser Options (Refer to Table 3.6) 


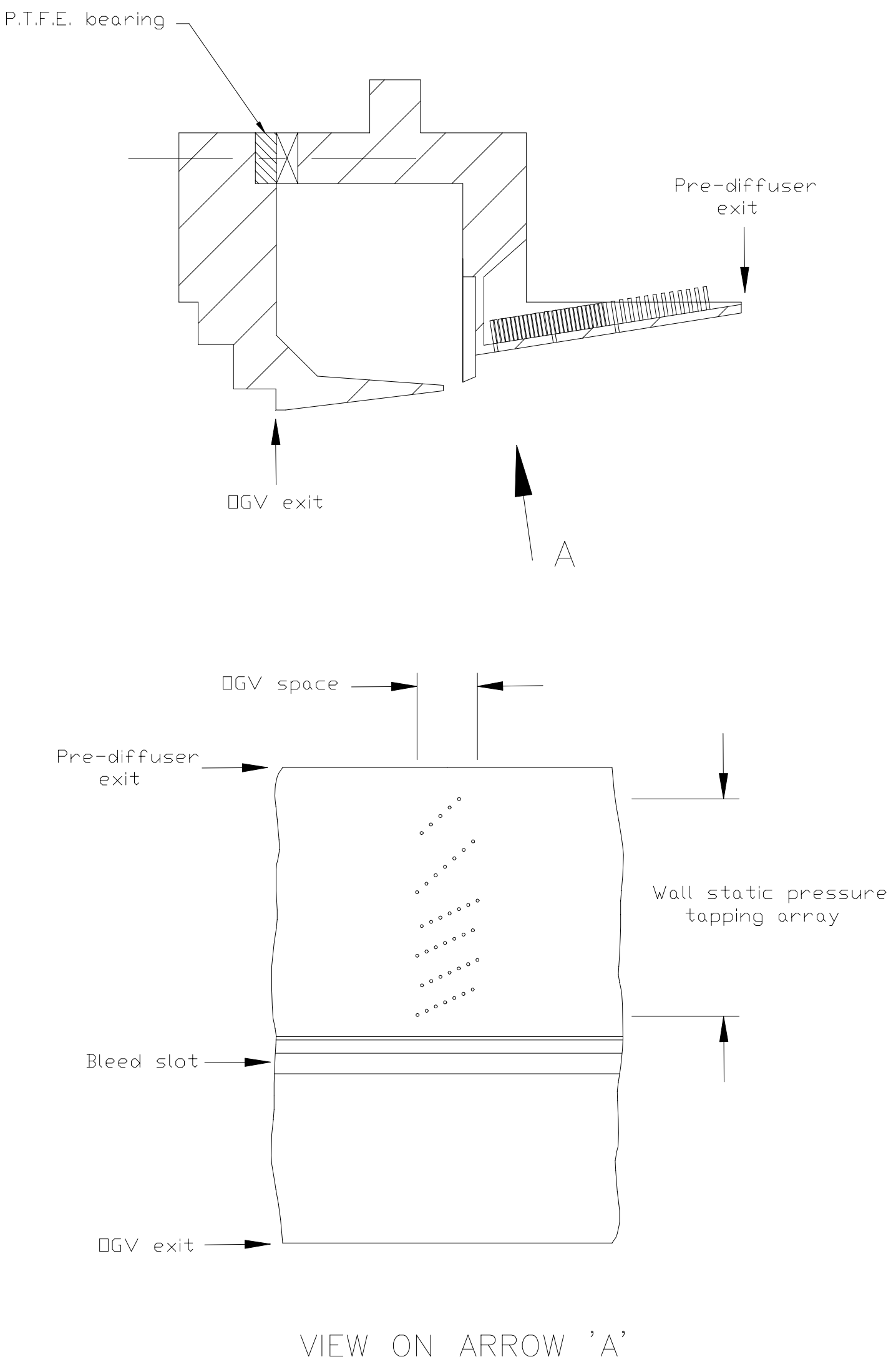

Figure 3.15 Static Pressure Tapping Arrangement on Stage 3 Outer Wall 


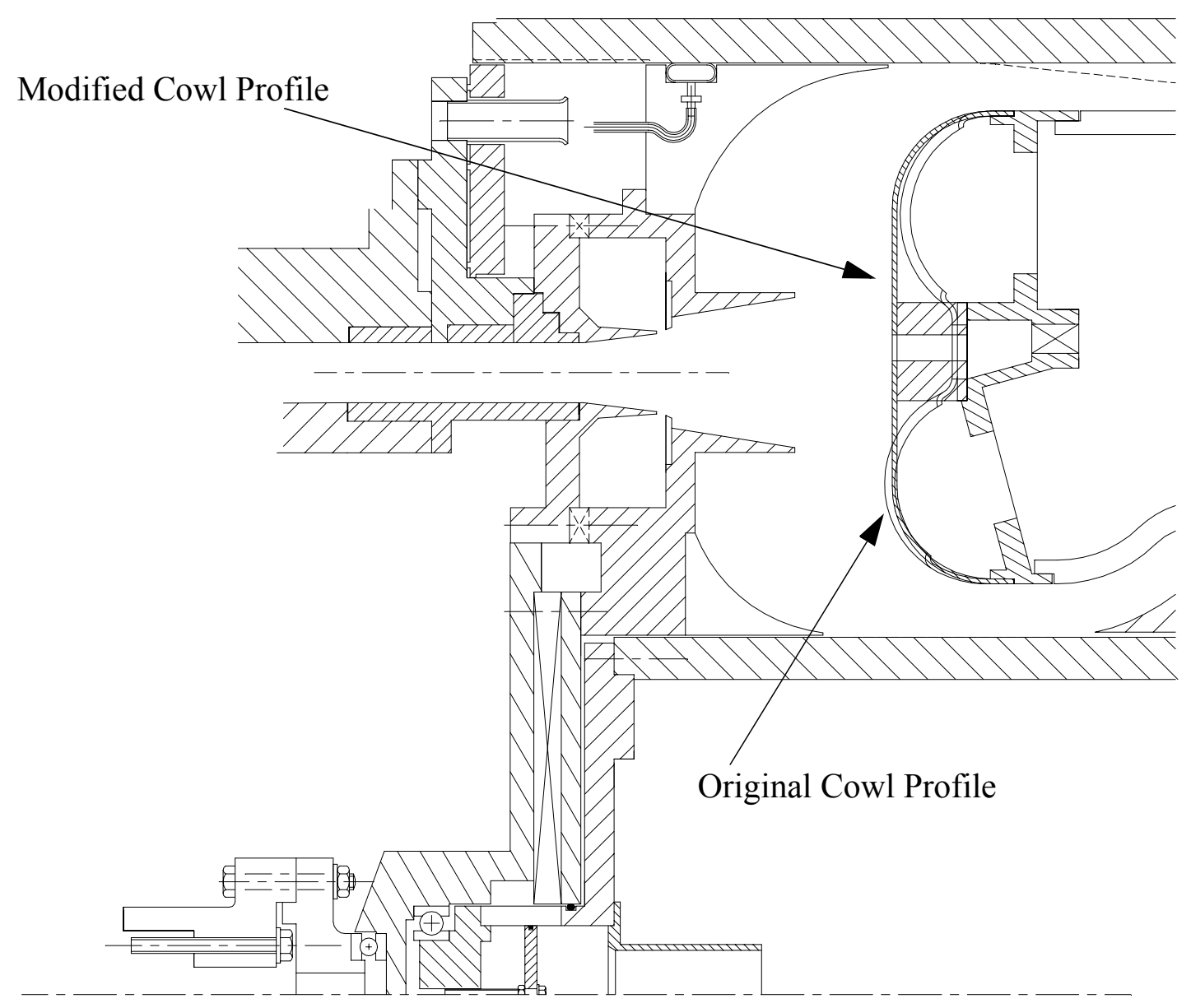

Figure 3.16 Modified (Two-Dimensional, Axi-Symmetric) Test Section

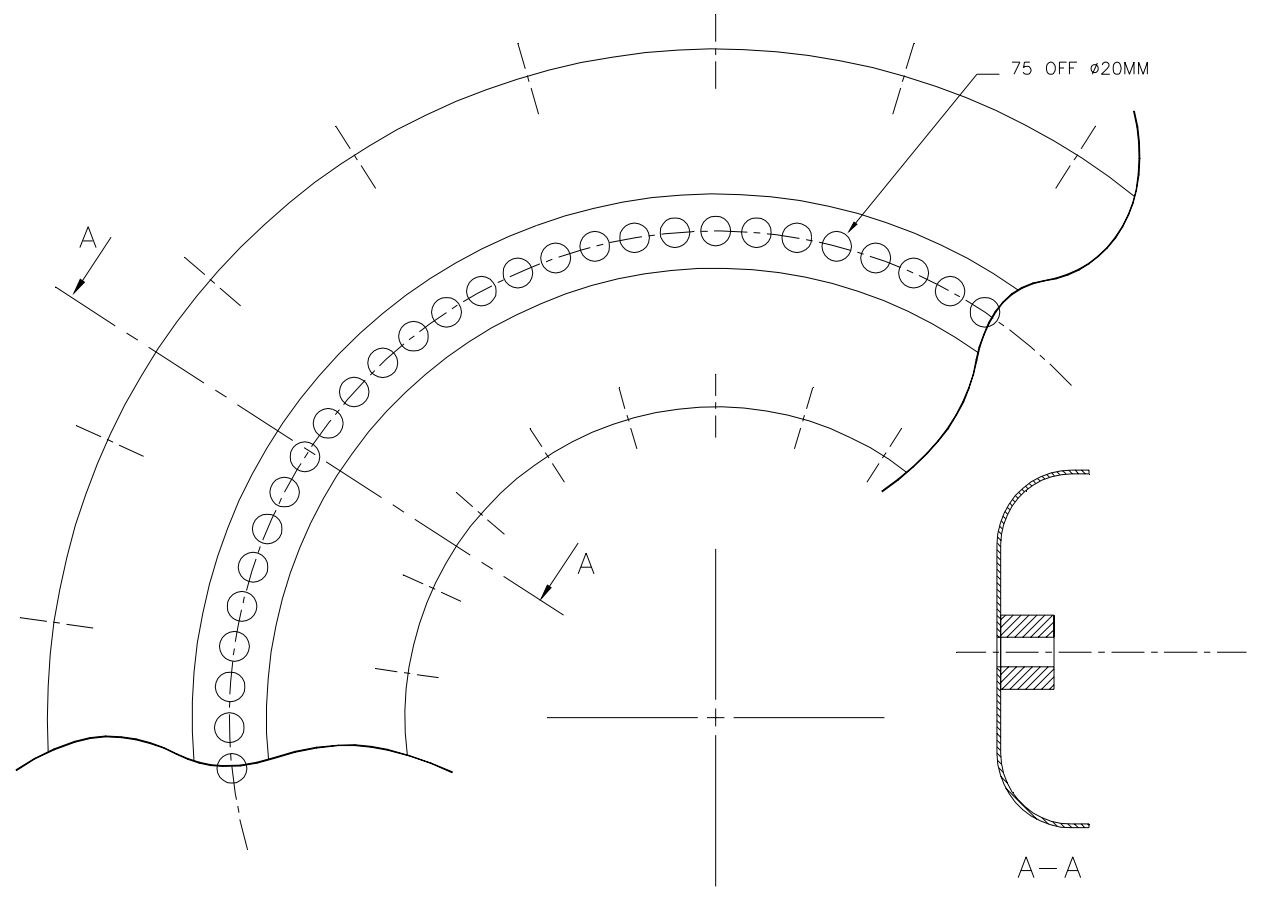

Figure 3.17 Modified Cowl Geometry 


\subsection{EXPERIMENTAL RESULTS AND DISCUSSION}

4.1 Build 1 Flame Tube $\ldots \ldots \ldots \ldots \ldots \ldots \ldots \ldots \ldots \ldots \ldots \ldots$

4.1.1 Diffuser Performance . . . . . . . . . . . . . . . . . . 117

4.1.2 Hybrid 4 Stage 3 Wall Static Pressure Tapping Data .... . . 122

4.1.3 Outer Feed Annulus Performance . . . . . . . . . . . . . . . 124

4.1.4 Splitter Feed Annulus Performance . . . . . . . . . . 126

4.1.5 Inner Feed Annulus Performance . . . . . . . . . . . . . 127

4.1.6 Burner Feed Performance . . . . . . . . . . . . . . 128

4.1.7 Dump Losses . . . . . . . . . . . . . . . . . . . . . . . . . . . . 129

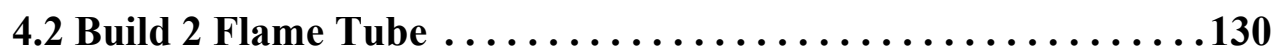

4.2.1 Diffuser Performance $\ldots \ldots \ldots \ldots \ldots \ldots \ldots \ldots \ldots \ldots \ldots$

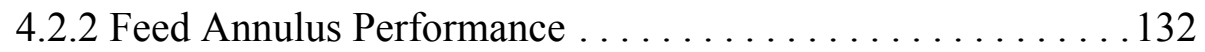

4.3 Bleed Flow Assessment $\ldots \ldots \ldots \ldots \ldots \ldots \ldots \ldots \ldots \ldots \ldots \ldots$

4.3.1 Effect of Bleed Rate on Diffuser Performance . . . . . . . . . 133

4.3.2 Vortex Chamber versus Duct Bleed ... . . . . . . . . . . . 134

4.3.3 Bleed Flow Total Pressure Loss . . . . . . . . . . . . . 135

4.4 Two-Dimensional, Axi-Symmetric Flame Tube Arrangement . . 137

4.4.1 Two-Dimensional Inlet (Clean) $\ldots \ldots \ldots \ldots \ldots \ldots \ldots \ldots$

4.4.2 Three-Dimensional Inlet Conditions (Rotor) . . . . . . . 138

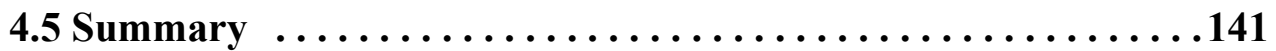

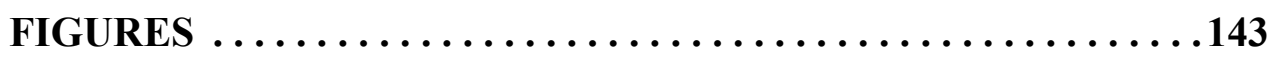




\subsection{EXPERIMENTAL RESULTS AND DISCUSSION}

\subsection{Build 1 Flame Tube}

\subsubsection{Diffuser Performance}

Diffuser performance was assessed using the methods described in Section 3 by conducting five-hole probe area traverses at rotor exit (station 2) and pre-diffuser exit (station 4). Due to the presence of the bleed chamber access for instrumentation was not available at OGV exit (station 3) and therefore, for all the diffusers, the reference/inlet plane for all performance parameters was rotor exit. Hybrid diffuser data are quoted for the minimum bleed requirement (unless otherwise stated). This corresponds to the minimum amount of bleed required for the hybrid diffuser to move from a state of stall into a stable flow regime (refer to Section 2.6). Work conducted by Adkins et al ${ }^{[1980]}$ and Myres et al ${ }^{[1994]}$ suggested that the minimum bleed requirement would be in the region of 1 to $3 \%$ of total inlet mass flow bled through each vortex chamber/bleed duct. It can be seen from Table 4.1 that the current results concur with this. The minimum bleed requirement for each hybrid diffuser was determined experimentally by systematically increasing the bleed levels and conducting an area traverse at diffuser exit. As an example of this Figure 4.1 illustrates the diffuser exit velocity profiles for Hybrid 4 at various bleed rates and indicates that the minimum bleed requirement was found to be $3.0 \%$ of the inlet mass flow bled through each chamber. At bleed rates below this the diffuser has either stalled or is close to stall. As suggested by Adkins et al ${ }^{[1980]}$, the minimum required bleed increases with diffuser loading such that, at an area ratio of 2.49 , the most heavily loaded hybrid diffuser (Hybrid 4) also has the largest minimum bleed requirement.

Table 4.1 details the main diffuser performance parameters (as defined in Section 2.2) for all the diffusers tested with the Build 1 flame tube. The Datum diffuser (Figure 3.8) represents current 'engine-in-service' technology and the Optimum Conventional diffuser (Figure 3.9) is a twin passage diffuser which represents the limit to which conventional technology can be taken. Thus, these two conventional diffusers offer a benchmark by which to measure any increase in performance from hybrid diffusers. The Datum diffuser has the lowest area ratio and as expected returns the smallest mass weighted static 
pressure recovery coefficient of 0.63 but with a low aerodynamic loading it has a low mass weighted total pressure loss coefficient of 0.16 . The Optimum Conventional diffuser has a much higher area ratio and yields a significant increase in performance over the Datum diffuser; the inner and outer passages have static pressure recovery coefficients of 0.72 and 0.71 respectively. As described in Section 2.5 a splitter vane increases the effective non-dimensional length of the resulting passages. This allows a higher overall area ratio to be achieved but increases skin friction losses due to a larger surface area. However, the final mass weighted total pressure loss coefficients of 0.15 and 0.17 for the inner and outer passages respectively are similar to that of the Datum diffuser.

The Hybrid 1 diffuser has similar performance figures to the Optimum Conventional diffuser with a static pressure recovery coefficient of 0.71 and a slightly increased loss coefficient of 0.18 . It is important to note when directly comparing the performance of the conventional and hybrid pre-diffusers that the conventional pre-diffusers contain struts whereas the hybrid pre-diffusers do not. The inclusion of struts will increase the loss because of increased skin friction but can, potentially, increase pressure recovery by removing swirl. Initially Hybrid 1 may seem to have a poor level of performance for a diffuser with an overall area ratio of 2.27. Certainly it is operating at a much lower effectiveness than the conventional diffusers. (Note it is difficult to calculate an ideal static pressure rise, and hence an effectiveness due to the presence of the OGVs between the measurement stations. The expression for an ideal diffuser, $\mathrm{C}_{\mathrm{p}}{ }^{\prime}=1-\mathrm{AR}^{-2}$, is not appropriate). However, Figure 3.11 shows that at this area ratio and non-dimensional length conventional diffusers would undoubtedly stall. Figure 3.11 is plot of area ratio versus non-dimensional length and shows an experimentally determined line of first stall for conventional diffusers (Sovran and Klomp ${ }^{[1967]}$ ). It is a notable fact that all the hybrid diffusers are well above this line and, with the correct amount of bleed, remain fully attached.

The change from Hybrid 1 to Hybrid 2 involved an increase in overall area ratio to 2.4 which was achieved by increasing the divergence angle of the Stage 3 diffuser from 7 to 9 degrees. This resulted in only a very slight increase in the static pressure recovery coefficient; it remained almost constant at 0.72. This suggests Hybrid 2 is less efficient than Hybrid 1 as an area ratio increase has not yielded a higher static pressure recovery. A fur- 
ther increase in Stage 3 wall angle to 11 degrees and overall area ratio to 2.5 (Hybrid 3) also resulted in very little change in static pressure recovery although the total pressure loss increases to 0.2. Increases in the Stage 3 loading had little effect on diffuser performance thus, for Hybrid 4, the Stage 2 area ratio was increased from 1.32 to 1.4 while maintaining an overall area ratio of 2.5 (i.e. diffuser loading was redistributed from Stage 3 to Stage 2).

\begin{tabular}{|l||c|c|c|c|c|c|}
\hline Diffuser & $\begin{array}{l}\text { Area } \\
\text { Ratio }\end{array}$ & $\mathrm{L} / \mathrm{h}_{1}$ & $\begin{array}{c}\text { Bleed Req. } \\
\text { (inner/outer) } \\
\dot{\mathrm{m}}_{\mathrm{b}} / \dot{\mathrm{m}}_{\text {tot }}(\%)\end{array}$ & $\mathrm{C}_{\mathrm{p} 2-4}$ & $\lambda_{2-4}$ & $\alpha_{4}$ \\
\hline \hline Datum* & 1.48 & 3.13 & - & 0.63 & 0.16 & 1.58 \\
\hline Opt. Conv. & 2.0 & 6.79 & - & 0.72 & 0.15 & 2.01 \\
(inner/outer) & & 4.95 & 1.5 & 0.71 & 0.18 & 1.25 \\
\hline Hybrid 1 & 2.27 & 3.5 & 1.5 & & 0.71 & 0.17 \\
\hline Hybrid 2 & 2.40 & 3.5 & 2.0 & 0.72 & 0.18 & 1.4 \\
\hline Hybrid 3 & 2.49 & 3.5 & 2.5 & 0.71 & 0.20 & 1.45 \\
& & & 2.5 & & & \\
\hline Hybrid 4 & 2.49 & 3.5 & 3.0 & 0.71 & 0.20 & 1.45 \\
& & & 3.0 & & & \\
\hline Hybrid 5* & 2.27 & 3.5 & 3.0 & 0.71 & 0.19 & 1.55 \\
& & & 3.0 & & & \\
\hline
\end{tabular}

Table 4.1 Pre-Diffuser Performance Data (* - indicates strutted diffusers)

In summary the total pressure loss in the hybrid diffusers is higher than for either of the conventional diffusers and also increases further with area ratio. This is due to the inherent behaviour of hybrid diffusers; the separation and reattachment process incurring higher intrinsic aerodynamic losses.

For any type of hybrid diffuser to be placed in an operating aero gas turbine combustion 
system the presence of load bearing radial struts would be necessary and an important issue is whether the presence of these struts will effect the flow mechanism of hybrid diffusers. The positioning of the struts is discussed in Section 4.1.2 but it can be seen from Table 4.1 that the presence of struts in Hybrid 5 has no detrimental effect on overall diffuser performance.

Looking solely at the diffuser performance parameters, it does not initially appear that hybrid diffusers offer any significant increase in performance over their conventional counterparts. As the Stage 2 and Stage 3 area ratios are increased from Hybrid 1 to 4 there is effectively no change in static pressure recovery. All the un-strutted hybrid diffusers provide a higher static pressure recovery than the Datum diffuser but only offer comparable values to the Optimum Conventional diffuser. However, it will be shown later, that the exit conditions presented to the dump and flame tube are more favourable. The increased area ratio and exit height means that the hybrid diffusers perform a much higher degree of controlled turning, directing the flow, at diffuser exit, towards the combustor feed annuli. The required diffusion and turning within the dump gap is reduced and, as reported by Fishenden and Stevens ${ }^{[1977]}$, this will reduce the overall total pressure loss to the feed annuli.

Some evidence of the improved flow condition at diffuser exit can be seen by examining the kinetic energy coefficient, $\alpha$, at diffuser exit. Essentially $\alpha$ is a measure of the flow distortion; a value of unity indicating a flat velocity profile with the value increasing with the level distortion. The affect of flow distortion on the diffuser performance was discussed in Section 2.0 and in summary an increased level of distortion is detrimental. With reference to Equation 2.23 (Stevens et $\mathrm{al}^{[1978]}$ ) an increase in the kinetic energy coefficient at diffuser exit potentially increases the total pressure loss. A highly distorted flow has a loss which is "locked into the profile". Such a flow, allowed to mix out to a uniform profile will incur a higher total pressure loss than a less distorted flow (i.e lower $\alpha$ ). The kinetic energy coefficient for each diffuser is given in Table 4.1. However, it must be noted that a strutted diffuser will generally have a higher value of $\alpha$ due to flow distortions caused by the strut wake. Thus, only the kinetic energy coefficient for Hybrid 5 can be sensibly compared to the Datum and Optimum Conventional diffusers. The Datum diffuser has an $\alpha$ value of 1.58 but this increases above a value of 2 for the Opti- 
mum Conventional diffuser. This is due to the extra curvature of the twin-passage diffuser introducing a biased and peaked velocity profile. However, at exit to the Hybrid 5 diffuser the kinetic energy coefficient has a value of 1.55 and is similar to that of the Datum diffuser. In addition to the Hybrid 5 diffuser achieving a significant increase in area ratio this relatively low level of flow distortion suggests that the feed to the flame tube annuli will be superior to the conventional diffusers.

Figure 4.3 shows the axial velocity contours, normalised by the average axial velocity, at exit to the various diffusers at the bleed rates indicated in Table 4.1. For the Datum diffuser the plot shows a complete burner sector (i.e. strut to strut) which equates to seven OGV spaces and represents a repeating sector. The struts wakes are clearly visible as areas of low velocity (dark grey regions) at the circumferential extremities of the sector. The OGV wakes are also seen as areas of locally lower velocity separated by the higher velocity non-wake flow (i.e. lighter contours). The different nature between adjacent OGV wakes is due to the existence of IGV wakes persisting through the rotor. Axial velocity contours for Hybrid 1-4 (Figure 4.3(b)-(e)) are shown for two OGV spaces only as these are un-strutted diffusers and flow phenomena are repeated every two OGV spaces.

Velocity vectors at diffuser exit (Figure 4.4) illustrate the effect of the high divergence angle in the Stage 3 section which encourages the flow to turn outboard and inboard towards the outer and inner feed annuli. The magnitude of the radial velocity component is relatively small and increases only marginally from the Datum diffuser through to Hybrid 5. However, recall that the hybrid diffusers, especially Hybrid 4 and 5, have a much larger exit height and as such are providing a much higher degree of controlled turning. Although increased performance is not obvious at diffuser exit, this is another indication that the hybrid diffusers will provide improved feed to the flame tube and feed annuli. This tendency to turn is shown more clearly in the circumferentially averaged pitch angle (Figure 4.5). At diffuser exit, close to the walls, the average flow is angled outboard and inboard at \pm 30 degrees thus directing it towards the feed annuli.

Velocity vectors also indicate that there is a higher residual swirl (persisting from the rotor) at diffuser exit for all non-strutted diffusers. This swirl will persist into the feed 
annuli and, due to conservation of angular momentum, its magnitude will diminish in the outer annulus but increase in the inner annulus as observed by Bailey and Carrotte ${ }^{[1996]}$. This will have a significant and detrimental effect on the feed to the flame tube. Although struts impose a loss penalty due to increased 'wetted' area they can be beneficial as they damp out residual swirl. This is clearly seen in the circumferentially averaged swirl angle profiles (Figure 4.6). The average diffuser exit swirl angle for the Datum and Hybrid 5 (both strutted) diffusers is small. However, with no struts present the other diffusers have much higher levels of swirl; approaching -5 degrees at mid height. Hybrid 4 is the most heavily loaded diffuser in the Stage 2 and 3 section and as such increased boundary layer growth and reduced axial velocity results in the increased swirl angle seen close to the outer wall (Figure 4.6).

\subsubsection{Hybrid 4 Stage 3 Wall Static Pressure Tapping Data}

In hybrid diffusers including a step expansion, the mainstream flow separates from the tip of the fence and, under the action of the bleed flow, reattaches at some point downstream on the Stage 3 diffuser wall. The low energy fluid close to the wall in the Stage 1 diffuser is unable to overcome the pressure gradient set up by the bleed flow. It is diverted and rapidly accelerates into the bleed as shown in Figure 4.7. Adkins et al ${ }^{[1980]}$ suggest the remaining air flows into the Stage 3 diffuser and will begin to decelerate and thus a shearing action is set up between the bleed flow and the mainstream flow. This creates a turbulent shear layer and re-energises the mainstream flow enabling it to negotiate the step expansion and reattach on the Stage 3 wall. A Coanda bubble is set up in the corner immediately downstream of the fence; the size and rotational strength of this is a function of the bleed rate.

It is the interaction of the bleed flow and mainstream flow which combine to cause the flow to reattach on the Stage 3 wall. Thus it is of interest to determine what effect the level of bleed has on this process. From the work of Adkins et al ${ }^{[1980]}$ it is already known that there is a minimum level of bleed below which the flow will not reattach. Experimentally it is very difficult to measure the effect of the bleed rate on the flow mechanisms near the transition from Stage 2 to Stage 3. Pressure probes are intrusive and can, by their very nature, destroy the flow mechanism under investigation. Furthermore, the 
test facility is complex and it is extremely difficult to position a pressure probe in the region immediately downstream of the step expansion (Stage 2 diffuser). Therefore, in an attempt to study the effect of the bleed experimentally, an array of static pressure tappings on the Stage 3 wall of the Hybrid 4 diffuser were employed (refer to Section 3.5.3 and Figure 3.11). These pressure tappings were located only on the outer wall of the Stage 3 diffuser and as such do not necessarily give a true indication of the condition of the air flow. For example, below the minimum bleed requirement, the diffuser could be in a state of transitory stall. It would then be possible for the flow to be attached to the outer wall of the diffuser but separated from the inner wall. This may not be clear from the static pressure readings. Thus these results were not assessed in isolation but with careful consideration of diffuser exit data from five-hole probe measurements.

Figure 4.8 illustrates the variation of circumferentially averaged wall static pressure recovery (with respect to rotor exit) along the Stage 3 diffuser outer wall. It is important to note that there is only a relatively small static pressure rise within the Stage 3 diffuser with $C_{p}$ increasing by only 0.06 from 0.61 to 0.67 . Assuming that the Stage 1 diffuser operates with a typical effectiveness of about $80 \%$ it would produce a static pressure recovery coefficient of 0.32 . The measured overall static pressure recovery coefficient of the diffuser is about 0.70 thus the majority of the static pressure recovery must, therefore, be attributed to the step expansion (Stage 2 diffuser).

It is not possible to derive the exact position at which the flow reattaches from the static pressure data. What is clear, from Figure 4.8, is that the most rapid increase in wall static pressure occurs immediately downstream of the step; i.e. in the region associated with flow reattachment. Figure 4.9 illustrates how the location of the maximum wall static pressure gradient varies with bleed. This does not reveal the actual reattachment point but gives a good indication of how it varies with bleed. Simple computational predictions (see Sections 5.0 and 6.0) indicate reattachment should occur in the region 10-20 mm downstream of the step and reduce with increasing bleed. The location of the maximum gradient in wall static pressure agrees with this as does the variation of its location with bleed rate.

Figure 4.10 shows a typical contour plot of wall static pressure recovery with a bleed rate 
of nominally $3.0 \%$ each side. Contour plots for all the bleed rates show similar trends and the three-dimensionality of the flow can be seen. There exists a periodic variation of the wall static pressure recovery across the three OGV spaces shown. This suggests that the reattachment length will also vary periodically. Obviously there is an interaction between the OGV wake flow and the bleed which locally affects the reattachment mechanism. It is not possible to give an exact explanation for the behaviour but a study to extract this type of information would lend itself to the use of Computational Fluid Dynamics (CFD). As described in Sections 5.0 and 6.0 CFD predicts a solution for the entire flow domain thus revealing much more information on the nature of the flow. However, a three dimensional CFD study is not a simple task.

Figure 4.9 and Figure 4.10 both suggest that the flow reattaches on the outer wall at, and beyond, a point some $10 \mathrm{~mm}$ downstream of the step. As mentioned earlier, for a hybrid diffuser to be used in a practical aero gas turbine the presence of load bearing radial struts would be required. A major point of concern is the placement of these struts, more specifically the location of the leading edge with respect to the reattachment point. It was argued that one reason for the failure of the planar test facility (Appendix A1) was the destruction of the flow mechanisms by the end walls. Placing the strut leading edge within the Coanda bubble could also have the same effect. Information from the static pressure tapping data suggest that the strut leading edge should be placed no closer than $10 \mathrm{~mm}$ to the step. Thus, the 22 radial struts for Hybrid 5 were located $12 \mathrm{~mm}$ downstream of the step. This extra $2 \mathrm{~mm}$ providing a small margin for error as time constraints only allowed a single set of struts to be designed and manufactured. Results presented in Table 4.1 demonstrate that the inclusion of such struts does not have a significant effect on diffuser performance.

\subsubsection{Outer Feed Annulus Performance}

Table 4.2 details performance figures for the flow to the outer combustor feed annuli (station 7) with reference to rotor exit (station 2). Adenubi ${ }^{[1976]}$ comments that the total pressure loss from OGV exit to the combustor feed annuli should be no more than $30 \%$ to $40 \%$ of the OGV exit dynamic pressure. As reported in Section 3.0 the reference plane throughout this experimental investigation was rotor exit, not OGV exit, because of 
access constraints imposed by the bleed chambers. However, with the Datum diffuser fitted measurements were taken which enabled OGV performance to be determined (Table 3.3). Incorporating this data, Adenubi's target loss of $35 \%$ of OGV exit dynamic was recalculated giving a new target loss equivalent to $30 \%$ of rotor exit dynamic pressure.

It is important that the flow delivered to the feed annuli has a sufficient static pressure recovery (reduction in velocity) with a minimum total pressure loss such that it in a good condition to adequately feed the primary ports and cooling rings etc. Immediately, it is evident from Table 4.2 that the mass weighted total pressure loss coefficient to the outer annuli for both conventional diffusers is above the target of 0.3. The Datum diffuser results in a loss coefficient to the outer annuli of 0.33 and the Optimised Conventional diffuser has a slightly smaller loss of 0.32 .

The total pressure loss to the outer annuli for all the hybrid diffusers is below the target value. For example, Hybrid 4 shows a reduction of $5 \%$ of rotor exit dynamic from the Optimum Conventional diffuser with a total pressure loss coefficient of 0.27 . The addition of radial struts in Stage 3 of the Hybrid 5 diffuser appears to have only a small effect on performance to the outer annuli. The loss for the strutted Hybrid 5 diffuser is slightly increased from Hybrid 1 which has the same overall area ratio with no struts, but the loss is nominally the same as for Hybrid 4 which, apart from struts, is geometrically identical to Hybrid 5.

\begin{tabular}{|l||c|c|c|}
\hline Diffuser & $\mathrm{C}_{\mathrm{p} 2-7}$ & $\lambda_{2-7}$ & $\alpha_{7}$ \\
\hline \hline Datum & 0.63 & 0.33 & 1.17 \\
\hline Opt. Conv. & 0.65 & 0.32 & 1.17 \\
\hline Hybrid 1 & 0.70 & 0.27 & 1.14 \\
\hline Hybrid 2 & 0.70 & 0.26 & 1.13 \\
\hline Hybrid 4 & 0.70 & 0.27 & 1.13 \\
\hline Hybrid 5 & 0.70 & 0.27 & 1.13 \\
\hline
\end{tabular}

Table 4.2 Outer Feed Annulus Performance Data

Figure 4.11 shows the circumferentially averaged velocity profiles in the outer annulus with axial velocity contours shown in Figure 4.12. All the diffusers show a similar shape 
profile with a slight outboard bias.

Velocity contours and vectors (Figure 4.13) also show that there exists, in all cases, a region of low velocity associated with the wake from the burner feed arm. For the Datum and Optimum Conventional diffusers this wake is in line with the burner feed arm and is seen in the middle of the sector. However, due to the greater swirl in the non-strutted prediffusers the burner feed arm wake is located circumferentially left of centre.

Outer annulus velocity vectors (Figure 4.13) and circumferentially averaged swirl angles (Figure 4.14) clearly show that there is higher degree of swirl in the outer annulus when strutted diffusers are not used. With reference to Figure 4.6, struts in the diffuser remove this swirl from the rotor. However, close to the inner casing there is a local increase in swirl due to the low axial velocity in this region (refer to Figure 4.11). A high degree of swirl within the combustor annuli is not desirable as it degrades the quality of the feed to the flame tube admission ports and cooling features.

On further examination of the velocity vectors in the outer feed annuli (Figure 4.13) pairs of contra-rotating vortices can be seen. These pairs of vortices have a periodicity which is set by the burner spacing and are generated as the annulus flow fills the injector/burner feed arm wake. It is also possible that the formation of these vortices is aided by a type of Goertler instability which is generated within a shear layer when it undergoes a significant amount of turning and results in the formation of Taylor-Goertler vortices (Schlichting $\left.^{[1979]}\right)$.

\subsubsection{Splitter Feed Annulus Performance}

Instrumentation in the splitter annulus was limited due to access restrictions as described in Section 3.0. However, the instrumentation was sufficient to obtain the performance figures shown in Table 4.3. The static pressure recovery and total pressure loss coefficients are again quoted with reference to rotor exit and target value of 0.3 for the total pressure loss coefficient is again applicable. Only the Datum diffuser supplies air to the splitter annulus with a loss greater than 0.3 , all the other diffuser configurations meet the performance requirements; the hybrid diffuser options returning loss coefficients of 0.21 
- 0.23. The Optimum Conventional diffuser only just meets the target loss coefficient with a value of 0.30 . Although the diffuser static pressure recovery is relatively high the presence of a vane (see Figure 3.8b) directs flow away from the splitter annulus and thus introduces an extra turning loss for the splitter flow. The series of hybrid diffusers do not suffer from this impediment and as such have significantly lower loss figures to the splitter annulus.

\begin{tabular}{|l||c|c|c|}
\hline Diffuser & $\mathrm{C}_{\mathrm{p} 2-6}$ & $\lambda_{2-6}$ & $\alpha_{6}$ \\
\hline \hline Datum & 0.62 & 0.33 & 1.16 \\
\hline Opt. Conv. & 0.67 & 0.30 & 1.16 \\
\hline Hybrid 1 & 0.76 & 0.21 & 1.12 \\
\hline Hybrid 2 & 0.74 & 0.22 & 1.12 \\
\hline Hybrid 4 & 0.74 & 0.22 & 1.11 \\
\hline Hybrid 5 & 0.74 & 0.23 & 1.12 \\
\hline
\end{tabular}

Table 4.3 Splitter Feed Annulus Performance Data8

\subsubsection{Inner Feed Annulus Performance}

Table 4.4 details the performance figures for the flow to the inner feed annulus (station 4) with respect to rotor exit conditions (station 2). Applying the same target criteria for the total pressure loss as before (i.e. $\lambda<0.3$ ) it is again evident that the flow from the Datum diffuser fails to meet this target, exceeding it by some $5 \%$ of rotor exit dynamic. However, the Optimum Conventional diffuser and all the hybrid diffusers produce inner annulus loss coefficients below the target value. Comparing the Optimum Conventional and hybrid diffusers there is very little difference in overall performance. All yield loss coefficients of $0.25-0.26$ and static pressure recovery coefficients of $0.68-0.70$.

Figure 4.15 illustrates the circumferentially averaged velocity profiles in the inner annulus and shows that for all the diffusers the flow has a slight bias towards the inner casing. This can also be seen in the axial velocity contour plots (Figure 4.16) in addition to the presence of wakes from the pilot injectors. Pairs of contra rotating vortices are, again, also present created by the annulus flow filling these wakes. The vortices are easily visi- 
ble on the velocity vector plots shown in Figure 4.17.

\begin{tabular}{|l||c|c|c|}
\hline Diffuser & $\mathrm{C}_{\mathrm{p} 2-5}$ & $\lambda_{2-5}$ & $\alpha_{5}$ \\
\hline \hline Datum & 0.61 & 0.35 & 1.07 \\
\hline Opt. Conv. & 0.68 & 0.27 & 1.10 \\
\hline Hybrid 1 & 0.71 & 0.25 & 1.15 \\
\hline Hybrid 2 & 0.70 & 0.26 & 1.11 \\
\hline Hybrid 4 & 0.70 & 0.26 & 1.11 \\
\hline Hybrid 5 & 0.70 & 0.25 & 1.16 \\
\hline
\end{tabular}

Table 4.4 Inner Feed Annulus Performance Data

Conservation of angular momentum dictates (Bailey and Carrotte ${ }^{[1996]}$ ) that the swirl velocity magnifies with decreasing radius. Figure 4.18 clearly show a large swirl component is present in the flow for the non-strutted diffusers (Hybrid 1, 2 and 4) but is reduced by the introduction of struts (Datum and Hybrid 5).

\subsubsection{Burner Feed Performance}

Burner feed data obtained from the test facility can be used for comparative analysis only due to the fact that dummy fuel injectors with representative porosity were used (refer to Figure 3.8). No attempt was made to model swirler flows; importance being placed on obtaining the correct mass flow only. However, the data obtained are sufficient to calculated total pressure loss figures and assess the relative performance of each diffuser in terms of their ability to feed the burners.

Table 4.5 details the total pressure loss coefficients (with respect to rotor exit) to the pilot and main burners. For both burners the flow with the lowest loss is delivered via the Optimum Conventional diffuser. The Datum diffuser has the highest losses with the hybrid diffusers showing an improvement but unable to match the Optimum Conventional diffuser. The reason why the Optimum Conventional diffuser results in the lowest burner feed losses is that its twin passages point the flow directly at the burners (see Fig- 
ure 3.10).

\begin{tabular}{|l||c|c|}
\hline Diffuser & Pilot (inner) & Main (outer) \\
\hline \hline Datum & 0.32 & 0.35 \\
\hline Opt. Conv. & 0.25 & 0.30 \\
\hline Hybrid 1 & 0.33 & 0.36 \\
\hline Hybrid 2 & 0.30 & 0.34 \\
\hline Hybrid 4 & 0.27 & 0.36 \\
\hline Hybrid 5 & 0.27 & 0.35 \\
\hline
\end{tabular}

Table 4.5 Burner Flow Total Pressure Loss Coefficient (w.r.t. Rotor Exit)

\subsubsection{Dump Losses}

Total pressure loss to the combustor feed annuli are, according to Fishenden and Stevens ${ }^{[1977]}$, attributed to two sources. Namely, losses in the pre-diffuser and losses in the dump region. Pre-diffuser losses and overall losses have been discussed in Sections 4.1.1 and 4.1.3 - 5 respectively. The dump gap total pressure loss coefficients are shown in Table 4.6 and defined (Equation 4.1) as the total pressure loss from diffuser exit (station 4) to the combustor feed annuli (station 5/7) normalised by the rotor exit dynamic pressure.

$$
\lambda_{4-5}=\frac{\tilde{\mathrm{P}}_{4}-\tilde{\mathrm{P}}_{5}}{\tilde{\mathrm{q}}_{2}}
$$

\begin{tabular}{|l||l|l|}
\hline Diffuser & $\lambda_{4-5}$ & $\lambda_{4-7}$ \\
\hline \hline Datum & 0.19 & 0.18 \\
\hline Opt. Conv. & 0.13 & 0.16 \\
\hline Hybrid 1 & 0.08 & 0.11 \\
\hline Hybrid 2 & 0.08 & 0.09 \\
\hline Hybrid 4 & 0.06 & 0.07 \\
\hline Hybrid 5 & 0.06 & 0.07 \\
\hline
\end{tabular}

Table 4.6 Total Pressure Loss Coefficients in the Dump 
The dump losses for the Datum diffuser are relatively high which is a function of the modest area ratio and low degree of turning offered by the diffuser. The flow must undergo a significant amount of turning within the dump in order to reach the feed annuli which leads to high dump losses and ultimately high losses to the feed annuli.

At exit to the Optimum Conventional diffuser the static pressure recovery is greater and the velocity lower than for the Datum diffuser and as such the flow incurs a slightly lower dump loss. However, because the Optimum Conventional diffuser is longer than all the other diffusers and the dump gap smaller, the flow has less room in which to turn and enter the feed annuli and therefore incurs a high turning loss. Thus the benefit of extra turning within the Optimum Conventional diffuser is somewhat offset by the reduction in dump gap size. The dump gap size is restored to that of the Datum diffuser for the series of hybrid diffusers and Table 4.6 shows that there is a marked improvement in dump gap performance. Now the benefit of increased diffusion and turning offered by the hybrid diffusers is realised and dramatically reduces the total pressure loss within the dump. The loss is reduced by almost half from the Datum to Hybrid 1 diffuser and continues to reduces, albeit only slightly, as the Stage 3 divergence angle is increased.

\subsection{Build 2 Flame Tube}

Section 3.4 has discussed the difference between the two flame tube builds. In summary the main difference is the fact that in the second design the pilot flame tube and burner were moved axially upstream of their location in Build 1 configuration (see Figure 3.7). This significantly reduced the size of the dump gap (by almost 50\%) and limits the available space for the pre-diffuser. As a result the Build 2 flame tube significantly magnifies the aerodynamic challenge facing the diffuser. Not only is the Optimum Conventional diffuser now too long for the available space but the hybrid diffusers also had to be radically modified. Section 3.5 has discussed how, to reduce length, the vortex chamber was replaced by a simple bleed duct in the Hybrid 6 diffuser (Figure 3.14). This represents a significant departure from the concept of the hybrid diffuser introduced by Adkins et $\mathrm{al}^{[1980]}$ but it also represents a much more attractive engineering option. This duct-bled concept reduces complexity, saves length and weight and potentially offers a method by which the bleed air can be recovered with a lower loss. Furthermore, the Build 2 flame 
tube and Hybrid 6 diffuser provide an experimental test case which can be used to assess the validity of a duct-bled hybrid diffuser.

\subsubsection{Diffuser Performance}

Diffuser performance was assessed as before using the methods described in Section 3.0. Table 4.7 details the main performance parameters for the Datum and Hybrid 6 diffusers. Note, also, that Datum diffuser performance data for the Build 1 flame tube are also quoted for ease of comparison.

\begin{tabular}{|l||c|c|c|c|c|c|}
\hline Diffuser & $\begin{array}{l}\text { Area } \\
\text { Ratio }\end{array}$ & $\mathrm{L} / \mathrm{h}_{1}$ & $\begin{array}{c}\text { Bleed Req. } \\
(\text { inner/outer }) \\
\left(\% \dot{\mathrm{m}}_{\text {tot }}\right)\end{array}$ & $\mathrm{C}_{\mathrm{p} 2-4}$ & $\lambda_{2-4}$ & $\alpha_{4}$ \\
\hline \hline $\begin{array}{l}\text { Datum } \\
\text { (Build 1) }\end{array}$ & 1.48 & 3.13 & - & 0.63 & 0.16 & 1.58 \\
\hline $\begin{array}{l}\text { Datum } \\
\text { (Build 2) }\end{array}$ & 1.48 & 3.13 & - & 0.65 & 0.14 & 1.68 \\
\hline Hybrid 6 & 2.06 & 3.0 & 3.0 & 0.69 & 0.20 & 1.59 \\
\hline
\end{tabular}

(NB all diffusers contain struts)

Table 4.7 Pre-Diffuser Performance Data (Build 2 Flame Tube)

Clearly a hybrid diffuser with a duct bleed is a viable option. With reference to Figure 3.11, Hybrid 6 has a high aerodynamic loading but remains attached under the action of bleed. Previous results suggested that the Hybrid 6 diffuser would require a bleed rate of about $3.0 \%$ of total inlet mass flow through each of the inner and outer bleed ducts. However, at this level of bleed the Hybrid 6 diffuser is seen to exhibit a small region of separated flow on the outer wall of the Stage 3 diffuser. This is evident on the axial velocity contour plot (Figure 4.20c) and the velocity vector plot (Figure 4.21c). In order to remove this separation the outer bleed was increased to $4.0 \%$ of the total inlet mass flow while maintaining the inner bleed at 3.0\%. Plots of axial velocity contours and velocity vectors, shown in Figure 4.20(d) and Figure 4.21(d) respectively, indicate that 
this was successful at eliminating the flow separation.

\subsubsection{Feed Annulus Performance}

Moving the pilot flame tube burner forward was expected to have a detrimental effect on the performance of the flow to the feed annuli. A higher degree of turning is now required in a shorter length which will ultimately cause higher turning losses. Table 4.8 and Table 4.9 detail the outer and inner annulus performance figures respectively. It is evident that performance with the Build 2 flame tube is severely reduced. For the Datum diffuser the total pressure losses to each annuli increase well in excess of the target value of 0.3 . Furthermore, the additional turning offered by the Hybrid 6 diffuser reduces the total pressure loss but it still remains above the target value. In summary the reduction of the dump gap coupled with the deep flame tube will result in high losses to the annuli irrespective of the diffuser option employed.

\begin{tabular}{|l||c|c|c|}
\hline Diffuser & $\mathrm{C}_{\mathrm{p} 2-7}$ & $\lambda_{2-7}$ & $\alpha_{7}$ \\
\hline \hline Datum (Build 1) & 0.63 & 0.33 & 1.17 \\
\hline Datum (Build 2) & 0.54 & 0.40 & 1.13 \\
\hline Hybrid 6 & 0.59 & 0.36 & 1.16 \\
\hline
\end{tabular}

Table 4.8 Outer Annulus Performance Figures - Build 2

\begin{tabular}{|l||c|c|c|}
\hline Diffuser & $\mathrm{C}_{\mathrm{p} 2-5}$ & $\lambda_{2-5}$ & $\alpha_{5}$ \\
\hline \hline Datum (Build 1) & 0.61 & 0.35 & 1.16 \\
\hline Datum (Build 2) & 0.44 & 0.44 & 1.30 \\
\hline Hybrid 6 & 0.52 & 0.36 & 1.52 \\
\hline
\end{tabular}

Table 4.9 Inner Annulus Performance Figures - Build 2 


\section{$\underline{4.3}$ Bleed Flow Assessment}

\subsubsection{Effect of Bleed Rate on Diffuser Performance}

The effect of bleed rate is documented by Adkins et al ${ }^{[1980]}$ who have shown that there is a step increase in performance once the minimum bleed requirement is reached (refer to Section 2.6). The relationship shown in Figure 2.33 has been difficult to verify experimentally. A minimum bleed requirement exists as detailed in Table 4.1 and below this value the hybrid diffuser was in a state of stall. Unfortunately, it was not possible to obtain sensible performance figures for the hybrid diffusers when they had stalled. The reversed flow associated with separation does not fall within the calibration range of the five-hole probe (refer to Appendix A3) and zero values are returned by the reduction software for the pressures and velocities; the latter certainly should be negative. These zero values are included in the final data reduction which leads to a false result for the performance parameters. Thus, it has not been possible to reproduce the relationship seen by Adkins et al ${ }^{[1980]}$. However, above the minimum bleed requirement the hybrid diffusers behaved as the literature predicted; the values of static pressure recovery and total pressure loss do not alter greatly with a further increase in bleed rate. This is illustrated in Table 4.10 which shows the performance parameters for Hybrid 4 for various bleed rates above the minimum bleed requirement.

\begin{tabular}{|c||c|c|}
\hline $\begin{array}{c}\text { Inner/Outer Bleed } \\
\left(\% \dot{\mathrm{m}}_{\text {total }}\right)\end{array}$ & $\mathrm{C}_{\mathrm{p} 2-4}$ & $\lambda_{2-4}$ \\
\hline \hline $2.0 / 2.0$ & 0.70 & 0.20 \\
\hline $2.5 / 2.5$ & 0.71 & 0.20 \\
\hline $3.0 / 3.0$ & 0.71 & 0.20 \\
\hline
\end{tabular}

Table 4.10 Variation of Diffuser Performance with Bleed Rate (Hybrid 4)

The method used for calculating the data presented in Table 4.10 does not take into account the bleed flow. The total pressure loss coefficient from rotor exit to diffuser exit is defined as: 


$$
\lambda_{2-4}=\frac{\tilde{\mathrm{P}}_{2}-\tilde{\mathrm{P}}_{4}}{\tilde{\mathrm{q}} 2}
$$

In Equation 4.2 the mass weighted total pressure and dynamic pressure at rotor exit include the bleed flow whereas the total pressure at diffuser exit includes only that flow which passes down the diffuser. It is difficult to say what effect this has on the final performance figures. It is the low energy, poor quality boundary layer flow, associated with the Stage 1 diffuser wall flow, that is bled off and if this is not accounted for Equation 4.2 may give an optimistic value for loss. A stream tube analysis using only the flow which passes down the diffuser to calculate rotor exit values may yield better results. However, due to the complex three dimensional nature of the flow it is difficult to define what flow is actually bled off. The low energy flow associated with the boundary layer is likely to be bled off but so is a proportion of the low energy OGV wake flow. Thus the traditional methods for presenting total pressure loss and static pressure recovery are the only viable option, and have therefore been used throughout this experimental study. Furthermore, from an engine performance perspective accounting for the bleed flow does not matter provided the bleed air can be used to replace component cooling flows usually removed elsewhere in the engine.

\subsubsection{Vortex Chamber versus Duct Bleed}

For many years the main driving force behind the development of diffuser technology has been the desire to decrease length and hence weight but maintain efficiency and effectiveness. Currently with the advent of low emission, radially staged combustion systems a significant driving force behind diffuser development is to increase the capability of diffusers to turn the flow around the deep flame tube and reduce total pressure losses to the feed annuli. Hybrid diffusers offer a potential solution to this problem but the issue of weight is still an important one. Hybrid diffusers studied by Adkins et $\mathrm{al}^{[1980]}$ and Myres et $\mathrm{a}^{[1994]}$ have all incorporated a vortex chamber as discussed in Section 2.6. However, a vortex chamber is a significant component and as such carries a length and weight penalty. Due to this it is unlikely that a vortex chamber would be included in any design of hybrid diffuser fitted in an aero gas turbine combustion system. It was shown in Section 4.2.1, however, that a vortex chamber is not necessary and exists 
only to provide a stable pressure gradient which will drive the bleed flow. A ducted bleed offers further advantages in terms of using the bleed air elsewhere in the engine. In a modern aero gas turbine air is usually bled from the dump region for use in turbine blade and disc cooling. Since this usually amounts to about $7-10 \%$ of the engine mass flow it has been suggested (Adkins et $\mathrm{al}^{[1980]}$ ) that the air bled form a hybrid diffuser could be used for this purpose. If this was to be the case then the bleed air must be recovered with the minimum of pressure loss. A duct bleed potentially offers lower losses than a vortex chamber as it can be used to eliminate the high loss vortex flow. Unfortunately without a back-to-back test of identical vortex and duct bled diffusers the results do not indicate what impact on overall diffuser performance a duct bleed has. This information cannot be derived from these results as the Hybrid 6 diffuser and Build 2 flame tube are significantly different form Hybrid 1-5 diffusers which contain a vortex chamber but are fitted with the Build 1 flame tube. A duct bleed and a vortex bleed will differ as Figure 4.22 illustrates. In a vortex chamber the vortex will expand to fill the entire chamber. However, with a duct a separation bubble will form on the upstream side of the duct. This separation bubble will present a blockage to the bleed flow affecting the manner in which mainstream flow is captured by the bleed. It will also cause an acceleration of the flow adjacent to the downstream wall of the duct. Furthermore, the size and shape of the duct will further alter this. The effect of substituting a duct for the vortex chamber and further modifications to the bleed geometry are computationally investigated and discussed in Sections 5.0 and 6.0 .

\subsubsection{Bleed Flow Total Pressure Loss}

No data exist defining a target total pressure loss for the bleed flow at which it would still be suitable for use in component cooling. However, during assessment of the Optimum Conventional diffuser a bleed from the outer dump region was simulated as illustrated in Figure 4.23. With reference to Figure 4.23 it can be seen that this bleed air flows through the same cavity and throttle set up as the outer hybrid diffuser bleed flow. Furthermore, the test rig reference pressure is also measured within this cavity (i.e. the pressure relative to which all other pressures are measured, see Section 3.0). Therefore, it is possible to define a bleed flow total pressure loss for both the dump bleed (Optimum Conventional Diffuser) and hybrid diffuser bleeds; 


$$
\lambda_{2-\mathrm{B}}=\frac{\tilde{\mathrm{P}}_{2}-\tilde{\mathrm{P}}_{\mathrm{B}}}{\tilde{\mathrm{q}}_{2}}
$$

where B is Outer Bleed and:

$$
\tilde{\mathrm{P}}_{\mathrm{ref}}=\tilde{\mathrm{P}}_{\mathrm{B}}
$$

Thus, the bleed loss coefficient becomes:

$$
\lambda_{2-\mathrm{B}}=\frac{\tilde{\mathrm{P}}_{2}-\tilde{\mathrm{P}}_{\mathrm{ref}}}{\tilde{\mathrm{q}}_{2}}
$$

The total pressure loss of the flow for the Optimum Conventional dump bleed was acceptable (Denamn ${ }^{[1996]}$ ) and thus it serves to provide a figure to use as a bench mark for hybrid diffuser bleed flow losses. Table 4.11 gives the total pressure loss coefficient for the outer bleed flow for the various diffusers. For the Optimum Conventional diffuser the loss coefficient amounts to $36 \%$ of rotor exit dynamic pressure but the losses for the hybrid diffuser bleed flows all exceed this. Thus it is likely that further work would be required to investigate this and perhaps reduce the bleed flow losses.

\begin{tabular}{|c||c|c|}
\hline Diffuser & $\begin{array}{c}\text { Outer Bleed } \\
\left(\% \dot{\mathrm{m}}_{\text {total }}\right)\end{array}$ & $\lambda_{2-\mathrm{B}}$ \\
\hline \hline Opt.Conventional & 6.7 & 0.36 \\
\hline Hybrid 1 & 1.5 & 0.48 \\
\hline Hybrid 2 & 2.0 & 0.50 \\
\hline Hybrid 3 & 2.5 & 0.53 \\
\hline Hybrid 4 & 3.0 & 0.55 \\
\hline Hybrid 5 & 3.0 & 0.55 \\
\hline Hybrid 6 & 4.0 & 0.61 \\
\hline
\end{tabular}

\section{Table 4.11 Bleed Flow Total Pressure Loss Coefficients}

It is apparent from the data that the loss coefficient increases with bleed rate. Figure 4.24 is a plot showing how the bleed flow total pressure loss coefficient varies with bleed rate for the Hybrid 4 diffuser. This would suggest that in any future design of hybrid diffuser 
a compromise may be necessary. It could be possible to design a diffuser with the maximum possible area ratio and Stage 3 divergence angle to maximise combustor feed annuli performance. However, the bleed rate required may incur unacceptable bleed flow losses. Thus it would be necessary to decrease the diffuser loading in order to reduce the amount of bleed flow and its total pressure loss.

\subsection{Two-Dimensional, Axi-Symmetric Flame Tube Arrangement}

\subsubsection{Two-Dimensional Inlet (Clean)}

The test facility was modified as described in Section 3.6 to remove the single stage axial compressor and replace the flame tube/burner arrangement with a simple two-dimensional, axi-symmetric flame tube. Furthermore, the test section was fitted with the Hybrid 4 diffuser thus producing an experimental arrangement which could be directly simulated using Computational Fluid Dynamics (CFD) as described in Section 5.0.

Initially the modified cowl had no porosity and the mass flow was split equally between the inner and outer feed annuli. Five-hole probe traverses at diffuser exit revealed that for this configuration, no matter what bleed rate was used, the diffuser stalled. The possibility of this being caused by leaks or geometrical irregularities was investigated and ruled out. Further measurements revealed that for a bleed rate of $3.0 \%$ each side the diffuser stalled from the outer wall. In an attempt to prevent this the outer bleed was increased but, although the outer wall flow reattached, the inner wall stalled. No combination of inner/outer bleed could be found such that the flow remained attached to both the inner and outer diffuser walls.

A contributing factor to this behaviour was thought to be instability effects caused by the stagnation point on the cowl moving randomly. With porosity added (refer to Figure 3.17 ) it was hoped that this would provide a feature to anchor the stagnation point and introduce a degree of stability. The flow split was set to 35:30:35 between the inner:splitter:outer annuli and the bleed set to $3.0 \%$ each side. Again the diffuser stalled on the outer wall. Increasing the outer bleed caused the outer wall flow to reattach but the inner wall flow then separated. Initial CFD predictions (see Section 5.0 and 6.0) indicated that 
a bleed of 3.0\% would result in a stable flow regime. However, these initial calculations were performed with an inlet turbulence intensity of 5\% which is representative of the flow downstream of a rotor and not the clean inlet used here. Stevens and Williams ${ }^{[1980]}$ demonstrated that increased levels of inlet turbulence are beneficial to diffuser performance. However, with clean inlet conditions the turbulence intensity is likely to be less than $1.0 \%$. Further CFD predictions with this level of inlet turbulence intensity indicated the diffuser would now stall. At this low level of inlet turbulence it was not possible, no matter what bleed rates were used, to prevent the flow from separating on the experimetal facility. Unfortunately the result of this is that the data are not of sufficient quality or quantity to satisfactorily evaluate and assess 2D CFD accuracy and validity. This must now rely on the three dimensional calculations

\subsubsection{Three-Dimensional Inlet (Rotor)}

The three-dimensional computational model is described in Section 5.0 and employs the same simplified flame tube geometry as the two-dimensional model but with inlet conditions taken from experimental measurements downstream of the single stage axial compressor. This configuration was easily recreated experimentally by simply returning the compressor to the facility as illustrated in Figure 4.25. Using the experimental techniques described in Section 3.0 this configuration was assessed with an initial inner and outer bleed rate of $2.5 \%$ and a downstream mass flow split of 35:30:35 between the inner, splitter and outer annuli.

Figure 4.26 shows the circumferentially averaged, normalised axial velocity profile at diffuser exit. The profile is inboard biased, due in part to the modified geometry and flow splits but also due to a thicker inlet boundary layer on the outer wall as shown in Figure 4.27. At diffuser exit this results in an outer wall flow which is close to separating

$\left(\mathrm{u} / \bar{U}_{\text {wall }}<0.1\right)$ and an inner wall flow which is well behaved $\left(\mathrm{u} / \bar{U}_{\text {wall }}>0.8\right)$. Before entering the diffuser the outer wall boundary layer is already thick and low in axial momentum. An adverse pressure gradient will further thicken the boundary layer having a negative effect on diffuser performance. In an attempt to improve outer wall performance the outer bleed was increased firstly to $3.0 \%$ and then to $3.5 \%$. By increasing the bleed rate the transfer of energy/momentum from the bleed flow to the mainstream flow 
will also increase thus improving the condition of the flow close to the outer wall. Figure 4.26 shows that increasing the bleed rate to $3.0 \%$ has only a small positive effect but further increasing the bleed to $3.5 \%$ causes the non-dimensionalised velocity close to the outer wall to increase by almost a factor of 3 . This supports the theory that the flow mechanisms of a hybrid diffuser are such that there is a transfer of positive momentum from the accelerating bleed flow to the diffusing mainstream flow enabling it to reattach on the Stage 3 wall. Furthermore, as the bleed rate is increased this transfer of energy is also increased. The fact that altering the bleed flow balance enables hybrid diffusers to overcome problems imposed by biased inlet profiles or thick developed boundary layers is important. In a gas turbine the compressor does not always run at the design condition and will therefore present the diffuser with a range of inlet conditions. However, it is clear from these results that by altering the bleed flow this should have no significant impact on diffuser and combustion system performance.

The effect of increasing the outer bleed on the condition of the flow at diffuser exit is more clearly seen on examination of the normalised axial velocity contours (Figure 4.28). Two OGV wakes are visible separated by a peak in the velocity. As the outer bleed rate is increased the wakes elongate, the contrast with the peak velocity reduces and the overall distortion $\left(\alpha_{4}\right)$ decreases. Furthermore, overall diffuser performance is seen to increase with a reduction in total pressure loss being accompanied by an increase in static pressure recovery as shown in Table 4.12.

\begin{tabular}{|c||c|c|c|}
\hline $\begin{array}{c}\text { Outer Bleed } \\
\dot{\mathrm{m}}_{\text {total }}(\%)\end{array}$ & $\mathrm{C}_{\mathrm{p} 2-4}$ & $\lambda_{2-4}$ & $\alpha_{4}$ \\
\hline \hline 2.5 & 0.70 & 0.21 & 1.63 \\
\hline 3.0 & 0.70 & 0.21 & 1.60 \\
\hline 3.5 & 0.71 & 0.20 & 1.45 \\
\hline
\end{tabular}

Table 4.12 Diffuser Performance (2D Flame Tube, 3D Inlet Conditions, $B_{i}=2.5 \%$ )

The main aim of measurements on this experimental configuration was not to further investigate hybrid diffuser performance or flow mechanisms but to provide a set of data 
which could be used to validate computational predictions. To aid this the flame tube was geometrically simple and used merely to present some representative blockage. Thus the actual annulus performance (Table 4.13) is not important other than to provide validation figures. It is clear from profiles of axial velocity within the annuli that attempting to turn $35 \%$ of the mass flow around the bluff flame tube and into the feed annuli presents a severe aerodynamic challenge to the flow. In the inner annuli (Figure 4.29) the flow is characterised by a very low velocity close to the flame tube casing. This is indicative of the presence of separation as the flow attempts to follow the cowl profile. The flow in the outer annuli (Figure 4.30) has not separated from the cowl but shows an inboard bias.

\begin{tabular}{|c||c|c|}
\hline $\begin{array}{c}\text { Outer Bleed } \\
\dot{\mathrm{m}}_{\text {total }}(\%)\end{array}$ & $\lambda_{2-5}$ & $\lambda_{2-7}$ \\
\hline \hline 2.5 & 0.24 & 0.29 \\
\hline 3.0 & 0.24 & 0.29 \\
\hline 3.5 & 0.23 & 0.29 \\
\hline
\end{tabular}

Table 4.13 Annuli Performance (2D Flame Tube, 3D Inlet Conditions, $B_{i}=2.5 \%$ )

The computational methodology is described and the results presented in the following two sections. It is not sensible to compare and contrast the experimental data with the CFD data until the latter has been fully introduced. Therefore, discussion of computational accuracy and validity will be presented in Section 6.0. 


\subsection{Summary}

In summary the experimental investigation has revealed the following main points:

- Hybrid diffusers demonstrated a significant increase in performance over conventional diffusers within an isothermal representation of a modern, low-emission, radially staged combustion system. Pre-diffuser area ratios were increased by over $60 \%$ from a conventional single passage diffuser with an associated rise in static pressure recovery. However, perhaps the most notable performance benefit demonstrated was a $30 \%$ reduction in the feed annuli total pressure loss. This was attributed to the higher rates of diffusion achieved by a hybrid diffuser which reduced dump gap losses and ultimately improved the annulus feed. Performance was increased such that a system incorporating a hybrid diffuser surpassed target total pressure losses in the feed annuli whereas a conventional diffuser could not.

- Additionally, operation downstream of an axial compressor, in the presence of OGV wakes, presented no problems. Similarly the inclusion of radial struts within the stage 3 section of the hybrid diffuser was not detrimental to overall performance and was seen to be slightly beneficial by removing residual swirl.

- Results demonstrated agreement with the literature (Adkins et al ${ }^{[1980]}$ ) such that hybrid diffusers were shown to require a certain minimum level of bleed before they will function. However, contrary to conclusions in the literature it was clearly shown that the presence of a vortex chamber is not necessary. A hybrid diffuser with bleed afforded via a simple duct achieved similar performance to a hybrid diffuser containing a vortex chamber. This represents a significant engineering advantage as it allows simplification of the bleed chamber geometry

- In general, bleed flow total pressure losses were seen to increase with bleed rate and at the minimum bleed requirement were approximately $50 \%$ of rotor exit dynamic. This is high and suggests more work is necessary to reduce this loss before the bleed air can be confidently employed for turbine blade/disc cooling. However, a duct bleed 
offers a greater potential for reducing bleed flow total pressure losses than a vortex chamber bleed.

- Finally, an attempt to simulate a 2D axi-symmetric configuration in order to provide validation data for computational work proved unsuccessful. The introduction of a clean inlet section reduced turbulence levels which had previously been beneficial to the operation of the hybrid diffuser, consequently the flow separated. However, comprehensive measurements were taken on a simplified 3D geometry in order to provide the necessary validation data. 


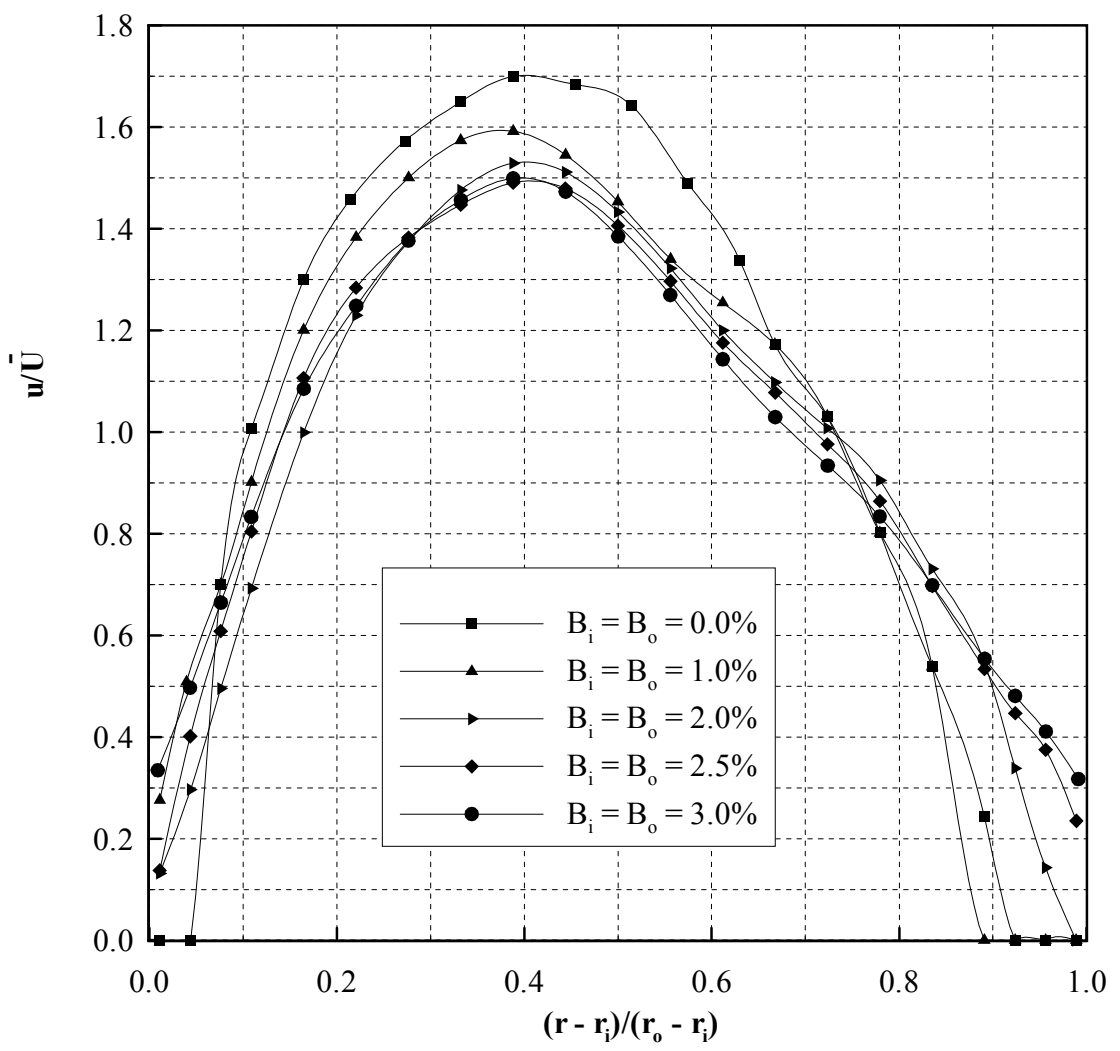

Figure 4.1 Variation of Diffuser Exit Profile with Bleed (Hybrid 4)

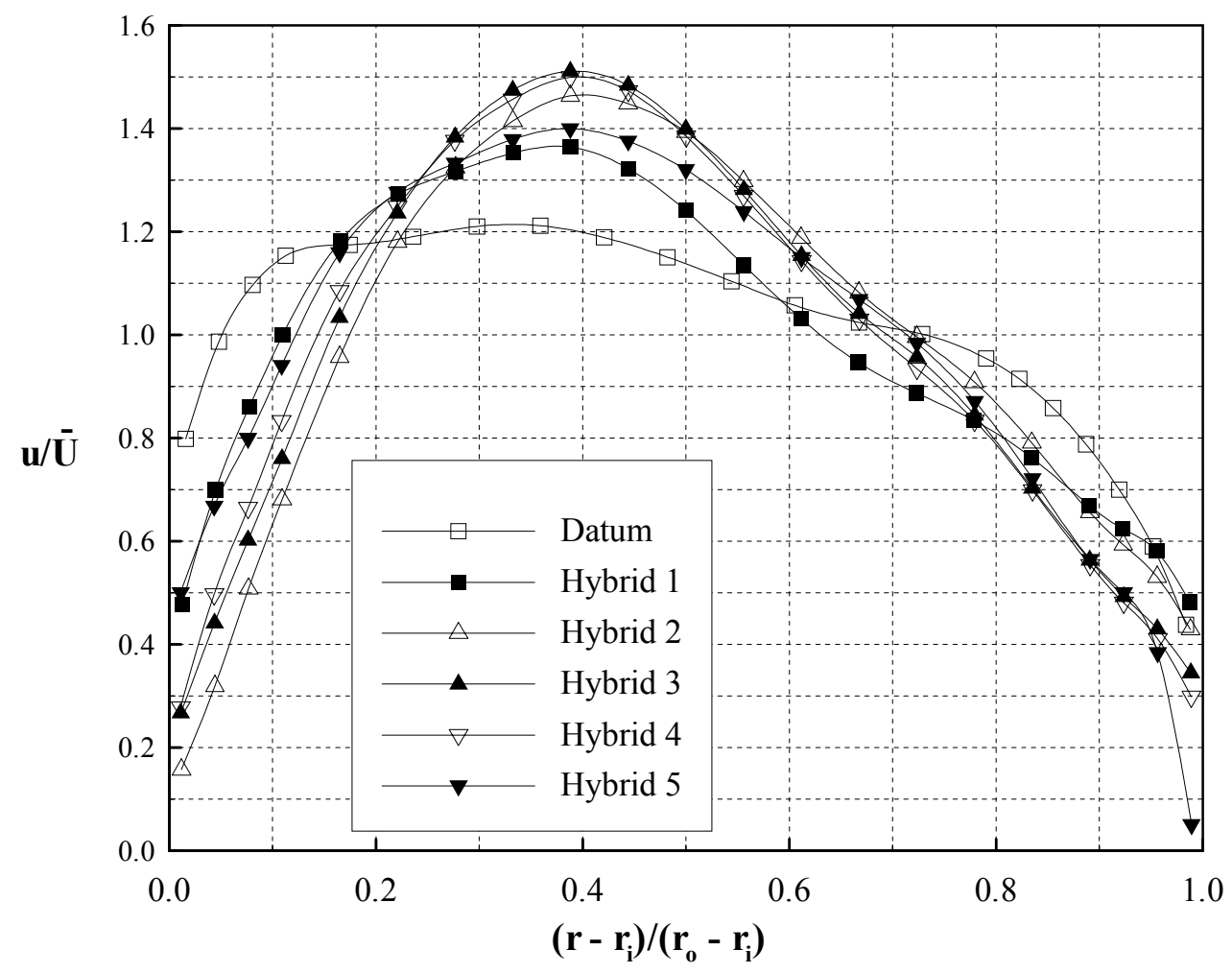

NB - Datum and Hybrid 5 diffuser contain struts

Figure 4.2 Circumferentially Averaged Axial Velocity Profiles at Pre-Diffuser Exit 
(a) Datum

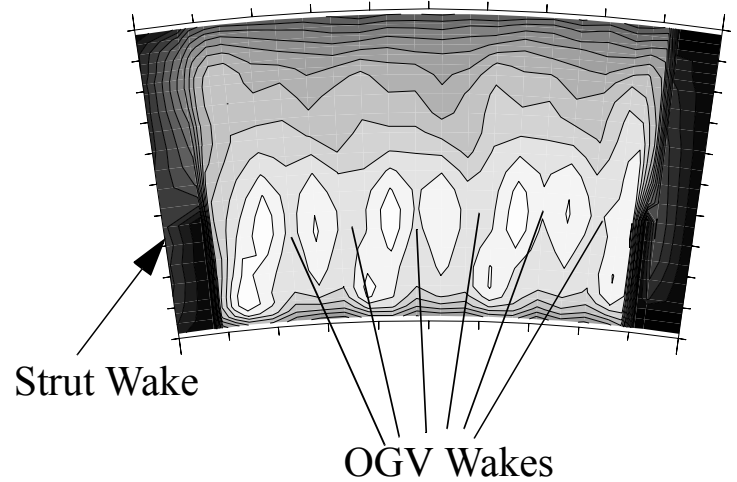

(c) Hybrid 2

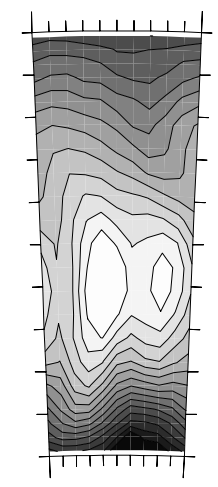

(e) Hybrid 4

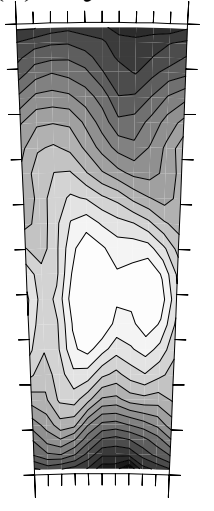

(b) Hybrid 1

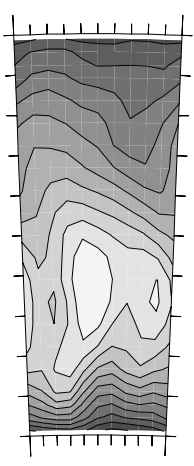

(d) Hybrid 3

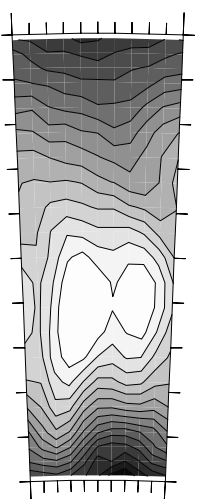

(f) Hybrid 5

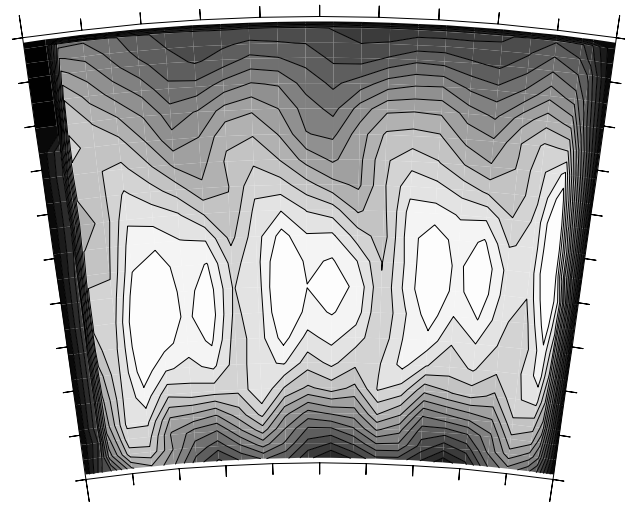

$\begin{array}{llllllllllllllllll}\mathrm{u} / \overline{\mathrm{U}} & 0.0 & 0.1 & 0.2 & 0.3 & 0.4 & 0.5 & 0.6 & 0.7 & 0.8 & 0.9 & 1.0 & 1.1 & 1.2 & 1.3 & 1.4 & 1.5\end{array}$

Figure 4.3 Axial Velocity Contours at Pre-Diffuser Exit 
(a) Datum

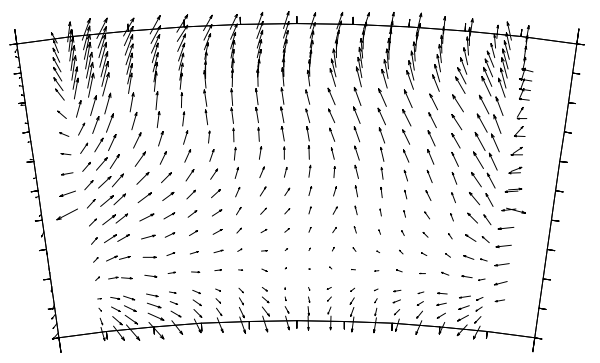

(c) Hybrid 2

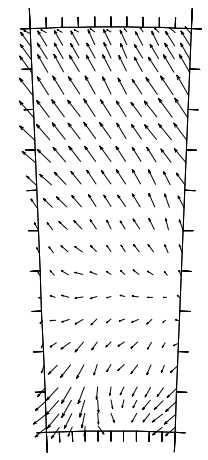

(e) Hybrid 4

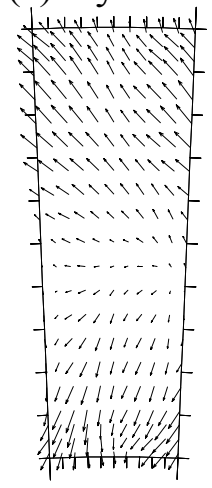

(b) Hybrid 1

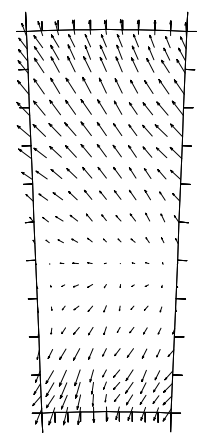

(d) Hybrid 3

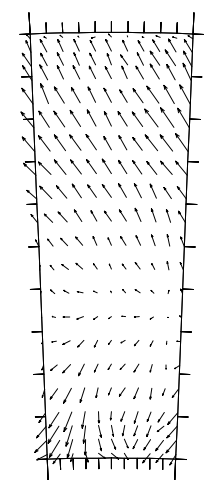

(f) Hybrid 5

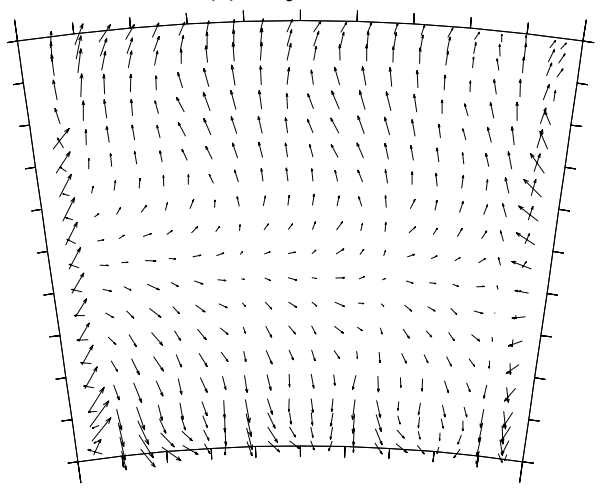

Figure 4.4 Projected Total Velocity Vectors at Pre-Diffuser Exit 


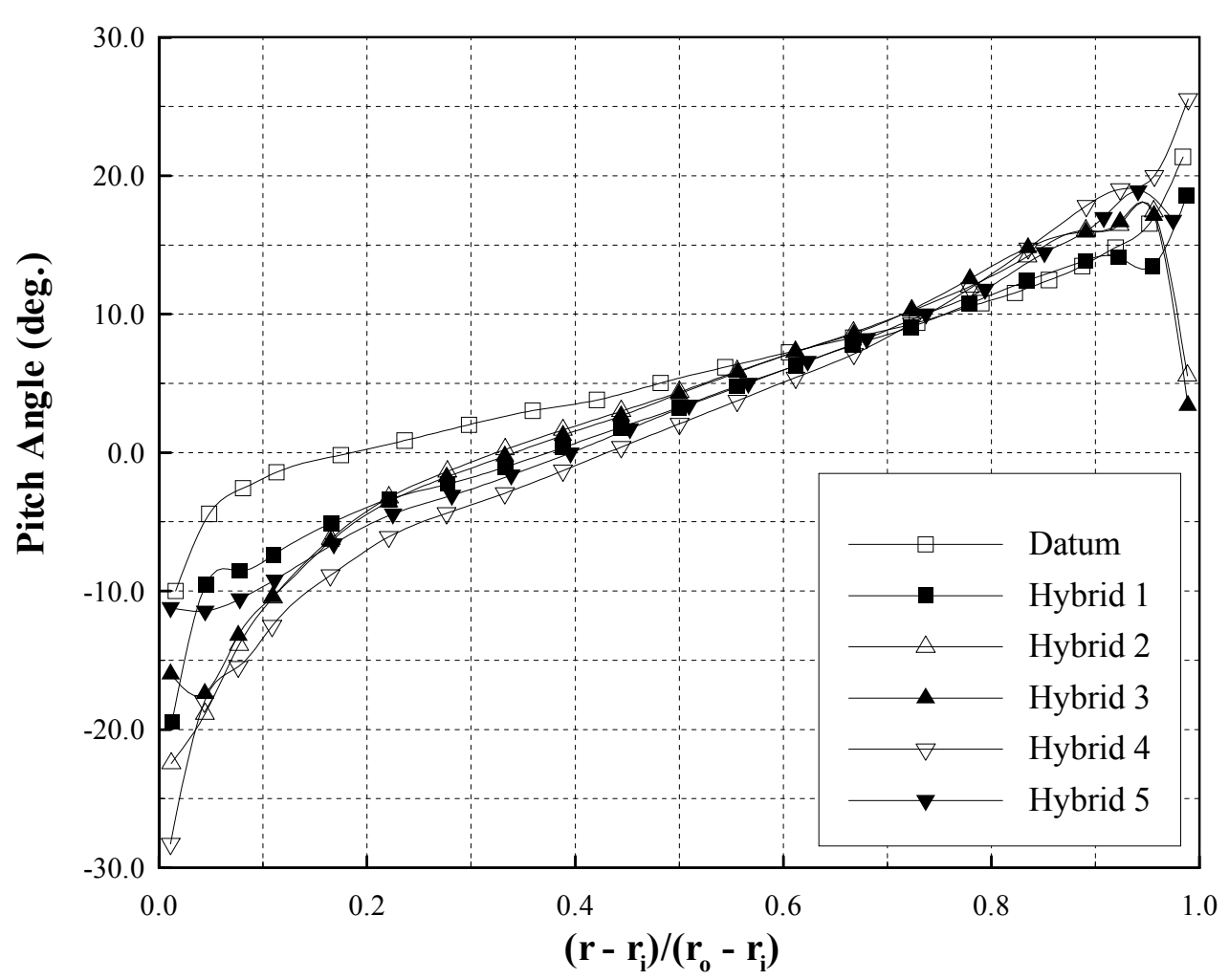

Figure 4.5 Circumferentially Averaged Pitch Angle at Pre-Diffuser Exit

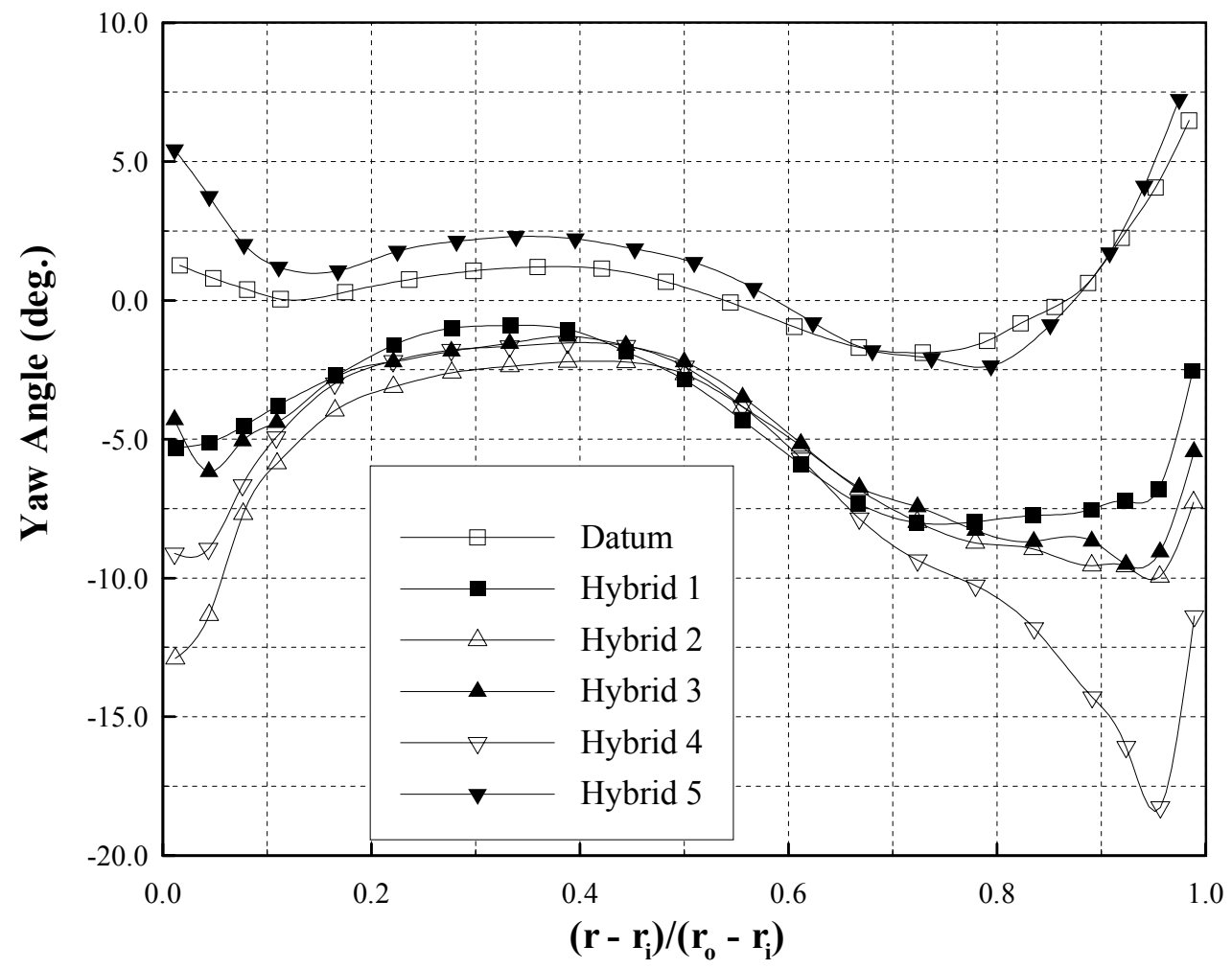

Figure 4.6 Circumferentially Averaged Swirl Angle at Pre-Diffuser Exit 


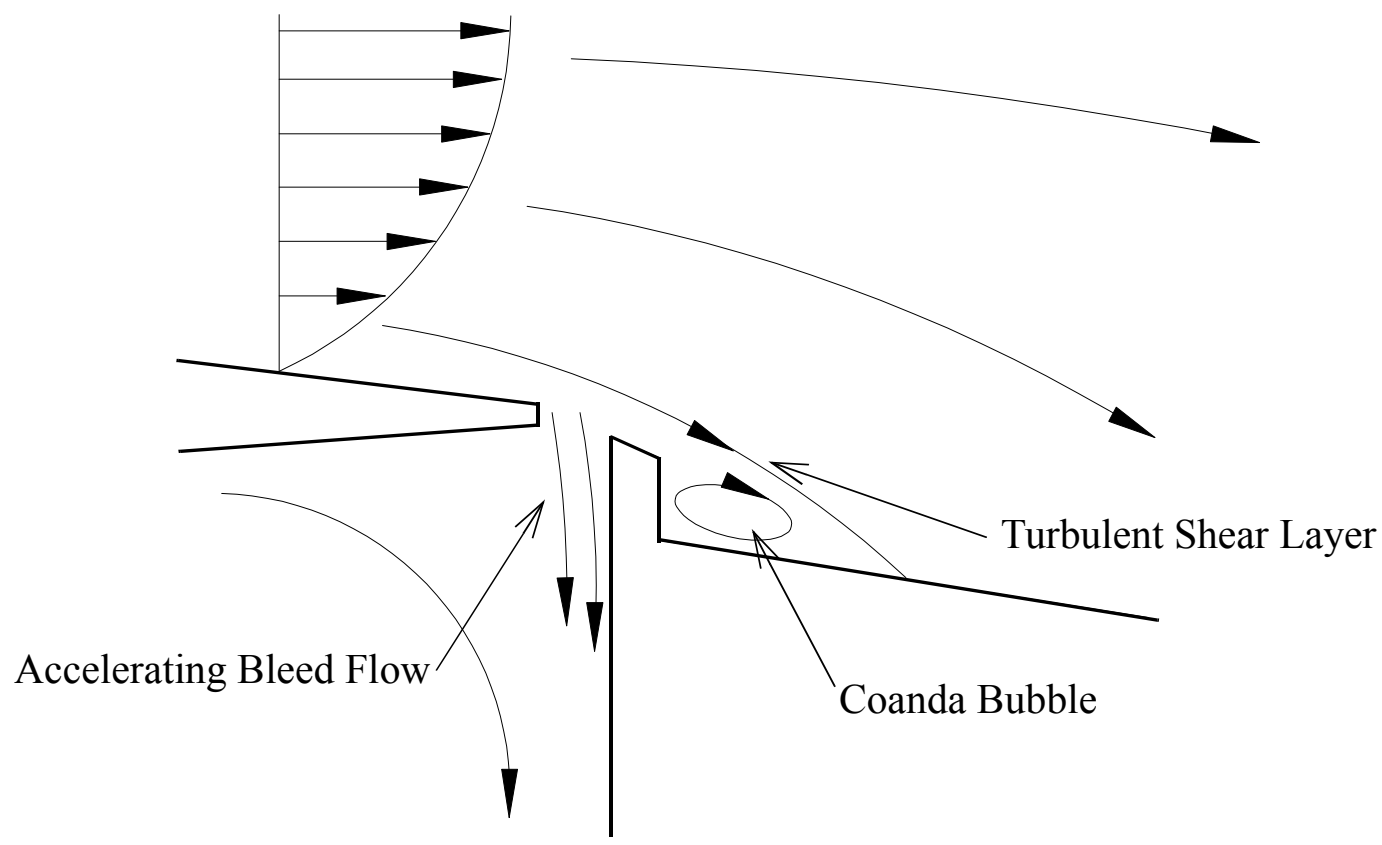

Figure 4.7 Flow Mechanism of a Hybrid Diffuser

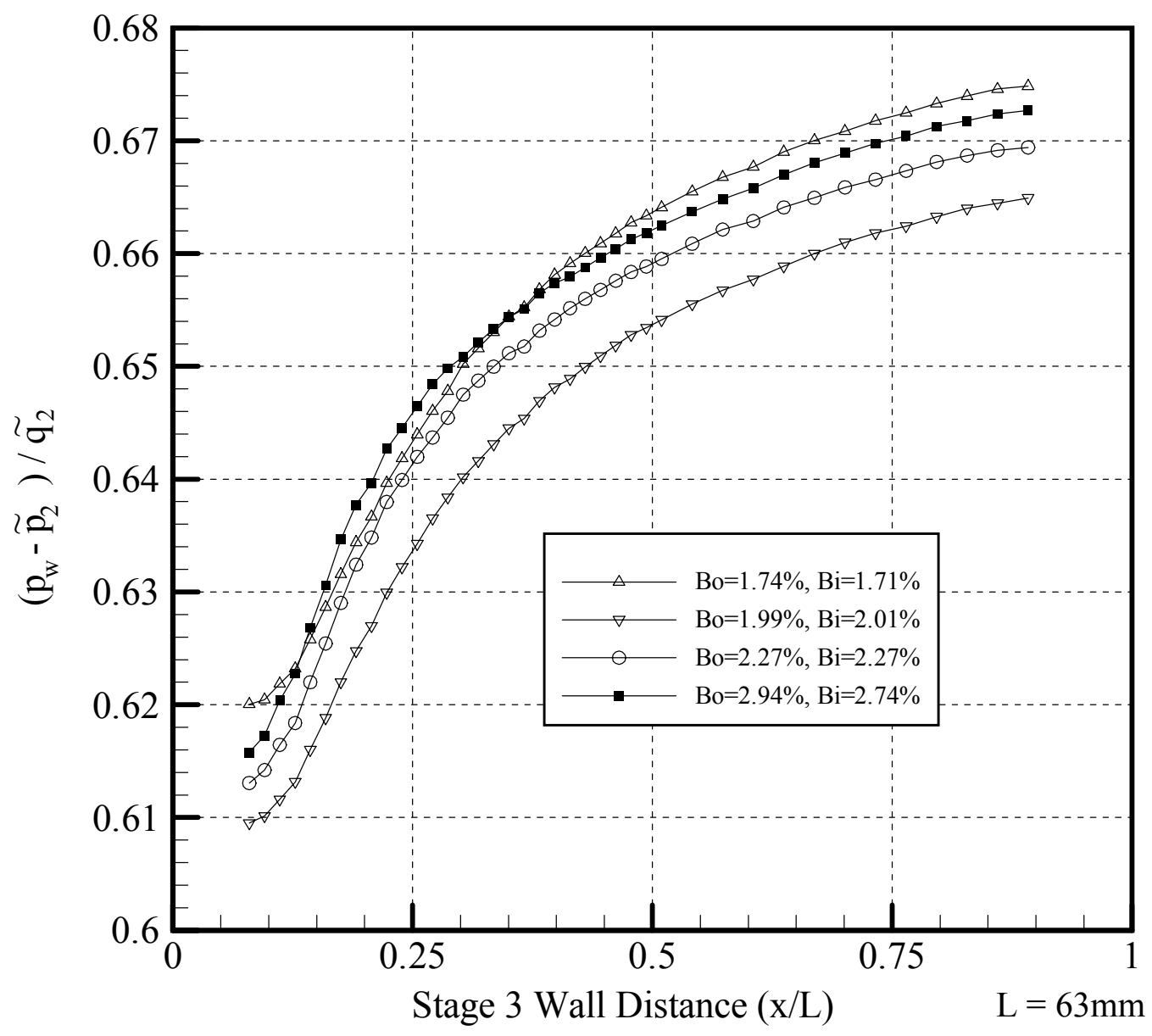

Figure 4.8 Circumferentially Averaged Wall Static Pressure Recovery (Hybrid 4) 


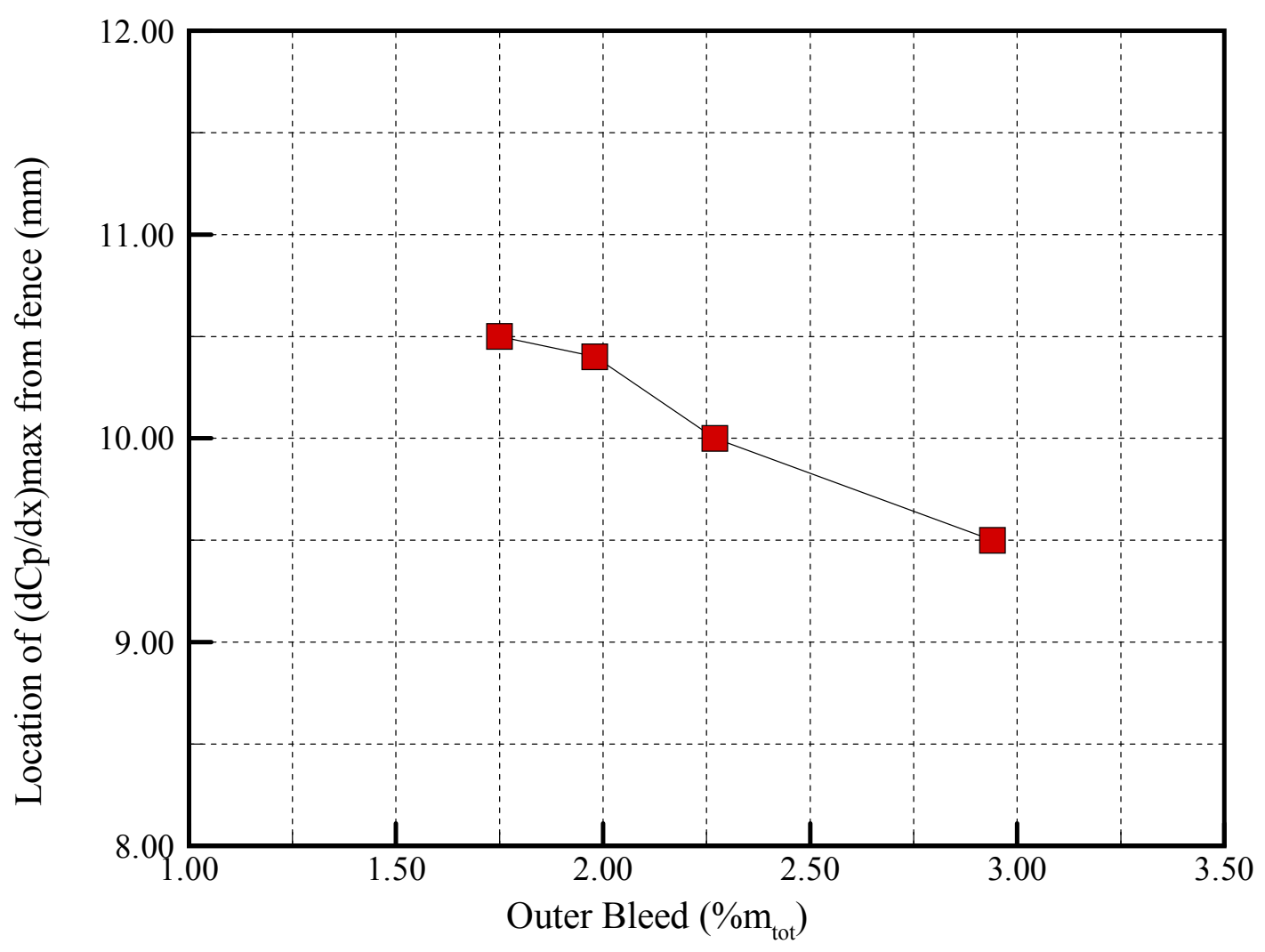

Figure 4.9 Variation of Maximum Static Pressure Recovery

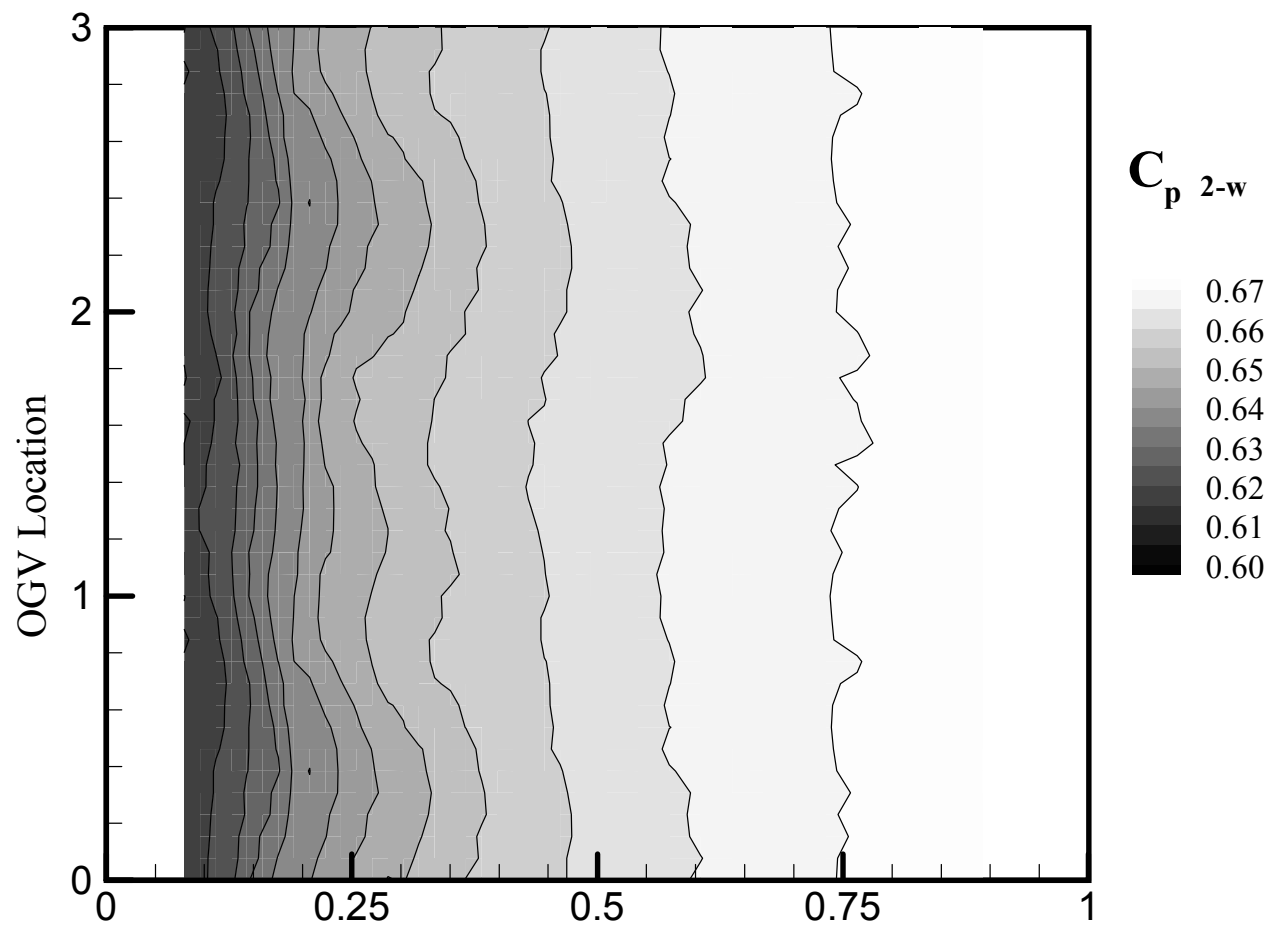

Satge 3 Wall Distance (x/L)

Figure 4.10 Contours of Wall Static Pressure Recovery (Hybrid 4, $B_{\text {inner }}=\mathbf{2 . 7 5 \%}$,

$$
B_{\text {outer }}=\mathbf{2 . 9 5 \% )}
$$




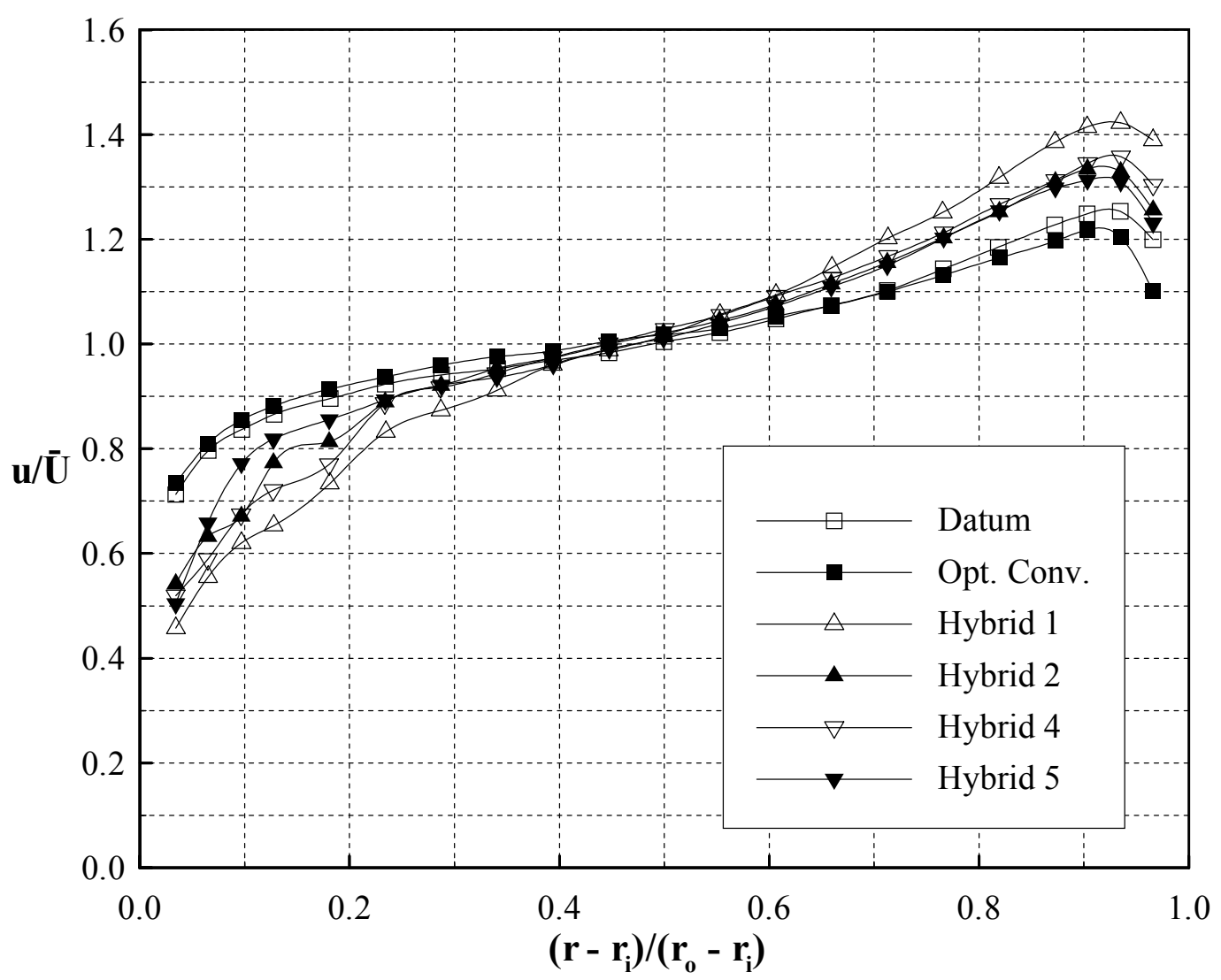

Figure 4.11 Circumferentially Averaged Axial Velocity Profiles in Outer Annulus 


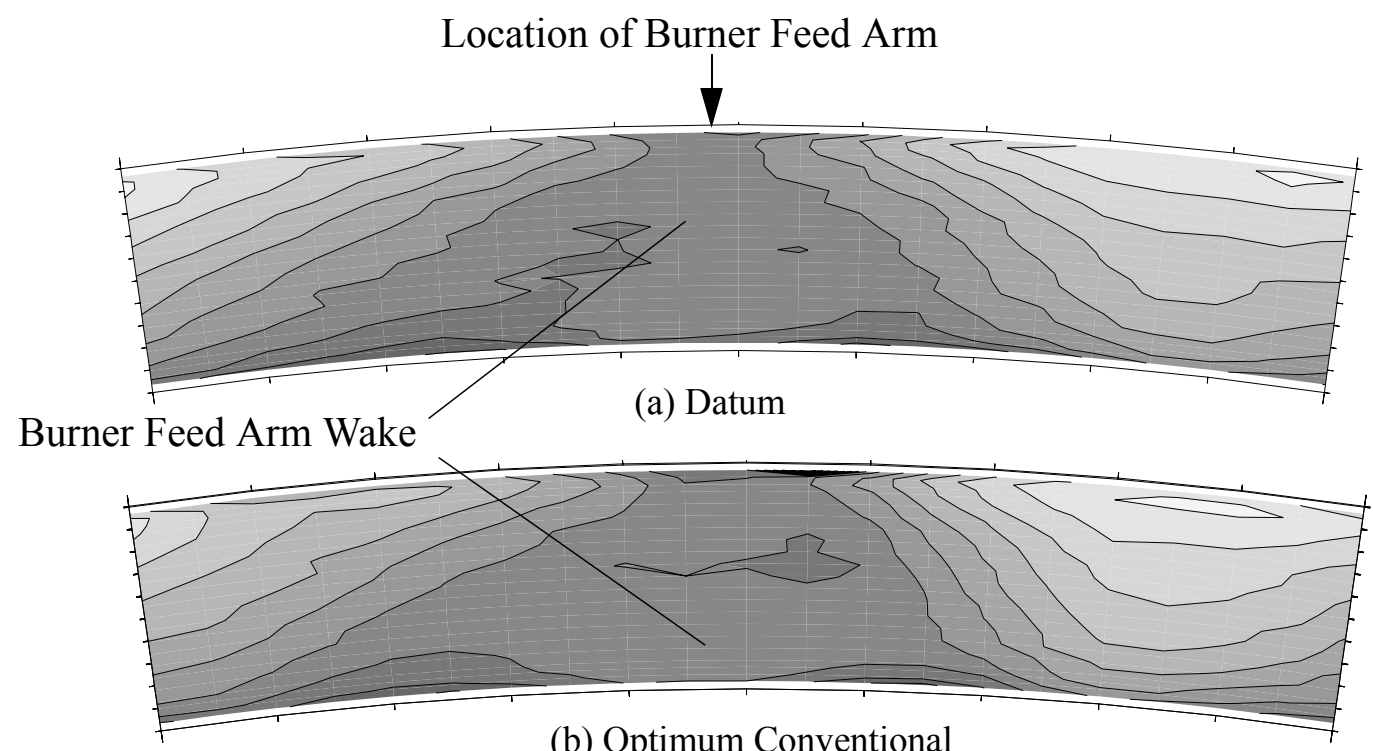

(b) Optimum Conventional

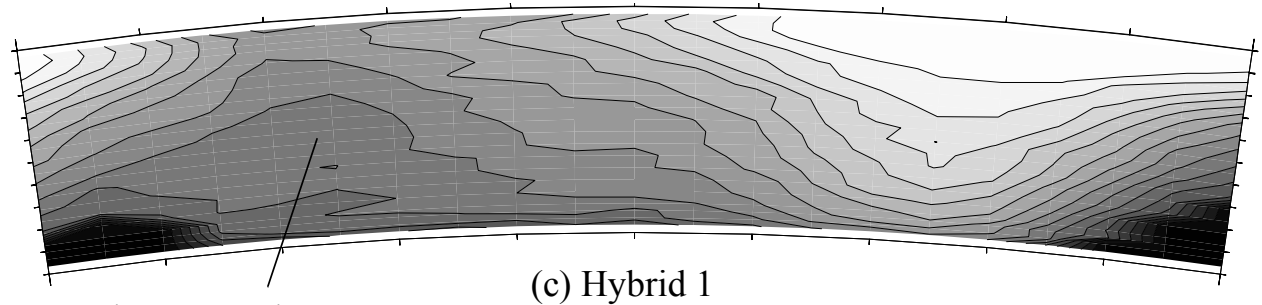

Burner Feed Arm Wake

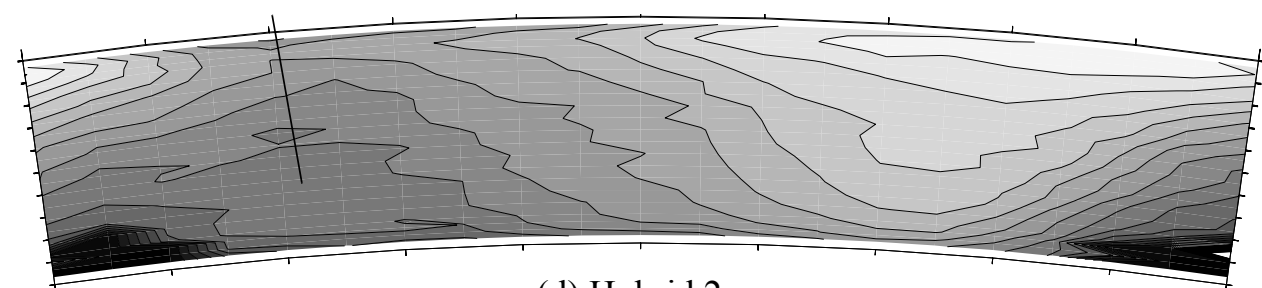

(d) Hybrid 2

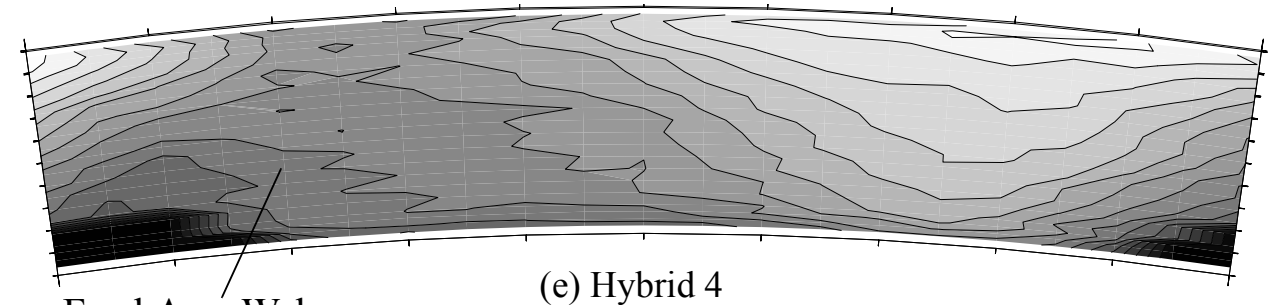

Burner Feed Arm Wake

(e) Hybrid 4
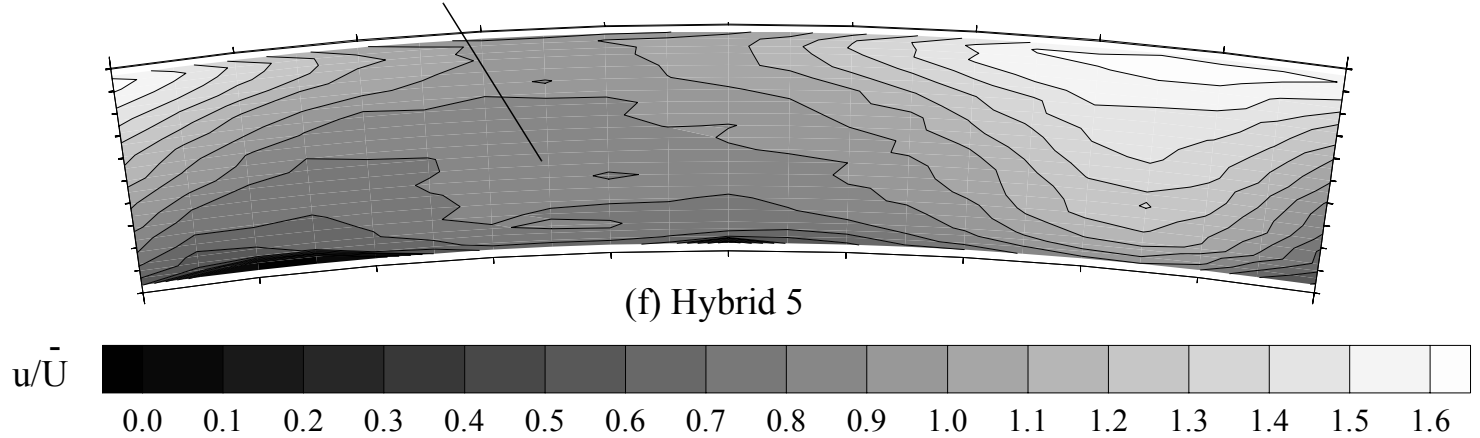

Figure 4.12 Axial Velocity Contours in the Outer Annulus 


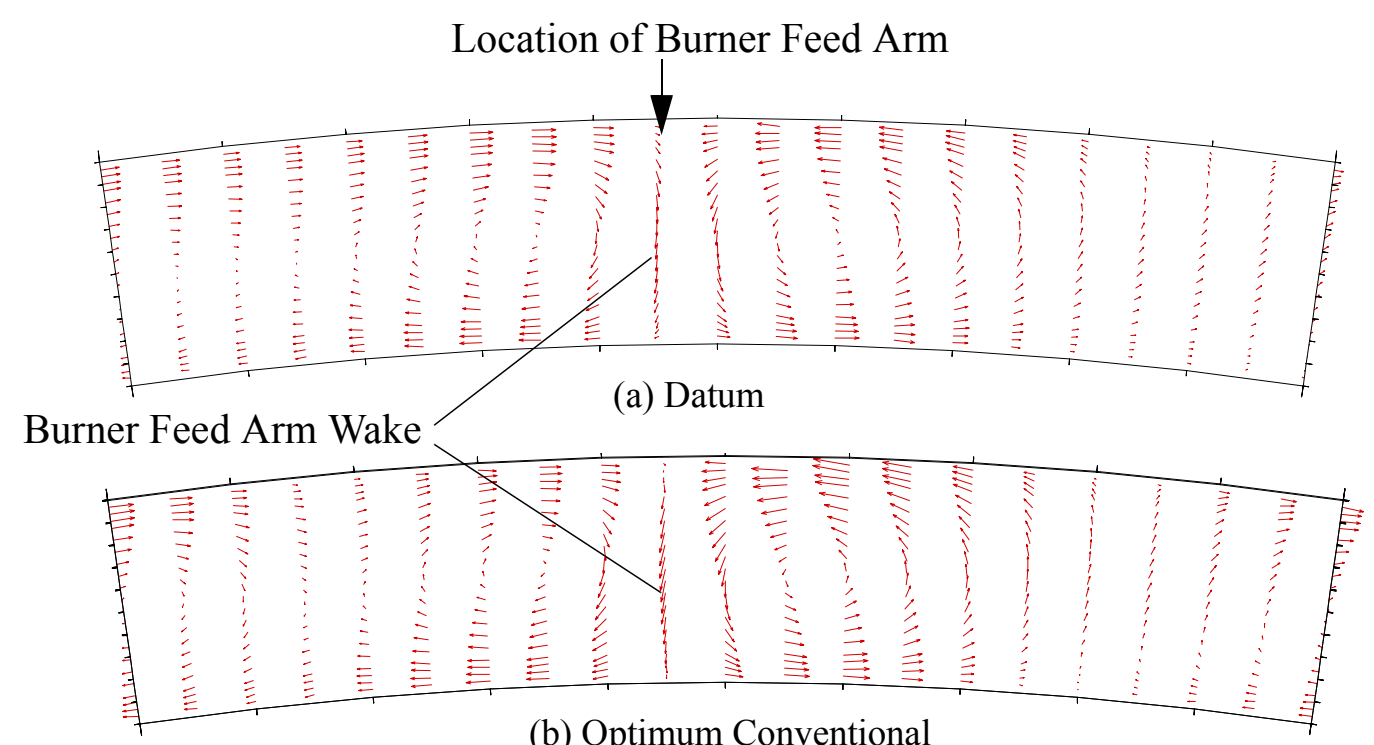

(b) Optimum Conventional

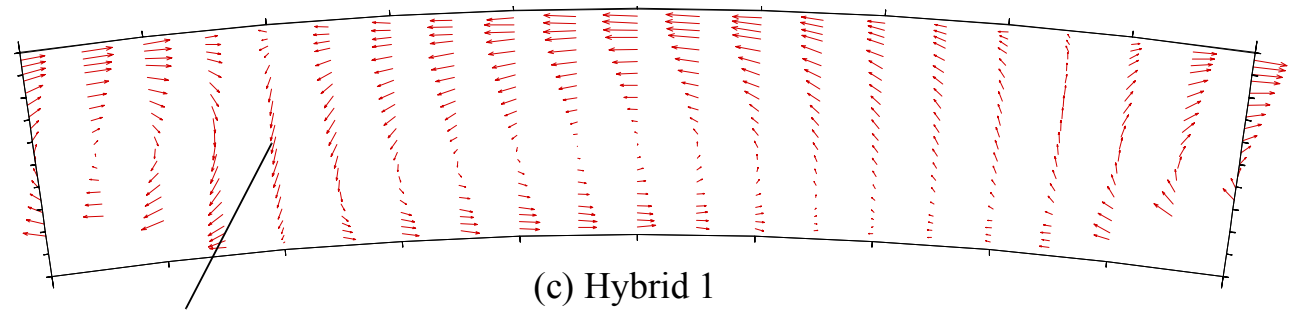

Burner Feed Arm Wake

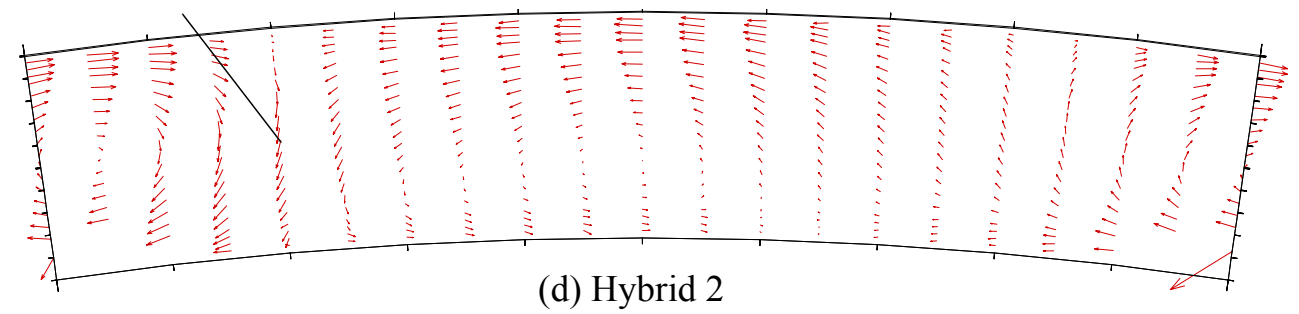

(d) Hybrid 2

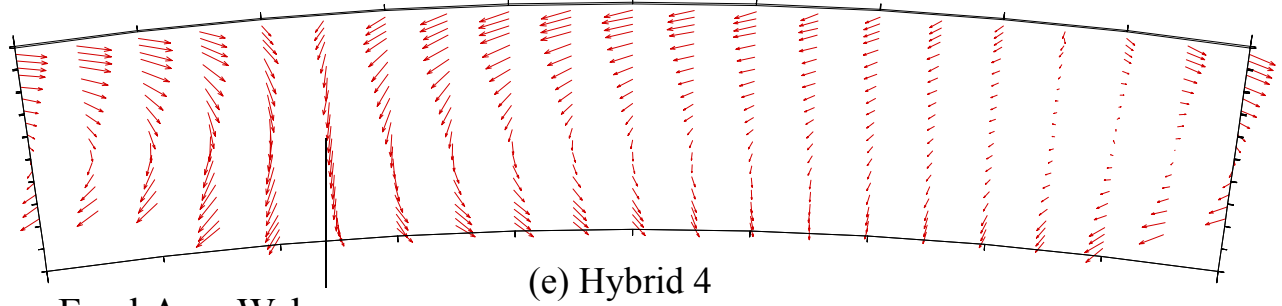

Burner Feed Arm Wake

e) Hybrid 4

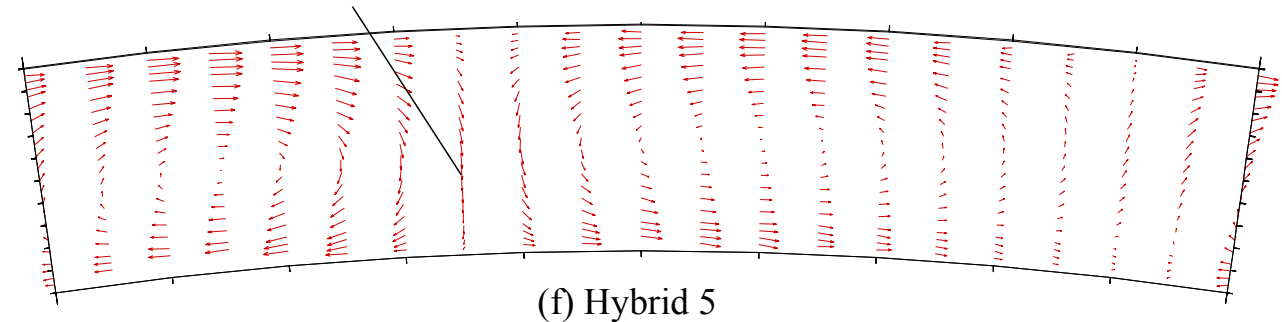

Figure 4.13 Projected Total Velocity Vectors in the Outer Annulus 


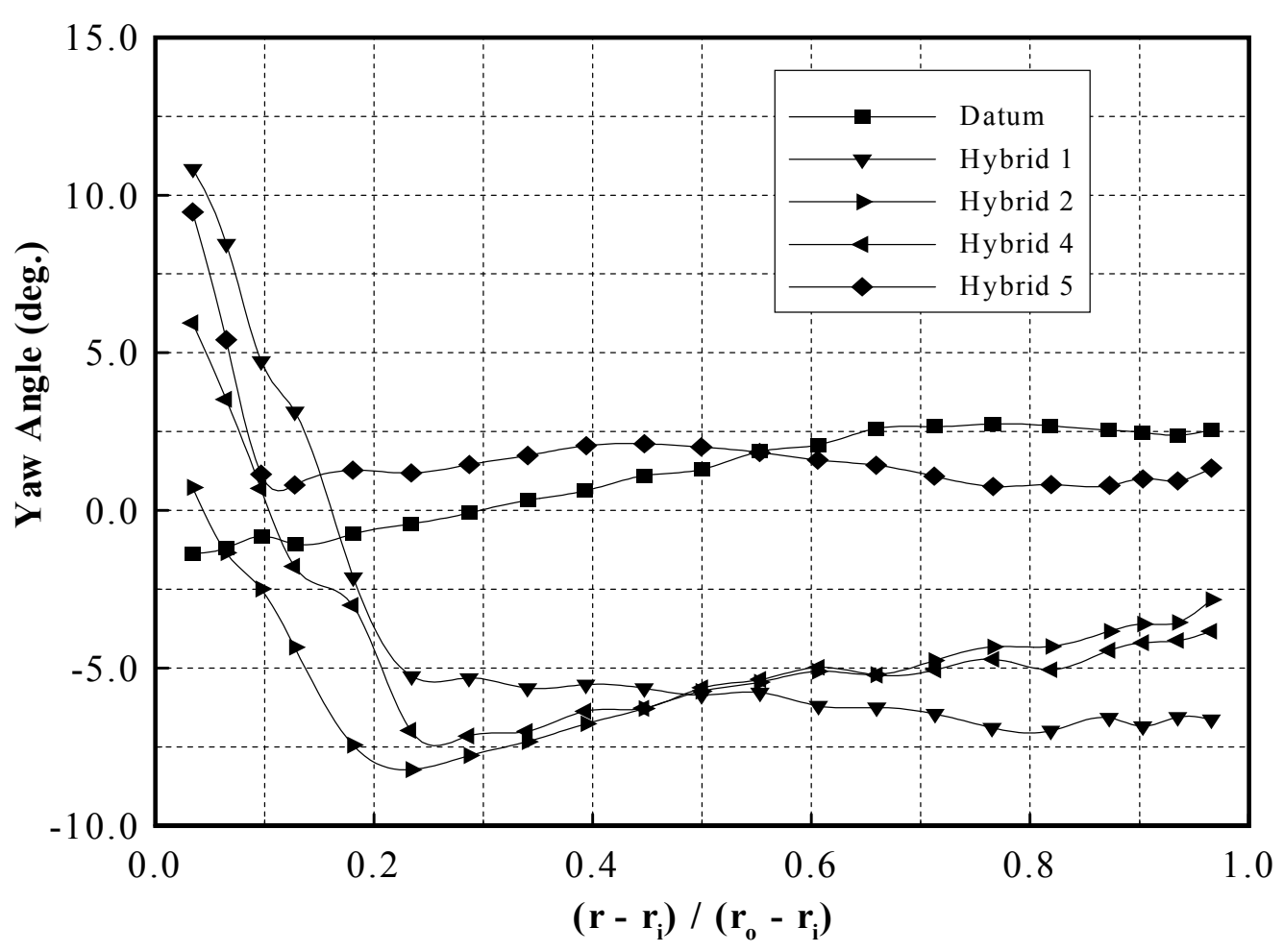

Figure 4.14 Circumferentially Averaged Swirl Angle in Outer Annulus

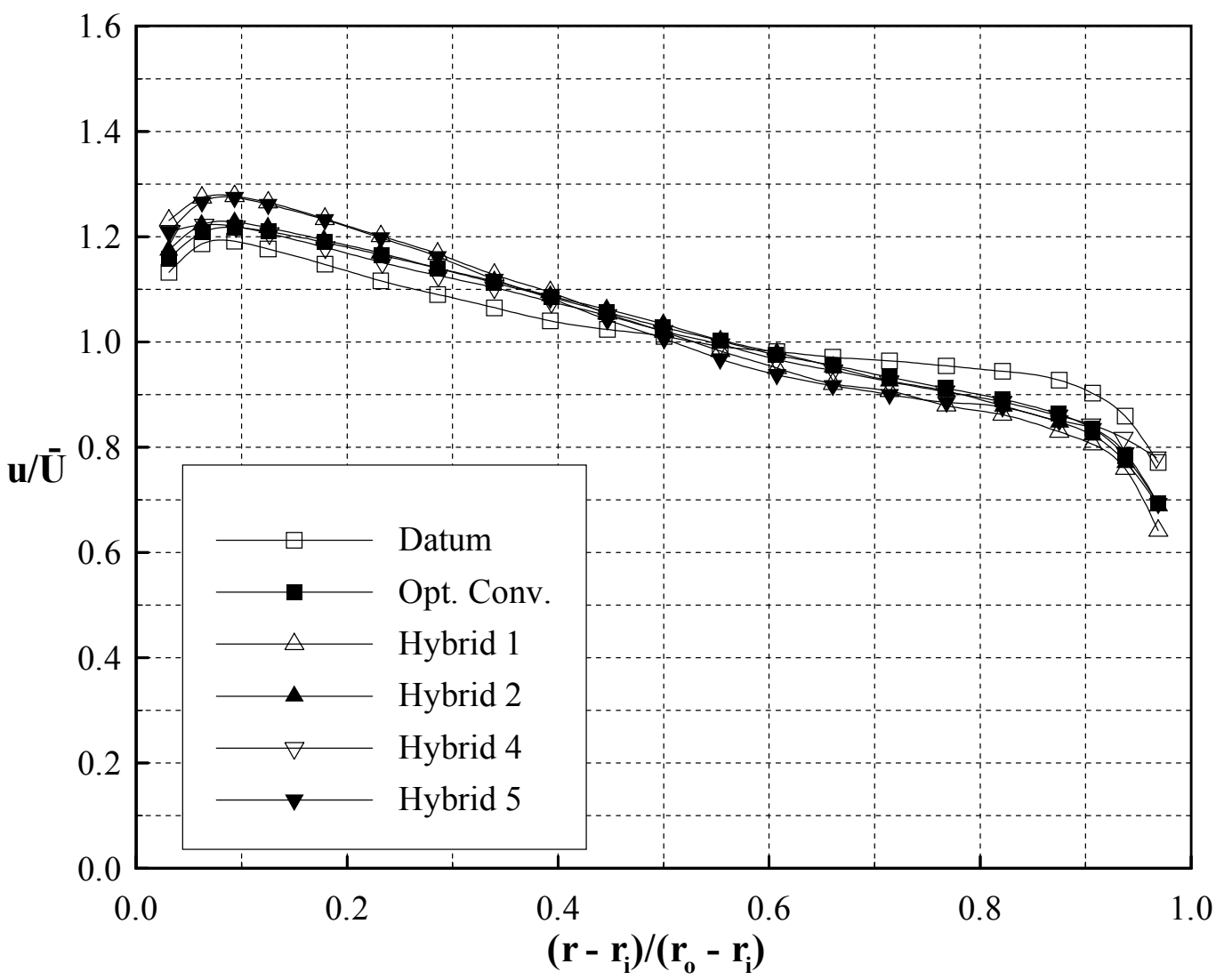

Figure 4.15 Circumferentially Averaged Velocity Profiles in Inner Annulus 


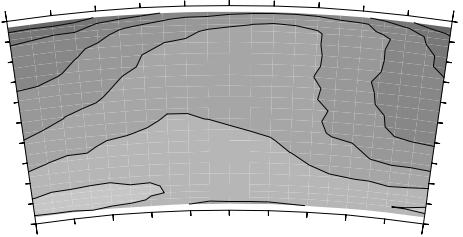

(a) Datum

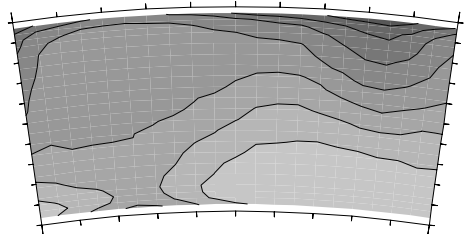

(b) Optimum Conventional

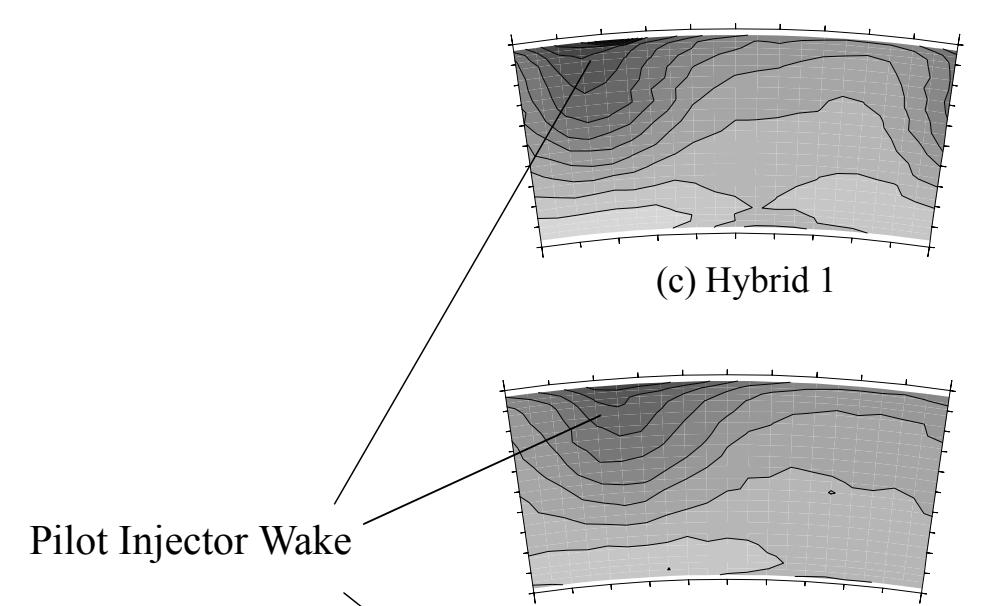

(d) Hybrid 2

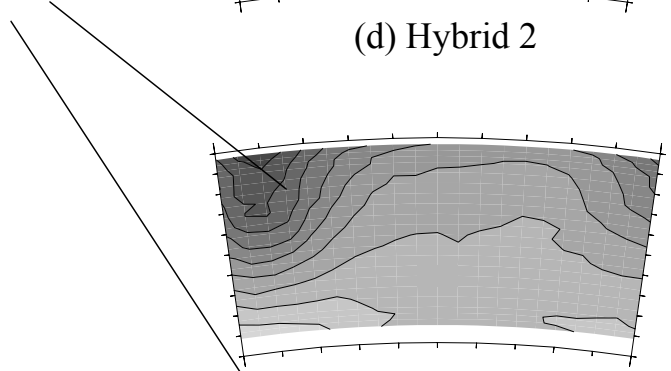

(e) Hybrid 4

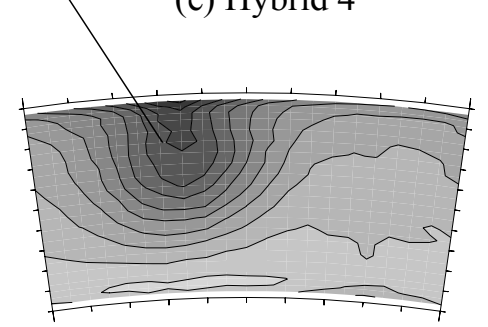

(f) Hybrid 5

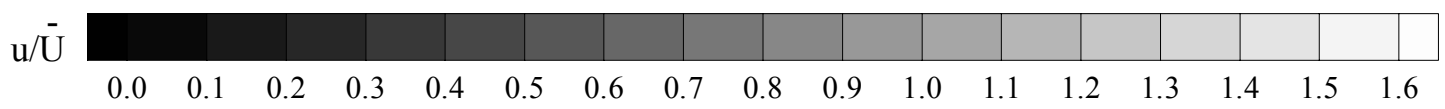

Figure 4.16 Axial Velocity Contours in Inner Annulus 


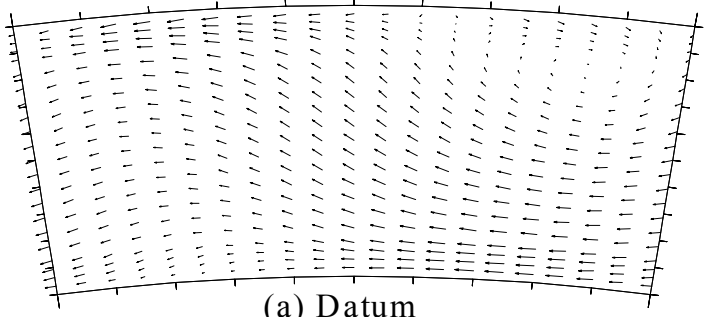

(a) Datum

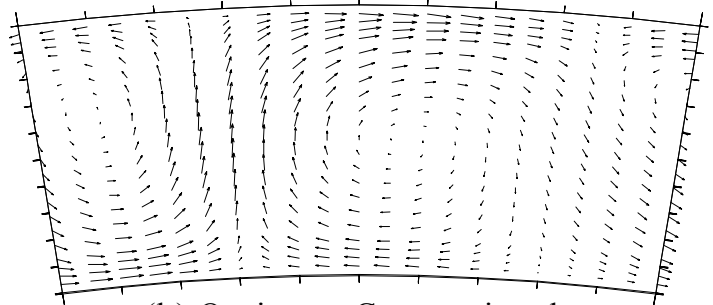

(b) Optimum Conventional
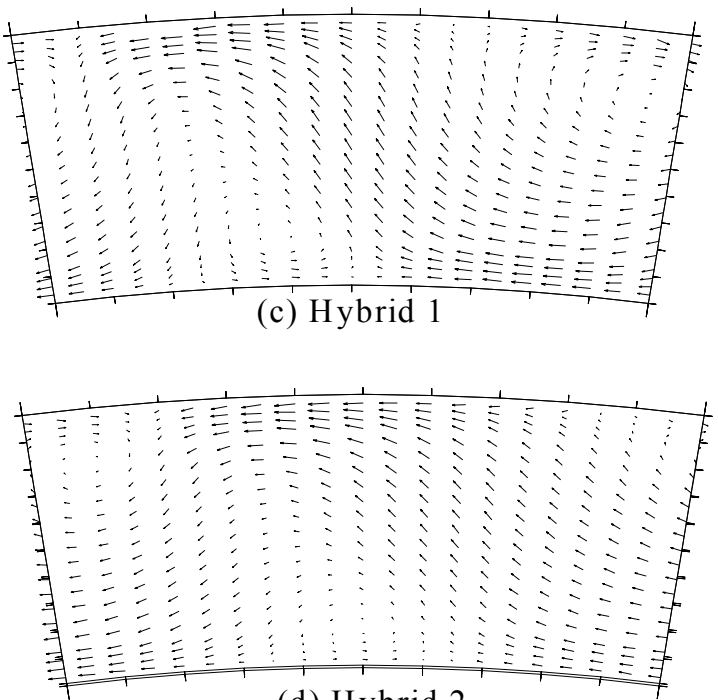

(d) Hybrid 2

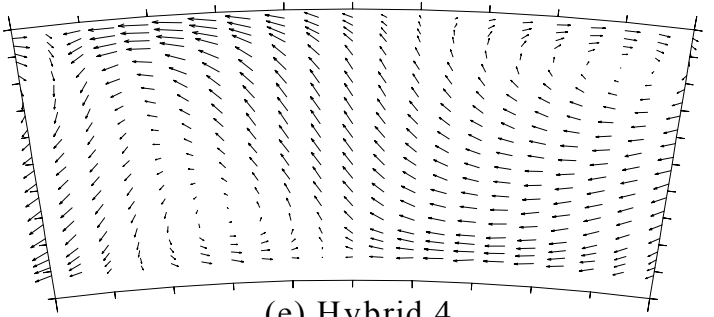

(e) Hybrid 4

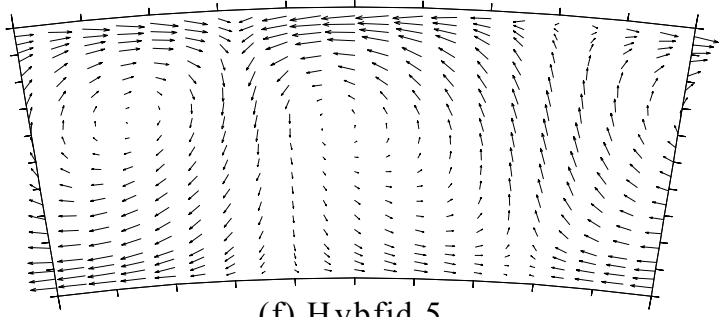

(f) Hybfid 5

Figure 4.17 Projected Total Velocity Vectors in Inner Annulus 


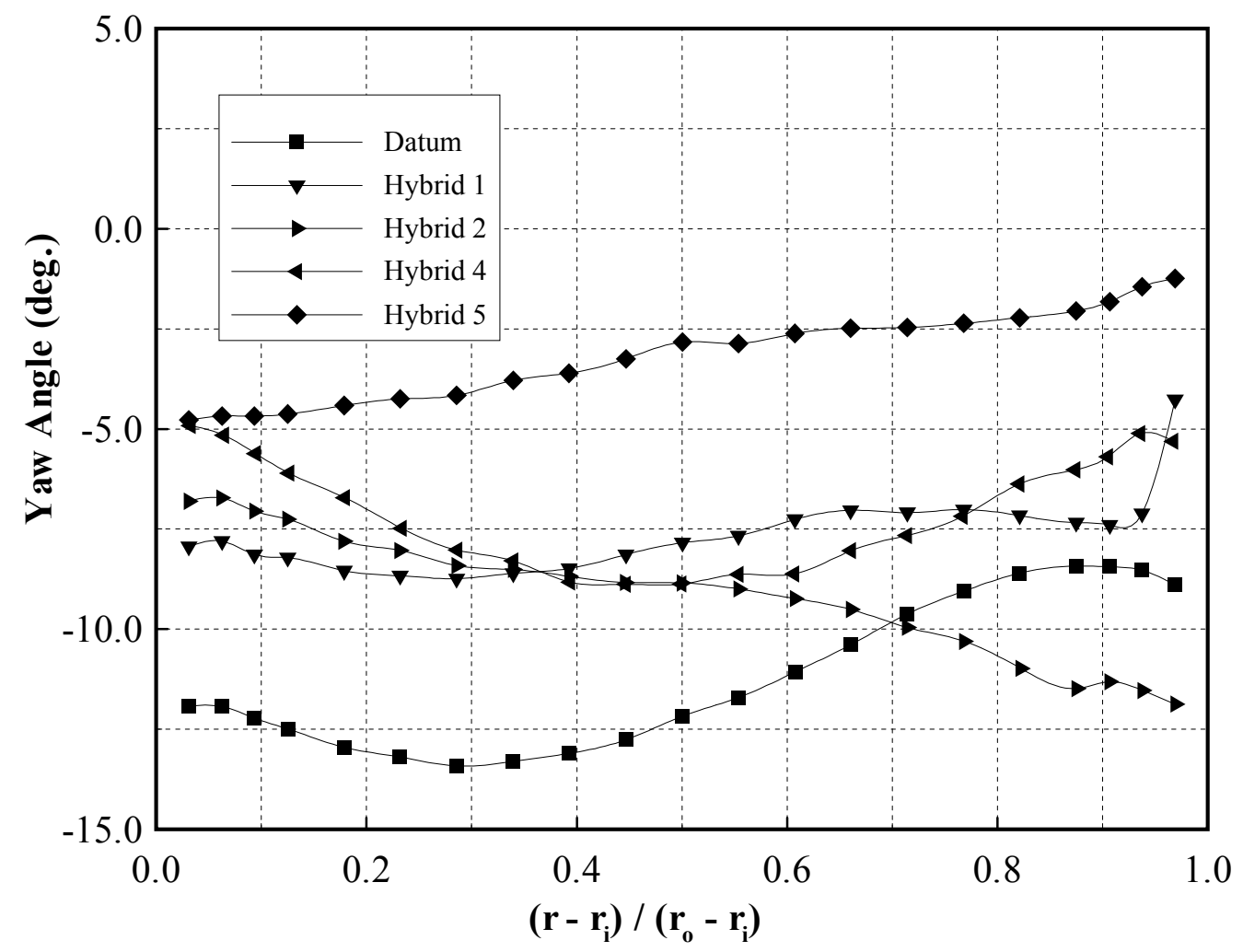

Figure 4.18 Circumferentially Averaged Swirl Angle in Inner Annulus

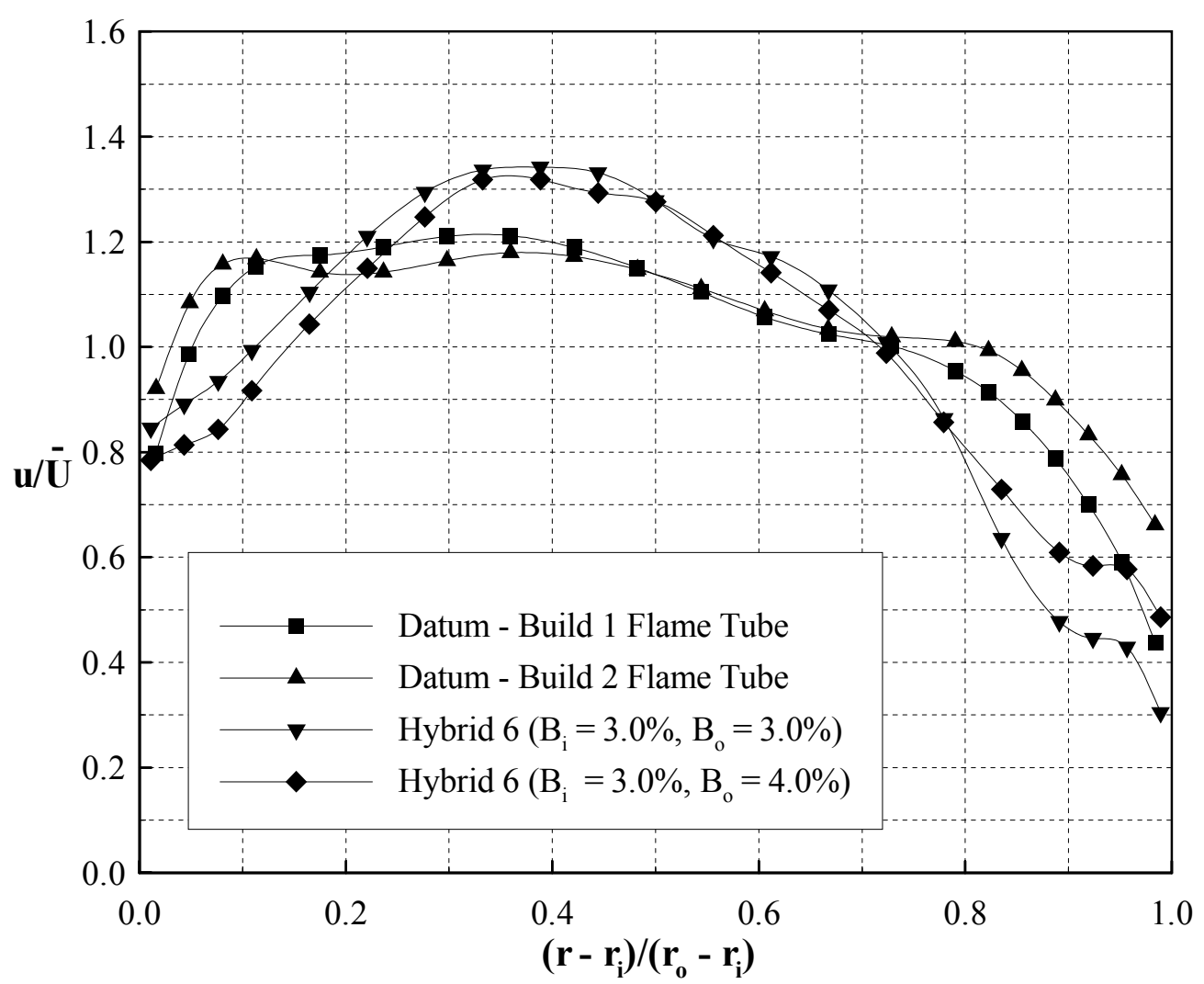

Figure 4.19 Circumferentially Averaged Axial Velocity Profiles at Pre-Diffuser Exit 
(a) Datum (Build 1 Flame Tube)

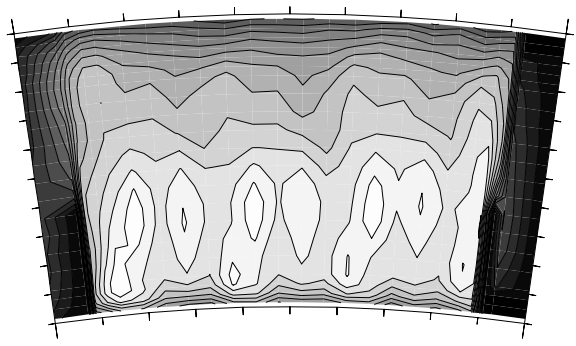

(b) Datum (Build 2 Flame Tube)

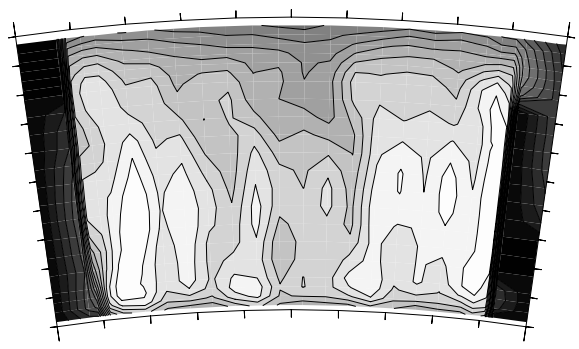

(c) Hybrid 6

$\mathrm{B}_{\mathrm{i}}=3.0 \%, \mathrm{~B}_{\mathrm{o}}=3.0 \%$

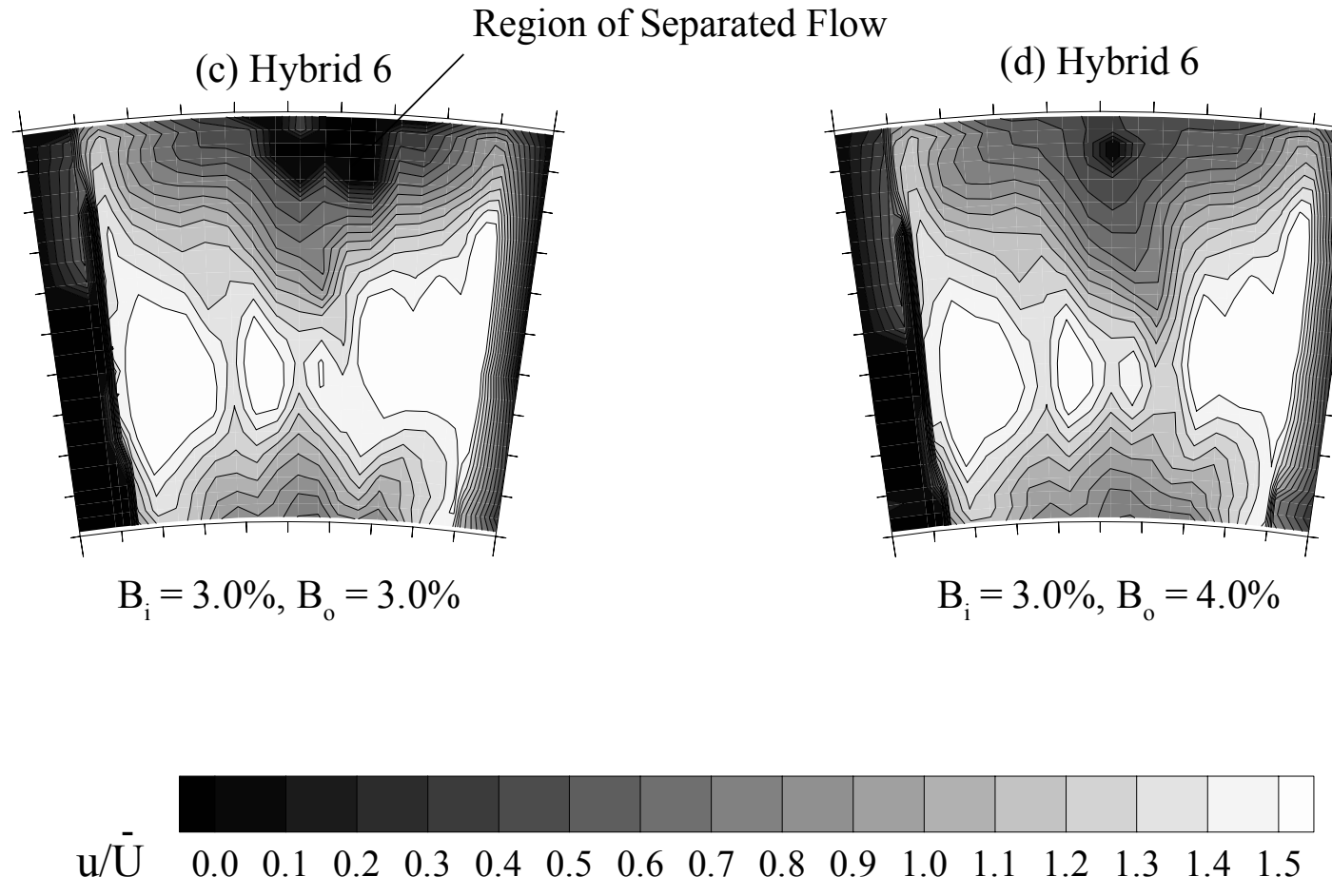

Region of Separated Flow

Figure 4.20 Axial Velocity Contours at Pre-Diffuser Exit (Build 2) 
(a) Datum (Build 1 Flame Tube)

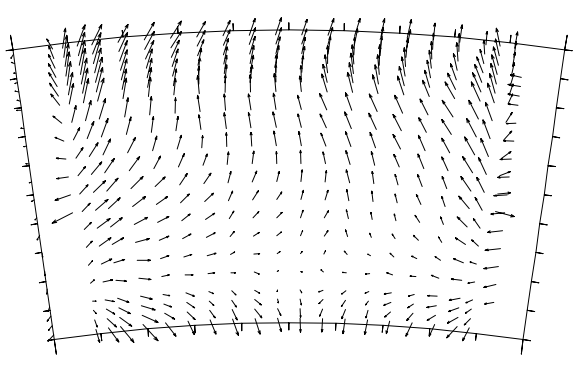

(c) Hybrid 6

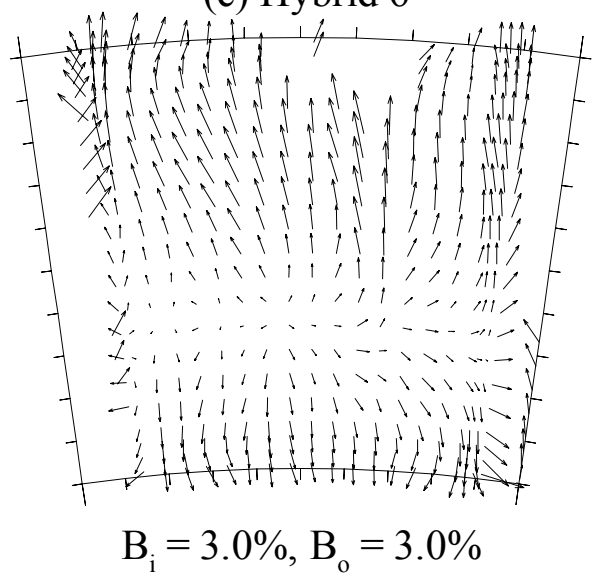

(b) Datum (Build 2 Flame Tube)

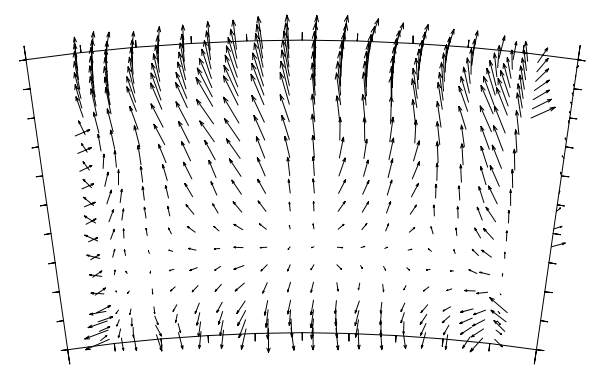

(d) Hybrid 6

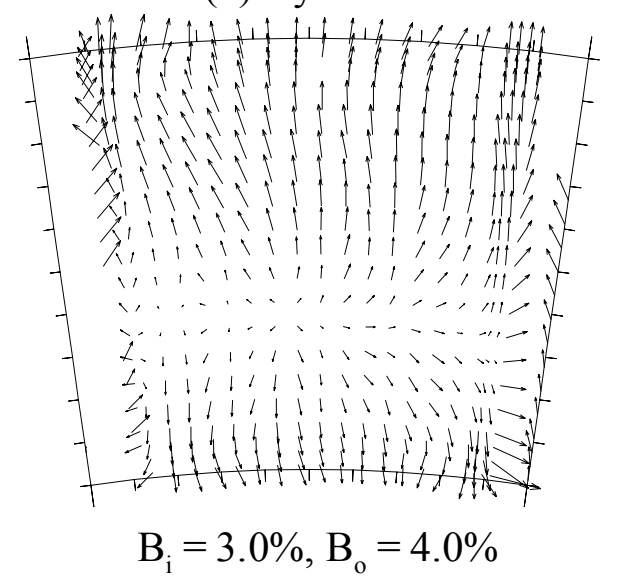

Figure 4.21 Projected Total Velocity Vectors at Pre-Diffuser Exit (Build 2) 


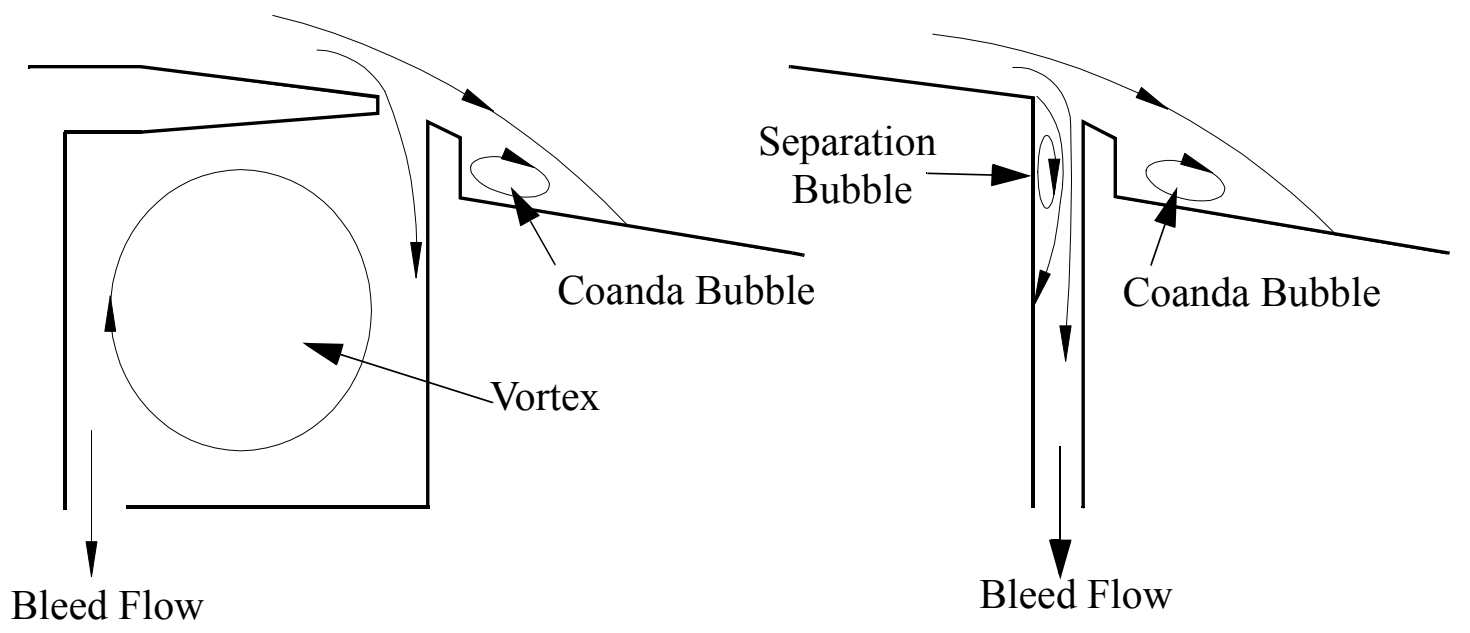

Figure 4.22 Differences between Vortex and Duct Bleed

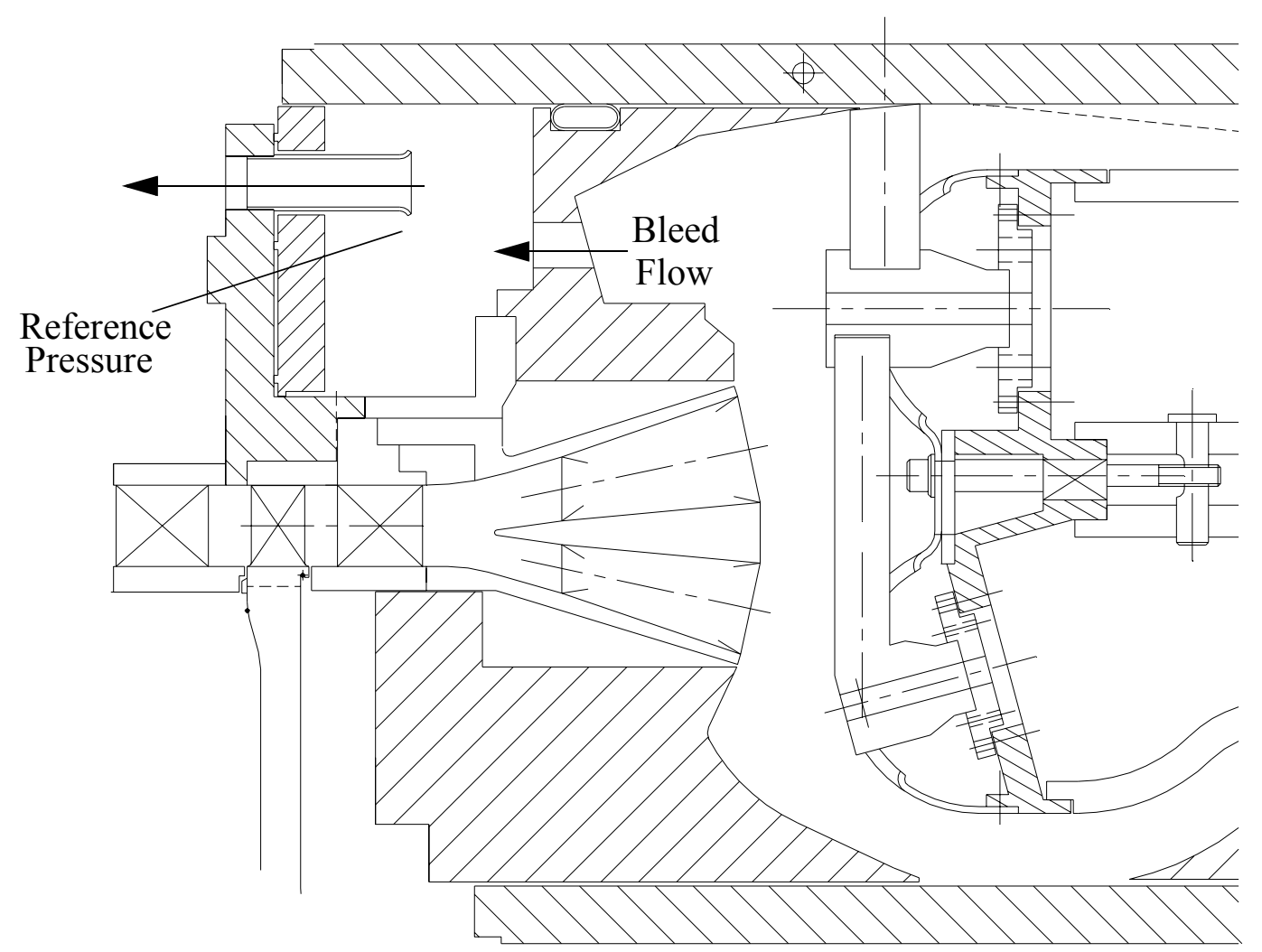

Figure 4.23 Optimum Conventional Dump Bleed 


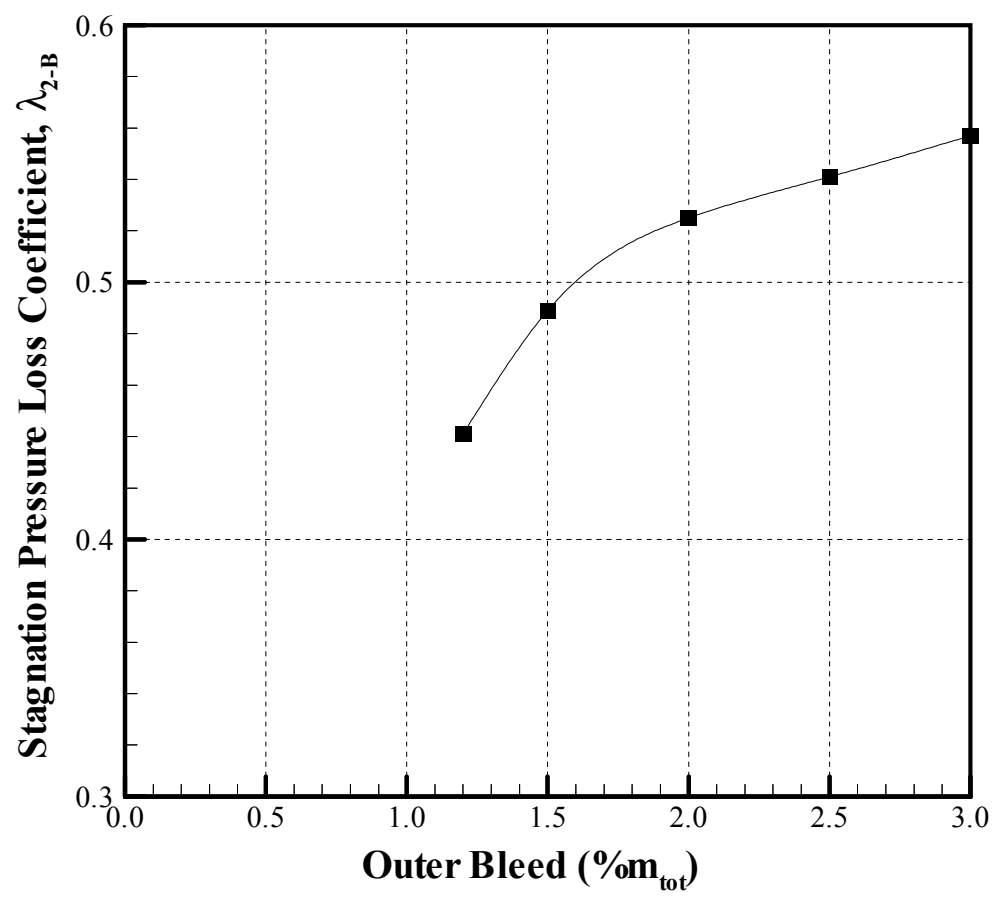

Figure 4.24 Variation of Outer Bleed Flow Loss with Bleed Rate (Hybrid 4)

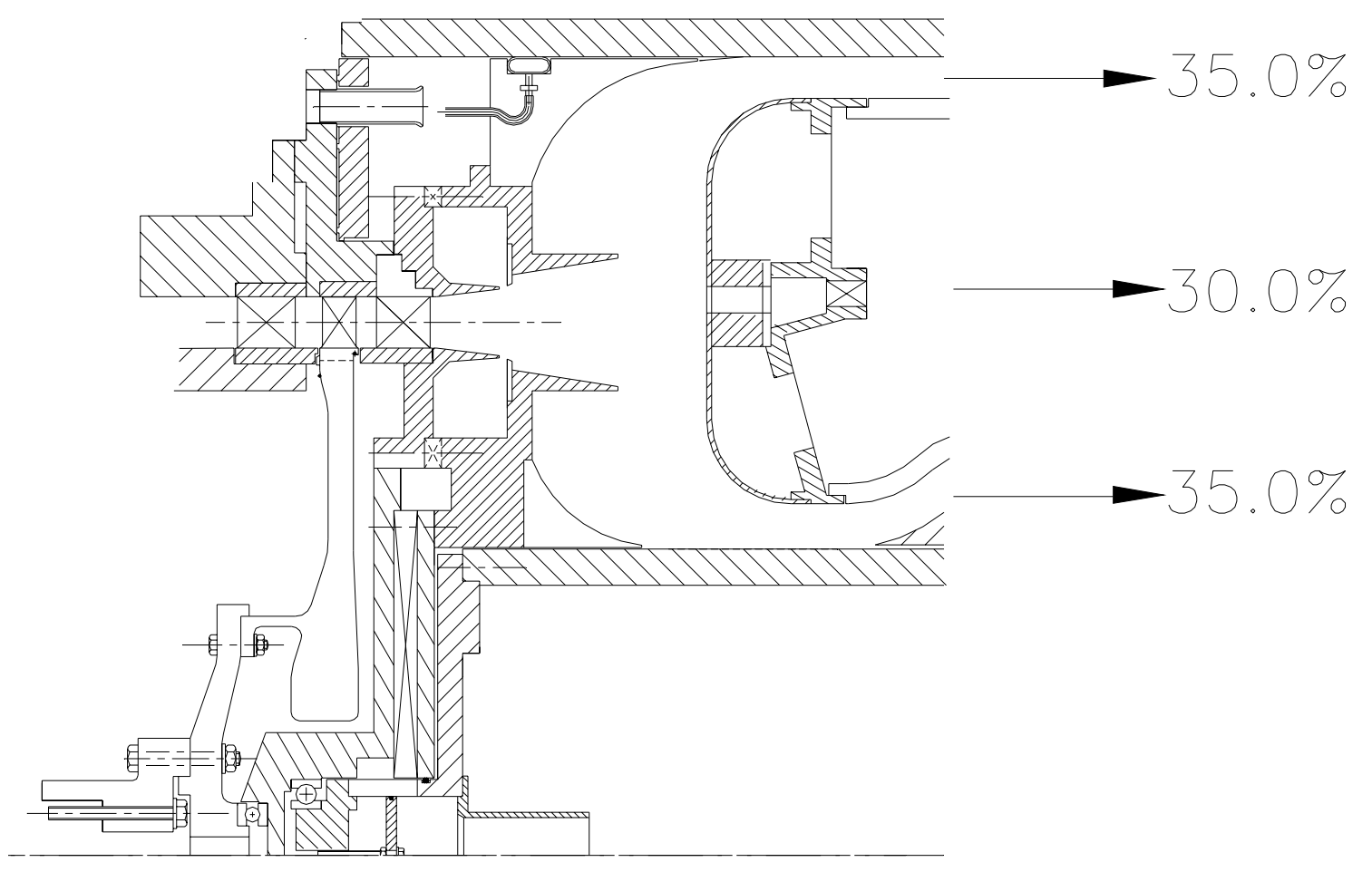

Figure 4.25 2D Flame Tube with 3D Inlet (Rotor) 


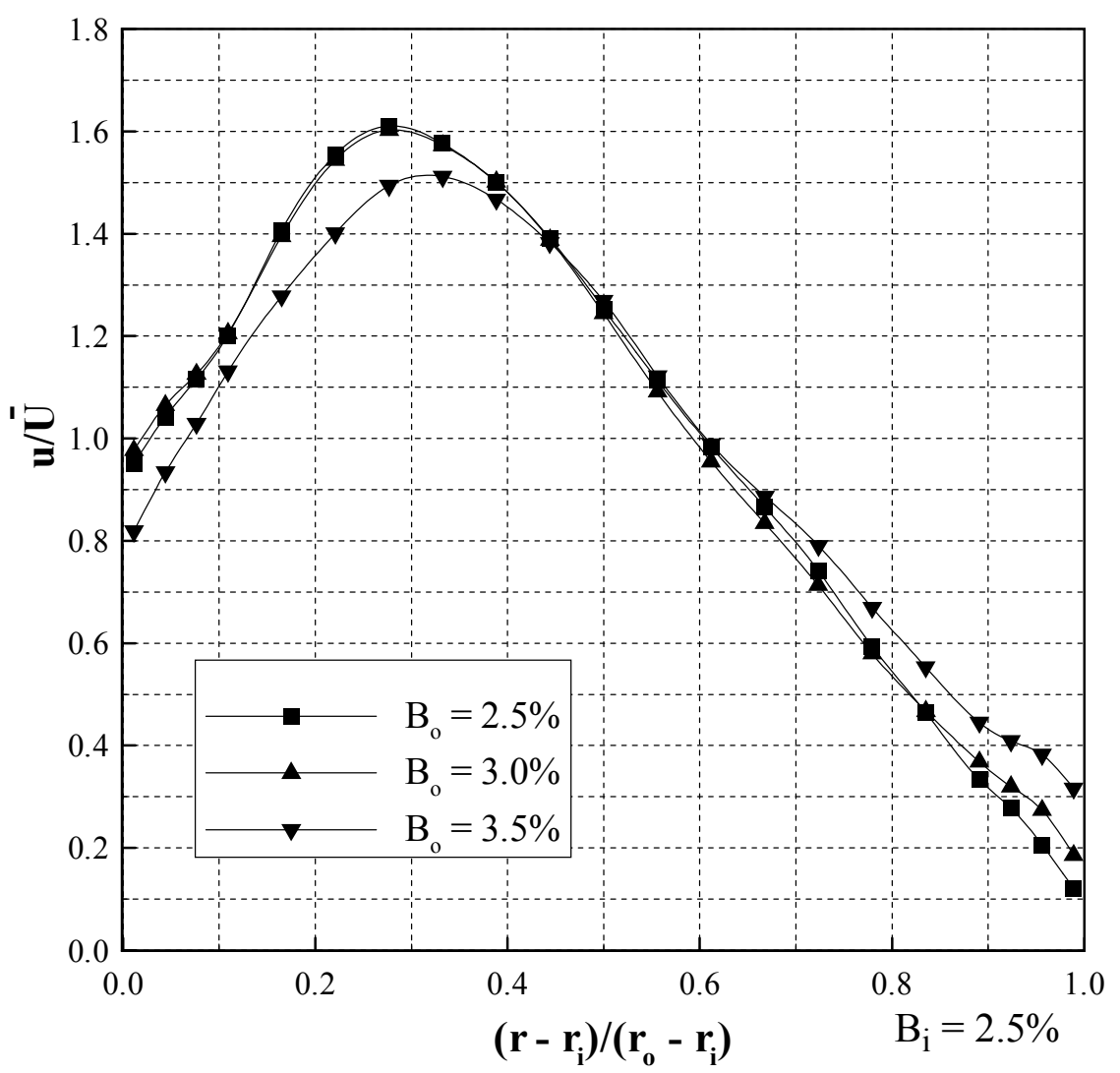

Figure 4.26 Circumferentially Averaged Axial Velocity Profile at Diffuser Exit

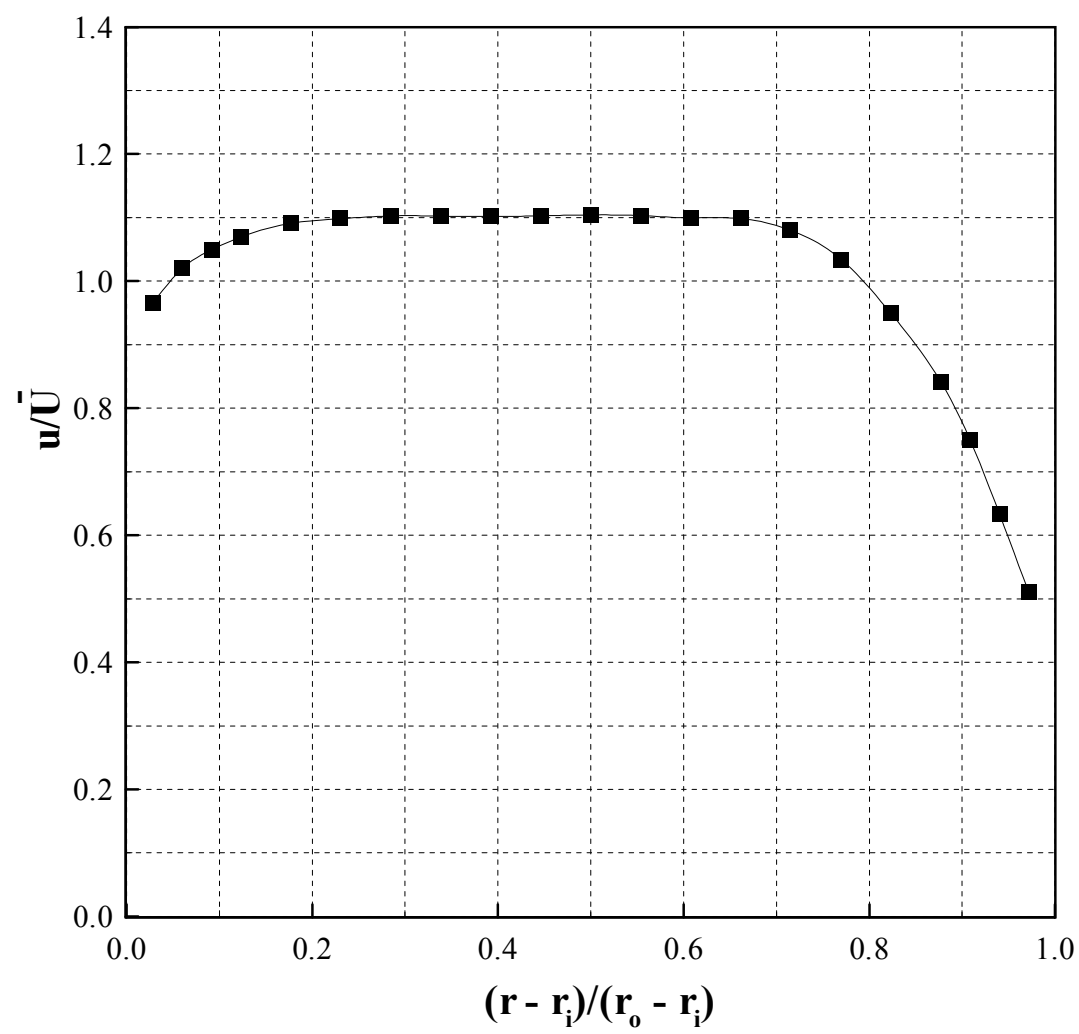

Figure 4.27 Circumferentially Averaged Axial Velocity Profile at Pre-Diffuser Inlet 


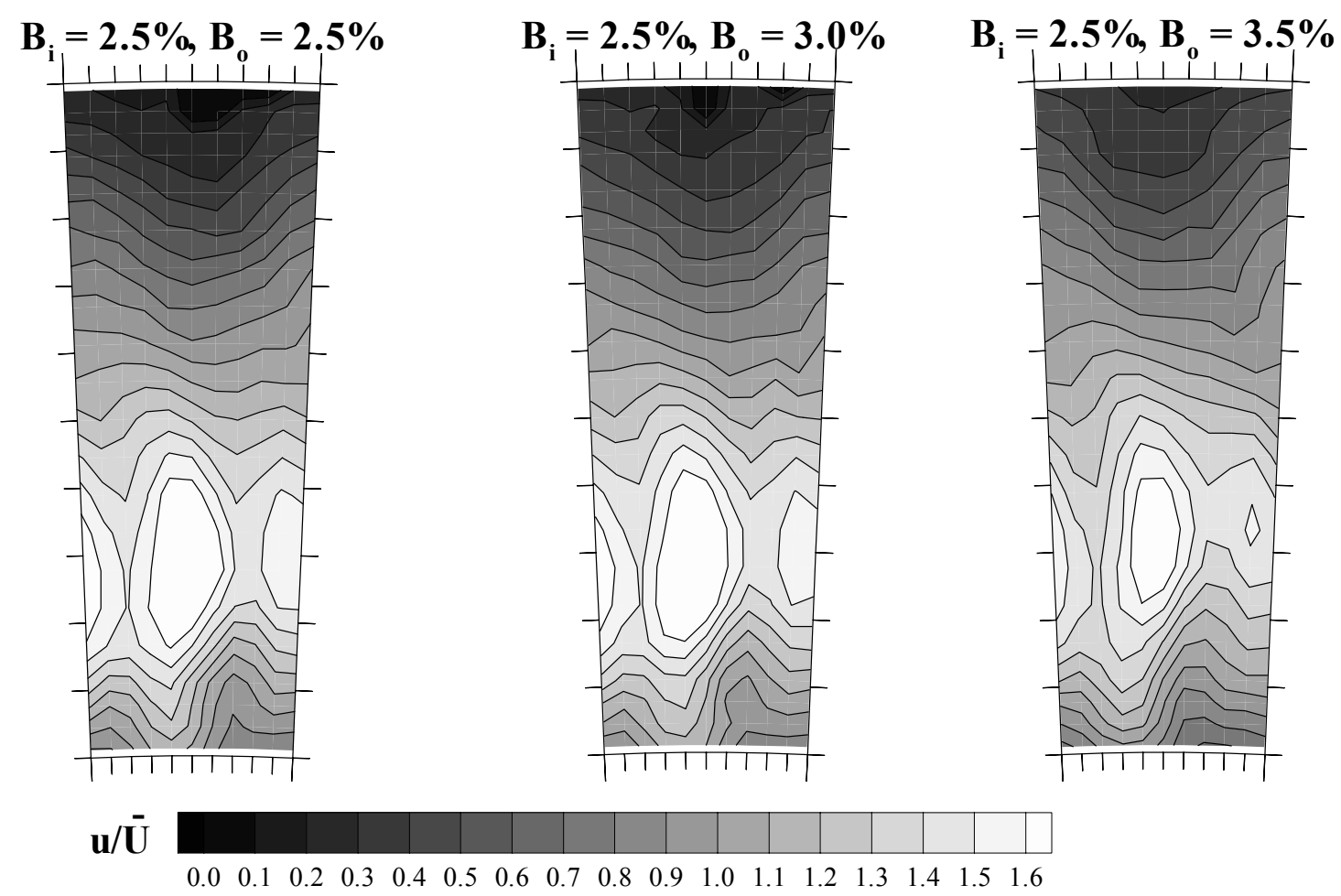

Figure 4.28 Axial Velocity Contours at Diffuser Exit

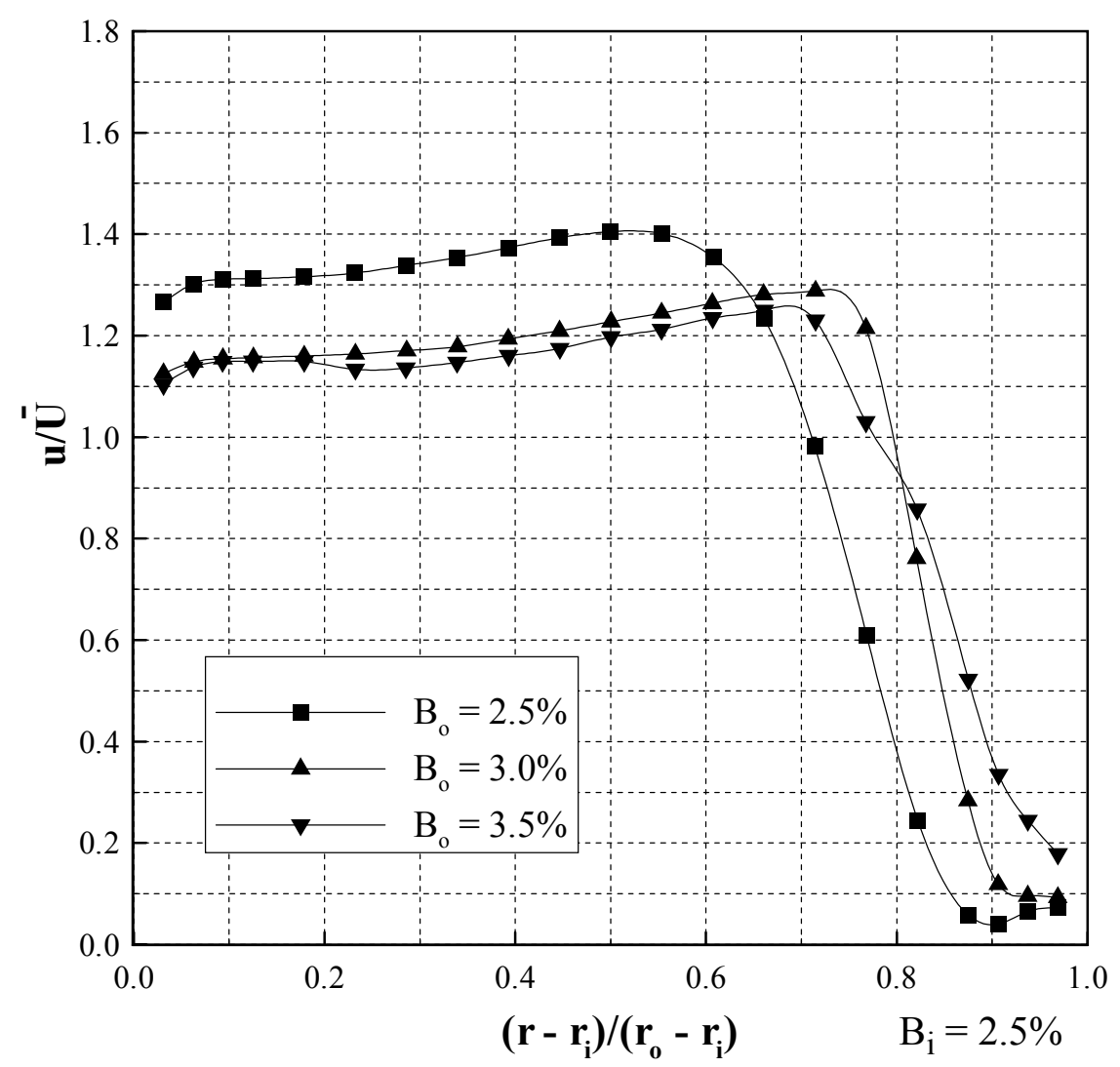

Figure 4.29 Circumferentially Averaged Axial Velocity Profiles in Inner Annulus 


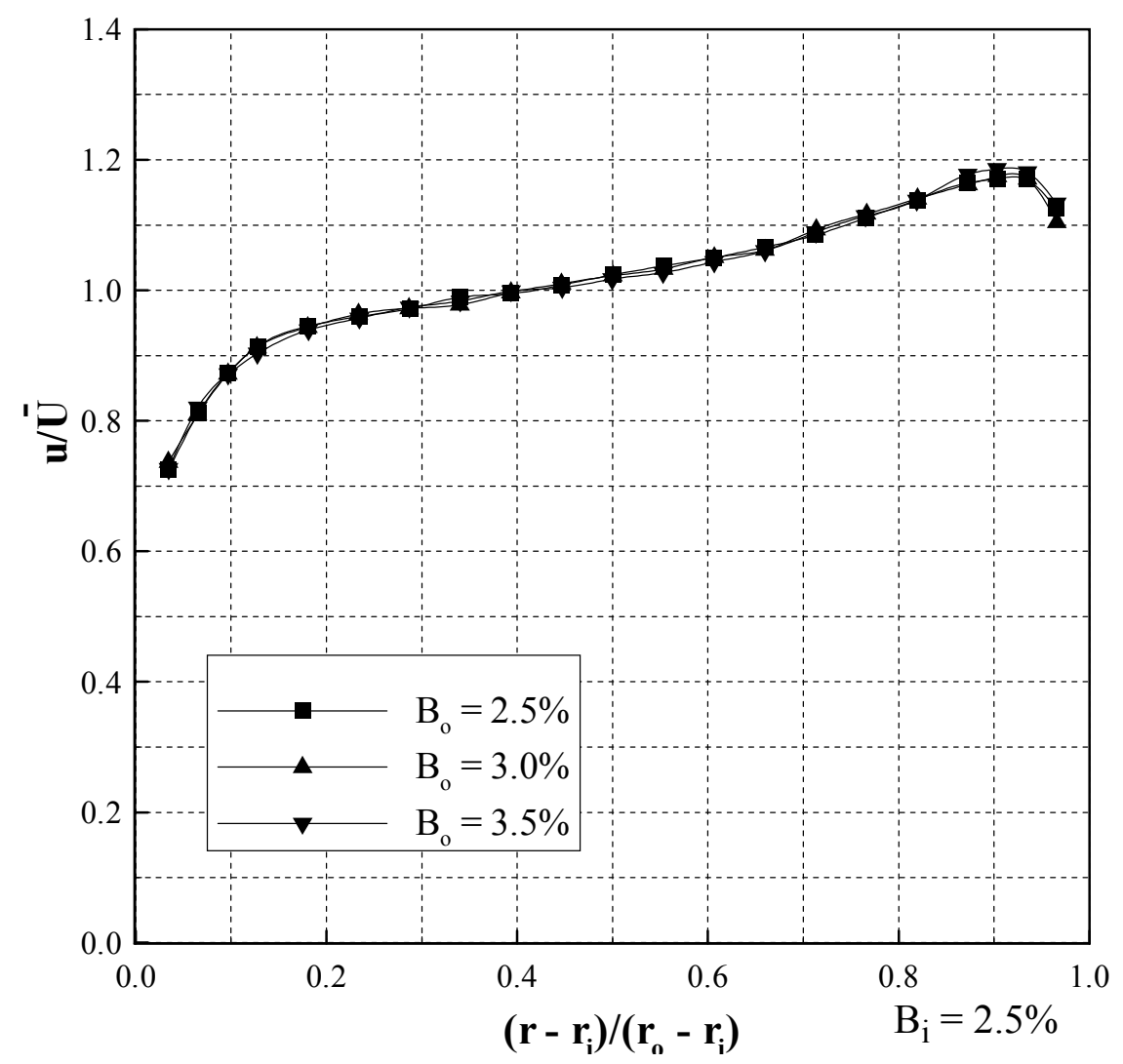

Figure 4.30 Circumferentially Averaged Axial Velocity Profiles in Outer Annulus 


\subsection{COMPUTATIONAL METHODOLOGY}

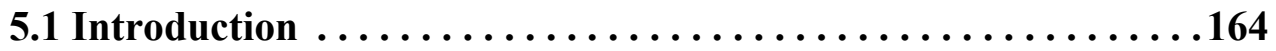

5.2 Governing Equations . . . . . . . . . . . . . . . . 165

5.3 Solution of the Governing Equations . . . . . . . . . . 166

5.4 Closure - Turbulence Modelling $\ldots \ldots \ldots \ldots \ldots \ldots \ldots$

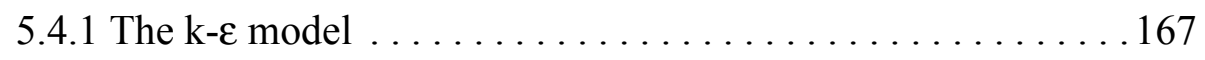

5.4.2 The Reynolds Stress Equation Model . . . . . . . . . . . . 172

5.5 Modelling of Near-Wall Flows for Wall-Bounded Flows $\ldots \ldots 175$

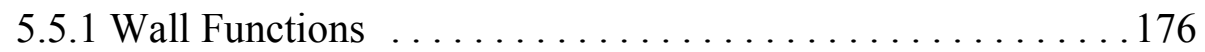

5.6 Numerical Implementation $\ldots \ldots \ldots \ldots \ldots \ldots \ldots \ldots \ldots \ldots$

5.7 Computational Investigation of Hybrid Diffusers $\ldots \ldots \ldots 183$

5.7.1 Geometry Definition and Boundary Conditions . . . . . . . . 184

5.7.1.1 Plane Two-Dimensional Hybrid Diffusers . . . . . . . . 184

5.7.1.2 Two-Dimensional Axi-Symmetric Hybrid Diffuser .188

5.7.1.3 Three-Dimensional, Fully Annular Hybrid Diffuser . 189

5.7.2 Post-Processing and Presentation of Results . . . . . . . 190

5.8 Grid Generation and Solution Development $\ldots \ldots \ldots \ldots \ldots 1$

5.8.1 Initial Grid Definition . . . . . . . . . . . . . . . . . 192

5.8.2 Grid Refinement and Near Wall Treatment ........... 193

5.8.3 Turbulence Model Assessment . . . . . . . . . . . . . . . . . . 194

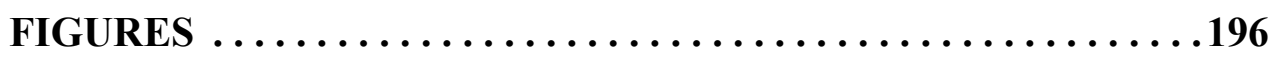




\subsection{COMPUTATIONAL METHODOLOGY}

\section{$\underline{5.1 \text { Introduction }}$}

Sections 3.0 and 4.0 have described and discussed how high quality data for hybrid diffusers were obtained using an experimental facility. However, the cost of such an experiment is often high preventing a large parametric study being undertaken. This is significant here as the effect of altering bleed gap geometrical parameters, although of considerable design interest, was not investigated experimentally due to the complexity and expense of test rig modifications. Computational Fluid Dynamics (CFD) offers a complementary approach to experimental methods and, although not problem free, provides several key advantages. Over the last couple of decades both CFD algorithms and computational power have increased immensely, thus allowing CFD techniques to be used to predict many complex aerodynamic flows. Ultimately, final proof of most designs is still determined using an experimental facility but CFD is an important tool in the design process and significantly increases understanding of the relevant flow mechanisms. It is in the nature of CFD that the flow field is predicted throughout the entire solution domain at a resolution limited only by the modelling techniques employed and the physical size of the computational grid. Thus, flow behaviour can be studied in areas in which it may not be possible to gain access with instrumentation. It is significant that this is used here to allow a detailed examination of the nature and behaviour of the flow in a hybrid diffuser; specifically in the Stage 2 diffuser and subsequent reattachment on the Stage 3 wall.

The software used for CFD predictions is usually classified in one of three areas: PreProcessor, Solver and Post-Processor. In order to perform this investigation a number of commercially available packages were used. Geometries and mesh definitions were generated using two packages; PreBFC (from Fluent Inc.) and the ICEM CFD suite (including DDN, DDN Mesher Interface and HEX Mesher). A popular and robust solver, Fluent, was used to define the physical constants, fluid properties and boundary conditions, and finally solve the Reynolds Averaged Navier Stokes Equations. Fluent is also capable of performing a significant amount of post-processing and has its own data visualisation tools. However, to maintain consistency with the experimental data, Tecplot 
(AMTEC Engineering Inc.) was used for final graphical visualisation of the CFD data.

Commercially available software was used with the aim of simplifying the computational study. However, this does mean that no further development of solution techniques to suit the particular flow physics was undertaken other than those already contained within the software. Nonetheless, the following presents, for completeness a summary of the modelling techniques used.

\subsection{Governing Equations}

Unfortunately CFD is not an easy tool to use and requires a sizable amount of knowledge and experience before accurate predictions can be achieved. The following presents, briefly, the governing equations of fluid motion for which direct numerical solution (DNS) is not presently computationally feasible at high Reynolds Numbers. The classical methods employed to enable solution of the governing equations will be discussed together with their applicability and accuracy.

The instantaneous continuity equation in Cartesian tensor notation is:

$$
\frac{\partial \rho}{\partial t}+\frac{\partial \rho U_{i}}{\partial x_{i}}=0
$$

The momentum equations can be written as:

$$
\frac{\partial \rho U_{i}}{\partial t}+\frac{\partial \rho U_{i} U_{j}}{\partial x_{j}}=-\frac{\partial p}{\partial x_{i}}+\frac{\partial \tau_{i j}}{\partial x_{j}}
$$

Equation 5.2 introduces further variables, namely the viscous stress components, $\tau_{\mathrm{ij}}$ and these can be expressed for a Newtonian fluid (Schlichting ${ }^{[1979]}$ ) as:

$$
\tau_{i j}=\mu\left[\frac{\partial U_{i}}{\partial x_{j}}+\frac{\partial U_{j}}{\partial x_{i}}\right]-\frac{2}{3} \mu \frac{\partial U_{i}}{\partial x_{i}}
$$


Substitution of these viscous stresses into the momentum equations yields the NavierStokes Equations which are a closed set of four non-linear equations with four unknowns; $\mathrm{U}, \mathrm{V}, \mathrm{W}$ and $\mathrm{P}$ (assuming that at low Mach numbers the fluid properties $\rho$ and $\mu$ may be considered constant and known).

\subsection{Solution of the Governing Equations}

Direct numerical solution of the Navier-Stokes equations is theoretically possible but currently computationally far too demanding for practical Reynolds numbers to be useful. Thus, the common practice is to decompose all of the instantaneous quantities into mean and fluctuating components:

$$
U_{\mathrm{i}}=\overline{\mathrm{U}}_{\mathrm{i}}+\mathrm{u}_{\mathrm{i}}
$$

$$
\text { instantaneous }=\text { mean }+ \text { fluctuating }
$$

Defining the mean value via a long time-averaging

$$
\bar{U}_{i}\left(x_{i}\right)=\lim _{T-\infty} \frac{1}{T} \int_{t}^{(t+T)} U_{i}\left(x_{i}, t^{\prime}\right) d t^{\prime}
$$

leads to the Reynolds Averaged form of the Navier-Stokes equations (quoted here for a constant density, Newtonian fluid under steady state flow conditions):

$$
\begin{gathered}
\frac{\partial \overline{\mathrm{U}}_{\mathrm{i}}}{\partial \mathrm{x}_{\mathrm{i}}}=0 \\
\frac{\partial \rho \overline{\mathrm{U}}_{\mathrm{i}} \overline{\mathrm{U}}_{\mathrm{j}}}{\partial \mathrm{x}_{\mathrm{j}}}=-\frac{\partial}{\partial \mathrm{x}_{\mathrm{i}}} \overline{\mathrm{p}}+\frac{\partial \tau_{\mathrm{ij}}}{\partial \mathrm{x}_{\mathrm{j}}}-\frac{\partial \overline{\rho \mathrm{u}_{\mathrm{i}} \mathrm{u}_{\mathrm{j}}}}{\partial \mathrm{x}_{\mathrm{j}}} \\
(1) \quad(2) \\
\tau_{\mathrm{ij}}=\mu\left[\frac{\partial \overline{\mathrm{U}}_{\mathrm{i}}}{\partial \mathrm{x}_{\mathrm{j}}}+\frac{\partial \overline{\mathrm{U}}_{\mathrm{j}}}{\partial \mathrm{x}_{\mathrm{j}}}\right]
\end{gathered}
$$

The various terms can be described as:
(1) convection
(2) static pressure gradient
(3) viscous diffusion
(4) turbulent transport 
This set of equations is no longer closed since six extra stress terms, $\left(\overline{\rho u_{i} u_{j}}\right)$, three normal and three shear, appear in the Reynolds equations. Note that there are nine stress terms but the stress tensor is symmetrical such that:

$$
\overline{\rho u_{i} u_{j}}=\overline{\rho u_{j} u_{i}}
$$

These turbulent stresses are termed the Reynolds stresses and physically represent the transport of momentum due to the turbulent fluctuations.

\subsection{Closure - Turbulence Modelling}

The Reynolds averaged form of the Navier-Stokes equations no longer represents a closed system due to the extra Reynolds stress terms. Turbulence modelling is a computational method employed to close the set of mean flow equations. The main task is to generate procedures which predict the Reynolds stress terms with sufficient accuracy from knowledge of the time-averaged flow field. There are various methods by which turbulence can be modelled with different amounts of success and accuracy. These methods fall into different categories depending upon the number of extra equations to be solved for turbulence related quantities. Only two methods have been employed in this investigation, so logically only these two turbulence models will be described. The k- $\varepsilon$ model (eddy-viscosity) and the Reynolds Stress model are two of the more established/ mature turbulence models and a full description of each can be found in most relevant texts (for example Versteeg and Malalasekera ${ }^{[1995]}$ ). However, to allow discussion of the turbulence characteristics of the flow in this investigation some of the underlying ideas will be briefly presented here.

\subsubsection{The k- $\varepsilon$ Model (Eddy-Viscosity)}

Turbulent stresses are found to increase as the mean rate of deformation increases and Boussinesq (Hinze ${ }^{[1975]}$ ) proposed that the Reynolds stresses could be linked to the mean rates of deformation in direct analogy with the Newtonian definition of molecular viscous stress. Here the Reynolds stresses are modelled using an extension of Boussin- 
esq's eddy-viscosity relationship:

$$
-\rho{\overline{u_{i}}}_{j}=\mu_{t}\left[\frac{\partial \bar{U}_{i}}{\partial x_{j}}+\frac{\partial \bar{U}_{j}}{\partial x_{i}}\right]-\frac{2}{3} \delta_{i j} \rho k
$$

Where the extra term contains the Kronecker delta $\left(\delta_{i j}=1\right.$ when $i=j$ and $\delta_{i j}=0$ when $i \neq$ j) which provides the formula with the correct tensorial properties.

It is a common misconception that the Boussinesq hypothesis implies isotropic turbulence of the normal Reynolds stresses which would be physically incorrect even for simple two-dimensional flows. Clearly, from Equation 5.10 $\overline{\mathrm{u}_{\mathrm{i}} \mathrm{u}_{\mathrm{i}}} \neq \overline{\mathrm{u}_{\mathrm{j}} \mathrm{u}_{\mathrm{j}}} \neq \overline{\mathrm{u}_{\mathrm{k}} \mathrm{u}_{\mathrm{k}}}$ as each term contains different spatial velocity gradients. However, the Boussinesq hypothesis does assume that the turbulent viscosity, $\mu_{\mathrm{t}}$, is a scalar quality, which is not true. The turbulent viscosity is a function of the local shear which is a tensor and varies with direction. Thus, when the anisotropy of the turbulence dominates the mean flow then inconsistencies in $\mu_{\mathrm{t}}$ will introduce errors in Equation 5.10. However, in flows of this type, such as highly swirling flows or stress-driven secondary flows, the Boussinesq relationship still accounts for the high velocity gradients.

The kinematic turbulent viscosity, which has units of $\mathrm{m}^{2} \mathrm{~s}^{-1}$ can be expressed as a function of the velocity and length scale. The turbulent kinetic energy, $\mathrm{k}$, and the isotropic disipation rate of the turbulent kinetic energy, $\varepsilon$, are used to define a velocity scale, $\vartheta$, and a length scale, $\ell$ :

$$
\begin{gathered}
\vartheta=\mathrm{k}^{\frac{1}{2}}, \ell=\frac{\mathrm{k}^{\frac{3}{2}}}{\varepsilon} \\
v_{\mathrm{t}}=\mathrm{C} \vartheta \ell=\mathrm{C}_{\mu} \frac{\mathrm{k}^{2}}{\varepsilon}
\end{gathered}
$$

and the dynamic turbulent viscosity as:

$$
\mu_{\mathrm{t}}=\mathrm{C} \rho \vartheta \ell=\mathrm{C}_{\mu} \rho \frac{\mathrm{k}^{2}}{\varepsilon}
$$

where $\mathrm{C}_{\mu}$ is a dimensionless constant. 
The standard high Reynolds Number k- $\varepsilon$ Model (Launder and Spalding ${ }^{[1972,74]}$ ) has two model equations, one for $\mathrm{k}$ and one for $\varepsilon$. Where:

$$
\begin{gathered}
k=\frac{\frac{1}{2}\left(\overline{u_{i} u_{i}}\right)}{\varepsilon=\frac{\mu}{\rho\left(\frac{\partial u_{i}}{\partial x_{j}}\right)\left(\frac{\partial u_{i}}{\partial x_{j}}\right)}}
\end{gathered}
$$

Exact steady state transport equations for $\mathrm{k}$ and $\varepsilon$ can be derived (Launder and Spalding ${ }^{[1974]}$ ) and expressed for $\mathrm{k}$ as:

$$
\mathrm{C}_{\mathrm{k}}=\mathrm{P}_{\mathrm{k}}+\mathrm{T}_{\mathrm{k}}+\mathrm{V}_{\mathrm{k}}+\Pi_{\mathrm{k}}+\varepsilon
$$

where:

$\mathrm{C}_{\mathrm{k}}$ - convection

$\mathrm{P}_{\mathrm{k}}$ - production

$\mathrm{T}_{\mathrm{k}}$ - turbulent transport

$\mathrm{V}_{\mathrm{k}}$ - viscous diffusion

$\Pi_{\mathrm{k}}-$ velocity-pressure fluctuation interactions

$\varepsilon-$ dissipation

and for $\varepsilon$ :

$$
\mathrm{C}_{\varepsilon}=\mathrm{T}_{\varepsilon}+\Pi_{\varepsilon}+\mathrm{V}_{\varepsilon}+\mathrm{P}_{\varepsilon}^{1}+\mathrm{P}_{\varepsilon}^{2}+\mathrm{P}_{\varepsilon}^{3}-\mathrm{P}_{\varepsilon}^{4}
$$

where:

$\mathrm{C}_{\varepsilon}$ - convection

$\mathrm{T}_{\varepsilon^{-}}$turbulent transport

$\Pi_{\varepsilon}-$ pressure transport

$\mathrm{V}_{\varepsilon}$ - viscous diffusion

$\mathrm{P}_{\varepsilon}^{1}, \mathrm{P}_{\varepsilon^{-}}^{2}$ creation by interaction of turbulence with mean flow

$\mathrm{P}_{\varepsilon}^{3}$ - creation by turbulent interaction

$\mathrm{P}_{\varepsilon}^{4}$ - viscous destruction 
The exact equations contain unknowns and unmeasureable terms but based on an understanding of the relevant process involved it is possible to model these terms. Beginning with the exact Reynolds averaged equations for continuity, momentum, turbulent kinetic energy and it's isentropic dissipation rate (at high Reynolds numbers) the following modelling assumptions are made to close the set of equations.

An eddy-diffusivity is used to model the correlation of any fluctuating scalar quantity, $\phi$ (e.g. fluctuations in $\mathrm{k}$ or $\varepsilon$ ) with the fluctuating velocities (these appear in the turbulent transport terms $\mathrm{T}_{\mathrm{k}}$ and $\mathrm{T}_{\varepsilon}$ ):

$$
-\rho \overline{u_{i} \phi_{j}}=\frac{\mu_{t}}{\sigma_{t}}\left[\frac{\partial \phi}{\partial x_{j}}\right]
$$

A turbulent Schmidt number (or turbulent Prandtl number), $\sigma_{t}$ has been introduced because $\phi$ may not diffuse at the same rate as momentum.

The pressure transport terms $\left(\Pi_{\mathrm{k}}\right.$ and $\left.\Pi_{\varepsilon}\right)$ are deemed small and neglected, although, Bradshaw et al ${ }^{[1981]}$ note that this is not generally true.

Finally, the remaining terms in the $\varepsilon$ equation are modelled with a largely empirical expression:

$$
\left(\mathrm{P}_{\varepsilon}^{1}+\mathrm{P}_{\varepsilon}^{2}+\mathrm{P}_{\varepsilon}^{3}\right)+\mathrm{P}_{\varepsilon}^{4}=\mathrm{C}_{\varepsilon 1} \frac{\varepsilon}{\mathrm{k}} \mathrm{P}_{\mathrm{k}}-\mathrm{C}_{\varepsilon 2} \rho \frac{\varepsilon^{2}}{\mathrm{k}}
$$

where $\mathrm{P}_{\mathrm{k}}$ is the production of turbulent kinetic energy given by (for constant density flow):

$$
P_{k}=-\overline{\rho u_{i} u_{j}} \frac{\partial U_{i}}{\partial x_{j}}=\mu_{t}\left\{\left[\frac{\partial U_{i}}{\partial x_{j}}+\frac{\partial U_{j}}{\partial x_{i}}\right]-\frac{2}{3} \rho_{i j} k\right\} \frac{\partial U_{i}}{\partial x_{j}}=\mu_{t}\left[\frac{\partial U_{i}}{\partial x_{j}}+\frac{\partial U_{j}}{\partial x_{i}}\right] \frac{\partial U_{i}}{\partial x_{j}}
$$

Including all the above modelling assumptions and for high Reynolds number flows the following set of equations is formed: 


$$
\begin{gathered}
\frac{\partial \mathrm{U}_{\mathrm{i}}}{\partial \mathrm{x}_{\mathrm{i}}}=0 \\
\frac{\partial}{\partial \mathrm{x}_{\mathrm{j}}}\left(\rho \mathrm{U}_{\mathrm{i}} \mathrm{U}_{\mathrm{j}}\right)=-\frac{\partial \rho}{\partial \mathrm{x}_{\mathrm{i}}}+\frac{\partial}{\partial \mathrm{x}_{\mathrm{j}}}\left[\left(\mu+\mu_{\mathrm{t}}\right)\left[\frac{\partial \mathrm{U}_{\mathrm{i}}}{\partial \mathrm{x}_{\mathrm{j}}}+\frac{\partial \mathrm{U}_{\mathrm{j}}}{\partial \mathrm{x}_{\mathrm{i}}}\right]\right] \\
\frac{\partial}{\partial \mathrm{x}_{\mathrm{i}}}\left(\mathrm{U}_{\mathrm{i}} \rho \mathrm{k}\right)=\frac{\partial}{\partial \mathrm{x}_{\mathrm{j}}}\left[\left(\mu+\frac{\mu_{\mathrm{t}}}{\sigma_{\mathrm{k}}}\right) \frac{\partial \mathrm{k}}{\partial \mathrm{x}_{\mathrm{j}}}\right]+\mathrm{P}-\rho \varepsilon \\
\frac{\partial}{\partial \mathrm{x}_{\mathrm{i}}}\left(\mathrm{U}_{\mathrm{i}} \rho \varepsilon\right)=\frac{\partial}{\partial \mathrm{x}_{\mathrm{j}}}\left[\left(\mu+\frac{\mu_{\mathrm{t}}}{\sigma_{\varepsilon}}\right) \frac{\partial \varepsilon}{\partial \mathrm{x}_{\mathrm{j}}}\right]-\frac{\varepsilon}{\mathrm{k}}\left(\mathrm{C}_{\varepsilon 1} \mathrm{P}-\mathrm{C}_{\varepsilon 2} \rho \varepsilon\right)
\end{gathered}
$$

The five empirical constants are determined in a variety of ways (Launder and Spalding $^{[1972,1974]}$ ) and the most common values used, also the default values in the Fluent code, are:

$$
\mathrm{C}_{\mu}=0.09, \mathrm{C}_{\varepsilon 1}=1.44, \mathrm{C}_{\varepsilon 2}=1.92, \sigma_{\mathrm{k}}=1.0, \sigma_{\varepsilon}=1.3
$$

The model equations are elliptic by nature and thus the following boundary conditions are needed:

1) inlet distributions of $\mathrm{k}$ and $\varepsilon$ must be supplied

2) outlet or symmetry axis $\partial \mathrm{k} / \partial \mathrm{n}=0$ and $\partial \varepsilon / \partial \mathrm{n}=0$

3) turbulent free stream $\quad \mathrm{k}=0$ and $\varepsilon=0$

4) solid walls Log-law for high Reynolds numbers and a reworking of the model equations for low Reynolds numbers

(see Section 5.5)

If values of $\mathrm{k}$ and $\varepsilon$ are not available at inlet then approximations for the inlet conditions can be obtained from an assumed turbulence intensity, $\mathrm{T}_{\mathrm{i}}$, and a characteristic eddy length, $\ell$ :

$$
\mathrm{k}=\frac{3}{2}\left(\mathrm{U}_{\mathrm{ref}} \mathrm{T}_{\mathrm{i}}\right)^{2} \quad \varepsilon=\frac{\mathrm{k}}{\ell}^{\frac{3}{2}}
$$


Versteeg and Malalasekera ${ }^{[1995]}$ report that, although the k- $\varepsilon$ model has achieved notable success in predicting general engineering flows it is not without its problems. It is well established and widely validated for many cases but has been observed to be inaccurate in unconfined flows such as far wakes and mixing layers as the rate of production of turbulent kinetic energy can be much less than the rate of dissipation. Problems are also encountered for flows with large extra strains such as curved boundary layers, diverging passages and swirling flows; where highly anisotropic turbulence has a dominant effect. Craft and Launder ${ }^{[1991]}$ reported that the $\mathrm{k}-\varepsilon$ model will overpredict the total pressure losses in impingement regions whereas Bradshaw ${ }^{[1973]}$ reported that the model fails to predict the large total pressure losses caused by streamline curvature. Little et al ${ }^{[1997]}$ predicted the flow in a dump diffuser combustion system and suggested that the latter failing is almost certainly the largest leading to an underprediction of total pressure loss.

\subsubsection{The Reynolds Stress Equation Model}

The Reynolds Stress Equation Model (RSM), also called the second-order or secondmoment closure model, is the most complex classical statistical turbulence model. The RSM involves abandoning the concept of an eddy-viscosity altogether (and hence the Boussinesq relationship for ${\overline{\mathrm{u}_{\mathrm{i}}}}_{\mathrm{j}}$ ) and solving separate transport equations for the individual Reynolds stresses. These can be derived from the momentum equations and contain triple order velocity correlations and pressure-velocity correlations that must be modelled to obtain closure. The methodology, in its most complex form, arises from Launder et $\mathrm{al}^{[1975]}$ and Launder ${ }^{[1989]}$. However, most commercial codes (including Fluent) have some simplification.

A transport equation for the Reynolds stresses $\left({\overline{u_{i}}}_{j}\right)$ can be written in the form:

$$
\mathrm{C}_{\mathrm{ij}}=\mathrm{P}_{\mathrm{ij}}+\mathrm{T}_{\mathrm{ij}}+\mathrm{V}_{\mathrm{ij}}+\Pi_{\mathrm{ij}}+\varepsilon_{\mathrm{ij}}
$$

where:

$$
\begin{aligned}
& \mathrm{C} \text { - convection } \\
& \mathrm{P} \text { - production } \\
& \mathrm{T} \text { - turbulent transport }
\end{aligned}
$$




$$
\begin{aligned}
& \text { V - viscous diffusion } \\
& \Pi \text { - velocity-pressure gradient interaction } \\
& \varepsilon \text { - dissipation }
\end{aligned}
$$

Within Equation 5.27 the convection, production and viscous diffusion terms require no modelling and are given by Equations 5.28 to 5.30:

$$
\begin{gathered}
C_{i j}=\frac{\partial}{\partial x_{k}}\left(\rho U_{k} \overline{u_{i} u_{j}}\right) \\
P_{i j}=-\left[\overline{\rho u_{i} u_{k}} \frac{\partial U_{j}}{\partial x_{k}}+\overline{\rho u_{j} u_{k}} \frac{\partial U_{i}}{\partial x_{k}}\right] \\
V_{i j}=v \frac{\partial^{2}}{\partial x_{k} \partial x_{k}}\left(\overline{\rho u_{i} u_{j}}\right)
\end{gathered}
$$

The turbulent transport term is given by:

$$
\mathrm{T}_{\mathrm{ij}}=-\frac{\partial}{\partial \mathrm{x}_{\mathrm{k}}}\left(\overline{\mathrm{u}_{\mathrm{i}} \mathrm{u}_{\mathrm{j}} \mathrm{u}_{\mathrm{j}}}\right)
$$

and requires modelling. This is achieved by assuming that the triple velocity correlations are directly related to the gradients of the Reynolds stresses through the generalised gradient-diffusion model of Daly and Harlow ${ }^{[1970]}$. However this can result in numerical instabilities so is simplified in Fluent to use a scalar diffusivity (Lien and Leschziner $\left.^{[1994]}\right)$ :

$$
\mathrm{T}_{\mathrm{ij}}=\frac{\partial}{\partial \mathrm{x}_{\mathrm{k}}}\left(\mathrm{C}_{\mathrm{s}} \frac{\mathrm{k}^{2}}{\varepsilon} \frac{\partial \overline{\mathrm{u}_{\mathrm{i}} \mathrm{u}_{\mathrm{j}}}}{\partial \mathrm{x}_{\mathrm{k}}}\right)
$$

The pressure-strain interactions $\left(\Pi_{\mathrm{ij}}\right)$ affect the Reynolds stresses in two ways; (1) via pressure fluctuations arising due to interactions of eddies with regions of flow with different mean velocity and (2) via pressure fluctuations caused by two eddies interacting with each other. These processes re-distribute energy between the Reynolds normal stresses making them more isotropic while reducing the Reynolds shear stresses. The influence of a wall on this process necessitates corrections, details of which are given in Launder et al ${ }^{[1975]}$, however, Fluent's RSM does not include the wall reflection terms 
resulting in a pressure-strain term modelled as:

$$
\Pi_{\mathrm{ij}}=-\mathrm{C}_{1} \frac{\varepsilon}{\mathrm{k}}\left[\overline{\mathrm{u}_{\mathrm{i}} \mathrm{u}_{\mathrm{j}}}-\frac{2}{3} \delta_{\mathrm{ij}} \mathrm{k}\right]-\mathrm{C}_{2}\left[\mathrm{P}_{\mathrm{ij}}-\frac{2}{3} \delta_{\mathrm{ij}} \mathrm{P}\right]
$$

where $\mathrm{P}=0.5 \mathrm{P}_{\mathrm{ii}}, \mathrm{C}_{1}=1.8$ and $\mathrm{C}_{2}=0.6$

The turbulent kinetic energy is computed by summing the normal Reynolds stresses:

$$
\mathrm{k}=\frac{1}{2} \overline{\mathrm{u}_{\mathrm{i}} \mathrm{u}_{\mathrm{i}}}
$$

The dissipation rate, $\varepsilon_{\mathrm{ij}}$, is approximated, for high Reynolds numbers, by the isotropic dissipation rate, $\varepsilon$, such that:

$$
\varepsilon_{\mathrm{ij}}=\frac{2}{3} \varepsilon \delta_{\mathrm{ij}}
$$

$\varepsilon$ is computed using a model transport equation. Launder et al ${ }^{[1975]}$ present a more exact form of this equation for $\varepsilon$ but like most commercial codes Fluent uses an equation identical to that used in the standard k- $\varepsilon$ model. $\delta_{\mathrm{ij}}$ is the Kronecker delta as before and thus affects normal Reynolds stresses only. This is a reasonable assumption since small scale motions responsible for the dissipation of turbulence are isotropic.

The model equations are elliptic by nature and the required boundary conditions are:

1) inlet distributions of $\overline{u_{i} u_{j}}$ and $\varepsilon$ must be provided

2) outlet or symmetry axis

$\partial \overline{u_{\mathrm{i}} \mathrm{u}_{\mathrm{j}}} / \partial \mathrm{n}=0$ and $\partial \varepsilon / \partial \mathrm{n}=0$

3) turbulent free stream

$\overline{\mathrm{u}_{\mathrm{i}} \mathrm{u}_{\mathrm{j}}}=0$ and $\varepsilon=0$

4) solid walls

Similar wall functions to the k- $\varepsilon$ model for high Reynolds numbers or a more detailed treatment is required at low Reynolds numbers

If exact distributions of $\overline{u_{i} u_{j}}$ and $\varepsilon$ are unavailable at flow inlets then approximations can 
be obtained from a turbulent intensity, $\mathrm{T}_{\mathrm{i}}$, and a characteristic length scale, $\mathrm{L}$, using the following assumptions:

$$
\begin{gathered}
\mathrm{k}=\frac{3}{2}\left(\mathrm{U}_{\mathrm{ref}} \mathrm{T}_{\mathrm{i}}\right)^{2} \quad \varepsilon=\frac{\mathrm{k}^{\frac{3}{2}}}{\ell} \quad \ell=0.07 \mathrm{~L} \quad \overline{\mathrm{u}_{1}{ }^{2}}=\mathrm{k} \\
\overline{\mathrm{u}_{2}^{2}}=\overline{\mathrm{u}_{3}{ }^{2}}=\frac{1}{2} \mathrm{k} \quad \overline{\mathrm{u}_{\mathrm{i}} \mathrm{u}_{\mathrm{j}}}=0(\mathrm{i} \neq \mathrm{j})
\end{gathered}
$$

\subsection{Modelling of Near-Wall Flows for Wall-Bounded Flows}

The two turbulence models discussed in Sections 5.4.1 and 5.4.2 are valid for mainstream flows only (i.e. flows which are not affected by the presence of a wall). The turbulent flow in regions close to a wall is affected greatly by the presence of this wall. Firstly, the mean velocity field is altered by the no-slip condition at the wall (i.e. $\mathrm{U}_{\text {wall }}=0$ ). Secondly, turbulence close to the wall is heavily damped. In the outer part of the near wall region turbulence is rapidly amplified by the production of turbulent kinetic energy due to the Reynolds stresses and the large gradient of mean velocity.

The near-wall region is classically separated into three layers; a laminar sub-layer, an inner region and an outer region. In the laminar sub-layer the flow, as the name suggests, is almost laminar in nature and momentum transfer is dominated by the molecular viscosity. In the outer layer the flow is fully turbulent with the turbulence levels playing a major role. Finally, in the inner layer (or buffer layer) turbulence and molecular viscosity are both equally important.

There are two methods which can be employed to model this near-wall region. Firstly, the viscous laminar sub-layer and the outer regions described above can be resolved completely in a method known as 'near-wall modelling'. This involves generating a mesh close to the wall that is fine enough to capture the sub-layer. The main drawback of this method is it results in a very high density mesh in the regions close to the wall. This can be computationally expensive and impractical for complex geometries. The second method uses semi-empirical formulae called 'wall-functions' to bridge the viscosity affected region between the wall and the outer region. The wall function approach is reasonably robust, economical and accurate and is thus more popular than near-wall mode- 
ling.

\subsubsection{Wall Functions}

There are two wall functions available within Fluent; a standard wall function and a nonequilibrium wall function and both comprise law-of-the-wall type functions for mean velocity and appropriate treatments for the near-wall turbulent quantities.

The basis of wall functions is illustrated in Figure 5.1. An experimentally determined boundary layer velocity profile can be closely approximated by a linear relationship in the laminar sub-layer and a log-law relationship in the outer fully turbulent region.

Firstly, non-dimensional velocity and distance (from the wall) parameters are derived:

$$
\begin{gathered}
\mathrm{U}^{+}=\frac{\overline{\mathrm{U}}}{\mathrm{U}_{\tau}} \\
\mathrm{y}^{+}=\mathrm{U}_{\tau} \frac{\mathrm{y}}{v}
\end{gathered}
$$

where $U_{\tau}=\left(\frac{\tau_{\mathrm{w}}}{\rho}\right)^{\frac{1}{2}}$ and is known as a 'friction velocity'.

Thus, in the laminar sub-layer (where, generally $\mathrm{y}^{+}<5$ ):

$$
\mathrm{U}^{+}=\mathrm{y}^{+}
$$

or:

$$
\frac{\overline{\mathrm{U}}}{\mathrm{U}_{\tau}}=\frac{\mathrm{U}_{\tau} \mathrm{y}}{\mathrm{v}}
$$

In the outer layer $\left(30<y^{+}<500\right)$ the velocity profile is approximated by a log-law relationship as described in Launder and Spalding ${ }^{[1974]}$, such that:

$$
\mathrm{U}^{+}=\frac{1}{\kappa} \ln \left(\mathrm{Ey}^{+}\right)
$$

where:

$\kappa$ - von Karman's constant (0.42) 
E - empirical constant (9.81)

In Fluent the Standard Wall Function employs a slightly different format of the log-law with the mean velocity given by:

$$
\mathrm{U}^{*}=\frac{1}{\kappa} \ln \left(\mathrm{E} \mathrm{y}^{*}\right)
$$

where:

$$
\begin{aligned}
\mathrm{U}^{*} & \equiv \frac{\mathrm{U}_{\mathrm{P}} \mathrm{C}_{\mu}^{1 / 4} \mathrm{k}_{\mathrm{P}}^{1 / 2}}{\tau_{\mathrm{w}} / \rho} \\
\mathrm{y}^{*} & \equiv \frac{\rho \mathrm{C}_{\mu}^{1 / 4} \mathrm{k}_{\mathrm{P}}^{1 / 2} \mathrm{y}_{\mathrm{P}}}{\mu}
\end{aligned}
$$

and:

$\mathrm{U}_{\mathrm{P}}$ - mean velocity at point $\mathrm{P}$

$\mathrm{k}_{\mathrm{P}}$ - turbulent kinetic energy at point $\mathrm{P}$

$\mathrm{y}_{\mathrm{P}}$ - distance from point $\mathrm{P}$ to the wall

$\mu$ - dynamic viscosity of the fluid

The logarithmic law for mean velocity is valid for $\mathrm{y}^{*}$ greater than $30-60$. To determine a point at which to switch from a linear relationship to a log-law Fluent uses the intersection of the two relationships, where:

$$
\mathrm{U}^{*}=\mathrm{y}^{*}=\frac{1}{\kappa} \ln (\mathrm{Ey} *)
$$

This yields a value for $\mathrm{y}^{*}$ of about 11.2. Therefore, Fluent employs the log law when $\mathrm{y}^{*}$ is greater than 11.225. In cells next to the wall with values of $\mathrm{y}^{*}$ less than 11.225 the standard wall function in Fluent applies the laminar stress-strain relationship:

$$
\mathrm{U}^{*}=\mathrm{y}^{*}
$$

The log-law is implemented computationally by using information at the near wall point to deduce the wall shear stress, $\tau_{\mathrm{w}}$, which is then used in the discretised momentum equation for the near wall point. 
The $\mathrm{k}$ equation is solved in the whole solution domain including the wall cells with the imposed boundary condition:

$$
\frac{\partial \mathrm{k}}{\partial \mathrm{n}}=0
$$

The production of kinetic energy $\left(\mathrm{P}_{\mathrm{k}}\right)$ and its dissipation rate in the near-wall cells are computed from the assumption of local equilibrium. That is to say that the production of $\mathrm{k}$ and its dissipation is assumed to be equal in the near-wall cells. The production is calculated from:

$$
\mathrm{P}_{\mathrm{k}} \approx \tau_{\mathrm{w}} \frac{\partial \mathrm{U}}{\partial \mathrm{y}}=\tau_{\mathrm{w}} \frac{\tau_{\mathrm{w}}}{\kappa \rho \mathrm{C}_{\mu}^{1 / 4} \mathrm{k}_{\mathrm{P}}^{1 / 2} \mathrm{y}_{\mathrm{P}}}
$$

The $\varepsilon$ equation is not solved in the near-wall cells. Equation 5.49 is used instead.

$$
\varepsilon_{\mathrm{P}}=\frac{\mathrm{C}_{\mu}^{3 / 4} \mathrm{k}_{\mathrm{p}}^{3 / 2}}{\kappa \mathrm{y}_{\mathrm{P}}}
$$

The Non-Equilibrium Wall Function (Kim and Choudhury ${ }^{[1995]}$ ) is an additional nearwall treatment offered by Fluent. Essentially, the main differences from the standard function are that Launder and Spalding's ${ }^{[1974]} \log$-law is sensitised to pressure gradient effects and a two-layer concept is used to apportion the turbulent kinetic energy in the cells adjacent to the wall.

The sensitised log-law for mean velocity results in the following relationship:

$$
\frac{\overline{\mathrm{U}} \mathrm{C}_{\mu}^{1 / 4} \mathrm{k}^{1 / 2}}{\tau_{\mathrm{w}} / \rho}=\frac{1}{\kappa} \ln \left[\mathrm{E} \frac{\rho \mathrm{C}_{\mu}^{1 / 4} \mathrm{k}^{1 / 2} \mathrm{y}}{\mu}\right]
$$

where:

$$
\overline{\mathrm{U}}=\mathrm{U}-\frac{1}{2} \frac{d \mathrm{p}}{d \mathrm{x}}\left[\frac{\mathrm{y}_{\mathrm{v}}}{\rho \kappa^{*} \mathrm{k}^{1 / 2}} \cdot \ln \left(\frac{\mathrm{y}}{\mathrm{y}_{\mathrm{v}}}\right)+\frac{\mathrm{y}-\mathrm{y}_{\mathrm{v}}}{\rho \kappa^{*} \mathrm{k}^{1 / 2}}+\frac{\mathrm{y}_{\mathrm{v}}^{2}}{\mu}\right]
$$

$\mathrm{y}_{\mathrm{v}}$ is the non-dimensional thickness of the laminar sub-layer given by: 


$$
\mathrm{y}_{\mathrm{v}} \equiv \frac{\mu \mathrm{y}_{\mathrm{v}}{ }^{*}}{\rho \mathrm{C}_{\mu}^{1 / 4} \mathrm{k}_{\mathrm{p}}^{1 / 2}}
$$

where $\mathrm{y}_{\mathrm{v}}{ }^{*}=11.225$.

In the two-layer approach the cells immediately adjacent to the wall are assumed to be made up of a viscous sub-layer and a fully turbulent layer. The following assumptions for the turbulent quantities are then made:

$$
\tau_{t}=\left\{\left(\begin{array}{cc}
0, y<y_{v} \\
\tau_{w}, y>y_{v}
\end{array}\right), k=\left\{\left(\begin{array}{ll}
\left(\frac{y}{y_{v}}\right)^{2} k_{p}, & y<y_{v} \\
k_{P}, & y>y_{v}
\end{array}\right), \varepsilon=\left\{\begin{array}{ll}
\frac{2 v k}{y^{2}}, & y<y_{v} \\
3 / 2 \\
\frac{k^{3 / 2}}{C_{1}}, & y>y_{v}
\end{array}\right)\right.\right.
$$

where $\mathrm{C}_{1}=\kappa \mathrm{C}_{\mu}^{-3 / 4}$

From these profiles the cell-averaged production and dissipation may be computed. Essentially the local equilibrium assumption (production = dissipation) has been relaxed in the near-wall cells. The turbulent kinetic energy budget has been altered to take into account the proportions of the laminar sub-layer and the fully turbulent outer layer.

The standard wall function has been used extensively and gives reasonably accurate predictions for the majority of high Reynolds number, wall bounded flows. Kim and Choudhury ${ }^{[1995]}$ recommend that the non-equilibrium wall function should be used for complex flows involving separation or reattachment. However, both functions rely on the same assumption that the boundary layer profile can be closely approximated by a linear relationship in the laminar sub-layer and a log-law relationship in the outer fully turbulent region. Therefore, when this is not the case both wall functions will be in error. Furthermore, the successful computation of wall bounded flows requires careful considerations during the grid generation process. Grid cell size and spacing of wall adjacent cells must be determined such that the log-law is valid. Distance from the wall is measured by the parameters $\mathrm{y}^{+}$and $\mathrm{y}^{*}$ (Equations 5.36 and 5.43) which are both solution dependant and not geometrically fixed. According to Equation 5.45 the linear and log- 
law relationships coincide at a $y+$ value of approximately 11.2. This sets a lower limit to the distance from the wall to the nearest grid cell. In the outer layer Versteed and Malalalsekera ${ }^{[1995]}$ state that the value of $\mathrm{y}^{+}$is generally between 30 and 500 and thus suggest a minimum value of 30 is preferable. However, this is often not possible to ensure in a real flow. For example in recirculating flow, close to the reattachment point, the velocity component parallel to the wall is zero with the result that the computation reverts to a laminar case.

\section{$\underline{5.6}$ Numerical Implementation}

Fluent uses a control volume technique to convert the differential equations to algebraic equations which can be solved numerically. This consists of integrating the differential equations about each control volume (each grid cell), yielding a finite-difference equation that conserves each quantity on a control-volume basis. Discretisation, using this finite volume approach, requires appropriate differencing of the convective and diffusive fluxes at each face of the control volume.

The methods used to obtain face pressure and mass fluxes are outlined in Fluent ${ }^{[1998]}$. The face fluxes are obtained such that the face velocities obey an averaged momentum balance and face pressures such that velocities stored at cell centre obey the mass balance. In Fluent this avoids oscillatory pressure or velocity fields (Patankar ${ }^{[1980]}$ ) and that the physical variation of pressure and momentum between cell centres is accurately represented.

\section{A Blended Second Order Upwind/Central Difference Scheme (Maruszewski ${ }^{[1992]}$ )} was used to determine cell face fluxes. For example the face value of an unknown, $\phi_{\mathrm{f}}$, can be expressed as follows: 


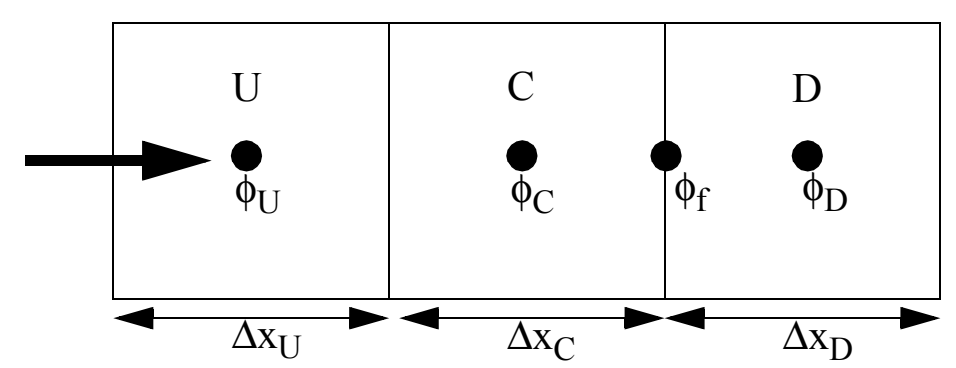

$$
\phi_{\mathrm{f}}=\theta\left[\frac{\Delta \mathrm{x}_{\mathrm{D}}}{\Delta \mathrm{x}_{\mathrm{C}}+\Delta \mathrm{x}_{\mathrm{D}}} \phi_{\mathrm{C}}+\frac{\Delta \mathrm{x}_{\mathrm{C}}}{\Delta \mathrm{x}_{\mathrm{C}}+\Delta \mathrm{x}_{\mathrm{D}}} \phi_{\mathrm{D}}\right]+(1-\theta)\left[\frac{\Delta \mathrm{x}_{\mathrm{U}}+2 \Delta \mathrm{x}_{\mathrm{C}}}{\Delta \mathrm{x}_{\mathrm{U}}+\Delta \mathrm{x}_{\mathrm{C}}} \phi_{\mathrm{C}}+\frac{\Delta \mathrm{x}_{\mathrm{C}}}{\Delta \mathrm{x}_{\mathrm{U}}+\Delta \mathrm{x}_{\mathrm{C}}} \phi_{\mathrm{D}}\right]
$$

Here boundedness is achieved through the use of $\theta$, which is computed from:

$$
\theta=\max \left(0, \min \left(\phi_{\mathrm{C}}, 1\right)\right) \text { where } \tilde{\phi}_{\mathrm{C}}=\frac{\phi_{\mathrm{C}}-\phi_{\mathrm{U}}}{\phi_{\mathrm{D}}-\phi_{\mathrm{U}}}
$$

\section{Pressure-Velocity Coupling}

In an incompressible flow prediction the pressure cannot be determined from the equation of state in the usual manner; the density is constant and by definition is not linked to the pressure. Furthermore, pressure is only introduced in each component of the momentum equation, which are used to determine each velocity component. The coupling between pressure and velocity introduces a constraint on the solution of the flow field: if the correct pressure field is applied in the momentum equations the resulting velocity field should satisfy continuity. Thus the problem which arises is how to determine the pressure field from the continuity equation. The pressure-correction technique used to do this is basically an iterative approach using some physical reasoning to construct the next iteration from the previous until the pressure and velocity filed are consistent and continuity is satisfied. The process can be summarised:

- Step 1 : start with a 'guess' for the pressure field ( $\left.\mathrm{p}^{*}\right)$.

- Step 2 : use $\mathrm{p}^{*}$ to solve for velocity from the momentum equations $\left(\mathrm{u}^{*}, \mathrm{v}^{*}\right)$.

- Step 3 : use the continuity equation to construct a pressure correction (p') which 
added to $\mathrm{p}^{*}$ will bring the velocity field closer to satisfying continuity. The 'corrected' pressure is $\mathrm{p}=\mathrm{p}^{*}+\mathrm{p}$ '. Corresponding velocity corrections can be obtained from p' such that $\mathrm{u}=\mathrm{u}^{*}+\mathrm{u}^{\prime}$ and $\mathrm{v}=\mathrm{v}^{*}+\mathrm{v}$ '

- Step 4 : use $p$ as the new value of $p^{*}$ and repeat the process from Step 2 until the velocity field satisfies continuity.

In this study the pressure correction was achieved using the SIMPLE algorithm which stands for Semi-Implicit Method for Pressure-Linked Equations and is described in detail by Patankar ${ }^{[1980]}$.

\section{The Iterative Solution Procedure}

The SIMPLE algorithm relates the velocity and pressure field which satisfy the momentum and continuity equations at a point. However, Fluent (and other CFD codes) do not solve the equations at all points simultaneously as it is too demanding on computational resources. Furthermore the equations are coupled and non-linear, therefore an iterative solution procedure is required with iterations continuing until all equations are satisfied at all points. 


\subsection{Computational Investigation of Hybrid Diffusers}

The main aim of this computational study was to provide a better understanding of the controlling flow mechanisms of a hybrid diffuser. This is a reasonably broad aim, and therefore the investigation initially concentrates on the bleed flow and reattachment process. To meet this aim flow predictions were made for a simple two-dimensional hybrid diffuser without a dump or flame tube. In undertaking any CFD calculations it is important to determine that the solution is grid independent and accurate within the bounds of the modelling assumptions used. A simple two-dimensional case not only provides an ideal vehicle with which to study the basic flow mechanisms but, importantly, it also provides a simple platform with which to:

- establish a grid methodology,

- ensure grid independency,

- assess turbulence model performance, and

- develop and assess boundary conditions.

Once the necessary methodology was established using this simple two-dimensional case it was then maintained throughout the CFD investigation including the results discussed in the following chapter.

Furthermore, a simple two-dimensional CFD investigation allows a more generic study to be undertaken. The experimental work described in Sections 3.0 and 4.0 employed hybrid diffusers similar to those described by Adkins et al ${ }^{[1980]}$ but due to hardware constraints the bleed gap geometry remained unaltered. However, it is reasonably straightforward to alter geometry using CFD and as such several different yet generic Stage 2 geometries were modelled and their relative performance compared.

A natural progression of the investigation leads to a prediction which matches the experimental test rig thus allowing direct comparison between experimental and computational data. However, a three-dimensional prediction including burners and a fully featured flame tube represents a very complex geometry. Grid definition and generation becomes a lengthy process and beyond the scope of this current investigation. It was not 
feasible to generate an exact computational model of the test facility and some geometrical simplification was necessary. Specifically this meant removing the burners and replacing the complex 'double-bubble' cowl with a simpler design. Furthermore, to facilitate the step from a plane two-dimensional geometry to three-dimensions an axisymmetric representation of the test rig was first investigated. Finally, this was extended into three-dimensional sector by rotation around the centre line thus allowing the inclusion of three-dimensional inlet conditions incorporating OGV wakes.

It is important for any CFD investigation that the solutions generated are shown to be accurate and valid and this is usually achieved by a grid refinement study and direct comparison with an identical experiment case. Thus, the experimental test facility was also modified by replacing the burners and cowl with a simple cowl (Figure 3.17, Section 3.6). Furthermore, two and three-dimensional inlet conditions were generated by removing the compressor (2D) or retaining it and its associated 3D downstream conditions (including OGV wakes).

\subsubsection{Geometry Definition and Boundary Conditions}

In this section the geometry definitions and applied boundary conditions for the CFD predictions will be discussed in detail. Eleven distinct diffuser geometries were studied, all based on an inlet height of $30.5 \mathrm{~mm}$, an overall area ratio of 2.5 and a non-dimensional length of 3.5. The first nine geometries model simple, plane, two-dimensional hybrid diffusers enabling a generic study of bleed gap geometry. The final two geometries were designed to model a simplified version of the test facility (refer to Section 3.6) firstly with two-dimensional inlet conditions and finally with representative three-dimensional inlet conditions including OGV wakes.

\subsubsection{Plane Two-Dimensional Hybrid Diffusers}

Computational models of nine plane two-dimensional diffusers were developed in order to study and reveal more information about hybrid diffuser flow mechanisms. For simplicity no downstream components were modelled and all the diffusers terminate in a constant area length of duct (see Figure 5.2). Furthermore, the two-dimensionality allows 
a symmetry boundary condition to be applied at mid inlet duct height, such that:

$$
\frac{\partial \mathrm{U}}{\partial \mathrm{n}}=\frac{\partial \mathrm{k}}{\partial \mathrm{n}}=\frac{\partial \varepsilon}{\partial \mathrm{n}}=0 \text { and } \mathrm{v}=0
$$

thus halving the number of grid cells and the computational load.

\section{a) Vortex Bled Hybrid Diffuser}

The first diffuser (Figure 5.2) is based on the type of diffuser proposed by Adkins et $\mathrm{al}^{[1980]}$ and has an identical geometry to the Hybrid4 diffuser (Section 3.0) used in the experimental study. This diffuser incorporates a bleed via a vortex chamber which is, again, identical in geometry to the vortex chamber used in conjunction with the Hybrid4 diffuser.

\section{b) Duct Bled Hybrid Diffuser}

Experimental results clearly indicated that it is not necessary to bleed the air via a vortex chamber as suggested by Adkins et al ${ }^{[1980]}$ and a ducted bleed performs a similar, if not the same, function. This is significant as it impacts the suitability of hybrid diffusers for use in aero gas turbine combustion systems. It is unlikely that a vortex chamber would be used due to its complexity and associated weight penalty but a ducted bleed is a much more viable option. Therefore, in order to further investigate this, a Duct Bled diffuser was studied. This is identical to the Vortex Bled diffuser except that, as shown in Figure 5.3 , the vortex chamber has been replaced by a simple duct of width $\mathrm{x}\left(\mathrm{x} / \mathrm{h}_{1}=0.15\right)$.

\section{c) Extended Duct Bled Hybrid Diffuser}

To further investigate the difference between a vortex chamber and a duct bleed a third hybrid diffuser was modelled with bleed afforded through an extended duct of width $2 \mathrm{x}$ (Figure 5.4). This then provided a geometry some way between a simple duct and a vortex chamber. 


\section{d) Conventional Diffuser}

In order to provide a benchmark the fourth diffuser represents a conventional unbled diffuser as shown in Figure 5.5. To maintain a measure of similarity between the conventional diffuser and the hybrid diffusers the wall angle is varied such that the Stage 1 geometry is unchanged.

\section{e) Alternative Bleed 1}

The importance of the combination of a step expansion and Coanda bubble is unclear. Thus to investigate this the Stage 2 fence was removed firstly by joining the tip of the fence to stage 3 diffuser exit as shown in Figure 5.6 and...

\section{f) Alternative Bleed 2}

... secondly by simply removing the fence (Figure 5.7)

\section{g) Alternative Bleed 3 - "Boundary Layer Bleed"}

It has been argued that the flow mechanisms of a hybrid diffuser do not simply remove the poor quality, energy deficient boundary layer flow but there is a more complex interaction between the bleed and mainstream flows. This diffuser, as shown in Figure 5.8, represents an attempt to model the conventional diffuser of Figure 5.5, but a portion of the boundary layer is "bled" off without any of the associated mechanisms of a hybrid diffuser in order confirm whether or not there is a more complex interaction.

\section{h) Alternative Bleed 4 - "Profiled Bleed Gap"}

The quality of the bleed flow will affect its suitability for use elsewhere in an engine and ultimately this will affect the potential use of hybrid diffusers in aero gas turbines. As the flow enters the bleed duct it will undoubtedly separate and there will exist a separation bubble (as shown in Figure 4.22). This process will inherently contain a high degree of loss and will contribute significantly to the overall bleed flow loss. Figure 5.9 illustrates 
one attempt to remove this separation by profiling the upstream corner of the duct.

\section{i) Alternative Bleed 5 - "Angled Bleed Duct"}

A second attempt to reduce bleed flow loss was made by angling the bleed duct with the aim of removing some of the turning loss as shown in Figure 5.10.

\section{Boundary Conditions}

The condition of the boundary layer prior to the bleed will influence the bleed flow and diffuser performance. Therefore, to ensure a boundary layer condition on the Stage 1 wall similar to the experimental case inlet conditions were inferred from OGV exit measurements on the experimental test facility. Taking the circumferentially averaged axial velocity profile (with zero swirl) gave the inlet axial velocity profile shown in Figure 5.11 with an average velocity of $50.5 \mathrm{~ms}^{-1}$. As no inlet turbulence data exist Equations 5.26 and 5.36 were used to approximate the relevant values for $\mathrm{k}, \varepsilon$ and Reynolds stress by specifying an inlet turbulence intensity and characteristic length. Using a hot wire anemometer to take measurements downstream of the rotor, the average inlet turbulence intensity was found to be about $5 \%$. Thus this value was used in conjunction with a characteristic length equal to the inlet passage height $(30.5 \mathrm{~mm})$ to define the inlet turbulence data. Additionally it was found that the final solution was relatively insensitive to variations in these values. For example, Table 5.1 shows that there was no change in overall diffuser static pressure recovery when the characteristic length was altered. Similarly Figure 5.12 illustrates that there was negligible difference in the axial velocity profile at stage 1 exit for changes in inlet turbulence intensity.

\begin{tabular}{|c||c|}
\hline Characteristic Length, $\mathbf{L}(\mathbf{m m})$ & $\mathbf{C}_{\mathbf{p 2 - 4}}$ \\
\hline \hline 15.0 & 0.779 \\
\hline 20.0 & 0.778 \\
\hline 30.5 & 0.778 \\
\hline
\end{tabular}

Table 5.1 Effect on Solution of Inlet Characteristic Length $\left(T_{i}=5.0 \%, B=3.0 \%\right)$ 
A fixed boundary condition was imposed at bleed flow exit and utilising continuity to calculate an appropriate velocity the mass flow rate could be set at the desired value. Finally a zero-gradient boundary condition was applied to the exit such that:

$$
\frac{\partial \mathrm{k}}{\partial \mathrm{n}} \text { and } \frac{\partial \varepsilon}{\partial \mathrm{n}}=0
$$

\subsubsection{Two-Dimensional Axi-Symmetric Hybrid Diffuser}

This geometry (Figure 5.13) comprises a two-dimensional, axi-symmetric representation of the test facility. The computational geometry has been somewhat simplified in order to make the CFD predictions possible. However, as discussed in Section 3.6 the test facility was also modified to produce a two-dimensional axi-symmetric configuration with the aim of using experimental data for CFD validation. Thus, many of the more complex features of the original test rig configuration have been removed but it is unlikely that these greatly affect the generic behaviour of the diffuser. The burners were omitted completely and the double cowl was replaced by a single entity with limited porosity modelled by a slot representing the splitter annulus. Finally, the dump liner profile was simplified in order to ease grid generation. A summary of the leading dimensions is given in Table 5.2. Removal of the burners necessitated a redistribution of the mass flow and simple area weighting was used to re-calculate the desired mass flow distribution such that the inner/splitter/outer annuli flow were divided in the ratio 35:30:35. An inlet velocity profile was taken from inlet measurements on the $2 \mathrm{D}$ axi-symmetric test rig (refer to Section 4.4.1) and the desired bleed flow rates set using fixed velocity boundary conditions at various flow rates.

\begin{tabular}{|c||c|c|c|}
\hline Feature & Station No. & Outer Radius $(\mathrm{mm})$ & Inner Radius $(\mathrm{mm})$ \\
\hline \hline Inlet & 3 & 332.0 & 301.5 \\
\hline Diffuser Exit & 4 & 354.9 & 278.6 \\
\hline Inner Annulus & 5 & 210.0 & 182.0 \\
\hline Splitter Annulus & 6 & 320.0 & 340.0 \\
\hline Outer Annulus & 7 & 474.5 & 450.0 \\
\hline
\end{tabular}

Table 5.2 2D Axi-Symmetric Geometry - Leading Dimensions 


\subsubsection{Three-Dimensional, Fully Annular Hybrid Diffuser}

The final geometry chosen for study computationally consists of essentially the same features as the $2 \mathrm{D}$ axi-symmetric geometry but extended by rotation in the circumferential direction. Experimental data illustrate that the inlet velocity varies in the circumferential $(\theta)$ direction in a cyclic/periodic nature. The periodicity of this variation is set by the OGVs and IGVs, therefore by applying periodic boundary conditions, a sector geometry was generated which computationally represented a full annulus. The sector geometry (Figure 5.14) encompasses two OGV spaces with the periodic boundary conditions subtending an angle of 4.62 degrees. By utilising this approach the demand on computational resources is significantly reduced.

The experimental facility was also modified by returning the single axial compressor stage to the two-dimensional axi-symmetric rig configuration, thus producing an experimental set up which was a good match for the computational prediction.

Three dimensional inlet velocity definitions were taken directly from five-hole probe measurements in the experimental facility. As described in Section 3.2.3 the experimental data is expressed in a polar $(\mathrm{x}, \mathrm{r}, \theta)$ co-ordinate system. Additionally Appendix A3 describes how the data acquisition system for a five-hole probe records data expressed in terms of a total velocity ( $\left.\mathrm{U}_{\text {tot }}\right)$, true yaw angle (YTR) and pseudo pitch angle (PPS) at specific radial (r) and circumferential $(\theta)$ locations. Fluent requires axial (u), radial (v) and tangential (w) velocities to be specified at inlet and these were easily computed for the $21 \times 21$ radial and circumferential locations corresponding to the five-hole probe area survey by application of the equations given in Appendix A3.

The resulting inlet axial velocity contours are shown in Figure 5.15 with a v-w vector plot shown in Figure 5.16. The three-dimensional nature of the inlet conditions is clearly visible with the presence of two OGV wakes (i.e. darker contours). Unfortunately, detailed information on the structure of the inlet turbulence was not available so, as before, an inlet turbulence intensity of 5\% and a characteristic length equal to the inlet passage height were used in conjunction with Equations 5.26 and 5.36 to approximate the distributions of $\mathrm{k}, \varepsilon$ and Reynolds stress. 
Boundary conditions for the bleed flows and combustor features were set as before using a fixed velocity condition to force the desired mass flow. The flow to the inner/splitter/ outer combustor feed annuli was divided, as for the experimental equivalent, in the ratio $35: 30: 35$.

\subsubsection{Post-Processing and Presentation of Results}

Once a CFD solution has been generated it is then necessary to extract data of the required type and form for analysis. Logically, since one aim was to compare the CFD data to experimental data, it is sensible that a consistent format is used. To achieve this is fairly straight forward as within Fluent there are several options which allow the user to extract solution variables and derived variables. Thus, mass weighted mean pressure data can be easily extracted at locations corresponding to the experimental measurements stations (refer to Figure 3.2).

Experimentally, for the hybrid diffuser, access was not available for instrumentation at OGV exit. Thus all experimental performance data are quoted with respect to rotor exit conditions (therefore including OGV performance). For example, the diffuser total pressure loss coefficient is:

$$
\lambda_{2-4}=\frac{\tilde{\mathrm{P}}_{2}-\tilde{\mathrm{P}}_{4}}{\tilde{\mathrm{P}}_{2}-\tilde{\mathrm{p}}_{2}}
$$

where 2 refers to rotor exit and 4 refers to diffuser exit

However, for the computational data the inlet corresponds to the OGV exit plane and performance data quoted with respect to this would not include OGV performance (e.g. loss). Thus to enable sensible comparisons with experimental data the computational data must be further processed to relate to a 'rotor-exit' condition.

In the experimental investigation (refer to Chapter 3.0 and 4.0) with either of the conventional diffusers fitted access became available for instrumentation at OGV exit. Hence OGV performance could be assessed and Equations 5.57 and 5.58 define the measured OGV total pressure loss and static pressure recovery respectively. 


$$
\begin{gathered}
\lambda_{2-3}=\frac{\tilde{\mathrm{P}}_{2}-\tilde{\mathrm{P}}_{3}}{\tilde{\mathrm{P}}_{2}-\tilde{\mathrm{p}}_{2}}=0.10 \\
\mathrm{C}_{\mathrm{p}_{2-3}}=\frac{\tilde{\mathrm{p}}_{2}-\tilde{\mathrm{p}}_{3}}{\tilde{\mathrm{P}}_{2}-\tilde{\mathrm{p}}_{2}}=0.40
\end{gathered}
$$

Using computational data at inlet (i.e. corresponding to OGV exit - station 3) and Equations 5.57 and 5.58 it becomes possible to calculate total, static and dynamic pressures at at 'pseudo' rotor exit plane. Using this information the computational performance data can be quoted with reference to this 'pseudo' rotor exit condition and hence compared directly with experimental data; both now including OGV performance.

Fluent also allows variables to be extracted at specific grid nodes which in turn enables the extraction of the necessary data to produce $\mathrm{X}-\mathrm{Y}$ profile plots, two-dimensional contour plots and velocity vector plots. Final graphical presentation of these data was achieved using Tecplot in an identical manner to that used for the experimental data as described in Section 3.2.4.

\subsection{Grid Generation and Solution Development}

To predict the flow field Fluent was used to solve for an incompressible flow regime in a polar co-ordinate system. The steady state solution to the equations of motion was found using a finite volume method, employing a blended second order upwind/central differencing scheme and the SIMPLE pressure correction algorithm. Both the k- $\varepsilon$ and Reynolds Stress turbulence models were used as were both available wall functions.

However, before any aerodynamic analysis of the CFD predictions can begin assessment and development of the computational grid and the solution techniques must be conducted. This involves examination of the solution to ensure that it is reasonable, represents a physical flow regime and grid independent (within practical limits). This is a complex issue as many of the factors involved are closely linked. For example, the accuracy of a CFD prediction is, in general, governed by the number of grid cells and a grid with too few cells may be unable to capture, effectively, some specific flow mechanism 
resulting in an inaccurate or un-physical solution. However, simply increasing the number of grid cells may not be computationally practical. Furthermore, the choice of turbulence model, wall model and boundary conditions will also effect the final solution. Therefore, it is important to investigate all of these effects and then decide on an optimum combination.

Here, development of the solution techniques was performed, for simplicity, on the plane two-dimensional, duct-bled hybrid diffuser (Figure 5.3) with the final methodology then being carried forward to the more complex predictions.

\subsubsection{Initial Grid Definition}

The important flow mechanisms for a hybrid diffuser are located in and around the Stage 2 section of the hybrid diffuser, specifically around the bleed off-take, step and fence. Therefore, it is important that the computational grid has a sufficient number of cells in these regions to successfully capture the physics of the flow. However, since the flow mechanisms weren't fully understood the initial grid involved a 'best-guess'. Thus 20 cells were used across the bleed gap. A cell aspect ratio close to unity was maintained around the bleed as shown in Figure 5.17. Uniform grid stretching was used to ensure cell aspect ratios and expansion ratios were kept within acceptable limits (i.e. ratios were close to unity in regions where there are strong gradients in the flow but relaxed in regions of the flow which were generally one dimensional). Overall the initial grid contained 300 x 100 cells which represents a relatively low computational workload given the facilities available.

Boundary conditions as described in Section 5.7.1 were applied in order to produce a bleed flow rate equal to $3.0 \%$ of the total inlet mass flow. A solution was generated using Fluent v 5 with, initially a k- $\varepsilon$ turbulence model in conjunction with a standard wall function to model the near-wall flow.

Experimental data (refer to Chapter 4.0) indicated that a bleed rate of 3.0\% would be above the minimum bleed requirement and the flow should rapidly reattach on the Stage 3 wall. However, a converged solution predicted a non-physical flow regime on the 
Stage 3 diffuser wall. The core flow appeared, initially, as expected with the bleed flow accelerating into the vortex chamber and a Coanda bubble present immediately downstream of the fence but on closer inspection it was clear that the predicted flow near the wall was physically incorrect. Along the length of the Stage 3 wall the predicted solution contained a long thin recirculation extending over the wall adjacent cell and its neighbour (Figure 5.18). This behaviour existed along the entire length of the Stage 3 diffuser wall with little or no positive momentum diffusing from the mainstream flow to the near wall cell. A second solution was generated but this time using Fluent's non-equilibrium wall function. No change was observed and a non-physical flow regime was still in existence along the Stage 3 wall. A third solution, this time employing a Reynolds Stress turbulence model and a standard wall function. However, Figure 5.19 illustrates that with a Reynolds Stress model the opposite was observed and the flow in the near wall cell was seen to accelerate. This 'wall-jet' also represents a non-physical solution as it is inconsistent with the 'no-slip' condition at the wall. A further solution was generated using Fluent's non-equilibrium wall function but the non-physical flow behaviour was still present and unchanged.

\subsubsection{Grid Refinement and Near Wall Treatment}

The standard and non-equilibrium wall functions both model the near-wall flow by employing a linear velocity-distance relationship in the laminar sub-layer and a log-law relationship in the fully turbulent layer as described in Section 5.5.1. The switch from linear (laminar) to log-law (fully turbulent) modelling occurs in Fluent at a value of $\mathrm{y}^{*}>11.225$, but the log-law is generally only valid for $\mathrm{y}^{+}$greater then $30-60$. On closer inspection of the initial solutions described above there was seen to be some discrepancy between the values of $\mathrm{y}^{+}$and $\mathrm{y}^{*}$ such that it appeared that a log-law relationship was being applied when it wasn't valid (i.e. $\mathrm{y}^{+}<<30-60$ ). This can occur when the near wall grid spacing is too dense and the laminar sub-layer extends over more than one cell. To avoid this situation the size of the near wall cell must be enlarged in a direction normal to the wall. The near wall cell height downstream of the fence in the initial grid corresponded to about $5 \%$ of the fence height. Three more grids were generated with the near wall cell height extended (along the Stage 3 wall only) to $16.5 \%, 25 \%$ and $50 \%$ of the fence height (Figure 5.20). 
Further solutions were computed for these new grids employing both turbulence models and both wall functions. For a k- $\varepsilon$ model it was immediately evident that the new solutions were much improved and, for the grid with a Stage 3 near wall cell height elongated to $16.5 \%$ of the fence height, the non-physical flow behaviour had been eliminated. However, with a Reynolds Stress model, although reduced, the non-physical flow was still evident to some extent for or all grids and both wall functions (refer to Section $5.8 .3)$.

The choice of wall function appears to make very little difference to the predicted solution. Figure 5.21 plots, for both wall functions, the axial velocity along the Stage 3 wall in the near wall cell for a $\mathrm{k}-\varepsilon$ model. Clearly, there is very little difference in the predicted velocity near the wall between the two wall functions. Both the standard and nonequilibrium wall functions are based on the same basic approximations (Section 5.5.1) and, therefore, since differences in the final solutions are small only the more classic standard wall function was employed for all successive predictions.

In order to finalise the grid it was necessary to ensure that the solution was grid independent. The overall accuracy of a CFD solution is related to the number of grid cells used, however, the final grid density is limited to a practical level by the complexity of the geometry. Two further grids were generated, maintaining the near wall spacing, but doubling the number of cells in each direction for the first, and similarly halving the number of cells in each direction for the second. Solutions were generated for each grid with both turbulence models and both wall functions. Figure 5.22 summarises the effect of grid density on the diffuser static pressure recovery and mean exit velocity. Effectively the solution appears grid independent above 30000 cells (300 x 100); any benefit gained by increasing grid density above this would be offset by increased computational load.

\section{$\underline{\text { 5.8.3 Turbulence Model Assessment }}$}

The final choice of turbulence model is not a simple one as there are many factors to take into account. Many CFD experts consider the more complex Reynolds Stress model to be a more viable way forward for a general purpose turbulence model. The k- $\varepsilon$ model is 
known to have problems for some unconfined flows and flows with large extra strains (e.g. curved boundary layers and swirling flows). Little et al ${ }^{[1997]}$ demonstrated that system loss in a dump diffuser was underpredicted due to the inability of the k- $\varepsilon$ model to predict the large increases in loss due to flow curvature over the head of a combustor. However, the k- $\varepsilon$ model has been widely used and validated and has achieved notable success in the prediction of engineering flows. Little et al ${ }^{[1997]}$ conclude that a $\mathrm{k}-\varepsilon$ model can be confidently used in the prediction of trends.

Predictions using the Reynolds Stress model also have problems in some flows involving unconfined recirculation owing to problems with the $\varepsilon$-equation modelling. Furthermore, the Reynolds Stress model can be restrictive as it is computationally more expensive due to the extra equations that must be solved. Also in this particular investigation there is very little inlet turbulence data available and as such it is difficult to determine and define a Reynolds Stress distribution at inlet.

Differences in solutions generated using a k- $\varepsilon$ and Reynolds Stress turbulence model occur in regions associated with the flow reattachment and subsequent boundary layer growth on the Stage 3 diffuser wall. Global parameters such as static pressure recovery are not notably different for the two turbulence models. Figure 5.23 shows the diffuser pressure recovery and mean exit velocity, illustrating the similarities.

However, close inspection of the reattachment region on the Stage 3 diffuser wall reveals differences and a non-physical behaviour for the Reynolds Stress case. Streamline plots for both models are shown in Figure 5.24 and 5.25 and clearly show differences in the formation of the Coanda bubble and reattachment lengths. Furthermore, Figure 5.26 shows that a wall-jet was again predicted along the Stage 3 using a Reynolds Stress model. Near wall treatments discussed in the previous were unable to eliminate this anomaly. The accurate prediction of the flow in this region is crucial to the aim of this study; understanding hybrid diffusers. The k- $\varepsilon$ prediction does not exhibit any non-physical behaviour. Additionally, it was envisaged that the final three-dimensional calculations would be very computationally expensive and possibly not feasible using the Reynolds Stress turbulence model. Therefore the decision was made to use a k- $\varepsilon$ turbulence model for all predictions discussed below. 


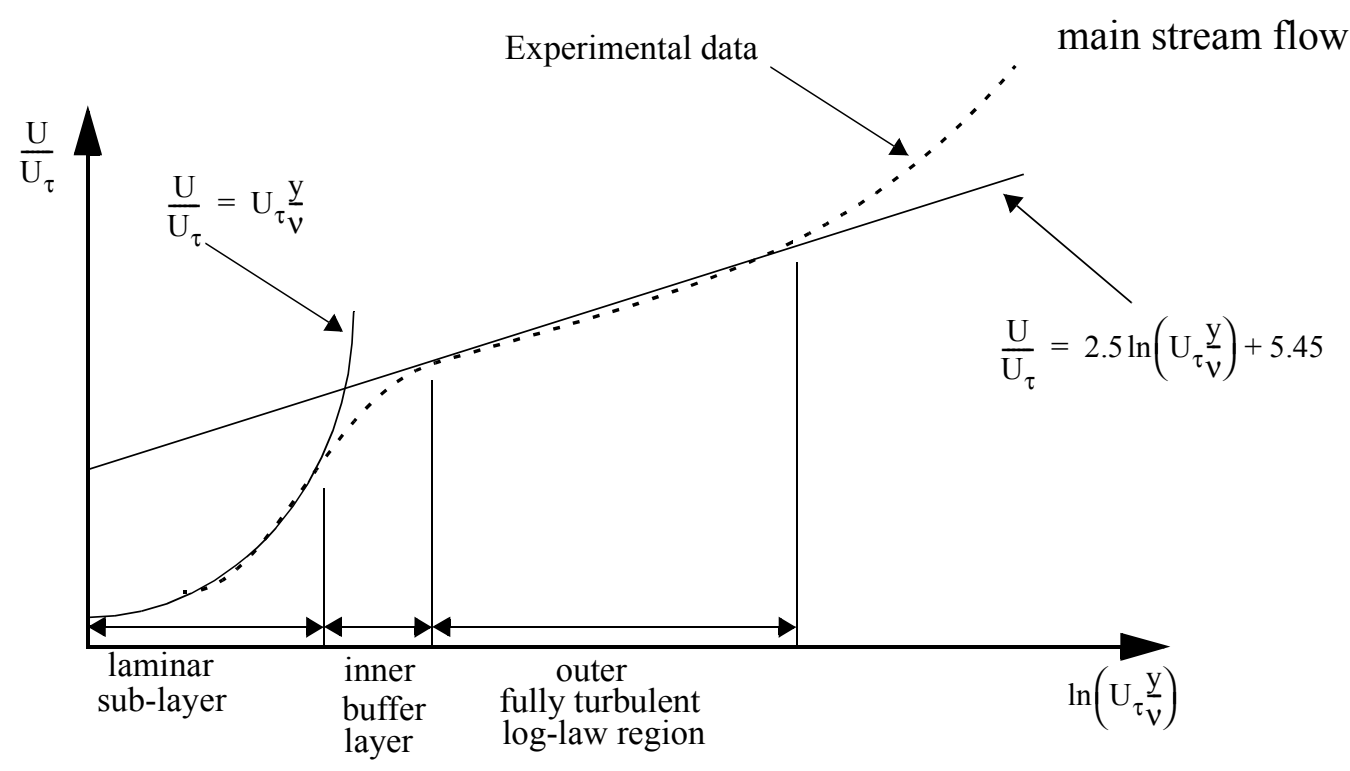

Figure 5.1 Near-Wall Region

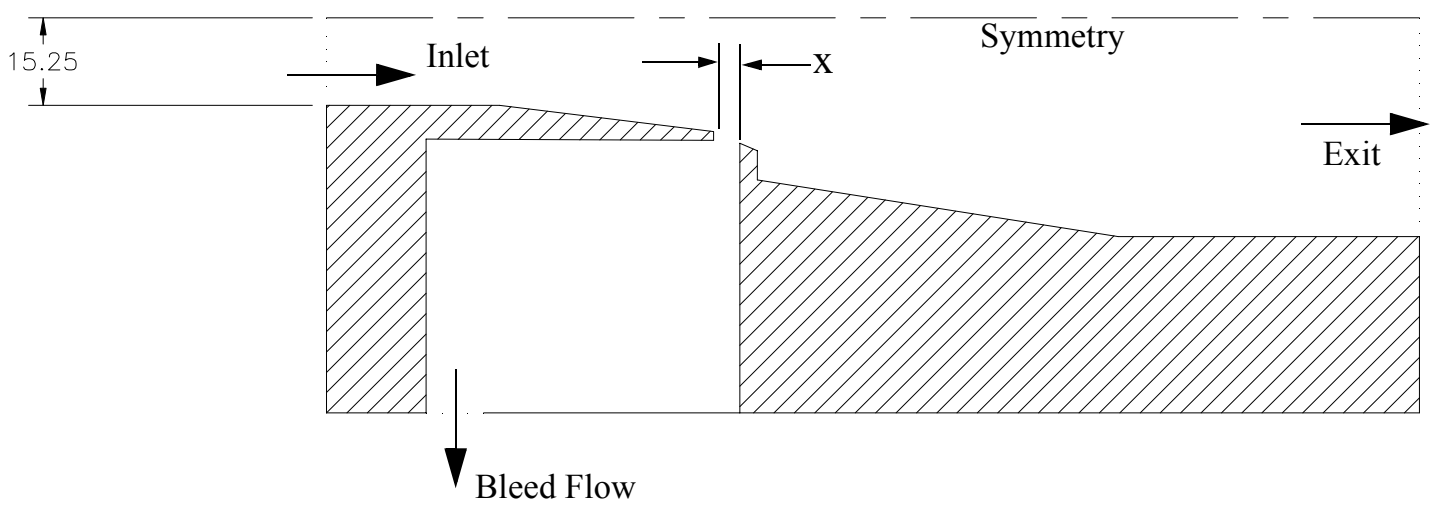
$\mathrm{AR}_{1}=1.3$
$\mathrm{AR}_{2}=1.4$
$\mathrm{AR}_{3}=1.37$
$\mathrm{AR}_{\mathrm{tot}}=2.5$
$\mathrm{L}_{1} / \mathrm{h}_{1}=1.4$
$\mathrm{L}_{3} / \mathrm{h}_{3}=1.11$
$\theta_{3}=9^{\circ}$
$\mathrm{L}_{\text {tot }} \mathrm{h}_{1}=3.5$

Figure 5.2 Vortex Bled Hybrid Diffuser

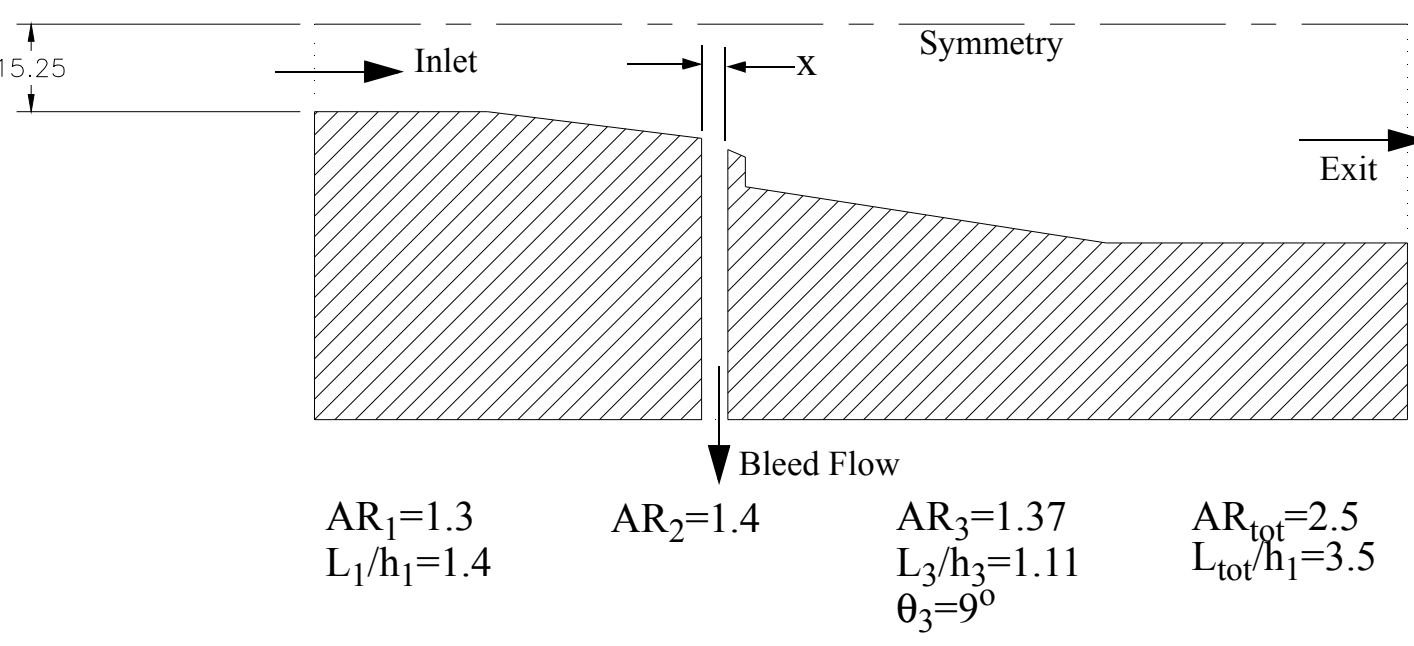

Figure 5.3 Duct Bleed Hybrid Diffuser 


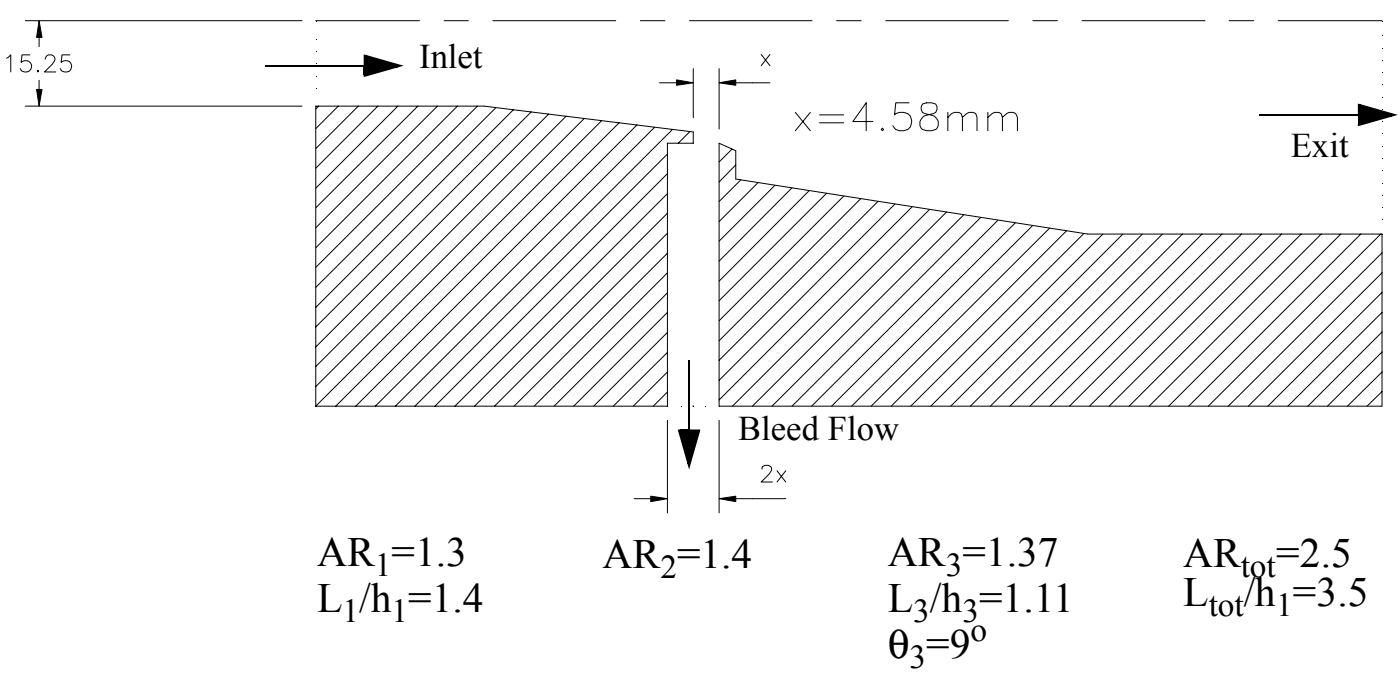

Figure 5.4 Extended Duct Bleed Hybrid Diffuser

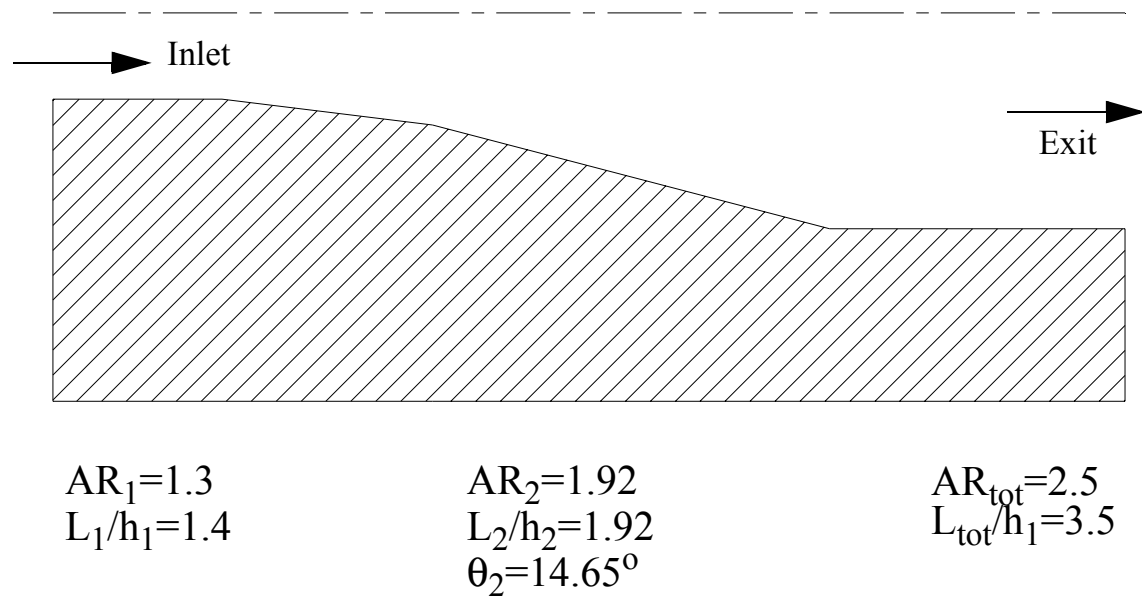

Figure 5.5 Conventional Diffuser

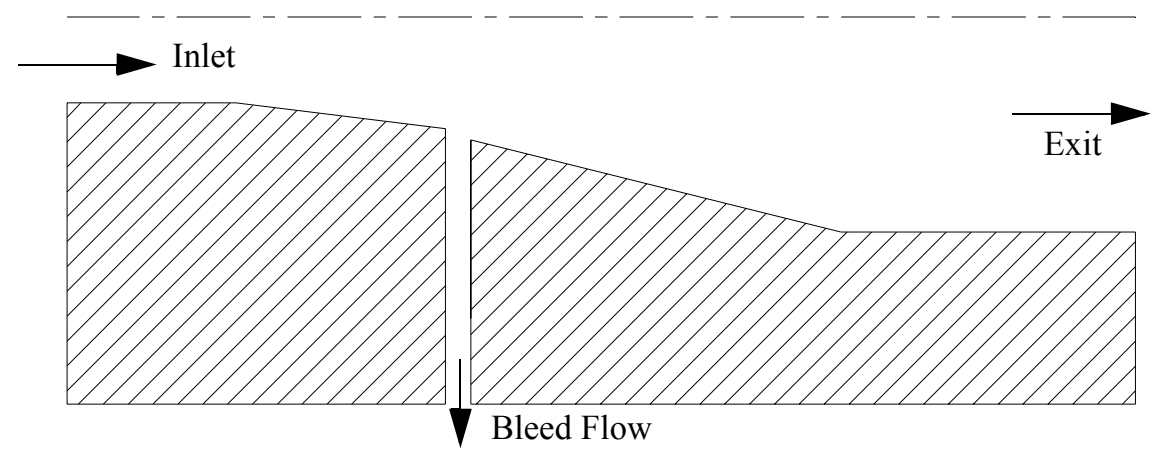
$\mathrm{AR}_{1}=1.3$
$\mathrm{AR}_{2}=1.07$
$\mathrm{AR}_{3}=1.8$
$\mathrm{L}_{3} / \mathrm{h}_{3}=1.55$
$\mathrm{AR}_{\mathrm{tot}}=2.5$
$\mathrm{L}_{1} / \mathrm{h}_{1}=1.4$
$\theta_{3}=14^{\mathrm{o}}$
$\mathrm{L}_{\text {tot }} h_{1}=3.5$

Figure 5.6 Alternative Bleed 1 


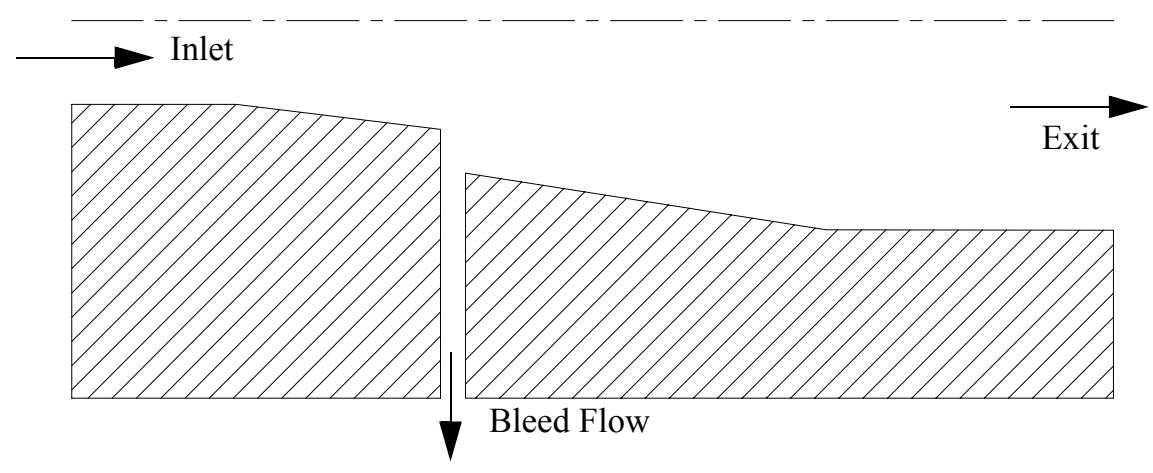

$$
\begin{array}{llll}
\mathrm{AR}_{1}=1.3 & \mathrm{AR}_{2}=1.4 & \mathrm{AR}_{3}=1.37 & \mathrm{AR}_{\mathrm{tot}}=2.5 \\
\mathrm{~L}_{1} / \mathrm{h}_{1}=1.4 & & \mathrm{~L}_{3} / \mathrm{h}_{3}=1.11 & \mathrm{~L}_{\text {tot }^{\prime}} / \mathrm{h}_{1}=3.5 \\
& \theta_{3}=9^{\mathrm{O}} &
\end{array}
$$

Figure 5.7 Alternative Bleed 2
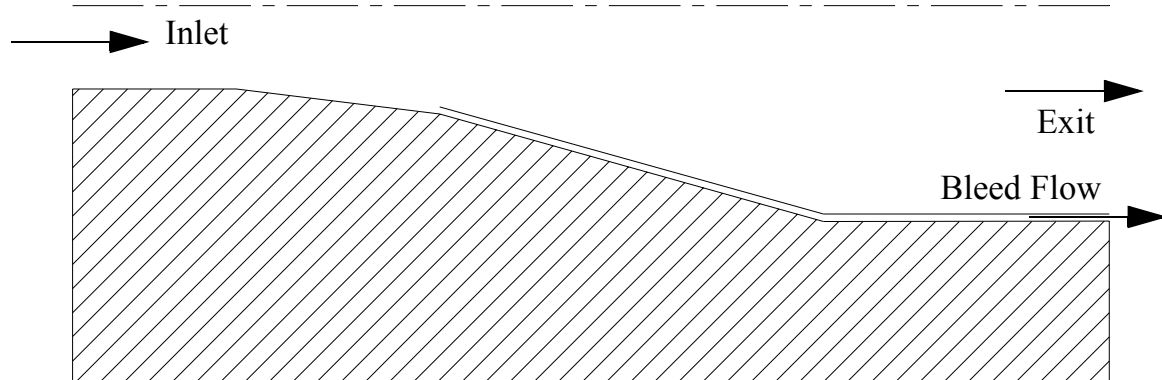
$\mathrm{AR}_{1}=1.3$
$\mathrm{AR}_{2}=1.92$
$\mathrm{L}_{1} / \mathrm{h}_{1}=1.4$
$\mathrm{L}_{3} / \mathrm{h}_{3}=1.77$
$\mathrm{AR}_{\mathrm{tot}}=2.5$
$\theta_{3}=15^{\circ}$

Figure 5.8 Alternative Bleed 3 - "Boundary Layer Bleed"

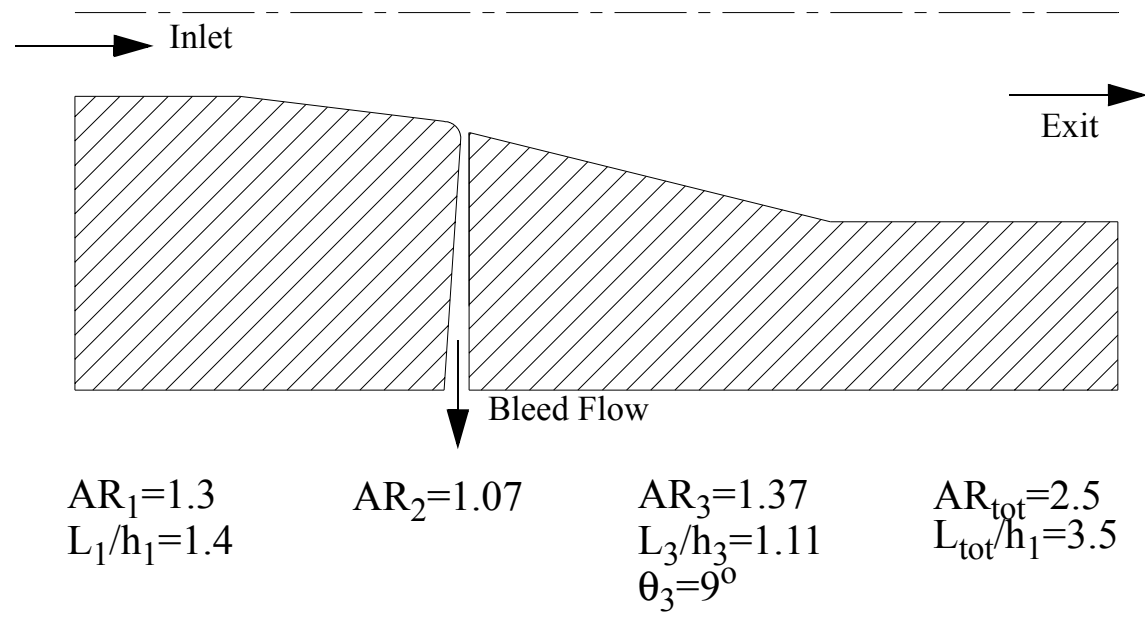

Figure 5.9 Alternative Bleed 4 - "Profiled Bleed Gap" 


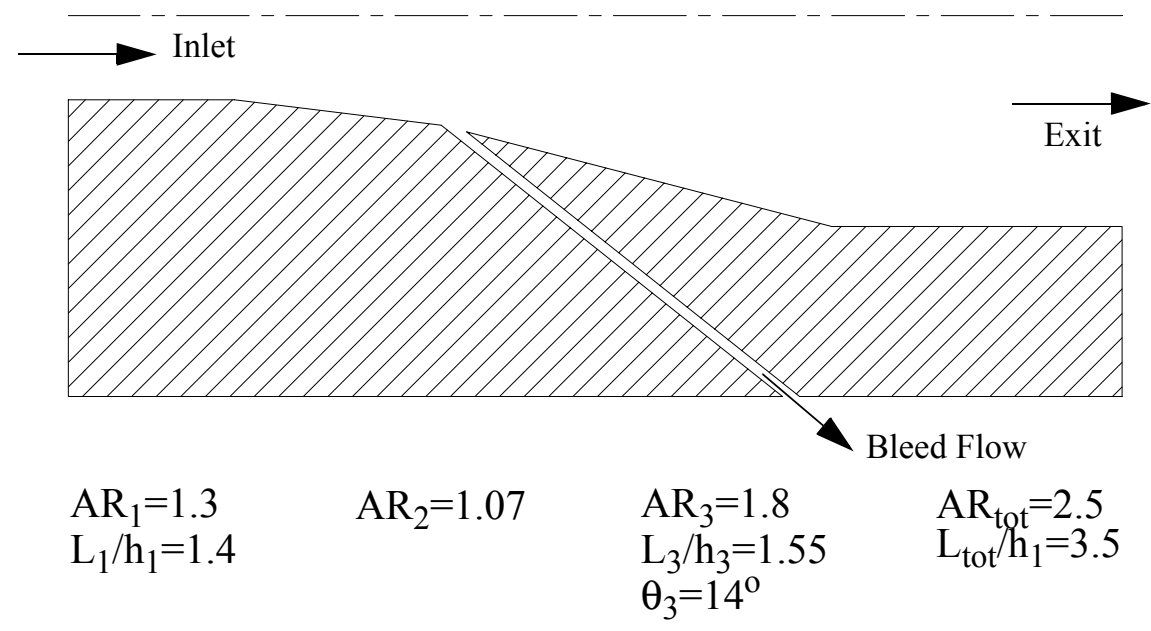

Figure 5.10 Alternative Bleed 5 - "Angled Bleed Duct"

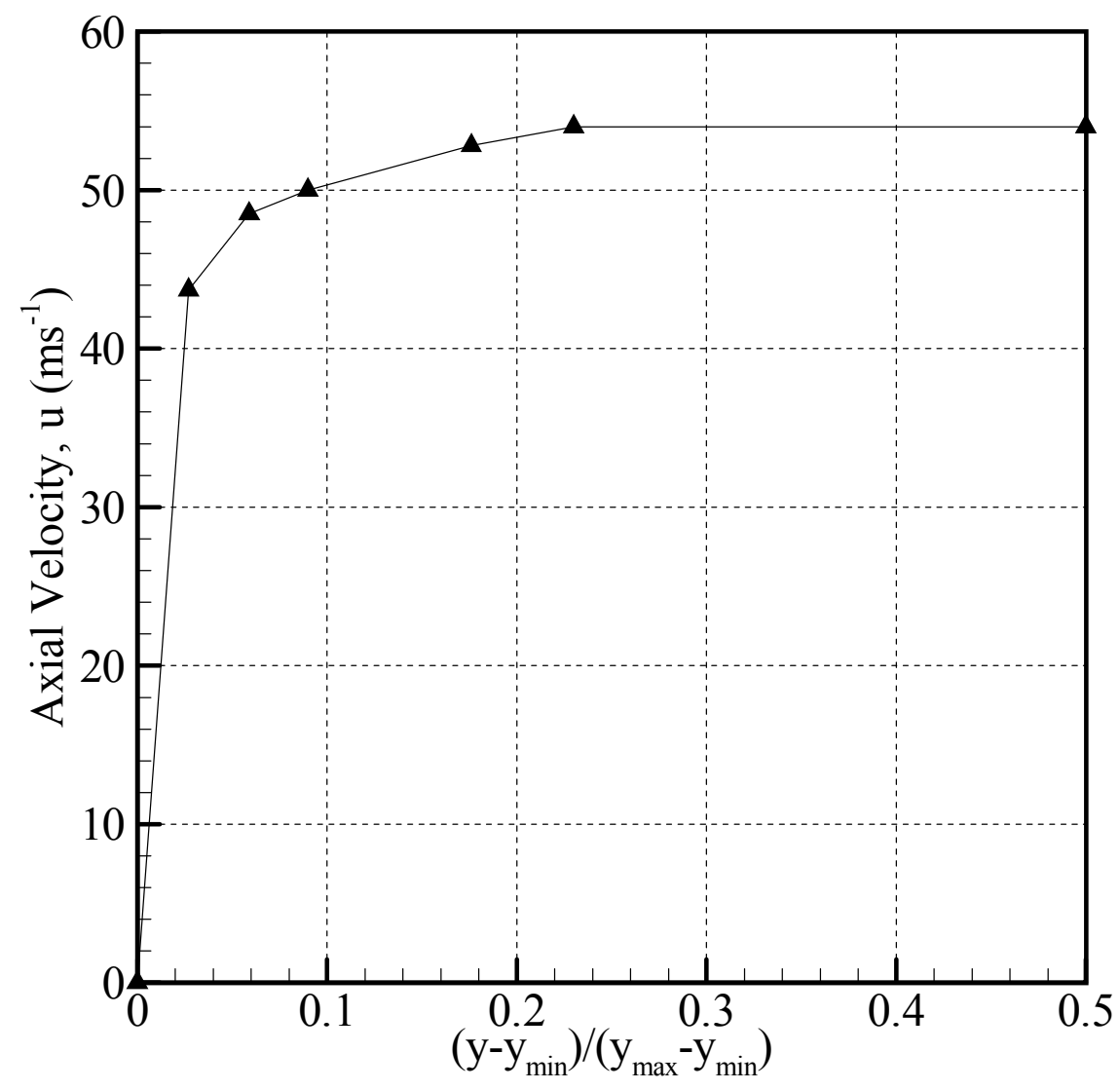

Figure 5.11 Inlet Velocity Profile for Plane Two-Dimensional CFD 


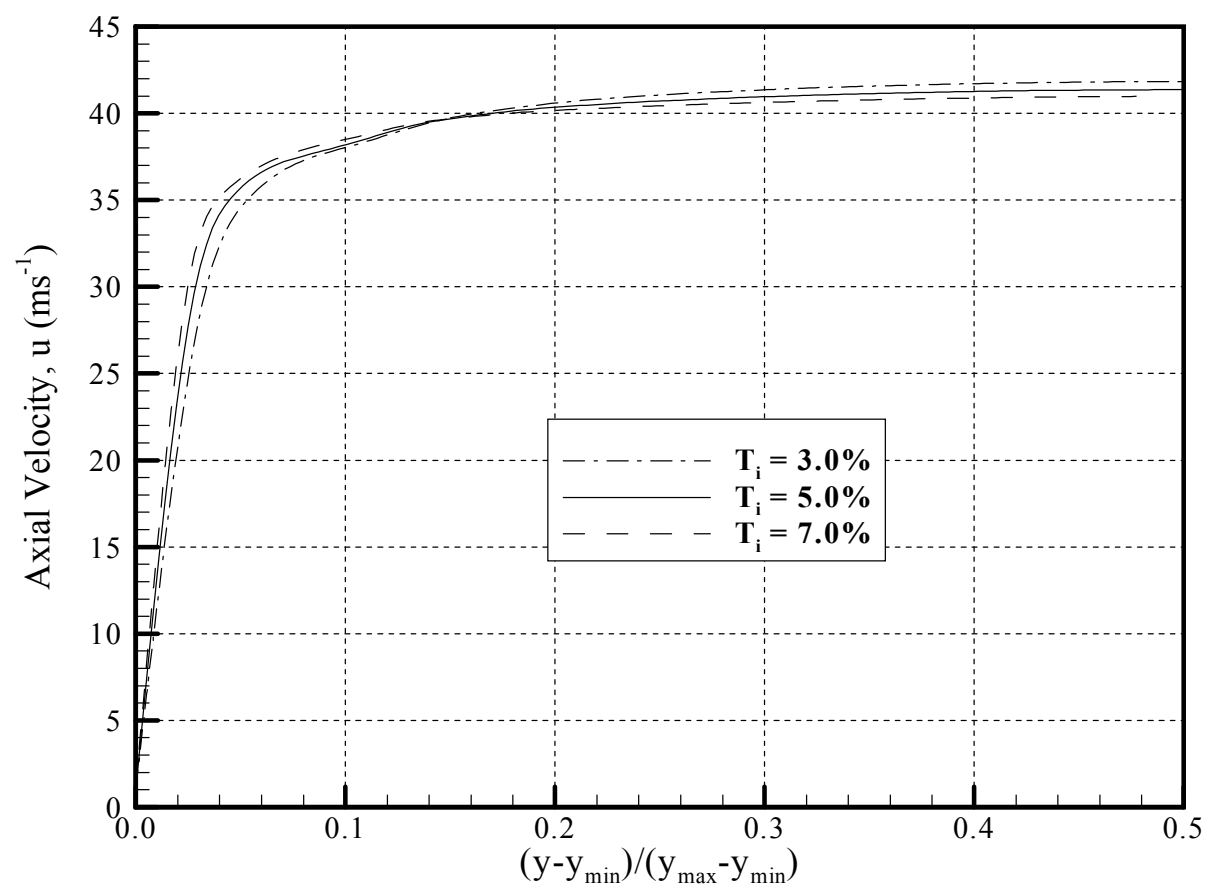

Figure 5.12 Effect of Inlet Turbulence Intensity on Solution $(L=30.5 \mathrm{~mm}, B=3.0 \%)$

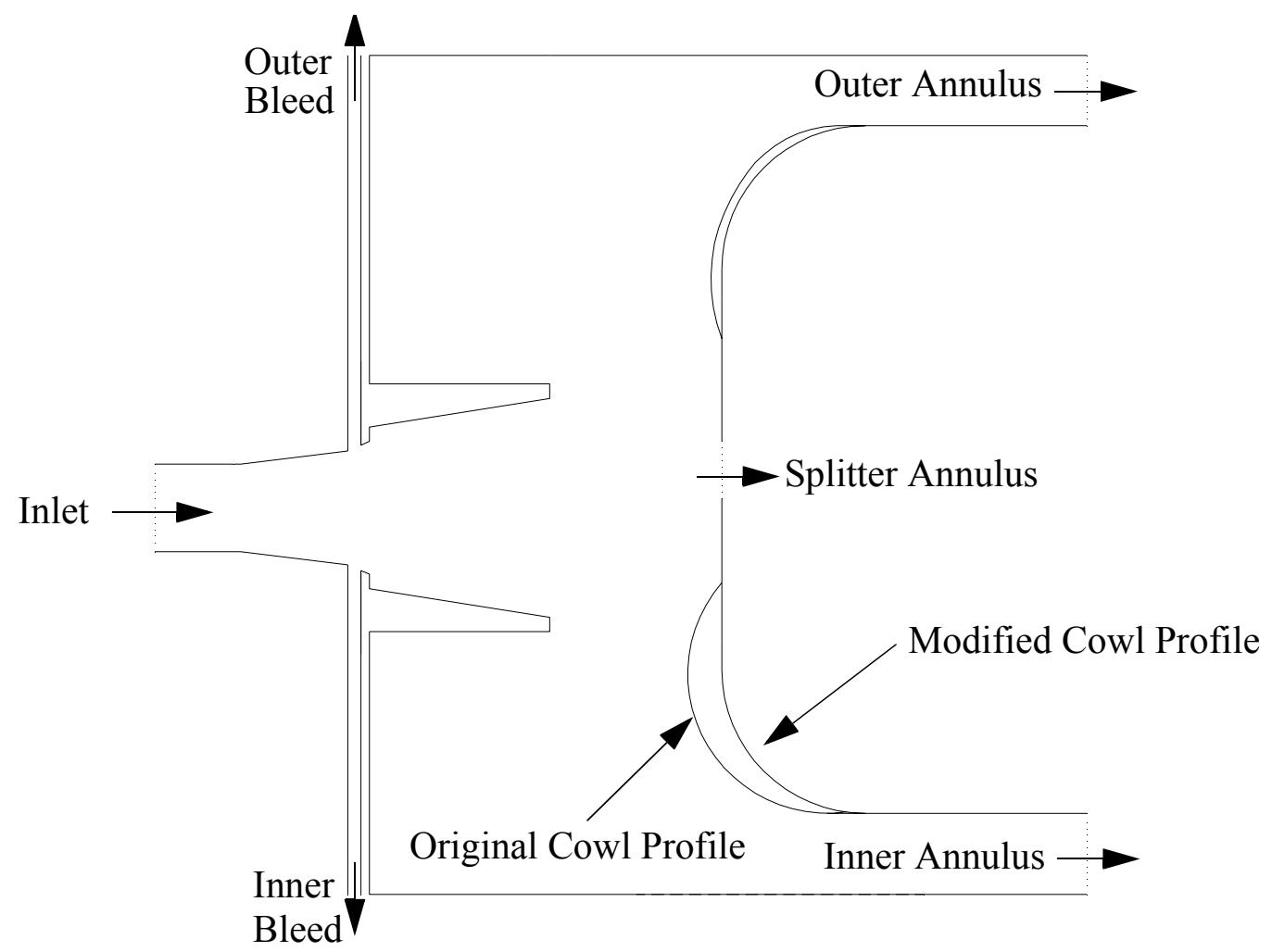

Figure 5.13 Two-Dimensional, Axi-Symmetric Hybrid Diffuser with Flame Tube 


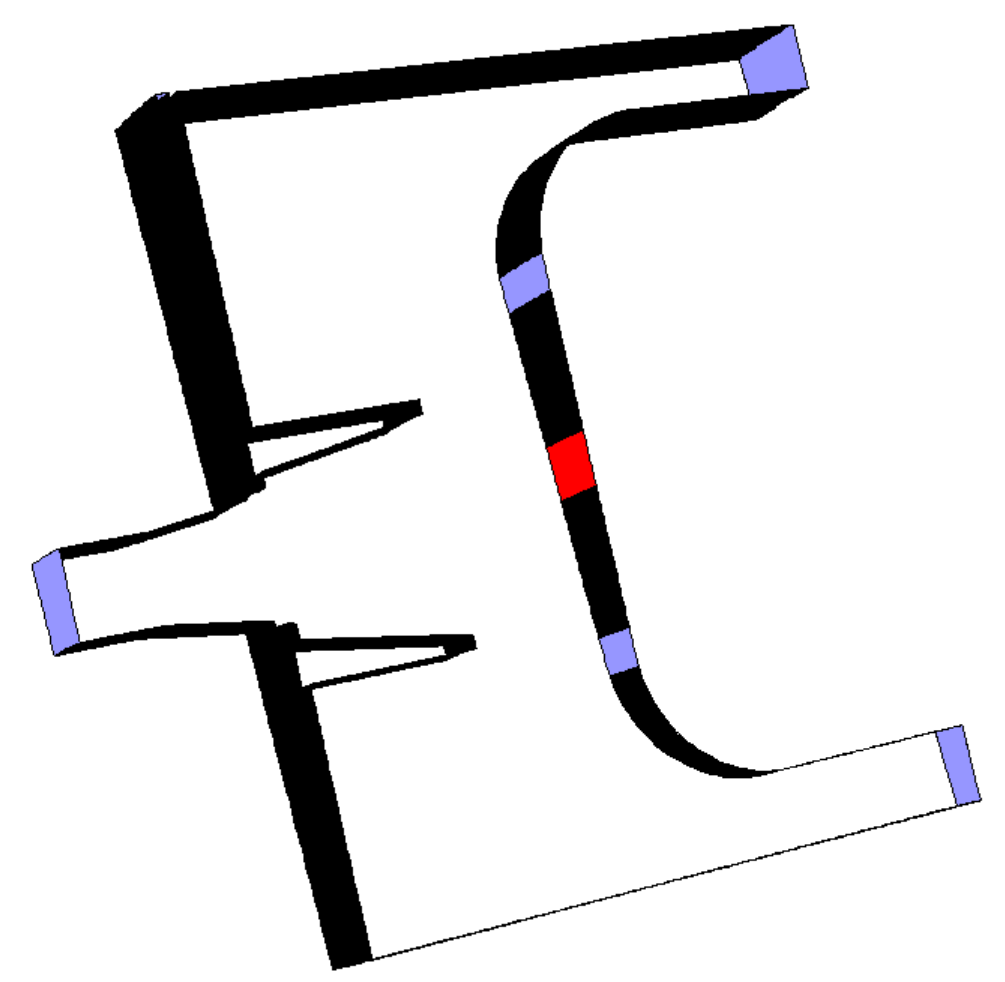

Figure 5.14 3D Computational Geometry

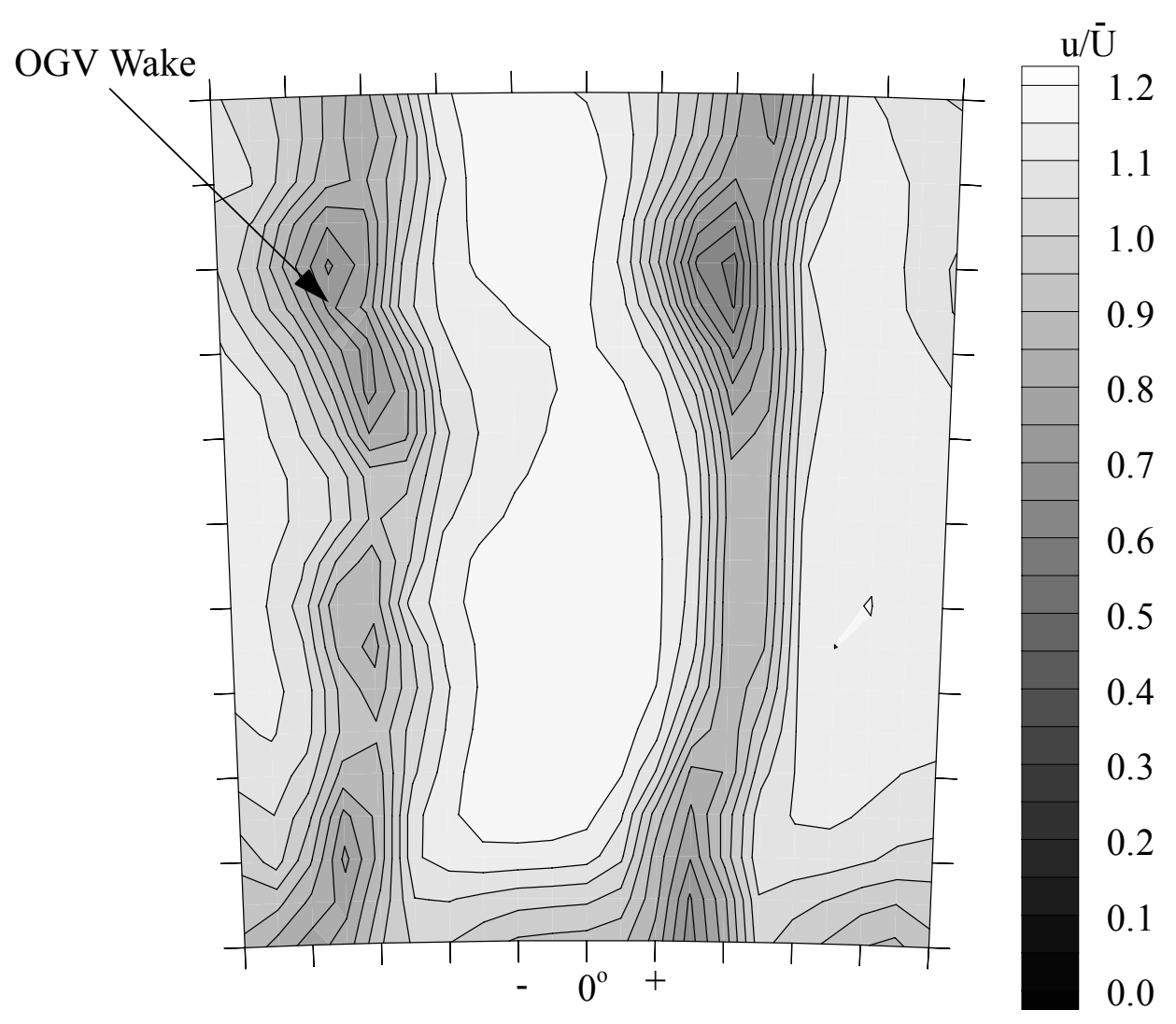

Figure 5.15 3D CFD Inlet Conditions - Normalised Axial Velocity Contours 


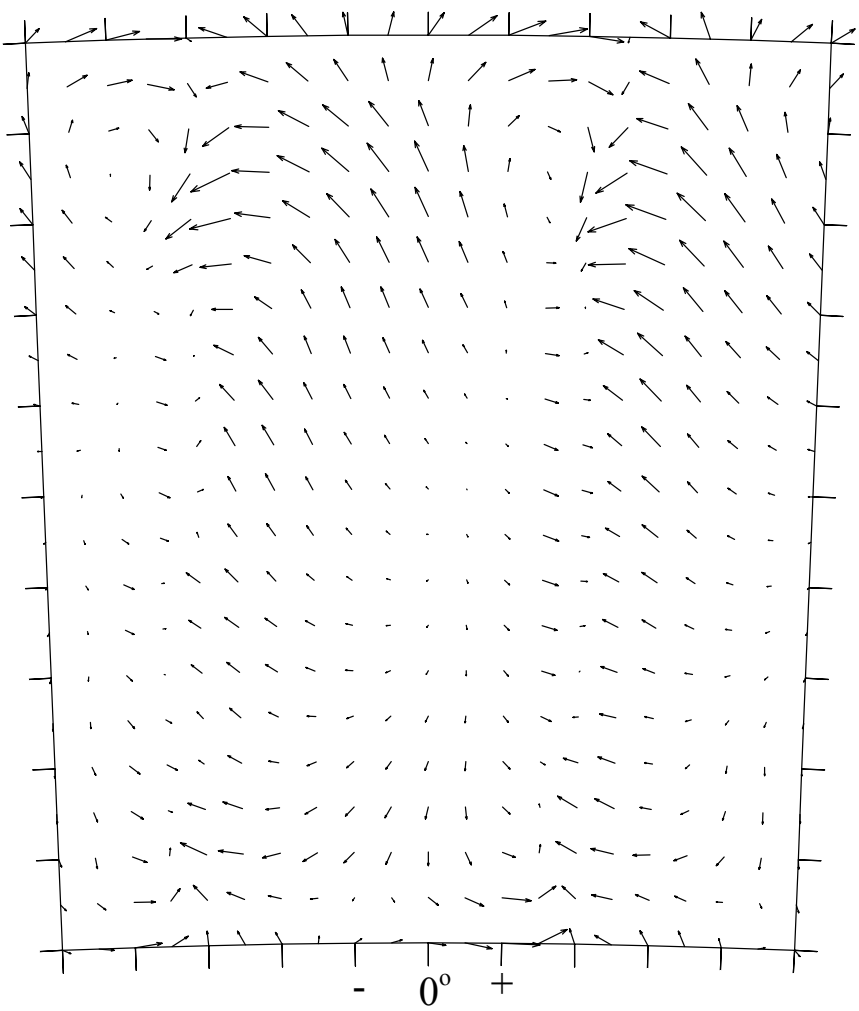

Figure 5.16 3D CFD Inlet Conditions - V-W Velocity Vectors

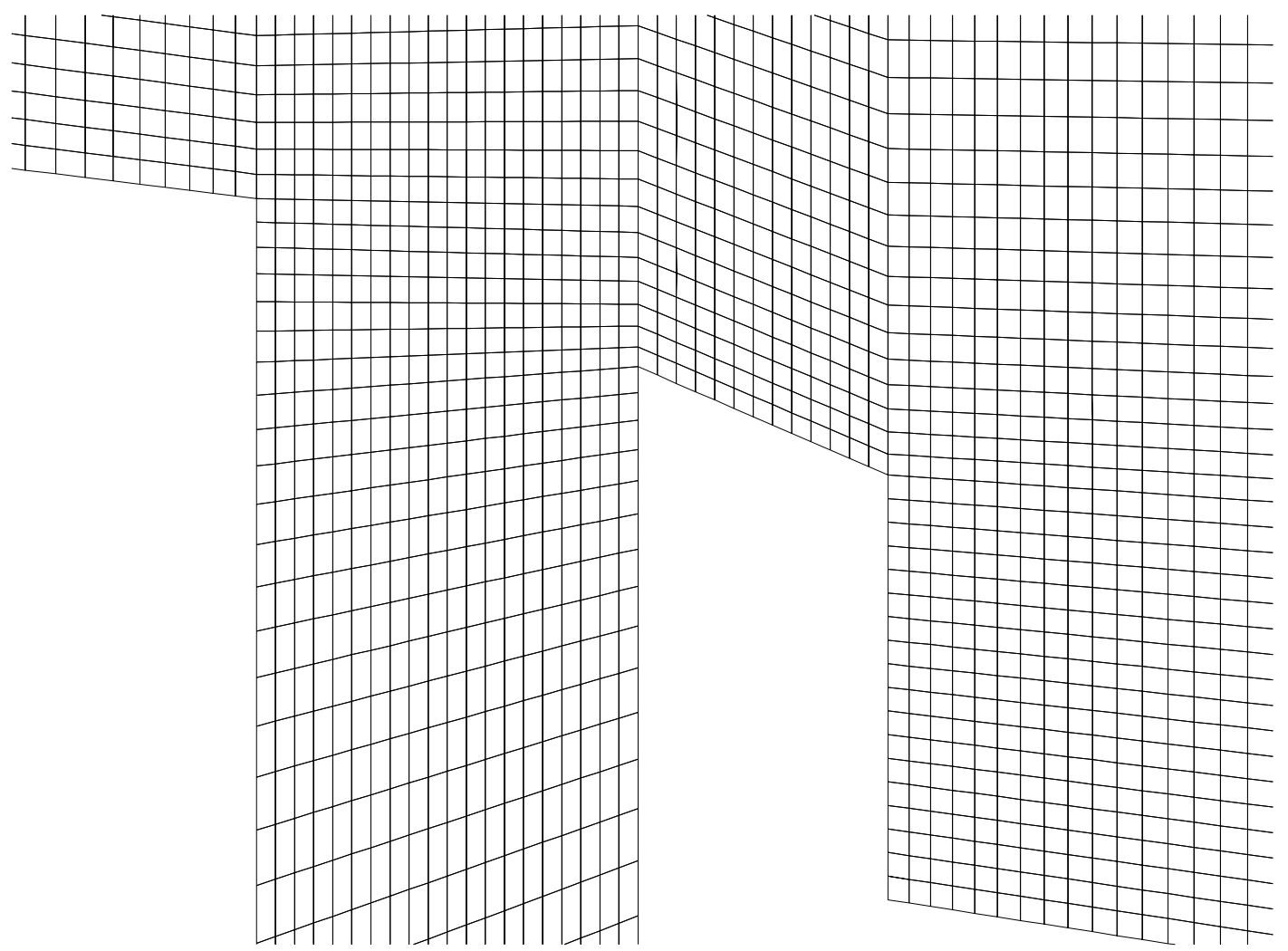

Figure 5.17 Initial 2D Grid in Stage 2 Diffuser (shown with Duct Bleed) 


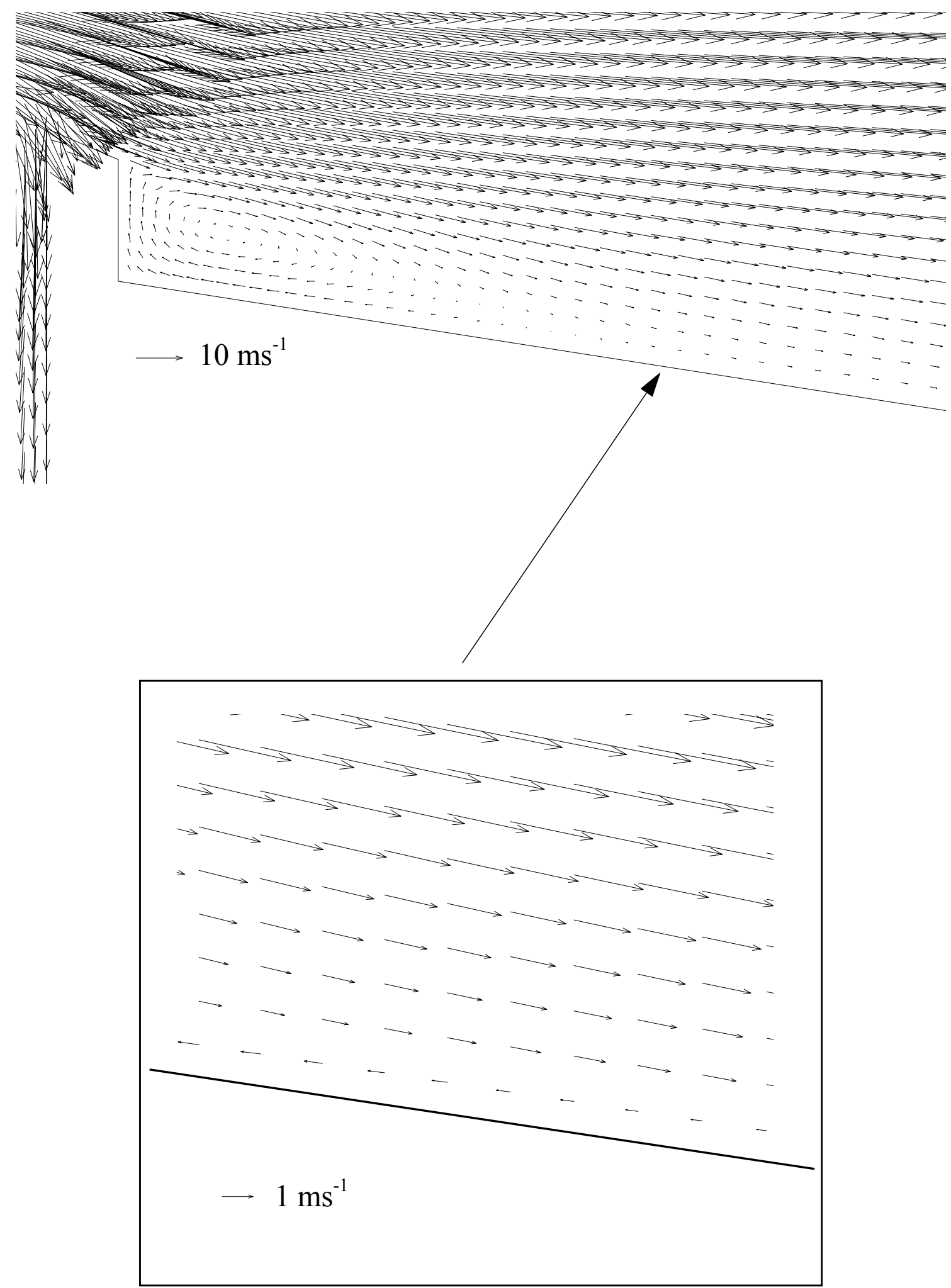

Figure 5.18 Non-Physical Near-Wall Flow (Initial 2D Grid, k- $\varepsilon$, Standard Wall Function, 3\% Bleed) 


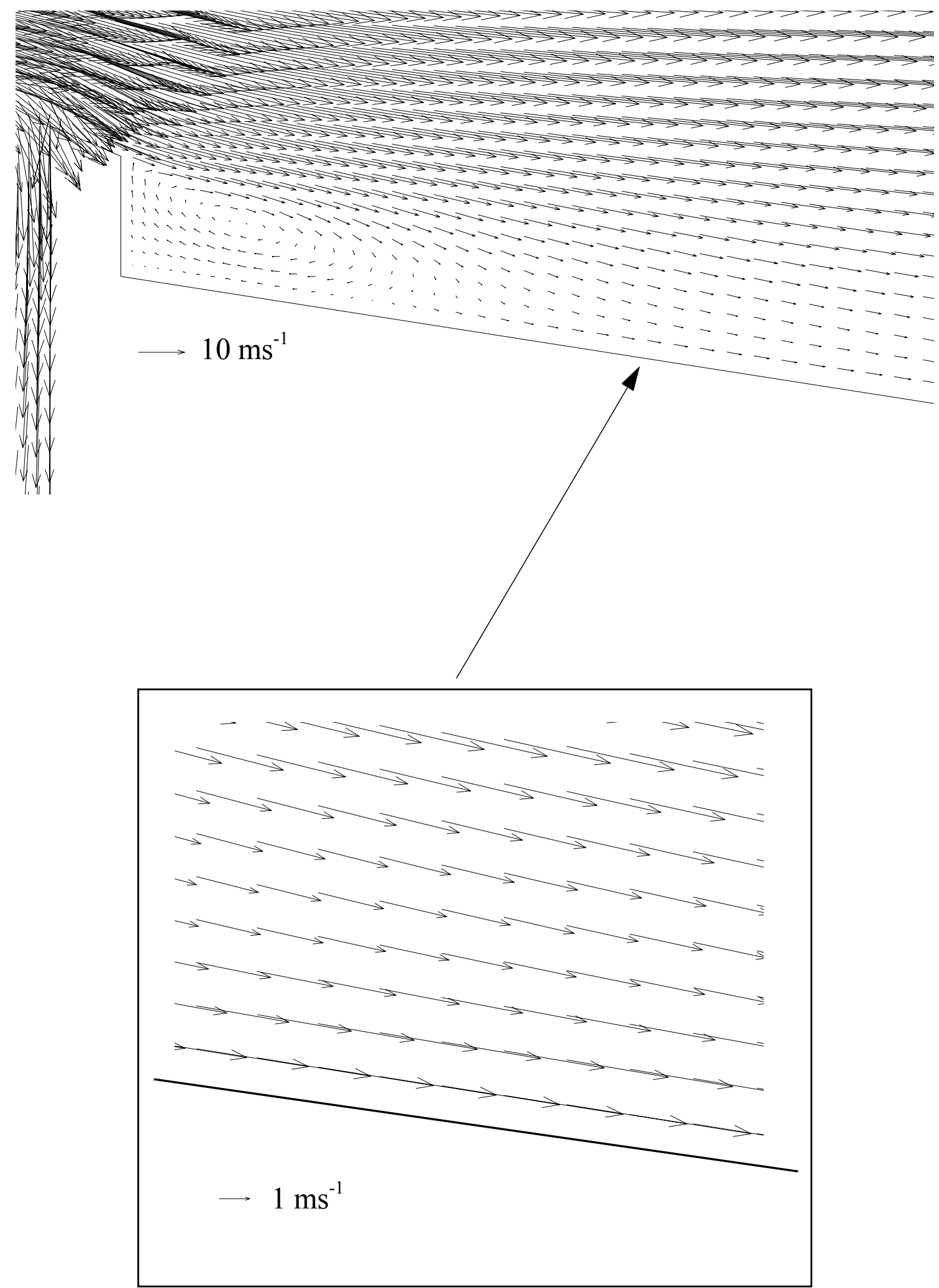

Figure 5.19 Non-Physical Near-Wall Flow (Initial 2D Grid, RNS, Standard Wall Function, 3\% Bleed) 

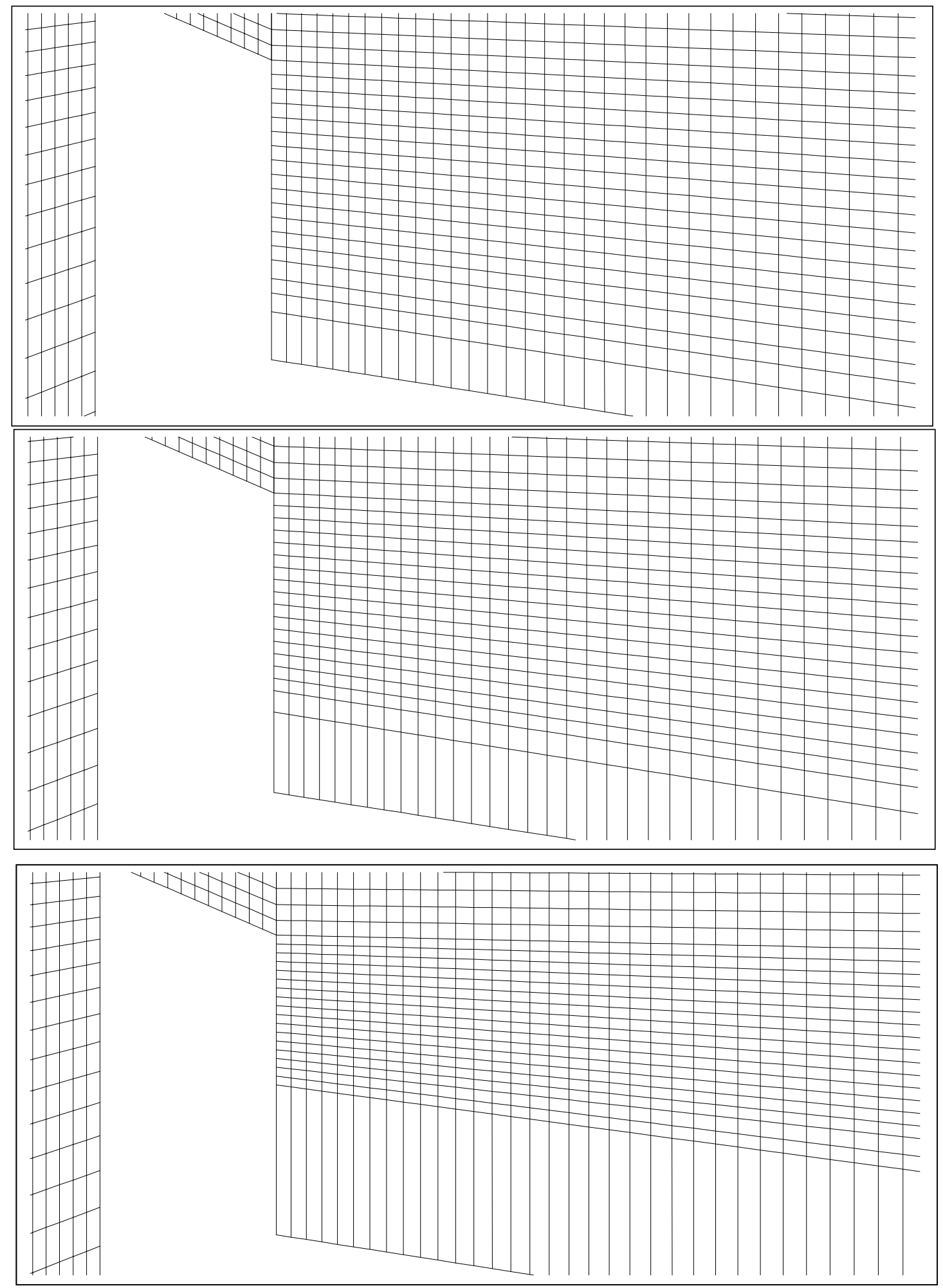

Figure 5.20 Near Wall Modified 2D Grids (Near Wall Cell 16.5\%, 25\% and 50\% of Fence Height) 


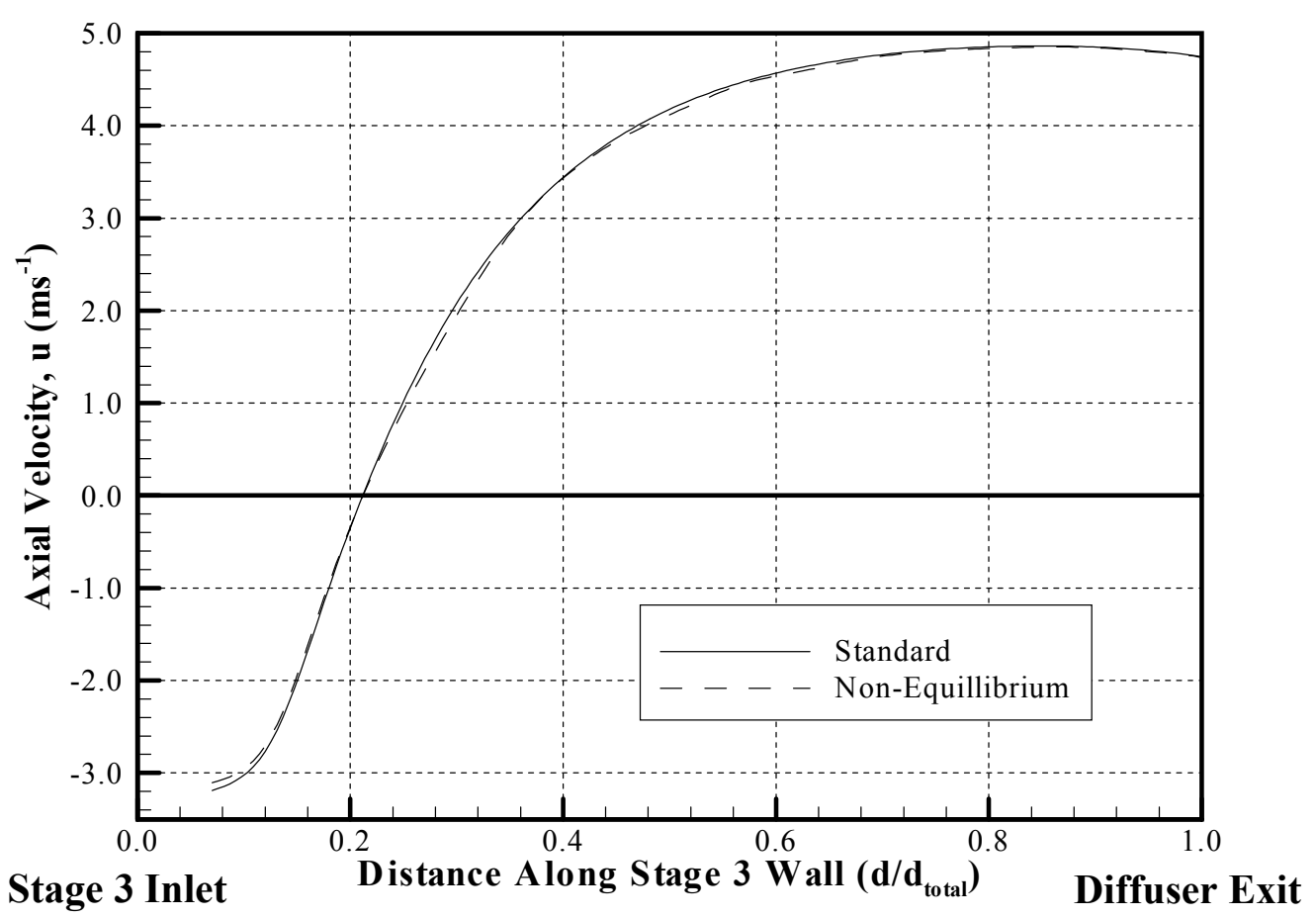

Figure 5.21 Axial Velocity in Stage 3 Near Wall Cell (k- $\varepsilon, 3 \%$ Bleed)

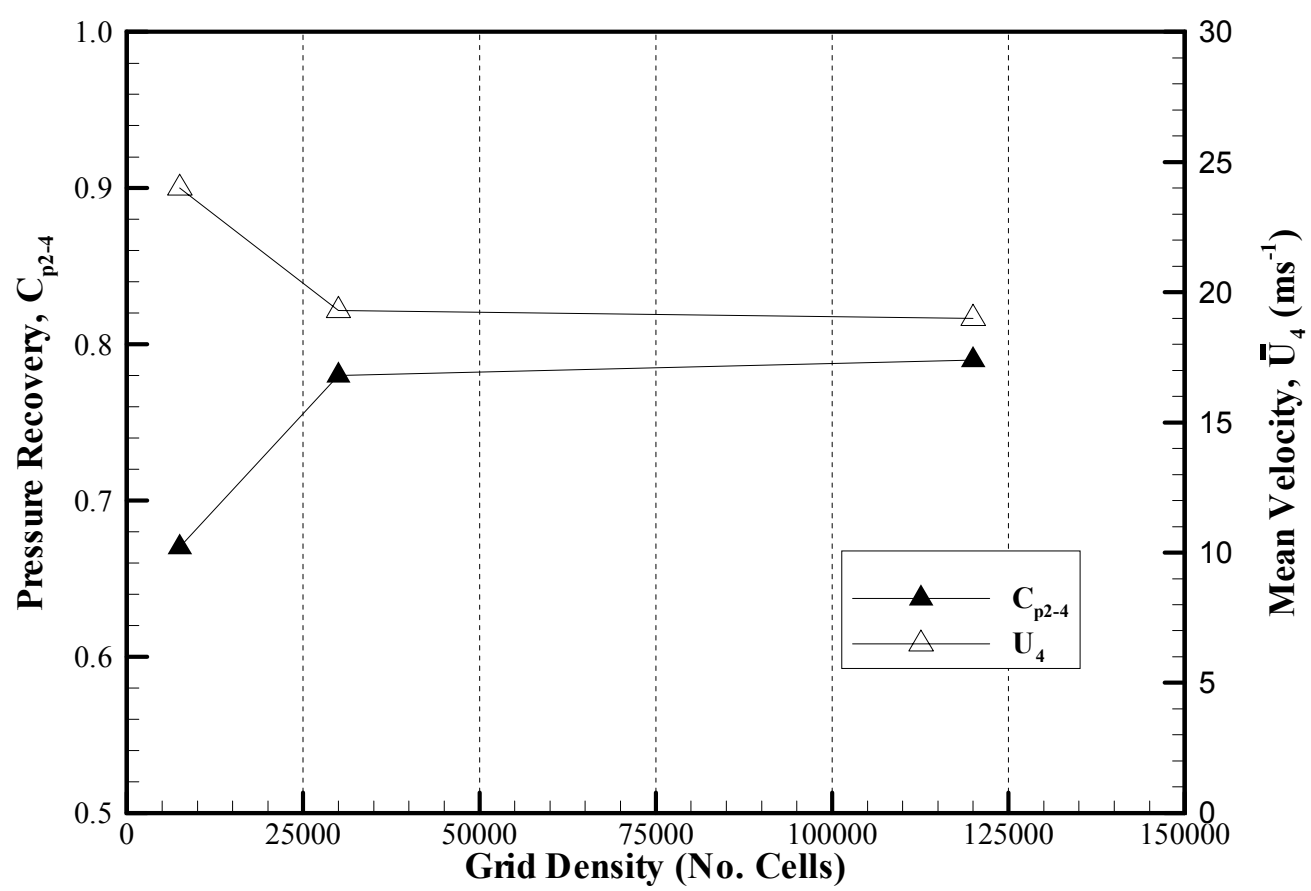

Figure 5.22 Effect of Grid Density on Solution (k- $\varepsilon$, Standard Wall Function, 3\% Bleed) 


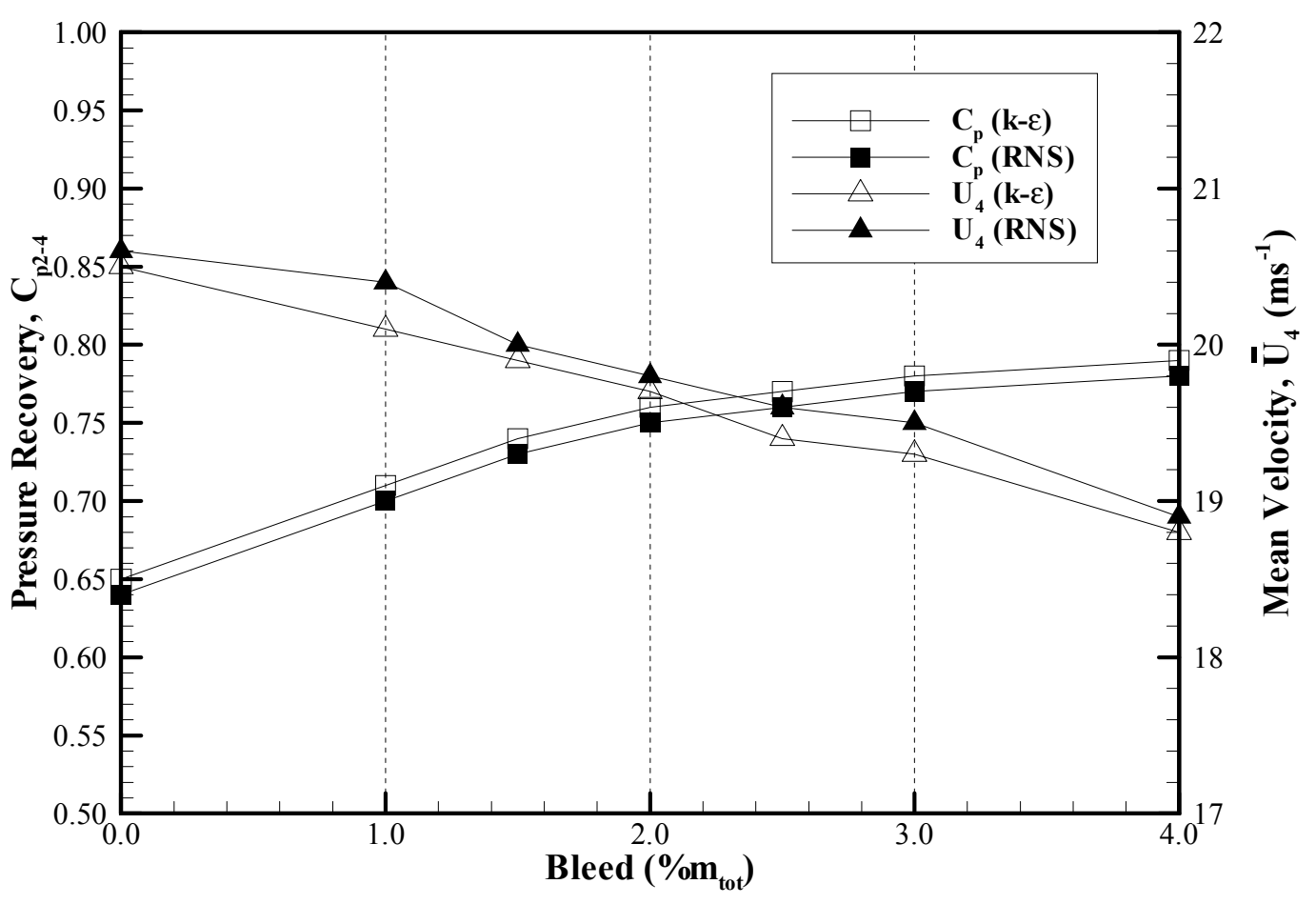

Figure 5.23 Effect of Turbulence Closure Method on Solution

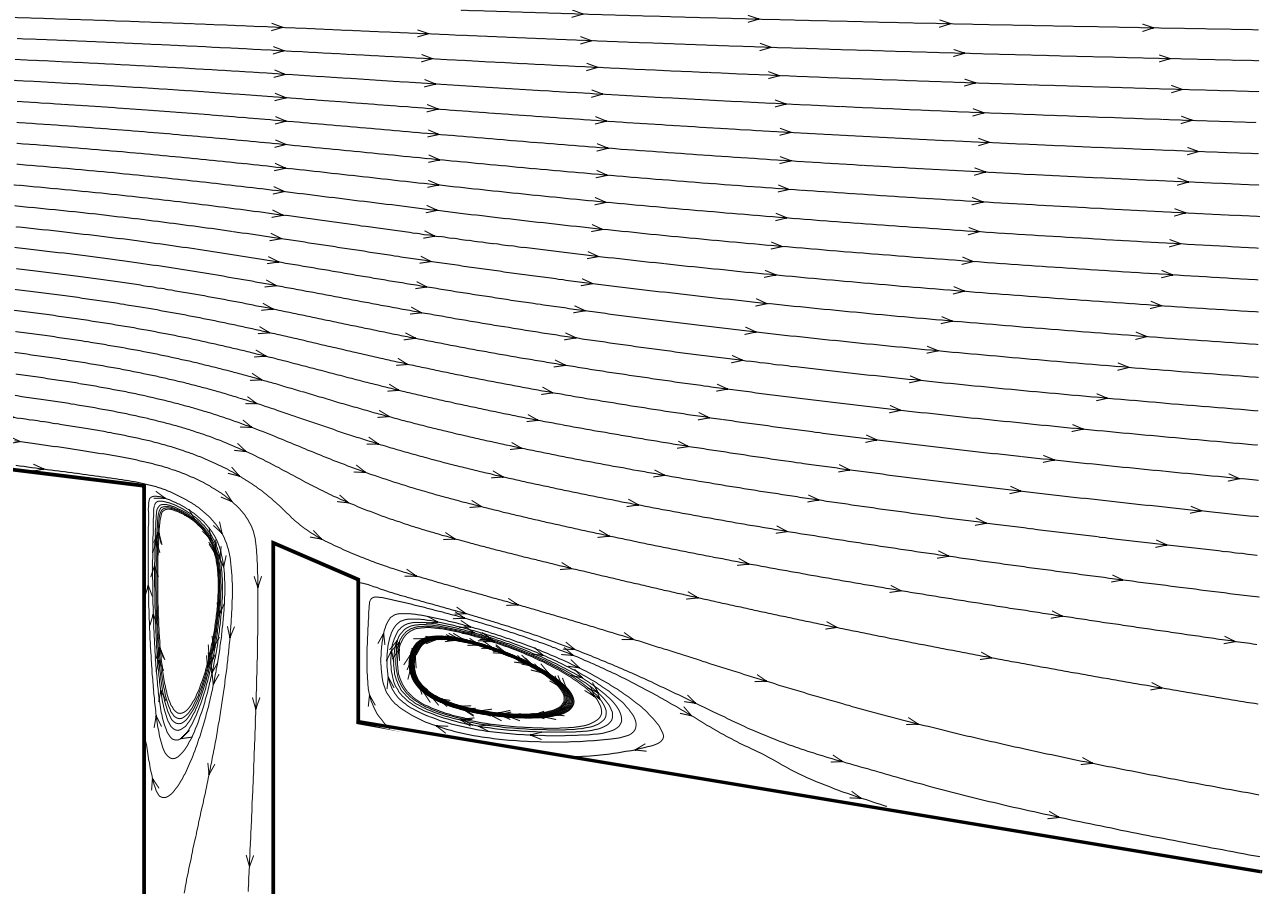

Figure 5.24 Streamlines at Reattachment (k- $\varepsilon$, Standard Wall Function, 3\% Bleed) 


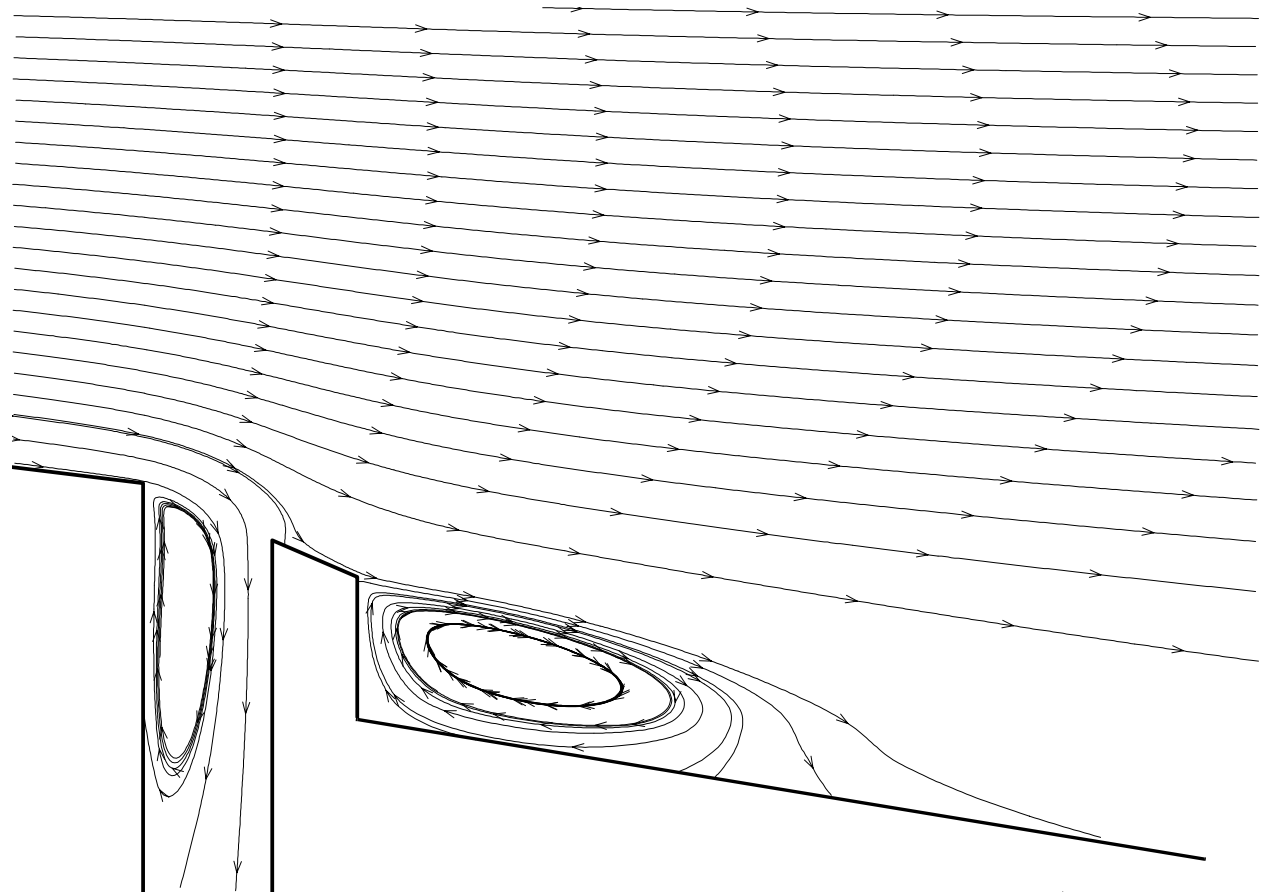

Figure 5.25 Streamlines at Reattachment (RNS, Standard Wall Function, 3\% Bleed)

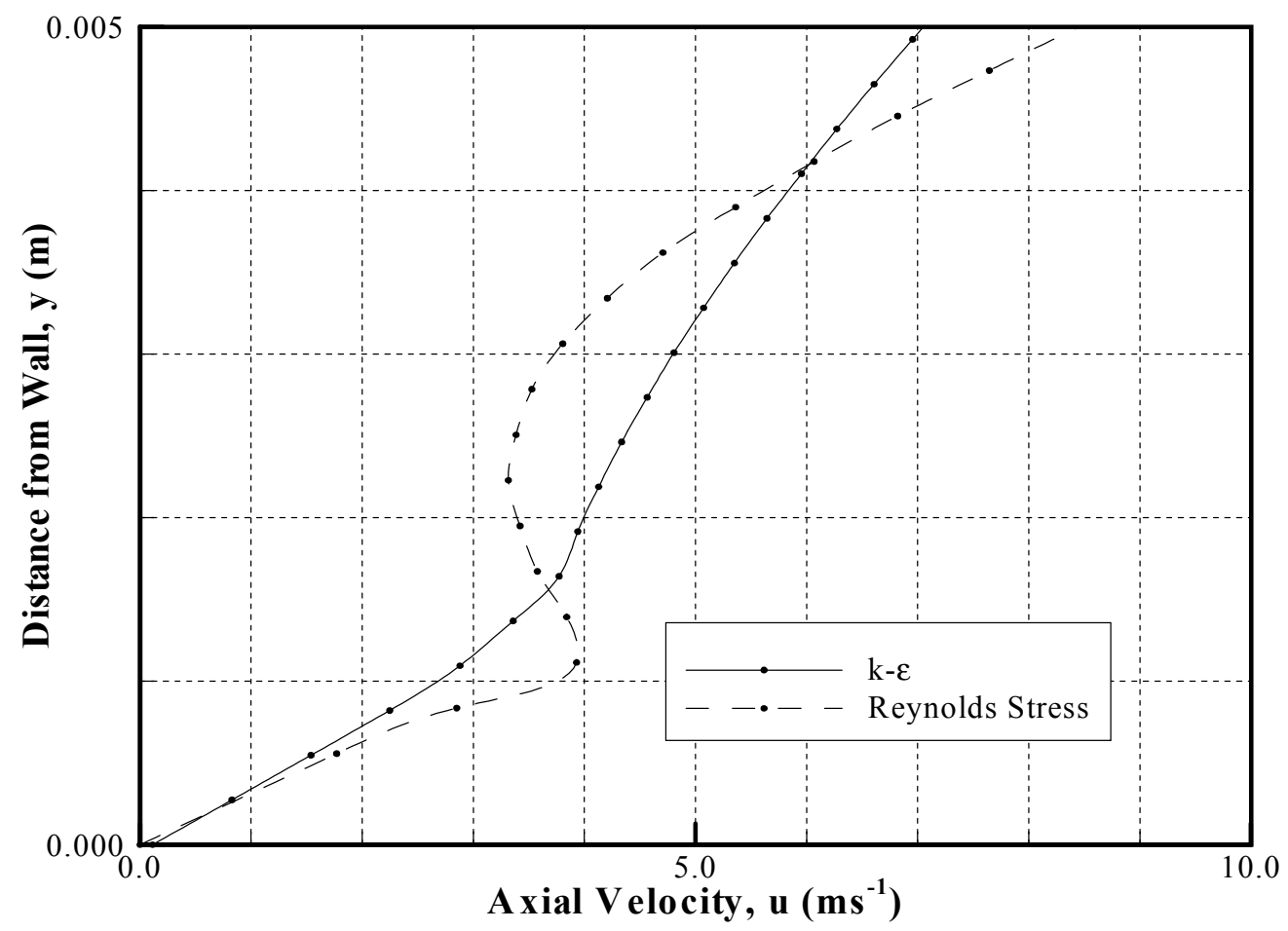

Figure 5.26 Anomaly in RNS Boundary Layer Profile on Stage 3 Wall (mid-way along walll) 


\subsection{COMPUTATIONAL RESULTS AND DISCUSSION}

6.1 Two-Dimensional, Plane Hybrid Diffuser Study $\ldots \ldots \ldots \ldots 210$

6.1.1 Overall Diffuser Performance . . . . . . . . . . . 210

6.1.1.1 Vortex Chamber and Duct Bled Hybrid Diffusers . . .212

6.1.1.2 Alternative Bleed Geometries . . . . . . . . . 216

6.1.2 Bleed Flow Assessment . . . . . . . . . . . . . . . . . . 220

6.1.3 Flow Mechanisms - Momentum Transfer Analysis .......222

6.1.3.1 The Momentum Equation . . . . . . . . . . . . . . . 224

6.1.3.2 Flow Field Discussion (2.5\% Bleed) . . . . . . . . 229

6.1.3.3 Flow Field Discussion $(0.0 \%$ Bleed $) \ldots \ldots \ldots .232$

6.2 Two-Dimensional, Axi-Symmetric Hybrid Diffuser with Flame Tube

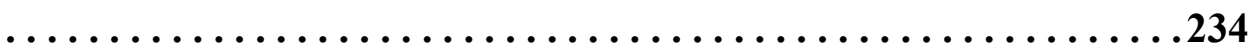

6.3 Hybrid Diffuser with OGV Wakes $\ldots \ldots \ldots \ldots \ldots \ldots \ldots 238$

6.3.1 Diffuser Performance and OGV Wake Effects ......... . 238

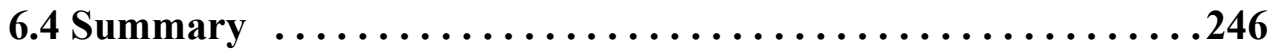

FIGURES $\ldots \ldots \ldots \ldots \ldots \ldots \ldots \ldots \ldots \ldots \ldots \ldots \ldots \ldots \ldots . \ldots . \ldots . \ldots . \ldots$ 


\subsection{COMPUTATIONAL RESULTS AND DISCUSSION}

The results presented and discussed in this section were all generated using a commercial CFD code, Fluent, with an eddy-viscosity turbulence model as described in Section 5.0. The various diffusers, described in Section 5.7.1, all comprise of the same overall area ratio (2.5) and non-dimensional length (3.5) as the Hybrid 4 diffuser used in the experimental study (Sections 3.0 and 4.0). Initially, the discussion will centre on the performance of a two-dimensional planar representation of the Hybrid 4 diffuser with a vortex chamber bleed, a duct bleed and an extended duct bleed. Results are presented for the $300 \times 100$ computational mesh which, as described in Section 5.8, produced a grid independent solution. Subsequently the discussion will move on to the performance of a series of two-dimensional diffusers with modified bleed off-take geometry. Logically, the next section discusses results from the simplified two-dimensional, axi-symmetric model of the test facility in conjunction with the relevant experimental data. Finally, the impact of three-dimensional inlet conditions including OGV wakes and residual swirl is discussed.

In line with the arguments presented in Section 5.7 data are only presented for an eddyviscosity $(\mathrm{k}-\varepsilon)$ turbulence closure used in conjunction with wall behaviour modelled by a standard log-law wall function.

\subsection{Two-Dimensional, Plane Hybrid Diffuser Study}

\subsubsection{Overall Diffuser Performance}

Overall diffuser performance data, as defined in Section 2.2, is presented in Table 6.1 Table 6.3. However, the question of correctly accounting for removing the bleed flow was first raised in Section 4.3.1. Traditionally, the mass weighted total pressure loss from rotor exit (measurement station 2) to diffuser exit (station 4) is defined as:

$$
\lambda_{2-4}=\frac{\tilde{\mathrm{P}}_{2}-\tilde{\mathrm{P}}_{4}}{\tilde{\mathrm{q}}_{2}}
$$


The question arose due to the fact that the mass weighted total and dynamic pressures $\left(\tilde{\mathrm{P}}_{2}, \tilde{\mathrm{q}}_{2}\right)$ at rotor exit include the bleed flow whereas at diffuser exit the mass weighted total pressure does not. It can be argued that removal of the energy deficient, poor quality boundary layer will, using Equation 6.1, result in an optimistic view of performance. Calculation of the mass weighted pressures using a streamtube analysis and hence including only the flow which passes down the diffuser would yield a more accurate result. However, for the experimental data only the traditional method of performance accounting was used. A streamtube approach was not possible due to the highly three dimensional nature of the inlet conditions. For example, OGV wake flow has low axial momentum and will be less able to overcome the radial pressure gradient and therefore more likely to be captured by the bleed. In the simple two-dimensional computational study, mass weighted pressures can easily be calculated which do not include the bleed flow. This was done by simply omitting the portion of flow, at inlet, which makes up the bleed streamtube. At inlet mass-weighted pressures were calculated using cell-centred values outside of the bleed streamtube, such that:

$$
\lambda_{2^{\prime}-4}=\frac{\tilde{\mathrm{P}}_{2^{\prime}}-\tilde{\mathrm{P}}_{4}}{\tilde{\mathrm{q}}_{2^{\prime}}}
$$

where $\tilde{\mathrm{P}}_{2^{\prime}}$ and $\tilde{\mathrm{q}}_{2^{\prime}}$ are computed from the inlet flow minus the bleed flow.

An assessment of the difference between $\lambda_{2-4}$ and $\lambda_{2}{ }^{\prime}-4$ was performed using the solution generated for the hybrid diffuser with a vortex chamber and the data are presented in Table 6.1. It can be seen that accounting for the bleed does, indeed, have an effect on the indicated diffuser performance. Corrected total pressure loss coefficients increase by 4 $5 \%$ and static pressure recovery coefficients reduce by about $1 \%$. Although this may not be a significant amount it is important to note that it exists and that performance data from the experimental investigation are slightly optimistic. However, to prevent confusion and maintain a consistent approach throughout this work, data will only be presented (unless otherwise stated) using the traditional methods without accounting for the effect of removing the bleed flow. Thus sensible comparisons can be made between all the configurations both experimental and computational. 
A reattachment length, $\mathrm{Xr}$, is also quoted and this is defined as the axial (x) distance along the Stage 3 wall at which the flow reattaches measured from the back face of the fence as shown in Figure 6.1. Reattachment is defined as the point at which:

$$
\left(\frac{\partial \mathrm{U}}{\partial \mathrm{y}}\right)_{\mathrm{wall}}=0
$$

\subsubsection{Vortex Chamber and Duct Bled Hybrid Diffusers}

Before considering the relative performance of the hybrid diffusers it is important to recognise that there is no difference in overall diffuser performance between the hybrid diffusers bled through a vortex chamber, a duct or an extended duct (refer to Section 5 for geometry description).

Tables 6.1 - 6.3 demonstrate that loss coefficients, pressure recovery coefficients and reattachment lengths are identical at the same bleed rates. For example, with a bleed rate of $2.5 \%$ all three hybrid diffusers have a mass weighted static pressure recovery coefficient of $0.77-0.78$, a mass weighted total pressure loss coefficient of 0.116 and the flow reattaches at a point $25 \%$ along the Stage 3 wall. This would suggest that the important parameter is the bleed mass flow rate, not the three different bleed geometry arrangements.

\begin{tabular}{|c|c|c|c|c|c|}
\hline $\begin{array}{c}\text { Bleed } \\
\left(\% \dot{\mathrm{m}}_{\text {tot }}\right)\end{array}$ & $\mathrm{C}_{\mathrm{p} 2-4}$ & $\lambda_{2-4}$ & $C_{p 2 '-4}$ & $\lambda_{2}{ }^{\prime}-4$ & $\mathrm{Xr} / \mathrm{L}_{3}$ \\
\hline 0.0 & 0.647 & 0.139 & 0.647 & 0.139 & stalled \\
\hline 1.0 & 0.724 & 0.124 & 0.717 & 0.129 & stalled \\
\hline 1.5 & 0.747 & 0.120 & 0.741 & 0.125 & 0.51 \\
\hline 2.0 & 0.764 & 0.118 & 0.758 & 0.123 & 0.33 \\
\hline 2.5 & 0.777 & 0.116 & 0.771 & 0.121 & 0.25 \\
\hline 3.0 & 0.786 & 0.115 & 0.780 & 0.120 & 0.21 \\
\hline 4.0 & 0.800 & 0.113 & 0.794 & 0.118 & 0.17 \\
\hline
\end{tabular}


Table 6.1 Diffuser Performance - Hybrid Diffuser with Vortex Chamber

\begin{tabular}{|c||c|c|c|}
\hline $\begin{array}{c}\text { Bleed } \\
\left(\mathbf{\%} \dot{\mathrm{m}}_{\text {tot }}\right)\end{array}$ & $\mathbf{C}_{\mathbf{p 2 - 4}}$ & $\boldsymbol{\lambda}_{\mathbf{2 - 4}}$ & $\mathrm{Xr} / \mathbf{L}_{\mathbf{3}}$ \\
\hline \hline $\mathbf{0 . 0}$ & 0.649 & 0.140 & stalled \\
\hline $\mathbf{1 . 0}$ & 0.713 & 0.125 & stalled \\
\hline $\mathbf{1 . 5}$ & 0.737 & 0.121 & 0.53 \\
\hline $\mathbf{2 . 0}$ & 0.756 & 0.121 & 0.33 \\
\hline $\mathbf{2 . 5}$ & 0.774 & 0.116 & 0.25 \\
\hline $\mathbf{3 . 0}$ & 0.778 & 0.115 & 0.21 \\
\hline $\mathbf{4 . 0}$ & 0.793 & 0.113 & 0.17 \\
\hline
\end{tabular}

Table 6.2 Diffuser Performance - Hybrid Diffuser with Duct Bleed

\begin{tabular}{|c||c|c|c|}
\hline $\begin{array}{c}\text { Bleed } \\
\left(\mathbf{\%}_{\text {tot }}\right)\end{array}$ & $\mathbf{C}_{\mathbf{p 2 - 4}}$ & $\boldsymbol{\lambda}_{\mathbf{2 - 4}}$ & $\mathbf{X r} / \mathbf{L}_{\mathbf{3}}$ \\
\hline \hline $\mathbf{0 . 0}$ & 0.656 & 0.138 & stalled \\
\hline $\mathbf{1 . 0}$ & 0.719 & 0.125 & stalled \\
\hline $\mathbf{1 . 5}$ & 0.744 & 0.121 & 0.53 \\
\hline $\mathbf{2 . 0}$ & 0.762 & 0.118 & 0.33 \\
\hline $\mathbf{2 . 5}$ & 0.775 & 0.116 & 0.25 \\
\hline $\mathbf{3 . 0}$ & 0.779 & 0.120 & 0.21 \\
\hline $\mathbf{4 . 0}$ & 0.799 & 0.114 & 0.17 \\
\hline
\end{tabular}

Table 6.3 Diffuser Performance - Hybrid Diffuser with Extended Duct Bleed

Normalised axial velocity profiles at diffuser exit, as shown in Figures 6.2 and 6.3, are also indistinguishable between a vortex chamber and a duct bleed. As are the variations of loss, pressure recovery and reattachment with bleed rate (Figures 6.4 - 6.6). Furthermore, a more detailed examination of the mainstream flow field reveals no difference between the three diffusers. Therefore, it is fair to conclude that the differences in the three methods of bleed employed do not effect the mainstream diffuser flow. The fact 
that a vortex chamber is unnecessary was initially confirmed during the experimental investigation (Section 4.0) and is now further confirmed here. This contradicts much of the previous research, including the work of Adkins et al ${ }^{[1980]}$, which suggested that a vortex chamber was a necessary component of a hybrid diffuser. Reiterating what has already been stated, this is significant in engineering terms as the inclusion of a vortex chamber increases the complexity of a hybrid diffuser and imposes a size and weight penalty. Replacing the vortex chamber by a simple duct immediately removes this problem making the hybrid diffuser more attractive for an engineering application, particularly in the aero industry. Furthermore, a duct has the potential to be developed to reduce aerodynamic losses to the bleed air thus enabling it to be used elsewhere in an engine for component cooling.

Initially at zero bleed the duct-bled hybrid diffuser stalls but as the bleed rate is increased diffuser performance begins to improve. At a bleed rate above $1.5 \%$ the flow reattaches on the Stage 3 wall but at a reattachment length corresponding to over half the length of the Stage 3 diffuser. Further increases in bleed reduce the reattachment length as illustrated in Figure 6.6 and with a bleed rate of $4.0 \%$ reattachment is rapid $\left(\mathrm{Xr} / \mathrm{L}_{3} \sim 0.17\right)$.

Figures 6.4 and 6.5 demonstrate that the total pressure loss reduces and the static pressure recovery increases as the point of reattachment moves upstream (i.e. increasing bleed). With no bleed the total pressure loss coefficient is 0.14 but this reduces to 0.113 for $4.0 \%$ bleed. Correspondingly, the static pressure recovery coefficient increases from 0.649 to 0.793 .

To quantify this level of performance, it is important to recognise that the overall area ratio of 2.5 is very high and is coupled with a relatively short non-dimensional length of 3.5. Nominal conventional diffuser design curves (refer to Figure 3.11) predict that at this loading a conventional diffuser would stall; a maximum area ratio of 1.5 being more realistic. A solution generated for a conventional diffuser $\left(\mathrm{AR}=2.5, \mathrm{~L} / \mathrm{h}_{1}=3.5\right)$ does indeed, show that at this level of loading a conventional diffuser will stall (Figure 6.7).

Figure 6.8 shows a streamline plot for the duct-bled hybrid diffuser with a $2.5 \%$ bleed rate. This represents a bleed rate above the minimum requirement and the flow reattaches 
on the Stage 3 wall after some $25 \%$ of its length. As the flow enters the bleed duct it can be seen to separate from the upstream side of the duct but rapidly accelerate on the downstream side. Clearly, in the Stage 2 diffuser, close to the bleed, there is a large amount of flow deflection. A Coanda bubble is formed on the leeward side of the fence and this feature is seen to decrease in size but increase in rotational strength as the bleed rate is increased.

Figure 6.9 shows a streamline plot for $1.5 \%$ bleed which is just below the minimum bleed requirement. Again the bleed flow accelerates into the duct and the mainstream flow undergoes some deflection. However, there exists a large recirculation and although the flow does eventually reattach to the Stage 3 wall the reattachment length is long $\left(\mathrm{X}_{\mathrm{r}} /\right.$ $\left.\mathrm{L}_{3}>0.5\right)$.

With no bleed, a streamline plot (Figure 6.10) shows that the Stage 3 flow is dominated by a large separation and the diffuser has clearly stalled presenting a non-viable configuration.

At exit to the Stage 1 section, before any bleed flow has been removed, profiles of axial velocity $(\mathrm{u})$ and radial velocity $(\mathrm{v})$ indicate that the flow is already feeling a significant influence of the bleed. Figure 6.11 shows the axial velocity profile at Stage 1 exit (immediately upstream of the bleed) for $0.0,1.5$ and $2.5 \%$ bleed and reveals that as the bleed rate increases the peak velocity decreases, the condition of the boundary layer improves (i.e. reduces in thickness) and there is a migration of mass flow towards the wall.

Similarly, the magnitude of the peak radial velocity (Figure 6.12) increases with bleed. At zero bleed there is only minimal flow deflection and there exists only a small radial component of around $-2 \mathrm{~ms}^{-1}$. However at $2.5 \%$ bleed this radial component increases to $-8 \mathrm{~ms}^{-1}$ indicating the flow has a much greater turning potential even before reaching the bleed.

At exit to the Stage 2 section a similar pattern of behaviour is observed. Again the magnitude of the peak axial velocity (Figure 6.13) decreases with increasing bleed rate. This is accompanied by a flattening of the profile and a migration of mass flow towards the 
wall. Alternatively this can be viewed as a transfer of positive momentum towards the wall, hence improving the condition of the boundary layer as it forms on the Stage 3 wall. However, with no bleed the axial velocity near the wall is negative and the diffuser is stalled.

The radial velocity at exit to Stage 2 with $2.5 \%$ bleed (Figure 6.14 ) is also significantly higher, demonstrating a much greater tendency for the flow to turn and expand into the Stage 3 diffuser. The radial component of the velocity is some four times greater at $2.5 \%$ bleed than for zero bleed.

Across the bleed gap itself there is also a notable variation of the radial velocity component for the various bleed rates. With no bleed (Figure 6.15) there is a negligible radial velocity component but with increased bleed the radial velocity increases rapidly, indicating the bleed flow must accelerate more quickly into the bleed duct. The high bleed flow velocity results in a region of low static pressure, thus creating a strong pressure gradient.

The static pressure gradient, dp/dy (computed by Fluent), across the bleed gap is plotted for $0.0,1.5$ and $2.5 \%$ bleed in Figure 6.16. At lower levels of bleed the pressure gradient is close to zero but increases rapidly with bleed such that this gradient becomes a strong factor in the deflection of the mainstream flow.

\subsubsection{Alternative Bleed Geometries}

The duct-bled hybrid diffuser represents a simple two-dimensional simulation of the experimental Hybrid 4 diffuser. The five alternative bleed geometries (Alt.1 to 5, refer to Figures 5.6 - 5.10) comprise of the same overall area ratio (2.5) and non-dimensional length (3.5) but represent distinct and logical extensions to the simple ducted bleed (refer to Section 5.5.6). Solutions generated for each of these geometries yielded the overall diffuser performance parameters presented in Table 6.4. 


\begin{tabular}{|c||c|c|c|}
\hline Diffuser & $\mathrm{C}_{\mathrm{p} 2-4}$ & $\lambda_{2-4}$ & $\mathrm{Xr} / \mathrm{L}_{3}$ \\
\hline \hline Conventional & 0.746 & 0.123 & Stall @ 0.56 \\
\hline Alt. 1 & 0.794 & 0.111 & 0 \\
\hline Alt. 2 & Stall & Stall & Stalled \\
\hline Alt. 3 & 0.785 & 0.116 & Stall @ 0.72 \\
\hline Alt. 4 & 0.795 & 0.109 & 0 \\
\hline Alt. 5 & 0.797 & 0.109 & 0 \\
\hline
\end{tabular}

Table 6.4 Diffuser Performance - Alternative Bleed Hybrid Diffusers (2.5\% Bleed)

By removing the fence the results for Alt.1 this configuration poses the question of whether the fence and Coanda bubble are a necessary combination and to what extent do their presence contribute to the performance of the hybrid diffuser. Indeed, with a bleed rate of $2.5 \%$ these features are not required and the Alt.1 diffuser has a slightly higher overall performance than the duct-bled hybrid diffuser. A streamline plot (Figure 6.17) for Alt. 1 shows that the flow reattaches immediately to the Stage 3 wall and no Coanda bubble is formed. This demonstrates that the Coanda bubble is not an essential component of the flow mechanisms of a hybrid diffuser. Furthermore, it is the elimination of this feature and the loss associated with the recirculating flow which results in the slight increase in performance. However, removal of the fence necessitates a higher Stage 3 wall angle if the overall area ratio is to be maintained. Figure 6.18 is a plot of the axial velocity in the wall adjacent cell and illustrates that for Alt.1 the near wall flow is positive and the diffuser is not close to stall. However, to achieve a higher overall area ratio a further increase in wall angle would be necessary and this may result in a stall. For Alt.1 it is clear that a divergence angle of 14 degrees is acceptable and the fence/step is not necessary. However the fence/step does offer an instantaneous area ratio increase with the added benefit of reducing Stage 3 loading. Nonetheless, for an aeronautical application a feature such as a fence is undesirable as it is not practical in an engineering sense, being difficult and expensive to manufacture and likely to fail (e.g. cracking at sharp corners) due to the harsh environment in which it operates. In summary, Alt.1 is an attractive option as it significantly simplifies the overall complexity of the diffuser without 
incurring any performance penalty.

The Alt.2 diffuser represents the opposite of Alt.1; removal of the fence but maintaining the sudden step expansion. Figure 6.19 shows a streamline plot for a $2.5 \%$ bleed rate revealing that the flow cannot now overcome the large unconfined expansion. The stresses in the free shear layer are too great and the flow separates resulting in a badly stalled diffuser and jet flow. It is unlikely that this configuration presents a viable option at whatever level of bleed rate is employed.

Hybrid diffusers operate by a more complex mechanism than simply removing the energy deficient boundary layer flow. It is argued here that hybrid diffusers essentially operate due to an additional mechanism, viz a transfer of positive momentum from the accelerating bleed flow to the diffusing mainstream flow. However, little evidence has been presented in the literature (refer to Section 2.6) to demonstrate this fact. The Alt.3 geometry slices off $2.5 \%$ of the near wall flow via a fence running parallel to the diffuser wall and effectively represents a boundary layer bleed, eliminating the accelerating bleed aspect of the hybrid diffuser flow mechanisms. A streamline plot (Figure 6.20) and near wall axial velocity plot (Figure 6.18) show that the flow separates from a point $72 \%$ along the Stage 3 wall. This is an improvement over the conventional diffuser (Figures 6.7 and 6.18 ) which separates at $56 \%$ but the Alt.3 diffuser, nevertheless, has stalled. Thus a simple boundary layer bleed, although beneficial, does not produce the same flow structure as the duct-bled (or Alt.1) hybrid diffuser. The streamline plot indicates that there is no flow acceleration associated with the bleed, the bleed flow is simply "sliced off". If the bleed flow does not accelerate into the bleed duct then it does not gain momentum and cannot transfer this positive momentum to the mainstream flow. The Stage 3 boundary layer flow is then unable to overcome the expansion and strong adverse pressure gradient. In conclusion, this qualitatively demonstrates that hybrid diffusers do not function by simply removing the low energy boundary layer flow, but by a more complex interaction between the bleed and mainstream flows.

The Alt.4 diffuser geometry is similar to Alt.1 but with the upstream corner of the bleed gap profiled in an attempt to eliminate the separation bubble in the bleed duct and hence reduce bleed flow loses. Performance figures (Table 6.4) clearly show that the alteration to the bleed gap geometry has no effect on overall performance. This is further supported 
by a streamline plot for a $2.5 \%$ bleed rate as shown in Figure 6.21 . Profiling the bleed gap merely replaces the dividing streamline associated with the separation bubble with a solid surface and removes the high loss flow. The bleed flow total pressure loss is indeed reduced (this is discussed in Section 6.1.2) but the overall diffuser performance is unaffected.

Performance figures (Table 6.4) and streamlines (Figure 6.22) for the Alt.5 diffuser with a $2.5 \%$ bleed rate illustrate that angling the bleed duct to reduce bleed flow losses also does not alter the mainstream flow or the pertinent mechanisms. The improvement in bleed flow performance is similar to that gained for Alt.4 (again refer to Section 6.1.2). It has not been investigated here but it is probable that the gain in bleed flow quality will increase as the angle of the bleed duct becomes more acute. However, at some angle the configuration will approach the case represented by Alt. 3 which has been shown to result in flow separation.

Figures 6.23 - 6.28 show contours of static pressure, normalised by inlet conditions, for the Duct-bled diffuser and the five alternative bleed geometries for a bleed rate of $2.5 \%$. The duct-bled, Alt.1, 4 and 5 diffusers all show very similar variations in static pressure with a modest recovery of static pressure in the Stage 1 section, followed by a rapid increase across Stage 2 with high gradients centred on the downstream side of the bleed. Examination of the contours reveal that the majority of the static pressure recovery can be attributed to the Stage 2 diffuser. This is supported by the experimental observations which, as discussed in Section 4.0, indicated that around half of the static pressure recovery occurred within this region. Interestingly, however, it would appear that the sudden step expansion does not contribute to the rapid static pressure recovery. Contour variations are almost identical regardless of whether a step expansion exists (duct-bled diffuser) or not (Alt.1). The highest gradients emanate from the downstream tip of the bleed gap and would appear to be a direct result of the interaction of the bleed flowwith the diffusing mainstream flow and not the sudden increase in area ratio.

Although not fully attached, Alt.3 (Figure 6.26) exhibits only a small region of separated flow and as such achieves a modest static pressure recovery. However, the influence of the bleed is marginal and the static pressure contours show that the recovery in the Stage 
2 diffuser is not rapid and compares poorly with the fully attached hybrid diffusers (Alt.1, 4 and 5).

The development of the mass weighted static pressure recovery coefficient $\left(\mathrm{C}_{\mathrm{p}}\right)$ along the entire length of the diffuser is shown in Figure 6.29. Initially the recovery is more or less identical for all the diffusers, as expected, since they all have identical Stage 1 geometry. However, for the functioning hybrid diffusers (duct-bled, Alt.1, 4, and 5) there is a rapid increase in $C_{p}$ at $x / L=0.35-0.4$ which corresponds to the location of the bleed. The remaining diffusers, which all contain some degree of flow separation, do not exhibit this phenomenon. The magnitude of recovery within the Stage 3 section is similar for all the diffusers with the gradient reducing as the boundary layer thickens under the action of the adverse pressure gradient. However, the final values of $\mathrm{C}_{\mathrm{p}}$ differ by a significant amount which can be attributed to the rapid increase in static pressure over the Stage 2 section.

The rapid recovery associated with the Stage 2 / bleed section is further illustrated in a plot of the axial gradient of the pressure coefficient against diffuser length (Figure 6.30). A high peak exists in the Stage 2 diffuser for the functioning hybrid diffusers (duct-bled, Alt.1, 4 and 5) but not for the remaining diffusers (Alt.2 and 3) which are non-functional and cannot truly be called hybrid diffusers.

\subsubsection{Bleed Flow Assessment}

The effect of bleed rate on diffuser performance has been discussed in Section 4.3 for the experimental data and in Section 6.1.1 for the two-dimensional computational model of the Hybrid 4 (duct-bled) diffuser. However, it is important to assess the condition of the bleed flow as current aero gas turbine engine cycles requires all the available air to be utilised. Air to be used for turbine blade cooling is usually bled off from within the combustion chamber and it is therefore logical (according to Adkins et al ${ }^{[1980]}$ ) that air bled from a hybrid diffuser could be used in place of this air.

The mass weighted total pressure loss coefficient from rotor exit to the bleed boundary condition is defined as: 


$$
\lambda_{2-\mathrm{B}}=\frac{\tilde{\mathrm{P}}_{2}-\tilde{\mathrm{P}}_{\mathrm{B}}}{\tilde{\mathrm{q}}_{2}}
$$

for station ' $\mathrm{B}$ ' mass-weighted pressure are evaluated across the exit of the bleed duct.

Although no definitive target is available for this parameter it is desirable that the loss is minimised.

\begin{tabular}{|c||c|c|c|}
\hline \multirow{2}{*}{\multicolumn{1}{|c||}{ Bleed }} & \multicolumn{3}{|c|}{$\lambda_{2-\mathrm{B}}=\frac{\tilde{\mathrm{P}}_{2}-\tilde{\mathrm{P}}_{\mathrm{B}}}{\tilde{\mathrm{q}}_{2}}$} \\
\cline { 2 - 4 } & Vortex Chamber & Ext. Duct & Duct \\
\hline \hline $\mathbf{1 . 0}$ & 0.495 & 0.494 & 0.467 \\
\hline $\mathbf{1 . 5}$ & 0.537 & 0.509 & 0.483 \\
\hline $\mathbf{2 . 0}$ & 0.541 & 0.524 & 0.489 \\
\hline $\mathbf{2 . 5}$ & 0.552 & 0.538 & 0.494 \\
\hline $\mathbf{3 . 0}$ & 0.572 & 0.552 & 0.499 \\
\hline $\mathbf{4 . 0}$ & 0.614 & 0.582 & 0.511 \\
\hline
\end{tabular}

Table 6.5 Bleed Flow Mass Weighted Total Pressure Loss

Table 6.5 details the total pressure loss for various bleed levels through the vortex chamber, extended duct-bled and duct-bled hybrid diffusers. The overall diffuser performance for these three configurations was identical, however, it is clear that the loss increases significantly as the bleed rate increases. Furthermore, the simple duct incurs a lower bleed flow loss than the extended duct and the vortex chamber at any given bleed rate. At $2.5 \%$ bleed the vortex chamber loss amounts to $53 \%$ of the rotor exit dynamic pressure and the extended duct loss is $54 \%$. Yet with a simple duct the bleed flow incurs a reduced loss amounting to $49 \%$ of rotor exit dynamic pressure.

The reason for this behaviour is not complex. In a vortex chamber the vortex will expand to fill the entire chamber. This process involves high loss mechanisms and will ultimately lead to a high total pressure loss for the bleed flow. A similar argument can be put forward for the extended duct as a vortex will form and expand to fill the available space. 
However, for a simple duct the separation bubble is much smaller and as a result the associated loss is reduced.

A bleed flow total pressure loss equivalent to $50 \%$ of the rotor exit dynamic pressure is a significant value. The Alt. 4 and 5 geometries represent two simple attempts to reduce this loss. Both aspire to do this by reducing the turning loss and removing the separation bubble by way of (1) profiling the bleed gap and, (2) angling the bleed duct.

\begin{tabular}{|c||c|}
\hline Hybrid Diffuser & $\lambda_{2-\mathrm{B}}=\frac{\tilde{\mathrm{P}}_{2}-\tilde{\mathrm{P}}_{\mathrm{B}}}{\tilde{\mathrm{q}}_{2}}$ \\
\hline \hline Duct Bleed & 0.494 \\
\hline Alt.1 & 0.456 \\
\hline Alt.4 & 0.384 \\
\hline Alt.5 & 0.387 \\
\hline
\end{tabular}

Table 6.6 Bleed Flow Mass Weighted Total Pressure Loss (2.5\% Bleed)

Table 6.6 details the bleed loss total pressure losses at $2.5 \%$ bleed for the duct-bled, Alt.1, 4 and 5 hybrid diffusers. The loss for the simple duct bled diffuser is greatest at $49.4 \%$ of rotor exit dynamic and is slightly reduced for Alt. 1 to $45.6 \%$. Thus, removal of the fence and Coanda bubble has a small effect on the bleed flow loss. However, Alt.4 and 5 achieve their aim of reducing the loss by around one fifth from a simple duct. Streamline plots (Figures 6.21 and 6.22) show that the separation bubble in the bleed duct has been eliminated hence the quality of the bleed flow increases making it more attractive for use in component cooling.

\subsubsection{Flow Mechanisms - Momentum Transfer Analysis}

It is clearly evident that the presence of the bleed has a strong influence on the performance of hybrid diffusers. It can been seen from streamline plots, velocity contours/profiles and mass-weighted pressure coefficients that without bleed a hybrid diffuser will stall. However, with increasing bleed the flow will reattach and the diffuser performance will improve. In a similar manner it has also been qualitatively demonstrated that the 
bleed mechanism is not just a simple boundary layer bleed, which merely removes energy deficient flow. When the same amount of mass flow was removed from the boundary layer using a simple flow-aligned slot, the pertinent flow mechanisms were not present and the diffuser stalled. In order to undertake a more quantitative examination of the flow mechanisms a momentum transfer analysis has been undertaken. Close inspection of the terms within the momentum equation can reveal where and how positive streamwise momentum is transported to the low energy flow, enabling it to overcome the high adverse pressure gradients and thus prevent separation.

To conduct this analysis only one geometry was considered. As discussed earlier, apart from two specific cases, the mainstream flow remains unchanged for the various hybrid bleed step geometries tested. These two cases were Alt. 2 and 3, both of which stall at relatively high bleed rates. However, of all the geometries studied, Alt. 4 presents the most simple case as the profiled bleed and removal of the large step expansion eliminate much of the complex flow associated with separation on the leeward side of the bleed duct and the Coanda bubble in the corner of the step. Such features contain extremely high gradients and as such have a tendency to overwhelm the data of interest. Hence the Alt. 4 geometry was selected for this detailed study. Furthermore, only three bleed rates were employed; $2.5 \%, 1.0 \%$ and $0.0 \%$ of the inlet mass flow. These represent, respectively, a rate above the minimum bleed requirement, an intermediate level of bleed just below the minimum required and no bleed.

As discussed in Section 6.1.1, overall diffuser performance improves with increasing bleed rate. This can be clearly seen in Table 6.7 which details the mass-weighted performance parameters as defined in Sections 2.2 and 6.1.1. Streamline plots for the three bleed rates are given in Figures $6.31-6.33$ and show that for 0.0 and 1.0\% bleed the flow separates from the Stage 3 wall. With no bleed the flow only remains attached for the first $40 \%$ of the Stage 3 wall. This increases to $90 \%$ for $1.0 \%$ bleed; only with $2.5 \%$ bleed does the diffuser remain fully attached with no evidence of stall. Profiles of axial velocity (Figure 6.34) at diffuser exit also reveal evidence of flow separation; the condition of the wall flow is clearly much improved for $2.5 \%$ bleed resulting in a much more uniform profile. 


\begin{tabular}{|l|l|l|}
\hline $\begin{array}{c}\text { Bleed } \\
\left(\% \mathrm{~m}_{\text {tot }}\right)\end{array}$ & \multicolumn{1}{|c|}{$\mathrm{C}_{\mathrm{p} 2-4}$} & \multicolumn{1}{|c|}{$\lambda_{2-4}$} \\
\hline \hline 0.0 & 0.738 & 0.122 \\
\hline 1.0 & 0.770 & 0.116 \\
\hline 2.5 & 0.795 & 0.111 \\
\hline
\end{tabular}

Table 6.7 Mass Weighted Diffuser Performance Figures - Alt. 4 Geometry

\subsubsection{The Momentum Equation}

The momentum equations are defined, derived and well-documented in several texts, such as Fletcher ${ }^{[1988]}$, in Cartesian or cylindrical polar co-ordinates. However, due to the diverging nature of a diffuser there will always exist a radial component of velocity and an associated momentum transfer. Therefore, for this analysis, it is useful to transform the momentum equations into a co-ordinate system defined using the inviscid streamlines. The reason being that if the flow development followed the inviscid behaviour exactly there would be zero velocity component or convective momentum transport normal to the local inviscid streamline. If the real viscous solution is transformed and analysed with respect to the inviscid streamline co-ordinate system the transverse component of momentum transport associated with viscous effects, such as boundary layer growth, is likely to be small. Hence, the viscous momentum transport due to the bleed flow will then be highlighted and can therefore be studied. Fluent was used to solve for an inviscid flow (as described in Fluent ${ }^{[1998]}$ ) and provide the co-ordinate system based on the inviscid streamlines. An example streamline plot of an inviscid solution obtained from Fluent for the Alt. 4 diffuser with 2.5\% bleed is shown in Figure 6.35.

With reference to Figure 6.36 the mean streamwise momentum equation (for incompressible, high Reynolds number, steady flow) applied along the inviscid streamlines can be written locally as:

$$
\mathrm{U}_{\mathrm{s}} \frac{\partial \mathrm{U}_{\mathrm{s}}}{\partial \mathrm{s}}+\mathrm{V}_{\mathrm{s}} \frac{\partial \mathrm{U}_{\mathrm{s}}}{\partial \mathrm{n}}=-\frac{1}{\rho} \frac{\partial \mathrm{p}}{\partial \mathrm{s}}-\left(\frac{\partial}{\partial \mathrm{s}} \overline{\mathrm{uu}}+\frac{\partial}{\partial \mathrm{n}} \overline{\mathrm{uv}}\right)
$$


Equation 6.5 assumes that locally the inviscid streamline-based co-ordinates $(\mathrm{s}, \mathrm{n})$ may be considered as a pair of orthogonal straight lines rotated by an angle $\beta$ to the Cartesian coordinate direction.

In Equation $6.5 \mathrm{U}_{\mathrm{s}}$ is aligned with the inviscid streamline, $\mathrm{V}_{\mathrm{s}}$ is normal to the inviscid streamline and $\overline{\mathrm{uu}}$, and $\overline{\mathrm{uv}}$ are now to be considered as the Reynolds stresses in the s-n co-ordinate system. The terms on the LHS of the equation are associated with the acceleration experienced by a fluid element, whereas the terms on the RHS represent the forces applied to the element of fluid. These forces arise due to the pressure gradient and/ or gradients in the Reynolds stresses. The flow path of an elemental fluid particle in the diffuser is therefore defined by the applied pressure and turbulent forces. At a given location the streamwise acceleration, $\mathrm{U}_{\mathrm{s}} \partial \mathrm{U}_{\mathrm{s}} / \partial \mathrm{s}$, in addition to depending on the applied forces, is also a function of the velocity component and streamwise velocity gradient normal to the direction being considered, $\mathrm{V}_{\mathrm{s}} \partial \mathrm{U}_{\mathrm{s}} / \partial \mathrm{n}$. This term represents the effects introduced due to the transverse convection of streamwise momentum by the normal velocity component $\left(\mathrm{V}_{\mathrm{s}}\right)$.

The various terms in Equation 6.5 are not immediately available and must be computed from the inviscid and turbulent solutions as predicted by Fluent. It now becomes necessary to express the cartesian velocity components of the turbulent soluyion with respect to the inviscid streamlines which, at any given point, subtend an angle $\beta$ with the axial, $x$, direction (refer to Figure 6.36). The computed flow field can then be resolved locally in the direction of the inviscid streamlines using the following transformation:

$$
\left(\begin{array}{c}
\mathrm{U}_{\mathrm{s}} \\
\mathrm{V}_{\mathrm{s}}
\end{array}\right)=\left(\begin{array}{cc}
\cos \beta & \sin \beta \\
-\sin \beta & \cos \beta
\end{array}\right) \cdot\left(\begin{array}{l}
\mathrm{u} \\
\mathrm{v}
\end{array}\right)
$$

where $\mathrm{u}$ and $\mathrm{v}$ represent the predicted, time-averaged Cartesian velocity components, and $\beta$ is obtained from the inviscid flow field such that:

$$
\beta=\operatorname{atan}\left(\frac{\mathrm{v}}{\mathrm{u}}\right)_{\text {inviscid solution }}
$$

A typical plot of $\beta$ is shown in Figure 6.37. Now, to obtain $V_{s} \partial U_{s} / \partial n$, for example, in 
terms of $\mathrm{x}, \mathrm{y}, \mathrm{u}$ and $\mathrm{v}$ the following steps are used:

Firstly, from Equation 6.6:

$$
\mathrm{V}_{\mathrm{s}}=-\mathrm{u} \sin \beta+\mathrm{v} \cos \beta
$$

Secondly, $\partial \mathrm{U}_{\mathrm{s}} / \partial \mathrm{n}$ can be re-written:

$$
\frac{\partial \mathrm{U}_{\mathrm{s}}}{\partial \mathrm{n}}=\frac{\partial \mathrm{U}_{\mathrm{s}}}{\partial \mathrm{x}} \cdot \frac{\partial \mathrm{x}}{\partial \mathrm{n}}+\frac{\partial \mathrm{U}_{\mathrm{s}}}{\partial \mathrm{y}} \cdot \frac{\partial \mathrm{y}}{\partial \mathrm{n}}
$$

or

$$
\frac{\partial \mathrm{U}_{\mathrm{s}}}{\partial \mathrm{n}}=\frac{\partial \mathrm{U}_{\mathrm{s}}}{\partial \mathrm{x}} \cdot-\sin \beta+\frac{\partial \mathrm{U}_{\mathrm{s}}}{\partial \mathrm{y}} \cdot \cos \beta
$$

Also, again from Equation 6.6,

$$
\mathrm{U}_{\mathrm{s}}=\mathrm{u} \cos \beta+\mathrm{v} \sin \beta
$$

therefore,

$$
\frac{\partial U_{s}}{\partial x}=\cos \beta\left(\frac{\partial u}{\partial x}+v \frac{\partial \beta}{\partial x}\right)+\sin \beta\left(\frac{\partial v}{\partial x}+u \frac{\partial \beta}{\partial x}\right)
$$

where,

$$
\frac{\partial \beta}{\partial x} \approx\left(\frac{u \frac{\partial v}{\partial x}-v \frac{\partial u}{\partial x}}{u^{2}+v^{2}}\right)_{\text {inviscid solution }}
$$

and similarly for $\partial \mathrm{U}_{\mathrm{s}} / \partial \mathrm{y}$.

$\mathrm{V}_{\mathrm{s}} \partial \mathrm{U}_{\mathrm{s}} / \partial \mathrm{n}$ can now be evaluated at any point in terms of the Cartesian components. $\mathrm{U}_{\mathrm{s}} \partial \mathrm{U}_{\mathrm{s}} / \partial \mathrm{s}$ can be found using a similar method and the pressure gradient term can be easily computed from:

$$
\frac{1}{\rho} \frac{\partial \mathrm{p}}{\partial \mathrm{s}}=\frac{1}{\rho} \frac{\partial \mathrm{p}}{\partial \mathrm{x}}+\frac{1}{\rho} \frac{\partial \mathrm{p}}{\partial \mathrm{y}}
$$

Although the individual Reynolds stresses can be computed using the Boussinesq relationship it was not considered necessary for the purposes of the present analysis to transform the Reynolds stresses in into the components in the $\mathrm{s}$ and $\mathrm{n}$ directions. Thus the final term in Equation 6.5 has not been separated into its individual components but is 
included in what follows as the 'closing term' in the momentum balance.

Returning to Equation 6.5 it is now possible to discuss the individual terms, their influence and contribution to streamwise momentum and how they are affected by the bleed. There are two classical methods of analysing the system; a Eulerian control volume technique or a Lagrangian approach which looks at the path of an elemental fluid particle. In a Eulerian approach Equation 6.15 represents a statement on the processes which potentially cause a change of the s-momentum content of an infinitesimal control volume surrounding any point. Since the flow is steady-state (time-invariant), then the change at any point is in fact zero, i.e. there is a balance between the various processes.

$$
0=\mathrm{U}_{\mathrm{s}} \frac{\partial \mathrm{U}_{\mathrm{s}}}{\partial \mathrm{s}}+\mathrm{V}_{\mathrm{s}} \frac{\partial \mathrm{U}_{\mathrm{s}}}{\partial \mathrm{n}}+\frac{1}{\rho} \frac{\partial \mathrm{p}}{\partial \mathrm{s}}+\left(\frac{\partial}{\partial \mathrm{s}} \overline{\mathrm{uu}}+\frac{\partial}{\partial \mathrm{n}} \overline{\mathrm{uv}}\right)
$$

A Eulerian method is useful to analyse how the control volume will gain or lose streamwise momentum due to each term. However, a Lagrangian approach is more useful when analysing the flow in a hybrid diffuser to demonstrate the effect on an elemental fluid particle as it passes through the control volume. This will highlight how the various processes contribute to the governing flow mechanisms of a hybrid diffuser.

For example, consider the effect of the static pressure gradient. A favourable streamwise pressure gradient (negative $\partial \mathrm{p} / \partial \mathrm{s}$ ) will, acting in isolation, give rise to a positive force in the s direction acting on the contents of the control volume. This may be interpreted as a positive source of streamwise momentum, tending to increase the s-momentum of the control volume (i.e. a gain process). To maintain the balance in Equation 6.15 the term $\mathrm{U}_{\mathrm{s}} \partial \mathrm{U}_{\mathrm{s}} / \partial \mathrm{s}$ must then be positive as the streamwise velocity increases tending to decrease the s-momentum of the control volume (i.e. a loss process). Alternatively, in a Lagrangian approach a fluid element passing through the control volume will gain s-momentum and accelerate due to the favourable pressure gradient.

Similarly, in a Eulerian approach, a negative value of $\mathrm{V}_{\mathrm{s}} \partial \mathrm{U}_{\mathrm{s}} / \partial \mathrm{n}$ can be interpreted as a gain process tending to increase the s-momentum of the control volume which will, in a Lagrangian approach, cause a fluid element passing through the control volume to gain 
positive streamwise momentum.

Secondly, consider a free shear layer such as the edge of a jet as illustrated in Figure 6.38. The figure shows the shape of the $U$ and V profiles in these flows. Physically, at the jet edge transverse convective transport leads to s-momentum gain as the higher momentum jet core region spreads into the surrounding lower velocity field. The term $\mathrm{V}_{\mathrm{s}} \partial \mathrm{U}_{\mathrm{s}} / \partial \mathrm{n}$ is negative illustrating that in a Eulerian approach a control volume will gain s-momentum due to this term. Thus in a Lagrangian approach a fluid particle passing through the control volume will also gain s-momentum (i.e. the term $U_{s} \partial U_{s} / \partial s$ is positive as the control volume will lose s-momentum to the this term).

In summary, with reference to Figures $6.40-6.63$ the terms in Equation 6.15 have the following characteristics:

\section{- $\mathbf{U}_{\mathbf{s}} \partial \mathbf{U}_{\mathbf{s}} / \partial \mathbf{s}$}

Dimensionally equivalent to an acceleration, $\mathrm{U}_{\mathrm{s}} \partial \mathrm{U}_{\mathrm{s}} / \partial \mathrm{s}$ can be considered as describing the acceleration (or deceleration) of the streamwise velocity component of the fluid in the direction of the inviscid streamlines. Within a diffusing flow this term will be positive (the flow is losing momentum) provided the flow is attached. The velocity in the direction of an inviscid streamline will be positive but reducing in magnitude $\left(\partial \mathrm{U}_{\mathrm{s}} / \partial \mathrm{s}\right.$ negative) due to the diffusing nature of the flow and the increasing static pressure.

\section{- $\mathbf{V}_{\mathbf{s}} \partial \mathbf{U}_{\mathrm{s}} / \partial \mathbf{n}$}

This term physically represents the transport of streamwise momentum across or in a direction normal to the inviscid streamlines by the normal velocity component $V_{\mathrm{s}}$. A negative value indicates a control volume will gain positive streamwise momentum in a transverse direction and this will therefore increase the s-momentum of a fluid particle.

\section{- $1 / \rho . \partial p / \partial s$}

This term represents the creation/destruction of s-momentum due to the pressure force 
exerted on a control volume. An increasing pressure produces an adverse pressure gradient and gives rise to a positive value of $1 / \mathrm{p} . \partial \mathrm{p} / \partial \mathrm{s}$ causing a reduction in streamwise velocity. In a diffuser the presence of an adverse pressure gradient acts to retard the axial velocity and causes rapid growth of the boundary layer which may lead to flow separation. Thus the transport of momentum associated with this term is an important feature in the flow mechanisms of a hybrid diffuser.

- $\left(\frac{\partial}{\partial \mathrm{s}} \bar{u} \mathrm{u}+\frac{\partial}{\partial \mathrm{n}} \overline{\mathrm{uv}}\right)$

This term physically represents gradients of the turbulent stresses acting on the control volume faces. The turbulent stresses may be interpreted as transporting streamwise momentum due to turbulent velocity fluctuations.

\subsubsection{Flow Field Discussion (2.5\% Bleed)}

With reference to Figure 6.39, at a bleed rate of $2.5 \%$ there are five distinct flow regions within the Alt. 4 hybrid diffuser. Thus, for ease of presentation, the flow field for each of these regions will be discussed separately. Region 1 corresponds to the inlet of the Stage 1 diffuser. Regions 2 and 3 are located immediately upstream and downstream of the bleed and hence describe the manner in which the bleed flow contributes to the mechanism of a hybrid diffuser. Finally, Region 4 represents the flow within the Stage 3 diffuser and Region 5 corresponds to overall diffuser exit.

\section{- Region 1}

Contour plots of the four terms in the momentum equation are presented, for Region 1, in Figures $6.40-6.43$, with the corresponding streamline plot in Figure 6.44. As the flow negotiates the constant area inlet duct the boundary layer develops normally. However, approaching the diffuser the flow begins to feel the influence of the downstream (increasing) pressure field with the result that the mainstream flow begins to slow and diverge. Upstream of the convex corner this gives rise to a negative $V_{\mathrm{s}}$ and $\mathrm{V}_{\mathrm{s}} \partial \mathrm{U}_{\mathrm{s}} / \partial \mathrm{n}$ as higher momentum fluid is transported into the boundary layer. Immediately upstream of the corner the turbulent solution has increasing velocity in the direction of the inviscid 
streamline which can be seen as a positive value of $\mathrm{U}_{\mathrm{s}} \partial \mathrm{U}_{\mathrm{s}} / \partial \mathrm{s}$. Immediately downstream of the corner the diverging passage gives rise to a strong adverse streamwise pressure gradient and a rapid retardation of the streamwise velocity $\left(\mathrm{U}_{\mathrm{s}}\right)$. Typically, as the flow diffuses $U_{s} \partial U_{s} / \partial s$ is negative as $U_{s}$ reduces and the local static pressure increases. Furthermore, as the boundary layer develops along the diffuser wall it begins to thicken under the action of the adverse pressure gradient.

\section{- Regions 2 and 3}

Figures $6.45-6.48$ show contour plots of the terms in the momentum equation as the flow enters the bleed, with streamlines shown in Figure 6.49. Significantly, as the flow approaches the bleed there is a notable upstream effect which improves the condition of the boundary layer on the Stage 1 diffuser. Positive regions of $U_{s} \partial U_{s} / \partial s$ (Figure 6.45) show firstly that the near wall flow accelerates towards the bleed and secondly that there is a strong acceleration of the flow as it enters the bleed duct. Negative contours of $\mathrm{V}_{\mathrm{s}} \partial \mathrm{U}_{\mathrm{s}} / \partial \mathrm{n}$ (Figure 6.46) demonstrate that positive s momentum is being transported from a normal direction into the near wall region. Additionally, contours of $-1 / \rho . \partial \mathrm{p} / \partial \mathrm{s}$ (Figure 6.47) show that there is a favourable streamwise pressure gradient set up by the bleed which is driving these effects.

As the flow negotiates the corner and flows into the bleed duct the pressure gradient then becomes strongly adverse and the boundary layer rapidly grows. However, the augmented condition of the boundary layer enables it to overcome this increased loading and prevents the flow from separating.

The rapidly increasing area within the main part of the diffuser coupled with the pressure gradient created by the bleed generates a strong adverse streamwise pressure gradient (high negative values of $-1 / \rho . \partial \mathrm{p} / \partial \mathrm{s}$ ). The velocity field responds to this pressure force and negative values of $U_{s} \partial U_{s} / \partial s$ indicate high levels of diffusion as the streamwise velocity component reduces.

The streamlines in Figure 6.49 show that the pressure gradient created by the bleed causes entrapment and deflection of the mainstream flow and also drives an acceleration 
of bleed flow (seen in highly positive values of $U_{s} \partial U_{s} / \partial s$ in the mouth of the bleed duct). Thus the bleed flow gains a significant amount of streamwise momentum

There exists a dividing streamline, as shown in Figure 6.49. On the LHS of (or below) this streamline the bleed flow turns and rapidly accelerates into the bleed duct. However, on the RHS of (or above) the dividing streamline the flow expands into the Stage 3 diffuser. Either side of this streamline contours of $-\left(\frac{\partial}{\partial \mathrm{s}} \bar{u}+\frac{\partial}{\partial \mathrm{n}} \overline{\mathrm{uv}}\right)$ (Figure 6.48) show that the fluid is subject to high turbulent forces of opposite sign. The result of this is a layer containing a high amount of shear between the accelerating bleed flow and diffusing mainstream flow.

Contours of $\mathrm{V}_{\mathrm{s}} \partial \mathrm{U}_{\mathrm{s}} / \partial \mathrm{n}$ (Figure 6.46) reveal that there is a high transverse transport of streamwise momentum from the accelerating bleed flow to the diffusing mainstream flow. This can be seen by the negative region of $\mathrm{V}_{\mathrm{s}} \partial \mathrm{U}_{\mathrm{s}} / \partial \mathrm{n}$ at the inlet to the Stage 3 diffuser which indicates this flow is gaining streamwise momentum from the bleed flow (i.e. from the positive region of $V_{s} \partial U_{s} / \partial n$ in the mouth of the bleed) which is high in streamwise momentum.

This is the important governing mechanism which allows a hybrid diffuser to function. The bleed flow accelerates into the bleed gaining positive streamwise momentum. A layer of high shear is setup between the accelerating bleed flow and diffusing mainstream flow. A transfer of positive streamwise momentum across this layer from the bleed flow enables the flow which forms the fresh boundary layer on the Stage 3 wall to remain attached and overcome the high adverse pressure gradient within the Stage 3 diffuser.

\section{- Region 4}

The flow in Region 4 (Figures 6.50 - 6.54) behaves typically as one would expect for a modest area ratio conventional diffuser even though at about 14 degrees the wall angle is much higher than normally acceptable. However, it is the augmented condition of the flow forming the boundary layer on the Stage 3 wall which enables it to remain attached to this high angle wall when under normal circumstances it would separate. 


\section{- Region 5}

Initially within Region 5 the static pressure continues to rise (Figure 6.58) and the axial velocity reduces (Figure 6.56) as the flow continues to diffuse. However, in the constant area duct the streamwise pressure gradient and $-1 / \rho . \partial \mathrm{p} / \partial \mathrm{s}$ (Figure 6.58) tend to zero. Coupled with this the streamwise velocity component approaches a constant value (i.e. $\mathrm{U}_{\mathrm{s}}$ becomes constant and $\partial \mathrm{U}_{\mathrm{s}} / \partial \mathrm{n}$ tends to zero) as the flow settles and becomes more uniform.

\subsubsection{Flow Field Discussion (Zero Bleed)}

In order to asses what effect the bleed has on the various terms of the momentum equation it is useful to examine the case with zero bleed. A streamline plot (Figure 6.31) demonstrated that without bleed the Alt. 4 hybrid diffuser stalls with the flow separating from the Stage 3 wall. Contour plots for the various terms of Equation 6.15 without bleed are presented in Figures 6.60 - 6.63.

\section{- Region 1}

Region 1 is too far upstream of the bleed to feel any significant effect thus the structure of the flow remains essentially unchanged for all bleed rates.

\section{- Regions 2 and 3}

Even without bleed the flow in Region 2 feels the influence of the bleed duct. However without bleed the effect of this is minimal. A small amount of flow enters the bleed duct giving rise to similar phenomenon seen with bleed. For example, there exists a small region immediately upstream of the bleed duct where the pressure gradient is favourable and the fluid accelerates slightly into the duct. However, the magnitude of this is greatly reduced from the case with $2.5 \%$ bleed and provides only a small benefit.

Within the main part of the diffuser, contours of $-1 / \rho . \partial \mathrm{p} / \partial \mathrm{s}$ (Figure 6.62) and $\mathrm{U}_{\mathrm{s}} \partial \mathrm{U}_{\mathrm{s}} / \partial \mathrm{s}$ (Figure 6.60) show that the adverse pressure gradient is not as strong as with bleed and the flow does not decelerate/diffuse to the same extent as when bleed is present. Data 
have shown that within a hybrid diffuser the majority of the static pressure recovery can be attributed to the Stage 2 section and it is clear that with zero bleed the level of recovery here is greatly reduced.

Contours for Region 3 demonstrate that without bleed the pertinent flow mechanisms are not present. Thus the flow forming the boundary layer is much lower in streamwise momentum than at a bleed rate of $2.5 \%$ and as such it cannot overcome the adverse pressure gradient within the Stage 3 diffuser and ultimately separates. Contours of $-1 / \rho . \partial \mathrm{p} / \partial \mathrm{s}$ show that the pressure gradient rapidly becomes strongly adverse and contours of $\mathrm{V}_{\mathrm{s}} \partial \mathrm{U}_{\mathrm{s}} /$ $\partial \mathrm{n}$ indicate that there is only a very small transfer of positive streamwise momentum to the flow forming the boundary layer.

\section{- Region 4}

Essentially the flow in Region 4 reflects the fact that the diffuser stalls. The adverse pressure gradient is strong resulting in rapid boundary layer growth. Furthermore, the flow forming the boundary layer is already low in streamwise momentum resulting in the fact that it cannot overcome this adverse pressure gradient and separates. 


\subsection{Two-Dimensional, Axi-Symmetric Hybrid Diffuser with Flame Tube}

The computational model of a two-dimensional, axi-symmetric hybrid diffuser with a flame tube is not only a logical extension of the planar work (Section 6.1) but is also a direct simulation of the modified experimental facility described in Section 3.6. In summary this geometry is a simplified two-dimensional, axi-symmetric representation of a radially staged combustion system incorporating the Hybrid 4 (Duct-bled) diffuser. The compressor stage was removed resulting in a "clean" inlet and the flame tube was somewhat simplified in addition to removing the burners (refer to Figure 3.16 for the experimental configuration and Figure 5.13 for the computational configuration).

The computational geometry, mesh, boundary conditions and solution techniques have been discussed in Section 5. The planar work (Section 6.1) employed an inlet turbulence intensity of 5\% which is typical of the average levels seen at OGV exit on the experimental facility when the single stage axial compressor is in place. However, with the compressor removed, as in the two-dimensional, axi-symmetric experimental configuration, inlet turbulence intensity would be much lower, typically $0.5 \sim 1.0 \%$. The results for this experimental configuration have been discussed in Section 4.4.1 and, in summary, it was found that this configuration was unstable and no matter what bleed rates or flow splits were employed the hybrid diffuser stalled.

A streamline plot (Figure 6.64) for a converged solution with an inlet turbulence intensity of $0.5 \%$ and bleed rates of $2.5 \%$ shows similar behaviour to the experimental case. The predicted flow clearly separates from the outer Stage 3 wall. (A stall occurs on the outer wall first due to radius effects giving rise to slightly higher levels of diffusion on the outer wall).

Several more solutions were generated with increasing inlet turbulence levels up to $5 \%$ (i.e. to a level equivalent to downstream of an axial compressor). Figure 6.65 shows a streamline plot for a converged solution with an inlet turbulence intensity of $5.0 \%$ and bleed rates of $2.5 \%$. The streamlines now show that the diffuser flow is attached and the system is stable. 
Authors such as Stevens and Williams ${ }^{[1980]}$ have shown that increased inlet turbulence intensity levels can be beneficial to diffuser performance (refer to Section 2.3.2). Also, Isomoto and Honami ${ }^{[1989]}$ showed that for a backward facing step the reattachment length has a strong negative correlation with the inlet turbulence intensity. Here, this effect is more pronounced and the hybrid diffuser is only viable with inlet turbulence above a certain level. To illustrate this Figure 6.66 shows axial velocity profiles at diffuser exit for solutions generated with various levels of inlet turbulence intensity. At intensities below $3.0 \%$ the diffuser stalls from the outer wall, however, above $3.0 \%$ the flow reattaches on the Stage 3 diffuser wall and the steady state solution is now stable.

Details of static pressure recovery and total pressure loss coefficients are given in Table 6.8 for varying levels of inlet turbulence intensity. Diffuser performance is seen to increase with inlet turbulence intensity. For example with an inlet turbulence intensity of $0.5 \%$ (i.e. similar to that in the "clean" experimental configuration) the diffuser is stalled and has a relatively low static pressure recovery coefficient. However, with an inlet turbulence intensity above $3.0 \%$, which is more typical of the conditions downstream of a compressor, the static pressure recovery coefficient rises to almost 0.8 .

\begin{tabular}{|c||c|c|c|c|c|c|}
\hline \multirow{2}{*}{$\begin{array}{c}\text { Inlet Turbulence, } \\
\mathbf{T}_{\mathbf{i}}(\mathbf{\%})\end{array}$} & \multicolumn{2}{c|}{ Diffuser Exit } & \multicolumn{2}{c|}{ Inner Annulus } & \multicolumn{2}{c|}{ Outer Annulus } \\
\cline { 2 - 7 } & $\mathbf{C}_{\mathbf{p 2 - 4}}$ & $\boldsymbol{\lambda}_{\mathbf{2 - 4}}$ & $\mathbf{C}_{\mathbf{p 2 - 5}}$ & $\boldsymbol{\lambda}_{\mathbf{2 - 5}}$ & $\mathbf{C}_{\mathbf{p 2 - 7}}$ & $\boldsymbol{\lambda}_{\mathbf{2 - 7}}$ \\
\hline \hline $\mathbf{0 . 5}$ & 0.744 & 0.122 & 0.628 & 0.201 & 0.721 & 0.246 \\
\hline $\mathbf{1 . 0}$ & 0.750 & 0.127 & 0.617 & 0.210 & 0.722 & 0.241 \\
\hline $\mathbf{2 . 0}$ & 0.760 & 0.129 & 0.612 & 0.213 & 0.730 & 0.234 \\
\hline $\mathbf{3 . 0}$ & 0.778 & 0.131 & 0.598 & 0.222 & 0.747 & 0.216 \\
\hline $\mathbf{5 . 0}$ & 0.789 & 0.131 & 0.639 & 0.210 & 0.755 & 0.203 \\
\hline
\end{tabular}

Table 6.8 Variation of Performance with Inlet Turbulence $\left(B_{i}=B_{0}=2.5 \%\right)$

The effect of inlet turbulence intensity on the static pressure field can be seen in Figures 6.67 and 6.68 which show static pressure recovery contours for an inlet turbulence intensity of $5.0 \%$ and $0.5 \%$ respectively. The distributions show a similar pattern but the level of static pressure recovery is globally greater for the higher inlet turbulence. 
Figure 6.66 also shows a line representing experimental data taken from the equivalent experimental arrangement (i.e. clean inlet and an inlet turbulence intensity of approximately $0.5 \sim 1.0 \%$ ). Although showing some differences the experimental and computational profiles agree well. The experimental profile is reasonably closely matched by the prediction (for $\mathrm{T}_{\mathrm{i}}=0.5 \%$ ) showing a stall of equal magnitude on the outer wall, a similar overall shape and an average velocity close to $26 \mathrm{~ms}^{-1}$. The profiles differ close to the inner wall over a distance corresponding to $10-15 \%$ of the diffuser exit height. However, it is known that the presence of a wall can introduce errors into five-hole pressure probe readings (Section 3.2.5). Thus it is possible that in reality the two profiles are more closely matched than indicated by Figure 6.66. Nonetheless, due to the low levels of inlet turbulence intensity in the two-dimensional test facility and the resulting unstable hybrid diffuser flow there is insufficient experimental data with which to compare computational predictions. However, at increased levels of inlet turbulence computational predictions can provide useful data. Although absolute accuracy cannot be established, some information on trends can be extracted. For example, Table 6.9 illustrates the effect of altering the bleed rate on diffuser and system performance with an inlet turbulence intensity of $5.0 \%$.

\begin{tabular}{|c||c|c|c|c|c|c|}
\hline \multirow{2}{*}{$\begin{array}{c}\text { Bleed }\left(\% \mathbf{m}_{\text {inlet }}\right) \\
\text { inner: outer }\end{array}$} & \multicolumn{2}{c|}{ Diffuser Exit } & \multicolumn{2}{c|}{ Inner Annulus } & \multicolumn{2}{c|}{ Outer Annulus } \\
\cline { 2 - 7 } & $\mathbf{C}_{\mathbf{p 2 - 4}}$ & $\boldsymbol{\lambda}_{\mathbf{2 - 4}}$ & $\mathbf{C}_{\mathbf{p 2 - 5}}$ & $\boldsymbol{\lambda}_{\mathbf{2 - 5}}$ & $\mathbf{C}_{\mathbf{p 2 - 7}}$ & $\boldsymbol{\lambda}_{\mathbf{2 - 7}}$ \\
\hline \hline $\mathbf{0 . 0}: \mathbf{0 . 0}$ & 0.712 & 0.144 & 0.579 & 0.241 & 0.694 & 0.263 \\
\hline $\mathbf{1 . 0}: \mathbf{1 . 0}$ & 0.736 & 0.128 & 0.605 & 0.222 & 0.718 & 0.241 \\
\hline $\mathbf{2 . 5}: \mathbf{2 . 5}$ & 0.789 & 0.131 & 0.639 & 0.210 & 0.755 & 0.203 \\
\hline $\mathbf{3 . 0}: \mathbf{3 . 0}$ & 0.796 & 0.131 & 0.689 & 0.186 & 0.759 & 0.191 \\
\hline
\end{tabular}

Table 6.9 Variation of Performance with Bleed $\left(T_{i}=5.0 \%\right)$

The two-dimensional, axi-symmetric computational geometry differs from the fully three dimensional experiment and as such will produce different values of performance parameters. However, the configurations are, to an extent, generically similar and it would therefore be fair to assume that levels of performance will also be similar. The experimental Hybrid4 diffuser, with inner and outer bleeds of $3.0 \%$, returned a diffuser 
pressure coefficient of $\mathrm{C}_{\mathrm{p} 2-4}=0.71$ and a loss coefficient of $\lambda_{2-4}=0.21$ (refer to Section 4.0). Table 6.9 shows that for the same bleed rate the two-dimensional, axi-symmetric predictions gave corresponding values of 0.80 and 0.13 . These values compare reasonably well considering the differences in the two configurations; the computational prediction does not include blade wakes and the simplified cowl geometry effectively increases the blockage at diffuser exit. Stevens et $\mathrm{al}^{[1970]}$ report that increased turbulence from OGV wakes can increase $C_{p}$. However, this is incidental here as the inlet turbulence, for the CFD, is set at a level representative of that downstream of the OGVs. Thus the inclusion of wakes would, here, only increase the loss due to increased mixing. Furthermore, an increased blockage at diffuser exit will reduce the velocity profile distortion and, according to Hestermann et $\mathrm{al}^{[1991]}$ augment diffuser performance. Thus it is possible to give reasons for the different performance figures, hence providing some measure of confidence in the two-dimensional prediction.

In summary, the effect of altering the bleed rate on the performance of the two-dimensional, axi-symmetric hybrid diffuser has been distinctly illustrated in Figures 6.64 6.70. Figure 6.69 shows a streamline plot for a bleed rate equal to $1.0 \%$ of inlet mass flow led through both the inner and outer bleeds. Clearly, this is below the minimum bleed requirement and the diffuser stalls from the outer Stage 3 wall. Figure 6.64 is the corresponding streamline plot for a bleed of $2.5 \%$ which is above the minimum requirement and shows a fully attached stable flow. The effect of bleed rate is also graphically illustrated in Figure 6.70 which shows normalised axial velocity profiles at diffuser exit for various bleed rates from $0.0-3.0 \%$. With $2.5 \%$ of the inlet mass flow bled through each side the profile demonstrates that the diffuser is fully attached. However, below this level of bleed the diffuser stalls from the outer Stage 3 wall resulting in a much higher peak velocity and profile distortion. Increasing the bleed rate above the minimum bleed requirement further improves the condition of the diffuser exit flow. At a bleed rate of $3.0 \%$ the profile becomes much more uniform with a greater outboard migration of mass flow which improves the condition of the boundary layers. The increased diffuser performance, at higher bleed rates, also beneficially effects the condition of the flow to the feed annuli. At higher bleed rates there is a greater degree of diffusion and the increased outboard migration of the mass flow within the diffuser reduces the amount of turning required within the dump gap. This reduces the dump gap losses and in turn reduces the 
losses to the feed annuli. For example at $0.0 \%$ bleed the outer annulus loss coefficient is 0.26 but this is reduced to 0.19 when the bleed rate is increased to $3.0 \%$.

\subsection{Hybrid Diffuser with OGV Wakes}

Directly extending the two-dimensional computational work discussed above into three dimensions enables the inclusion of three-dimensional inlet conditions incorporating the flow structure associated with OGV wake flow. Section 2.3 outlines the effect that inlet conditions have on diffuser performance, highlighting the importance of reproducing engine representative inlet conditions containing such features as OGV wakes. The geometrical configuration, grid generation and applied boundary conditions for the threedimensional predictions are described and discussed in Section 5.7. Significantly the inlet velocity definition is taken directly from five-hole probe measurement on the experimental facility (refer to Section 4.4) and thus exactly matches the experimental case. However, as highlighted in Section 5.7, the description of the inlet turbulence is less exact as these type of data cannot be obtained from a five-hole probe.

\subsubsection{Diffuser Performance and OGV Wake Effects}

Measurements from the experimental facility and data from two-dimensional, axi-symmetric computational work suggest that an inlet turbulence intensity of 5\% is both a realistic and necessary level for the diffuser flow to remain attached. Furthermore, measurements from the modified test facility (Section 4.4) indicate that an unbalanced bleed rate would also be required due to the diffuser outer wall being more prone to separation. Thus for the first computational prediction the inlet turbulence intensity was set to $5.0 \%$ in conjunction with inner and outer bleed rates equal to $2.5 \%$ and $3.0 \%$ of the inlet mass flow, respectively.

Figure 6.71 shows the normalised axial velocity contours at diffuser exit for the experimental case. The velocity profile has a slight inboard bias with the peak velocity located at some $35 \%$ of the annulus height. More notably, however, is that Figure 6.71 shows that velocity peaks and troughs associated with the OGV wakes are still present at diffuser exit. 
Figure 6.72 shows a corresponding axial velocity contour plot for the initial computational prediction (i.e. $\mathrm{T}_{\mathrm{i}}=5.0 \%, \mathrm{~B}_{\mathrm{i}}=2.5 \%$ and $\mathrm{B}_{\mathrm{o}}=3.0 \%$ ). It is immediately evident that the OGV wakes have not persisted through to diffuser exit as they do in the experimental case and, additionally to the wakes mixing out, the peak velocity has shifted and is located more centrally.

Velocity vectors at diffuser exit are shown in Figures 6.73 and 6.74 for the experimental and computational cases respectively. The experimental case exhibits a slightly higher swirl component but the pitch component appears lower than the computational prediction. Overall the predicted flow field at diffuser exit differs from the experimental case due, in part, to the more rapid mixing out of the wake flow. The rate at which OGV wakes will mix out is dependant upon the turbulent structure of the flow. Wolf and Johnston $^{[1969]}$ reported that mixing is promoted by higher turbulence intensities and larger scale eddies.

There are two potential reasons why the predicted flow field differs from the experimental case. Firstly, the inlet turbulence definition may be in error, and secondly, the turbulence model chosen may be unable to satisfactorily model the flow. Both these reasons are, however, closely linked as the turbulence model used dictates the method by which inlet turbulence must be defined. Furthermore, if the inlet turbulence definition is incorrect the solution may be in error regardless of the turbulence model used.

Here, for this computational prediction, an eddy-viscosity $(\mathrm{k}-\varepsilon)$ turbulence closure was used for reasons outlined in Section 5.0. This model requires the description of the inlet turbulent flow field by means of specifying distributions of $\mathrm{k}$ and $\varepsilon$; the Reynolds stresses are then computed form the Boussinesq hypothesis (Equation 5.16). Velocity data at inlet were specified directly from measurements on the experimental facility but distributions of $\mathrm{k}$ and $\varepsilon$ were not available and had to be approximated using Equation 5.26. Thus the structure of the inlet turbulence is not entirely correct; clearly from Equation 5.26 distributions of $\mathrm{k}$ and $\varepsilon$ calculated from a single turbulence intensity and characteristic length are uniform. In reality this is not the case downstream of OGVs where the distribution of intensity and length scale will be far from uniform. Therefore, although velocities and pressures are correct at inlet, the turbulence definition contains 
errors.

Additionally differences in the predicted solution may also be due to the eddy-viscosity turbulence model being unable to predict the evaluation of the Reynolds stresses satisfactorily. Many authors have commented that the Boussinesq hypothesis implies isotropic turbulence. This is not strictly correct; in reality it assumes that the turbulent viscosity is isotropic and can be expressed as a scalar ( $\mu_{\mathrm{t}}$ in Equation 5.10). This is generally a valid assumption but when the turbulence is strongly anisotropic, such as downstream of OGV wakes, the turbulent viscosity should definitely be a tensor quantity. Hence it is feasible that the calculated stresses may be incorrect which will, in turn, effect the turbulent mixing of the OGV wake flow.

A further turbulence model was available for use within Fluent, namely the Reynolds Stress Model (RSM) as described in Section 5.4. The RSM solves transport equations for the individual Reynolds stresses directly, avoiding the use of the Boussinesq hypothesis, and is therefore often considered a more accurate model for the prediction of turbulent flows. However, this model requires that the individual Reynolds stresses are specified at inlet and again this information was not available necessitating the use of an approximation as defined in Equation 5.36. Again the specification of a single turbulence intensity and characteristic length will result in uniform distributions of $\mathrm{k}, \varepsilon$ and the Reynolds stresses which will not be representative of the flow field downstream of OGV's. Thus, even if the Reynolds Stress model is more accurate, the prediction of the wake flow will still contain errors due to the method used to specify the inlet turbulence. The RSM was not used for the three-dimensional predictions due to the reasons discussed in Section 5.8 including the fact that the RSM is very demanding on computational resources.

Although the inlet turbulence structure is defined using a uniform distribution it is still possible to alter the wake mixing process by simply changing the inlet turbulence intensity. It has been shown in Figure 6.72 that an inlet intensity of $5.0 \%$ encourages vigorous mixing such that the OGV wakes have mixed out by the time the flow reaches diffuser exit. According to Wolf and Johnston ${ }^{[1969]}$ reducing the turbulence intensity should reduce the level of turbulent mixing. Figure 6.75 shows non-dimensionalised axial velocity contours with the inlet turbulence reduced to only $1.0 \%$. This is much lower 
than levels seen typically downstream of an axial compressor but it does result in a strong wake flow still being present at diffuser exit. Figure 6.75 clearly shows two OGV wakes but when compared to the experimental case (Figure 6.71) there remains distinct differences in the overall flow field. For the experimental case the peak velocity is around 1.6 times the average but for the predicted flow with an inlet turbulence intensity of $1.0 \%$ this falls to a factor of 1.4 . A slightly higher inlet turbulence intensity of $2.0 \%$ encourages a higher degree of mixing and, as shown in Figure 6.76, the wake flow is less pronounced but still differs from the experimental case.

In addition to changing the nature of the wake mixing by altering the inlet turbulence intensity it should also be possible to influence this process by altering the characteristic turbulence length scale used at inlet. In all the predictions here the characteristic length was set as the OGV passage height but it may be the case that a length based on OGV spacing or wake width is more appropriate. Unfortunately, due mainly to time constraints, this was not investigated.

In summary, the combination of the turbulence model and the method by which the inlet turbulent conditions are specified cannot reproduce the correct physical flow field. However, altering the level of inlet turbulence does demonstrate the effect of this parameter on the development of the OGV wake flow. As the inlet turbulence intensity increases there is increased mixing of the OGV wakes such that at a turbulence intensity of $5.0 \%$ the wake flow is completely mixed out at diffuser exit. Table 6.10 details some diffuser and system performance parameters for the experimental case and the computational predictions. Predicted diffuser static pressure recovery is $15 \%$ higher and loss $45 \%$ lower than measured on the experimental facility. Similarly, total pressure losses to the feed annuli are reduced by a comparable amount. Although many of these differences are caused by the differing inlet turbulence structure these observations are also consistent with those of Little et al ${ }^{[1997]}$ and Bradshaw ${ }^{[1973]}$ who suggest that the eddy-viscosity (k$\varepsilon)$ turbulence model fails to predict the high total pressure losses associated with flow curvature in the dump region and over the flame tube cowl. 


\begin{tabular}{|c||c|c|c|c|c|c|}
\hline Diffuser & $\mathrm{C}_{\mathrm{p} 2-4}$ & $\lambda_{2-4}$ & $\mathrm{C}_{\mathrm{p} 2-5}$ & $\lambda_{2-5}$ & $\mathrm{C}_{\mathrm{p} 2-7}$ & $\lambda_{2-7}$ \\
\hline \hline Experiment & 0.70 & 0.21 & 0.55 & 0.23 & 0.67 & 0.29 \\
\hline $\mathrm{CFD}, \mathrm{T}_{\mathrm{i}}=5.0 \%$ & 0.813 & 0.114 & 0.392 & 0.225 & 0.793 & 0.165 \\
\hline $\mathrm{CFD}, \mathrm{T}_{\mathrm{i}}=2.0 \%$ & 0.807 & 0.118 & 0.410 & 0.241 & 0.790 & 0.172 \\
\hline $\mathrm{CFD}, \mathrm{T}_{\mathrm{i}}=1.0 \%$ & 0.802 & 0.120 & 0.427 & 0.254 & 0.789 & 0.172 \\
\hline
\end{tabular}

Table 6.10 Performance Data, Three-Dimensional Predictions, $B_{i}=2.5 \%, B_{0}=3.0 \%$

The predicted wake flow may not be entirely representative of the physical case due to the inlet turbulence definition and the turbulence model but it does provide an opportunity to qualitatively asses the effect OGV wakes may have on the flow mechanisms of hybrid diffusers. For the hybrid diffuser studied here (Hybrid 4 - refer to Section 3.0) it seems sensible to suggest that the effect will be centred on two areas.

Firstly the presence of wakes will change the nature of the bleed as the lower axial momentum wake flow will be unable to overcome the radial pressure gradient setup by the bleed thus producing locally higher bleed rates. However, Figure 6.75 shows that the OGV wakes are not distributed evenly and do not occur in a simple circumferential plane. They have a complex ' $\mathrm{S}$ ' shape making it difficult to identify the actual circumferential location of each wake, especially since the swirl component of the inlet flow shifts this location out of line with the physical position of the OGV's. Notwithstanding this regions of low axial momentum fluid can clearly be identified close to the walls and it is here local bleed rate may be augmented.

Secondly, the structure of wake flow may also affect the reattachment process. Findings reported in Section 6.1 suggest that a locally increased bleed rate will result in a locally reduced reattachment length but the complex nature of wake flow may, itself, have a greater effect. For example, the OGV wake flow will, in general, have a lower axial momentum than the average value. In a deep wake this may result in the fact that the wake flow would be unable to overcome the shear stresses and adverse pressure gradient within the Stage 2 diffuser. This flow would then be more likely to stall and flow reat- 
tachment would be delayed or not occur and diffuser performance will fall.

Figure 6.77 plots the circumferential variation of reattachment on both the inner and outer walls of the Stage 3 diffuser with inner and outer bleed rates of $2.5 \%$ and $3.0 \%$ respectively and an inlet turbulence intensity of 5.0\%. (The plot is not symmetrical about the centre plane due to the fact that the inlet conditions are not symmetrical; adjacent OGV wakes differ due to interactions of the 77 IGVs with 154 OGVs and repeatability occurs over $2 \mathrm{OGVs}$ ). For this computational configuration the $\mathrm{OGV}$ wakes were almost entirely mixed out at diffuser exit (Figure 6.75) but Figure 6.77 clearly shows that the presence of OGVs alters the point of reattachment; defined as the point at which:

$$
\left(\frac{\partial U}{\partial y}\right)_{\text {wall }}=0
$$

Firstly, Figure 6.77 shows that reattachment is slightly more rapid on the outer wall due to the fact that the outer bleed rate is higher than the inner bleed. However, the reattachment length on both walls varies with a periodicity matched to the OGVs. This was also seen in the experimental observations as reported in Section 4.1.2. Figure 4.10 shows that wall static pressure was also seen to exhibit a periodic variation matched to the frequency of the OGVs. Experimentally it was not possible to determine a reattachment point but it was argued that it would vary circumferentially in a periodic manner, in line with the wall static pressure, due to the influence of the OGVs. Figure 6.78 shows a similar plot to Figure 4.10 but for the computational configuration. The inclusion of a line indicating the reattachment point confirms that the wall static pressure variation does indeed mimic the variation of reattachment.

It is difficult, however, to ascertain from Figure 6.77 if the reduction in reattachment length is associated with wake or non-wake flow because the residual swirl at inlet causes the wake flow to shift circumferentially from the physical OGV locations. Figure 6.79 is a plot of the axial velocity contours on an axial plane corresponding to a distance $60 \%$ across the mouth of the bleed duct. Although mixed considerably there are still OGV wakes present and, close to upper and lower extremes, regions of low axial velocity can be seen to periodically occur just to the left of the OGV locations. Comparing Figure 6.77 with Figure 6.79 it is now clear that downstream of these regions of low axial velocity (i.e. OGV wake flow) the reattachment length is increased. 
Figures 6.80 and 6.81 are plots of radial velocity contours across the mouth of the inner and outer bleed gaps and show higher radial velocities and thus higher bleed rates associated with the lower axial momentum regions. Work reported in Section 6.1 demonstrated that for two-dimensional systems higher bleed rates reduce the reattachment length. However this contradicts what is observed here as, although local bleed rates are increased, reattachment is delayed within the OGV wake flow.

Plots of velocity contours on circumferential planes corresponding to positions within and between OGV wake fluid allow the flow field to be further studied. Figures 6.82 and 6.83 are plots of axial and radial velocity contours in the Stage 2 diffuser in a region within the low axial momentum wake fluid whereas Figures 6.84 and 6.85 show corresponding plots in a region between wakes.

Firstly, the plots of axial velocity contours confirm that reattachment is less rapid within the low axial momentum OGV wake fluid. The reattachment point corresponds to the intersection of the zero contour level with the Stage 3 wall and the length required for the flow to reattach increases by some $30 \%$ within the wake.

Secondly, Figures 6.82 and 6.84 show that the axial velocity is globally reduced within the wakes (as would be expected) when compared to the non-wake flow. This is especially true in the near wall region approaching the bleed. Although bleed rate is locally increased within the wakes it is not sufficient to greatly affect the mainstream wake fluid. The reattachment length is, therefore, locally increased resulting in the periodic variation seen in Figure 6.77.

A lower inlet turbulence intensity will, according to Wolf and Johnston ${ }^{[1969]}$, reduce OGV wake mixing. With more pronounced, deeper OGV wakes the regions of low axial momentum fluid close to the inner wall increase in magnitude. Figure 6.75 shows axial velocity contours at diffuser exit for a solution generated with an inlet turbulence intensity of only $1.0 \%$ and clearly shows a very pronounced wake flow. The two-dimensional computational work reported in Section 6.2 suggested that such a low turbulence intensity flow would not reattach on the Stage 3 wall but, as Figure 6.75 clearly shows, this is not the case when OGV wakes are present. Barker and Carrotte ${ }^{[2000]}$ report that the 
highly three-dimensional OGV wake structure promotes radial movement of flow and encourages the transfer of axial momentum to the boundary layer flow and it is this phenomenon which enables the flow to reattach. Nevertheless this combination of low turbulence intensity and deep wakes results in the fact that within the low energy wake flow the reattachment length is greatly increased. Figure 6.86 shows the circumferential variation of reattachment point for an inlet turbulence intensity of $1.0 \%$ and shows that, although the outer wall reattachment is unchanged, the inner wall reattachment is greatly effected resulting in a large separation bubble. Within the more pronounced wake the flow is much lower in axial momentum and even with radial transfer of positive momentum under the action of the bleed the flow cannot rapidly reattach. Between the wakes the near wall flow, in conjunction with the bleed, still has sufficient momentum to over come the shear stress and adverse pressure gradient and more rapidly reattach on the Stage 3 wall. 


\section{$\underline{\text { 6.4 Summary }}$}

In summary the computational investigation has revealed the following main points:

- Predictions of the flow in a two-dimensional, plane hybrid diffuser have, in support of the experimental findings, both qualitatively and quantitatively demonstrated that a vortex chamber is not a necessary component of hybrid diffusers. Significantly this contradicts much of the early work on hybrid diffusers carried out by Adkins et $\mathrm{al}^{[1980]}$ and Myres et $\mathrm{al}^{[1995]}$. The performance of a hybrid diffuser incorporating a simple ducted bleed was seen to be the same as when a vortex chamber was present.

- Moreover, these predictions also demonstrated that the step/fence arrangement of Adkins et $\mathrm{a}^{[1980]}$ is unnecessary and does not contribute to the main flow mechanisms of a hybrid diffuser. In addition to simplifying the geometry, several alternative bleed configurations exhibited a diffuser performance unchanged from that of the original configuration.

- Furthermore, a momentum transfer analysis revealed that the pertinent flow mechanisms of a hybrid diffuser are centred on a transfer of streamwise momentum from the accelerating bleed flow to the diffusing mainstream flow. This the enables a fresh boundary layer to be formed on the stage 3 wall which is sufficiently energetic to overcome the high rates of diffusion and high adverse pressure gradient.

- Realisation that a vortex chamber could be replaced by a ducted bleed enabled the bleed geometry to be modified in an attempt to reduce bleed flow total pressure loss. Although not an optimised bleed geometry a profiled bleed (Alt. 4) reduced the total pressure loss to $70 \%$ of it original value (for $2.5 \%$ bleed). However, potential for further improvements need to be investigated before conclusions can be made about the suitability of the bleed air for use in component cooling.

- A prediction incorporating OGV wakes in the inlet conditions showed a level of diffuser and system performance that was slightly above that seen on the full experimental facility. Additionally, predicted and measured wakes at diffuser exit had some 
similarities but differed due to an incomplete three-dimensional description of the inlet turbulence ( $\mathrm{k}$ and $\varepsilon$ ). However, an investigation into the effect of $\mathrm{OGV}$ wakes revealed that they have an impact on the reattachment process, locally increasing reattachment length. 
Diffuser Exit

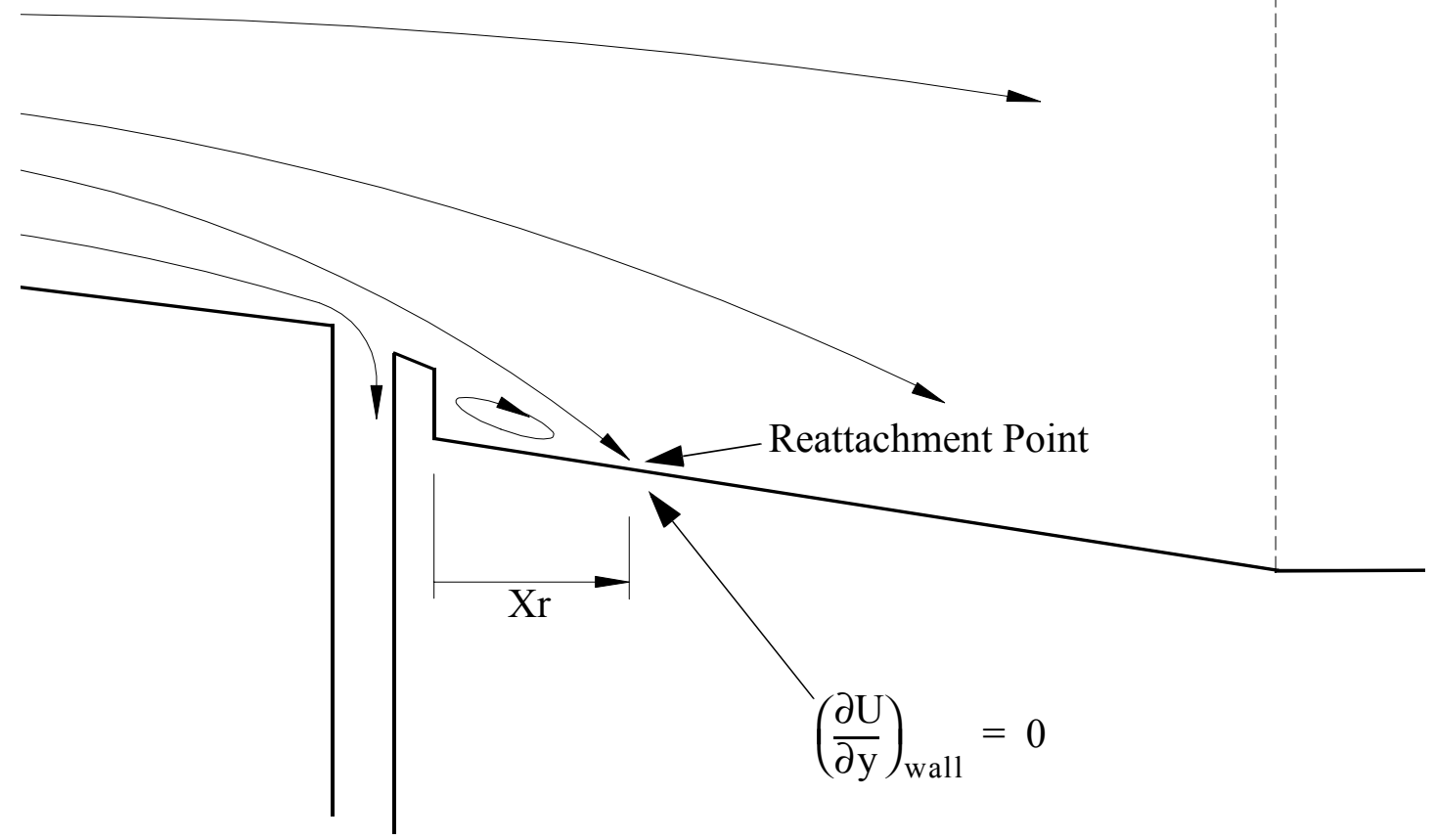

Figure 6.1 Definition of Reattachment Length, Xr

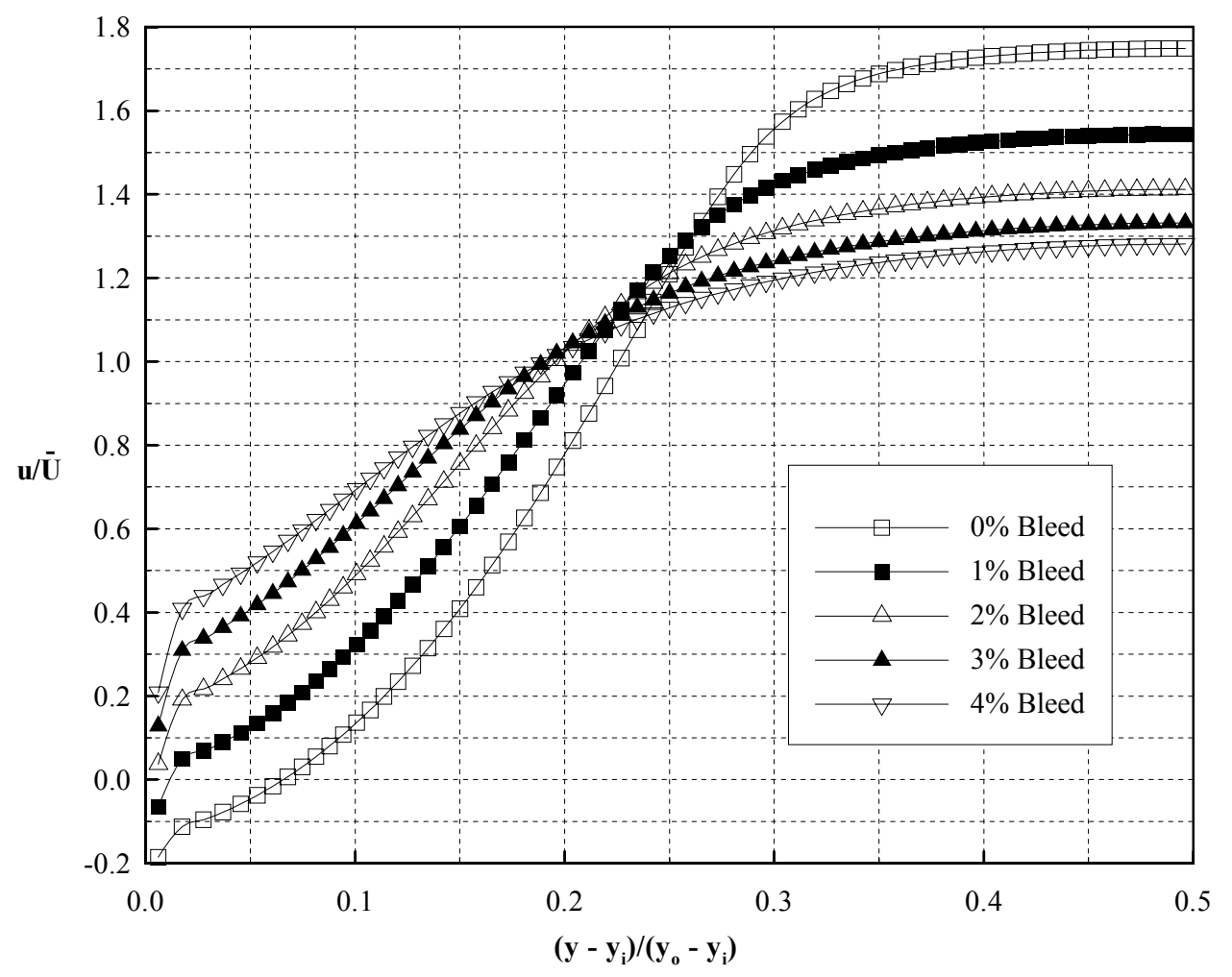

Figure 6.2 Axial Velocity Profiles at Diffuser Exit (Vortex Chamber) 


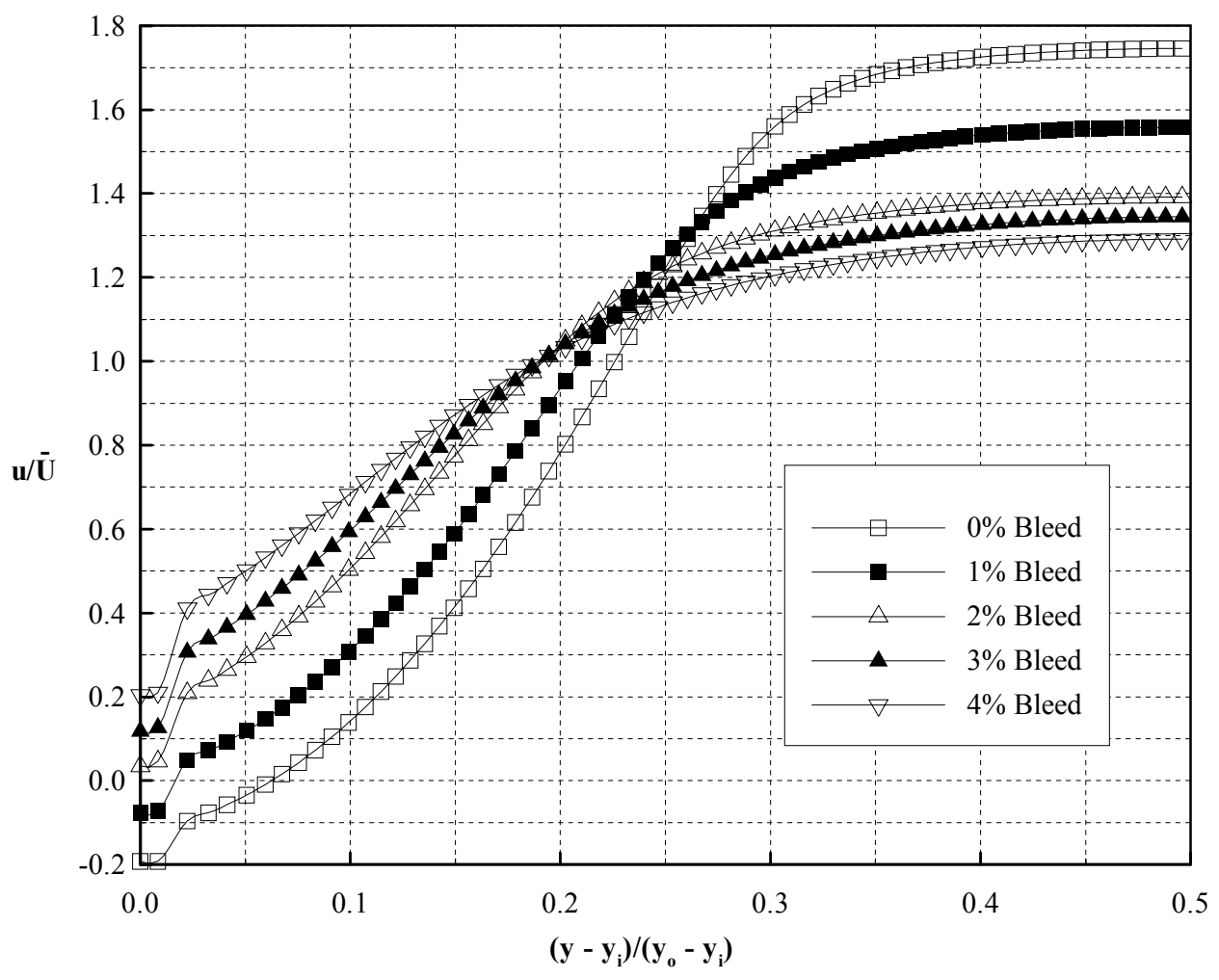

Figure 6.3 Axial Velocity Profiles at Diffuser Exit (Duct Bleed)

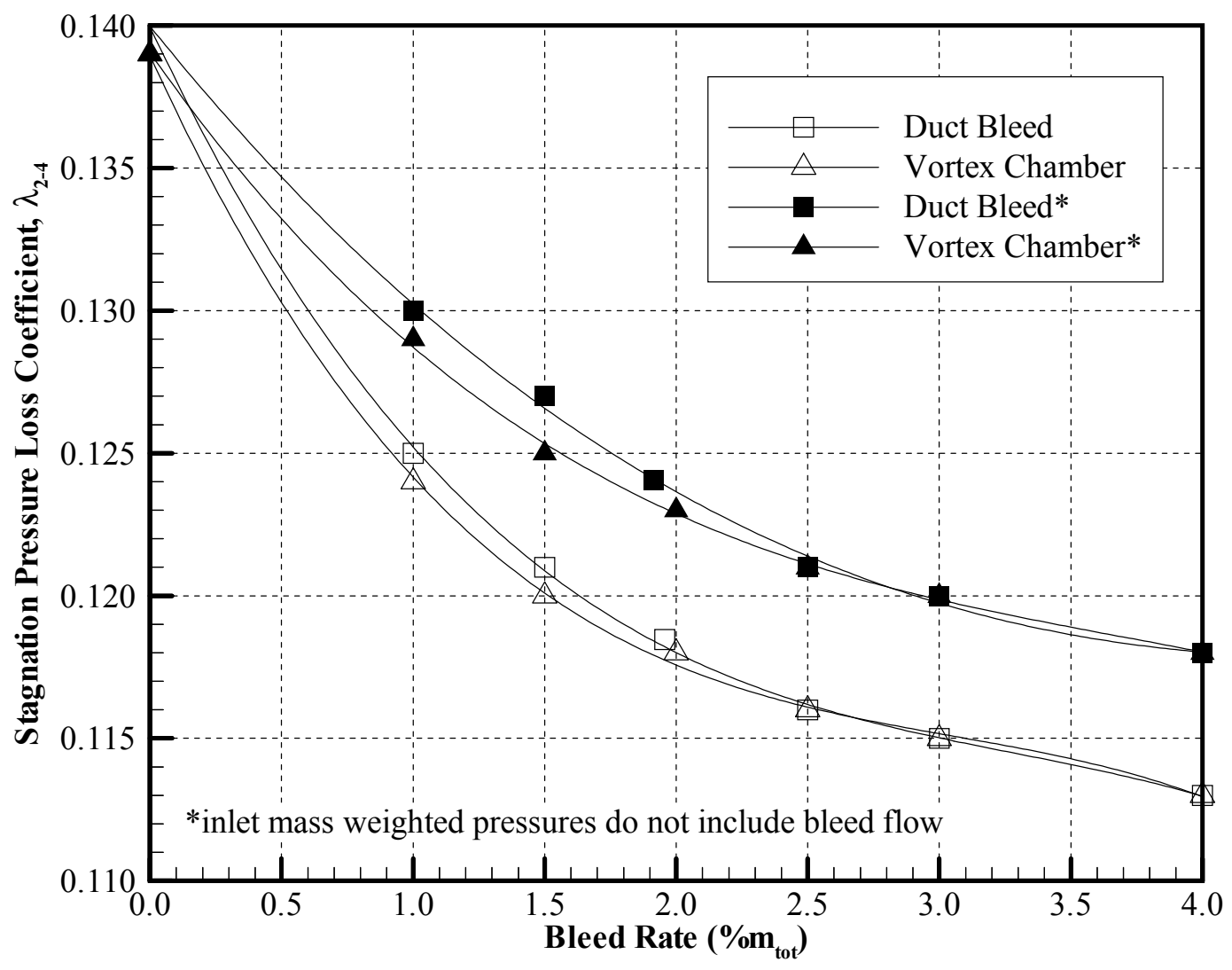

Figure 6.4 Variation of Diffuser Total Pressure Loss with Bleed 


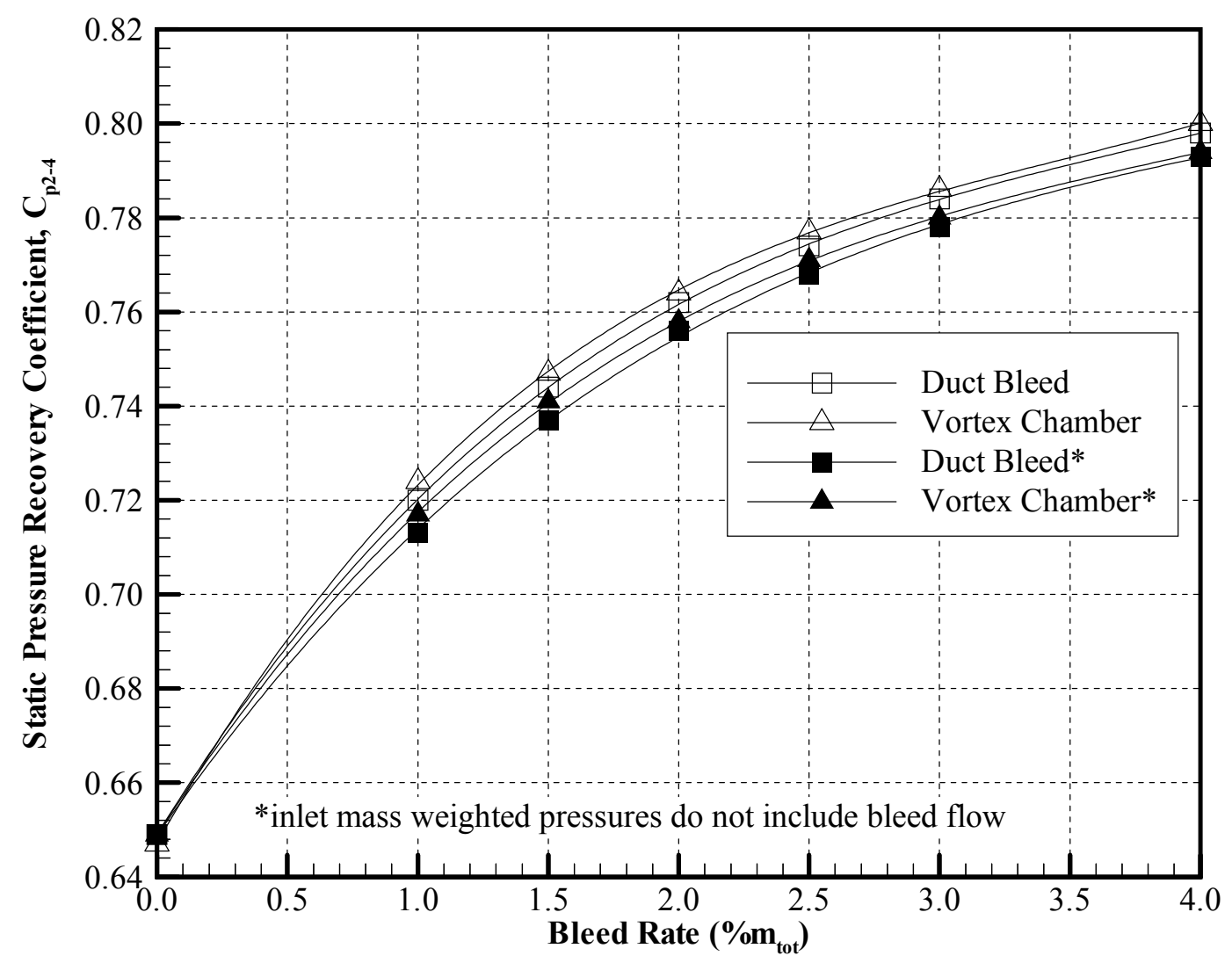

Figure 6.5 Variation of Diffuser Static Pressure Recovery with Bleed

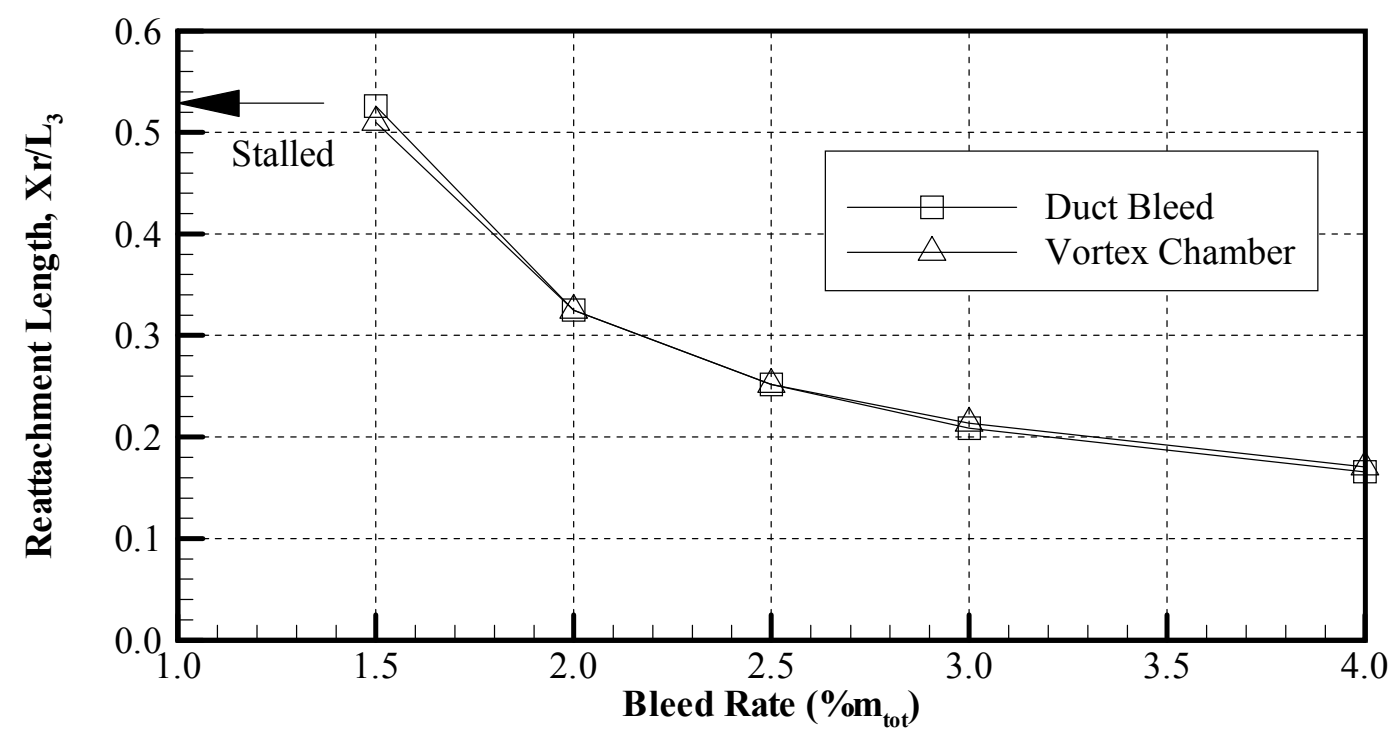

Figure 6.6 Variation of Reattachment Length with Bleed 


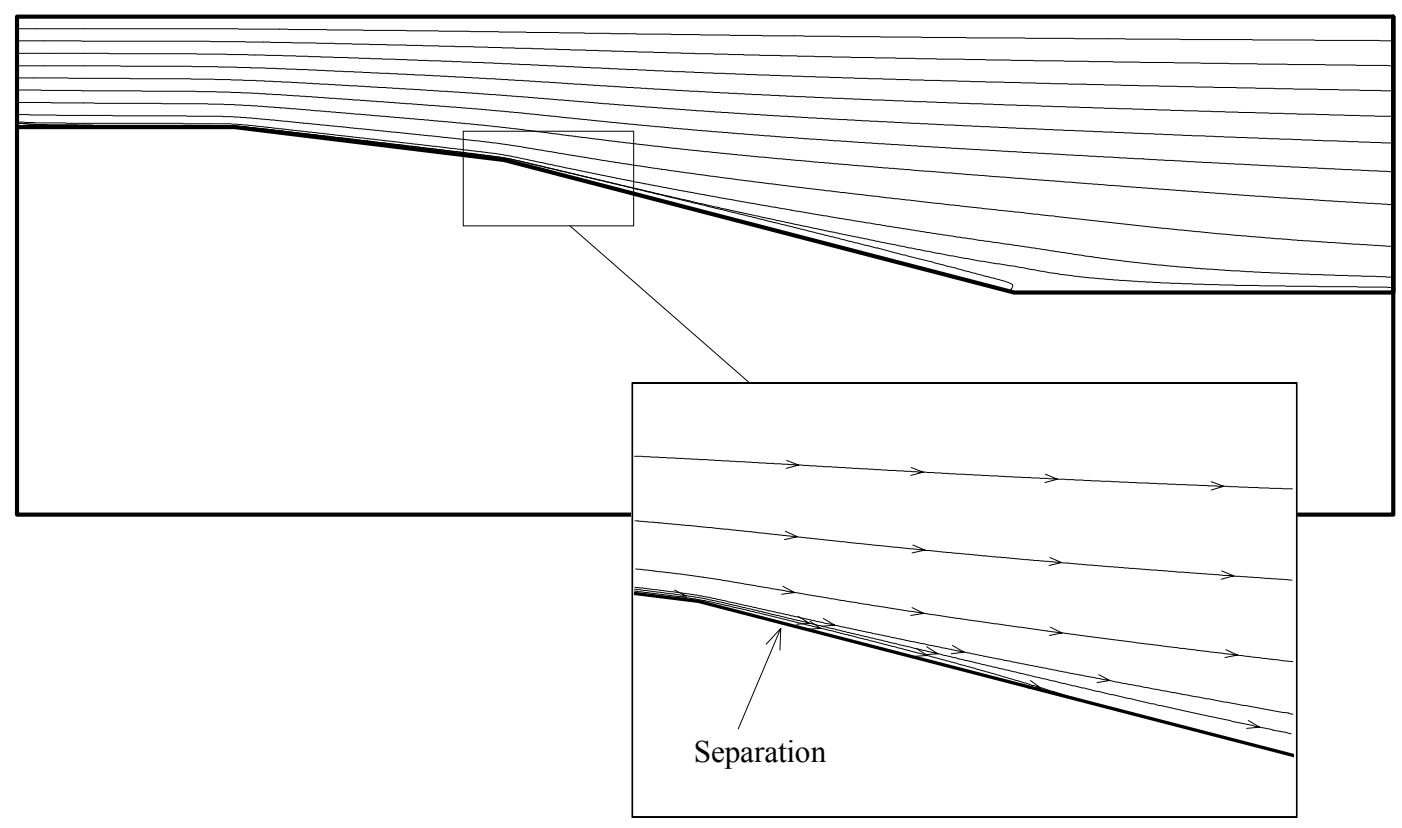

Figure 6.7 Streamline Plot - Conventional Diffuser

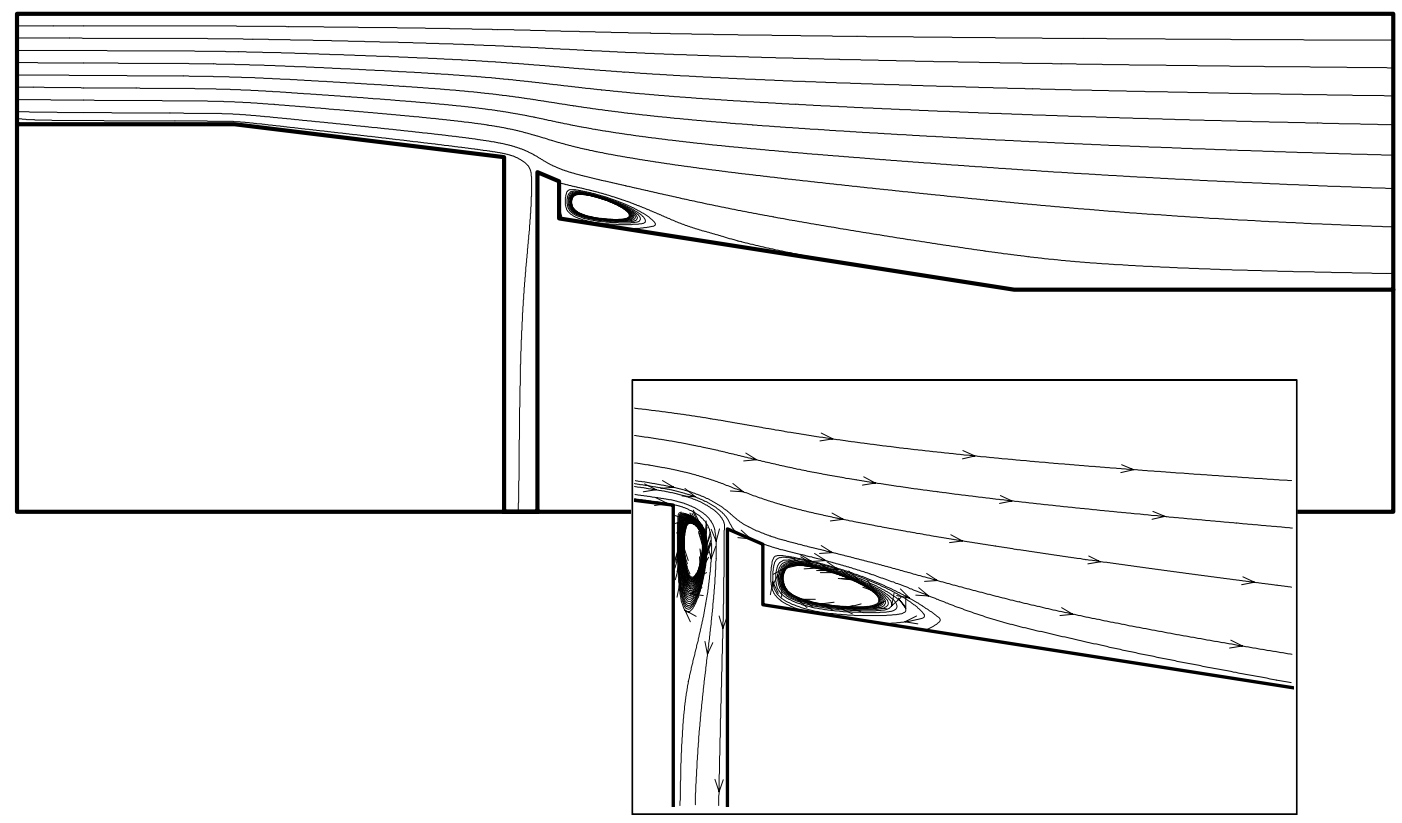

Figure 6.8 Streamline Plot - Duct-bled Diffuser, 2.5\% Bleed 


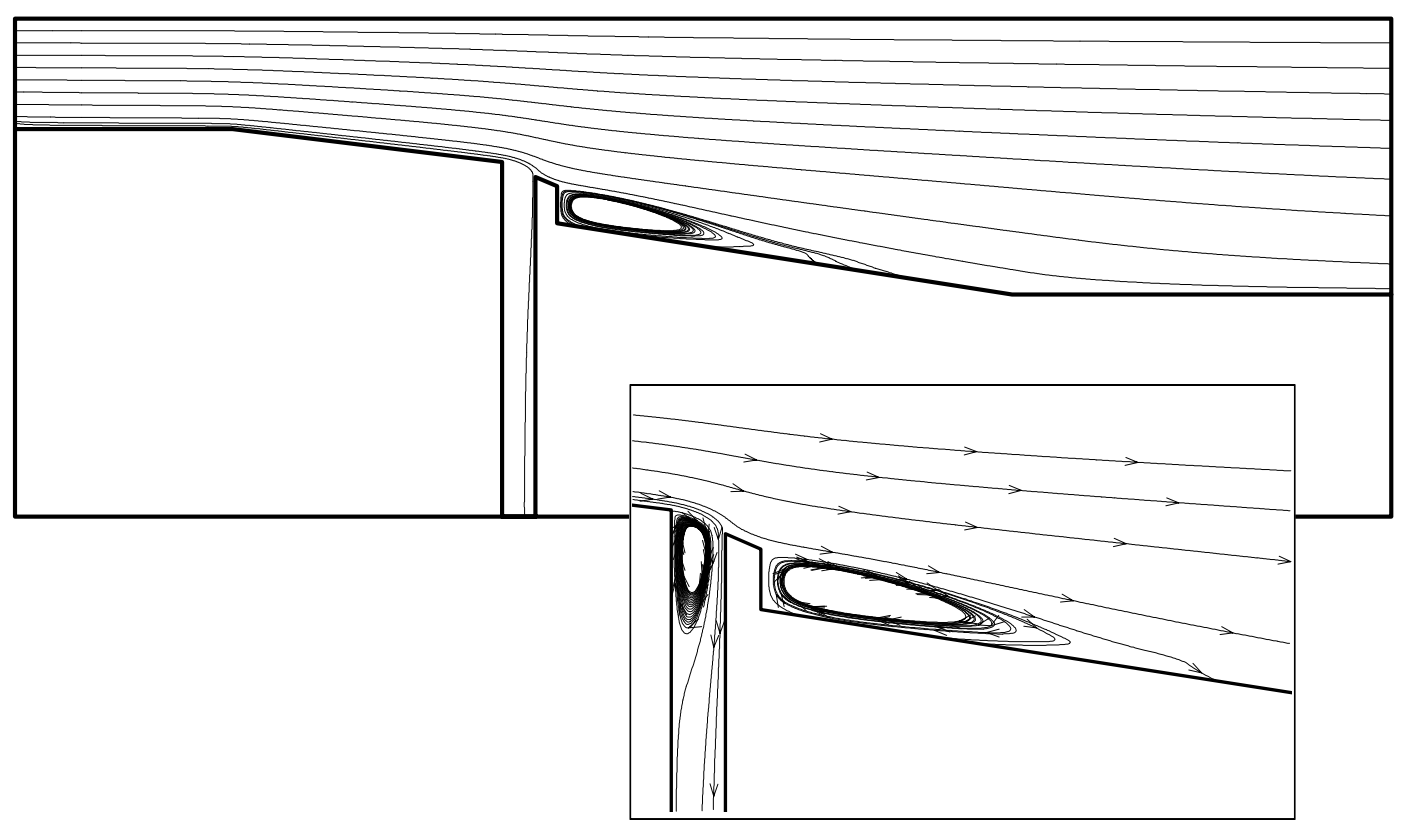

Figure 6.9 Streamline Plot - Duct-bled Diffuser, 1.5\% Bleed

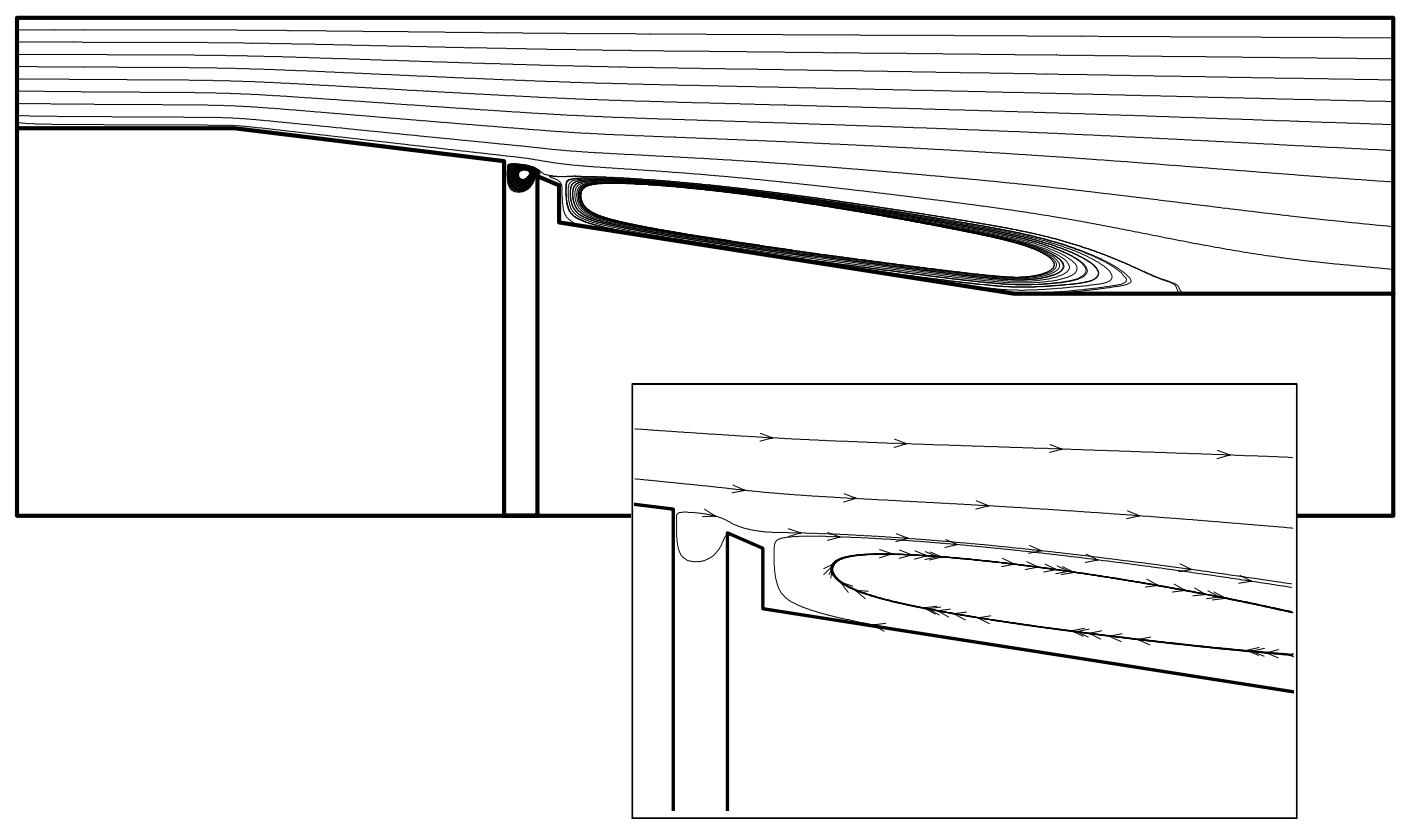

Figure 6.10 Streamline Plot - Duct-bled Diffuser, 0.0\% Bleed 


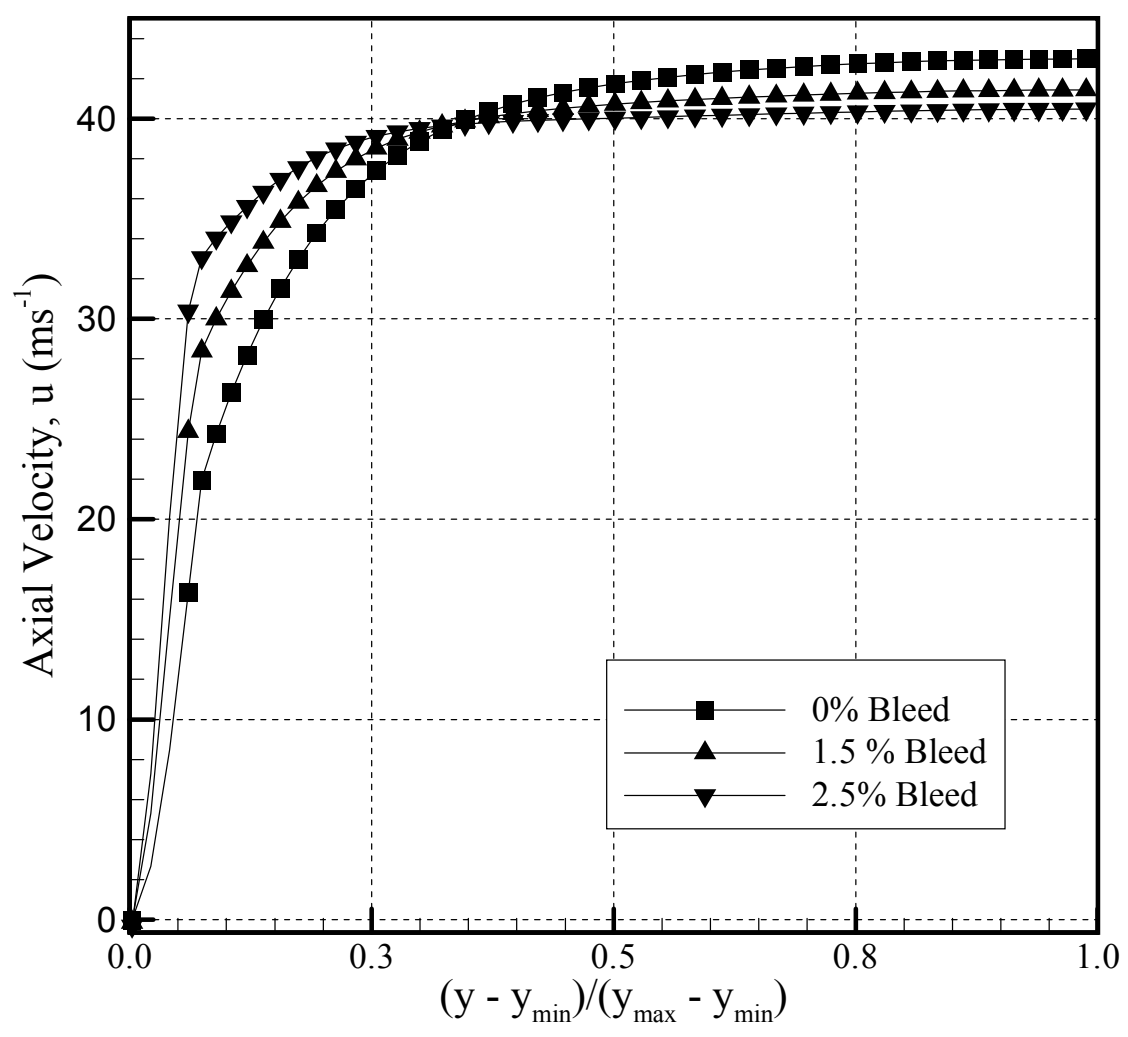

Figure 6.11 Axial Velocity Profile at Stage 1 Exit (Duct-bled Diffuser)

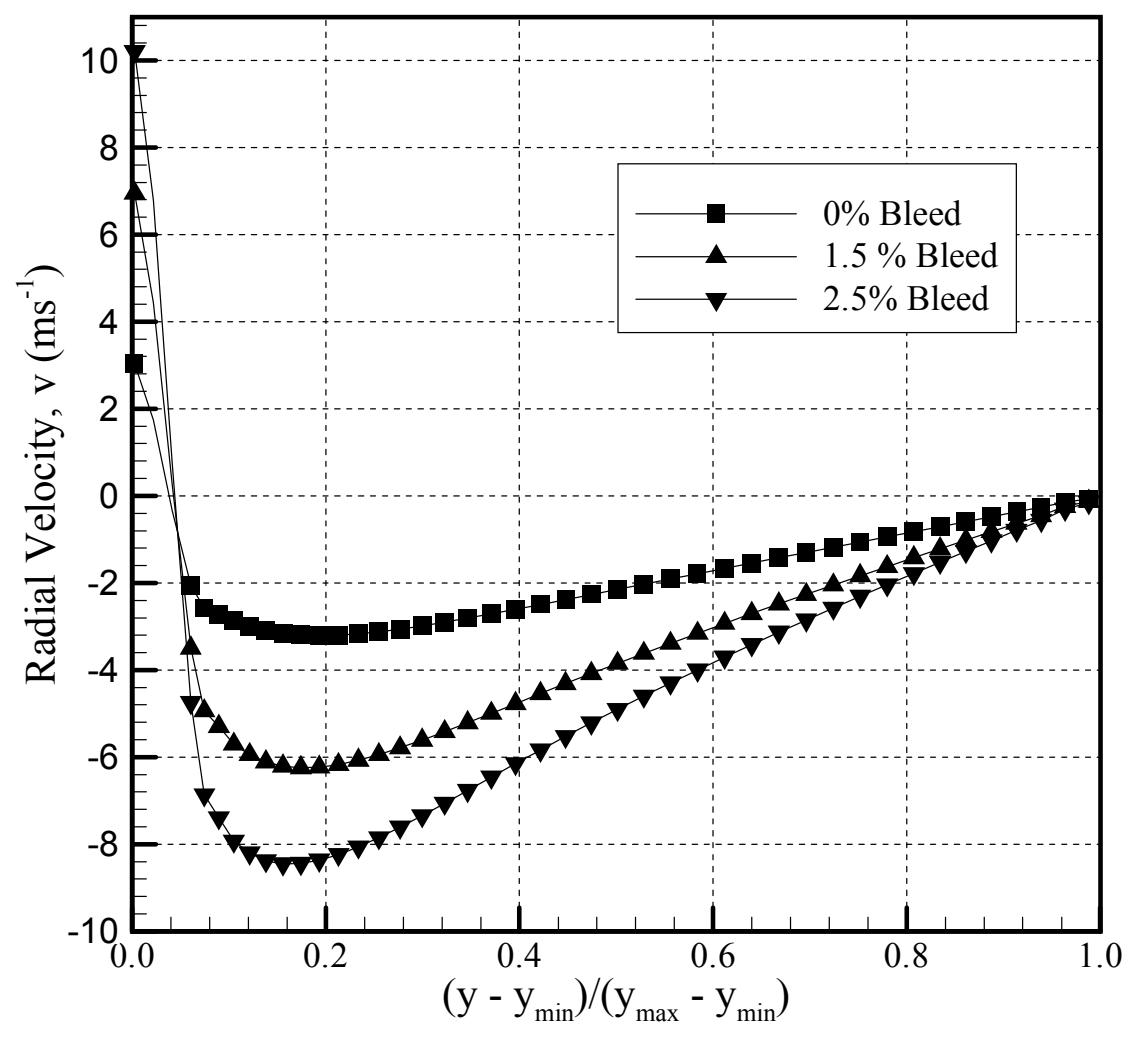

Figure 6.12 Radial Velocity Profile at Stage 1 Exit (Duct-bled Diffuser) 


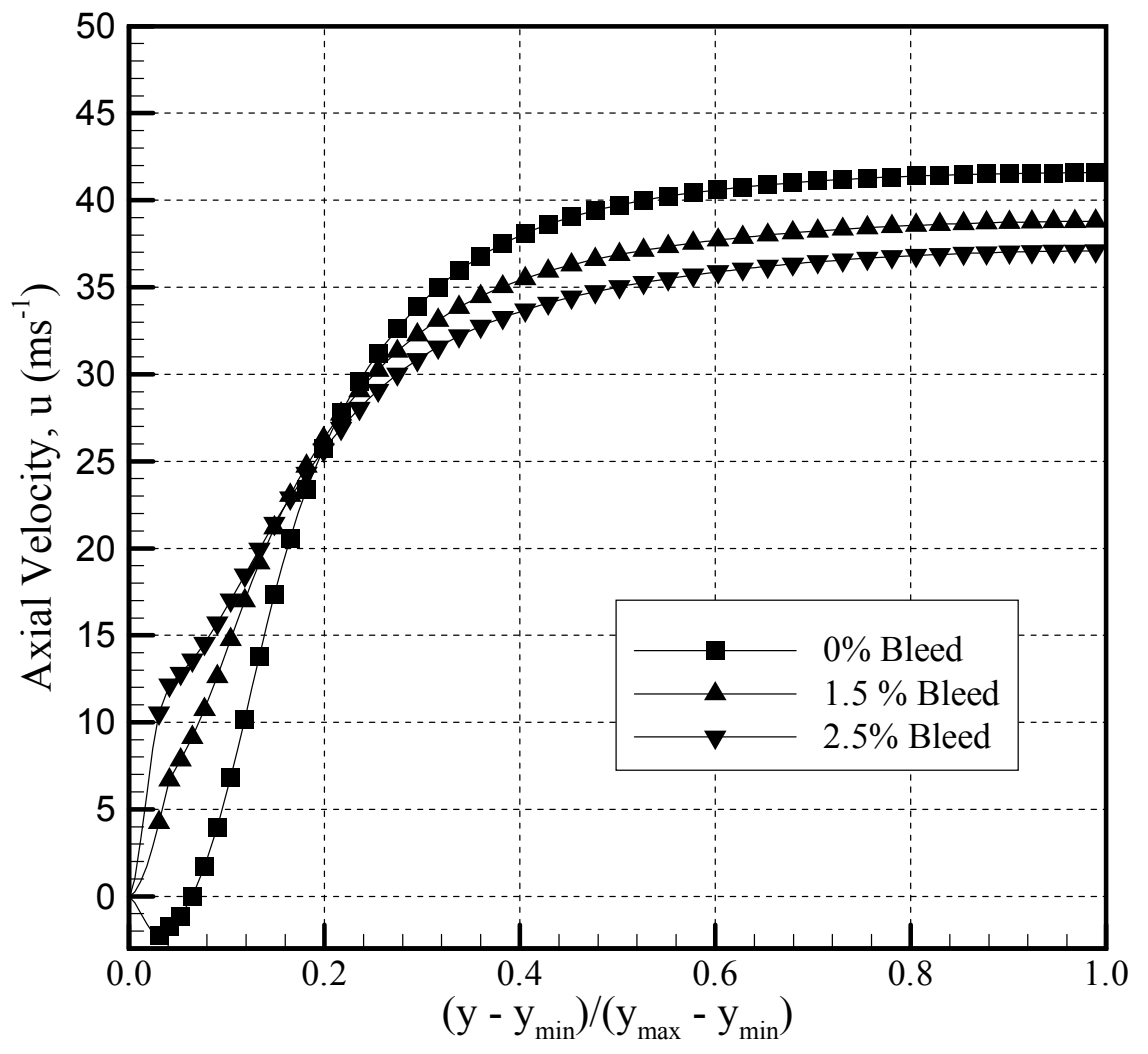

Figure 6.13 Axial Velocity Profile at Stage 2 Exit (Duct-bled Diffuser)

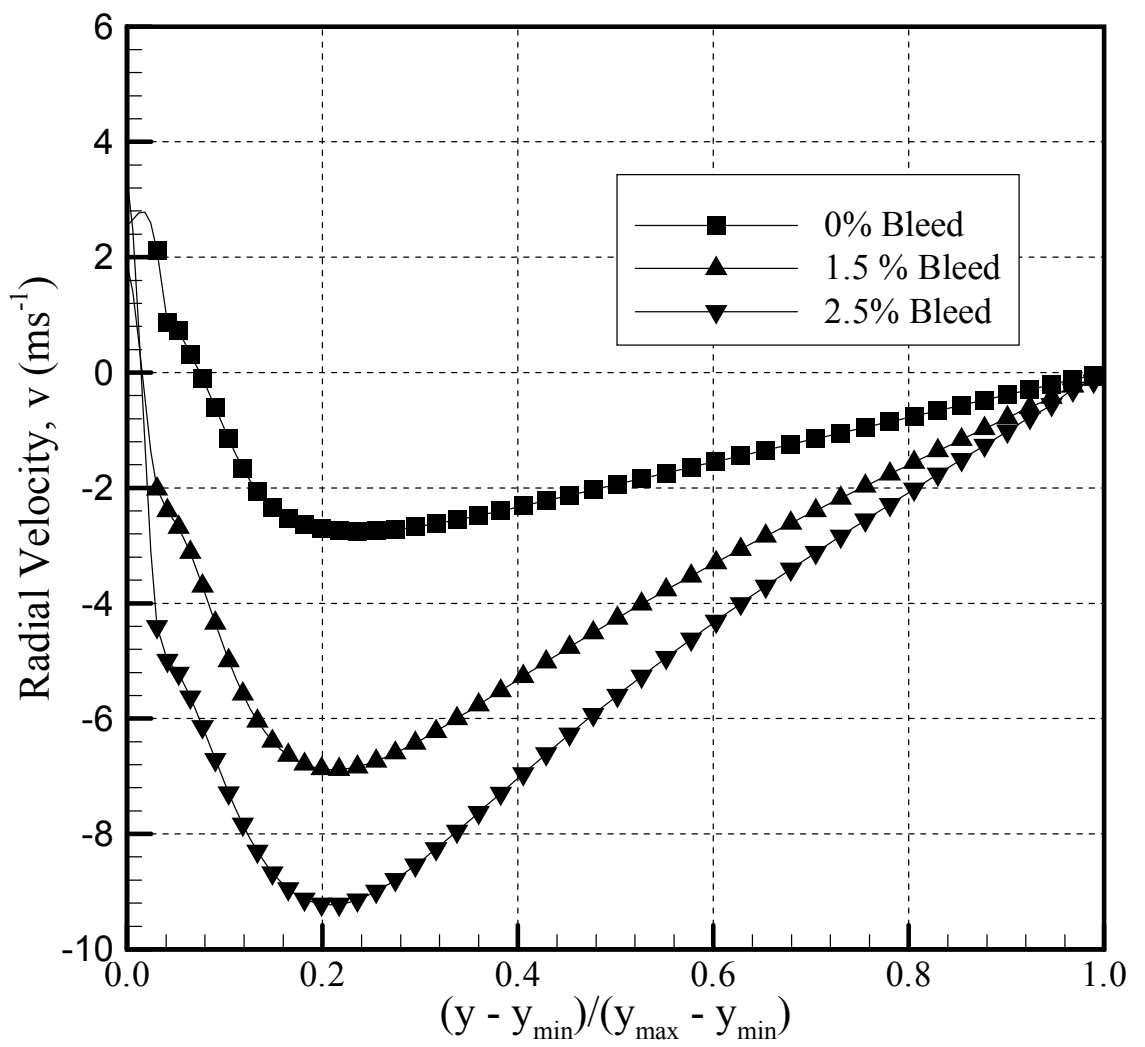

Figure 6.14 Radial Velocity Profile at Stage 2 Exit (Duct-bled Diffuser) 


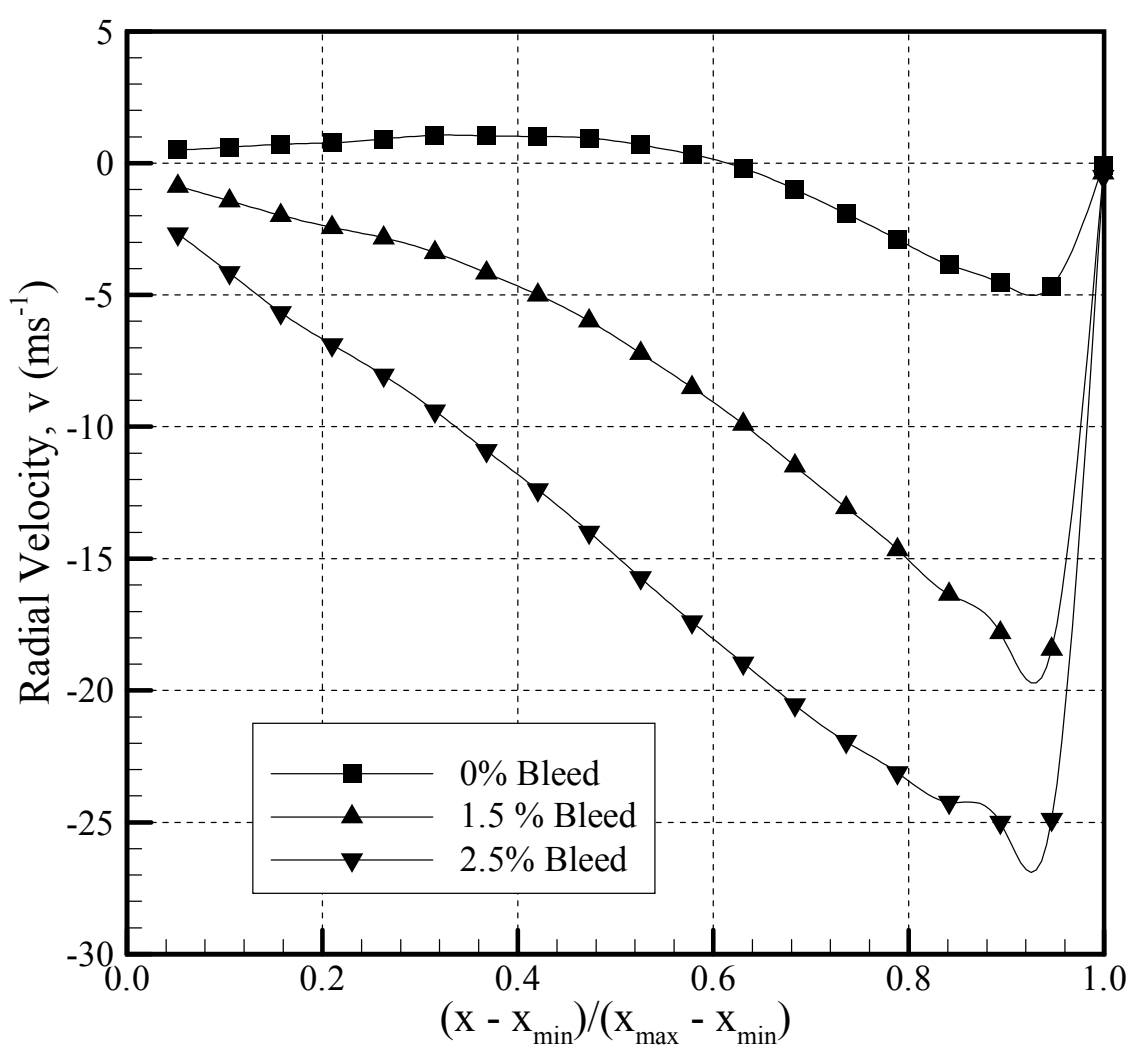

Figure 6.15 Radial Velocity Across Bleed Gap (Duct-bled Diffuser)

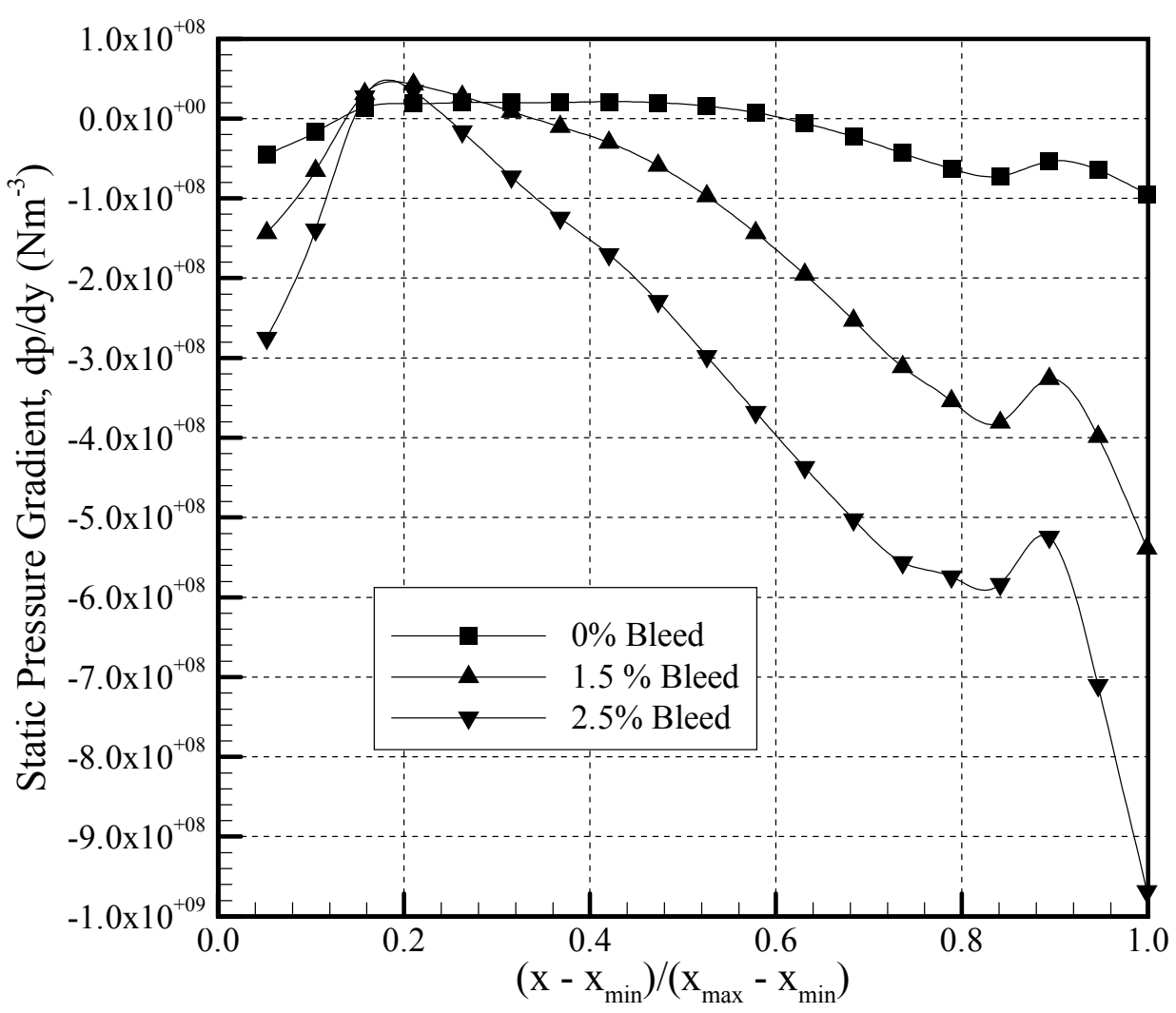

Figure 6.16 Static Pressure Gradient Across Bleed Gap (Duct-bled Diffuser) 


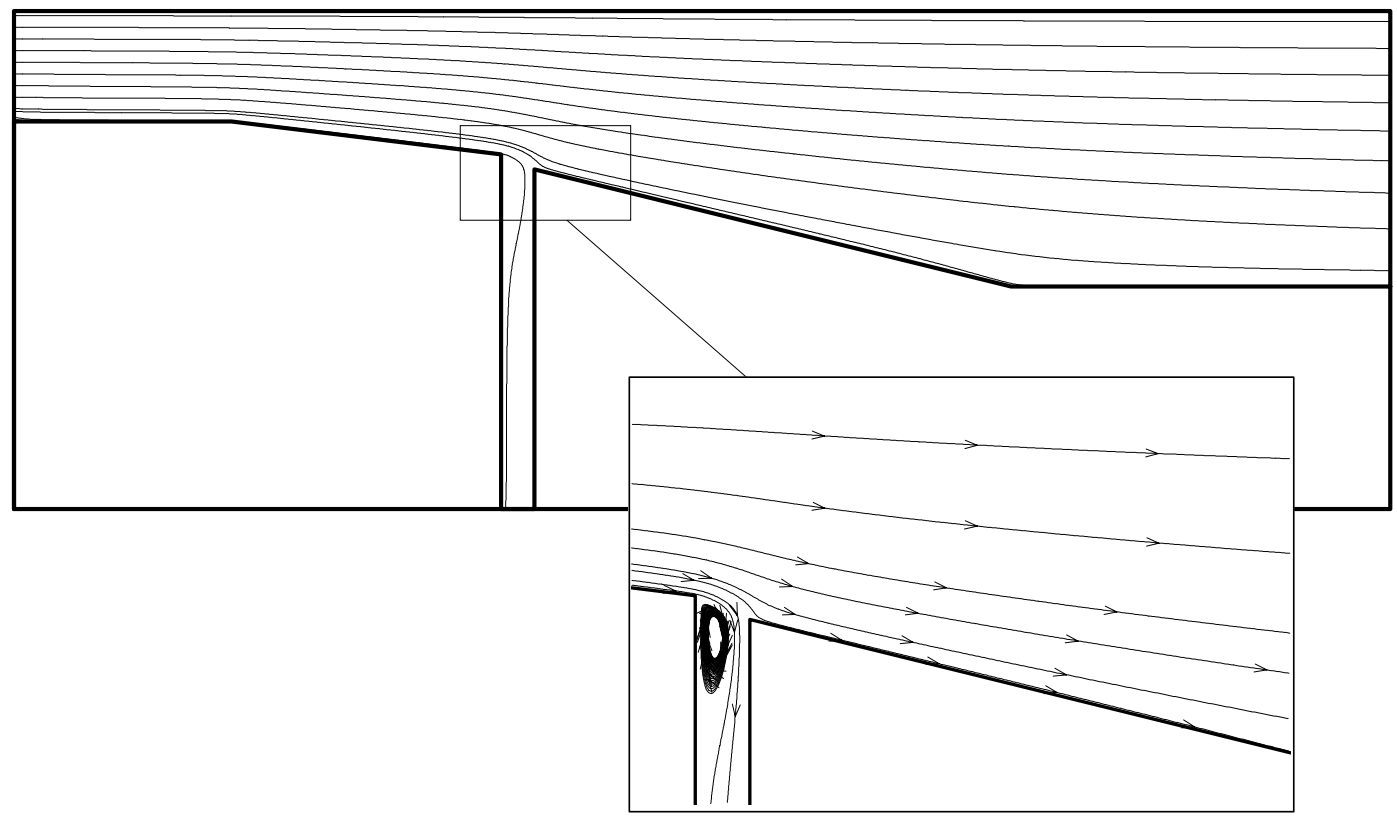

Figure 6.17 Streamline Plot - Alt. 1, 2.5\% Bleed

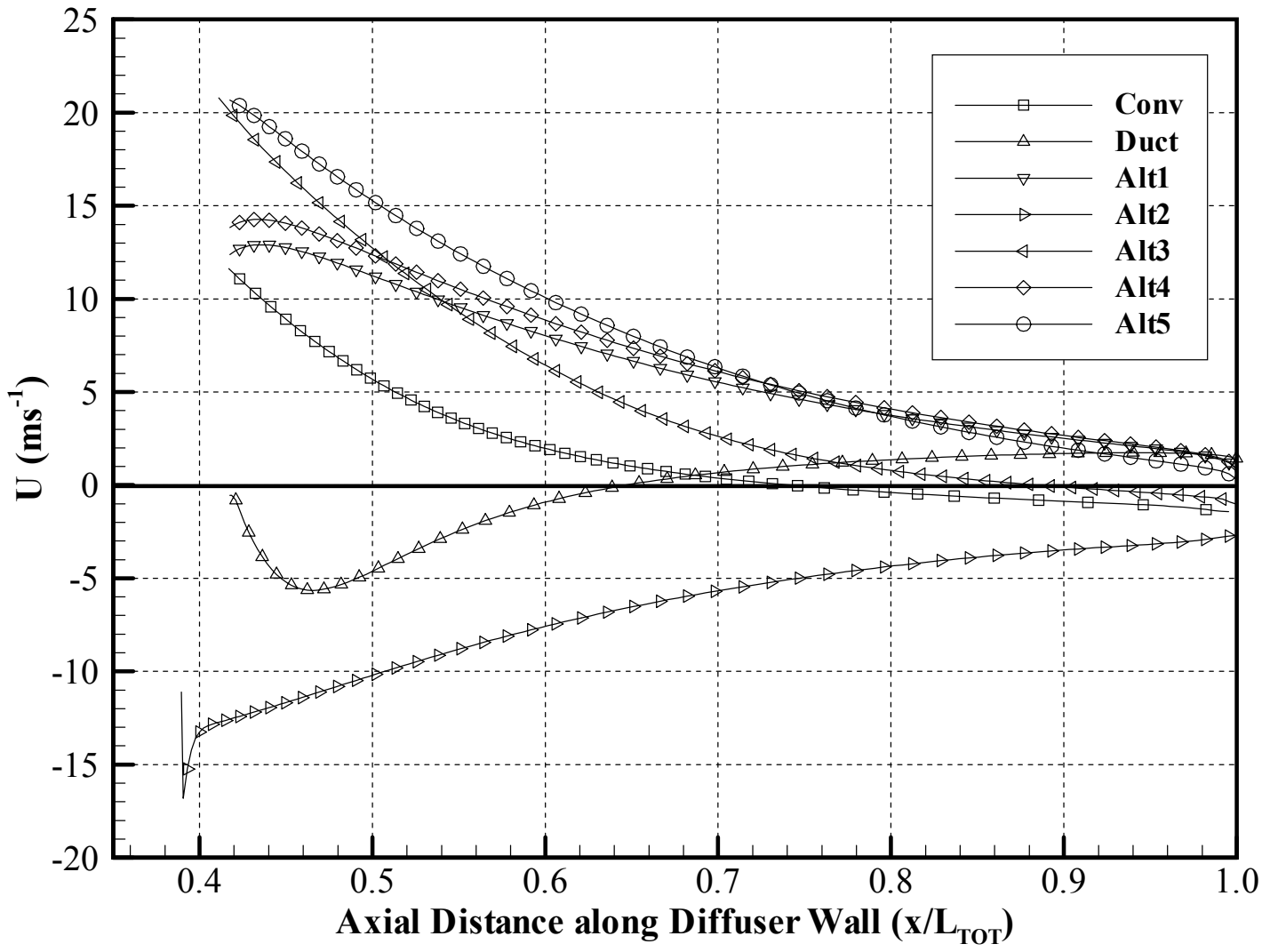

Figure 6.18 Cell Centred Axial Velocity in Wall Adjacent Cell, 2.5\% Bleed 


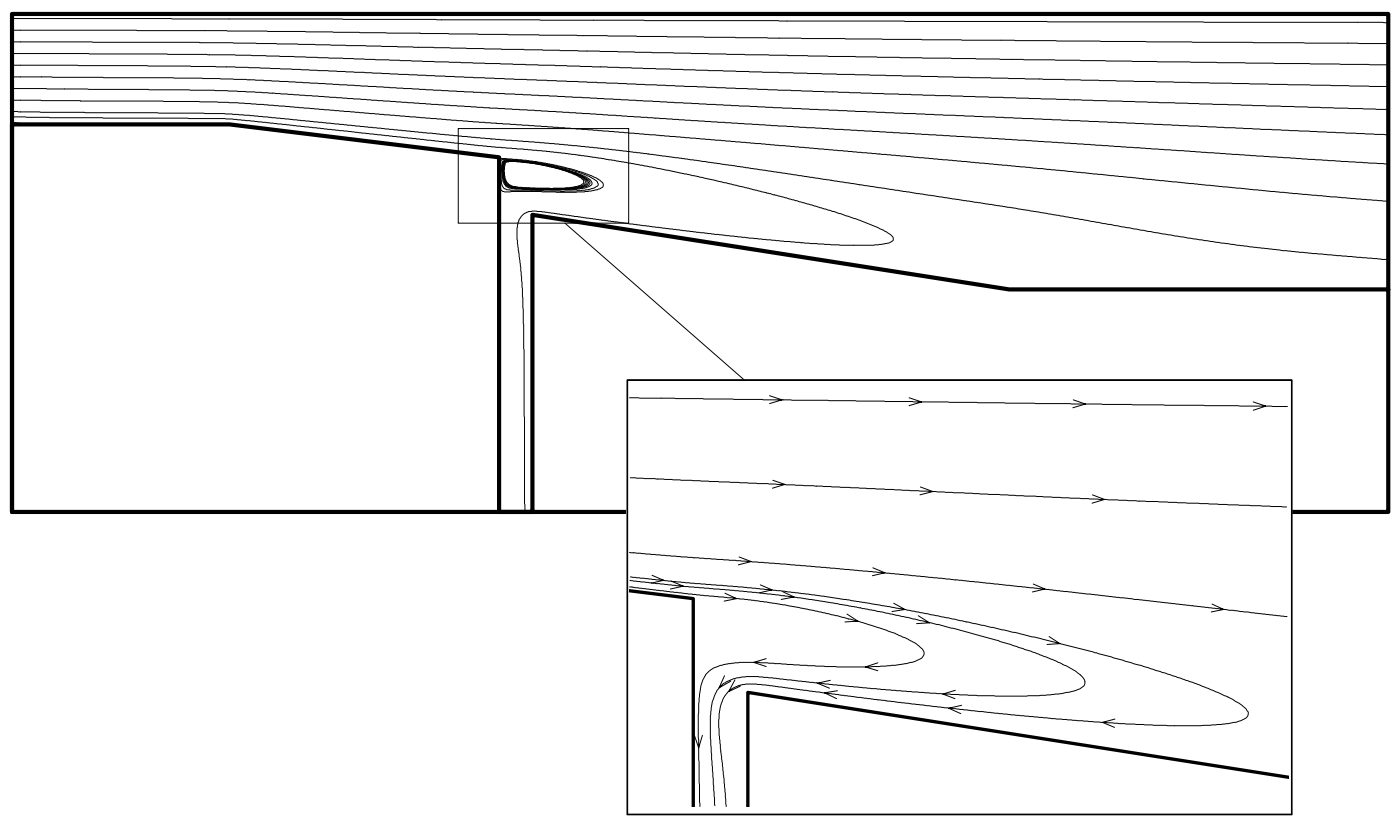

Figure 6.19 Streamline Plot - Alt.2, 2.5\% Bleed

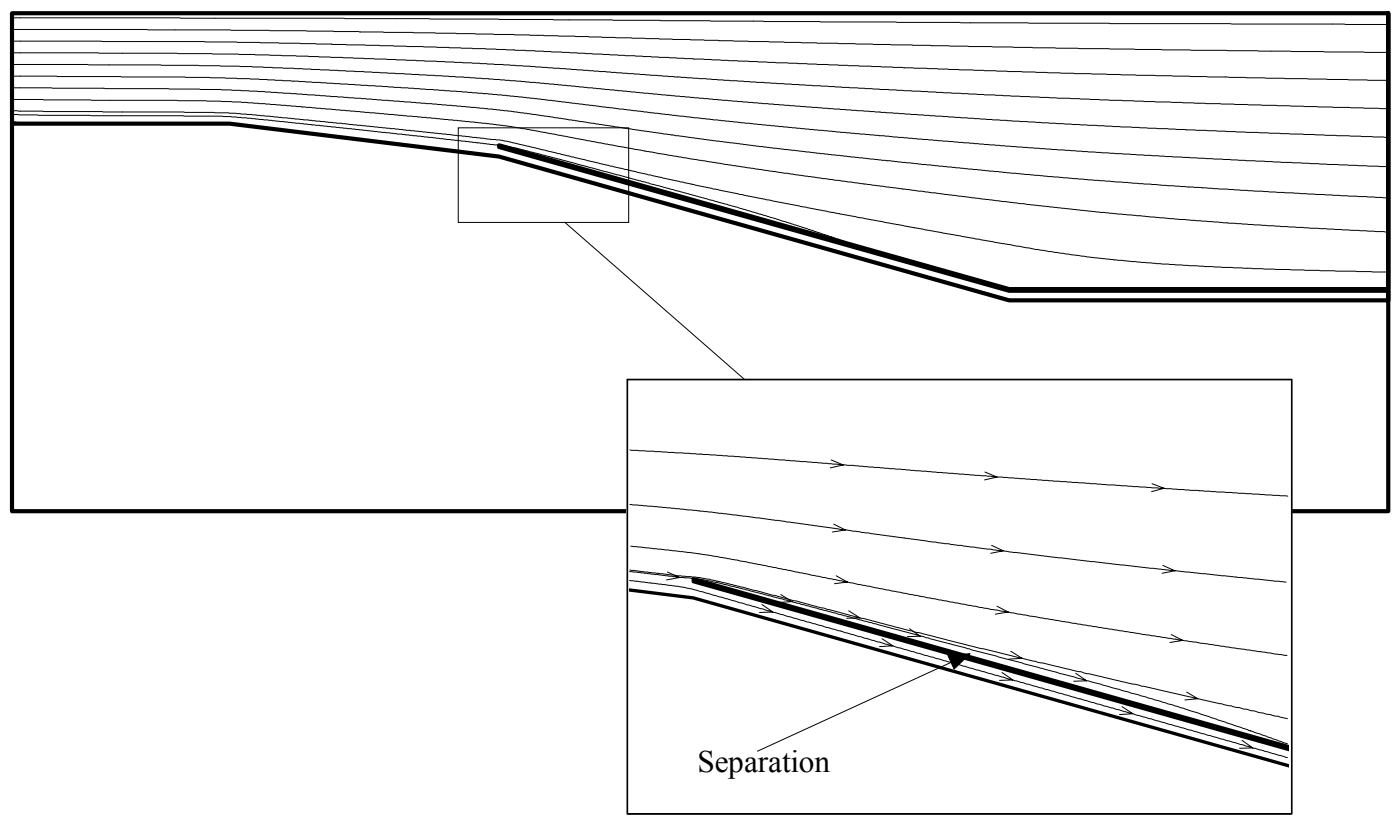

Figure 6.20 Streamline Plot - Alt.3, 2.5\% Bleed 


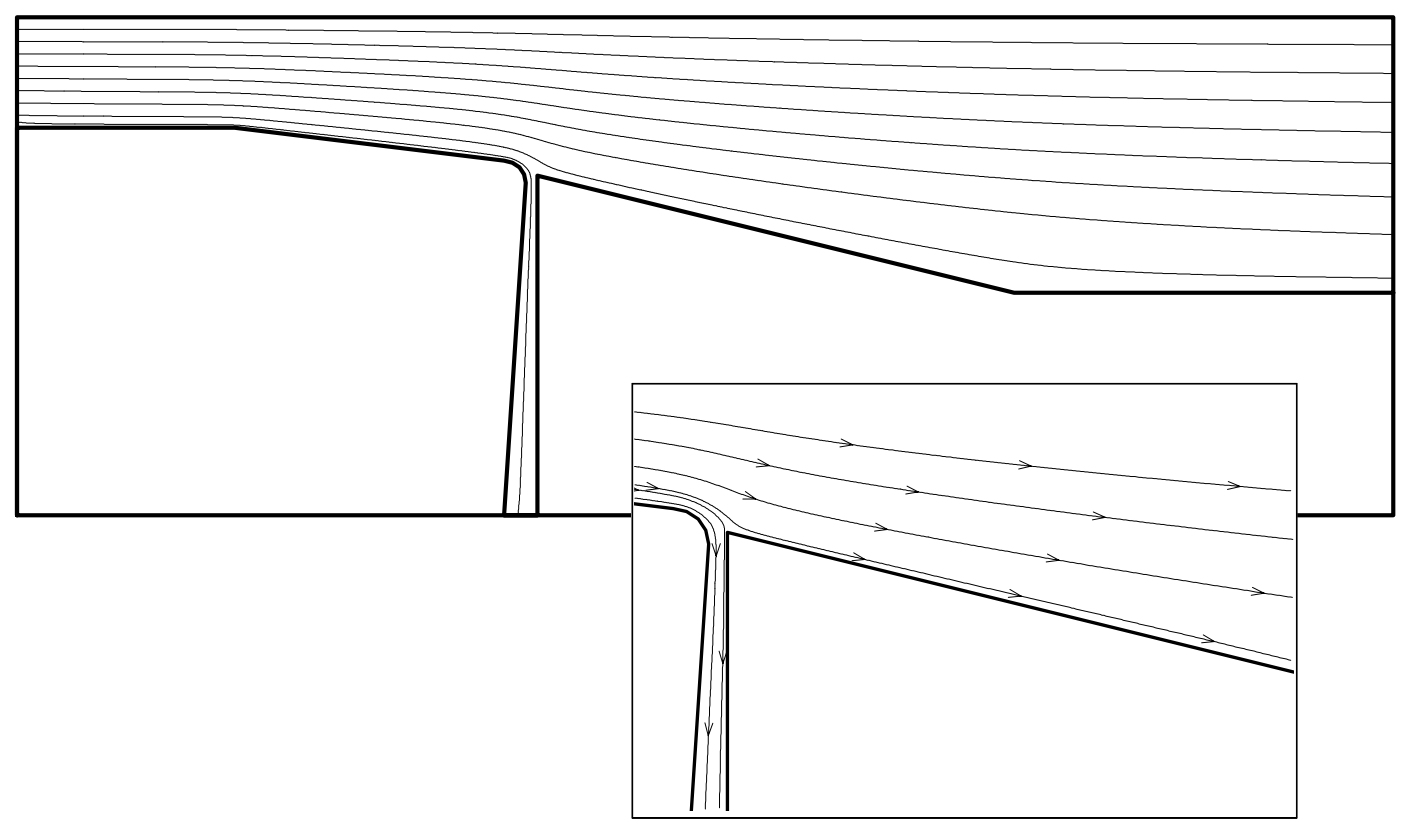

Figure 6.21 Streamline Plot - Alt.4, 2.5\% Bleed

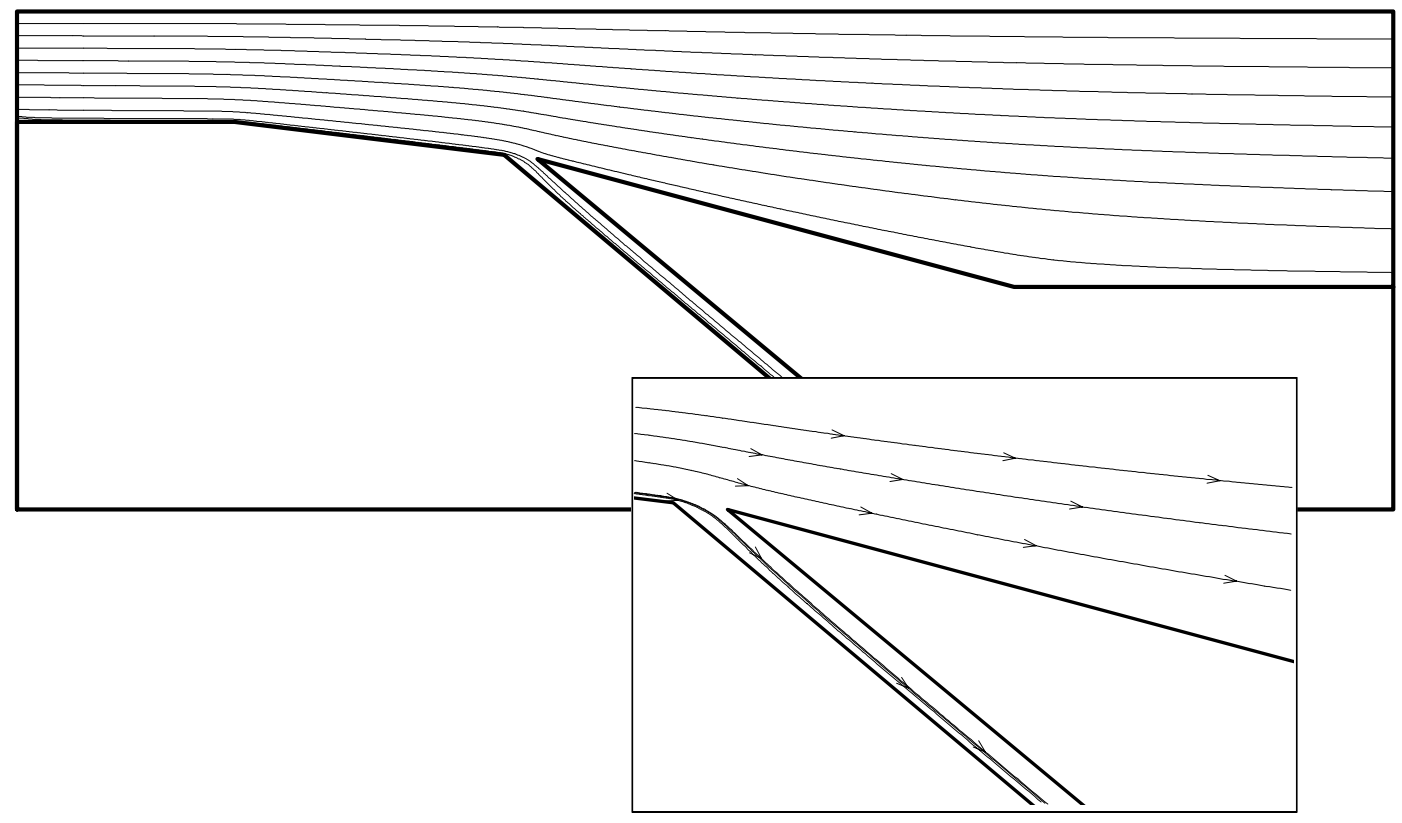

Figure 6.22 Streamline Plot - Alt.5, 2.5\% Bleed 


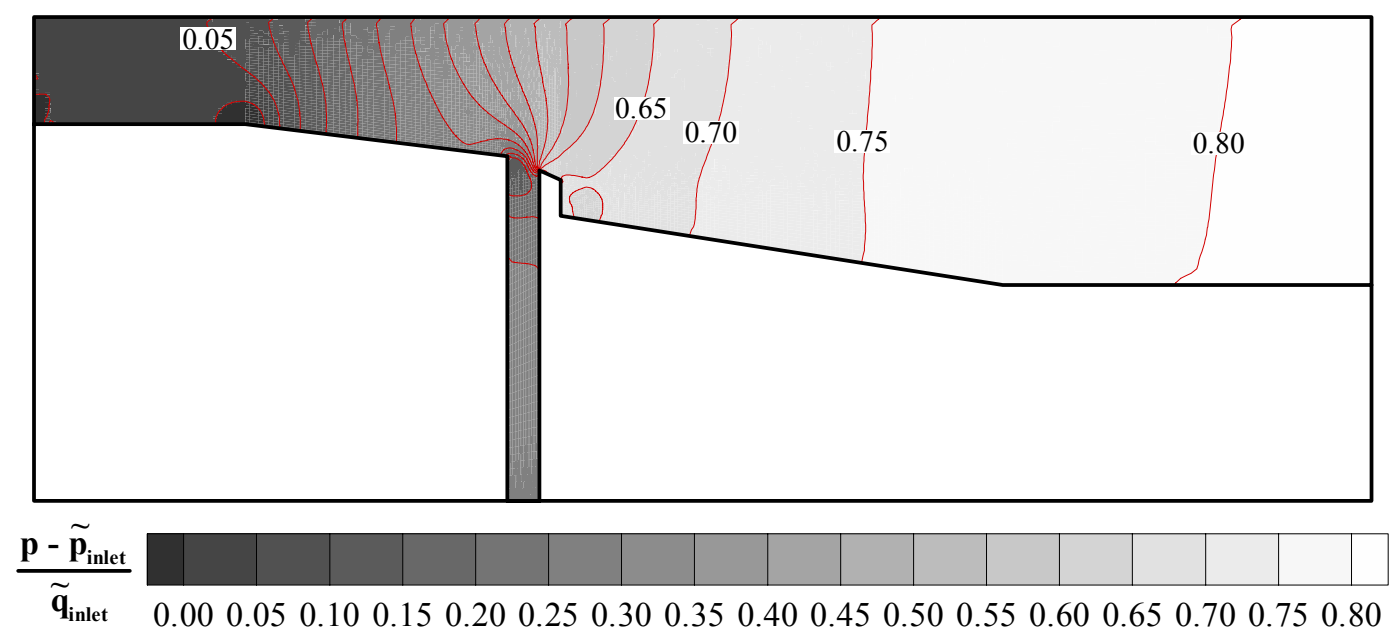

Figure 6.23 Static Pressure Contours - Duct, 2.5\% Bleed

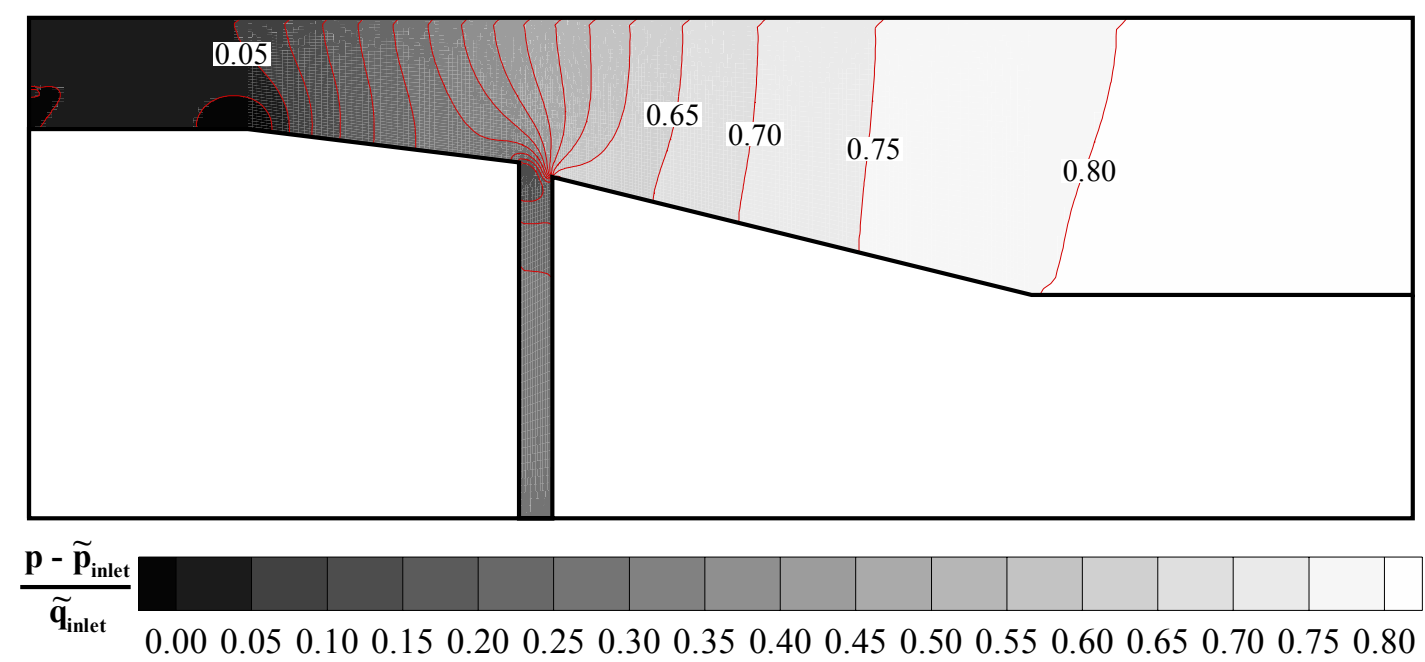

Figure 6.24 Static Pressure Contours - Alt.1, 2.5\% Bleed 


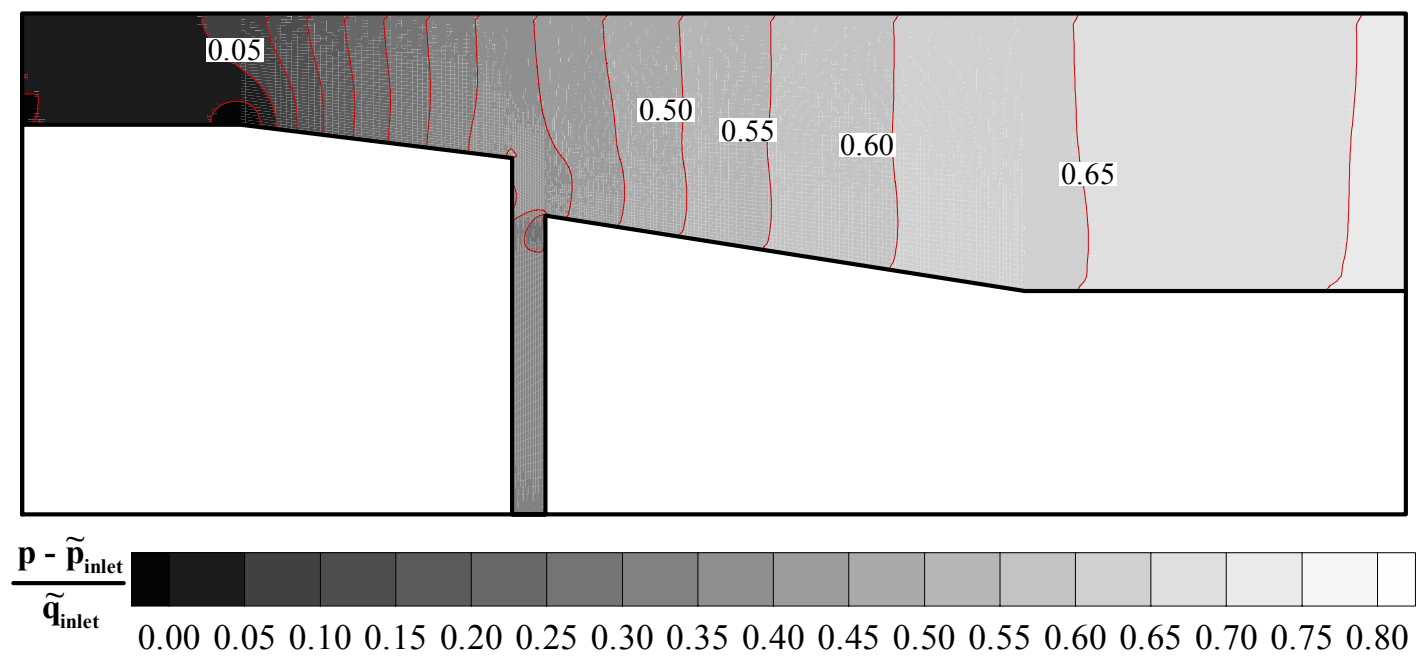

Figure 6.25 Static Pressure Contours - Alt.2, 2.5\% Bleed

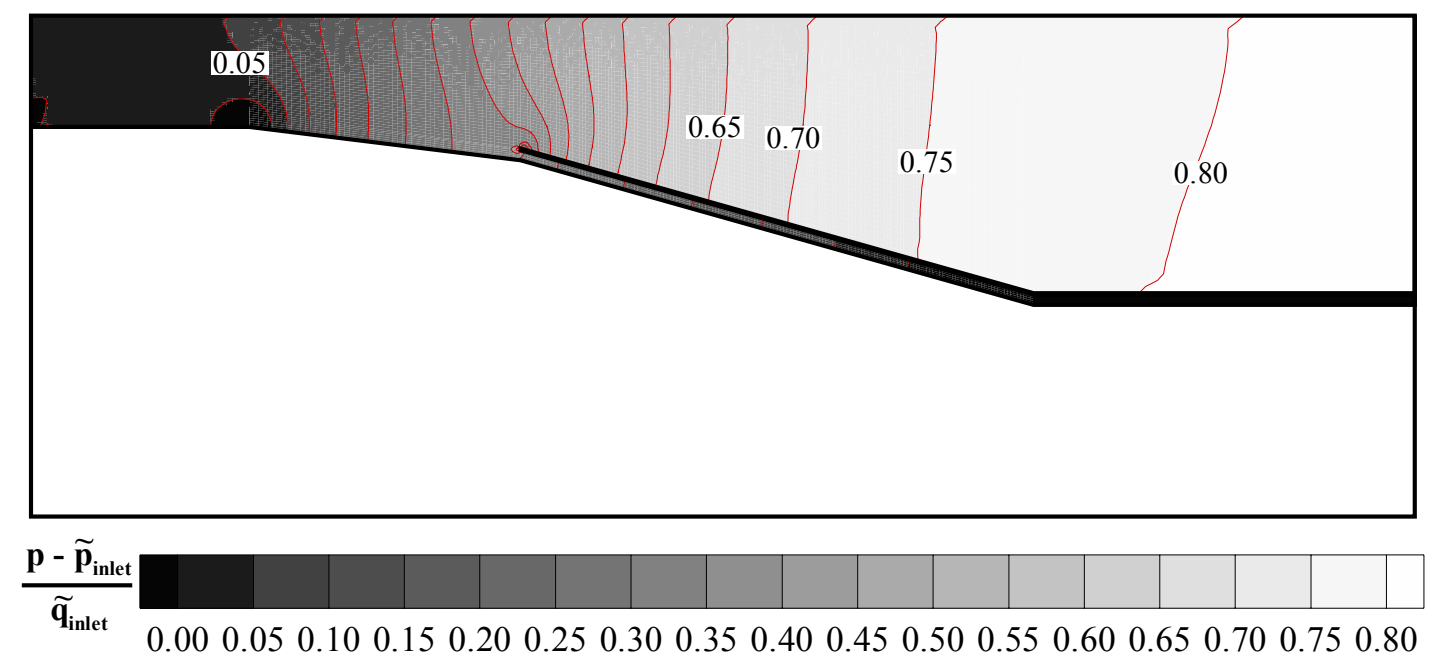

Figure 6.26 Static Pressure Contours - Alt.3, 2.5\% Bleed 


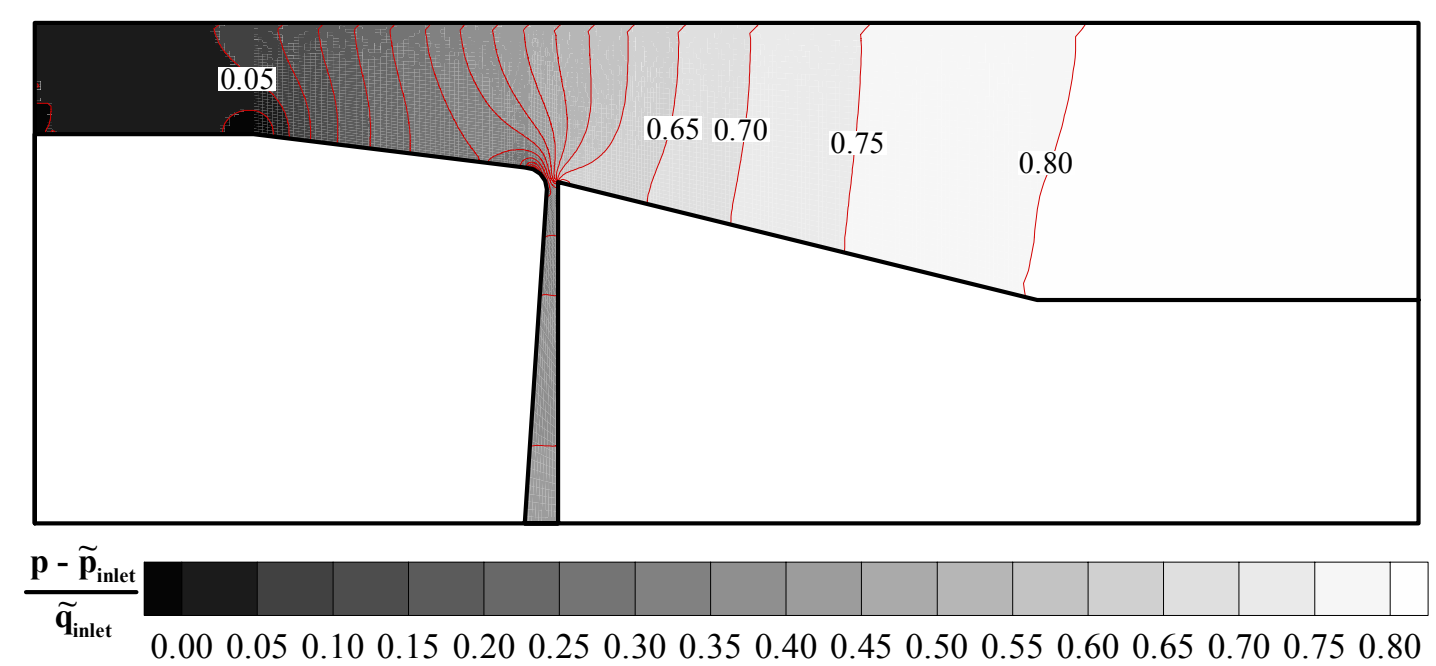

Figure 6.27 Static Pressure Contours - Alt.4, 2.5\% Bleed

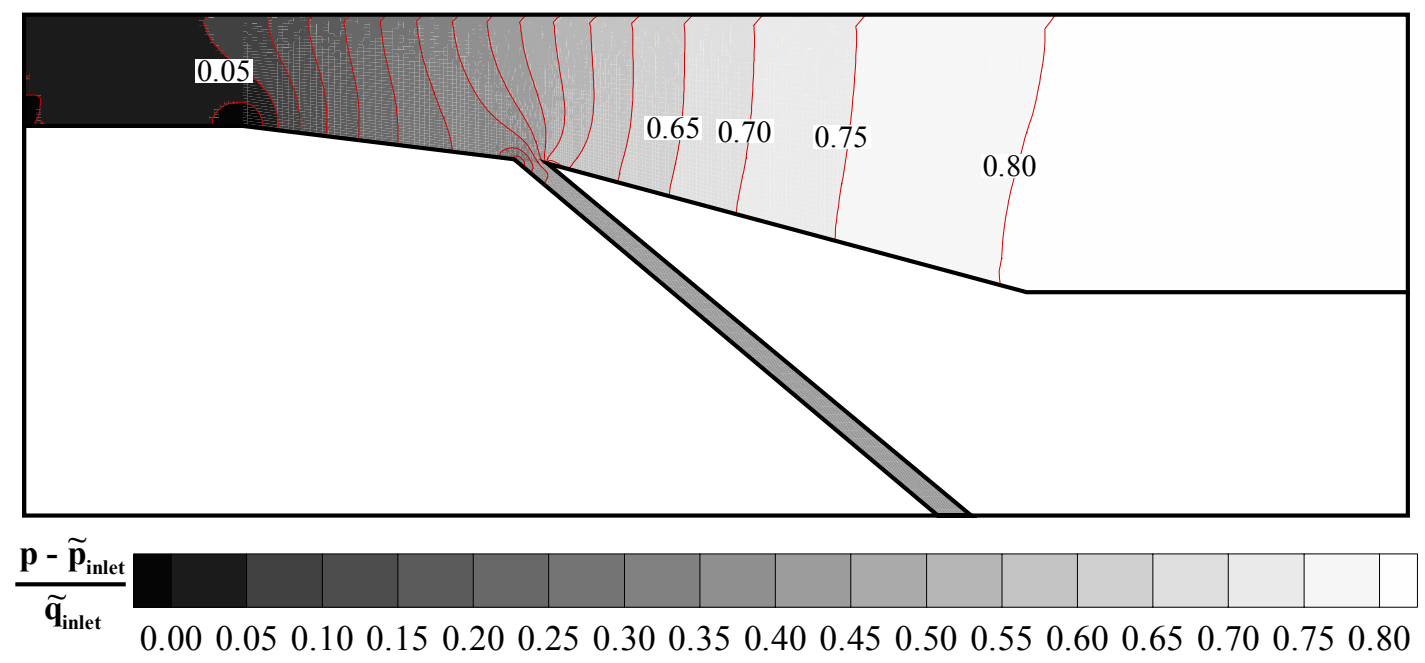

Figure 6.28 Static Pressure Contours - Alt.5, 2.5\% Bleed 


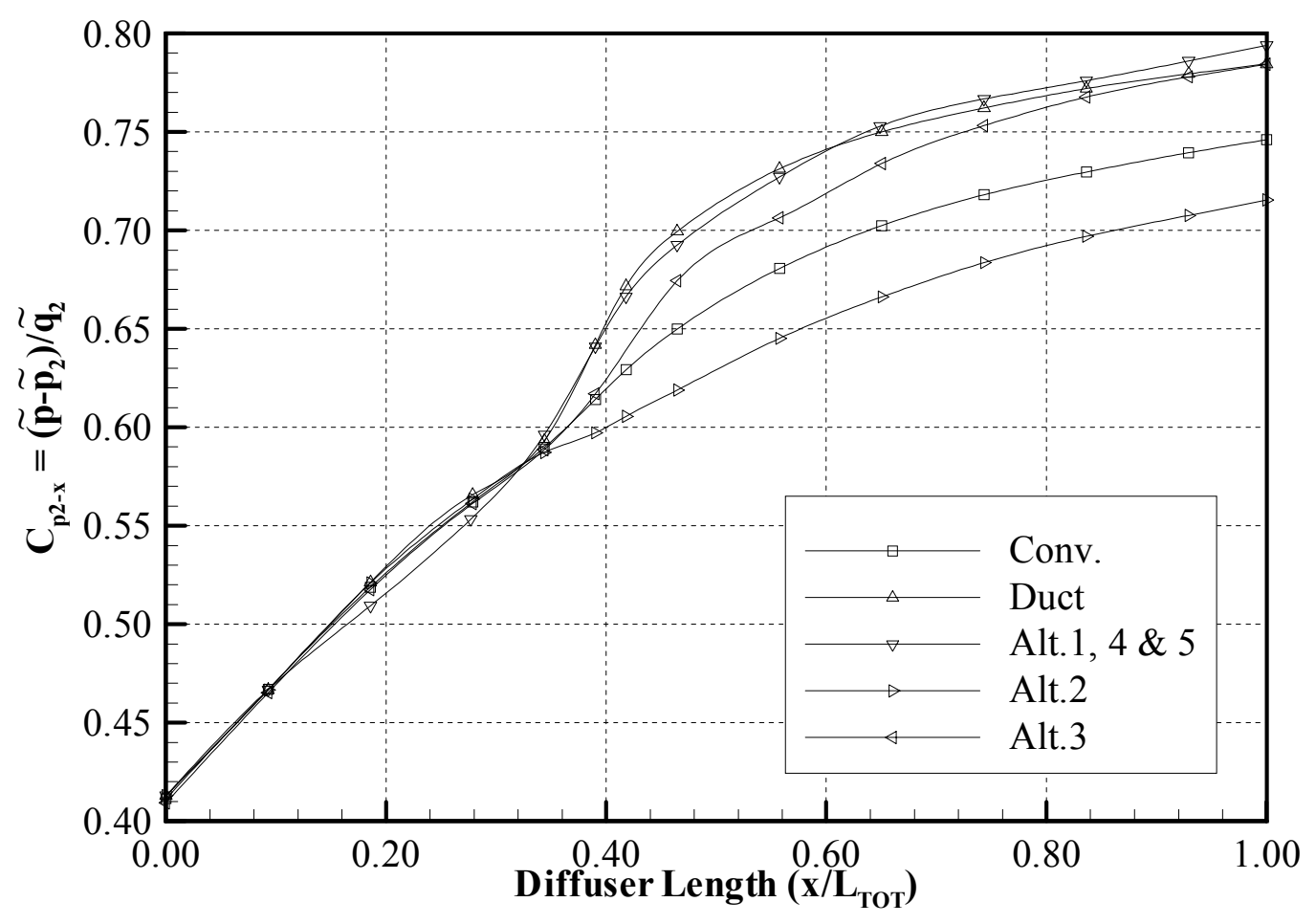

Figure 6.29 Development of Mass Weighted Static Pressure Recovery

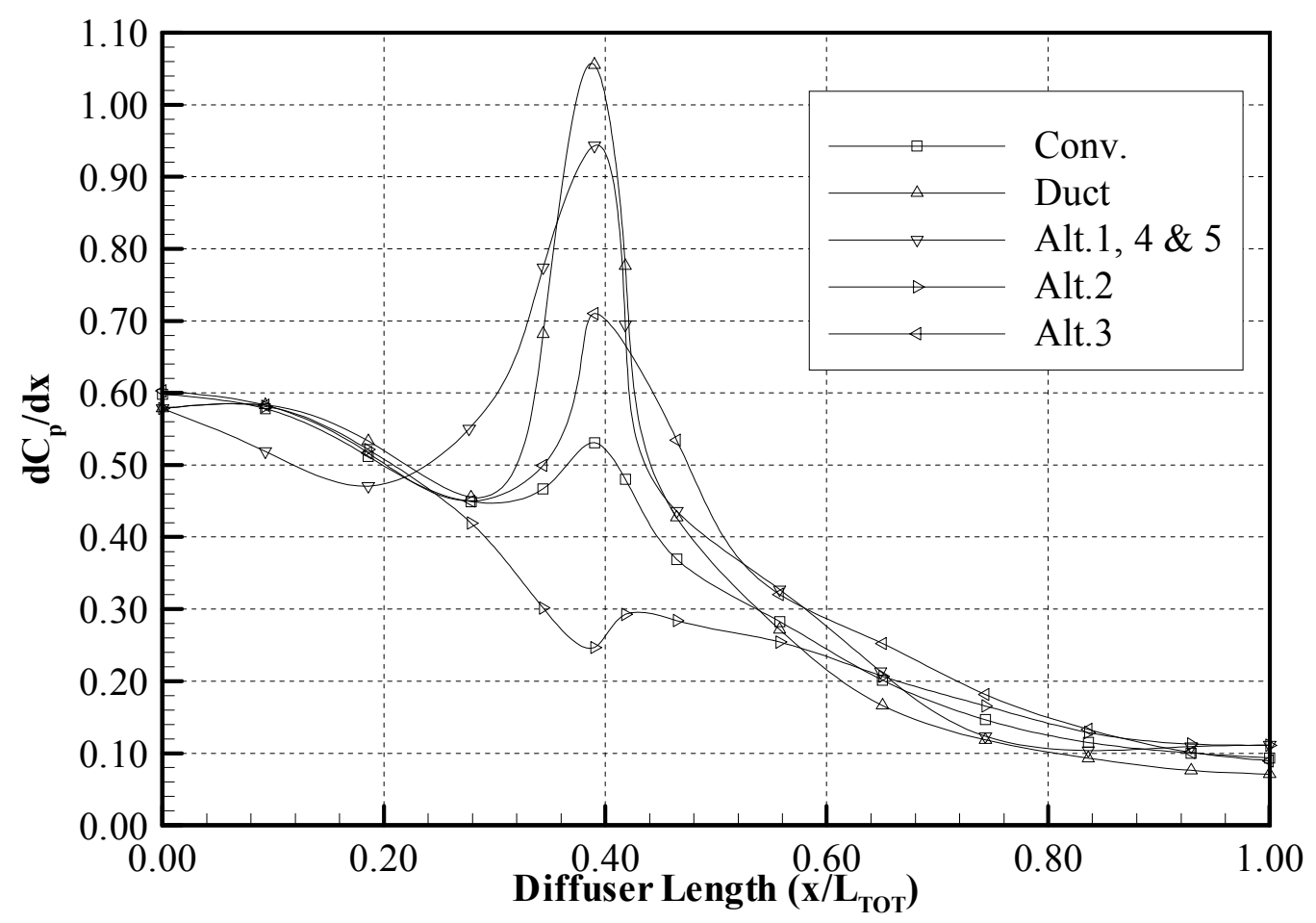

Figure 6.30 Rate of Mass Weighted Static Pressure Recovery 


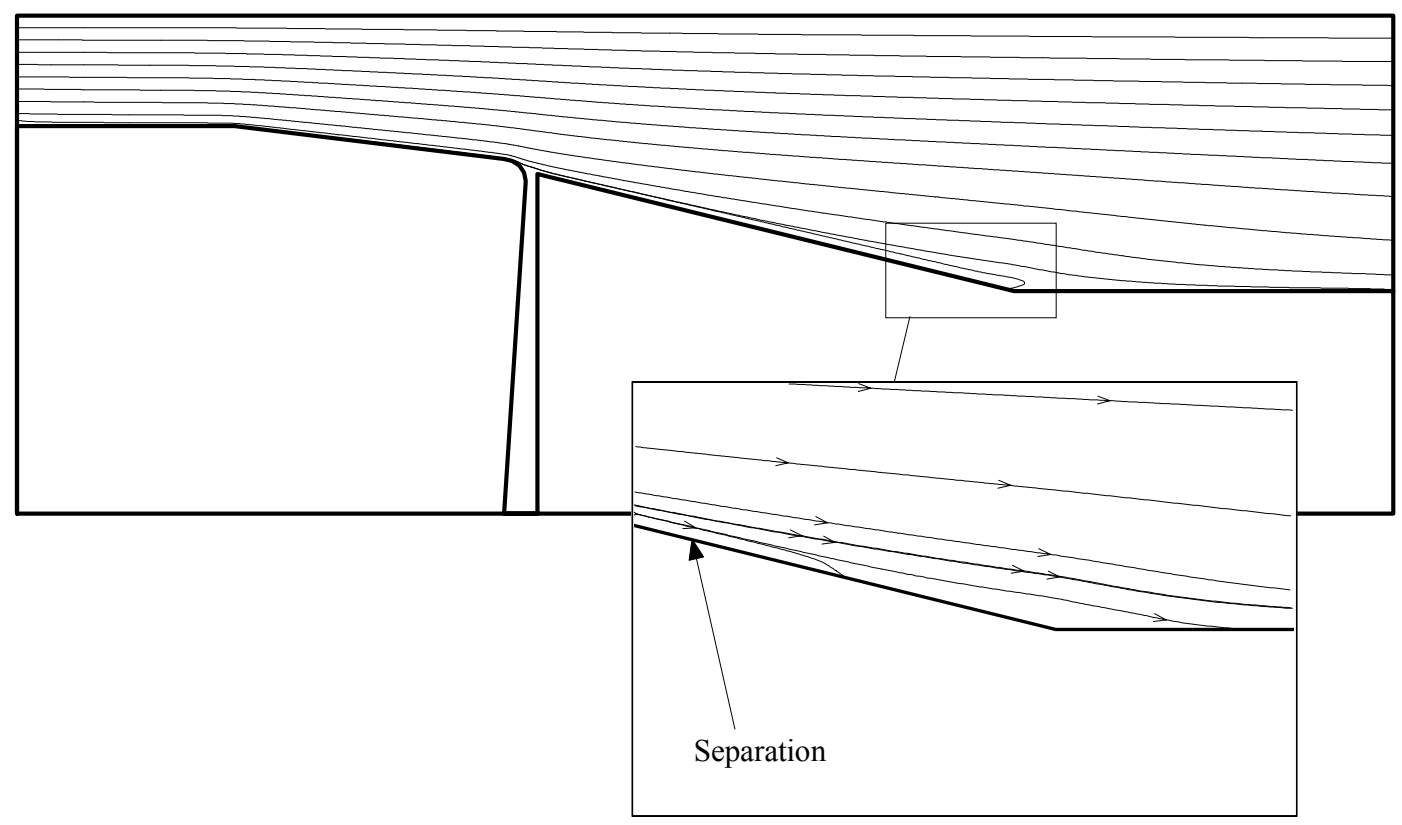

Figure 6.31 Streamline Plot - Alt.4, 0\% Bleed, Stalled

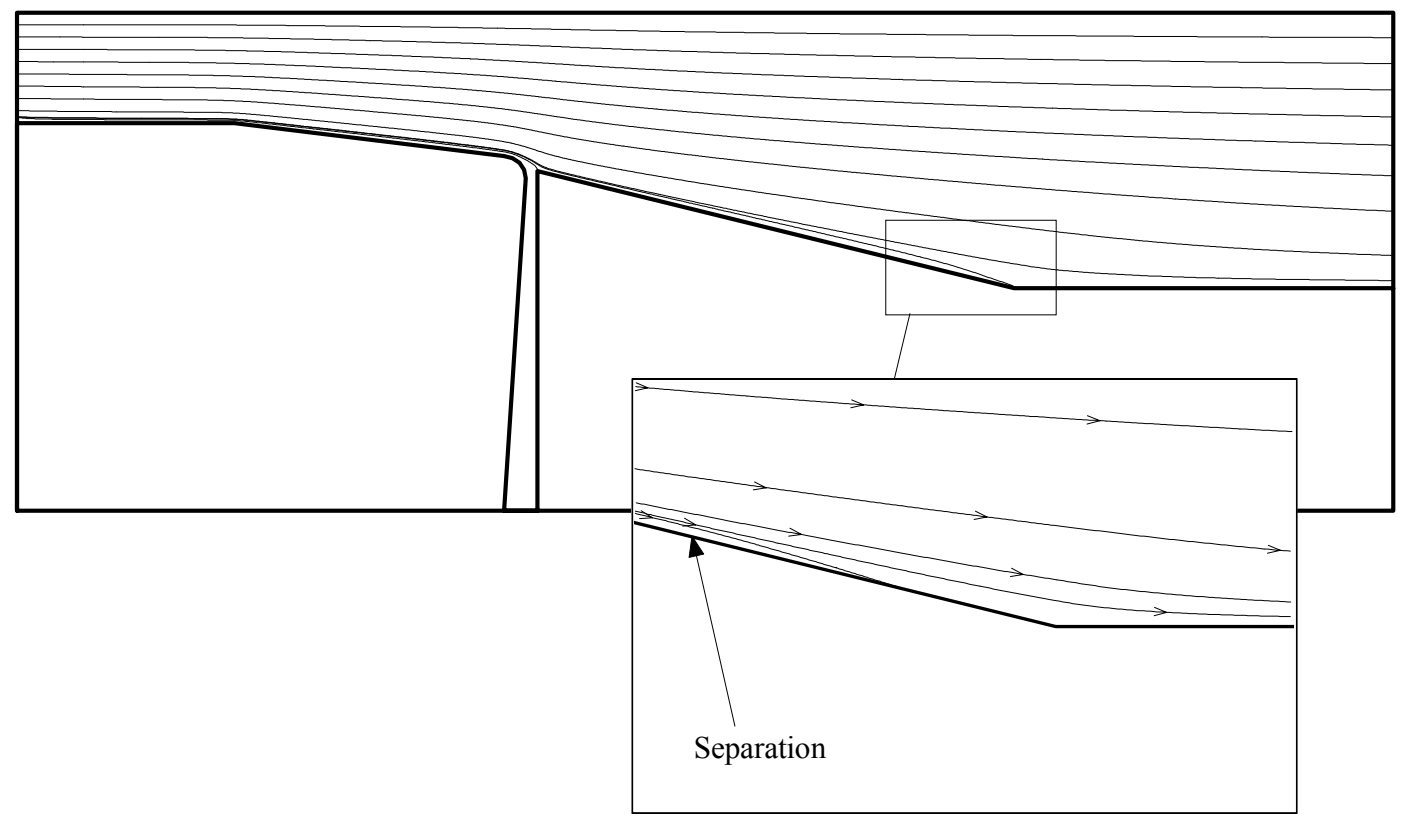

Figure 6.32 Streamline Plot - Alt.4, 1.0\% Bleed, Stalled 


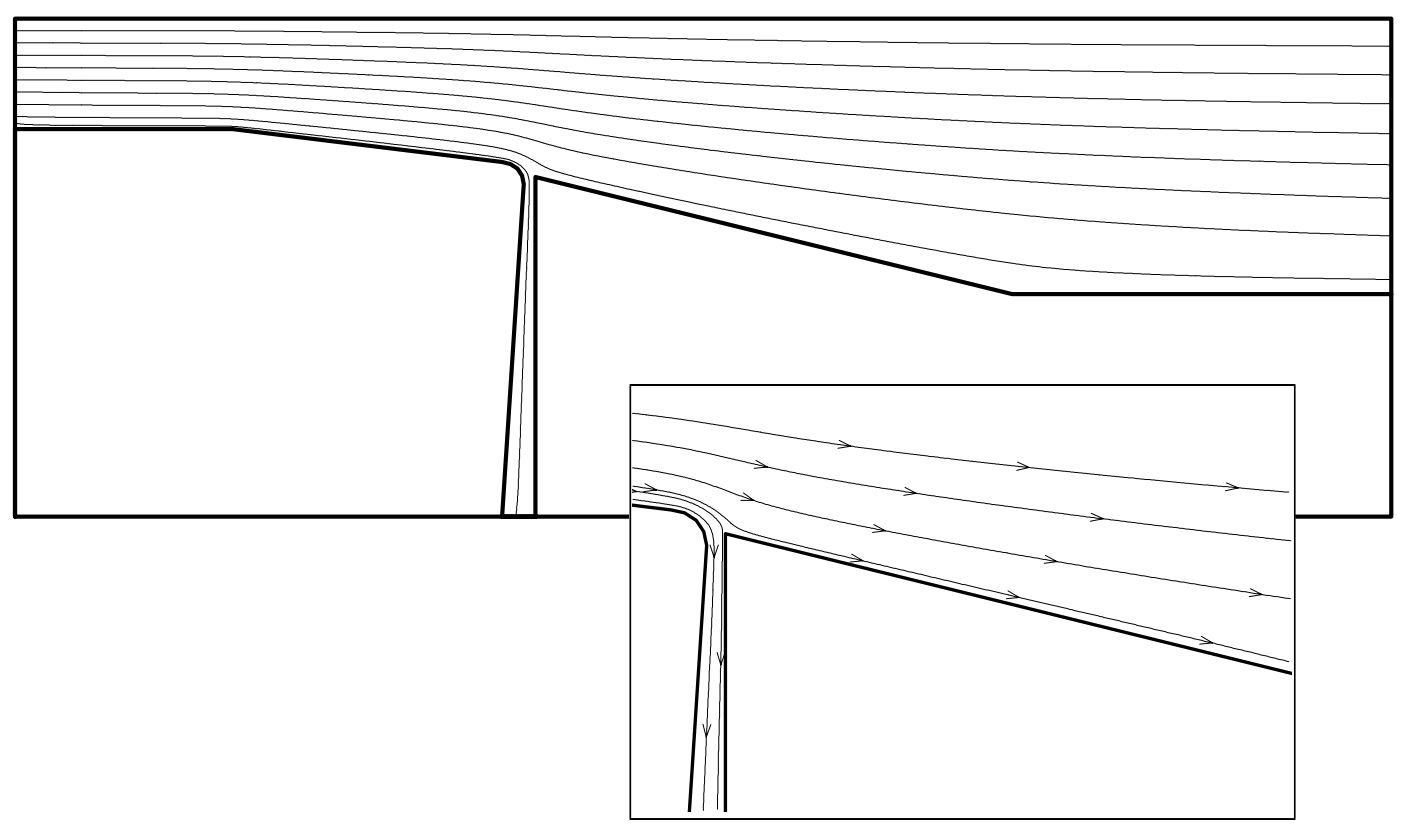

Figure 6.33 Streamline Plot - Alt.4, 2.5\% Bleed

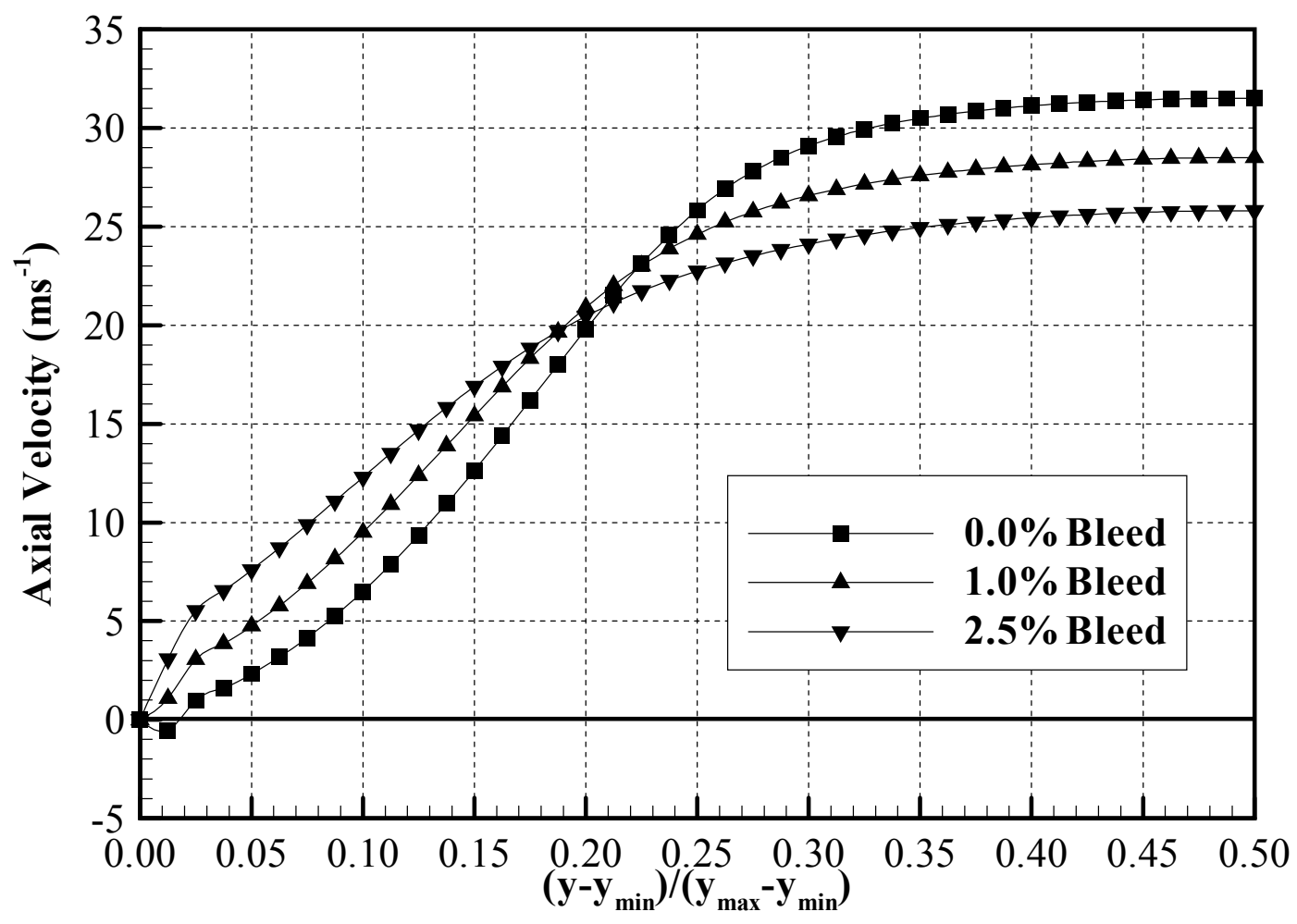

Figure 6.34 Axial Velocity Profiles at Diffuser Exit, Alt.4 


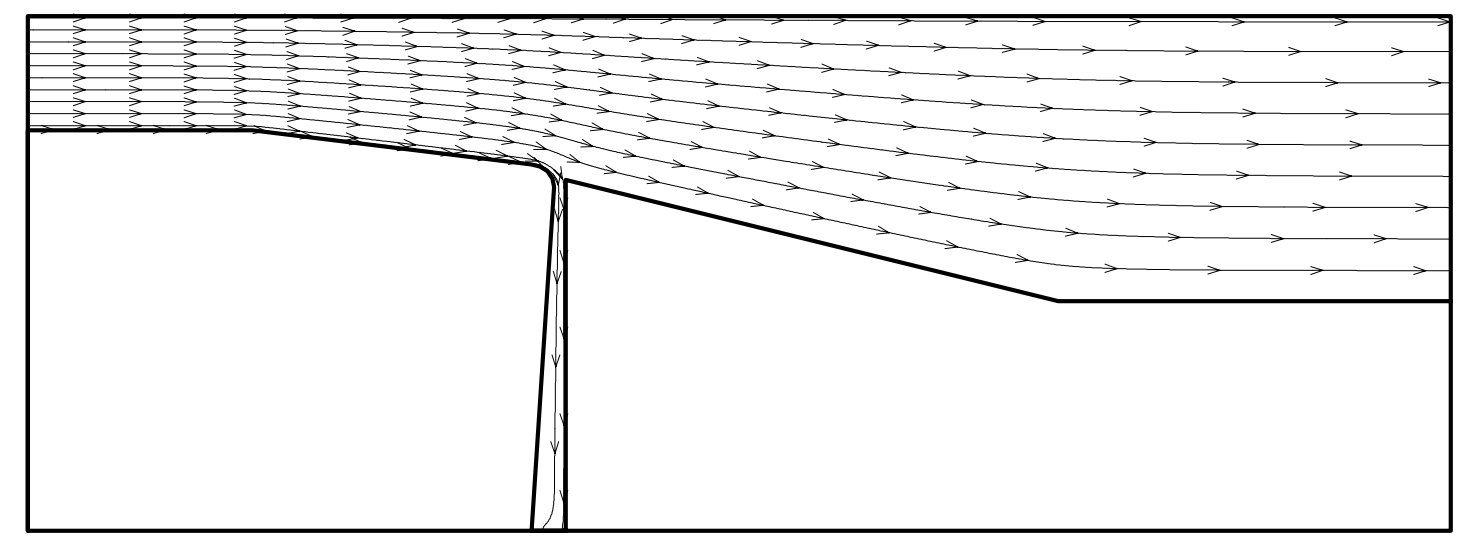

Figure 6.35 Inviscid Streamline Plot (2.5\% Bleed)
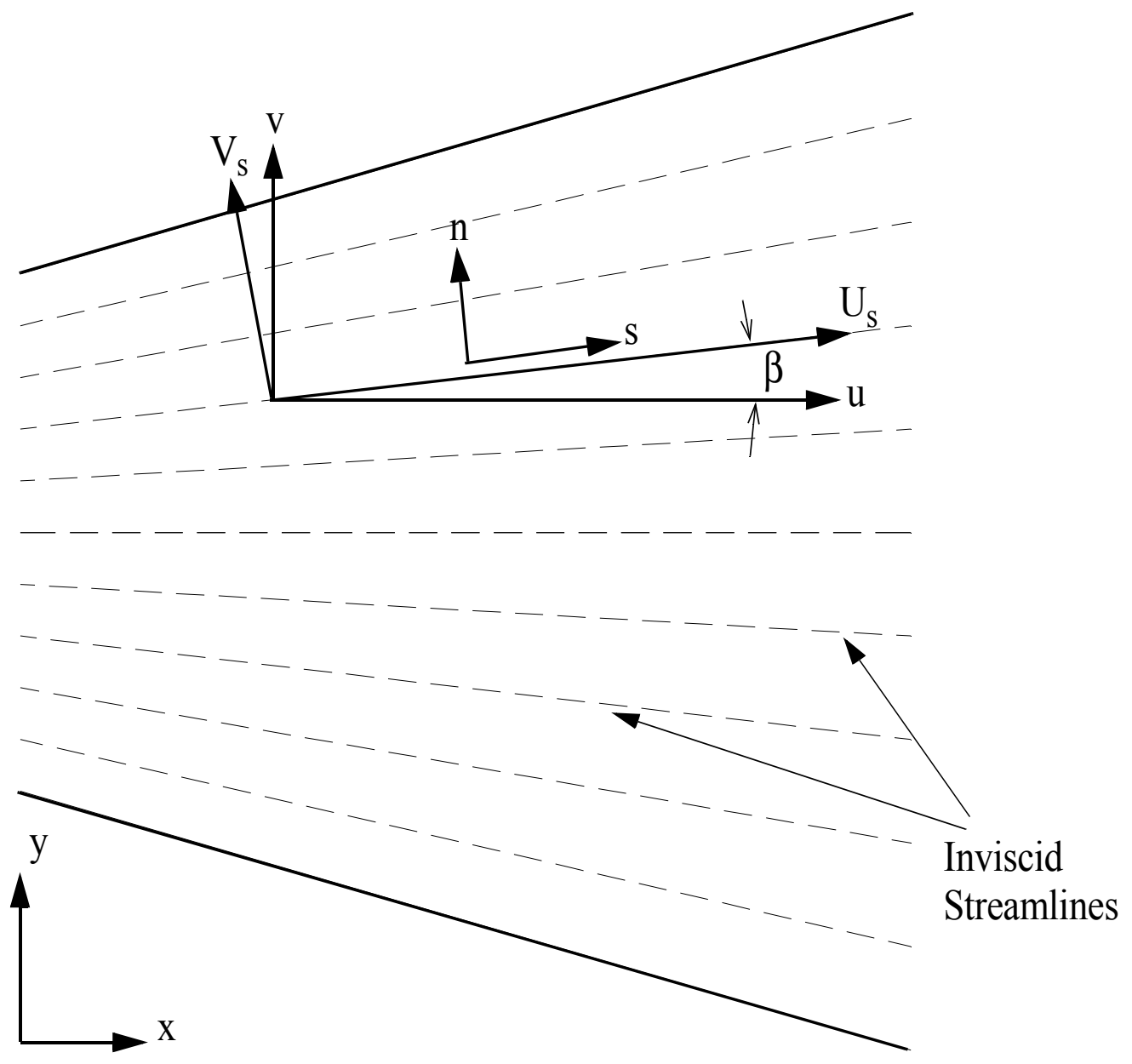

Figure 6.36 Diffuser Nomenclature (Momentum Analysis) 


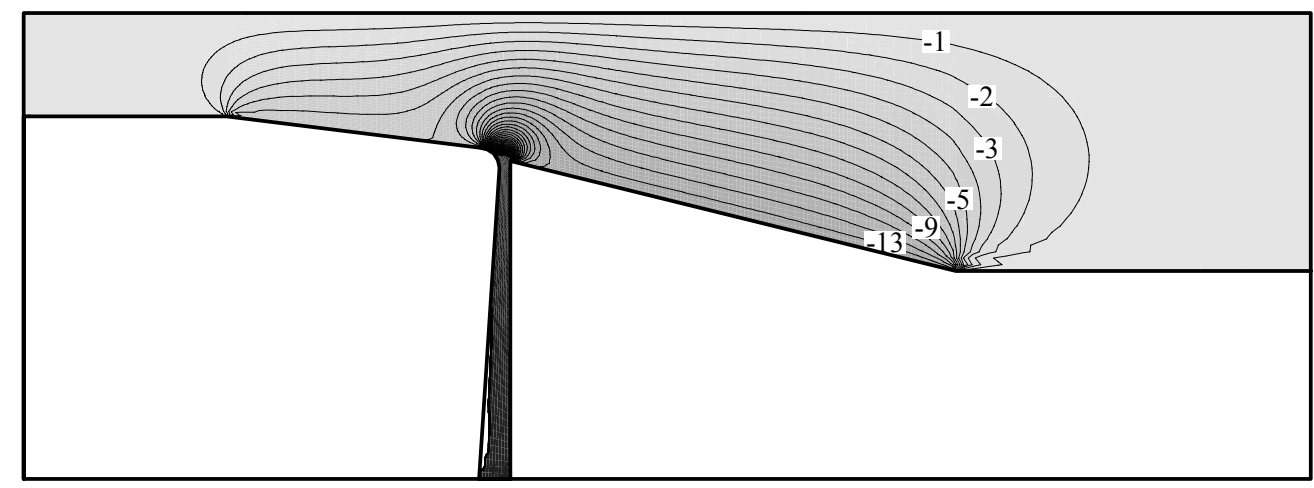

$\beta$ (deg.)

$\begin{array}{lllllllllll}-45 & -40 & -35 & -30 & -25 & -20 & -15 & -10 & -5 & 0 & 5\end{array}$

Figure 6.37 Typical Variation of $\boldsymbol{\beta}$ from Inviscid Solution

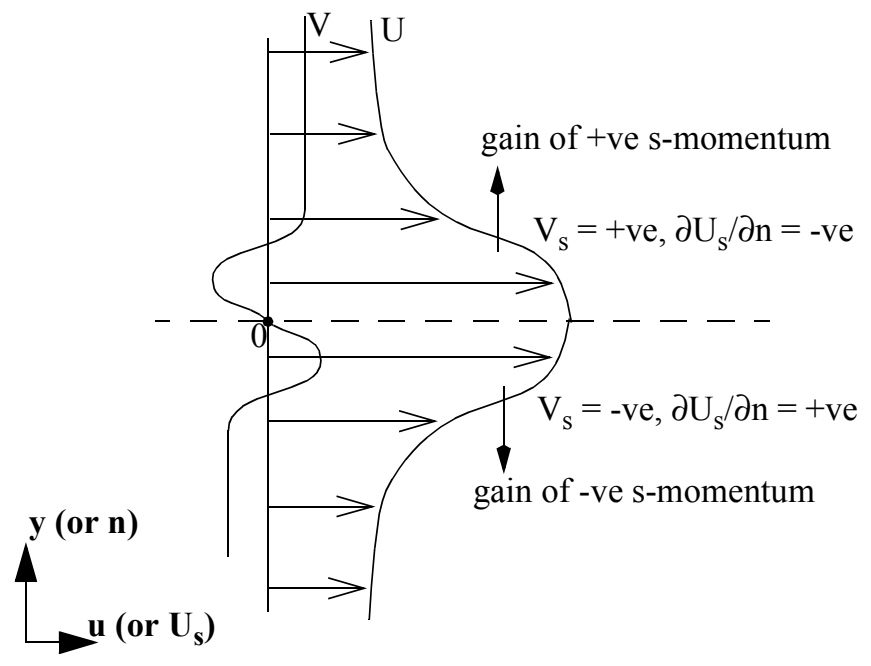

Figure 6.38 Gain/Loss Processes of Streamwise Momentum

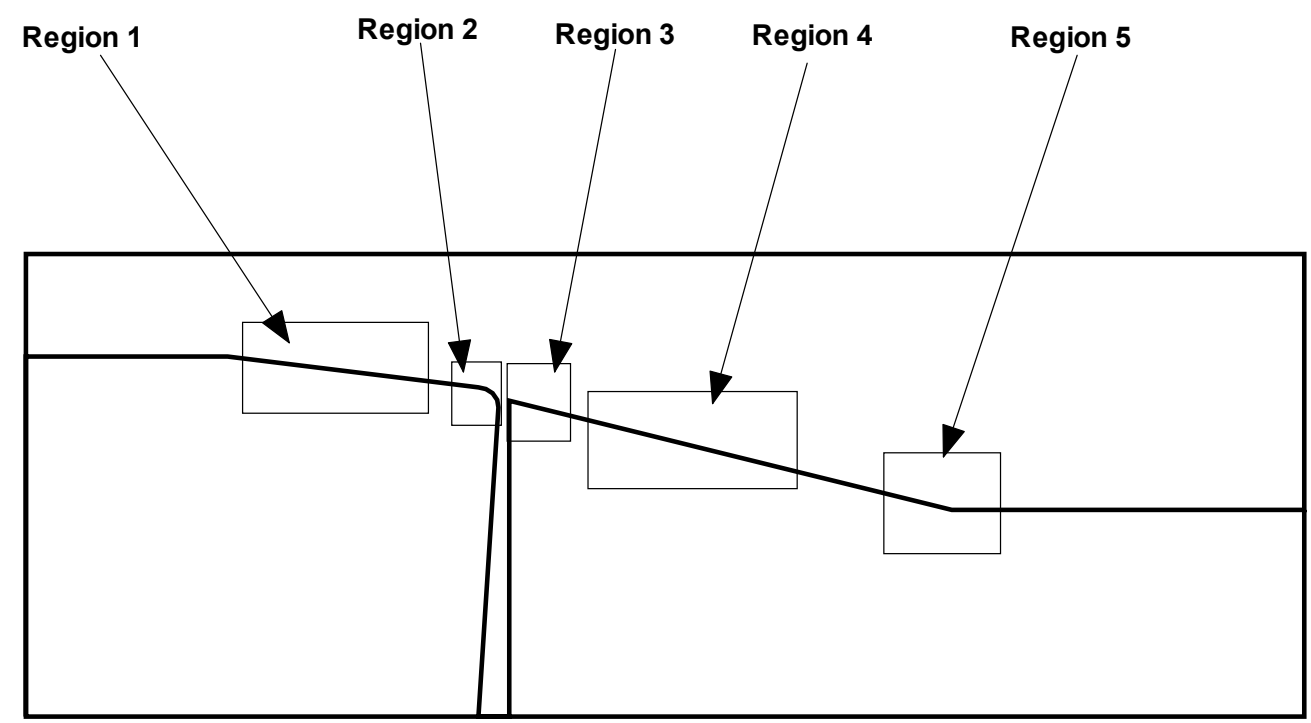

Figure 6.39 Flow Regions Within Alt.4 Hybrid Diffuser 

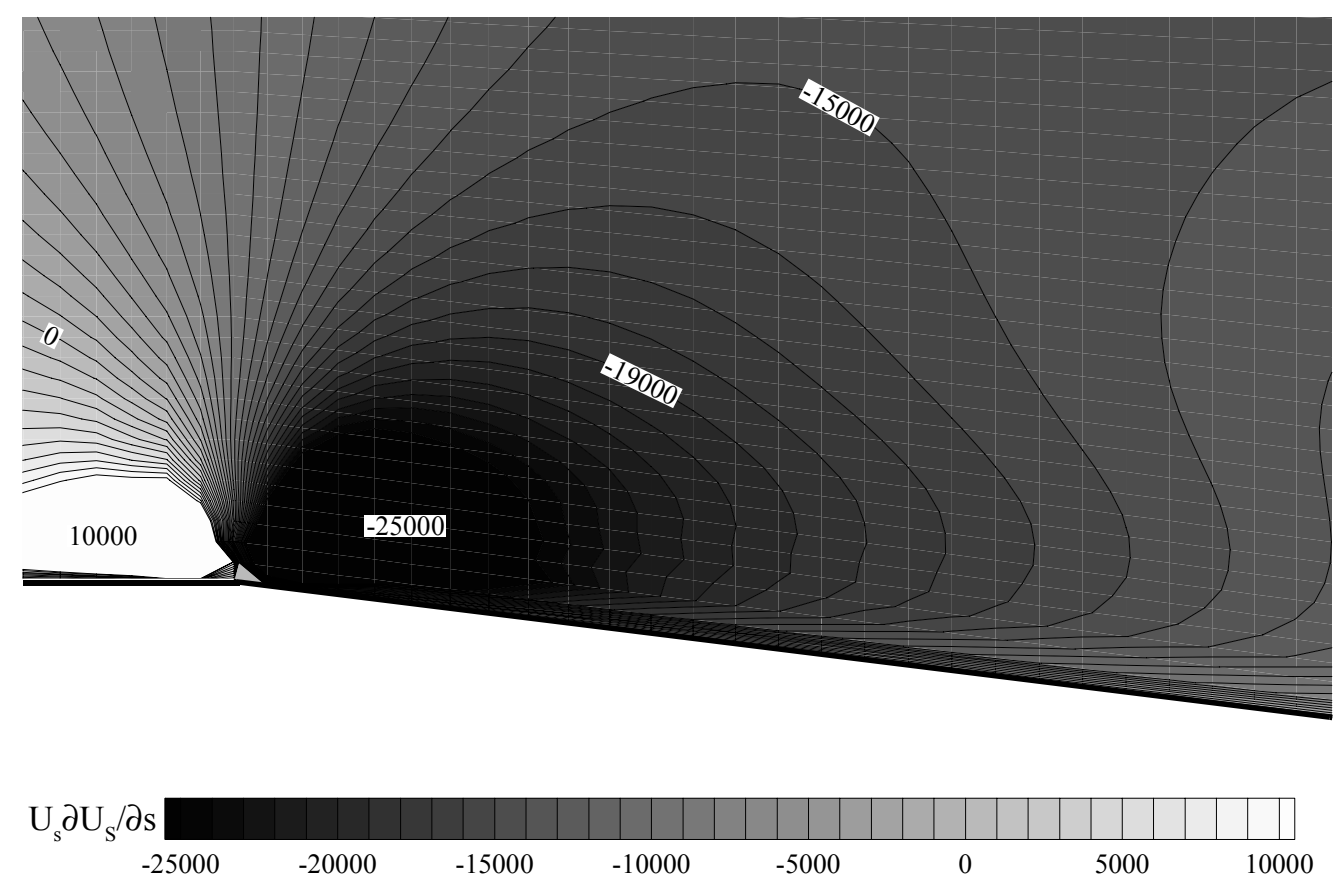

Figure 6.40 Contours of $U_{s} \frac{\partial U_{s}}{\partial s}$, Alt. 4, Region 1, 2.5\% Bleed
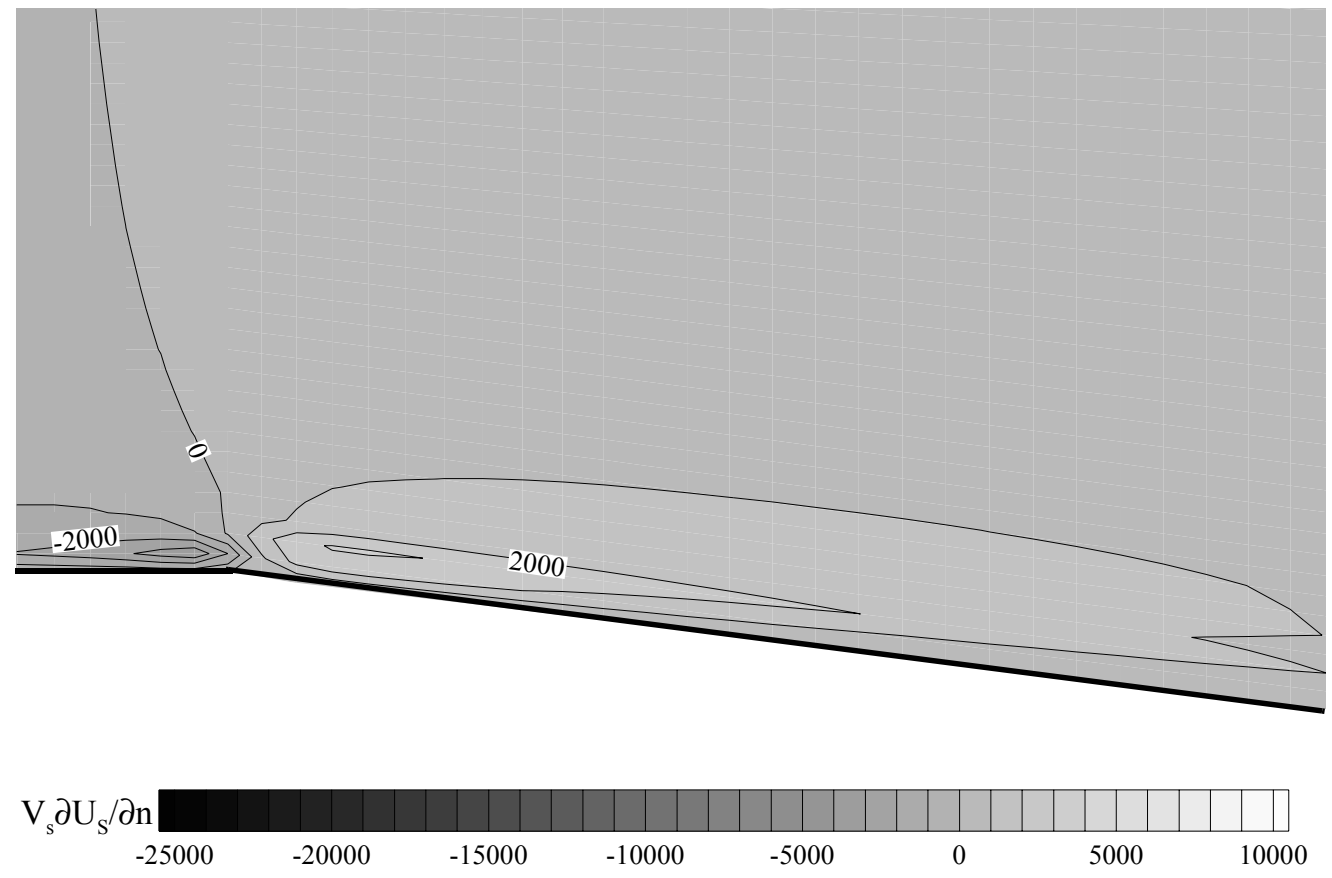

Figure 6.41 Contours of $v_{s} \frac{\partial U_{s}}{\partial n}$, Alt. 4, Region 1, 2.5\% Bleed 
$-18000$

$-15000$

$-25000$

$-13000$

$-1 / \rho . \partial \mathrm{p} / \partial \mathrm{s}$

$-25000$

$-20000$

$-15000$

$-10000$

$-5000$

5000

10000

Figure 6.42 Contours of $-\frac{1}{\rho} \frac{\partial p}{\partial \mathrm{s}}$, Alt. 4, Region 1, 2.5\% Bleed

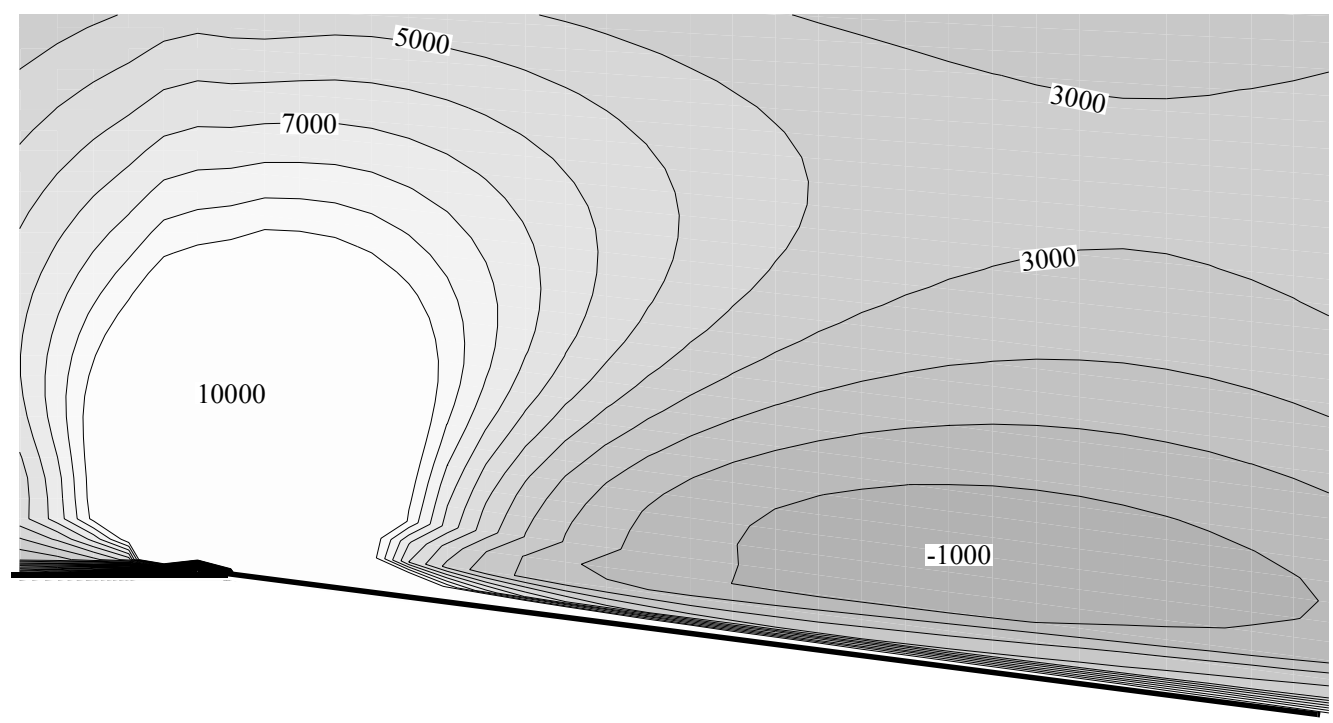

$-(\partial \overline{u u} / \partial s+\partial \overline{u v} / \partial n)$

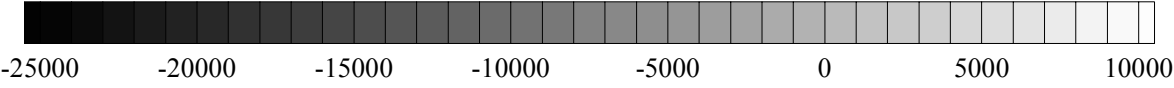

Figure 6.43 Contours of $-\left(\frac{\partial}{\partial \mathrm{s}} \overline{\mathrm{u}}+\frac{\partial}{\partial \mathrm{n}} \overline{\mathrm{uv}}\right)$, Alt. 4, Region 1, 2.5\% Bleed 


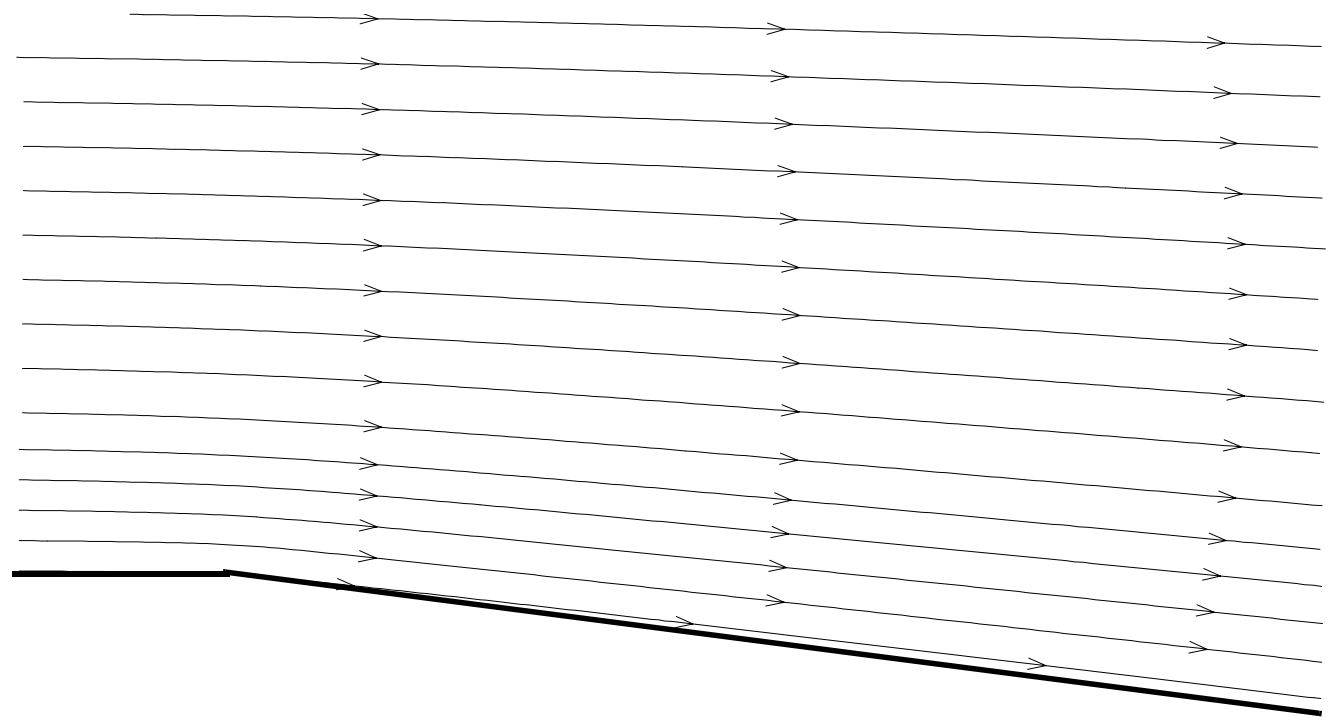

Figure 6.44 Turbulent Flow Streamlines, Alt. 4, Region 1, 2.5\% Bleed

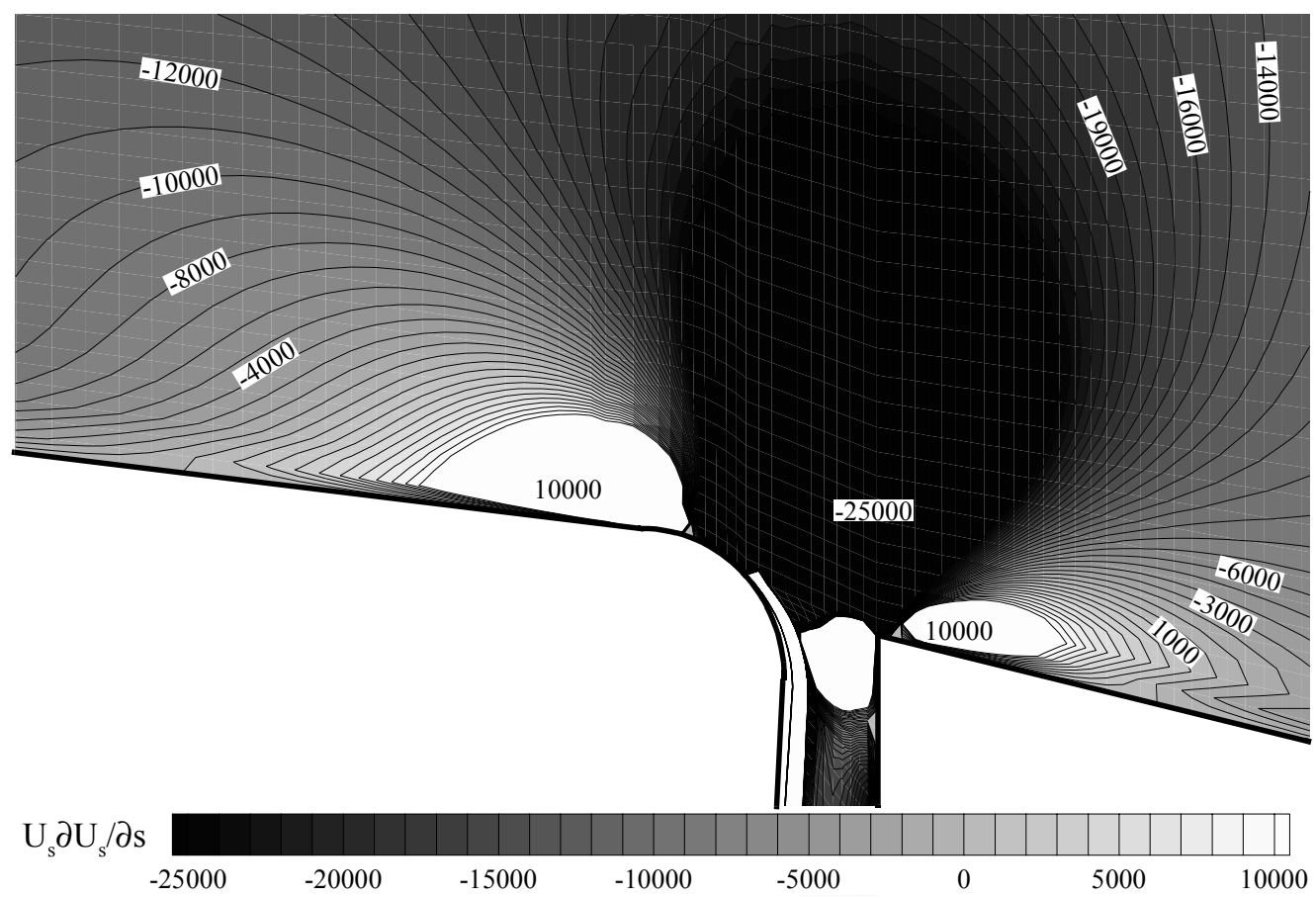

Figure 6.45 Contours of $U_{s} \frac{\partial U_{s}}{\partial s}$, Alt. 4, Region $2 \& 3,2.5 \%$ Bleed 


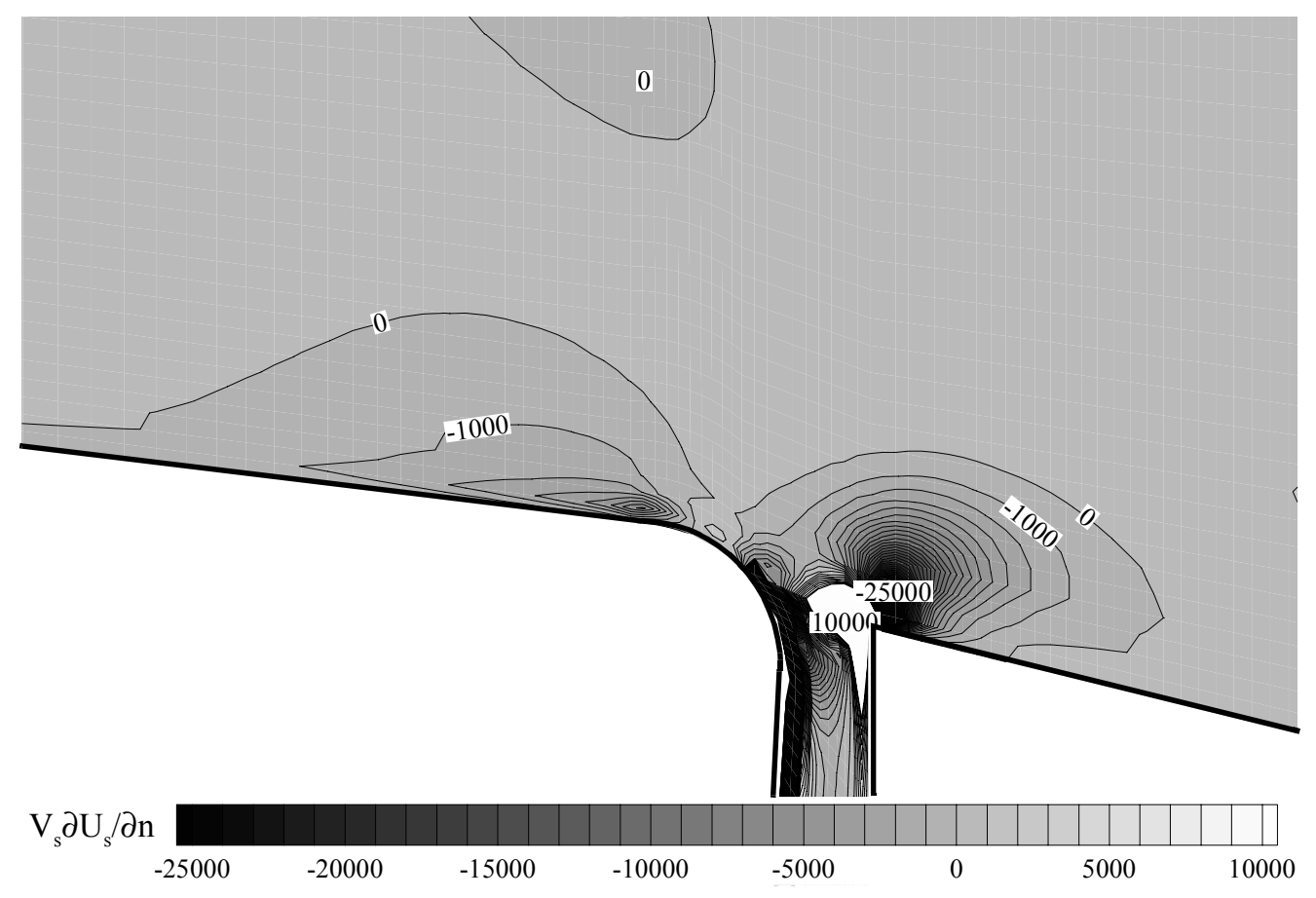

Figure 6.46 Contours of $V_{s} \frac{\partial U_{s}}{\partial n}$, Alt. 4, Region $2 \& 3,2.5 \%$ Bleed

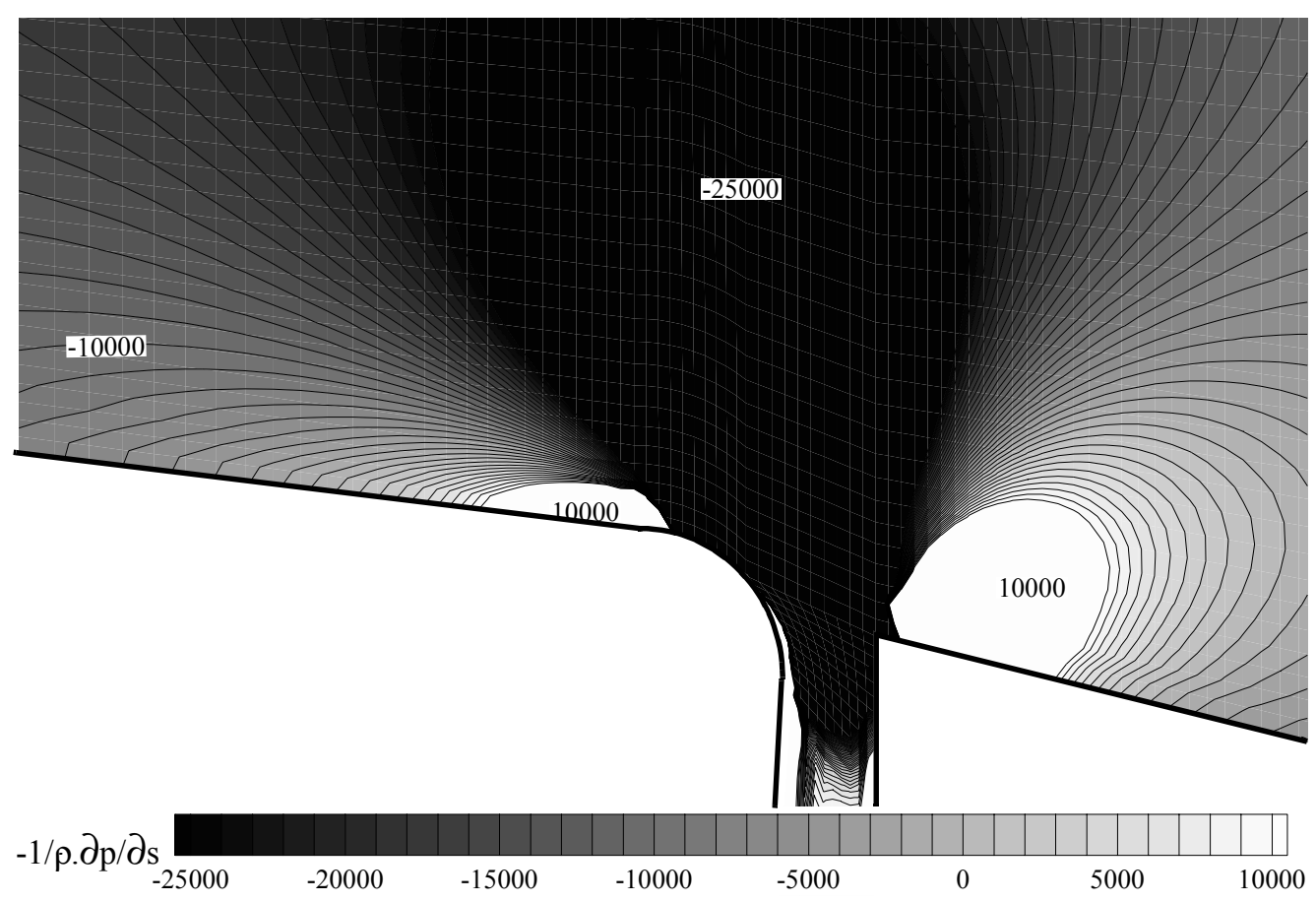

Figure 6.47 Contours of $-\frac{1}{\rho} \frac{\partial p}{\partial s}$, Alt. 4, Region $2 \& 3,2.5 \%$ Bleed 


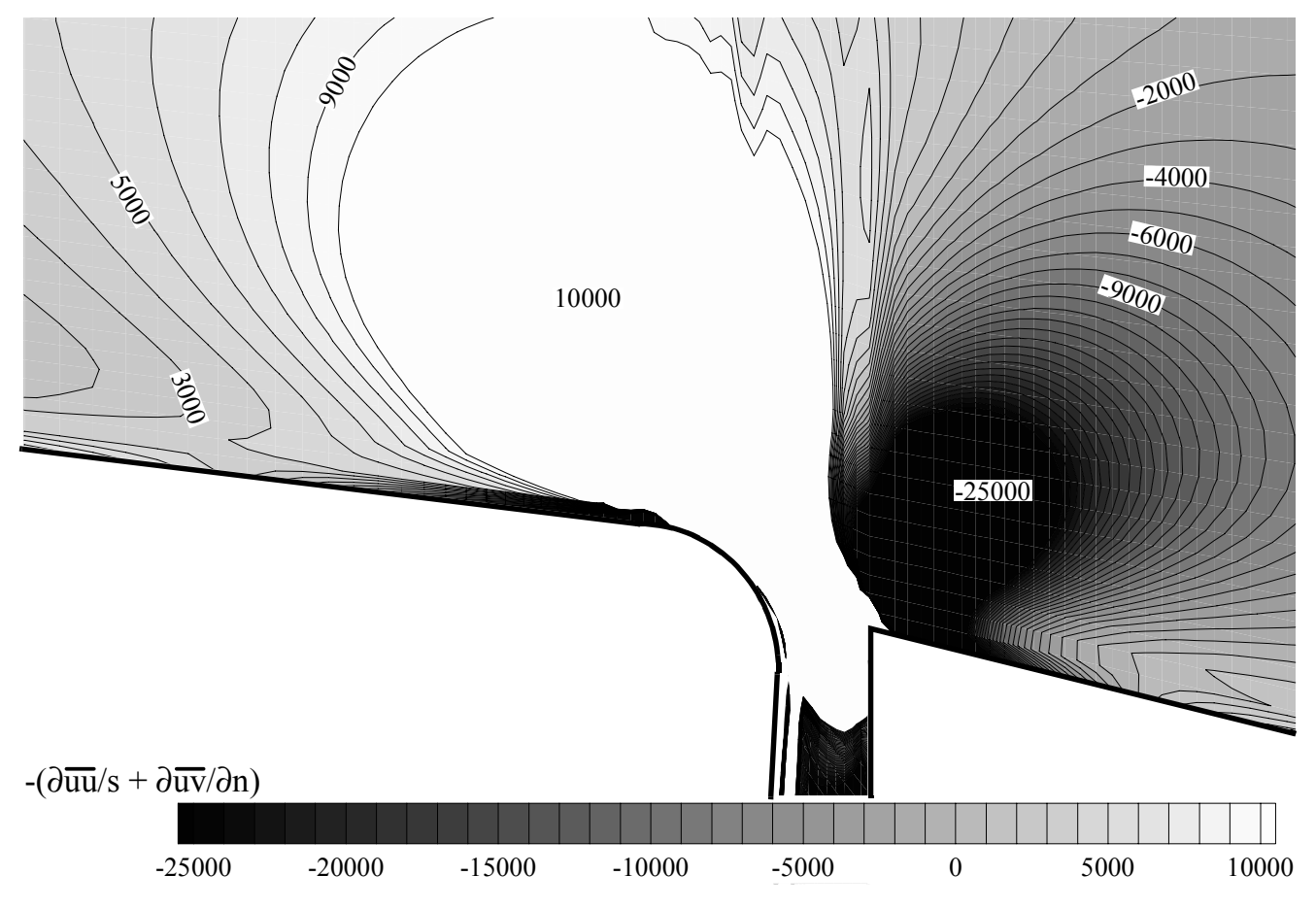

Figure 6.48 Contours of $-\left(\frac{\partial}{\partial \mathrm{s}} \overline{\mathrm{uu}}+\frac{\partial}{\partial \mathrm{n}} \overline{\mathrm{uv}}\right)$, Alt. 4, Region $2 \& 3,2.5 \%$ Bleed

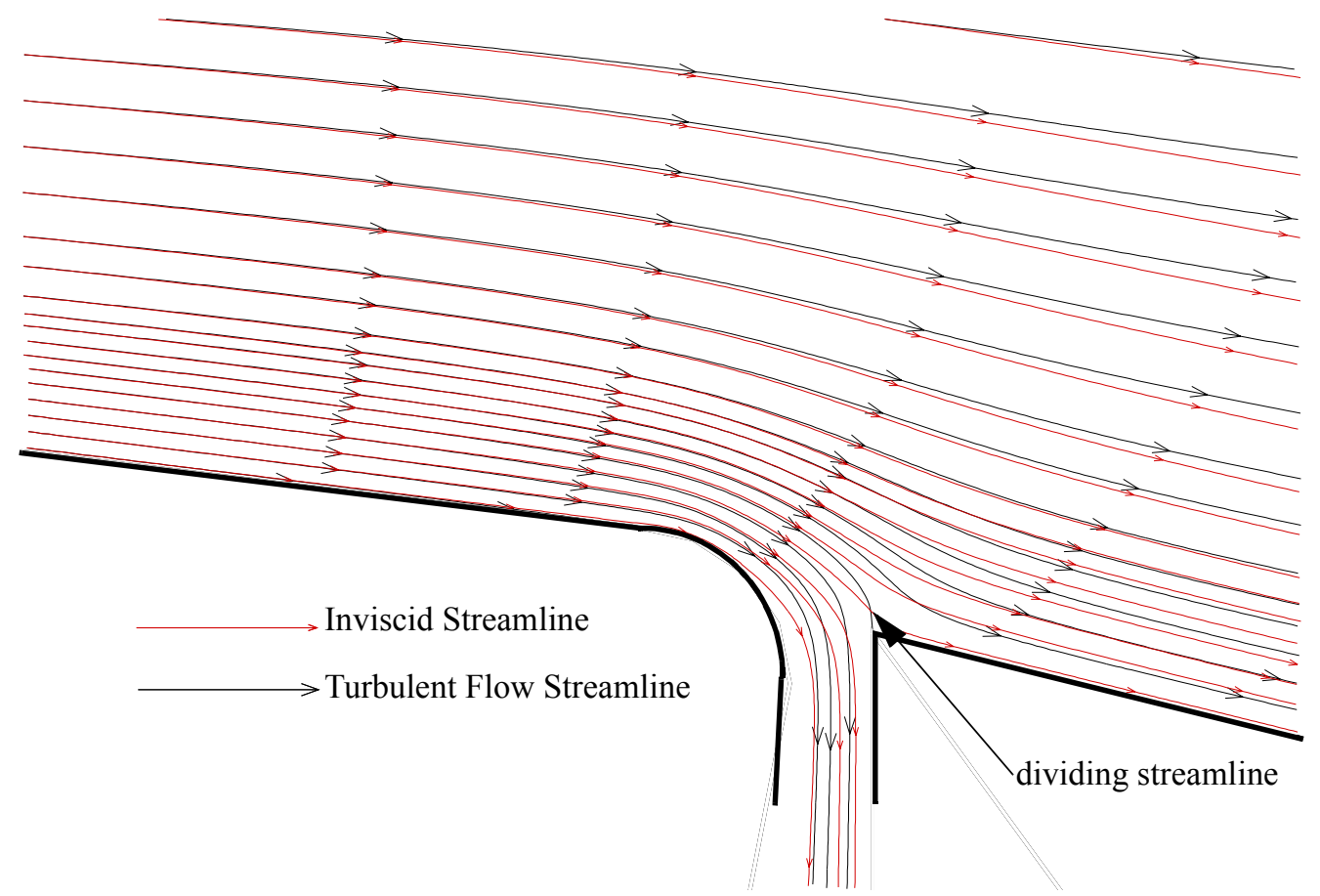

Figure 6.49 Streamlines, Alt. 4, Region 2 \& 3, 2.5\% Bleed 


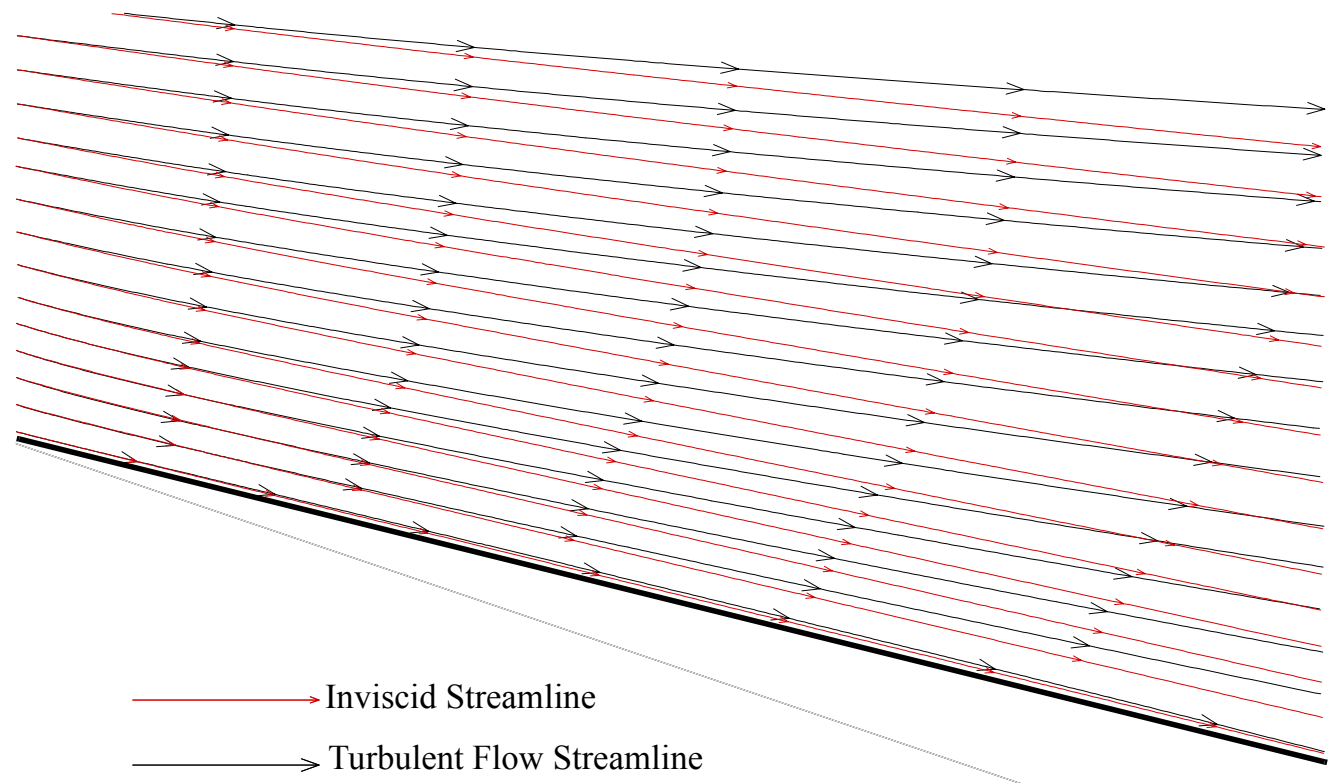

Figure 6.50 Streamlines, Alt. 4, Region 4, 2.5\% Bleed

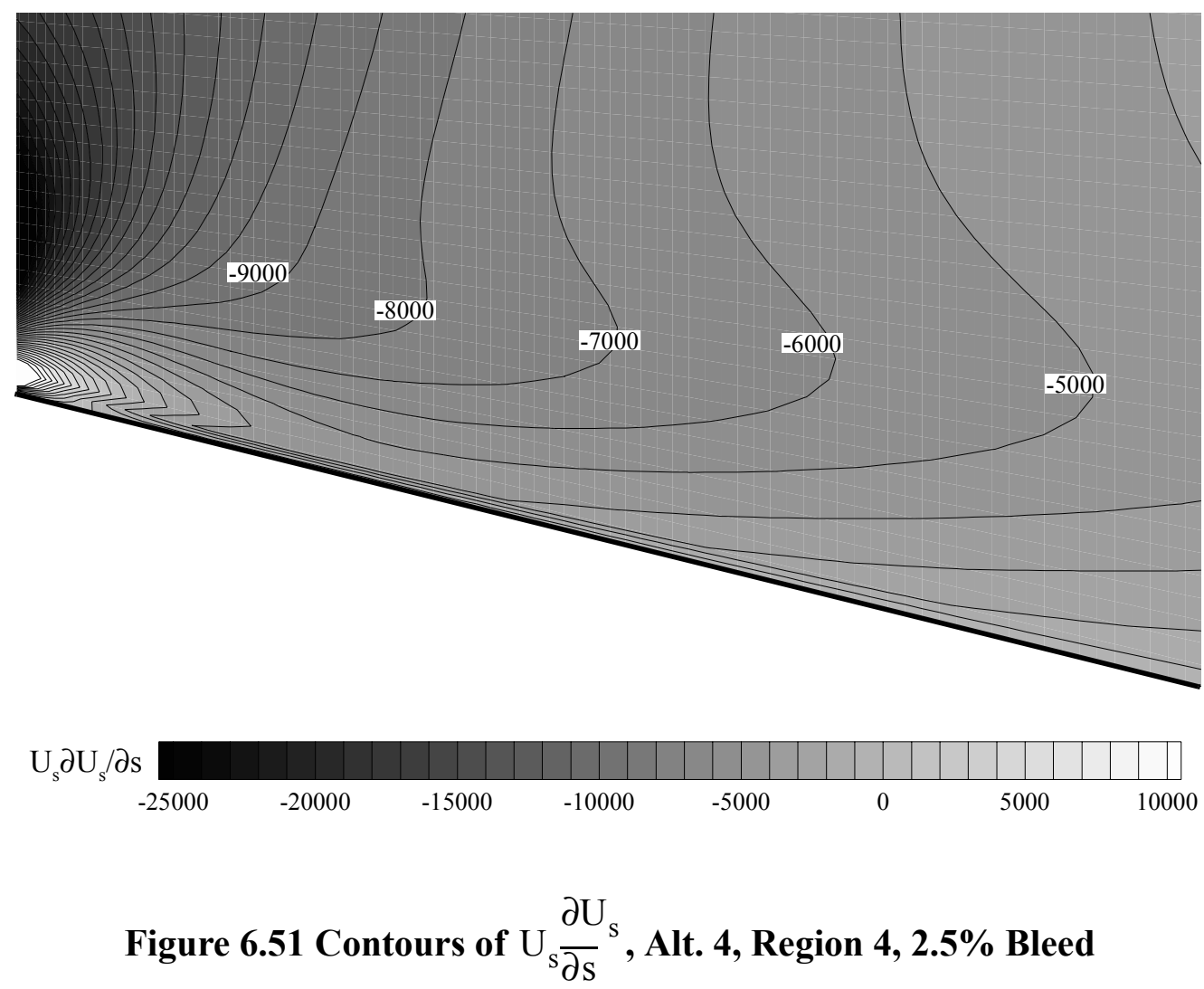




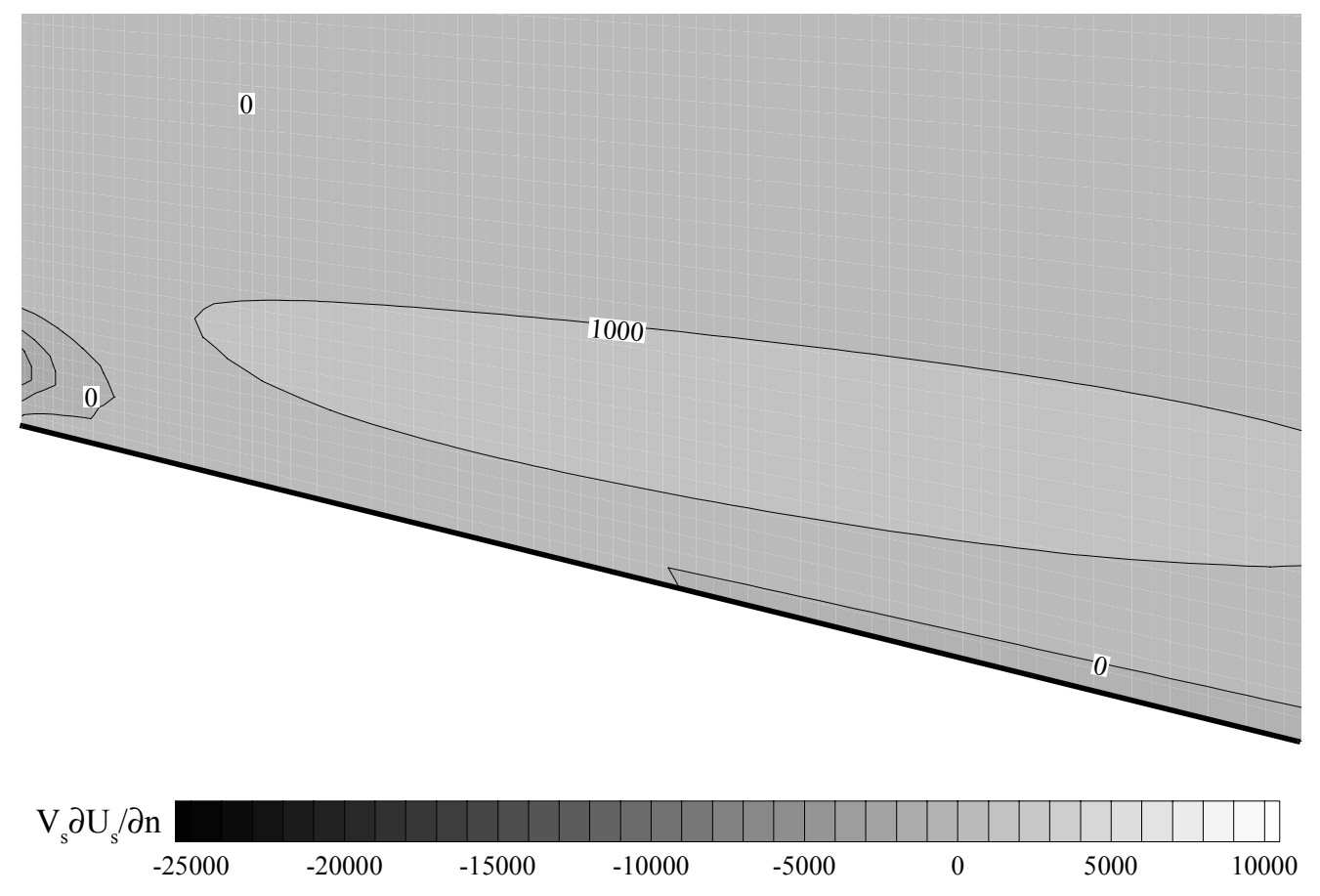

Figure 6.52 Contours of $V_{s} \frac{\partial U_{s}}{\partial n}$, Alt. 4, Region 4, 2.5\% Bleed
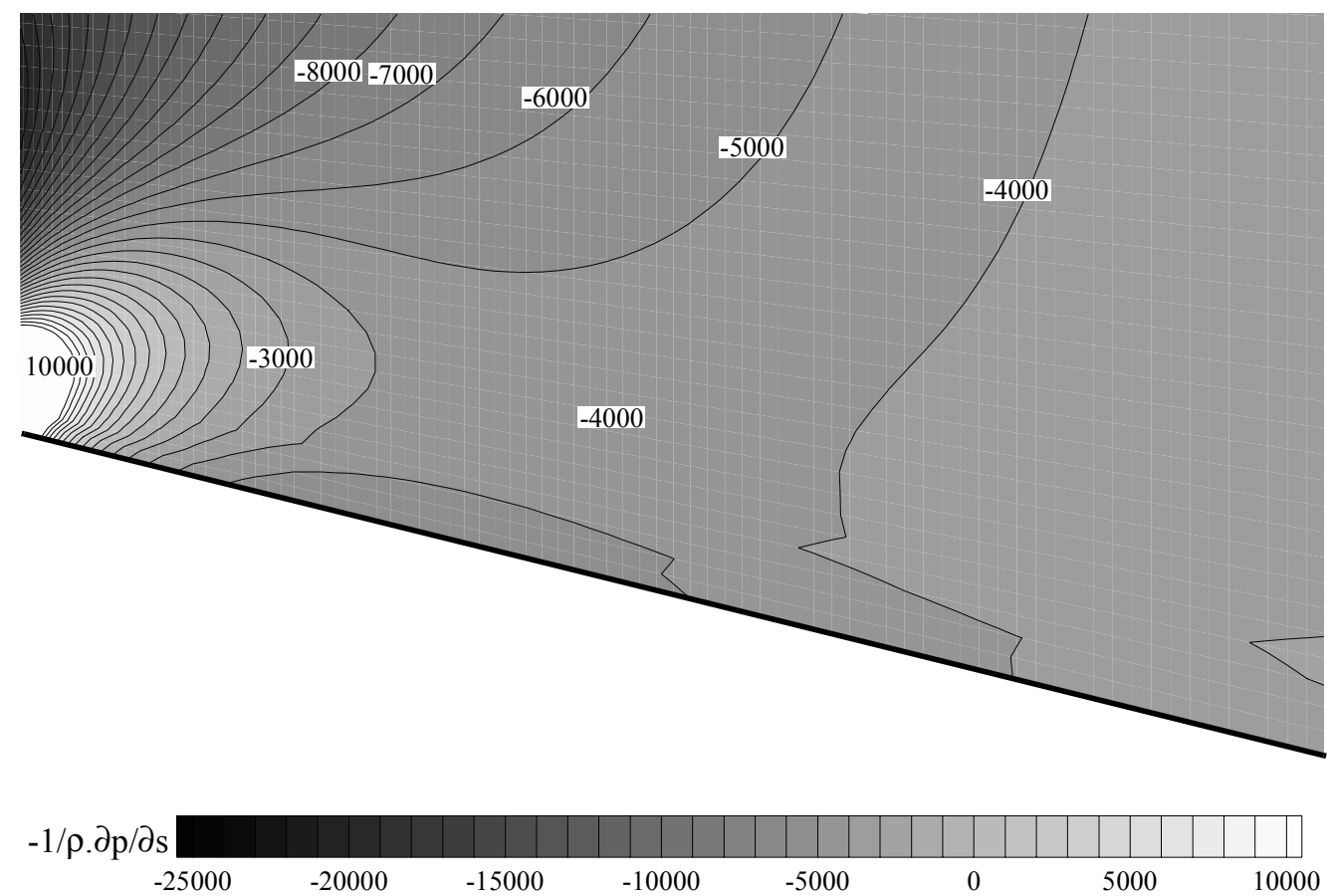

Figure 6.53 Contours of $-\frac{1}{\rho} \frac{\partial p}{\partial s}$, Alt. 4, Region 4, 2.5\% Bleed 


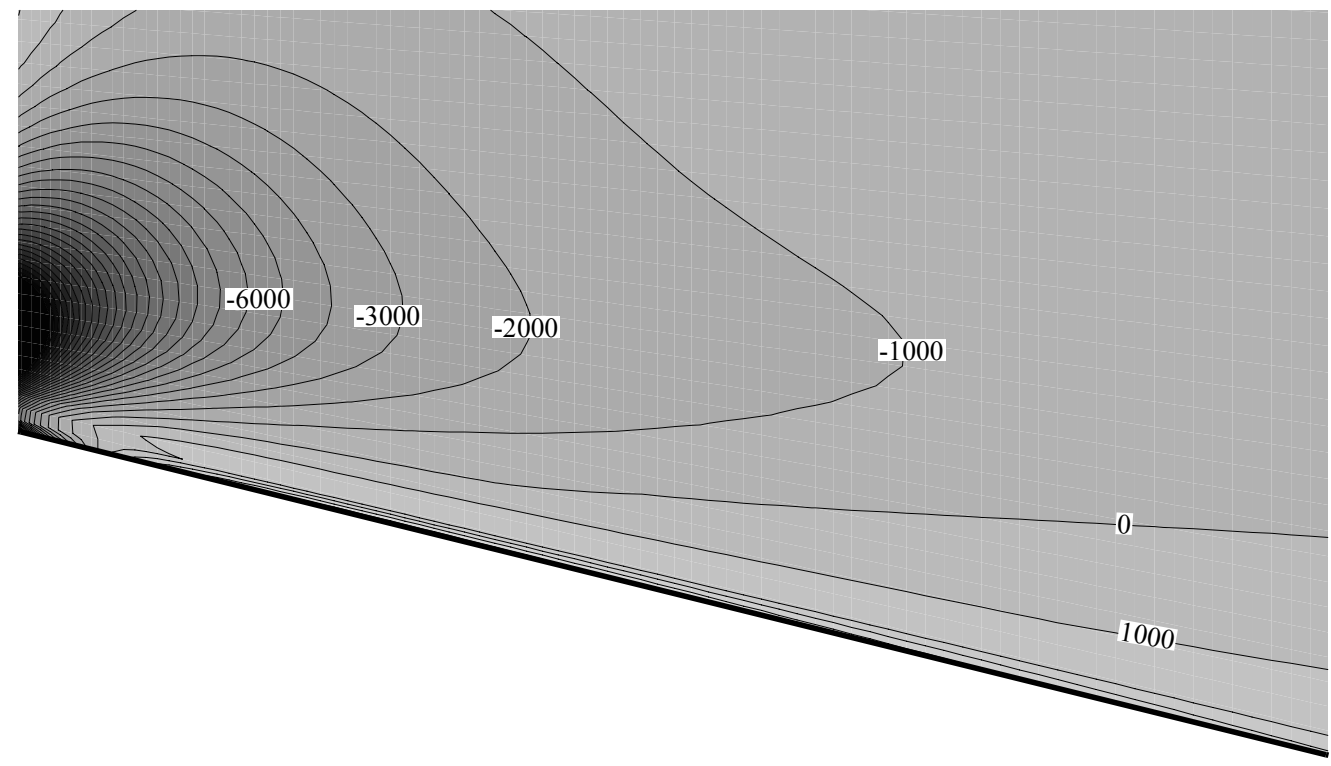

$-(\partial \overline{\mathrm{uu}} / \partial \mathrm{s}+\partial \overline{\mathrm{uv}} / \partial \mathrm{n})$

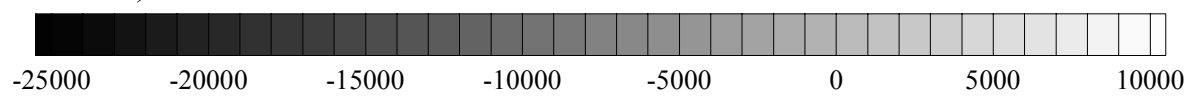

Figure 6.54 Contours of $-\left(\frac{\partial}{\partial \mathrm{s}} \overline{\mathrm{uu}}+\frac{\partial}{\partial \mathrm{n}} \overline{\mathrm{uv}}\right)$, Alt. 4, Region 4, 2.5\% Bleed

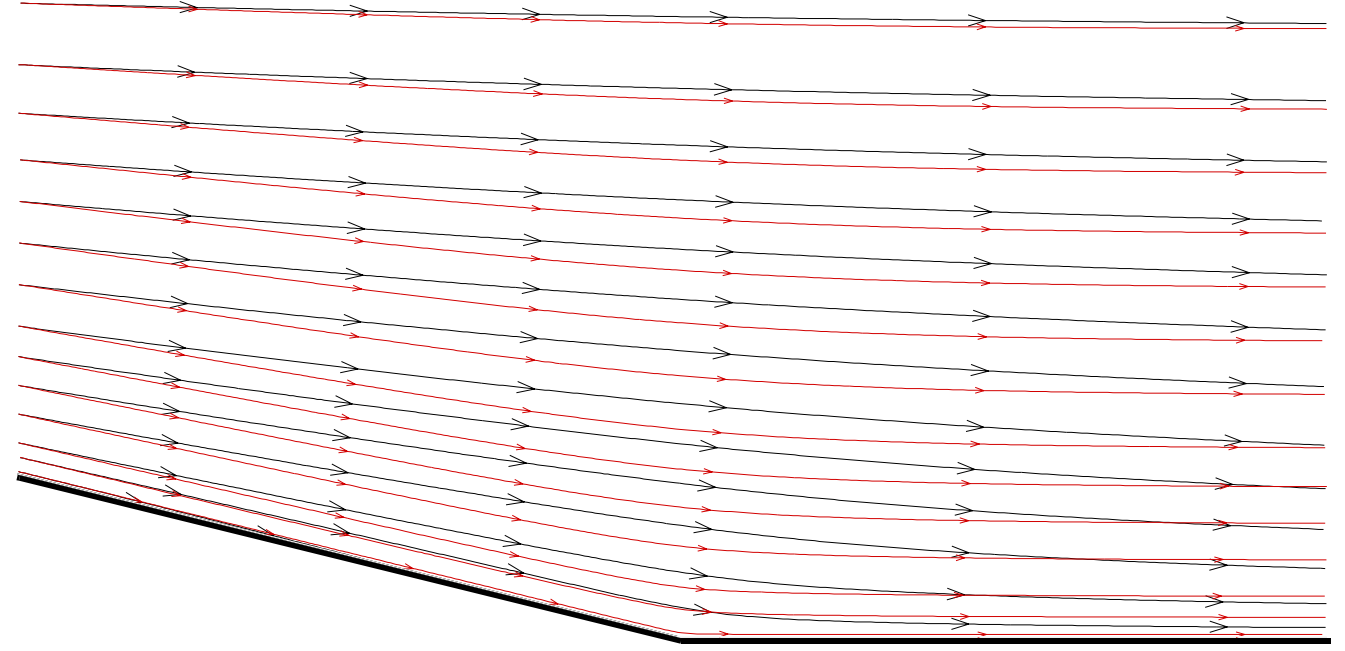

Inviscid Streamline

Turbulent Flow Streamline

Figure 6.55 Streamlines, Alt. 4, Region 5, 2.5\% Bleed 

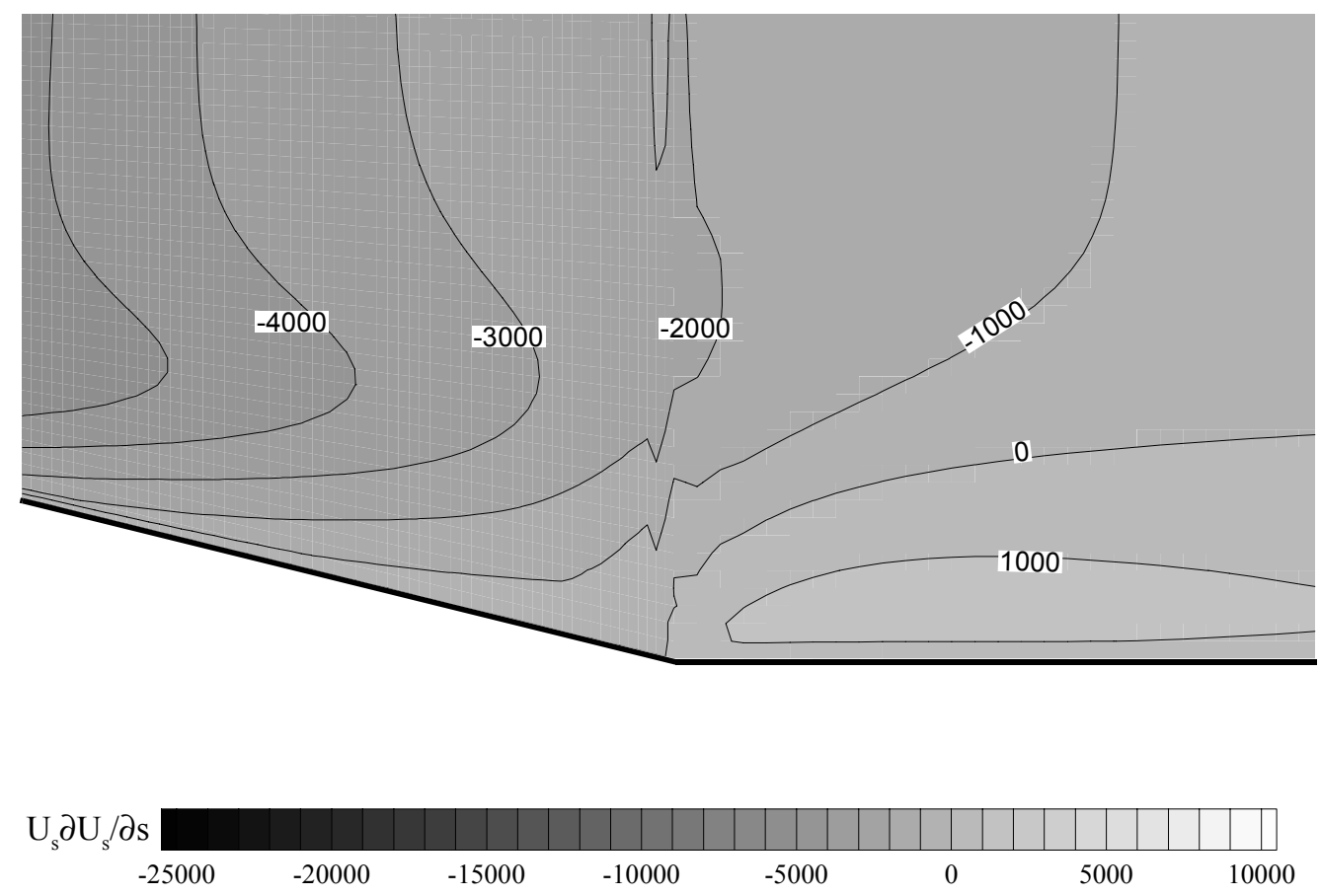

Figure 6.56 Contours of $U_{s} \frac{\partial U_{s}}{\partial s}$, Alt. 4, Region 5, 2.5\% Bleed
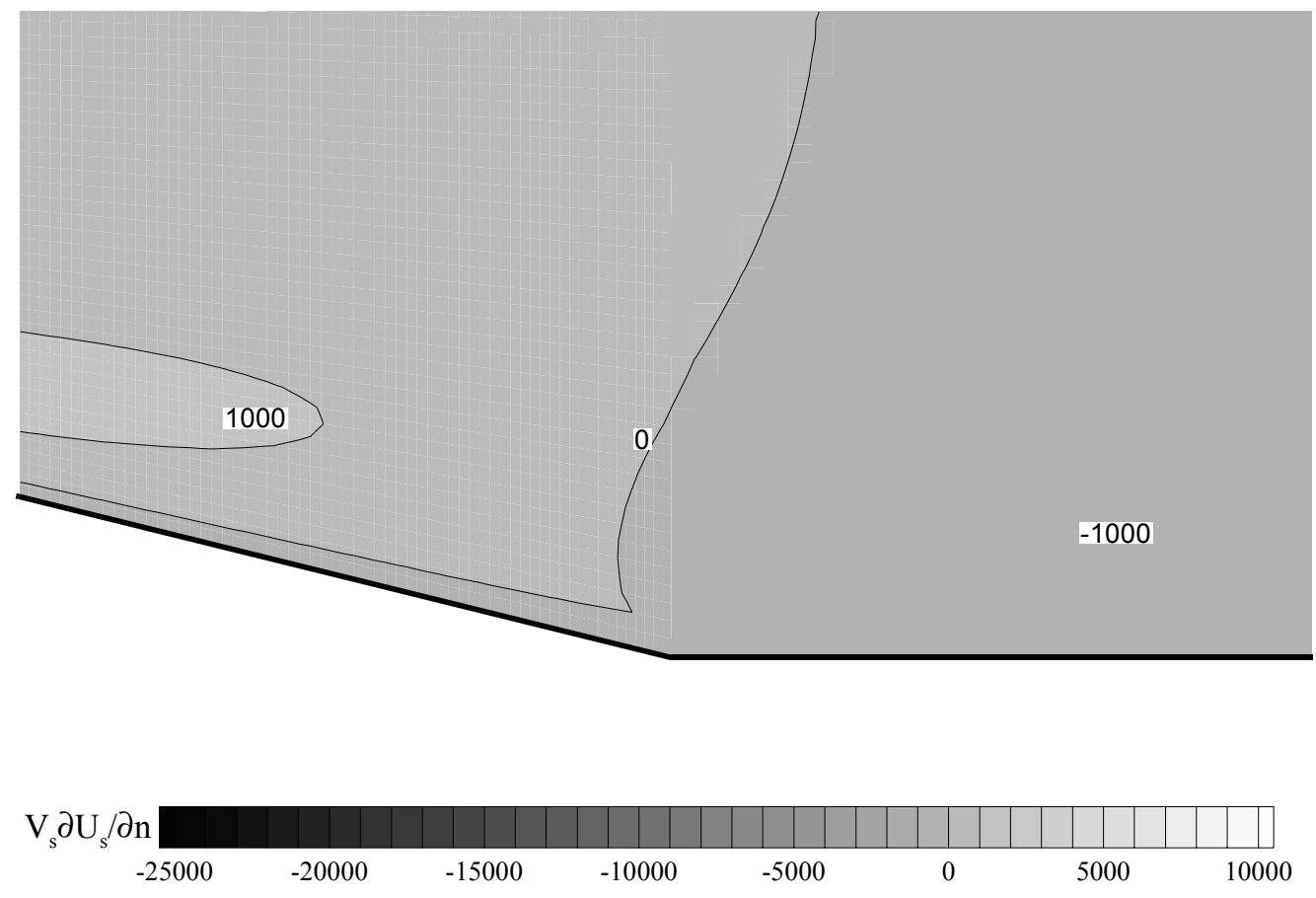

Figure 6.57 Contours of $v_{s} \frac{\partial U_{s}}{\partial n}$, Alt. 4, Region 5, 2.5\% Bleed 

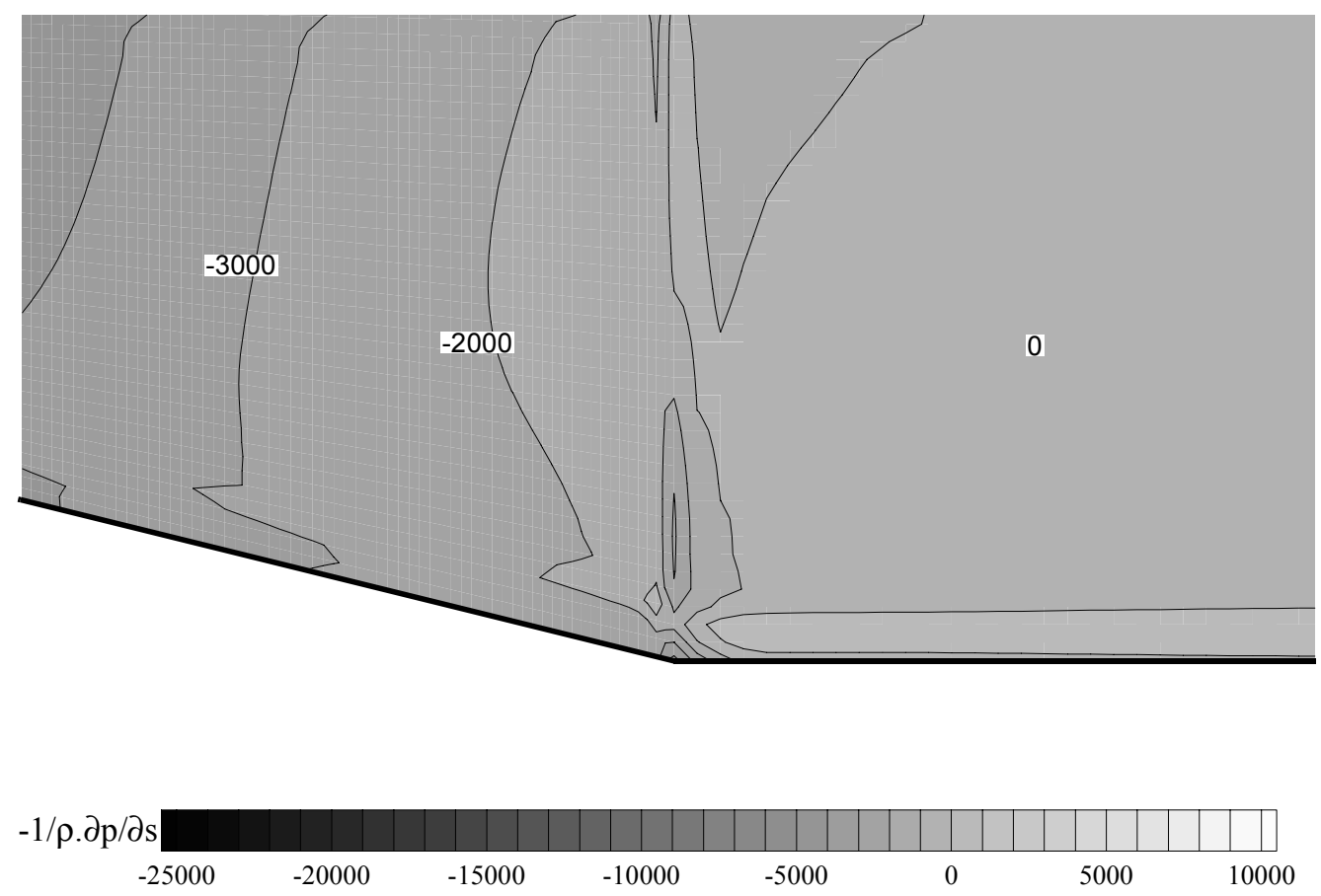

Figure 6.58 Contours of $-\frac{1}{\rho} \frac{\partial p}{\partial s}$, Alt. 4, Region 5, 2.5\% Bleed

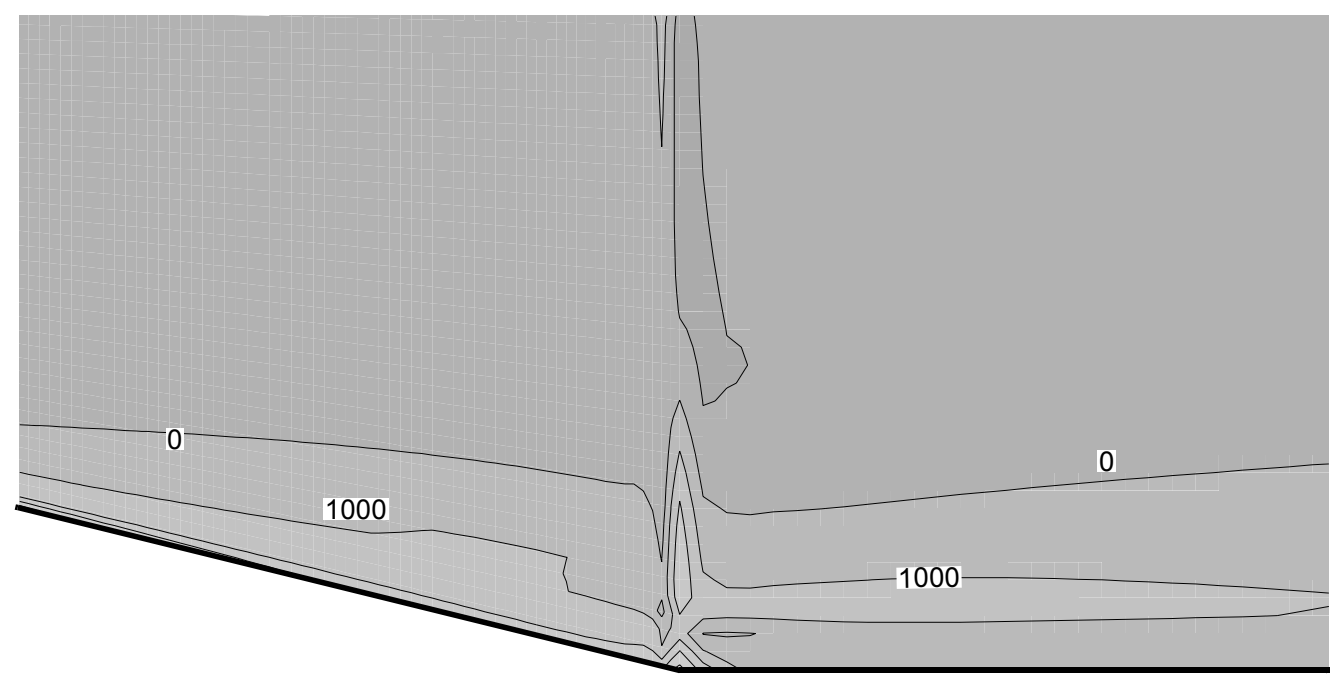

$-(\partial \overline{u v} / \partial s+\partial \overline{u v} / \partial n)$

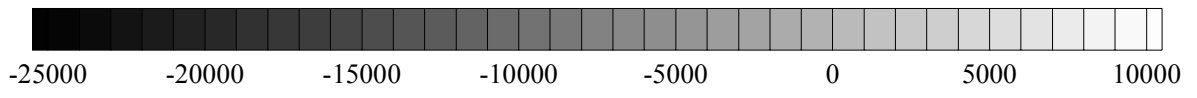

Figure 6.59 Contours of $-\left(\frac{\partial}{\partial \mathrm{s}} \overline{\mathrm{uu}}+\frac{\partial}{\partial \mathrm{n}} \overline{\mathrm{uv}}\right)$, Alt. 4, Region 5, 2.5\% Bleed 


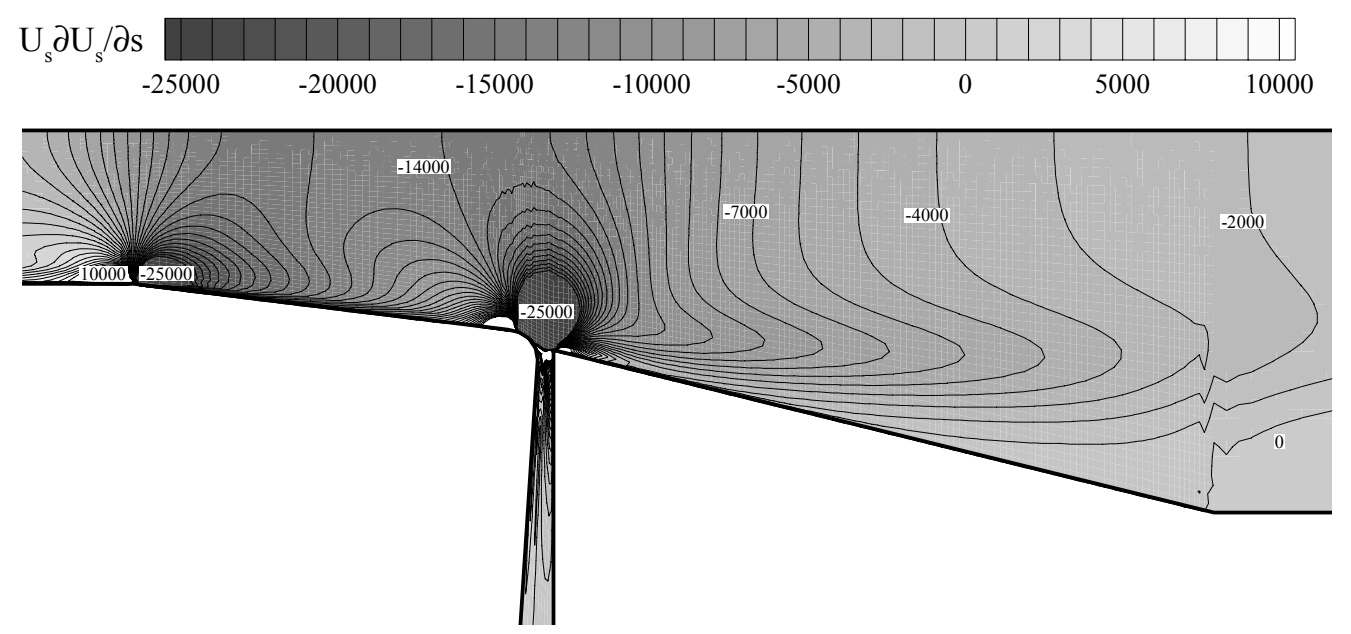

Figure 6.60 Contours of $U_{s} \frac{\partial U_{s}}{\partial s}$, Alt. 4, 0.0\% Bleed

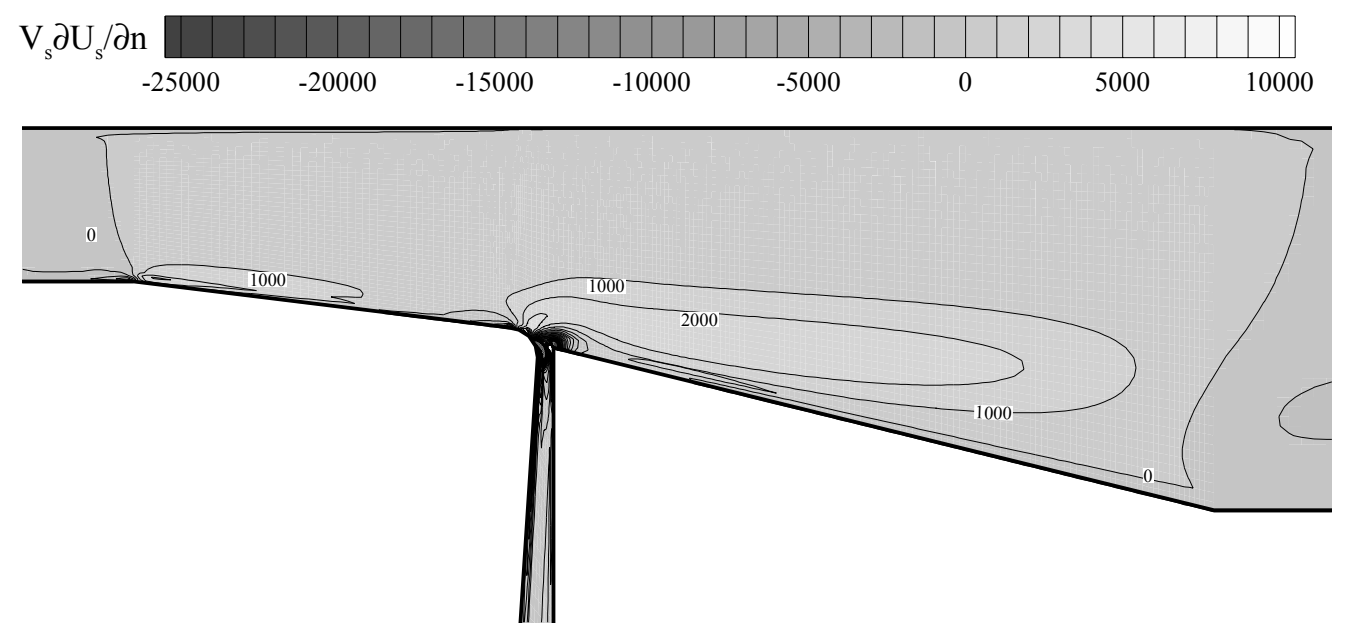

Figure 6.61 Contours of $V_{s} \frac{\partial U_{s}}{\partial n}$, Alt. 4, 0.0\% Bleed 


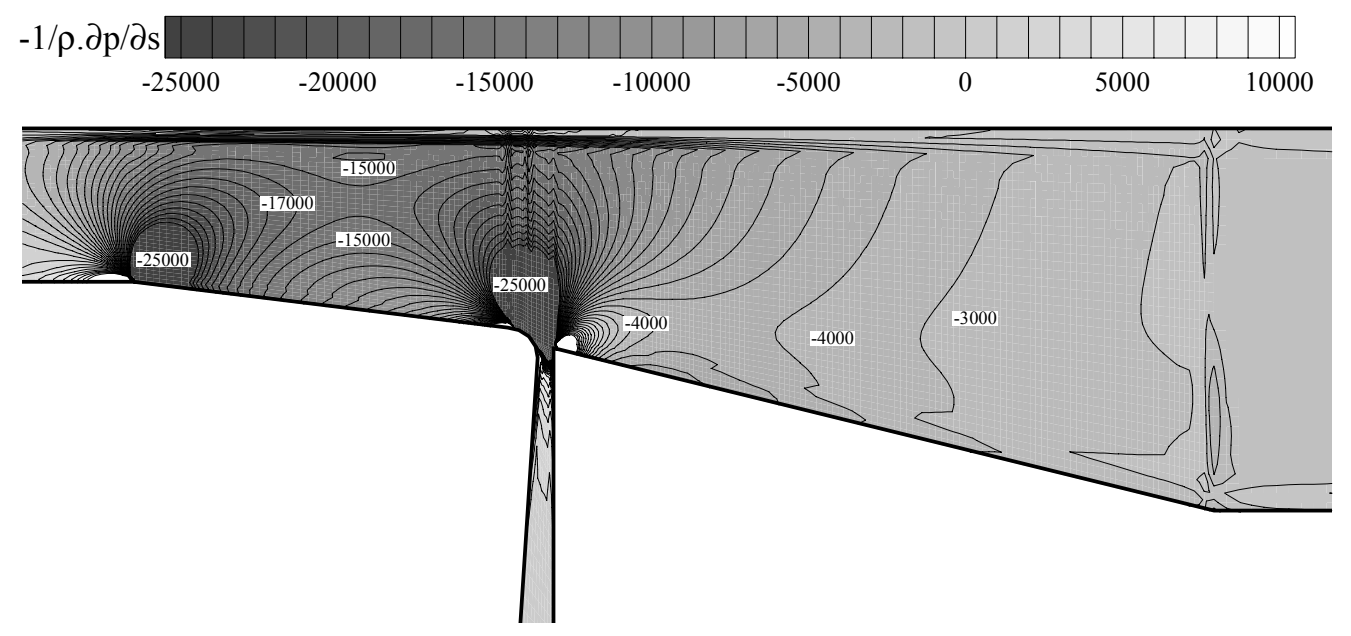

Figure 6.62 Contours of $-\frac{1}{\rho} \frac{\partial p}{\partial s}$, Alt. 4, 0.0\% Bleed

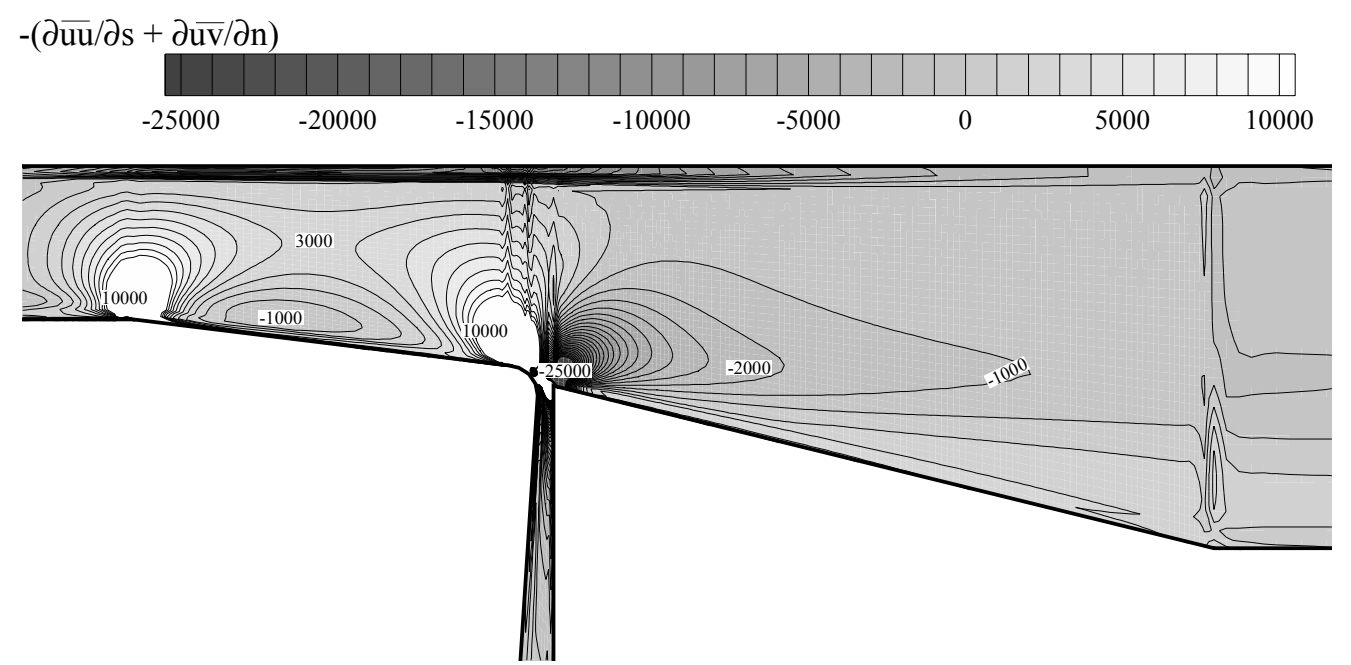

Figure 6.63 Contours of $-\left(\frac{\partial}{\partial \mathrm{s}} \overline{\mathrm{uu}}+\frac{\partial}{\partial \mathrm{n}} \overline{\mathrm{uv}}\right)$, Alt. 4, 0.0\% Bleed 


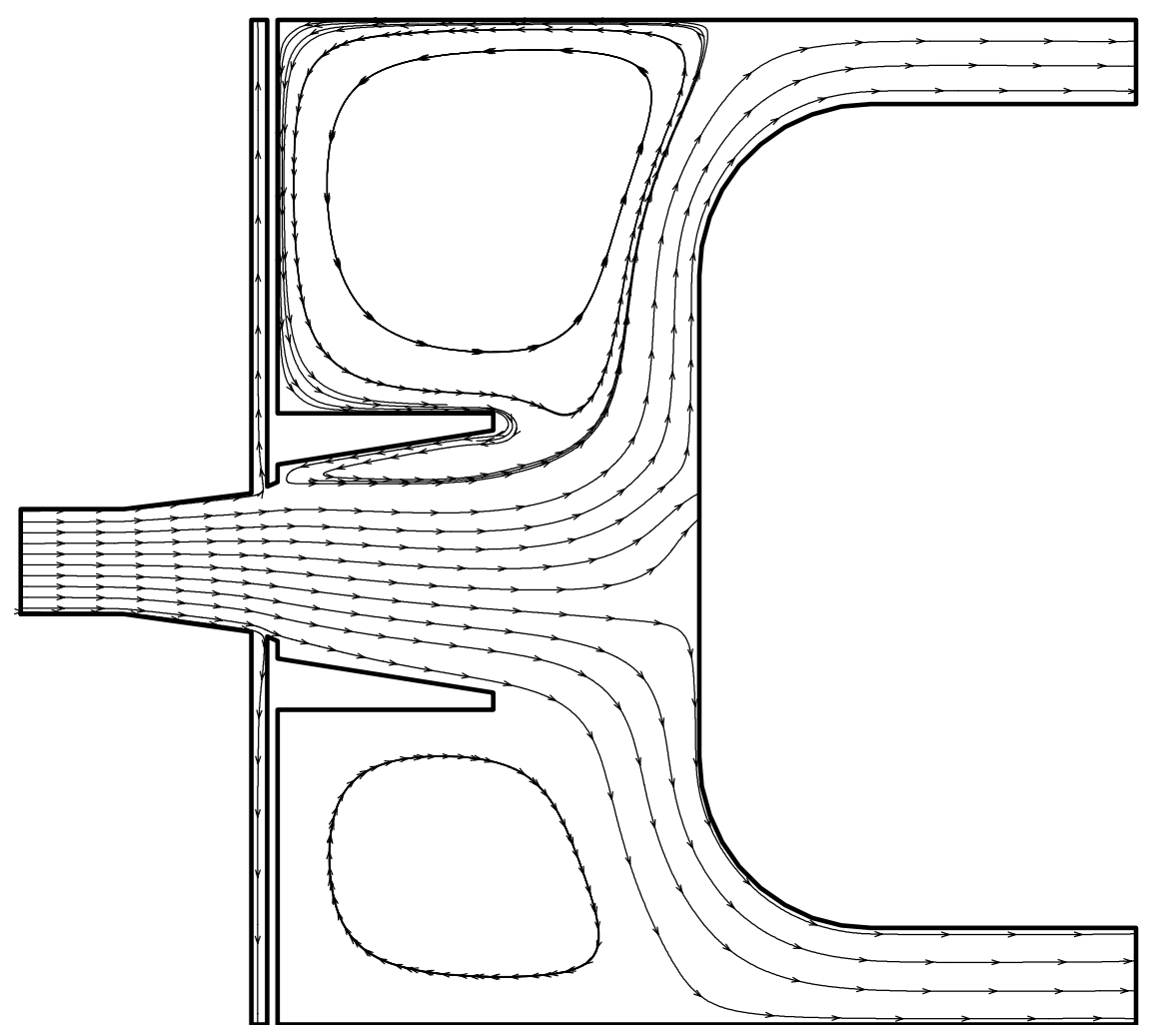

Figure 6.64 Streamline Plot, 2D, Axi-Symmetric, $B_{i}=B_{0}=2.5 \%, T_{i}=0.5 \%$

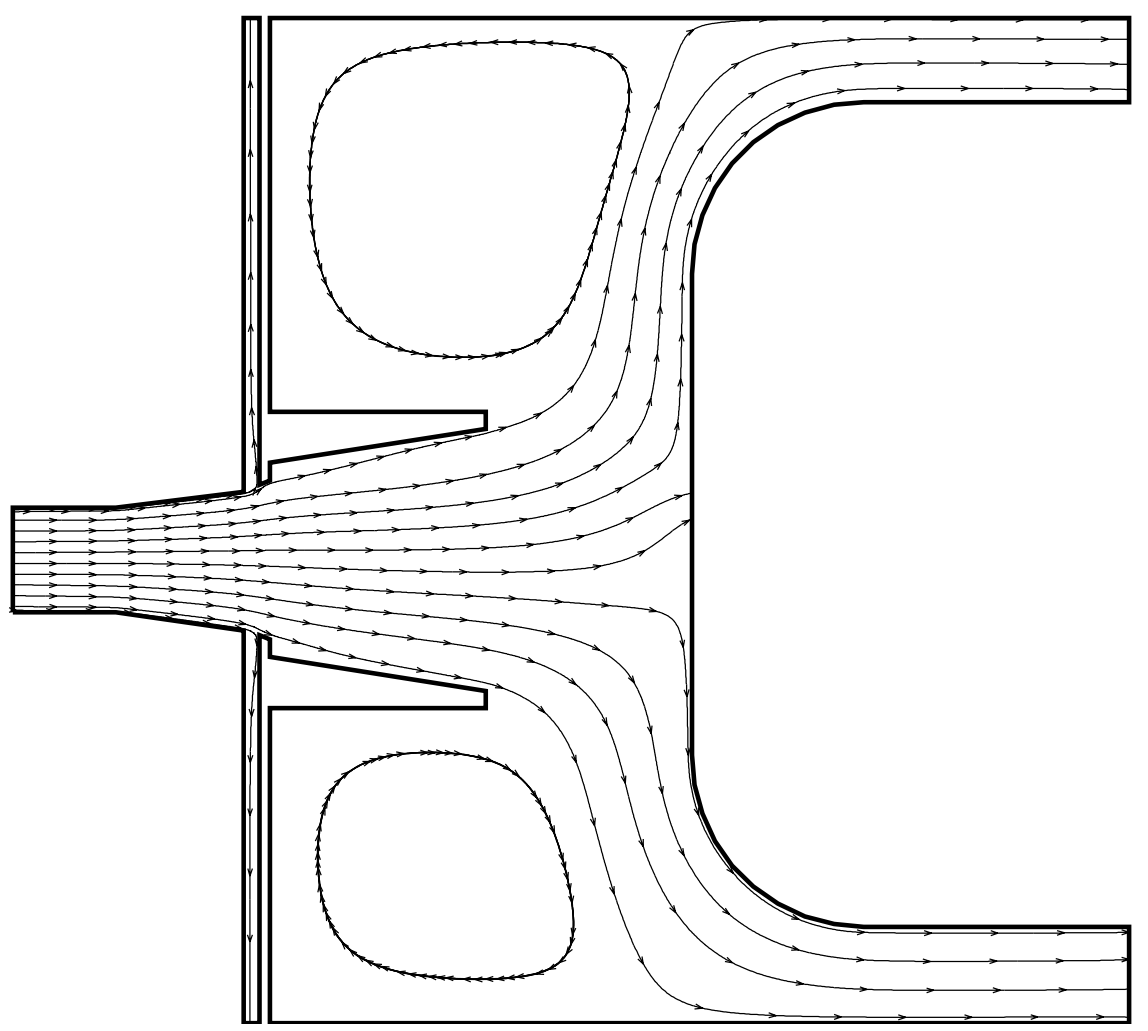

Figure 6.65 Streamline Plot, 2D, Axi-Symmetric, $B_{i}=B_{0}=2.5 \%, T_{i}=5.0 \%$ 


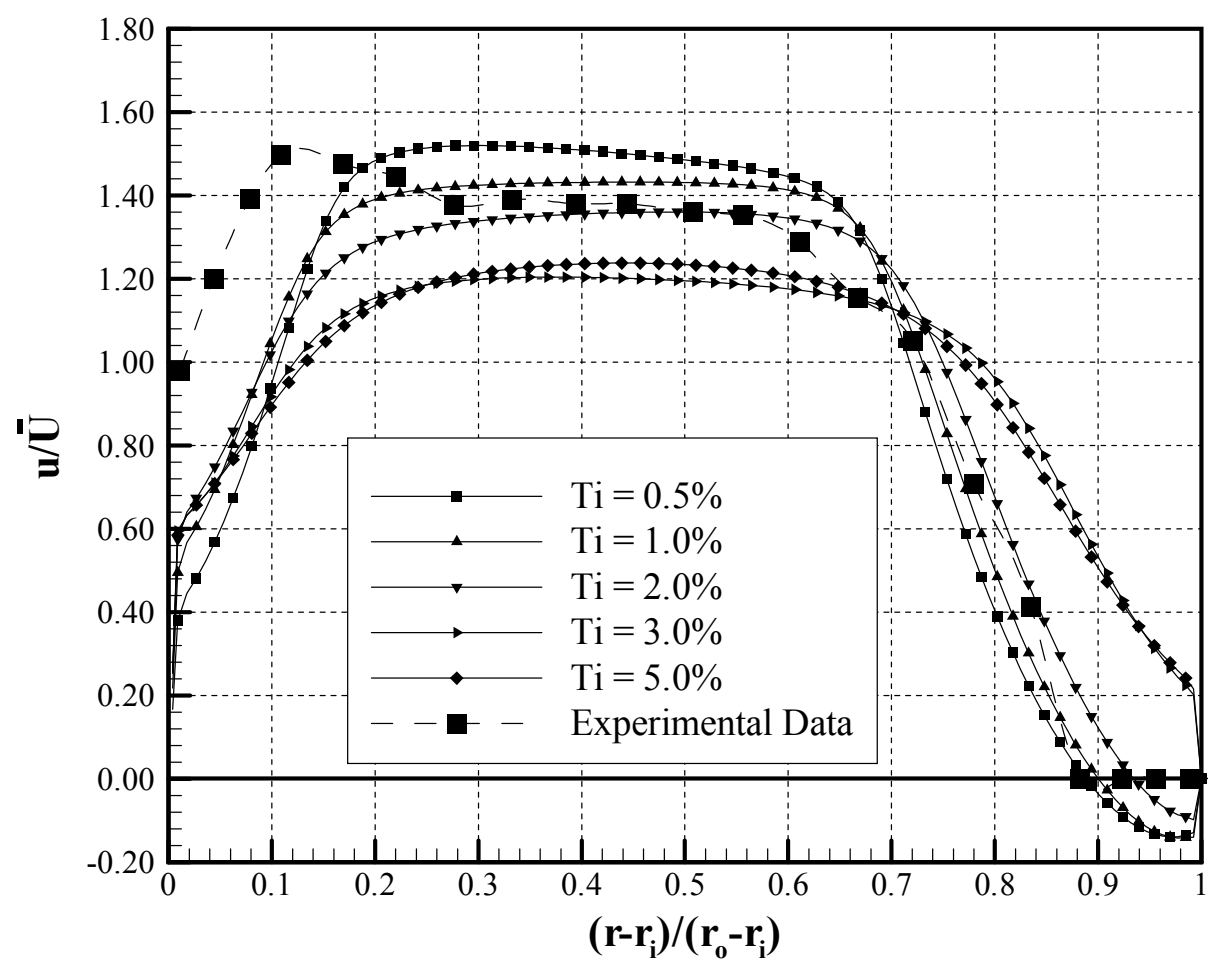

Figure 6.66 Effect of Inlet Turbulence on Axial Velocity Profile at Diffuser Exit, 2D, Axi-Symmetric, (Experimental Data, $T_{i}<1.0 \%$ )

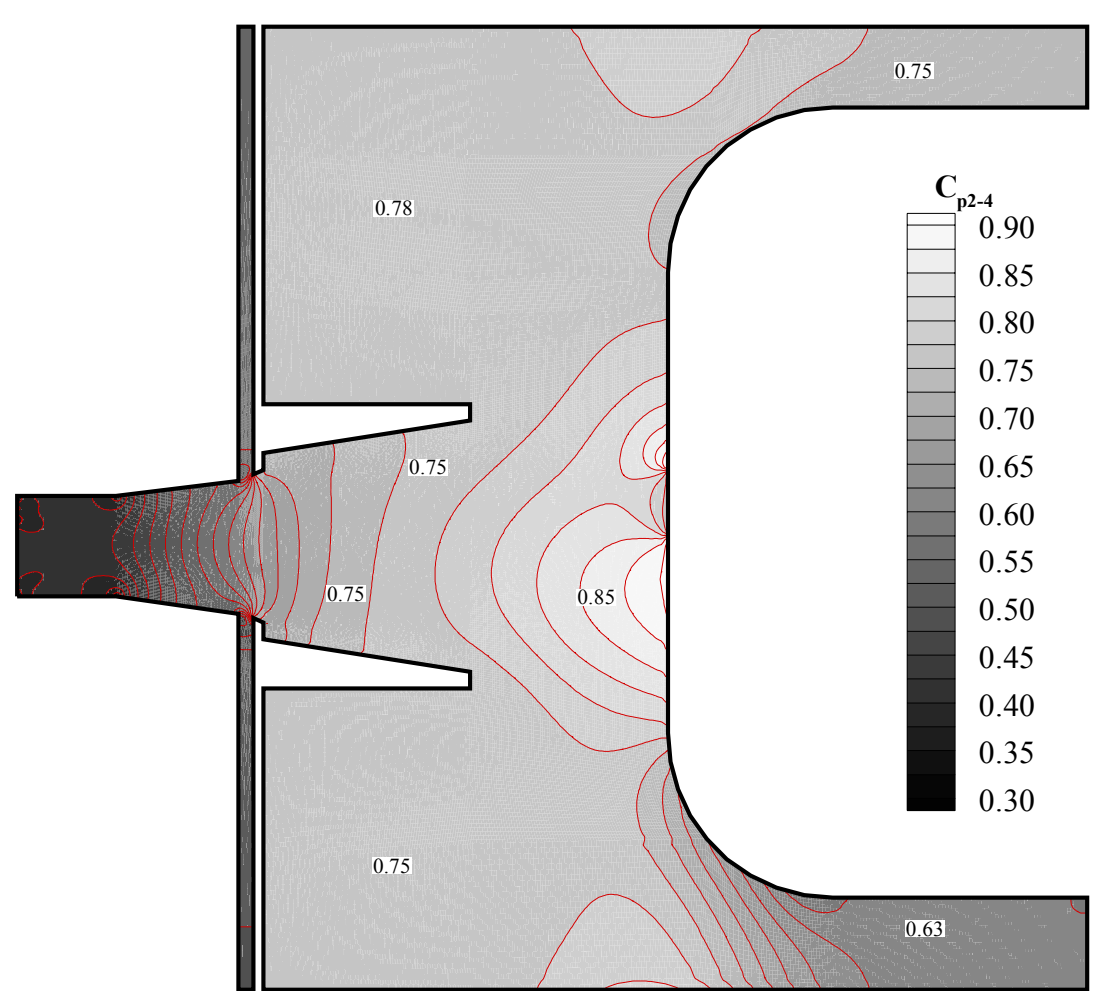

Figure 6.67 Static Pressure Recovery, 2D, Axi-Symmetric, $T_{i}=5.0 \%, B_{i}=B_{0}=2.5 \%$ 


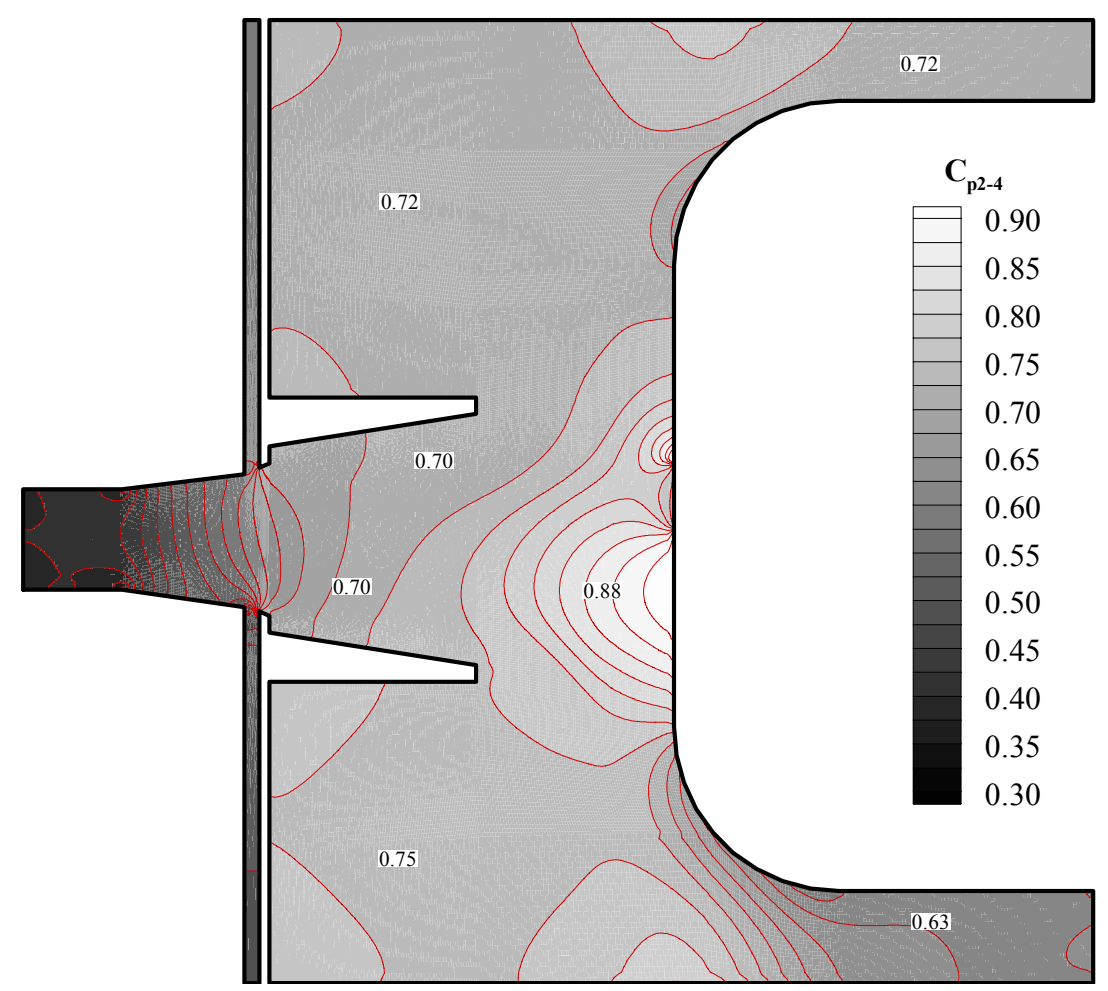

Figure 6.68 Static Pressure Recovery, 2D, Axi-Symmetric, $T_{i}=0.5 \%, B_{i}=B_{0}=2.5 \%$

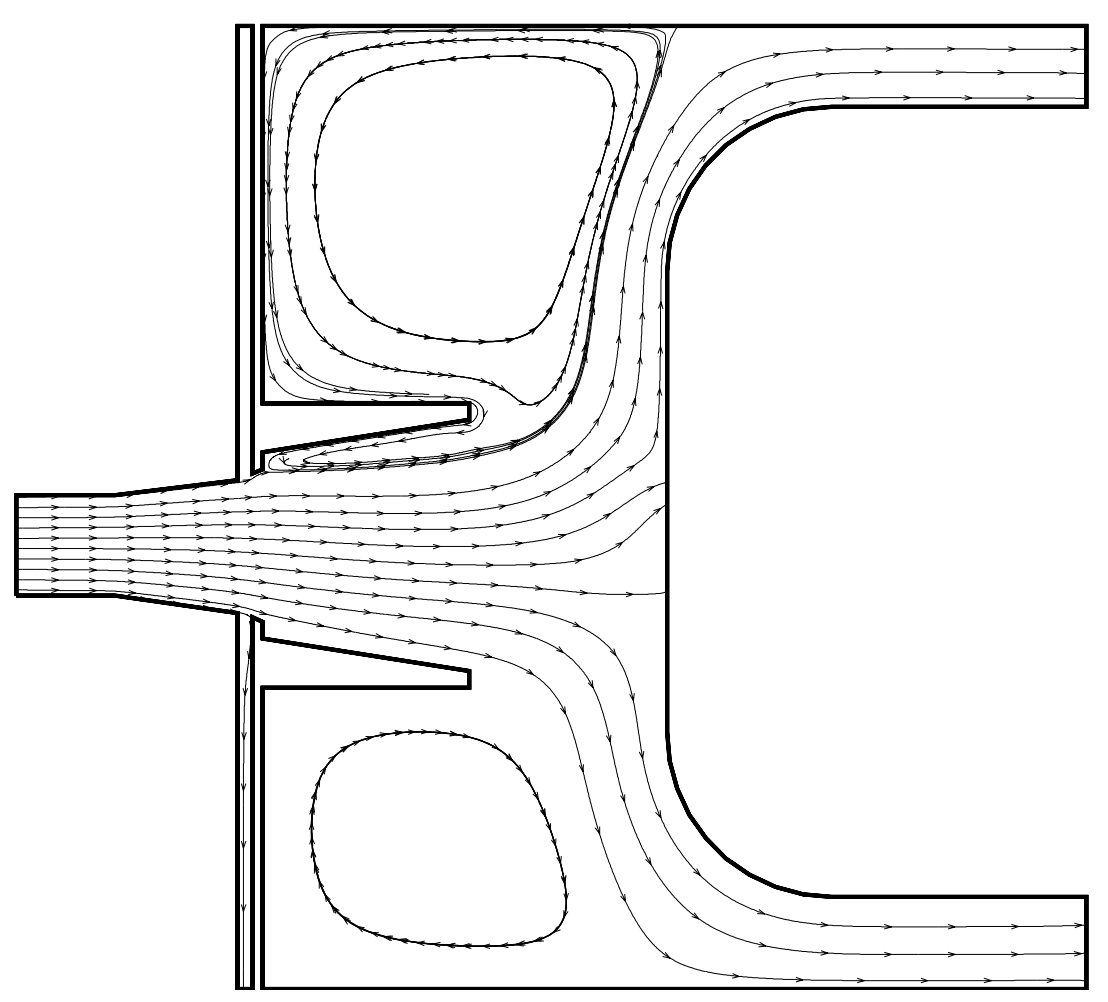

Figure 6.69 Streamline Plot, 2D, Axi-Symmetric, $T_{i}=5.0 \%, B_{i}=B_{0}=1.0 \%$ 


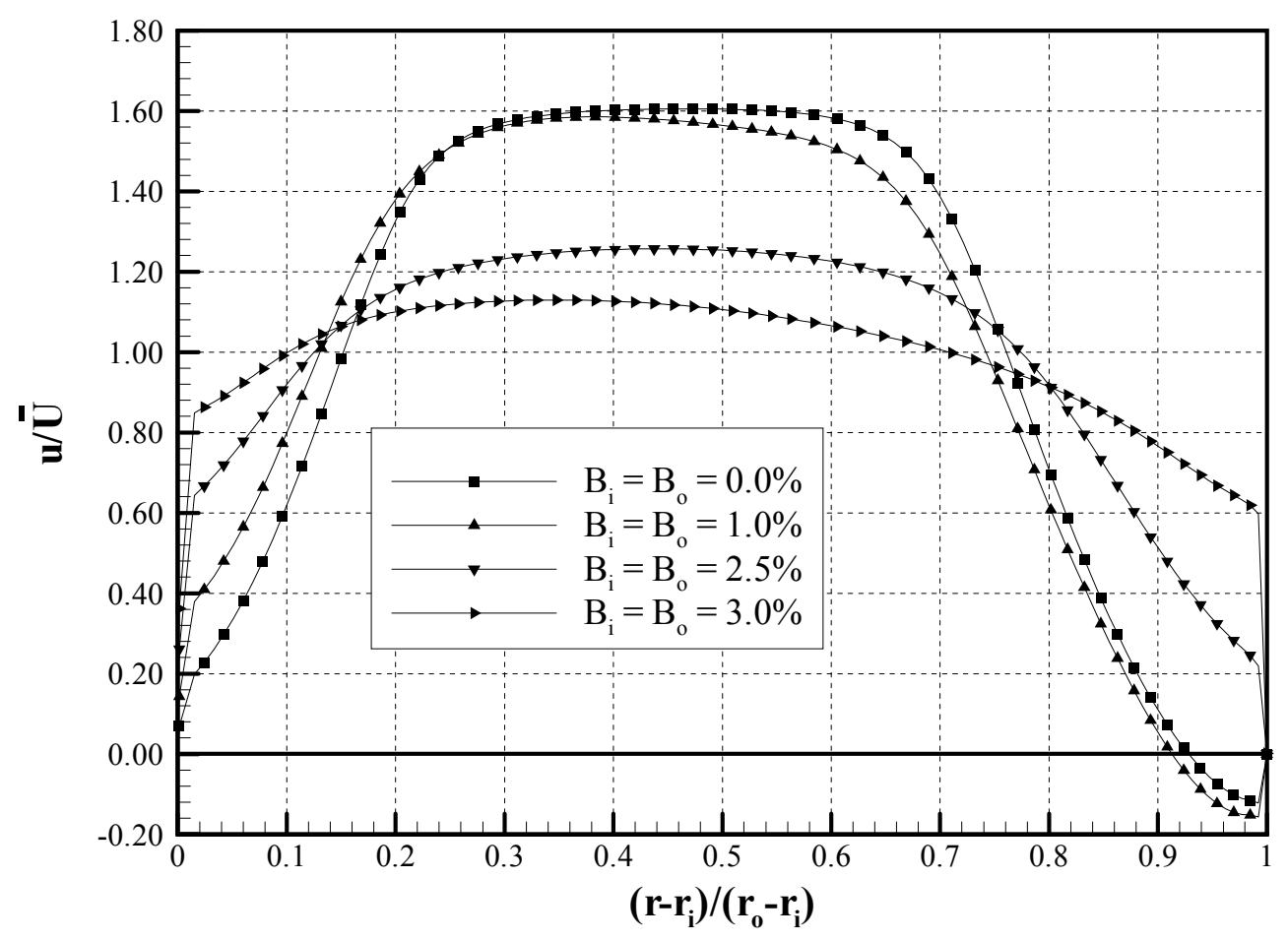

Figure 6.70 Effect of Bleed Rate on Axial Velocity Profile at Diffuser Exit, 2D, Axi-Symmetric, $T_{i}=\mathbf{5 . 0} \%$

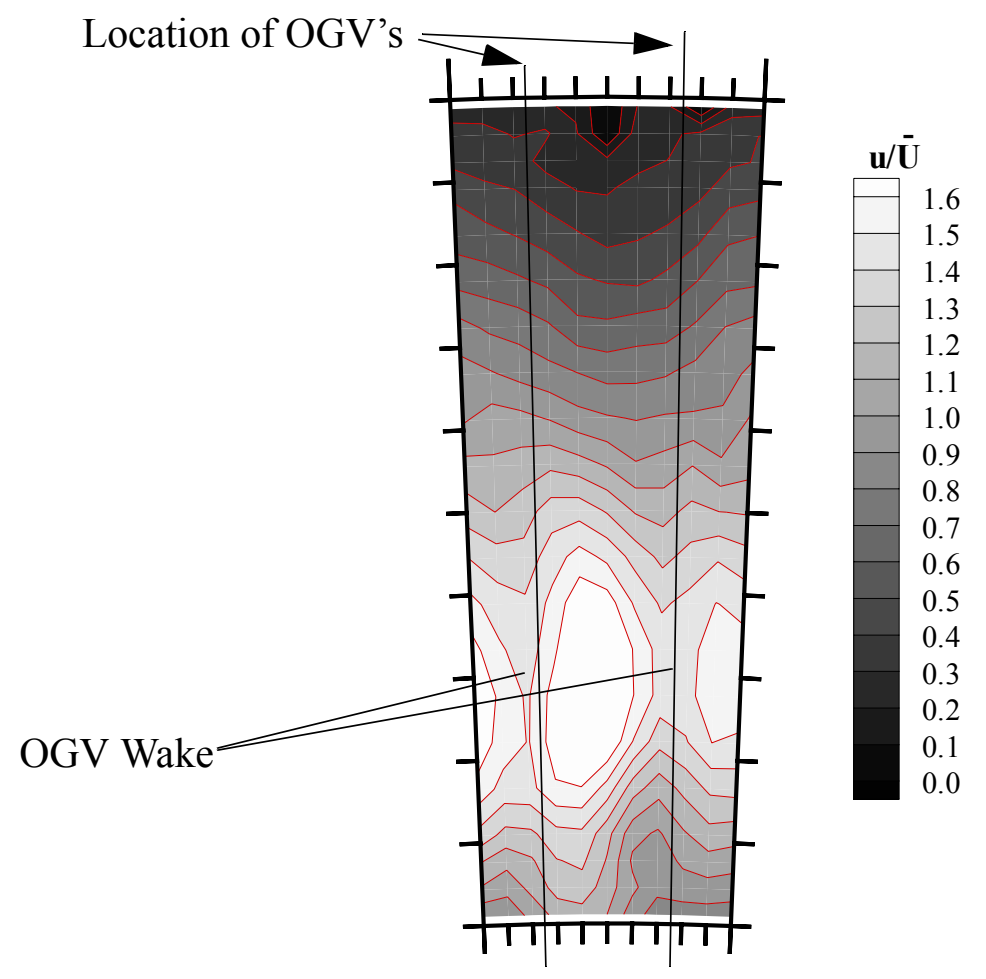

Figure 6.71 Normalised Axial Velocity Contours at Diffuser Exit (Experimental Data, $B_{i}=2.5 \%, B_{0}=3.0 \%$ ) 


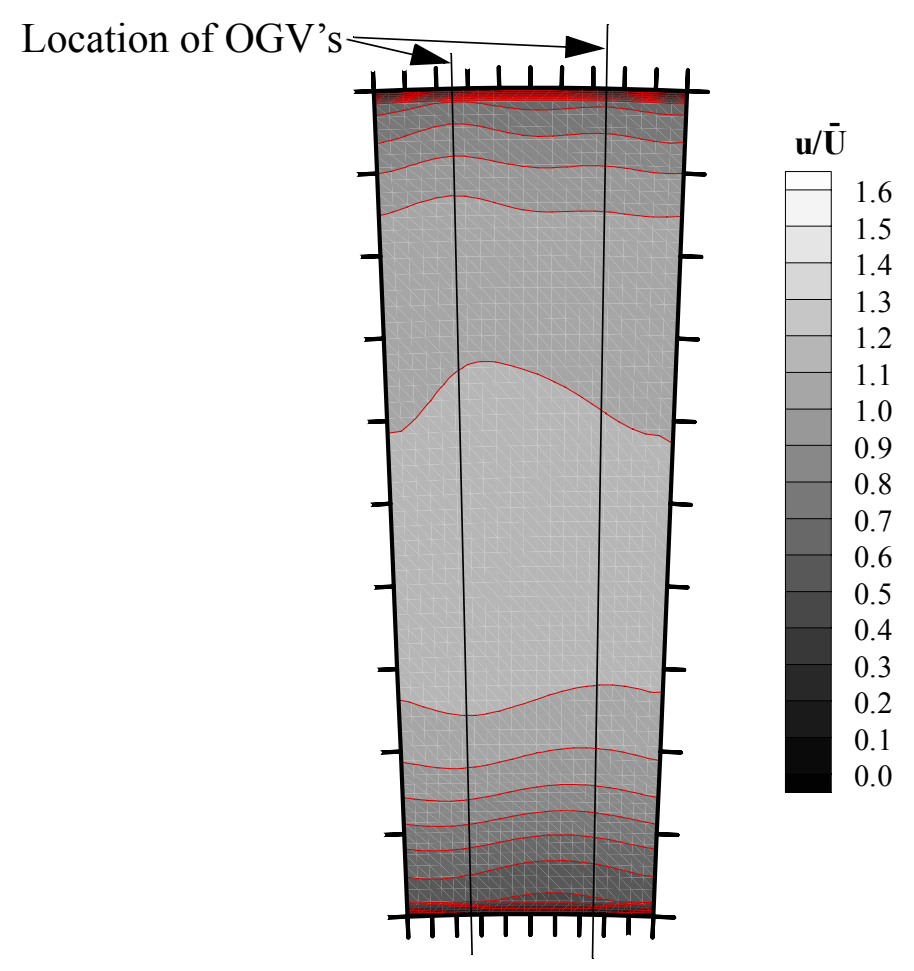

Figure 6.72 Normalised Axial Velocity Contours at Diffuser Exit (CFD Data, $T_{i}=$ $\left.5.0 \%, B_{i}=2.5 \%, B_{0}=3.0 \%\right)$

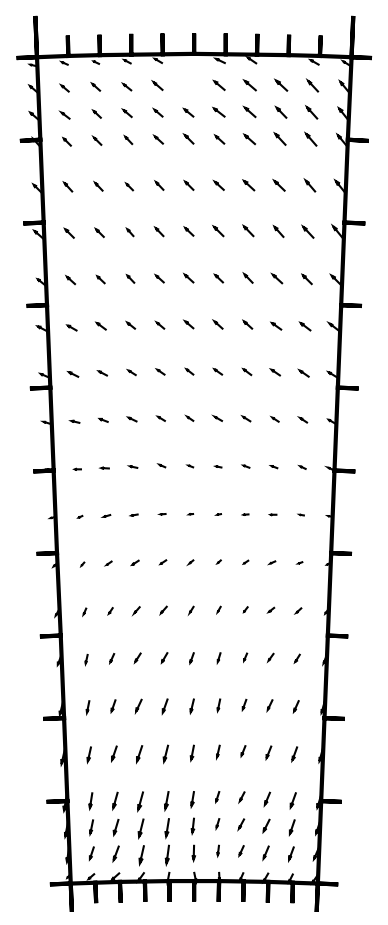

Figure 6.73 Velocity Vectors at Diffuser Exit (Experimental Data, $B_{i}=2.5 \%, B_{0}=$ $3.0 \%)$ 


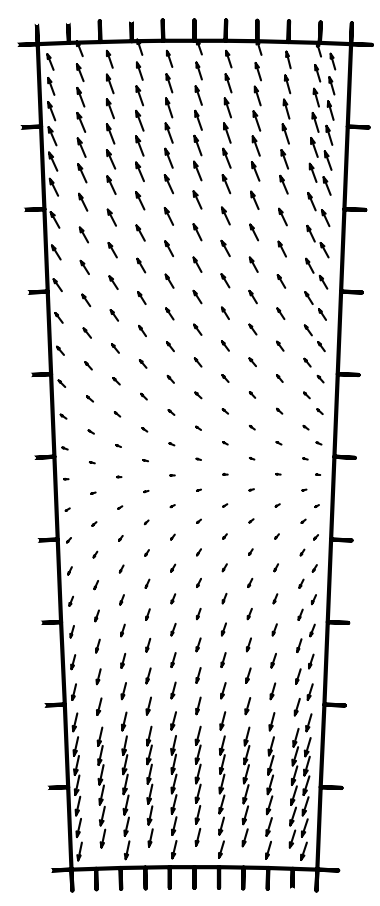

Figure 6.74 Velocity Vectors at Diffuser Exit (CFD Data, $T_{i}=5.0 \%, B_{i}=2.5 \%, B_{0}=$ $3.0 \%)$

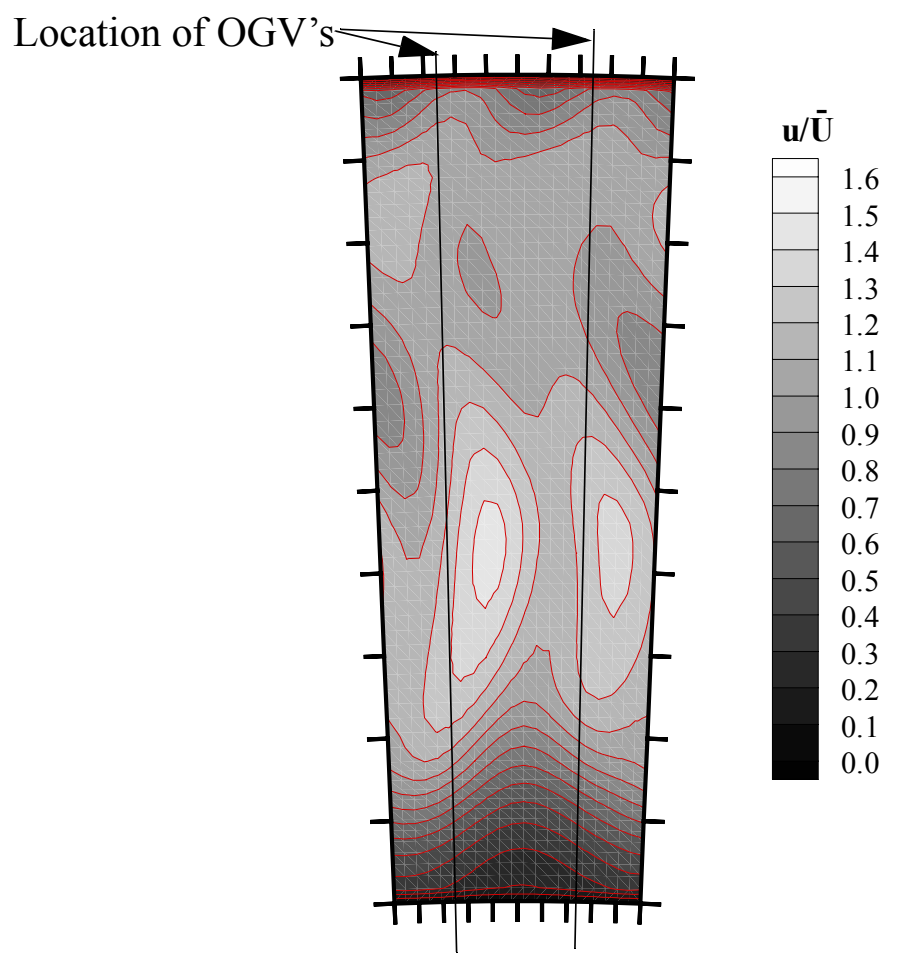

Figure 6.75 Normalised Axial Velocity Contours at Diffuser Exit (CFD Data $\mathbf{T}_{\mathbf{i}}=$

$$
\left.1.0 \%, B_{i}=2.5 \%, B_{0}=3.0 \%\right)
$$




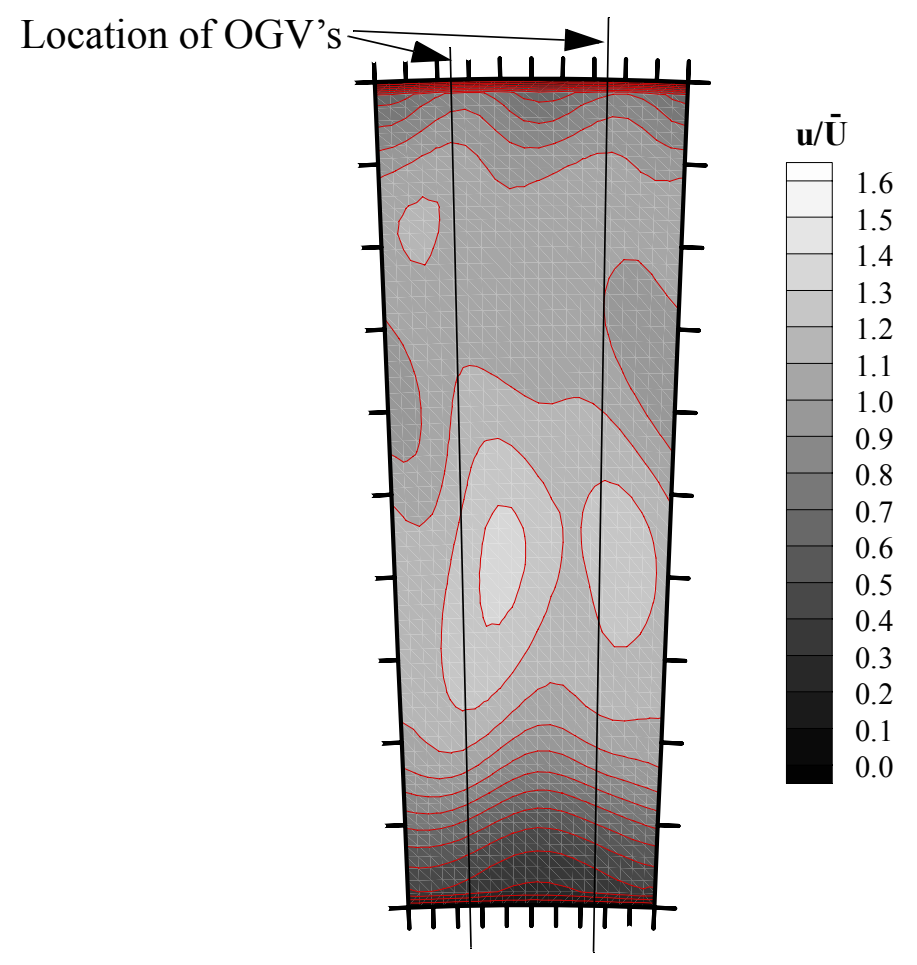

Figure 6.76 Normalised Axial Velocity Contours at Diffuser Exit (CFD Data $\mathbf{T}_{\mathbf{i}}=$ $\left.2.0 \%, B_{i}=2.5 \%, B_{0}=3.0 \%\right)$

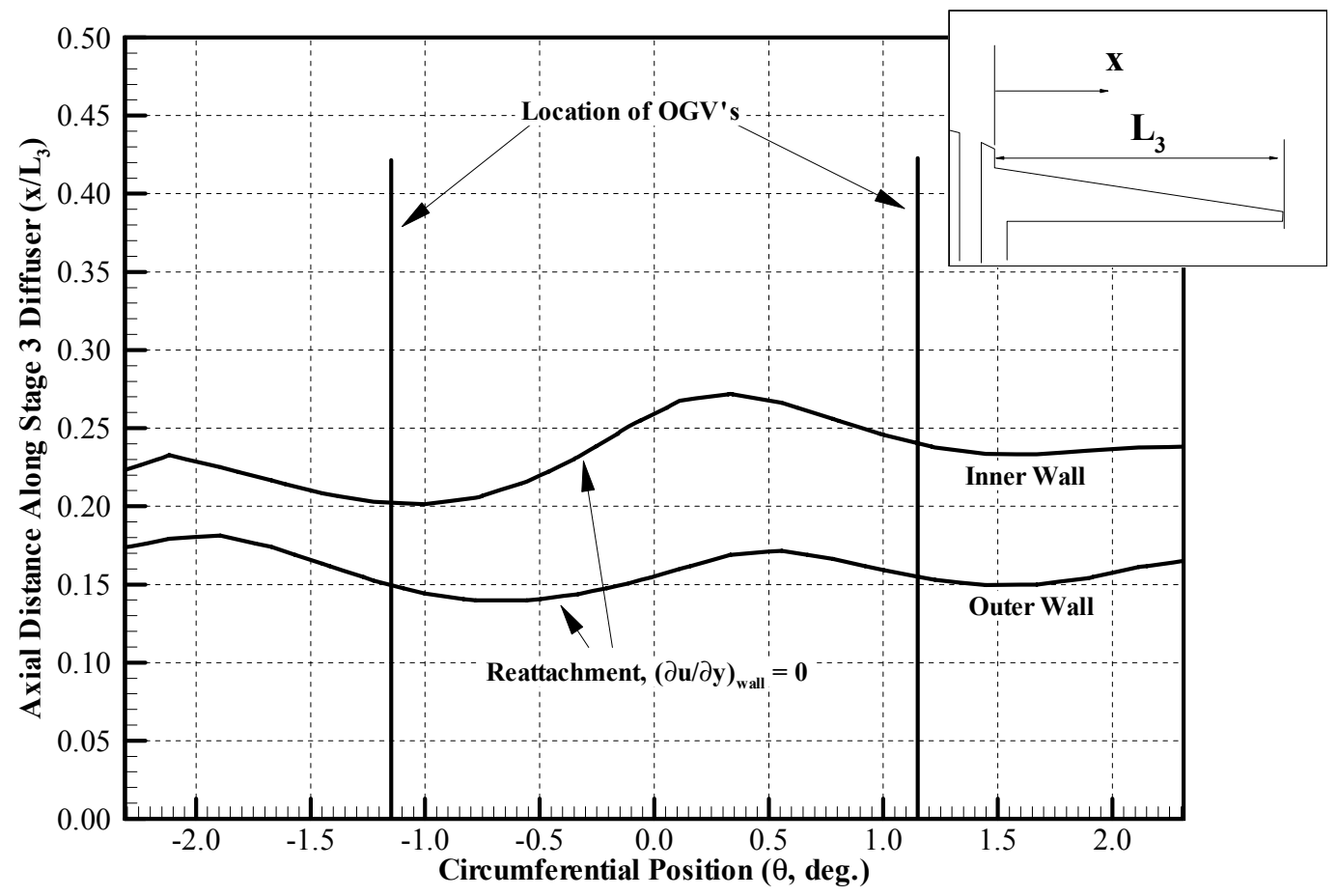

Figure 6.77 Circumferential Variation of Reattachment

$$
\left(T_{i}=5.0 \%, B_{i}=2.5 \%, B=3.0 \%\right)
$$




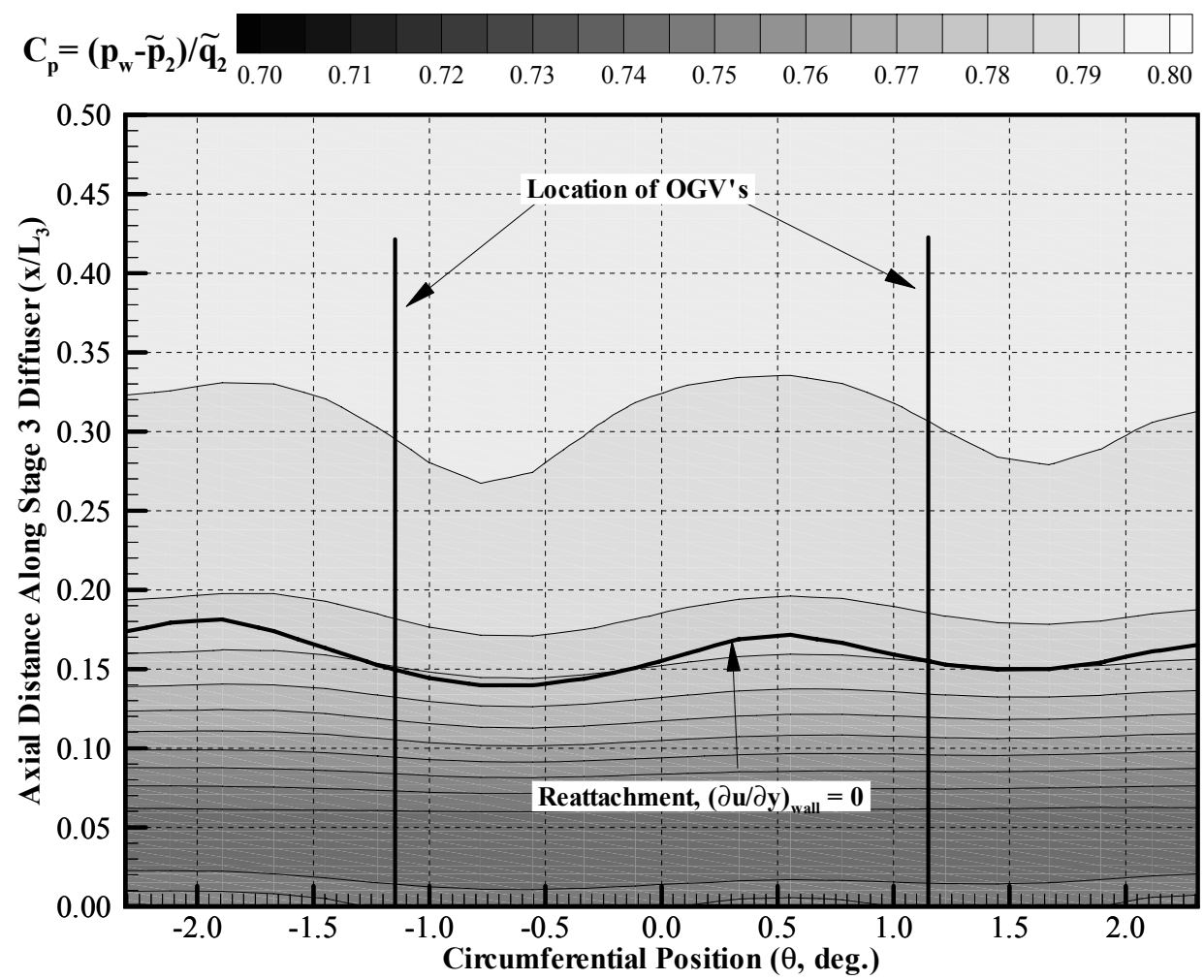

Figure 6.78 Outer Wall Static Pressure Contours $\left(T_{i}=5.0 \%, B_{i}=2.5 \%, B=3.0 \%\right)$

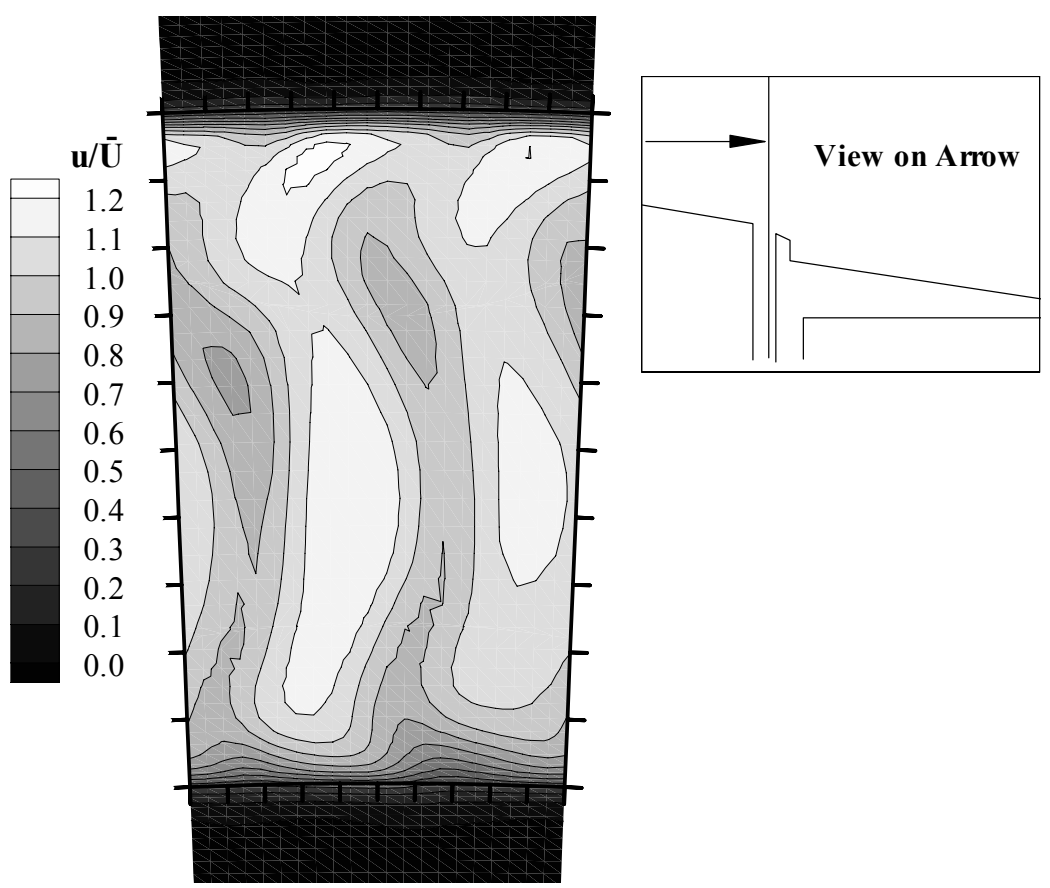

Figure 6.79 Axial Velocity Contours Plane at $x=70 \mathrm{~mm}$

$$
\left(T_{i}=5.0 \%, B_{i}=2.5 \%, B=3.0 \%\right)
$$




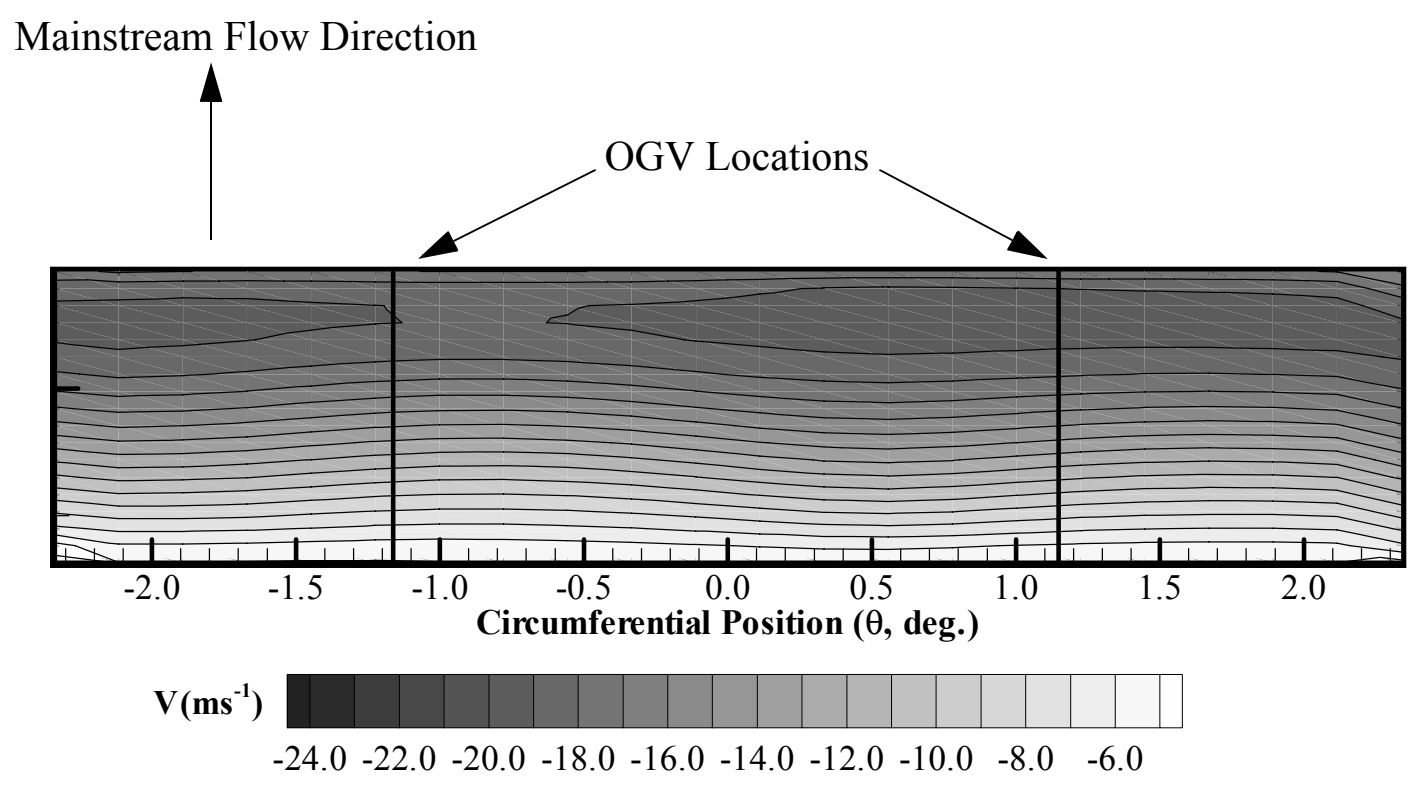

Figure 6.80 Inner Bleed Gap Radial Velocity Contours

$$
\left(T_{i}=5.0 \%, B_{i}=2.5 \%, B=3.0 \%\right)
$$

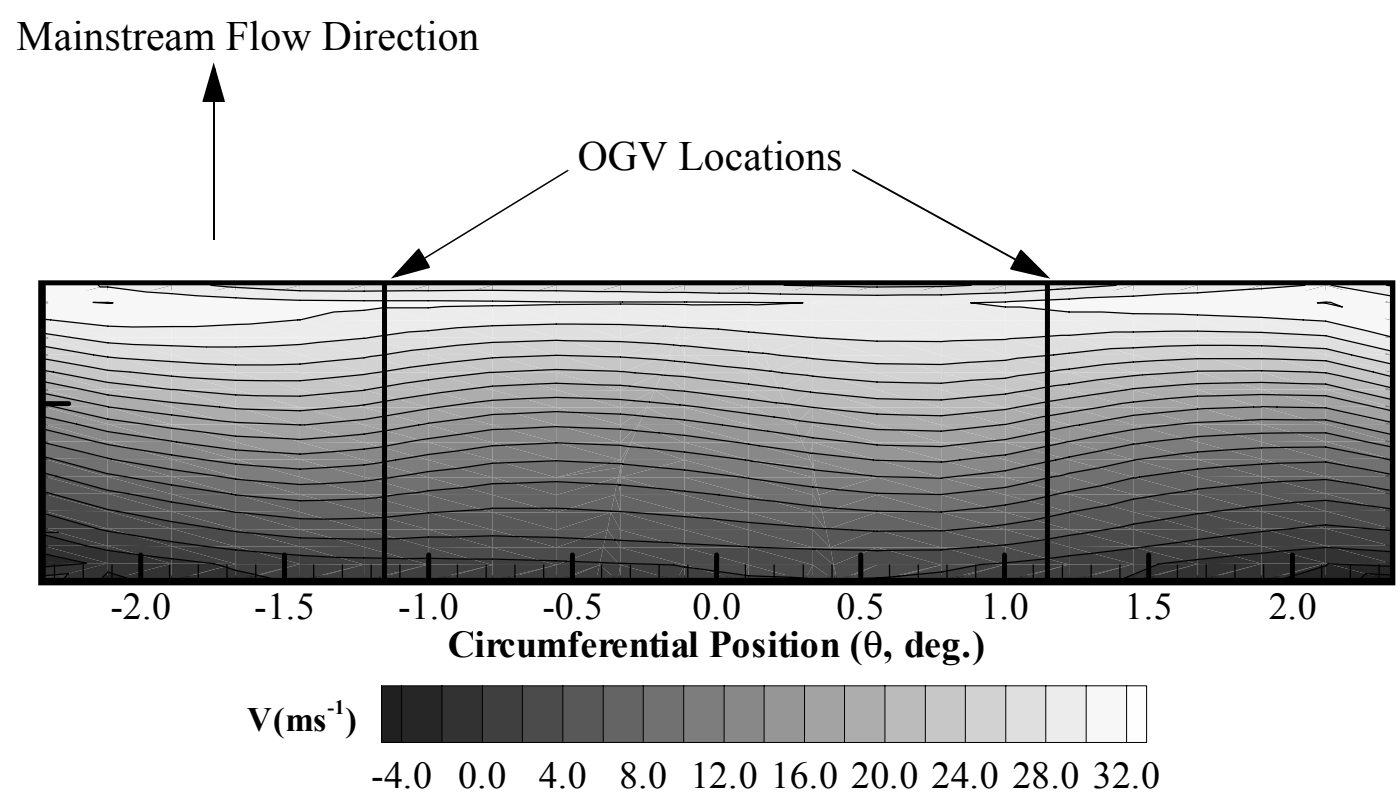

Figure 6.81 Outer Bleed Gap Radial Velocity Contours

$$
\left(T_{i}=5.0 \%, B_{i}=2.5 \%, B=3.0 \%\right)
$$




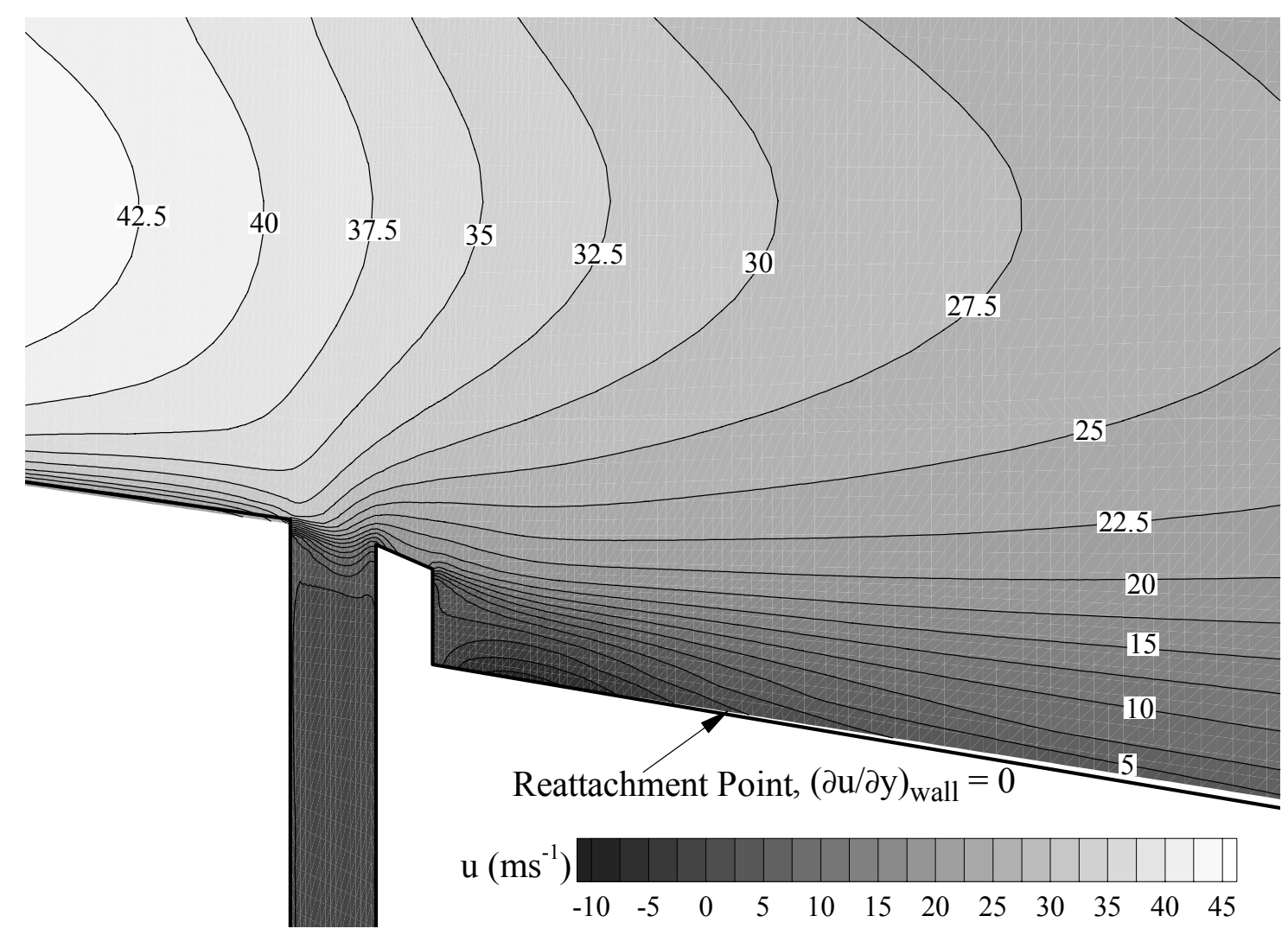

Figure 6.82 Stage 2Axial Velocity Contours within OGV Wake Fluid

$$
\left(T_{i}=5.0 \%, B_{i}=2.5 \%, B=3.0 \%\right)
$$

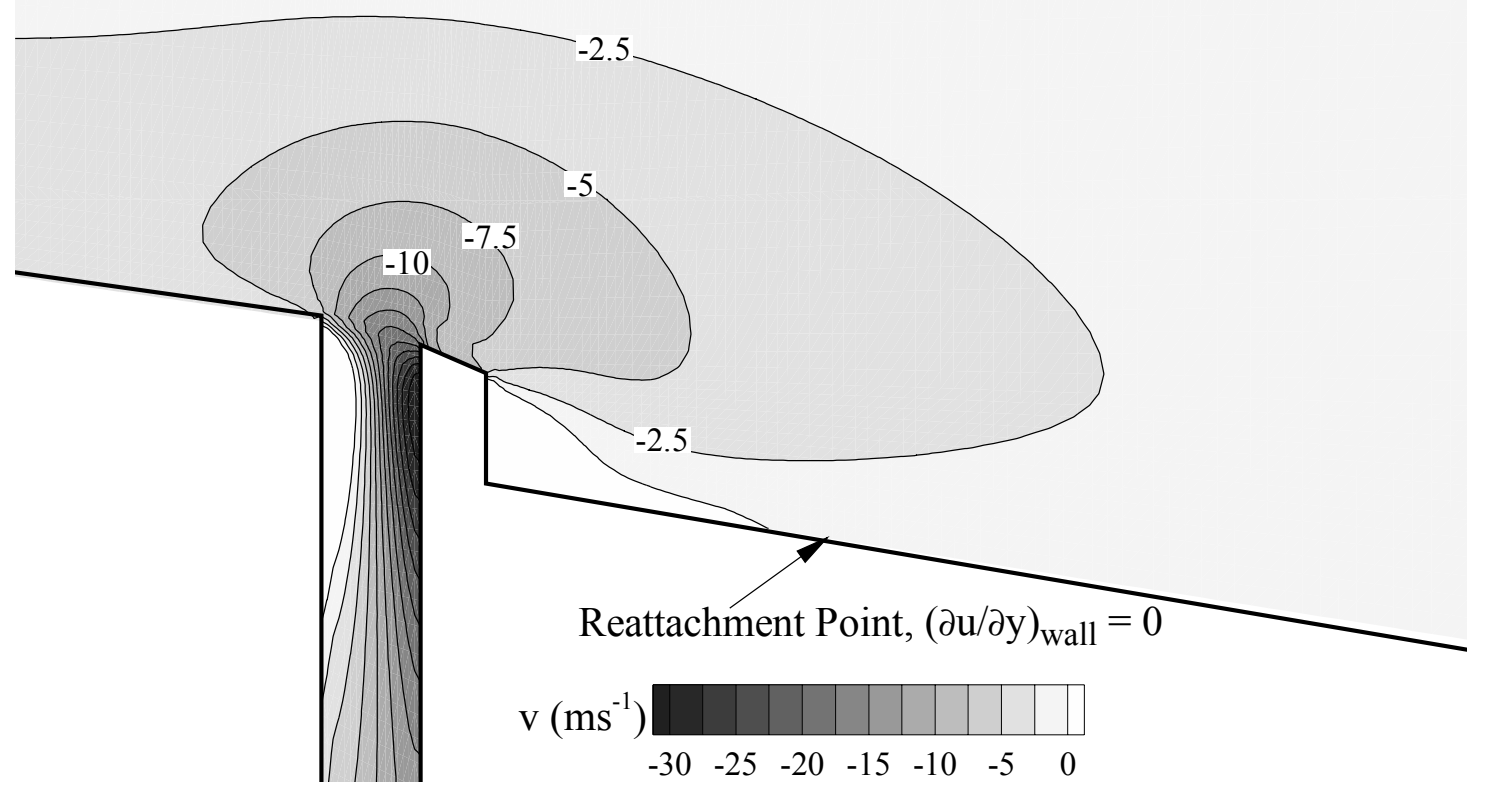

Figure 6.83 Stage 2 Radial Velocity Contours within OGV Wake Fluid

$$
\left(T_{i}=5.0 \%, B_{i}=2.5 \%, B=3.0 \%\right)
$$




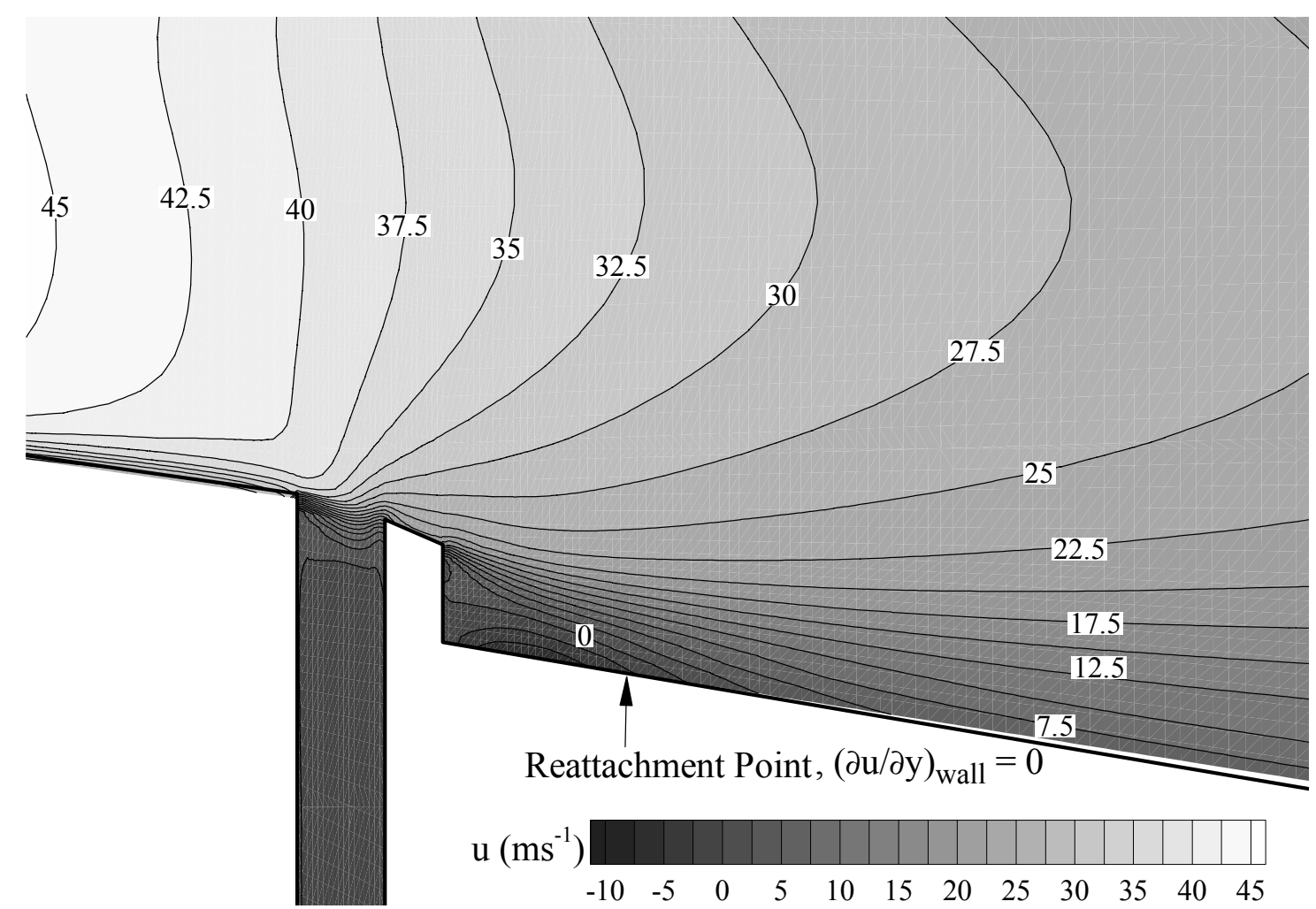

Figure 6.84 Stage 2 Axial Velocity Contours Between OGV Wake Fluid

$$
\left(T_{i}=5.0 \%, B_{i}=2.5 \%, B=3.0 \%\right)
$$

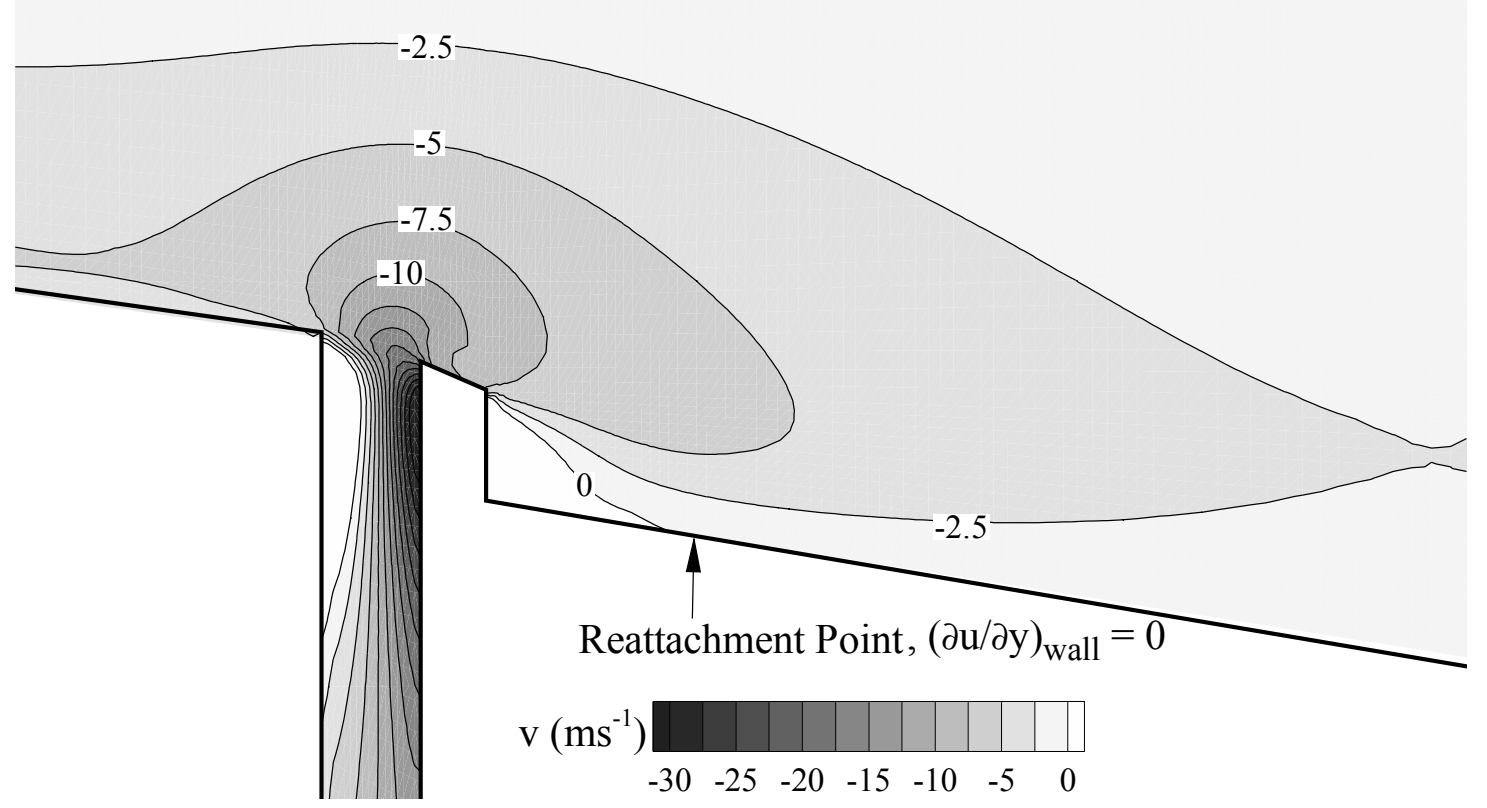

Figure 6.85 Stage 2 Radial Velocity Contours Between OGV Wake Fluid

$$
\left(T_{i}=5.0 \%, B_{i}=2.5 \%, B=3.0 \%\right)
$$




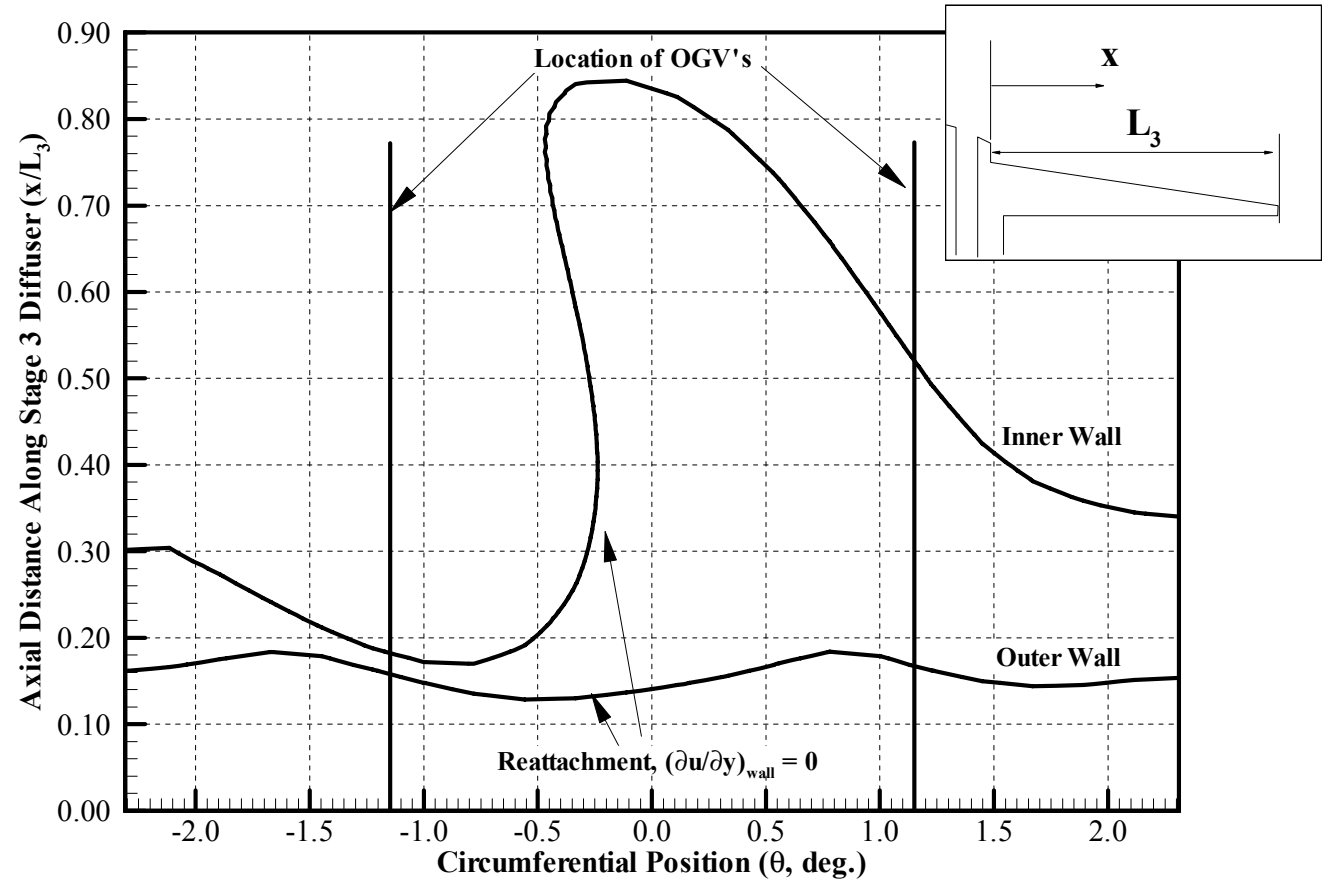

Figure 6.86 Circumferential Variation of Reattachment

$$
\left(T_{i}=5.0 \%, B_{i}=2.5 \%, B=3.0 \%\right)
$$




\subsection{CONCLUSIONS AND RECOMMENDATIONS}

7.1 Summary and Conclusions $\ldots \ldots \ldots \ldots \ldots \ldots \ldots 2$

7.2 Recommendations for Further Work $\ldots \ldots \ldots \ldots \ldots \ldots$. . . 297

FIGURES $\ldots \ldots \ldots \ldots \ldots \ldots \ldots \ldots \ldots \ldots \ldots \ldots \ldots . \ldots \ldots$ 


\subsection{CONCLUSIONS AND RECOMMENDATIONS}

\subsection{Summary and Conclusions}

Within the past few years strict legislation has come into force governing the emissions of pollutants from aircraft engines. Of prime importance to gas turbine designers is the reduction of $\mathrm{NO}_{\mathrm{x}}$ emissions and this has led to the possible use of staged combustion systems in an attempt to maintain combustion temperature within a low emission band. Staged combustion, however, demands high levels of diffusion and turning of the compressor efflux in order to avoid excessive aerodynamic losses which have a negative effect on engine specific fuel consumption. Furthermore, these high rates of diffusion are accompanied by large adverse pressure gradients and an associated risk of flow separation. A review of the relevant published data revealed that current conventional, modest area ratio diffuser technology is unable to meet these performance requirements. However, hybrid diffusers, as proposed by Adkins et $\mathrm{al}^{[1980]}$, can achieve high rates of efficient diffusion in far shorter lengths than conventional faired or dump pre-diffuser, representing a potential performance gain and weight saving. Notwithstanding this designers have been reluctant to employ such diffusers due to a lack of understanding of the pertinent flow mechanisms and how hybrid diffusers might be optimised for use in gas turbine combustors.

An experimental study has been carried out utilising an existing isothermal test facility comprising of a fully annular representation of a staged combustor downstream of a single stage axial compressor incorporating engine representative outlet guide vanes. Initial experimental work lead to rig modifications which allowed a range of hybrid diffuser systems to be studied. To act as a benchmark the performance of a conventional, singlepassage, dump pre-diffuser and a conventional, twin-passage, optimised dump pre-diffuser were first studied. Additionally, to provide a better understanding of the flow mechanisms, a computational investigation was also undertaken using a commercial CFD code (Fluent) employing finite volume methodology and an eddy-viscosity turbulence model.

Large amounts of data were gathered experimentally and it was concluded that these 
were of high quality. Similarly, a large amount of computational data were also produced. However, derived performance parameters such as mass weighted total pressure loss and static pressure recovery coefficients revealed that the computational data contained some degree of error. Predicted total pressure loss and static pressure recovery coefficients were slightly lower and higher, respectively, than those determined in an equivalent experiment. Notwithstanding this, it was shown that a valid parametric study could be performed to the extent that the same conclusions about parametric changes could be made from the experimental or predicted data. It is believed that the source of the error originated from two areas. Firstly, insufficient data were available to completely and accurately describe the flow's turbulence structure at inlet. Secondly, although the eddy-viscosity turbulence model is able to capture the main flow physics it is known to have some problems, for example under predicting turning losses.

On the whole, measured and predicted diffuser performance data agreed well with published data. For example, hybrid diffusers were shown, in a given length, to achieve much higher area ratios, controlled flow turning and static pressure recoveries than conventional diffusers. A strutted hybrid diffuser demonstrated a $53 \%$ increase in area ratio over a conventional, single passage "datum" diffuser designed using accepted nominal design rules and typical of current single annular engine technology. This increased area ratio realised a resultant static pressure recovery increase of $13 \%$. However, the type of hybrid diffuser proposed by Adkins et al ${ }^{[1980]}$ was seen to incur a higher diffuser total pressure loss due to the flow separation and reattachment process and the higher degree of overall turning.

The inclusion of radial struts within the Stage 3 section of a hybrid diffuser was shown to have only a minimal impact on the measured diffuser or system performance. Using measured data from an unstrutted hybrid diffuser the leading edge of the strut was positioned downstream of the reattachment point to prevent interactions with the reattachment process. Additionally, the inclusion of struts was also shown to remove residual swirl from the flow which in turn should have a beneficial effect on feed to the various combustor features.

In terms of overall system performance it is significant that both the conventional diffus- 
ers tested failed to deliver air to the combustor feed annuli without incurring unacceptably high total pressure losses. This further confirms the fact that conventional diffusers cannot achieve the required performance and are therefore unattractive for use with radially staged combustion systems.

In comparison, the increased diffusion and turning afforded by the hybrid diffusers greatly reduced the dump losses and resulted in feed annuli total pressure losses well within target values (i.e. below $30 \%$ of OGV exit dynamic pressure). This suggests that, in overall performance at least, hybrid diffusers represent a viable solution for the requirements of staged combustors.

Further agreement with previously published data was demonstrated in as much that the minimum required level of bleed was both dependant on, and increased with, diffuser area ratio. In addition, for a given configuration, levels of bleed similar to those employed in the published data were required such that at the maximum area ratio $(\approx 2.5)$ tested a bleed equal to $3.0 \%$ of the inlet mass flow was removed through both bleed ducts/chambers.

As the minimum bleed requirement is reached static pressure recovery rapidly increases while loss and reattachment length both rapidly reduce but all three then remain almost constant thereafter. Increasing the bleed rate above the minimum required level resulted in only a small increase in diffuser performance. However, increases in bleed rate were seen to be detrimental to total pressure losses within the bleed flow itself.

Levels of bleed required throughout this study were typical of the quantity of air used for turbine blade/disk cooling ( $6 \%$ of total inlet flow) and therefore the option of using the bleed air for component cooling remains a reality. Unfortunately, air bled through a vortex chamber incurs a high total pressure loss making it unattractive for component cooling purposes. However, it was demonstrated that a vortex chamber is not a necessary feature, and does not contribute to the flow mechanism of a hybrid diffuser, other than providing a stable bleed. A simple duct was shown to perform the same function and is not only attractive from a practical engineering perspective but was also seen to significantly reduce the bleed flow total pressure loss by reducing the size of the flow recircula- 
tion. For a bleed flow of $3.0 \%$ the total pressure loss through a vortex chamber was equal to $60 \%$ of the rotor exit dynamic pressure whereas for a simple duct this figure was reduced to $50 \%$. Additionally, modifications to the bleed duct, including profiling the upstream corner (Figure 7.1), removed any trace of flow separation and further reduced the bleed flow total pressure loss to $39 \%$ of the rotor exit dynamic head. This level of loss suggests that the air bled from a hybrid diffuser is sufficient in quality to be used for component cooling elsewhere in the engine.

Furthermore, a generic study of hybrid diffuser geometry revealed that the step/fence arrangement was also not necessary. Although providing an immediate increase in area the step does not contribute to the governing flow mechanisms. Moreover, the presence of the Coanda bubble is a result of the step/fence and also not a necessary component of hybrid diffusers. Thus the name Hybrid Vortex Controlled Diffuser becomes somewhat of a misnomer as the control is not afforded by the presence of any vortex. The term Hybrid Diffuser is more applicable.

Detailed examination of the flow field revealed that the controlling flow mechanisms are centred on the bleed flow. It is not a simple boundary layer bleed and involves a much more complex interaction between the accelerating bleed flow and the diffusing mainstream flow. Essentially, two processes occur as shown in Figure 7.2. Firstly, momentum is transferred from the accelerating bleed flow to the diffusing mainstream flow, re-energising it and enabling it to remain attached on the high angle Stage 3 wall under the action of a strong adverse pressure gradient. Secondly, the radial pressure gradient created by the bleed causes deflection of the mainstream flow which also transports higher momentum fluid into the boundary layer.

Understanding this allows the geometry of a hybrid diffuser to be optimised for use in a staged gas turbine combustion system. Figure 7.1 shows such a geometry with the step/ fence removed thus making the diffuser a much less complex engineering component. This type of hybrid diffuser was shown to achieve the same area ratio, static pressure recovery and loss in an equivalent length using comparable bleed rates as the type of hybrid "vortex controlled" diffuser proposed by Adkins at al ${ }^{[1980]}$ and others (Figure 7.3). Yet it is not only more attractive in engineering terms but modifications to the bleed 
duct were shown to reduce bleed flow losses making this air available for component cooling.

The condition of the flow at inlet to hybrid diffusers influences the performance of the diffuser and the ability of the flow to remain attached. With a clean inlet, a developed boundary layer and low levels of inlet turbulence hybrid diffusers were observed to stall. However, increased levels of inlet turbulence, such as those generated by the single stage axial compressor, for an identical hybrid diffuser prevented flow separation.

Additionally, a biased inlet velocity profile with a thick boundary layer on one wall does not present a problem to hybrid diffusers. Increasing the bleed on the wall in question has been shown to overcome any difficulties caused and returns a more uniform profile at diffuser exit. The ability of hybrid diffusers to overcome this type of inlet profile distortion may be significant in terms of operation downstream of a compressor functioning at an off-design condition.

The presence of OGV wakes in the inlet flow has been clearly shown to influence the performance of hybrid diffusers. The flow structure within OGV wakes redistributes high energy mainstream flow to the near wall regions thus preventing or delaying flow separation. Furthermore, the low energy fluid associated with wakes affects both the bleed and reattachment process. Local bleed rates are augmented because the low energy wake flow cannot overcome the radial pressure gradient set-up by the bleed. However, any benefit this may produce is negated by the fact that within the wake the axial momentum is relatively low and this fluid cannot easily overcome the high adverse pressure gradient. For a hybrid diffuser including a step this results in periodically increased reattachment lengths and regions, within the wakes, where the flow is more prone to separation.

Overall, this study has revealed a more detailed understanding into the pertinent flow mechanisms of hybrid diffusers. This has opened the path for modifications which can optimise this type of diffuser for a specific task such as use in a staged combustion system. Thus the final conclusion is that the performance of hybrid diffusers is more than satisfactory for use within low-emission, staged, gas turbine combustion systems. 


\subsection{Recommendations and Further Work}

Recommendations for further work forthcoming from this study can be separated into several main, but interconnected, areas:

- A greater understanding of the principles involved may have lead to a more simple design of hybrid diffuser but there remains no definitive data to define optimum bleed gap geometries. Several authors have inconclusively studied the effect of the relative size of the axial and radial bleed gaps but only on the type of hybrid "vortex controlled" diffuser proposed by Adkins et al ${ }^{[1980]}$. Therefore there exists a need to study the impact of alteration to bleed gap geometry in order to fully optimise hybrid diffusers. This type of parametric study would be ideally suited to the use of two-dimensional Computation Fluid Dynamics making it relatively straightforward and inexpensive.

- With the above in mind a logical extension to the work reported here would be to design, manufacture, fit and test an optimised hybrid diffuser to the experimental test facility. Furthermore, it is clear that twin-passage diffusers potentially offer many performance benefits over their single passage counterparts. Thus optimisation of a hybrid diffuser for use in a staged combustor may require such a feature leading to the need to investigate designs such as Figure 7.4.

- Further work could also be undertaken in an attempt to model, more accurately, a hybrid diffuser and staged combustor in three dimensions. Firstly, work would need to be undertaken to investigate and improve on the turbulence closure and, secondly, a more detailed description of the inlet turbulence ( $\mathrm{k}, \varepsilon$ and Reynolds Stress distributions) would have to be obtained. However, this type of study should not be undertaken lightly and would require a considerable amount of knowledge regarding advanced turbulence modelling. 


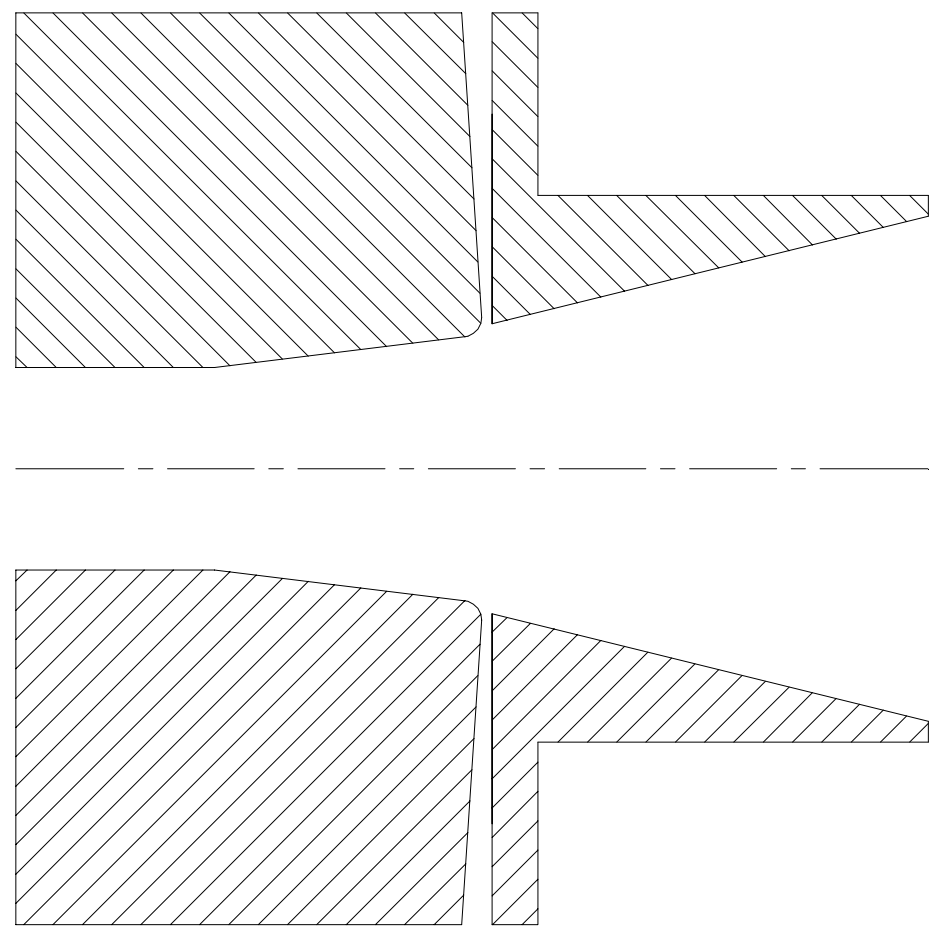

Figure 7.1 “Optimum” Hybrid Diffuser

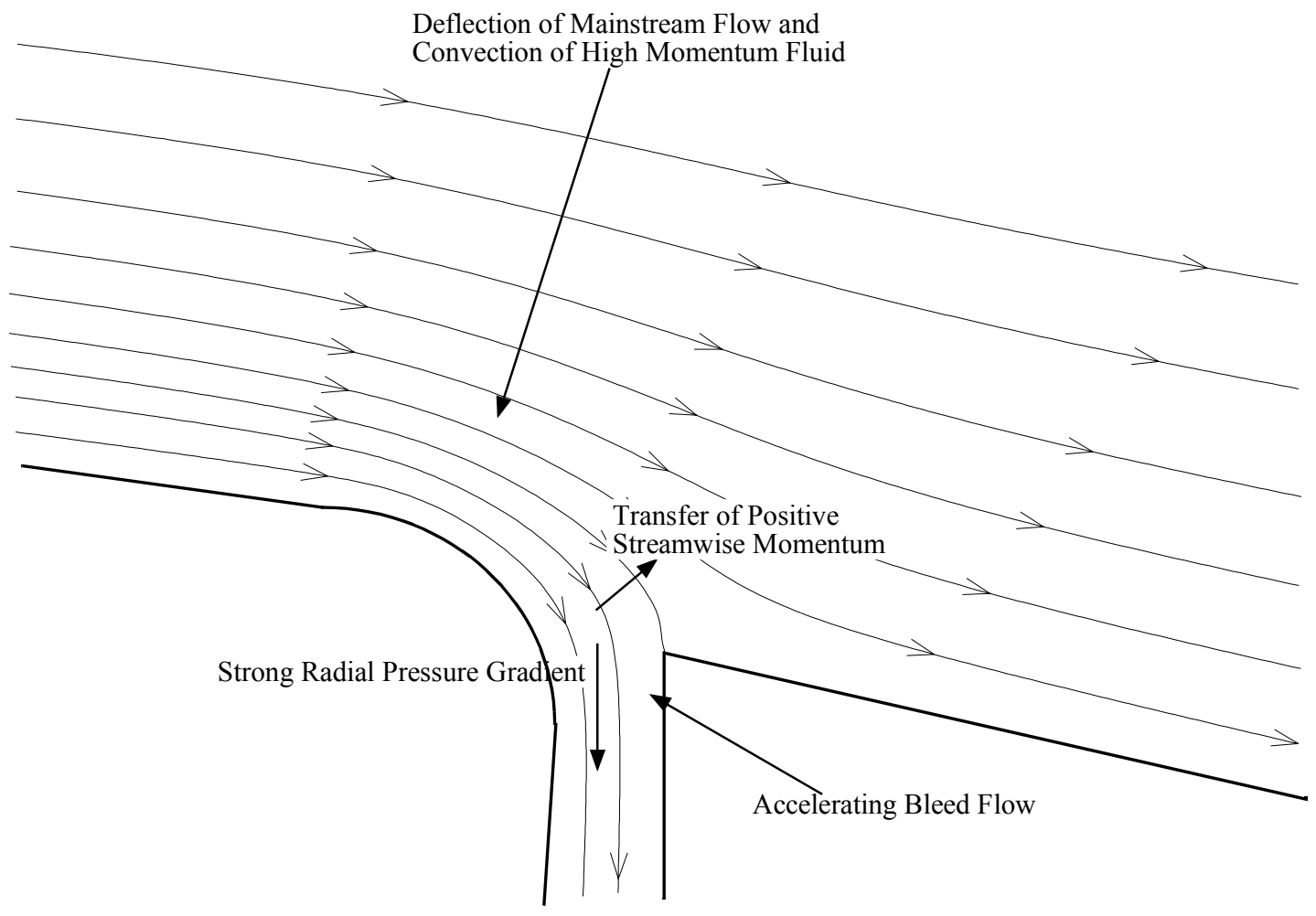

Figure 7.2 Main Flow Mechanisms 

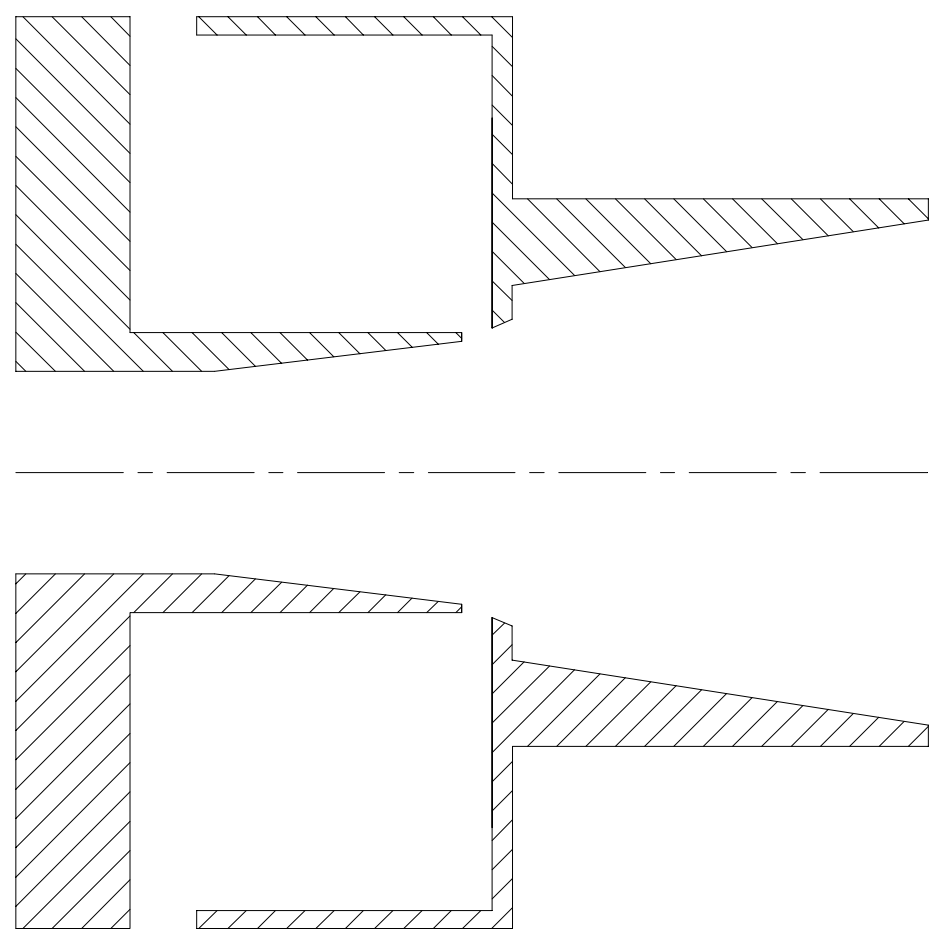

Figure 7.3 "Old" Hybrid "Vortex Controlled" Diffuser
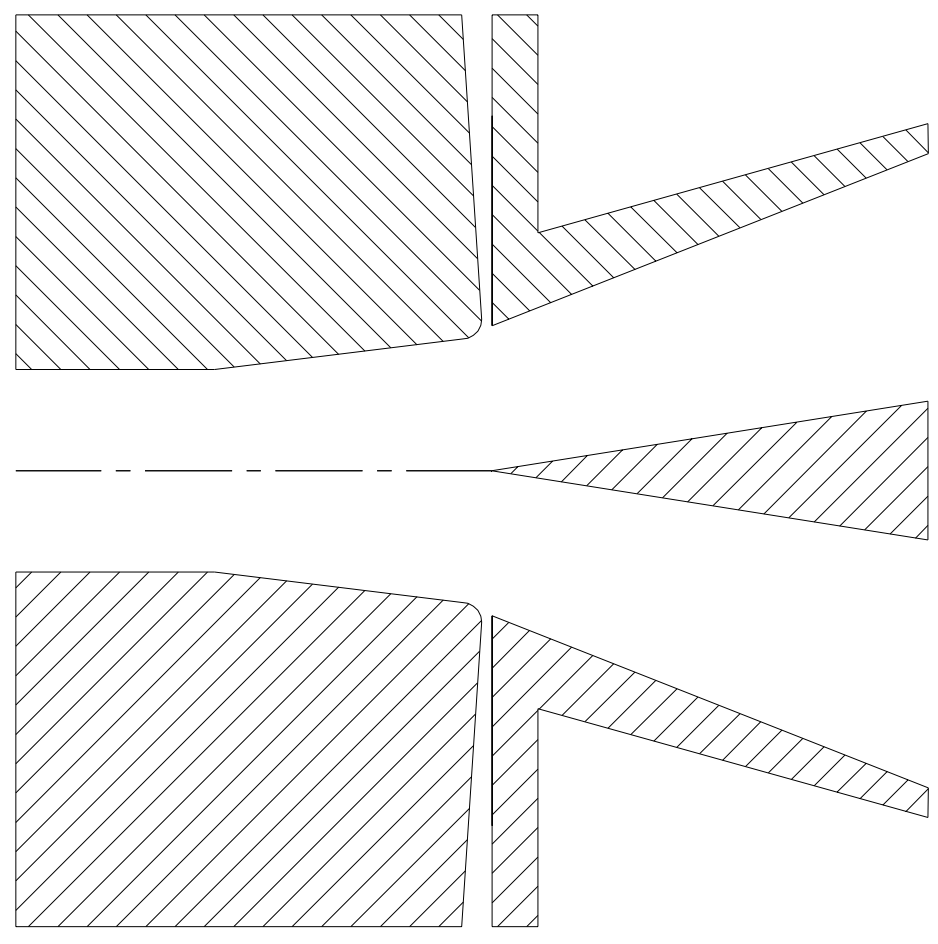

Figure 7.4 Further Hybrid Diffuser Development 
REFERENCES

\section{REFERENCES}


Adkins, R. C., 1975, “A Short Diffuser with Low Pressure Loss”, ASME Journal of Fluids Engineering, Vol. 97, pp 297-302.

Adkins, R. C., Matharu, D. S. and Yost, J. O., 1980, “The Hybrid Diffuser”, ASME Journal of Engineering for Gas Turbines and Power, 80-GT-136.

Adkins, R. C. and Montazerin N., 1985, “A Hybrid Diffuser with Distorted Inflow”. ASME Journal of Engineering for Gas Turbines and Power, 85-GT-109.

Adkins, R. C. and TerKuile, J.W.W., 1985, "Preliminary Aerodynamic Tests on a Canted Hybrid Diffuser”, Rolls-Royce Purchase Order T103994, Cranfield Project No. 615.

Adkins, R. C. and Yost, J. O., 1979, “A Combined Diffuser Arrangement”, Turbo and Jet Engine Technology, IGTA 19809, Part 1.

Adkins, R. C. and Yost, J. O., 1983, “A Compact Diffuser for Annular Combustors”, ASME Journal of Engineering for Gas Turbines and Power, 83-GT-43.

Bailey, D. W., 1997, "The Aerodynamic Performance of an Annular S-Shaped Duct”, PhD Thesis, Loughborough University.

Bailey, D. W. and Carrotte, J. F., 1996, “The Influence of Inlet Swirl on the Flow within an Annular S-Shaped Duct", ASME Journal of Engineering for Gas Turbines and Power, 96-GT-60.

Bahr, D. W., 1987, “Technology for the Design of High Temperature Rise Combustors”, AIAA Journal of Propulsion and Powerm Vol. 3, No. 2, pp 179-186.

Bahr, D. W., 1991, “Aircraft Engines $\mathrm{NO}_{\mathrm{x}}$ Emissions - Abatement Progress and Prospects", Proceedings of Tenth Inetrnational Symposium on Air-Breathing Engines, Nottingham, England.

Bahr, D. W., 1992, "Aircraft Turbine Engine $\mathrm{NO}_{\mathrm{x}}$ Emission Limits - Status and rends", 
ASME Paper No. 92-GT-415.

Bayle-Labouré, G., 1991, "Pollutant Emissions Frrom Aircraft Engines: a Situation Under Control”, Revue Scientifique SNECMA, 2nd Ed.

Barker, A. G. and Carrotte, J. F., 2000, “The Influence of Compressor Exit Conditions on Combustor Annular Diffusers, Part II: Flow Redistribution within the Diffuser", Dept. AAE, Loughborough University.

Beaty, C. G., 1970, "Diffusion Control by Trapped Vortices”, MSc Thesis, Cranfield Institute of Technology.

Birch, N. T., 2000, “2020 Vision : The Prospects for Large Civil Aircraft Propulsion”, The Aeronaut. J., Vol. 104, No. 1038, pp 347-352.

Bradshaw, P., 1973, "Effects of Streamline Curvature on Turbulent Flow”, AGARDograph AG 169.

Bradshaw, P., Cebeci, T. and Whitelaw, J. H., 1981, "Engineering Calculation Methods for Turbulent Flows", Academic Press, London.

Carrotte, J. and Bailey, D., 1994, “Annular Dump Diffuser Systems, Part I : Datum Configuration and the Effects of Dump Gap Reduction", Internal Report TT94R06, Dept. AAE, Loughborough University.

Carrotte, J. and Barker, A. G., 1994, “Annular Dump Diffuser Systems, Part II : The Combined Effects of Shortened Pre-Diffusers and Dump Gap Variation”, Internal Report TT94R07, Dept. AAE, Loughborough University.

Carrotte, J. and Barker, A. G., 1995, “Annular Dump Diffuser Systems, Part III : The Influence of Cowl Saape, Dump Liners and Mass Flow Splits", Internal Report TT95R03, Dept. AAE, Loughborough University.

Carrotte, J., Denman, P. A., Wray, A. P. and Fry, P., 1994, “Detailed Performance Com- 
parison of a Dump and Short Faired Combustor Diffuser", ASME Journal of Engineering for Gas Turbines and Power, Vol. 116, pp 517-526.

Craft, T. M. and Launder, B. E., 1991, “Computational Impinging of Flows Using Second Moment Closures", Paper 8-5, Eighth Symposium on Turbulent Shear Flows, Technical Universiy of Munich.

Cohen, H., Rogers, G. F. C. and Saravanamutto, H. I. H., 1996, “Gas Turbine Theory”, 4th Ed., Longman Group Ltd.

Daly, B. J. and Harlow, F. H., 1970, Phys. Fluids, Vol. 13, pp 2634-2649.

Denman, P. A., 1996, Private Communication, Dept. AAE, Loughborough University.

Elkersh, A. M., Elgammal, A. H. and Maccallum, N. R. L., 1985, “An Experimental Invetsigation of the Performance of Equiangular Annular Diffusers with Swirld Flow", Proceedings of the Institute of Mechanical Engineers, Vol. 199, No. C4, pp 293-297

Ferguson, T. B., 1962, "Stagnation Pressure and Losses in Ducts", Bulletin of Mechanical Engineering Education, July-Dec.

Fishenden, C. R. and Stevens, S. J., 1977, "Performance of Annular Combustor-Dump Diffusers”, Journal of Aircraft, Vol. 14, pp 60-67.

Fluent User's Guide, 1998, Flunet Inc.

Furuya, Y., Sato, T. and Hushida, T., 1966, “The Loss of Flow in the Connical Diffuser with Suction at the Entrance", JSME, Vol. 9, No. 33, pp 131-137.

Hay, N. and Spencer, A.,1992, "Discharge Coefficients of Cooling Holes with Radiused and Chamfered Inlets", ASME Journal of Turbomachinery, Vol. 114, pp 701-706.

Heskestad, G., 1966, “A Suction Scheme Applied to Flow Through a Sudden Enlarge- 
ment”, ASME Journal of Basic Engineering, June, pp 539-549.

Heskestad, G., 1968, "Remarks on Snow Cornice Theory and Related Experiments with Sink Flows", ASME Journal of Basic Engineering, December, pp 541-554.

Hestermann, R., Kim, S. and Wittig, S., 1991, "Geometrical Dependence of the Fluid Dynamic Parameters of Plane Combustor Model Diffusers", Proceedings of the International Symposium on Air Breathing Engines, ISABE 91-7105, pp 995-1001.

Hinze, J. O., 1975, “Turbulence”, McGraw-Hill Publishing Company, New York.

Hoffmann, J. A., 1981, "Effects of Free-Stream Turbulence on Diffuser Performance”, ASME Jourbal of Fluids Engineerin, Vol. 103, pp 385-390.

Hoffmann, J. A. and Gonzalez, G., 1983, "Inlet Free-Stream Turbulence Effacts on Diffuser Performance", NASA-CR-166516.

Hoffmann, J. A. and Gonzalez, G., 1994, "Effects of Small Scale, High Intensity Inlet Turbulence on Flow in a Two-Dimensional Diffuser", ASME Journal of Fluids Engineering, Vol. 106, pp 121-124.

Howard, J. H. G., Henseler, H. J. and Thornton-Trump, A. B., 1967, "Performance and Flow Regimes for Annular Diffusers”, ASME PAper 67-WA/FE-21.

Hung, W. S. Y., 1974, “Accurate Method of Predicting the Effect of Humidity or Injected Water on $\mathrm{NO}_{\mathrm{x}}$ Emissions From Industrial Gas Turbines”, ASME Paper No. 74-WA/GT6.

Hung, W. S. Y. and Agan, D. D., 1985, “The Control of $\mathrm{NO}_{\mathrm{x}}$ and CO Emissions From 7MW Gas Turbines with Water Injection as Influenced by Ambient Conditions", ASME Paper No. 85-GTY-50.

Intergovernmaental Panel on Climate Change, 1999, "Aviation and the Global Atmos- 
phere", IPCC.

Isomoto, K. and Honami, S., 1989, "The Effect of Inlet Turbulence Intensity on the Reattachment Process Over a Backward-facing Step”, ASME Journal of Fluids Engineering, Vol. 111, pp 87-93.

Japikse, D., 1984, “Turbo Machinery Diffuser Technology”, Concepts ETI.

Kim, S. E. and Choudhury, D., 1995, “A Near Wall Treatment Using Wall Functions Sensitised to Pressure Gradient", ASME FED Vol. 217, Separated and Complex Flows, ASME.

Klein, A., Pucher, P. and Rohiffs, M., 1980, “The Effect of Blade Wakes on the Performance of Short Dump-Diffuser Type Combustor Inlets", ASME Journal of Fluids Engineering, Vol. 102, pp 236-242.

Klein, A., 1981, "Effects of Inlet Conditions on Connical Diffuser Perofrmance", ASME Journal of Fluids Engineering, Vol. 103, pp 250-257.

Klein, A., "Characteristics of Combustor Diffusers", 1995, Prog. Aerospace Science, Vol. 31, pp 171-271.

Kline, S. J., Abbott, D. E. and Fox, R. W., 1959, “Optimum Design of Straight Walled Diffusers", ASME Journal of Basic Engineering, Vol. 81, pp 321-331.

Launder, B. E. and Spalding, D. B., 1972, "Lectures in Mathematical Models of Turbulence", Accademic Press, London.

Launder, B. E. and Spalding, D. B., 1974, "The Numerical Computation of Turbulent Flows". Comput. Methods Appl. Mech. Eng., Vol. 3, pp 269-289.

Launder, B. E., Reece, G. J. and Rodi, W., 1975, "Progress in the Development of a Reynolds-Stress Turbulence Closure”, Journal of Fluid Mech., Vol. 68 (Part 3), pp 537-566. 
Launder, B. E., 1989, “Second-Moment Closure: Present ... and Future?”, International Journal of Heat Fluid Flow, Vol. 10, No. 4, pp 282-300.

Lien, F. S. and Leschzine, M. A., 1994, "Assesment of Turbulent Transport Models Including Non-Linear RNG Eddy-Viscocity Formulation and Second Moment Closure", Computers and Fluids, Vol. 23, pp 983-1004.

Lefebvre, A. H., 1983, “Gas Turbine Combustion”, McGraw-Hill.

Lefebvre, A. H., 1995, “The Role of Fuel Preparation in Low-Emission Combustion”, ASME Journal of Engineering for Gas Turbines and Power, Vol. 117, pp 617-654.

Leipe, F., 1963, "Investigation into the Behaviour of Flows with Rotation in Conical Diffusers", Marchinenbautchnich, Vol. 12, No. 3.

Leonard, G. and Stegmier, J., 1994, "Development of an Aeroderivative Gas Turbine Dry Low Emissions Combustion System”, ASME Journal of Engineering for Gas Turbines and Power, Vol. 116, pp 542-546.

Little, A. R., Denman, P. A. and Manners, A. P., 1997, "Prediction and Measurement of the Total Pressure Loss in an Engine Representative Diffuser System", ASME Journal of Turbomachinery, Vol. 119, pp 390-396.

Livesey, J. L., 1972, “Duct Performance Parameters Considering Spacially Non-Uniform Flows", AIAA Paper, pp 72-85.

Livesey, J. L. and Hugh, T., 1966, "Suitable Mean Values in One-Dimensional Gas Dynamics”, Journal of Mechanical Engineering Science, Vol. 8, No. 4, pp 374-383.

Livesey, J. L. and Turner, J. T., 1964, "The Generation of Symmetrical Duct Velocity Profiles of High Uniform Shear", Journal of Fluid Mechanics, Vol. 20.

Lohmann, R. P., Markowski, S. J. and Brookman, E. T., 1979, “Swirling Flows Through 
Annular Diffusers with Conical Walls", ASME Journal of Fluids Engineering, Vol. 101, pp 224-229.

Maruszewski, J.P., 1991, "Bounded QUICK Scheme in FLUENT”, Fluent Inc. Tecnical memo, TM-049.

McDonald, A. T. and Fox, R.W., 1966, “An Experimental Investigation of Incompressible Flow in Conical Diffusers", International Journal of Mechanical Science, Vol. 8.

Montazerin, N. and Adkins, R. C., 1985, "Preliminary Tests on an Annular Hybrid Diffuser", ASME Journal of Engineering for Gas Turbines and Power, 85-GT-108.

Moore, A., 1976, "The Use of Vanes to Improve Wide Angle Diffuser Performance", RR1377, BHRA Fluid Engineering, Cranfield.

Mortimer, L., 1998, "Commitee Reaches Consensus on Need for Action to Further Protect the Environment", ICAO Journal, Vol. 53, No. 6.

Mosier, S. A. and Pierce, R. M., 1980, “Advanced Combustion Systems forStationary Gas Turbine Engines", Vol. I, EPA Contract 68-02-2136.

Myres, G., Cardenas, M., Srinivasan, R. and Arans, C., 1993, "Effect of Geometry, Bleed Rates and Flow Splits on the Pressure Recovery of a Canted Hybrid Vortex Controlled Diffuser", American Institute of Aeronautics Inc.

Oates, G. C. (ed.), 1985, “Aerodynamics of Aircraft Engine Components”, AIAA Eductation Series, New York.

Patankar, S. V., 1980, "Numerical Heat Transfer and Fluid Flow”, Hemisphere Publishing Corp., Washington, DC.

Pidcock, A. A., 2000, Private Communication, Rolls-Royce Plc.

Pierce, R. M., Smith, C. E. and Hinton, B. S., 1980, “Advanced Combustion Systems for 
Stationary Gas Turbine Engines", Vol. III , EPA Contract 68-02-2136.

Poeschl, G., Ruhkamp, W. and Pfost, H., 1994, "Combustion with Low Pollutant Emissions of Liquid Fuels in Gas Turbines by Premixing and Prevaporisation", ASME Paper No. 94-GT-443.

Ringleb, F. O., 1955, "Flow Control by Generation of Standing Vortices and the Cusp Effect”, Princeton University, Aeronautical Engineering Report No. 317.

Roberts, P. B., Kubasco, A. J. and Sekas, N. J., 1982, "Development of a Low $\mathrm{NO}_{\mathrm{x}}$ Lean Premixed Annular Combustor", ASME Journal of Engineering for Power, Vol. 104, pp 28-35.

Sagi, C. J. and Johnston, J. P., 1967, “The Design and Performance of Two-Dimensional Curved Diffusers", ASME Journal of Basic Engineering, pp 715-731.

Salba, P. E., Taylor, J. R. and Gaunter, D. J., 1982, "Design and Developement of teh Combustor Diffuser for NASA/GE Energy Efficient Engine" Journal of Energy, Vol. 6, pp 275-282.

Sasaki, M., Kumakura, H. and Suzuki, D., 1991, "Low NO $\mathrm{x}_{\mathrm{x}}$ Combustors for Automotive Ceramic Gas Turbine - Conceptual Design”, ASME Paper No. 91-GT-369.

Seaton, A., MacNee, W., Donaldson, K and Godden, E., 1995, "Particuate Air Pollution and Acute Health Effects", Lancet, Vol. 345, pp 176-178.

Schlitchting, H., 1979, "Boundary Layer Theory”, 7th ed. McGraw-Hill.

Shedden, P. S. R., 1993, “An Experimental Study of the Aerodynamic Performance of a Double Annular Combustor System”, M.Phil. Thesis, Loughborough University.

Sitaram, N., Lakshminarayana, B. and Ravidranath, A., 1981, "Conventional Probe for the Relative Flow Measurements in a Turbo Machinery Rotor Blade Passage", Trans. 
ASME Journal of Engineering and Power, Vol. 103.

Slingsby, B., 1967, “The Effect of Boundary Layer Control on Two-Dimensional Wide Angle Subsonic Diffusers", Thesis, Cranfield College of Aeronautics.

Sovran, G. and Klomp, E. D., 1967, “ Experimentally Determined Optimum Geometries for Rectilinear Diffusers with Rectangular, Connical or Annular Cross-Section", Fluid Mechanics of Internal Flow, pp 270-319, Elsevier, New York.

Srinivasan, R., Freeman, G., Mozumdar, S. and Grahmann, J.,1990, "Measurements in an Annular Combustor-Diffuser System”, AIAA 90-2162.

Srinivasan, R., Freeman, G., Grahmann, J. and Coleman, E., 1990, "Parametric Evaluation of the Aerodynamic Performance of an Annular Combustor-Diffuser System", AIAA 90-2163.

Stevens, S. J., Nayak, U. S. L., Preston, J. F., Robinson, P. J. and Scrivener, C. T. J., 1978, "Influence of Compressor Exit Conditions on Diffuser Performance", Journal of Aircraft, Vol. 15, No. 8.

Stevens, S. J. and Williams, G. J., 1980, “The Influence of Inlet Conditions on the Performance of Annular Diffusers", ASME Journal of Fluids Engineering, Vol. 102, pp 357 363.

Stevens, S. J., Harasgama, S. P. and Wray, A. P., 1984, "The Influence of Blade Wakes on the Performance of Combustor Shortened Pre-Diffusers”, Journal of Aircraft, Vol. 21, No. 9.

Sullerey, R. K, Ashok, V. and Shantharam. K. V., 1992, "Effect of Inlet Flow Distortion on Performance of Vortex Controlled Diffusers", ASME Journal of Fluids Engineering, Vol. 114, pp 191-197.

Sutherland, G. C., 1972, “An Investigation into the Flow of Vortex Diffusers”, MSc The- 
sis, Cranfield Institute of Technology.

Tamigniaux, T. L. B. and Oates, G. G., 1986. "Effects of a Nearby Solid Surface on a Five-Hole Probe Measurement”, AIAA Journal, Vol. 24.

Versteeg, H. K. and Malalasekera, W., 1995, "Introduction to computational fluid dynamics:the finite volume method", Harlow-Longman,1995.

Wen, P. R. and Jienkiewicz, H. K., 1957, "The Production of Shear Flow in a Wind Tunnel”, Journal of Fluid Mechanics, Vol. 2.

Wolf, S. and Johnston, J. P., 1969, "Effects of Non-Uniform Inlet Velocity Profiles On Flow Regimes and Performance in Two-Dimensional Diffusers", ASME Journal of Basic Engineering, Vol. 91, pp 462-473.

Wray, A. P., 1995, Private Communication, Dept. AAE, Loughborough University.

Young, K. F., 1988, “The Performance of Compressor OGV's and Downstream Diffuser", Ph.D. Thesis, Loughborough University. 


\section{A1 PRELIMINARY EXPERIMENTAL INVESTIGATION}

A1.1 Introduction $\ldots \ldots \ldots \ldots \ldots \ldots \ldots \ldots \ldots \ldots \ldots \ldots \ldots \ldots \ldots \ldots \ldots \ldots$

A1.2 Experimental Arrangement ................A1-2

1.2.1 Swirl - Deswirl Inlet $\ldots \ldots \ldots \ldots \ldots \ldots \ldots \ldots \ldots \ldots \ldots \ldots \ldots \ldots$

1.2.2 Test Section Geometry $\ldots \ldots \ldots \ldots \ldots \ldots \ldots \ldots \ldots \ldots$

1.2 .3 Instrumentation $\ldots \ldots \ldots \ldots \ldots \ldots \ldots \ldots \ldots \ldots \ldots \ldots \ldots \ldots$

A1.3 Results and Discussion $\ldots \ldots \ldots \ldots \ldots \ldots \ldots \ldots \ldots \ldots \ldots \ldots \ldots \ldots$

A1.4 Conclusion $\ldots \ldots \ldots \ldots \ldots \ldots \ldots \ldots \ldots \ldots \ldots \ldots \ldots \ldots \ldots \ldots$

A1.5 FIGURES ........................A1-8 


\section{A1 PRELIMINARY EXPERIMENTAL INVESTIGATION}

\section{$\underline{\text { A1.1 Introduction }}$}

The design of a research test facility for the study of hybrid diffusers involves consideration of several important factors and ultimately some kind of trade-off. On review of the relevant literature it was clear that there existed only a limited understanding of the controlling flow mechanisms of hybrid diffusers. Therefore, it was considered important to isolate these mechanisms by removing complex geometry influences and revert to a 'generic' geometry. An extra advantage of this is a reduction of the physical complexity of a test facility thus aiding access for instrumentation and minimising overall cost. However, it is also important that a test facility can adequately represent any real application under investigation. Here, for example, the potential use of hybrid diffusers in radially staged aero gas turbine combustion systems is an important factor. This raises the question of whether the test facility needs to be fully annular, include a flame tube, burners, a rotor, IGVs or OGVs.

For this project it was envisaged that the initial stages of the investigation would be conducted using a simple rectangular/planar test rig. A fully annular facility (refer to Section 3.0) would be used for the latter stages of the investigation once a set of design rules had been established. A simple rectangular/planar test rig would allow for modular construction which in turn enables easy modification of the diffuser geometry. Such a test rig was designed incorporating only a very basic and simple representation of a flame tube and a set of swirl-deswirl vanes to simulate reasonably representative inlet conditions.

\section{$\underline{\text { A1.2 Experimental Arrangement }}$}

The rectangular test facility (Figure A1.1) was designed to be of similar non-dimensional geometry (based on OGV exit height) as the fully annular test facility described in Section 3.0. The reason for maintaining a similar geometry was to allow for a back-to-back comparison between the two test facilities. The rig was supplied with air via an underground plenum chamber from a centrifugal fan driven by a variable speed, $75 \mathrm{~kW}$ d.c. motor at a rate of about $1.6-1.7 \mathrm{kgs}^{-1}$. 
The overall size of the rig was a compromise between several factors. A high aspect ratio rig is preferable in terms of reducing end wall boundary layer effects and improving accessibility for pressure probes and other instrumentation. Two limiting factors were the capacity of the centrifugal fan and the size of the plenum chamber. However, by far the most important factor in determining the rig size was the aspect ratio. This is extremely important because of the boundary layer growth on the end walls of a rectangular/sector rig. It is usual to quote the aspect ratio as the ratio between rig width and inlet height, i.e. $\mathrm{W}_{\mathrm{R}} / \mathrm{h}_{1}$, and a value of around 10 is typical. If the width of the rig is insufficient then the effect of the end wall boundary layers will be felt throughout the rig including the central measurement section. An aspect ratio quoted with respect to the diffuser exit height is perhaps more important because the adverse pressure gradient within the diffusing flow will cause the boundary layer to grow more rapidly. The final design of the test rig had an inlet height of $45.75 \mathrm{~mm}$ which is a 1.5 scale up from the fully annular facility. Rig width was physically limited to $1000 \mathrm{~mm}$ giving an aspect ratio, based on the inlet height, of around 20 and based on the diffuser exit height of around 8 .

\section{$\underline{\text { A1.2.1 Swirl - Deswirl Inlet }}$}

The effect of inlet conditions on the performance of diffusers is a subject which has been thoroughly investigated by Stevens, Harasgama and Wray ${ }^{[1984]}$, Stevens and Williams ${ }^{[1980]}$, Stevens et $\mathrm{al}^{[1978]}$ for conventional diffusers and Adkins et $\mathrm{al}^{[1980]}$ for Hybrid diffusers. The above authors all conclude that the condition of the inlet flow greatly effects the performance of the diffuser. Thus for a project concerned with aero gas turbine combustion systems it is important that diffuser inlet conditions are representative of those found in aircraft engines, i.e. the presence of residual swirl and outlet guide vane wakes. For a fully annular test facility this is usually achieved by placing a single stage axial compressor upstream of the diffuser. However, achieving entirely engine representative inlet conditions on a rectangular/planar test rig is not possible. Several authors including Myres et al ${ }^{[1993]}$ have attempted to simulate the presence of a rotor by using a cascade of blades to introduce some swirl followed by a row of outlet guide vanes to remove the swirl. These 'swirl-deswirl' blades produce wakes but generally do not produce the same distribution of swirl or the same turbulence structure as a rotor. 
The air flow enters the test rig from the plenum chamber via a contoured intake and then proceeds through a short length of flow straightener. A set of 'swirl-deswirl' vanes were used in an attempt to model compressor outlet conditions and provide blade wakes. The inlet guide vanes comprised of a set of 53 simple uncambered vanes which turned the flow from an axial direction through $45^{\circ}$. The outlet guide vanes were modelled on those used downstream of the rotor in the fully annular test facility. These returned the flow to an axial direction and produced the desired wakes. The 53 OGVs were untapered, untwisted, had an axial chord of $47.25 \mathrm{~mm}$ with a spacing of $19.35 \mathrm{~mm}$.

The inlet length was kept short to try to achieve a fairly flat velocity profile at diffuser inlet which is typical of actual aero gas turbine combustion systems. A relatively short inlet duct also ensured minimal boundary layer growth on the end walls.

\section{A1.2.2 Test Section Geometry}

Immediately after passing through the OGV row the air flows through a hybrid diffuser into the main test section. The hybrid diffuser, shown in Figure A1.2, is most easily described by separating it into three individual components; Stage 1 (conventional) diffuser, Stage 2 step expansion and Stage 3 (conventional) diffuser. The diffuser's total non-dimensional length $\left(\mathrm{L} / \mathrm{h}_{1}\right)$ was 3.5 and is non-dimensionally the same as the Datum diffuser used in the fully annular test facility. The Stage 1 diffuser has a modest area ratio of 1.3, a wall angle of $7^{\circ}$ and a non-dimensional length of 1.3. It was designed as a small insert so that its geometry could be easily changed.

The geometry of the Stage 2 diffuser was based, non-dimensionally on the work of Adkins et $\mathrm{al}^{[1980]}$ and Myres et $\mathrm{al}^{[1993]}$, giving a step expansion ratio of 1.3 and axial and radial bleed gaps ( $\mathrm{x}$ and $\mathrm{y}$ in Figure 1.25) of $7.1 \mathrm{~mm}$ and $1.8 \mathrm{~mm}$ respectively. The bleed was controlled via a slot and slider arrangement and a calibrated orifice (see Appendix A4) which enabled up to $3 \%$ of the total inlet mass flow to be bled through each vortex chamber.

Finally, the conventional Stage 3 diffuser was designed to hinge at inlet and have a variable angle of divergence thus facilitating rapid modification of the Stage 3 and overall 
area ratio.

To maintain the philosophy of a simple test rig the combustor flame tube profile was extensively simplified and did not incorporate a double cowl as in the fully annular rig. This was replaced by a flat cowl with the outer radii modelled, non-dimensionally, on the fully annular facility. Flame tube porosity was modelled only very approximately by a series of drilled holes in the cowl (not shown in Figure A1.1).

\section{$\underline{\text { A1.2.3 Instrumentation }}$}

The instrumentation and control techniques were identical in nature to those used with the fully annular facility. A description of the hardware, software and relevant techniques are presented in detail in Section 3. In summary, the instrumentation system is based around an IBM compatible Personal Computer (PC) linked to a data acquisition system and a DC servo traverse mechanism. Operating conditions and data were provided by pneumatic pressure probes and converted to voltages using Furness FC044 differential pressure transducers.

Rig inlet conditions were monitored using a pitot probe at OGV entry in conjunction with a wall static pressure tapping. Calibration of this combination was carried out by comparing the inlet dynamic pressure and mass flow with results from a button hook probe across the OGV entry plane in the centre of the rig. A control loop enabled use of this information to govern the speed of the centrifugal fan to achieve and maintain the desired inlet conditions $\left(\operatorname{Re}_{\mathrm{OGV}}=1 \times 10^{5}\right)$.

\section{$\underline{\text { A1.3 Results and Discussion }}$}

During calibration of the inlet it became apparent that there was a problem with the test rig. The mass flows indicated by the pitot-static combination and that calculated from the button hook probe traverse were significantly different.

At the design process it was recognised that the boundary layer growth on the end walls would present a problem. Due to the adverse pressure gradient in a diffuser a boundary 
layer on an end wall grows rapidly. This effect is more pronounced in this case because of the large area ratios and high amount of diffusion. The boundary layer growth on the end walls was causing a mass flow migration towards the centre of the test rig. This was confirmed by traversing a five hole probe from each end wall at diffuser exit towards the central region of the rig. Figure A1.3 illustrates that a large boundary layer exists causing an increase in the local velocity in the central portion of the rig at diffuser exit and thus an increase in the indicated mass flow.

The effect of this flow migration is twofold;

1) there is an increased mass flow in the central portion of the test rig where detailed measurements are taken. Indicated mass-weighted pressures be will greater resulting in an over-optimistic measure of performance.

2) the outboard regions of the test facility are deficient of mass flow and will be more prone to flow separation under the action the adverse pressure gradient.

To try to solve this problem an attempt was made to bleed off the end wall boundary layer through a series of small holes. By a process of trial an error enough holes were drilled in each end wall to remove the boundary layer effect and prevent an excessive amount of mass flow migration. Comparing the mass flow between OGV entry and diffuser exit indicated that this appeared to cure the mass flow migration problem. Previously the mass flow in the central section at diffuser exit was seen to be $25 \%$ higher than measured at OGV entry. With the end wall boundary layer bleed in place only an error of $+5 \%$ was seen and, although not ideal, was considered acceptable.

The studies of Adkins et al ${ }^{[1980]}$ suggested that a divergence angle in the Stage 3 diffuser of 15 degrees would remain attached. However, a single five hole probe traverse at diffuser exit indicated that this geometry produced separated flow in the rectangular rig. In fact a transitory stall existed even when the divergence angle was reduced to 7 degrees. Using flow visualisation techniques such as 'wool-tufts' and smoke it became apparent that the flow was initially separating in the corner formed by the end wall and this separation was migrating throughout the diffuser. The question now became; why does the 
diffuser separate at much lower divergence angles than suggested by the literature? The answer to this was the interaction between regions of vorticity and the end walls. Flow visualisation techniques (wool tufts and smoke) revealed that these vortices roll up along the end walls as illustrated in Figure A1.4.

In a hybrid diffuser there are two distinct vortices as illustrated in Figure A1.5. The first, most obvious, vortex is in bleed chamber. The second area of high vorticity which exists behind the fence and may be described as a Coanda bubble. If this Coanda bubble rolls up at the end wall this would almost certainly cause flow separation in the corner where the Stage 3 diffuser meets the end wall. The presence of this separation will be felt throughout the diffuser causing a drastic drop in performance if not a complete stall across the test section. Furthermore, the interaction of the bleed chamber vortex with the end wall may reduce the effectiveness of the bleed. Thus, locally, the bleed could be below the minimum requirement also resulting an stall.

\section{$\underline{\text { A1.4 Conclusion }}$}

It is clear from the problems encountered that a rectangular test rig is not a suitable test vehicle with which to investigate hybrid vortex controlled diffusers. The interaction of areas of high vorticity with the end walls appears to destroy the key flow mechanisms. The three dimensional nature of the flow is not suited to an investigation in a pseudo two dimensional environment. To investigate a hybrid diffuser the integrity of the vortex on the leeward side of the fence must be maintained at all times. Thus without complex flow management at the end walls the only suitable test vehicle for the investigation of hybrid diffusers is a fully annular test facility. 


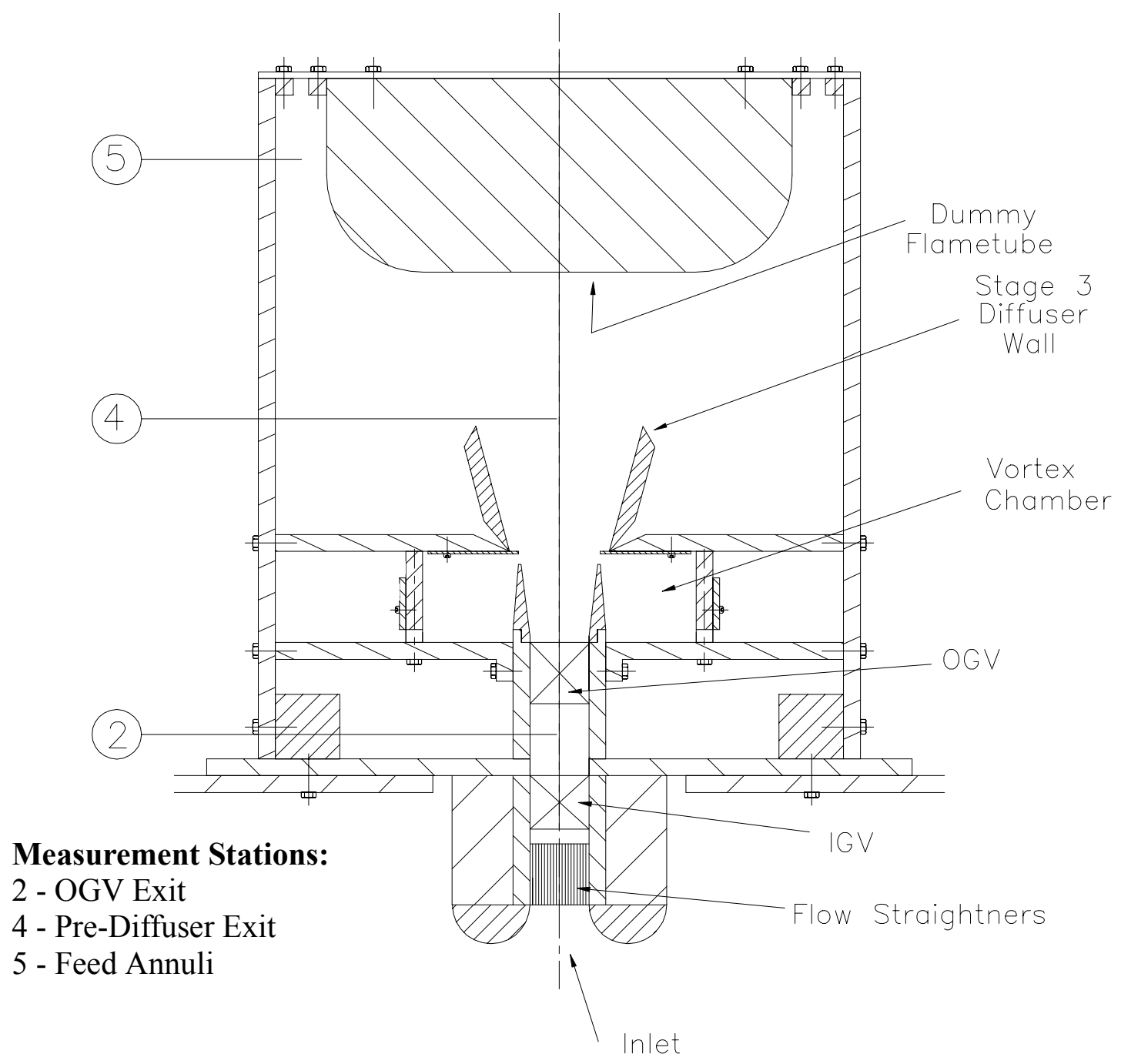

Figure A1.1 Rectangular/Planar Test Facility

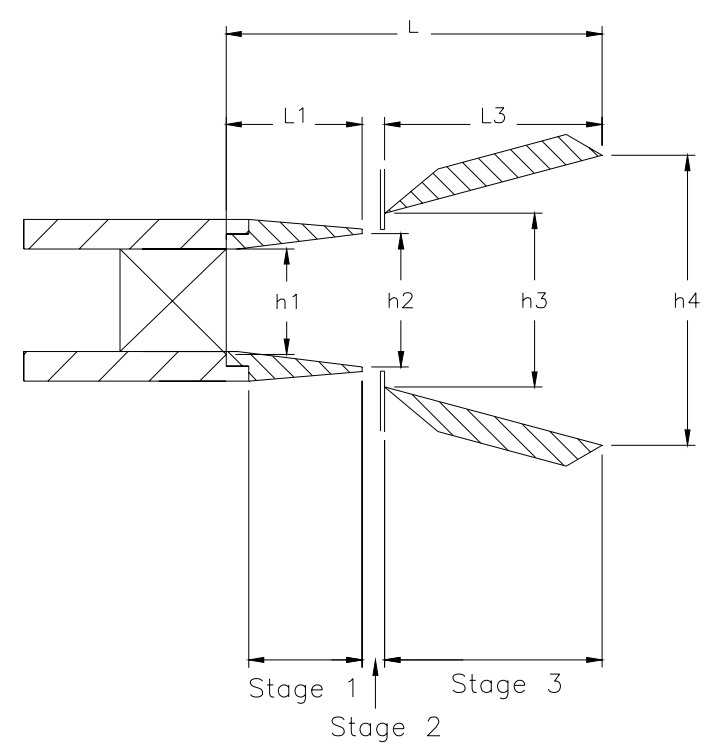

Figure A1.2 Hybrid Diffuser Nomenclature 


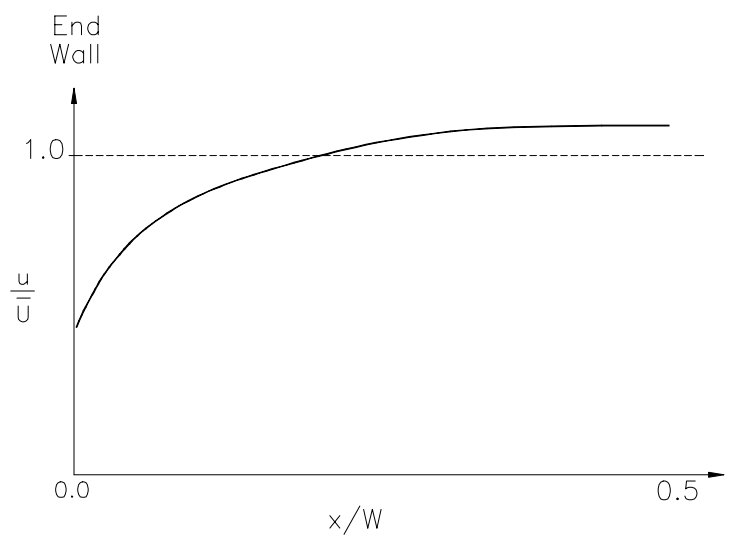

Figure A1.3 End Wall Boundary Layer

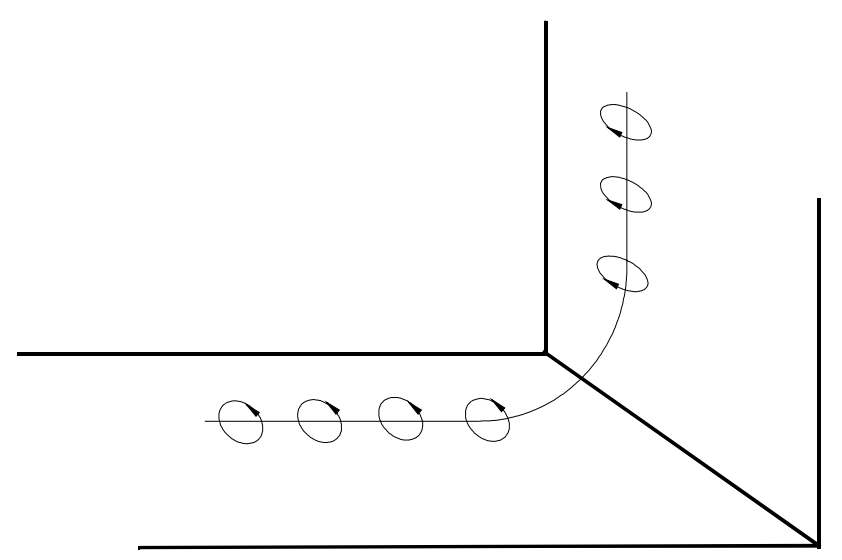

Figure A1.4 Vortex 'Roll-Up' at End Wall

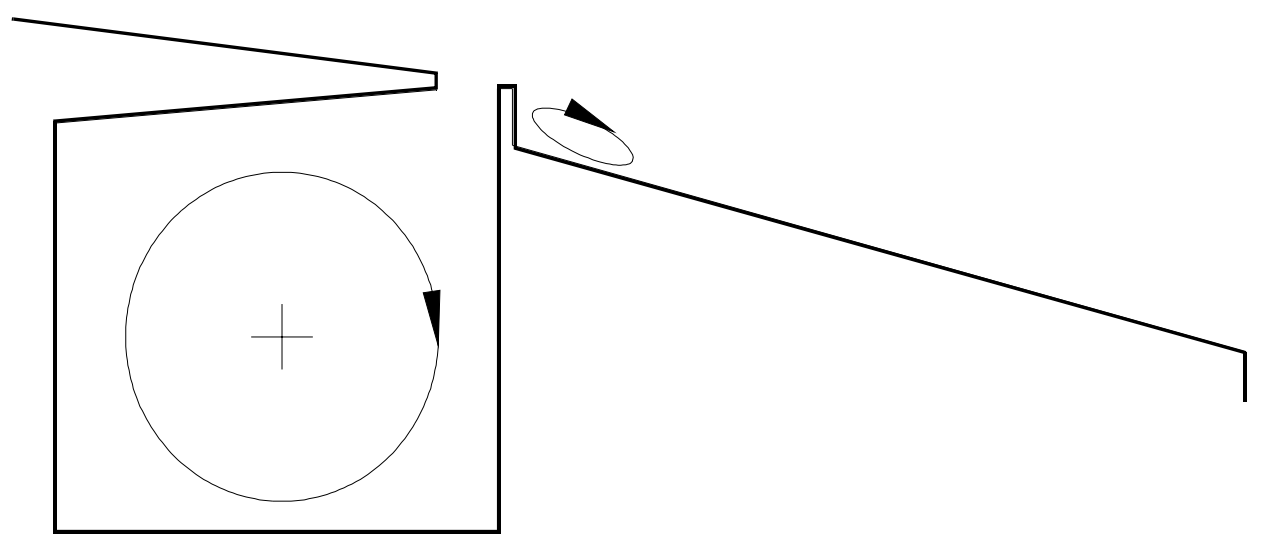

Figure A1.5 Regions of Vorticity 
A2 BUTTON HOOK PROBE THEORY

\section{A2 BUTTON HOOK PROBE THEORY}




\section{A2 BUTTON HOOK PROBE THEORY}

A button hook probe is a useful piece of equipment as, in addition to being used as a conventional pitot probe, it can also provide static pressure data. The two probe configurations are illustrated in Figure A2.1. To obtain a true total pressure reading the probe is aligned with the flow direction and to measure a pseudo static pressure the probe is rotated through $180^{\circ}$. The 'button hook' geometry ensures the entry to the probe remains in essentially the same spacial location when the probe is rotated $180^{\circ}$ about its stem. Therefore, the true total pressure and pseudo static pressure are recorded at the same location.

A calibration factor, $\mathrm{K}$ is defined as:

$$
K=\frac{\left(P-p_{p s}\right)}{(P-p)}
$$

where:

$\mathrm{P}$ total pressure of the flow, as measured by the probe when facing in the upstream direction

$\mathrm{p}_{\mathrm{ps}} \quad$ pseudo static pressure as recorded by the probe when rotated through $180^{\circ}$

$\mathrm{p} \quad$ static pressure of the flow

A value of $\mathrm{K}$ (typically 1.31) is obtained by placing the probe in a calibration tunnel and subjecting it to various dynamic pressures $(q=P-p)$ of known magnitude while taking readings for $\left(\mathrm{P}-\mathrm{p}_{\mathrm{ps}}\right)$. The value of $\mathrm{K}$ does vary slightly with Reynolds Number. However, for the velocity range over which the calibration was performed (typically 30$70 \mathrm{~ms}^{-1}$ ) the value of $\mathrm{K}$ varies by less than $\pm 0.6 \%$. 


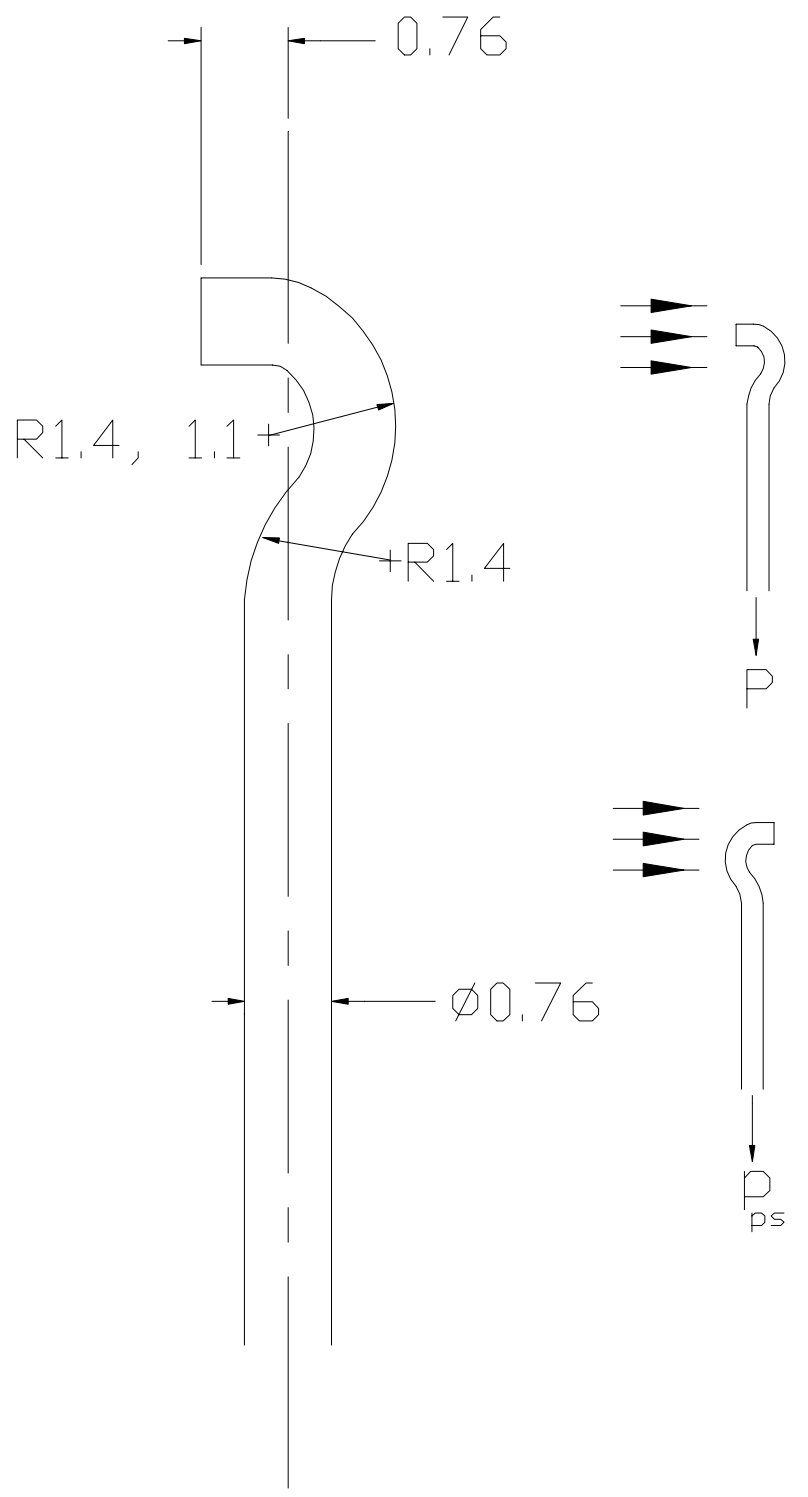

Figure A2.1 Button Hook Probe Geometry 


\section{A3. FIVE-HOLE PROBE THEORY}

A3.1 Introduction $\ldots \ldots \ldots \ldots \ldots \ldots \ldots \ldots \ldots \ldots \ldots \ldots \ldots \ldots \ldots \ldots$

A3.2 Theory for Non-Nulled Probes $\ldots \ldots \ldots \ldots \ldots \ldots \ldots \ldots$. $\ldots \ldots$

3.2.1 Pitch and Yaw Pressure Parameters, $X$ and $Y \ldots \ldots$. A3-3

3.2.2 Dynamic Pressure Parameter, $D_{p} \ldots \ldots \ldots \ldots \ldots$ A3-4

3.2.3 Stagnation Pressure Parameter, $\mathrm{S}_{\mathrm{p}} \ldots \ldots \ldots \ldots \ldots$ A3-4

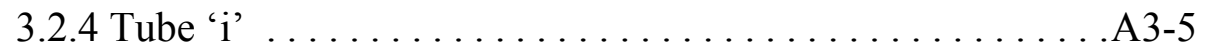

A3.3 Calibration of Five-Hole Probes $\ldots \ldots \ldots \ldots \ldots \ldots \ldots \ldots$ A3-5

A3.4 Data Reduction .........................

FIGURES $\ldots \ldots \ldots \ldots \ldots \ldots \ldots \ldots \ldots \ldots \ldots \ldots \ldots \ldots \ldots \ldots \ldots \ldots \ldots$ 


\section{A3 FIVE-HOLE PROBE THEORY}

\section{$\underline{\text { A3.1 Introduction }}$}

The five-hole probe consists of five individual pressure sensing holes - a central hole around which four chamfered holes are arranged. A typical five-hole probe is illustrated in Figure A3.1 and A3.2, both showing a central tube around which four chamfered tubes are arranged. This type of probe can be used in one of two different methods to determine local values of flow direction, velocity and total pressure within an air flow. The two methods are normally referred to as 'nulled' or 'non-nulled'.

\section{(i) Nulled Five-Hole Probe}

In this method the probe is aligned in the flow such that each pair of opposing holes is balanced. In this configuration the central hole acts as a pitot probe and registers the local stagnation pressure. The dynamic pressure is a function of the difference between the central hole and the side holes and is obtained from a calibration chart. The 'nulled' method of operation requires the probe to be mounted in some mechanism which allows it to be rotated in both yaw and pitch. It is relatively easy to obtain the flow direction as this is given by measuring the amount of rotation, from some datum, before the probe achieves the 'balanced' condition. Unfortunately the 'nulled' method has some disadvantages, namely;

(i) Rotation of the probe within the test facility is usually very difficult due to the typically limited access.

(ii) Even if (i) is not a major factor the actual process of nulling the side holes can be difficult and time consuming, even with the aid of computers.

Added to this there is also significant effort required, during calibration and data reduction, to obtain the static pressure and flow velocities. 


\section{(ii) Non-nulled Probe}

In this method the five-hole probe is introduced into the flow in a fixed and known attitude, usually in alignment with the rig access. Figure A3.1 illustrates this with a velocity vector approaching the probe from a direction which can be described by a pitch and a yaw angle in the conventional sense. In this configuration it is possible to determine, using suitable calibration data, the total pressure, the flow velocity, the pitch and yaw angle from the five pressures. A set of typical calibration data is shown in Figures A3.2 A3.6 and comprises of four two-dimensional graphs showing the pitch angle, yaw angle, Dynamic and Static Pressure Parameters $\left(D_{p}\right.$ and $\left.S_{p}\right)$ plotted against the Pitch and Yaw Pressure Parameters ( $\mathrm{X}$ and $\mathrm{Y}$ ). The latter two parameters ' $\mathrm{X}$ ' and ' $\mathrm{Y}$ ' are mathematical combinations of the five hole pressures. The four pressure parameters are, for most practical purposes, independent of the flow velocity so it is possible to use a single set of calibration data.

\section{A3.2 Theory for Non-Nulled Probes}

The basic theory is based on the mathematical concept that when aligned to the flow the pressure registered by any tube, $\mathrm{P}_{\mathrm{n}}$, is the sum of the local static pressure, $\mathrm{p}_{\mathrm{s}}$, and some fraction of the dynamic pressure, q.

$$
\mathrm{P}_{\mathrm{n}}=\mathrm{p}_{\mathrm{s}}+\mathrm{K}_{\mathrm{n}} \mathrm{q}
$$

where $K_{n}$ is the fractional part of the dynamic pressure $q$ sensed by hole $n$.

Assuming that the effects of Mach Number and Reynolds Number are negligible then the value of $K_{n}$ depends only on the flow direction relative to hole $n$. This relationship can then be used to define the values of the Pitch and Yaw Pressure Parameters, the Dynamic Pressure Parameter and the Stagnation pressure Parameter. 


\section{A3.2.1 Pitch and Yaw Pressure Parameters, $X$ and $Y$}

An indication of the flow pitch and yaw can be reasonably obtained by comparing the pressure difference between opposing holes, i.e. $\left(\mathrm{p}_{1}-\mathrm{p}_{3}\right)$ and $\left(\mathrm{p}_{2}-\mathrm{p}_{4}\right)$. To obtain the Pitch and Yaw Pressure Parameters these are non-dimensionalised by some function of dynamic pressure;

$$
\begin{aligned}
& X=\frac{p_{1}-p_{3}}{p_{5}-p_{i}} \\
& Y=\frac{p_{2}-p_{4}}{p_{5}-p_{i}}
\end{aligned}
$$

The tube denoted by ' $i$ ' is chosen carefully in order to maximise the sensitivity (A3.2.4)

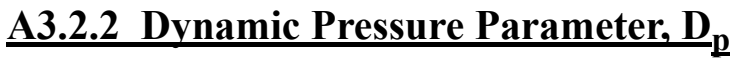

The difference between the pressure sensed by the central hole and any of the other four holes is a function of the flow velocity. Using Equation A3.1

$$
\mathrm{p}_{5}-\mathrm{p}_{\mathrm{i}}=\mathrm{q}\left(\mathrm{K}_{5}-\mathrm{K}_{\mathrm{i}}\right)
$$

Defining $\mathrm{D}_{\mathrm{p}}$ as $\left(\mathrm{K}_{5}-\mathrm{K}_{\mathrm{i}}\right)$ gives the Dynamic Pressure Parameter and this, like $\mathrm{X}$ and $\mathrm{Y}$, is a function of flow direction alone.

$$
\mathrm{q}=\frac{\mathrm{p}_{5}-\mathrm{p}_{\mathrm{i}}}{\mathrm{D}_{\mathrm{p}}}
$$

\section{A3.2.3 Stagnation Pressure Parameter, $S_{p}$}

By combining the incompressible Bernoulli equation with Equation A3.1 for hole 5:

$$
\mathrm{P}_{\mathrm{t}}-\mathrm{p}_{5}=\mathrm{q}\left(1-\mathrm{K}_{5}\right) \text {, and } \frac{\mathrm{p}_{\mathrm{t}}-\mathrm{p}_{5}}{\mathrm{p}_{5}-\mathrm{p}_{\mathrm{i}}} \equiv \frac{1-\mathrm{K}_{5}}{\mathrm{~K}_{5}-\mathrm{K}_{\mathrm{i}}}
$$

The Stagnation Pressure Parameter, $\mathrm{S}_{\mathrm{p}}$, is defined as the group on the right hand side, such that:

$$
\mathrm{P}_{\mathrm{t}}=\mathrm{p}_{5}+\mathrm{S}_{\mathrm{p}}\left(\mathrm{p}_{5}-\mathrm{p}_{\mathrm{i}}\right)
$$




\section{$\underline{\text { A3.2.4 Tube 'i' }}$}

The choice of tube ' $\mathrm{i}$ ' is varied as a function of flow direction in order to obtain 'sensible' values of the Pitch and Yaw Pressure Parameters, X and Y. With reference to Figure A3.1 consider a flow incident on a probe of cone angle $\phi$ (see Figure A3.2) at a positive yaw angle, YTR. As YTR $\rightarrow \phi / 2$ then $\mathrm{p}_{4} \rightarrow \mathrm{p}_{5}$ and $\left(\mathrm{p}_{5}-\mathrm{p}_{4}\right) \rightarrow 0$ resulting in both $\mathrm{X}$ and $\mathrm{Y}$ tending to infinity. Thus ' $\mathrm{i}$ ' is chosen as 2 to give finite values of $\left(\mathrm{p}_{5}-\mathrm{p}_{\mathrm{i}}\right)$ until YTR reaches $\phi / 2$ when hole 2 is in the wake of the probe tip. With real flows there are variations in both pitch and yaw so it is common practice to take hole 'i' as the most leeward hole with the lowest pressure. However, if the same methodology is used during calibration and analysis then hole ' $\mathrm{i}$ ' can realistically be any side hole 1 to 4 . To significantly simplify the analysis of results it is beneficial to limit the choice of hole ' $i$ ' to just two holes. Flows within combustion systems, such as those studied in this project, generally have much larger variations in pitch than yaw direction. Thus all work conducted presented here assumes the convention where $\mathrm{p}_{\mathrm{i}}$ is chosen from the lesser value of $\mathrm{p}_{2}$ or $\mathrm{p}_{4}$.

\section{$\underline{\text { A3.3 Calibration of Five-Hole Probes }}$}

Calibration of a five-hole probe to be used in the non-nulled mode involves subjecting the probe to a flow of known dynamic pressure while holding the probe in a known orientation. This must be done over a range of pitch and yaw angles which cover all combinations likely to be encountered during any flow study (typically $\pm 36^{\circ}$ for both yaw and pitch). The mechanism used to calibrate the probes is shown in Figure A3.7. The gimbals are accurate to within $\pm 0.1^{\circ}$ and can orient the probe at yaw and pitch angles up to $\pm 45^{\circ}$ from the flow direction. The direction of the flow vector is most sensibly described by 'true' yaw and pitch angles However, without the ability to achieve compound movement of each gimbal it is mechanically difficult to enable independent variation of these angles. Thus, with reference to Figure A3.1, the probe is positioned by the gimbals in a true yaw and a pseudo pitch plane.

The positioning of the gimbals is afforded by two d.c. servo motors controlled and monitored by a PC. The five hole pressures are recorded by a data acquisition system similar to that described in Section 3.0. To ensure continuity the same PC, analogue-to-digital 
convertor and pressure transducers were used for the calibration process and for recording of experimental data. Finally, a calibration file is written detailing the values of X, Y, Pseudo Pitch, True Yaw, $\mathrm{D}_{\mathrm{p}}$ and $\mathrm{S}_{\mathrm{p}}$.

\section{A3.4 Data Reduction}

Raw experimental data consists of a file containing positional data and the corresponding pressure readings for the five holes. A series of computer programs are used to post process this data and extract local and average values of pressure, velocity components and flow angles. The first stage in this process uses the five hole pressures to calculate the non-dimensional parameters $\mathrm{X}$ and $\mathrm{Y}$ (Equations A3.2 and A3.3). Although the size of the five hole probe is small only hole 5 is situated at the exact measurement point with holes 1 - 4 all being slightly offset. Thus, to correct for this, the holes 1 - 4 are interpolated onto the centre hole before calculating $\mathrm{X}$ and $\mathrm{Y}$. These values of $\mathrm{X}$ and $\mathrm{Y}$ can then be compared to the calibration file to recover values of PPS, YTR, $D_{p}$ and $S_{p}$. In order to do this a least square, bi-quadratic surface is fitted to each of the four parameter arrays using the closest 25 points. The dynamic pressure, q, is then calculated from the Dynamic Pressure Parameter, $\mathrm{D}_{\mathrm{p}}$ (Equation A3.5) and hence a total velocity, $\mathrm{U}_{\text {tot }}$, can be calculated. Velocity components $\mathrm{u}, \mathrm{v}$ and $\mathrm{w}$ are then calculated from the following equations:

$$
\begin{aligned}
& \mathrm{u}=\mathrm{U}_{\mathrm{tot}} \cos (\mathrm{PPS}) \cos (\mathrm{YTR}) \\
& \mathrm{v}=\mathrm{U}_{\mathrm{tot}} \sin (\mathrm{PPS}) \\
& \mathrm{w}=\mathrm{U}_{\mathrm{tot}} \cos (\mathrm{PPS}) \sin (\mathrm{YTR})
\end{aligned}
$$

If desired, the true pitch angle is given by:

$$
\mathrm{PTR}=\frac{\mathrm{v}}{\mathrm{u}}=\frac{\tan (\mathrm{PPS})}{\cos (\mathrm{YTR})}
$$




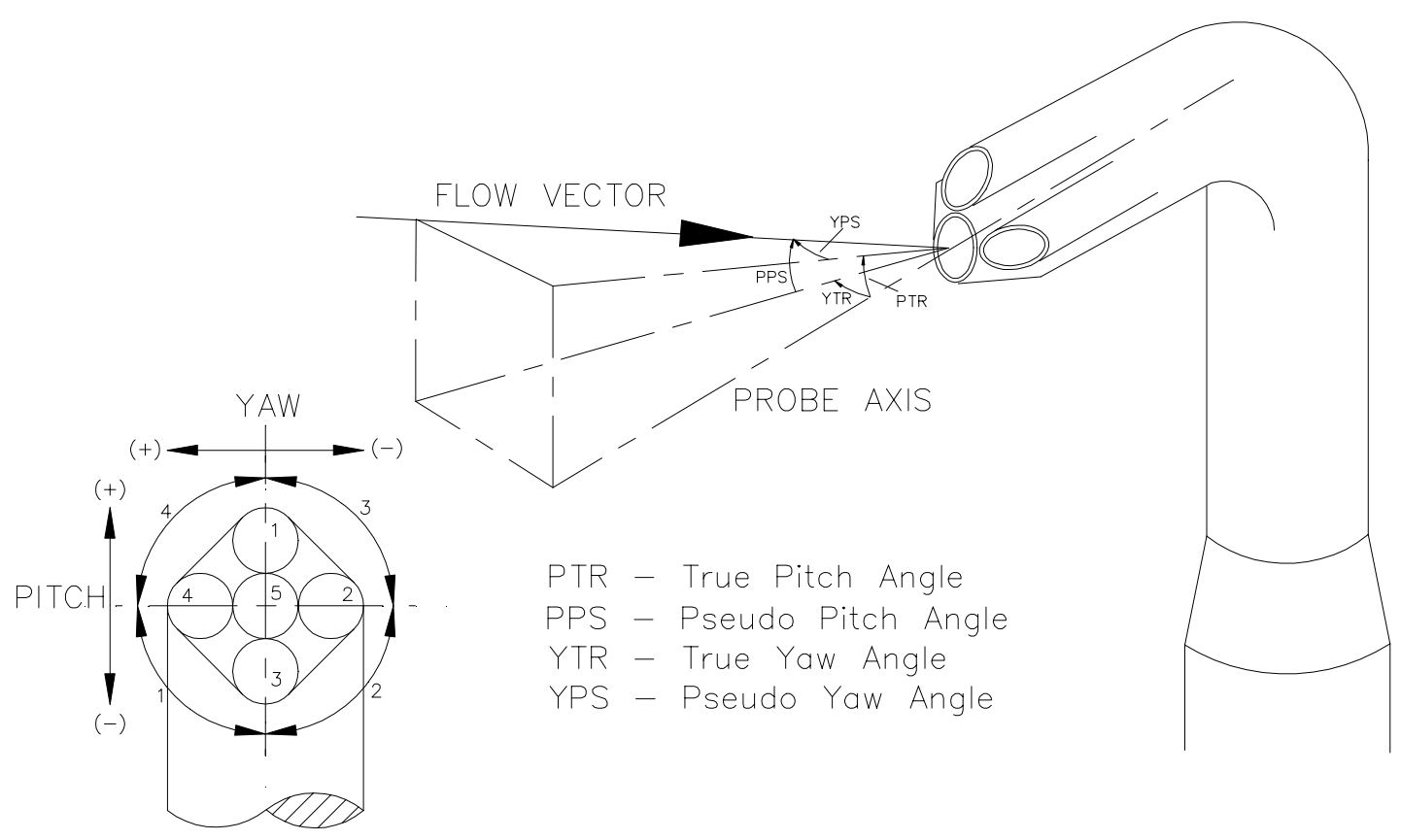

Figure A3.1 Five Hole Probe
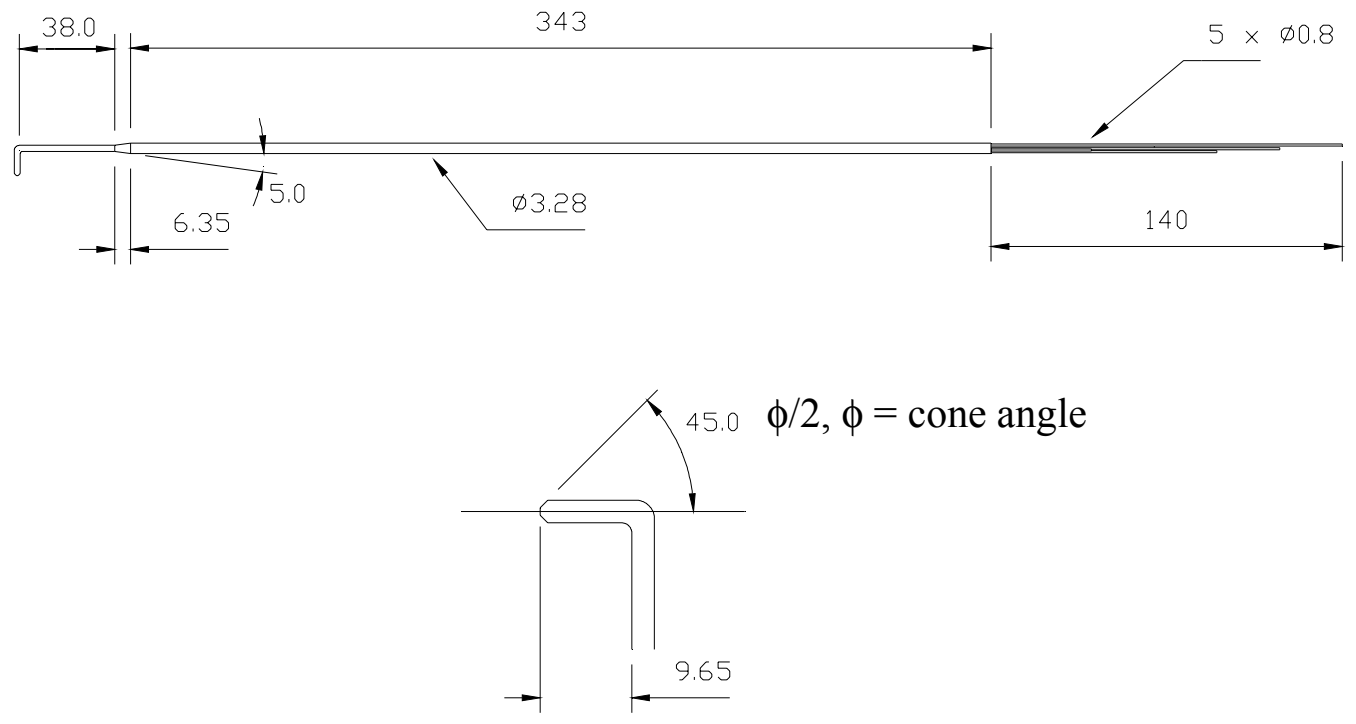

Figure A3.2 Typical Five Hole Probe Dimensions 


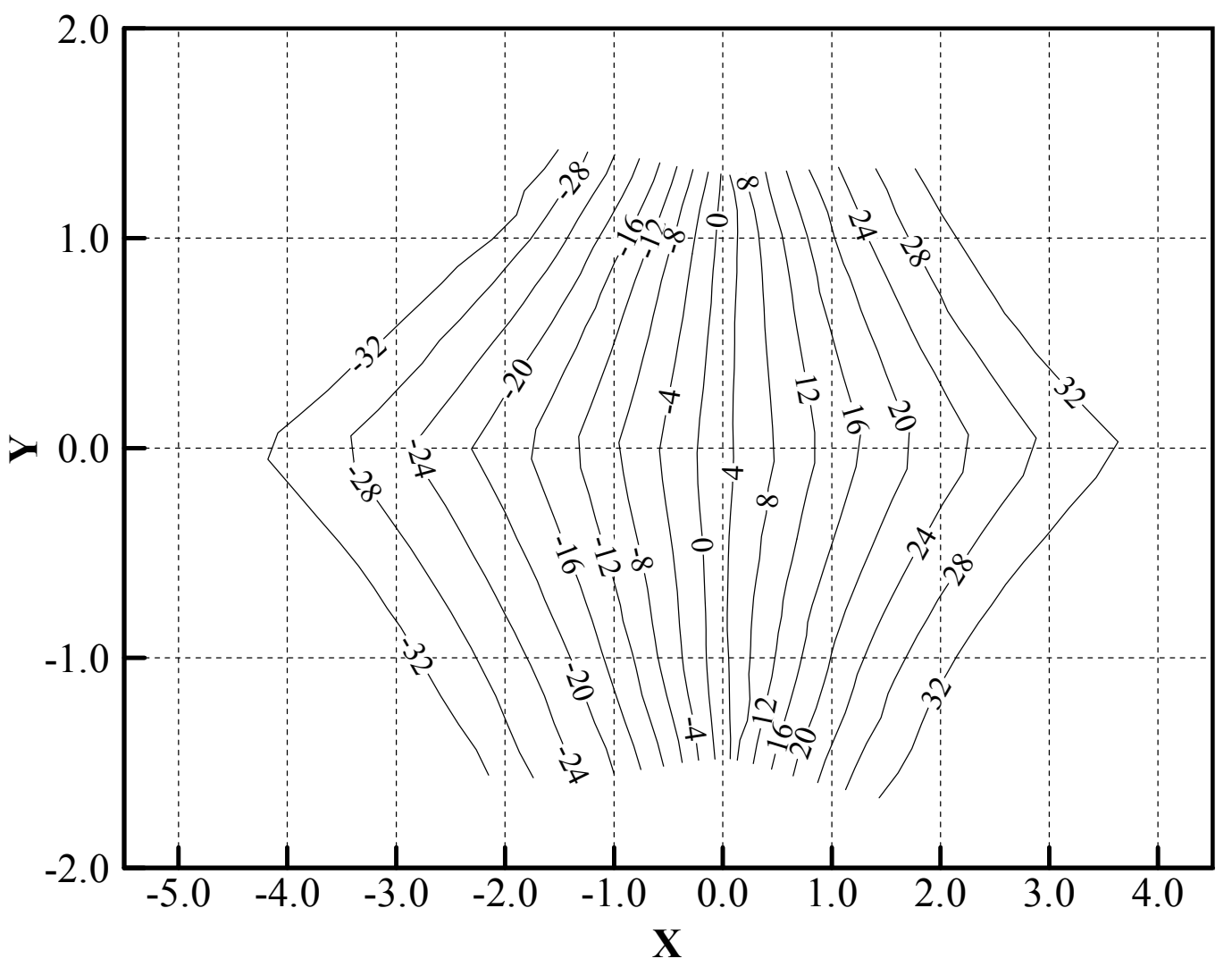

Figure A3.3 Contours of Constant Pseudo Pitch Angle, PPS

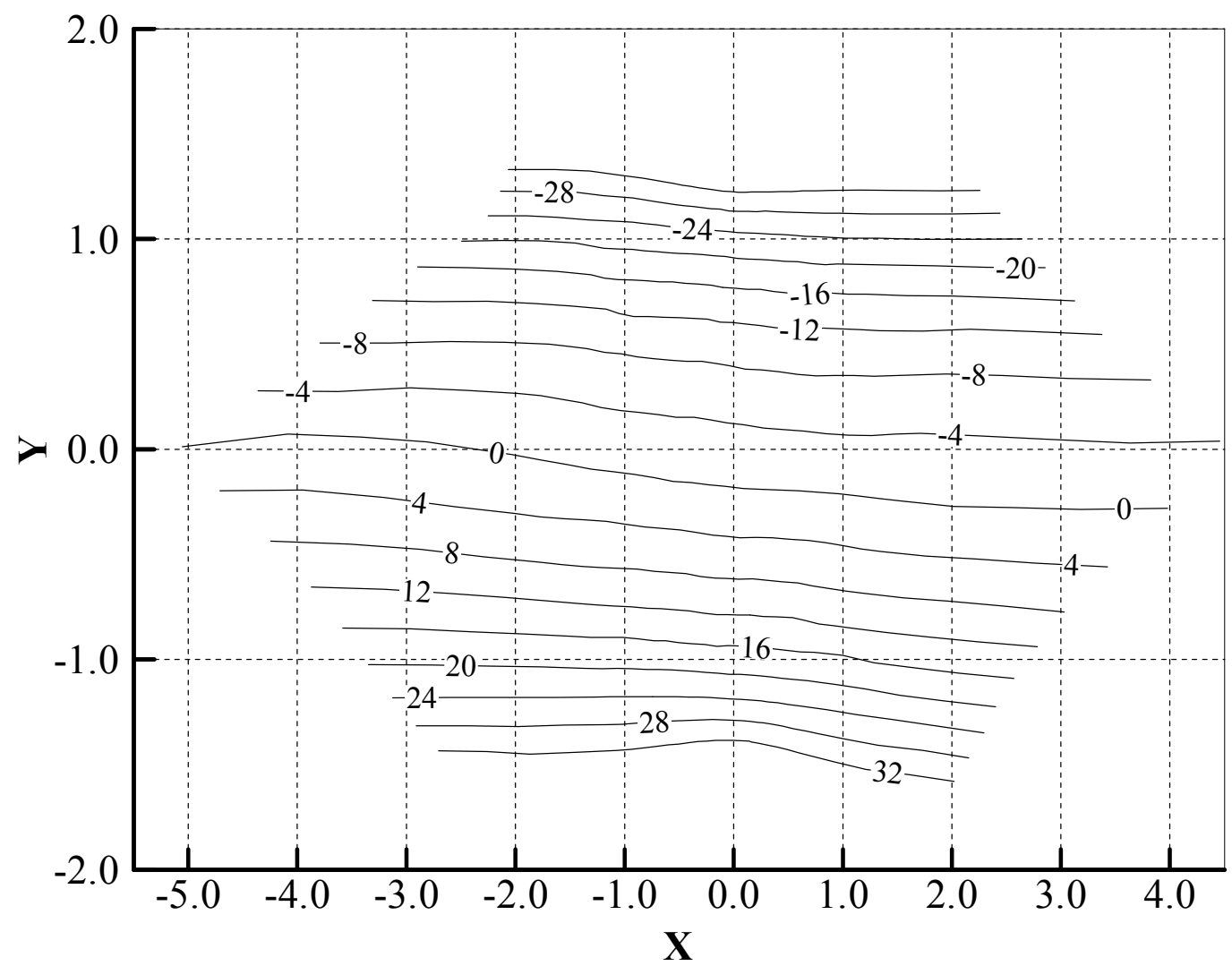

Figure A3.4 Contours of Constant Yaw Angle, YTR 


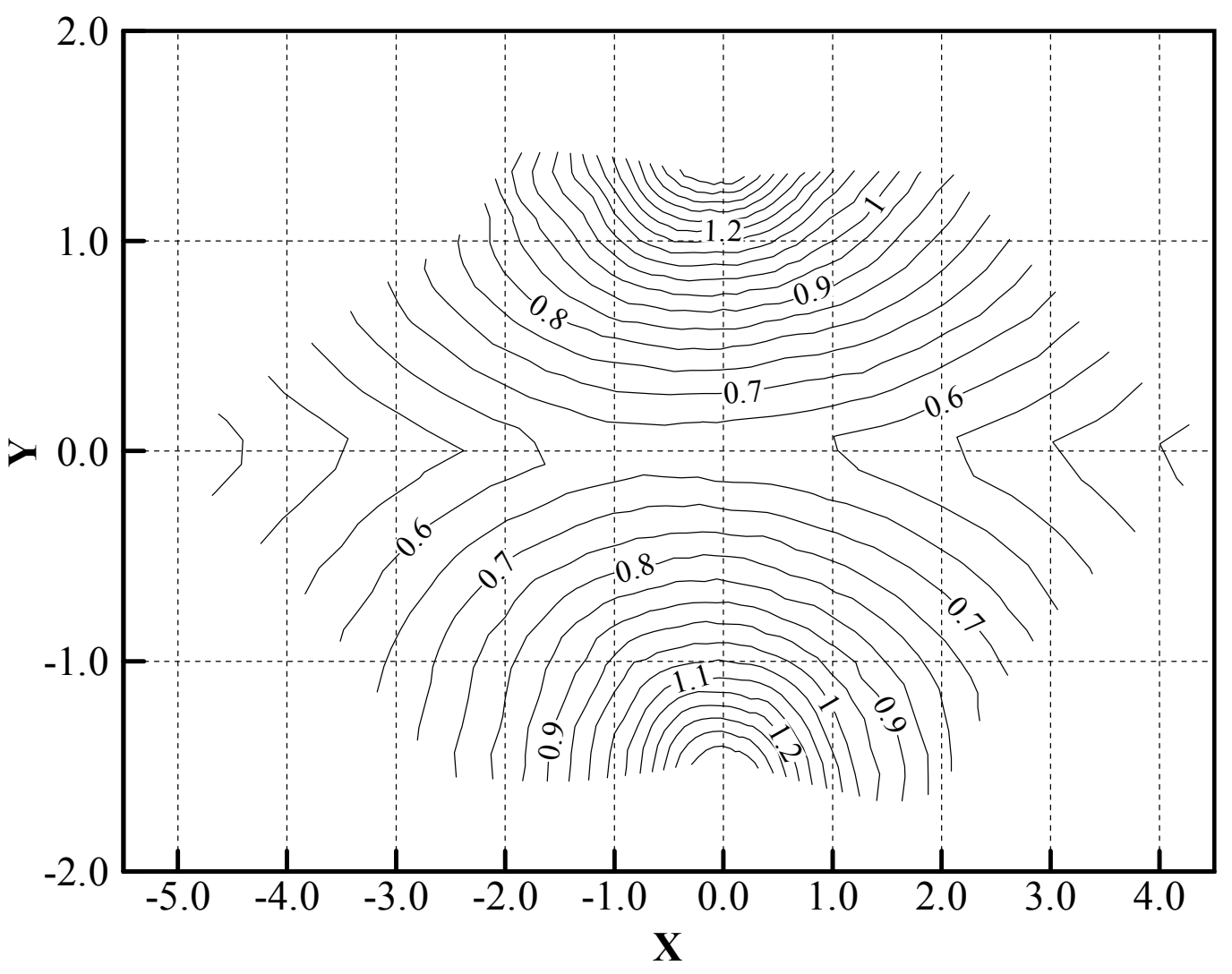

Figure A3.5 Contours of Constant Dynamic Pressure Parameter, Dp

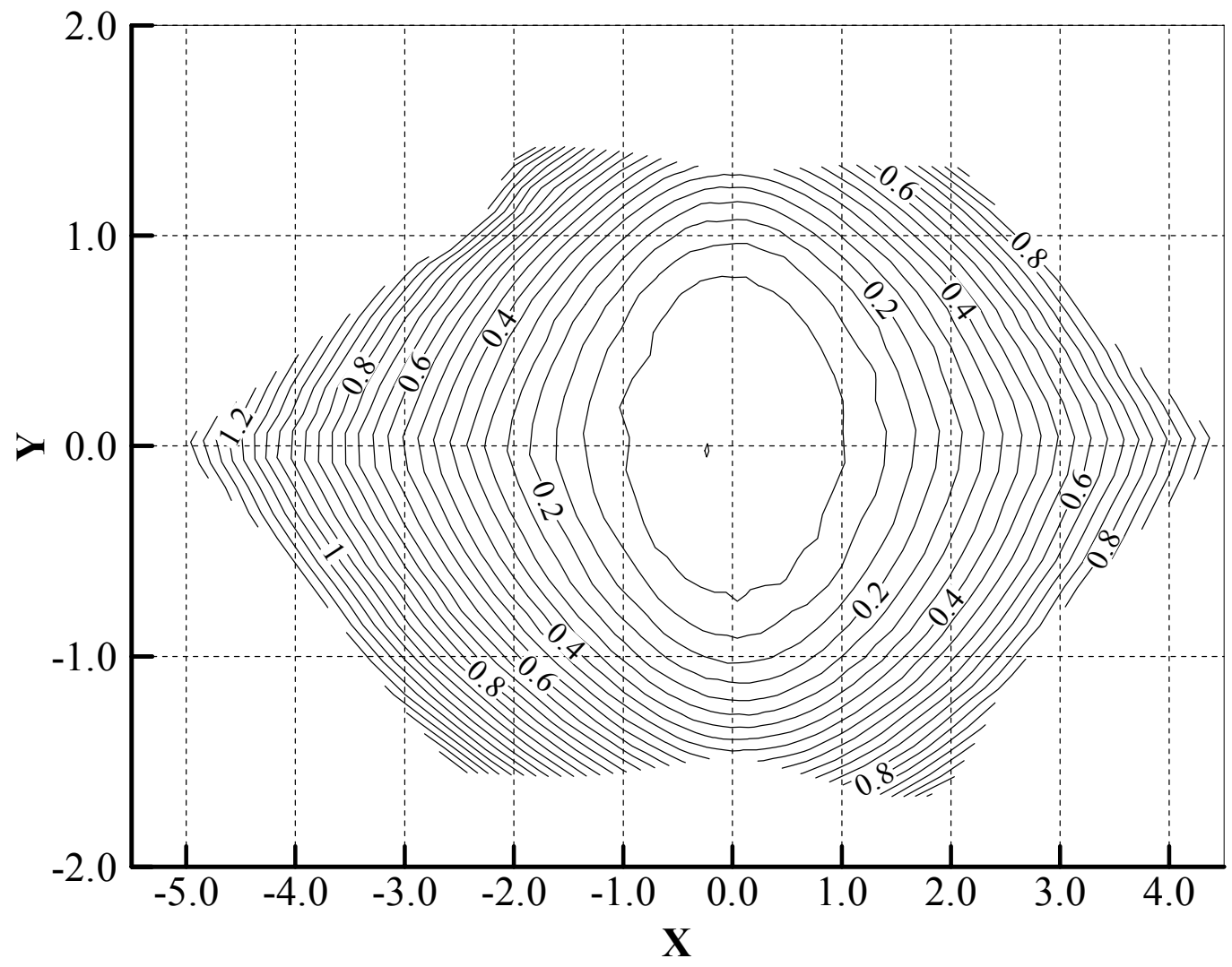

Figure A3.6 Contours of Constant Stagnation Pressure Parameter, Sp 


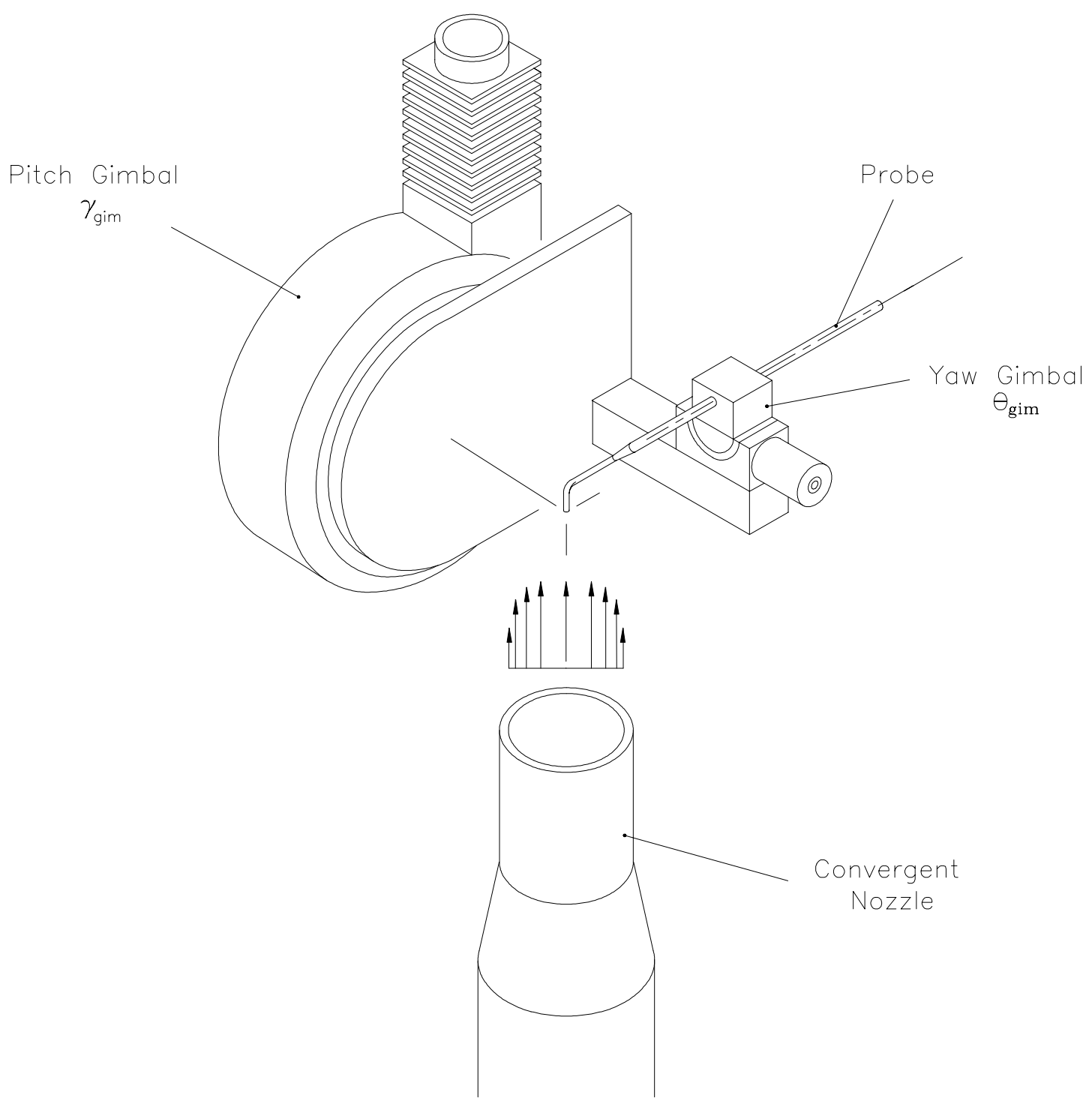

Figure A3.7 FIve Hole Probe Calibration Rig 


\section{A4 BLEED FLOW; CALIBRATION AND CONTROL}

A4.1 Bleed Flow; Calibration and Control ..............44-2

FIGURES ............................44-5 


\section{A4 BLEED FLOW; CALIBRATION AND CONTROL}

In any test facility where airflow is under investigation it is essential to accurately measure the air mass flow rate. This is of prime importance when one of the parameters under study is a bleed flow such as in a hybrid diffuser. Thus the problem posed is how can the flow be controlled and measured with sufficient accuracy. For the sake of brevity only the mechanisms employed to control the hybrid diffuser bleed in the fully annular test facility will be described here. Although the same principles were employed on the planar test facility.

The majority of the fully annular test facility existed before conception of this current project and was designed to allow the modifications for bleed flow extraction to be relatively easy. With reference to the test rig, shown in Figure 3.2, the inner bleed flows from the Stage 2 diffuser into the bleed chamber and then via a vaned passage into the central hub of the rig. The flow is throttled by a slotted drum containing a disc (Figure A4.1) which can be moved up and down, on a lead screw, to reduce or increase flow respectively. The air is then exhausted to atmosphere via a calibrated orifice hole designed in line with Hay and Spencer ${ }^{[1992]}$. The latter allowing the mass flow rate to be set to $\pm 0.05 \%$ of the total inlet mass flow rate. The outer bleed is controlled in a similar manner with the flow from the bleed chamber entering a cavity under the dump liner and then exhausting to atmosphere via 12 calibrated bell mouth trumpets (see Figure 3.2 and Figure A4.2). The outer bleed is throttled by rotating a wooden ring containing 12 holes which align with the exit to the trumpets.

The main advantage of using orifice plates/holes is that they are very simple and easy to use and if suitably calibrated they are also accurate. The basic theory is that the mass flow rate through an orifice meter is directly proportional to the square root of the static pressure drop across the orifice, or:

$$
\dot{\mathrm{m}}=\mathrm{K} \sqrt{\rho \Delta \mathrm{p}}
$$

where $\Delta \mathrm{p}=\mathrm{p}_{2}-\mathrm{p}_{1}$ and is the drop in static pressure across the orifice and $\mathrm{K}$ is a constant of proportionality. Both the inner and outer bleeds exhaust to atmosphere or more accurately to the pressure of the lab within which the test rig is housed. Therefore, 


$$
\Delta \mathrm{p}=\mathrm{p}_{\text {orifice }}-\mathrm{p}_{\text {lab }}
$$

$\mathrm{p}_{\text {orifice }}$ is the static pressure at some point in the orifice (refer to Figure A4.1 and Figure A4.2) and $\mathrm{p}_{\mathrm{lab}}$ is the lab pressure, measured by the rig control instrumentation.

Calibration is relatively easy and involves measuring the flow though the orifice with a calibrated probe and comparing directly with the measured pressure drop. This was achieved for the inner bleed by mounting the whole throttle assembly on top of a bell mouth supplied with air from a centrifugal fan via a plenum chamber (Figure A4.3). The fan speed and throttle setting were varied to produce mass flow rates close to those expected in the test rig (i.e. around 3\% of total rig inlet flow). A calibrated button hook probe was traversed across the outlet to the orifice and using a simple computer algorithm this was integrated up to give the mass flow. The pressure drop was also recorded and thus a suitable calibration coefficient was calculated. A similar procedure was used to calibrate a single trumpet from the outer bleed. Figure A4.4 shows plots of $\sqrt{\rho \Delta p}$ against mass flow rate and show good linear relationships. The equations for these relationships were then programmed into the rig control software thus enabling accurate control $/$ metering of the bleed flows.
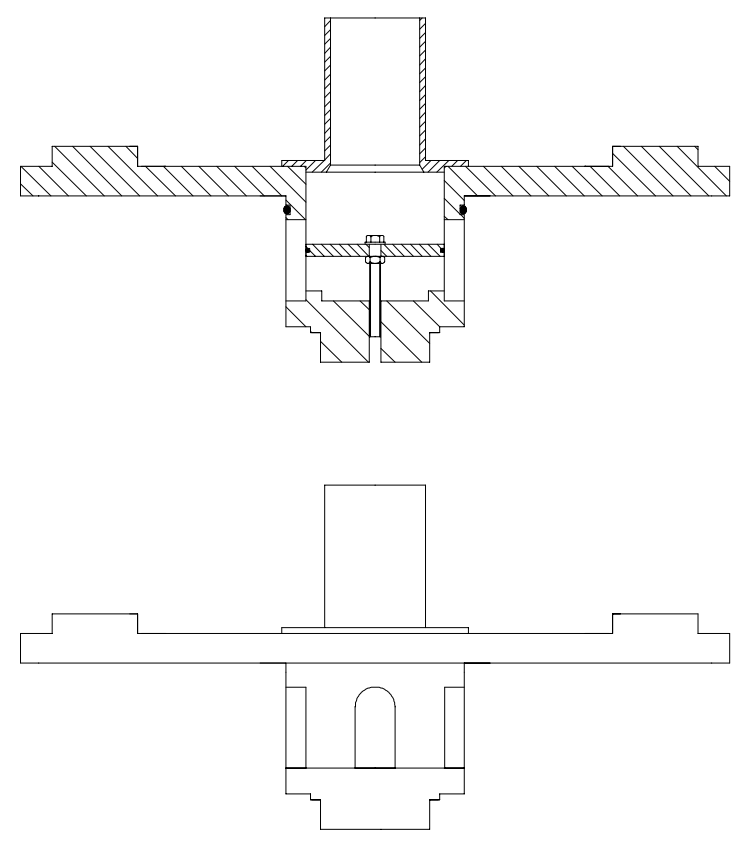

Figure A4.1 Inner Bleed Throttle and Orifice 


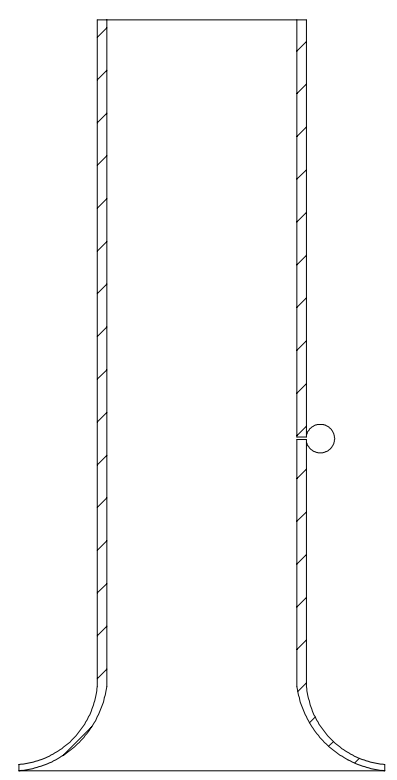

Figure A4.2 Outer Bleed Bell Mouth Trumpet

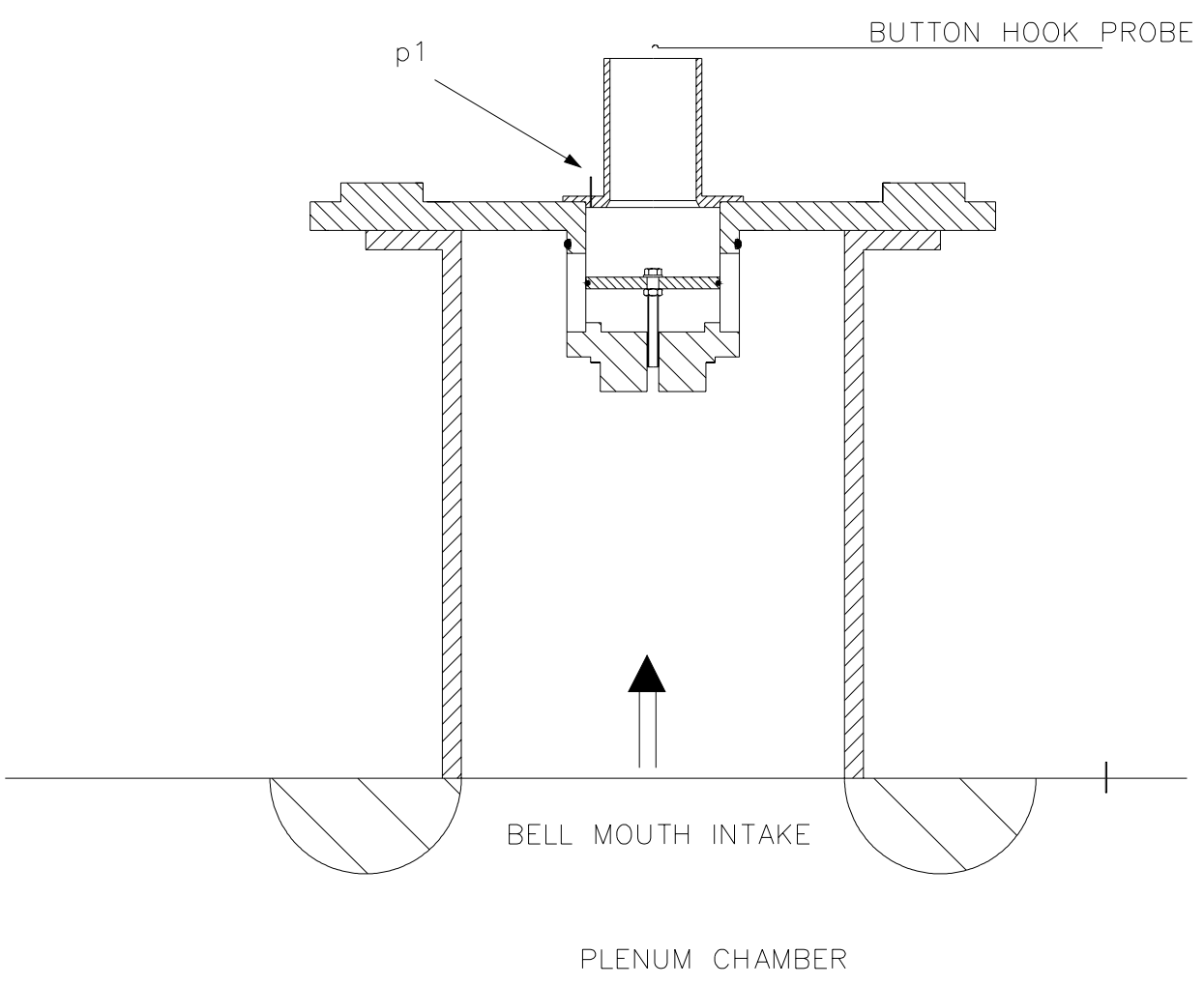

Figure A4.3 Inner Bleed Calibration 


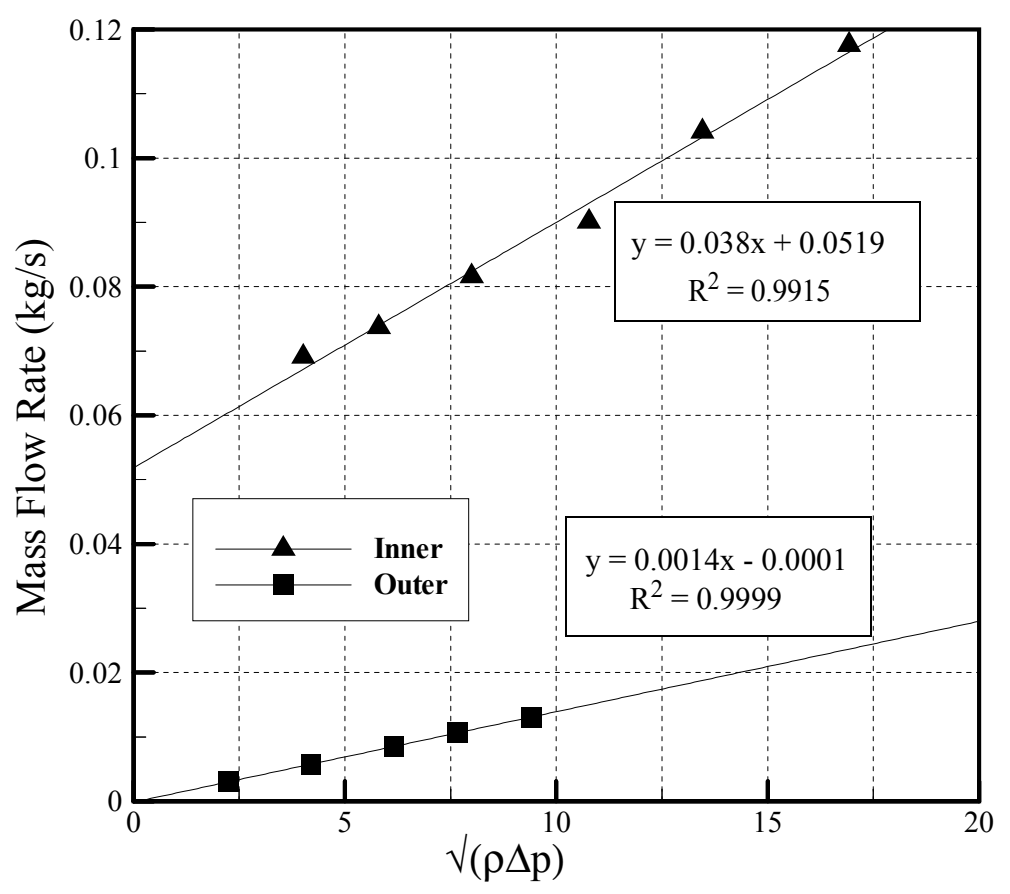

Figure A4.4 Vortex Bleed Calibration Curves 


\section{A5 PROBE LINEAR TRAVERSE MECHANISM}




\section{A5 PROBE LINEAR TRAVERSE MECHANISM}

The probe linear traverse mechanism enables a five hole or button hook probe to be accurately traversed across any passage such as a combustor feed annuli. It was specially designed and manufactured for this project and comprises of a linear guide, a stepper motor and a lead screw. The mechanism is controlled by PC (see Section 3.2) and has a positional accuracy of $\pm 0.025 \mathrm{~mm}$.

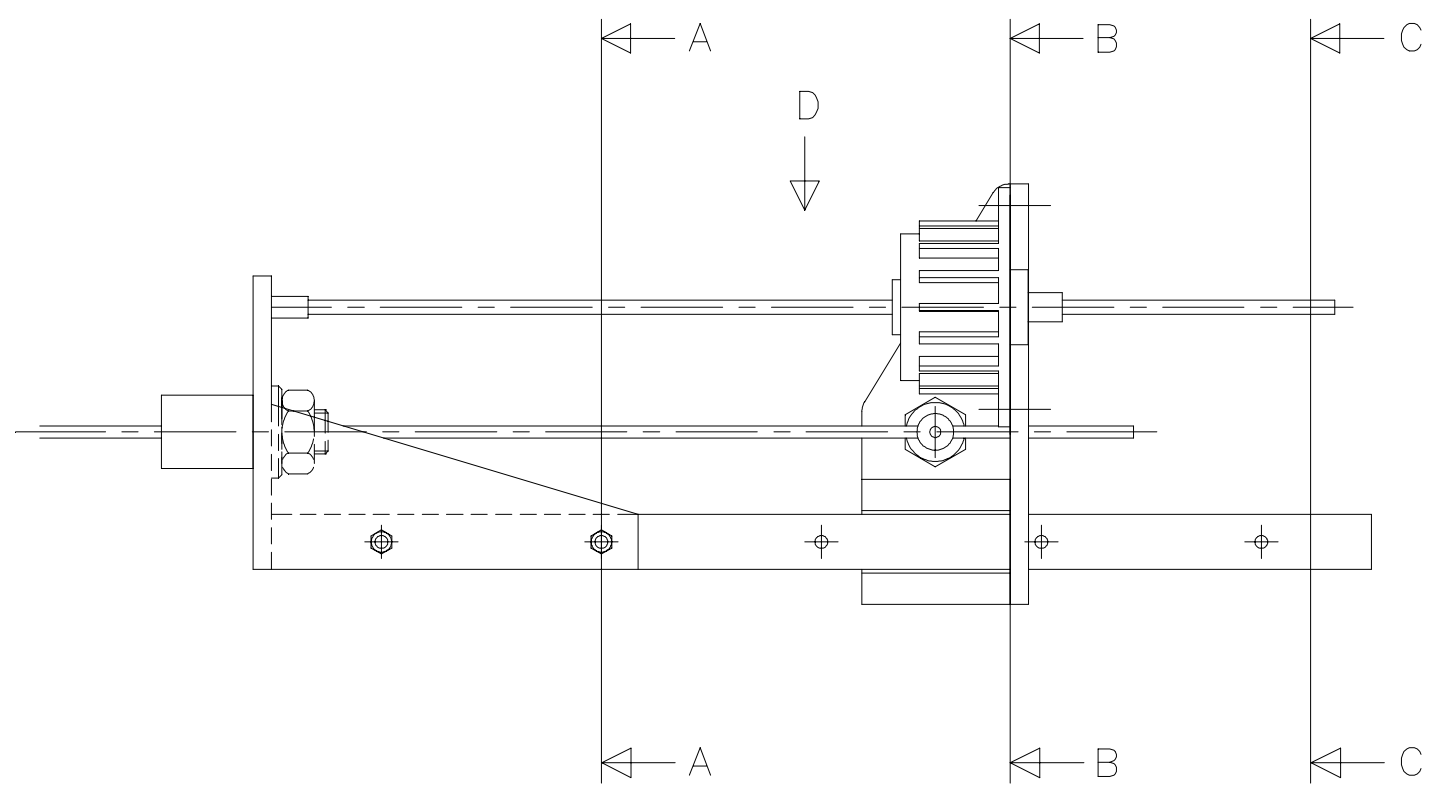

Figure A5.1 Probe Linear Traverse Mechanism

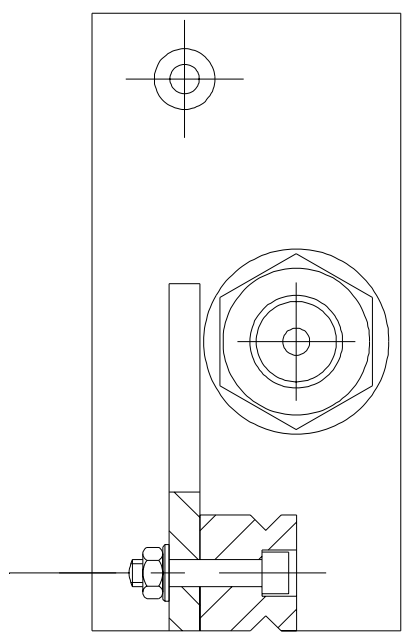

Figure A5.2 Section A-A 


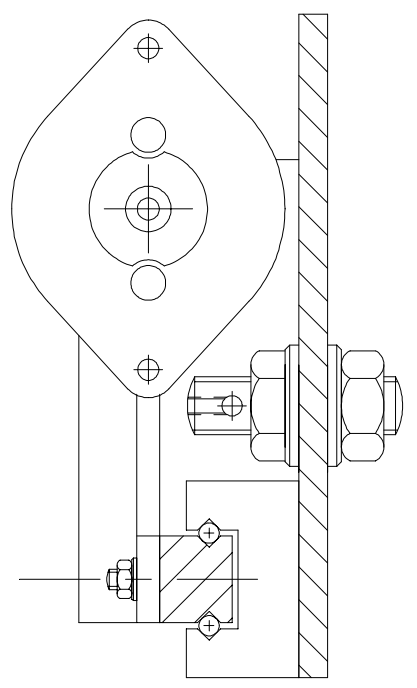

Figure A5.3 Section B-B

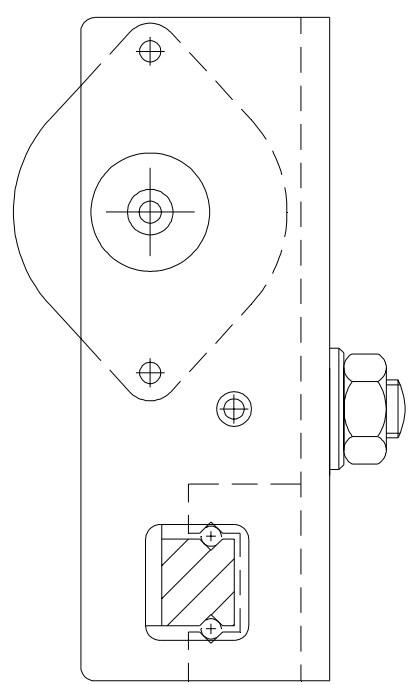

Figure A5.4 Section C-C 